\title{
METCO ENVIRONMENTAL
}

Data Reprocessed On 11/02/2005 15:42:10

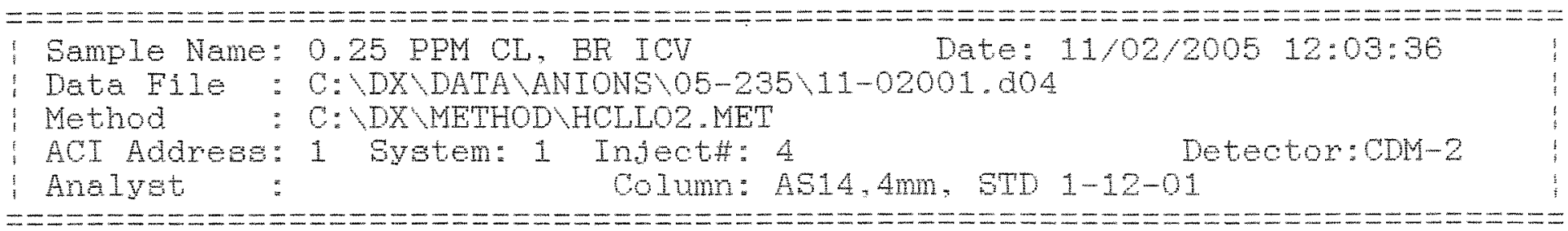

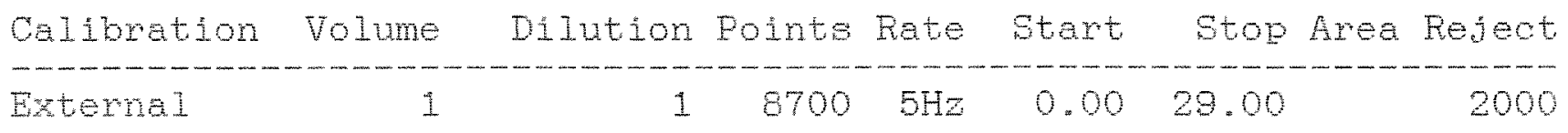

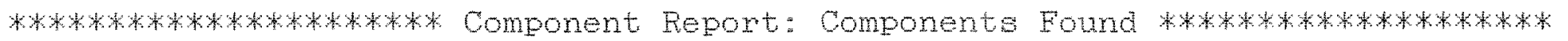

Ret Component Area Height Concentration

Time Name ppm

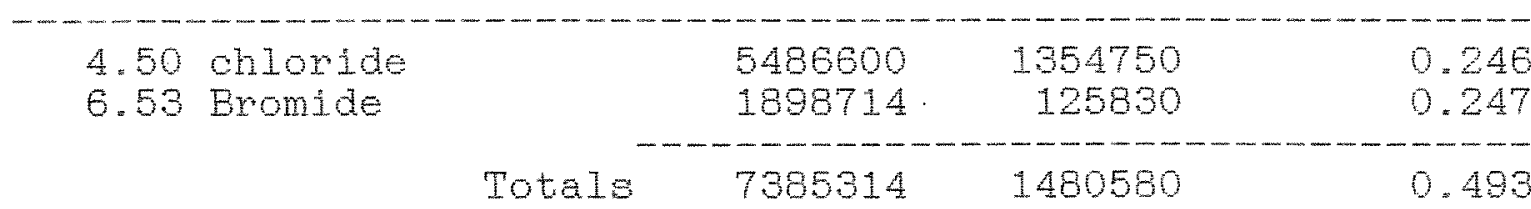

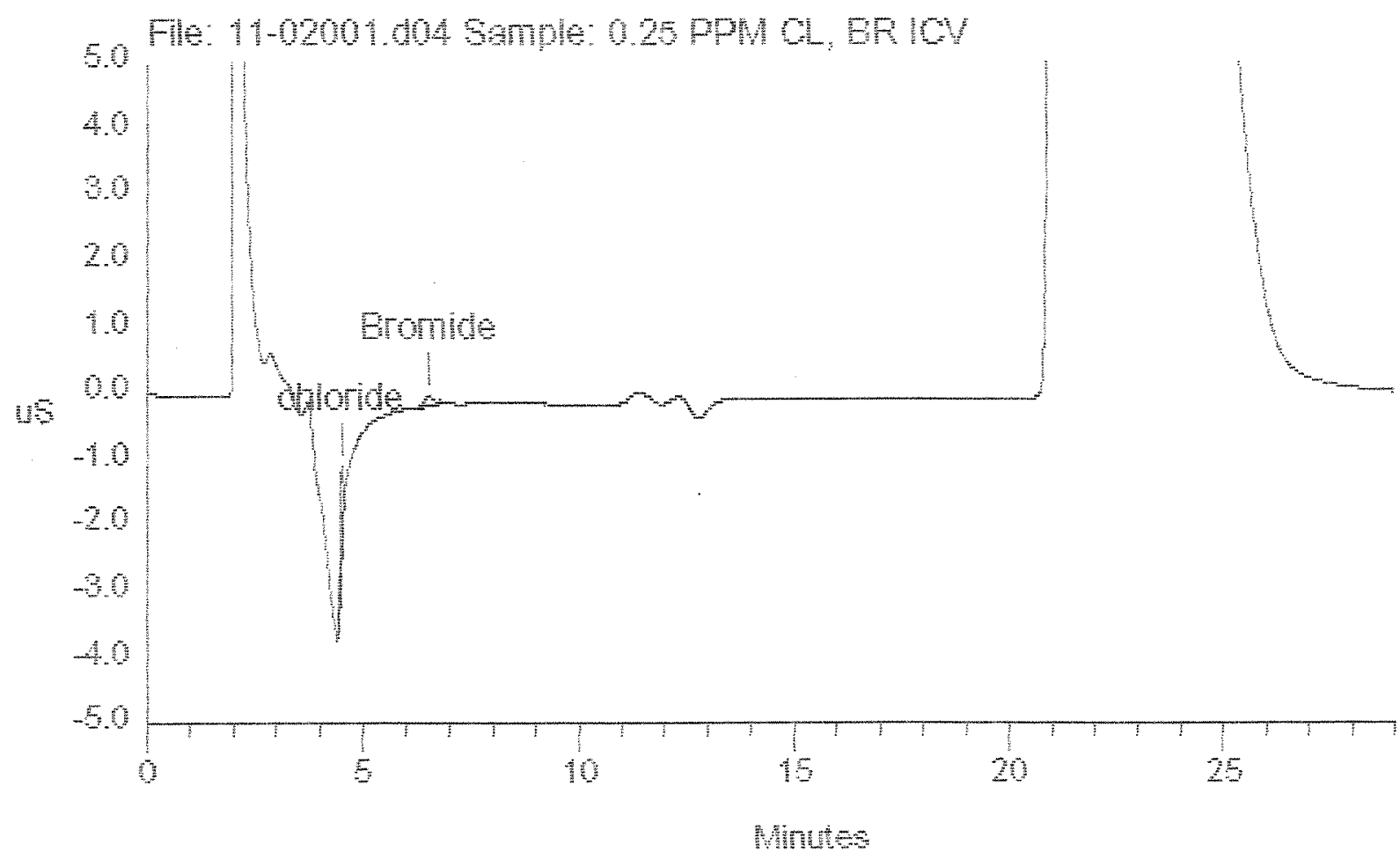




\section{METCO ENVIRONMENTAL}

Data Reprocessed on 11/03/2005 13:47:42

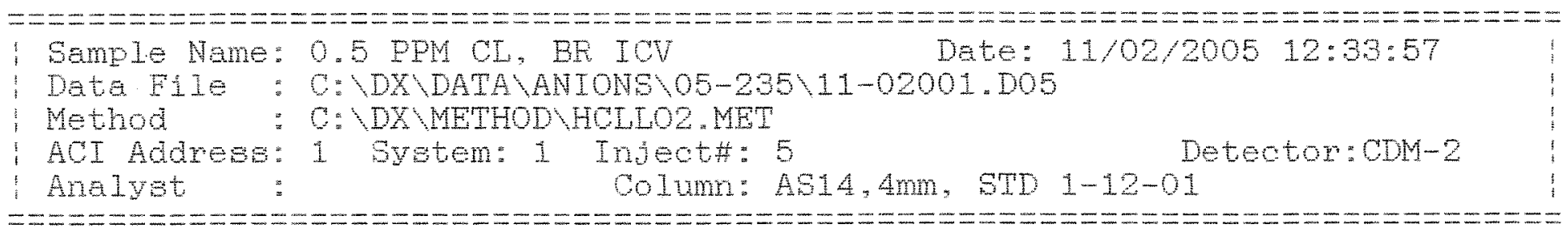

Calibration Volume Dilution Points Rate Start Stop Area Reject

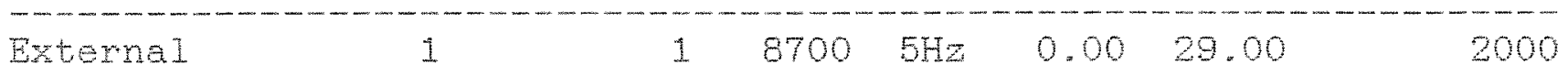

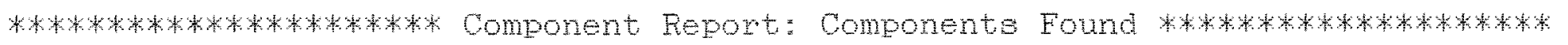

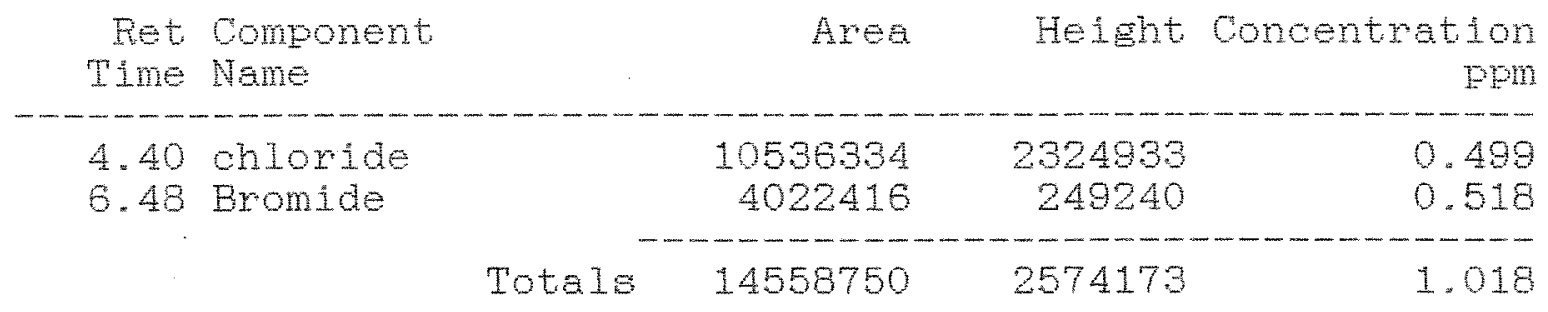

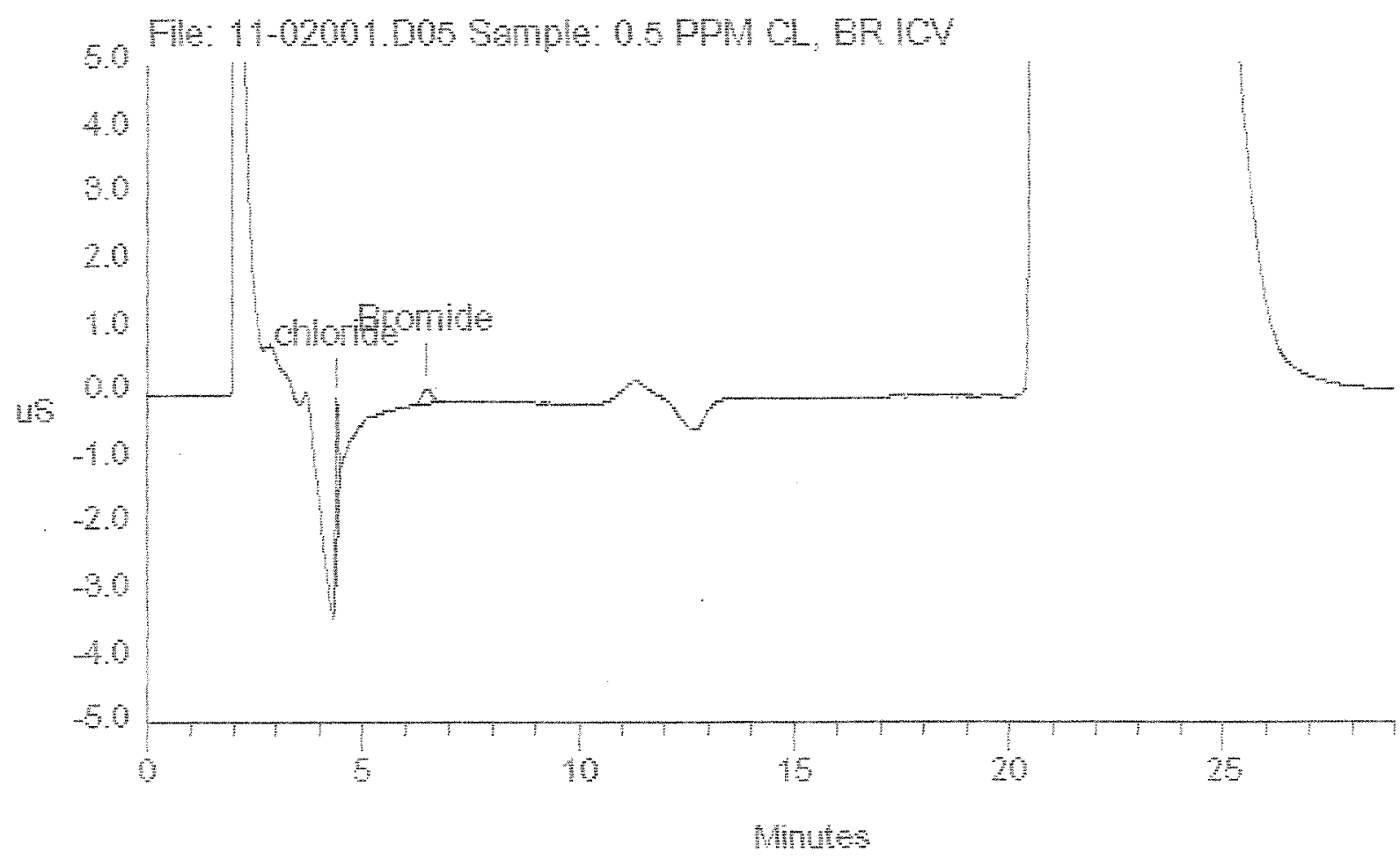




\section{METCO ENVIRONMENTAL}

Data Reprocessed on $11 / 02 / 200515: 42: 12$

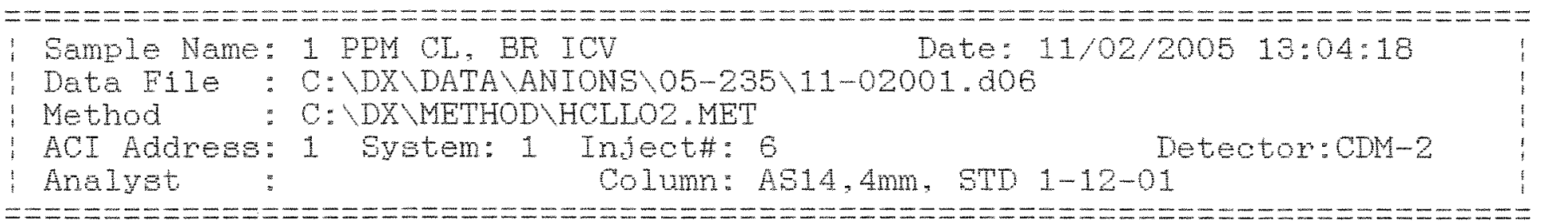

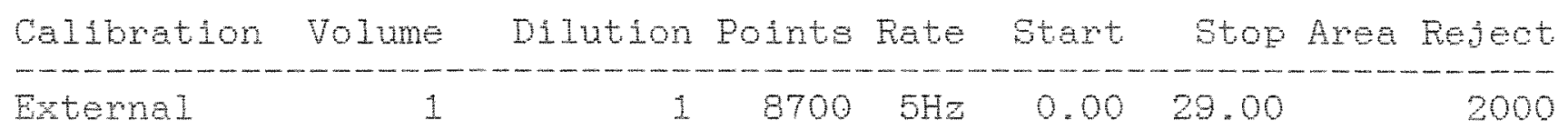

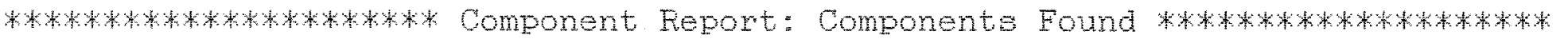

Ret Component Area Height Concentration

Time Name Ppm

4. 40 ohloride

6.47 Bromide

\begin{tabular}{|c|c|c|c|}
\hline & $\begin{array}{r}20074164 \\
8542370\end{array}$ & $\begin{array}{r}4097537 \\
511108\end{array}$ & $\begin{array}{l}0.079 \\
1.094\end{array}$ \\
\hline Totalo & 28616534 & 4608645 & 2.07 \\
\hline
\end{tabular}

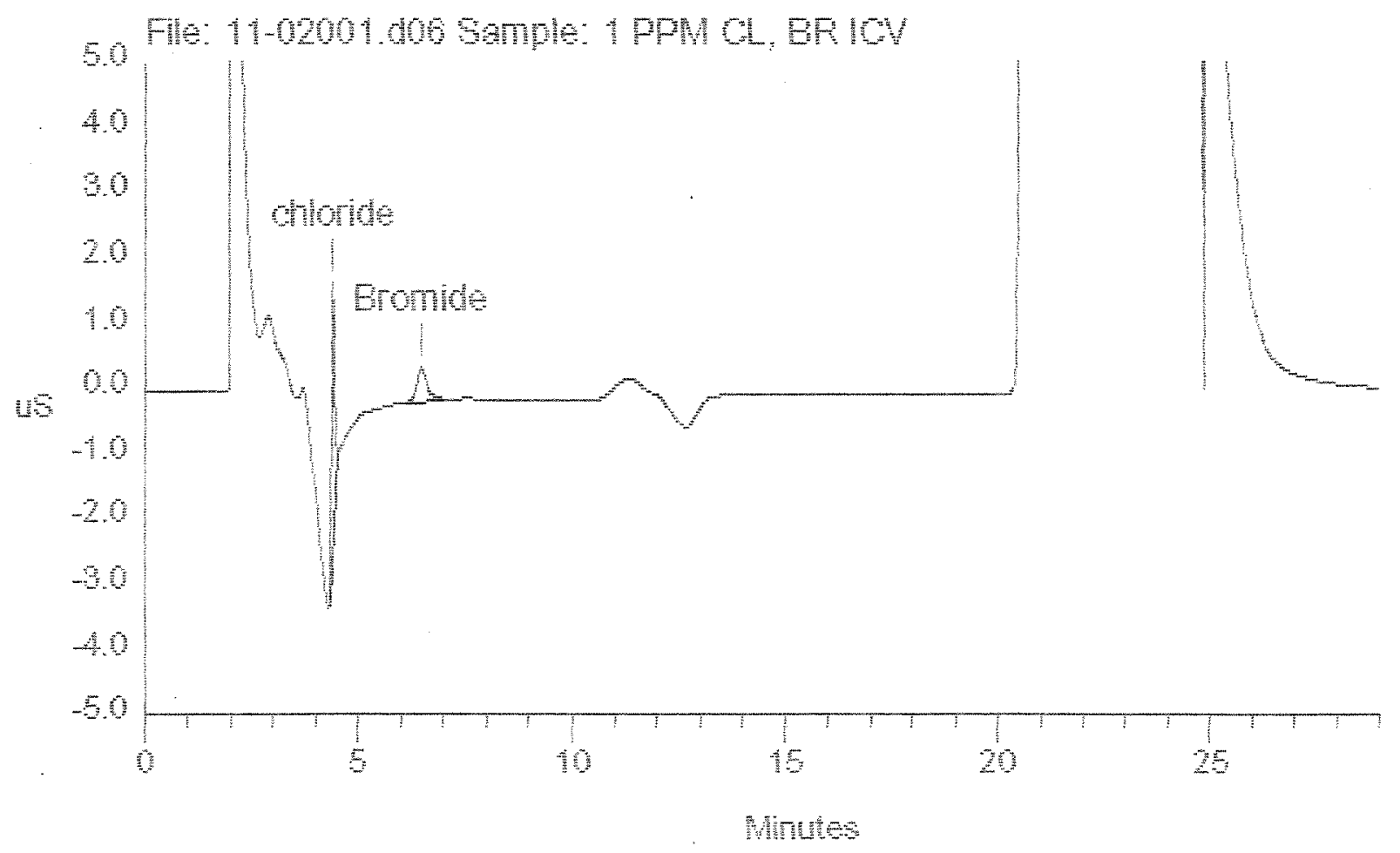




\section{METCO ENVIRONMENTAL}

Data Reprocessed On 11/02/2005 15:42:13

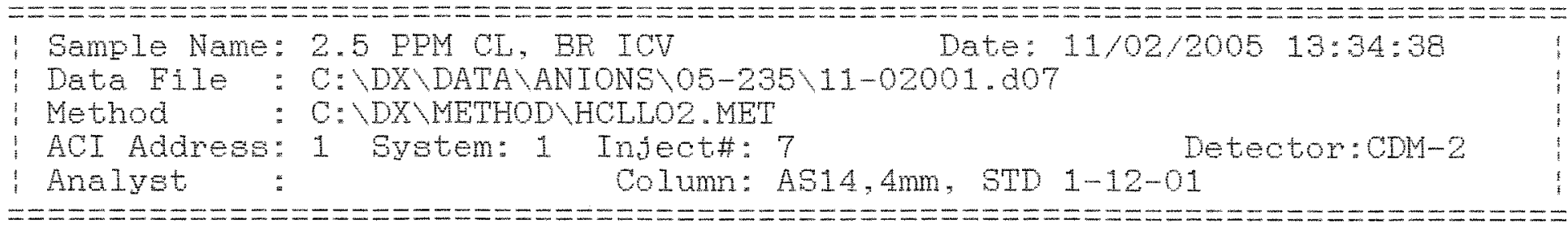

\begin{tabular}{|c|c|c|c|c|c|c|}
\hline External & 1 & 1 & 8700 & $5 \mathrm{~Hz}$ & 0.00 & 29.00 \\
\hline
\end{tabular}

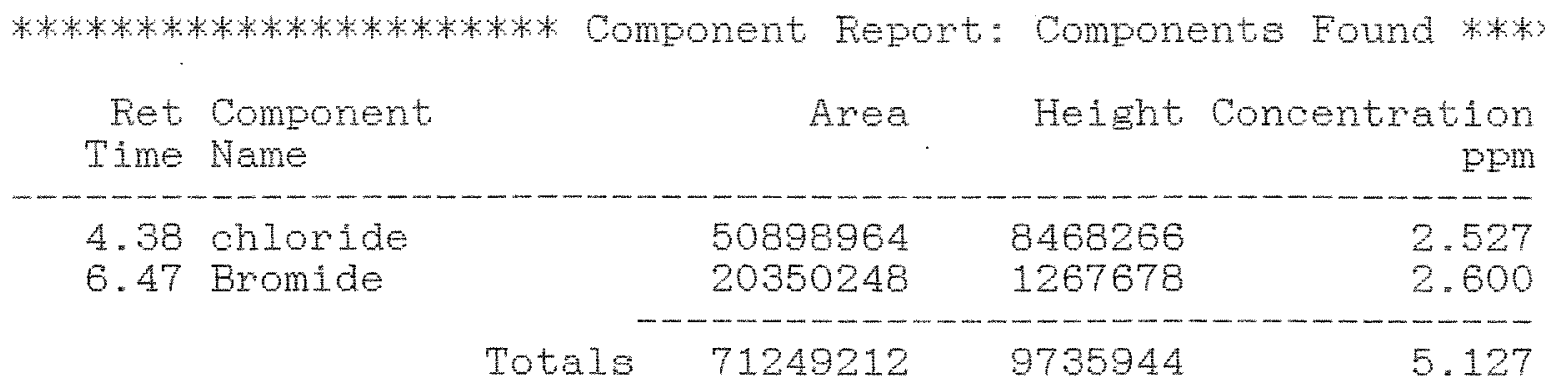

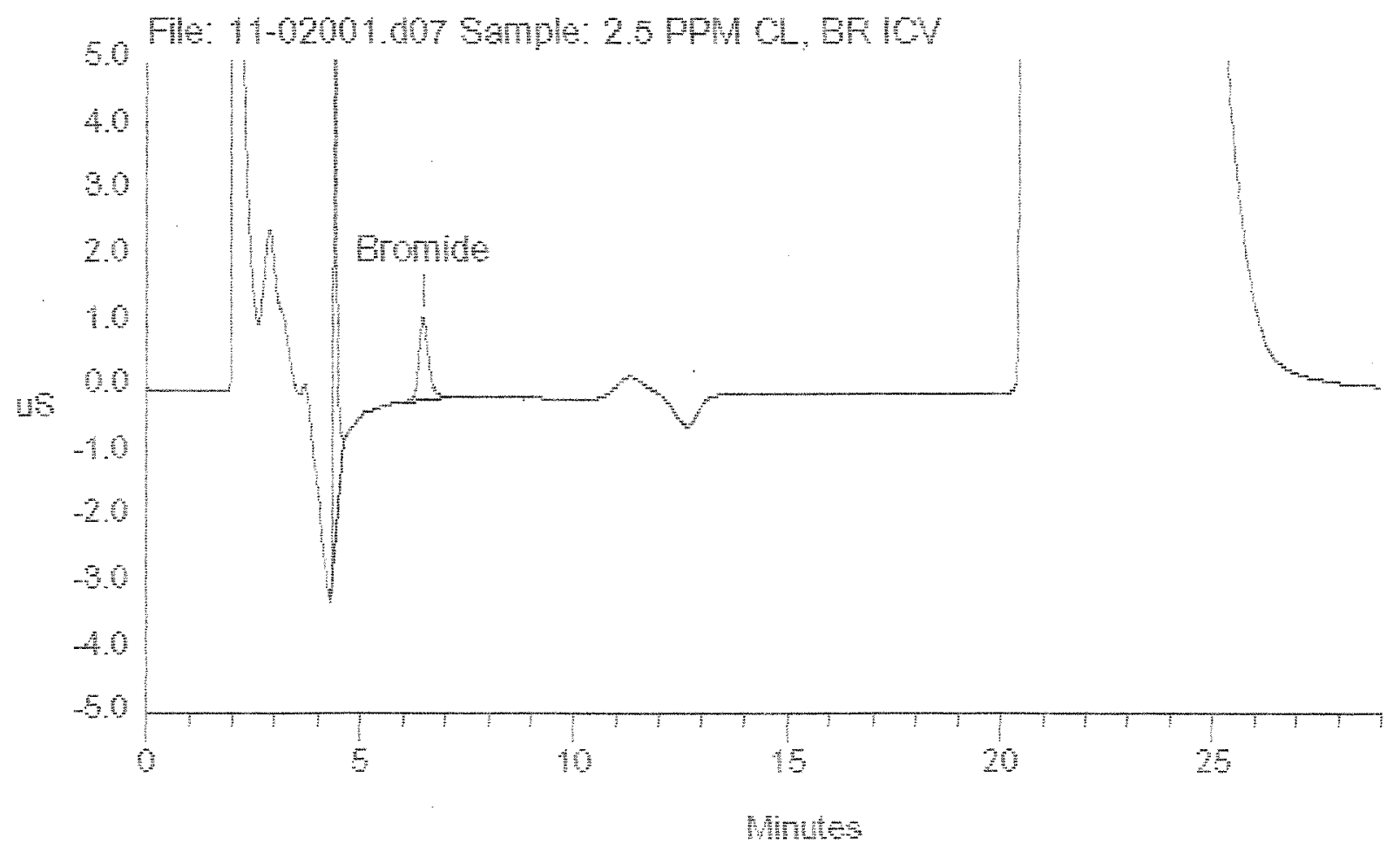




\section{METCO ENVIRONMENTAL}

Data Reprocessed on 11/02/2005 15:42:18

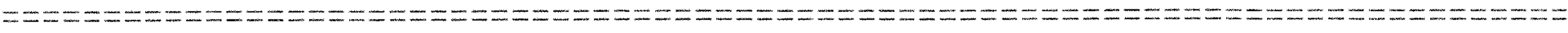

Sample Name: 2 PPM CL, BR CHK STD Date: 11/02/2005 15:05:43

Data File: C: DX DATA $\backslash A N I O N S \backslash 05-235 \backslash 11-02001 . d 10$

Method : C: $\backslash D X \backslash M E T H O D \backslash H C L L O 2 . M E T$

ACI Address: 1 System: 1 Inject\#: 10

Analyet: Colum: AS14,4mm, GTD 1-12-01

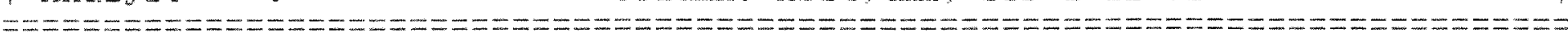

\begin{tabular}{|c|c|c|c|c|c|c|c|}
\hline Calibration & Volume & Dilution & Points & Rate & start & Stop Area & Reject \\
\hline Extemal & 1 & 1 & 8700 & $5 \mathrm{~Hz}$ & 0.00 & 29.00 & 2000 \\
\hline
\end{tabular}

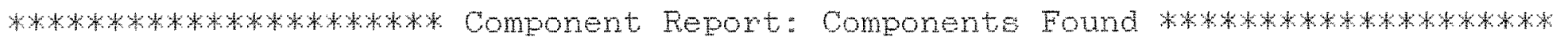

Ret Component Area Height Concentration

Time Name

\begin{tabular}{|c|c|c|c|c|c|}
\hline \multirow{2}{*}{\multicolumn{2}{|c|}{$\begin{array}{l}4.57 \text { chloride } \\
6.65 \text { Bromide }\end{array}$}} & & $\begin{array}{l}40742970 \\
15599270\end{array}$ & $\begin{array}{l}8177676 \\
1120853\end{array}$ & $\begin{array}{l}2.017 \\
1.994\end{array}$ \\
\hline & & Totals & 56342240 & 9298529 & 4.011 \\
\hline
\end{tabular}

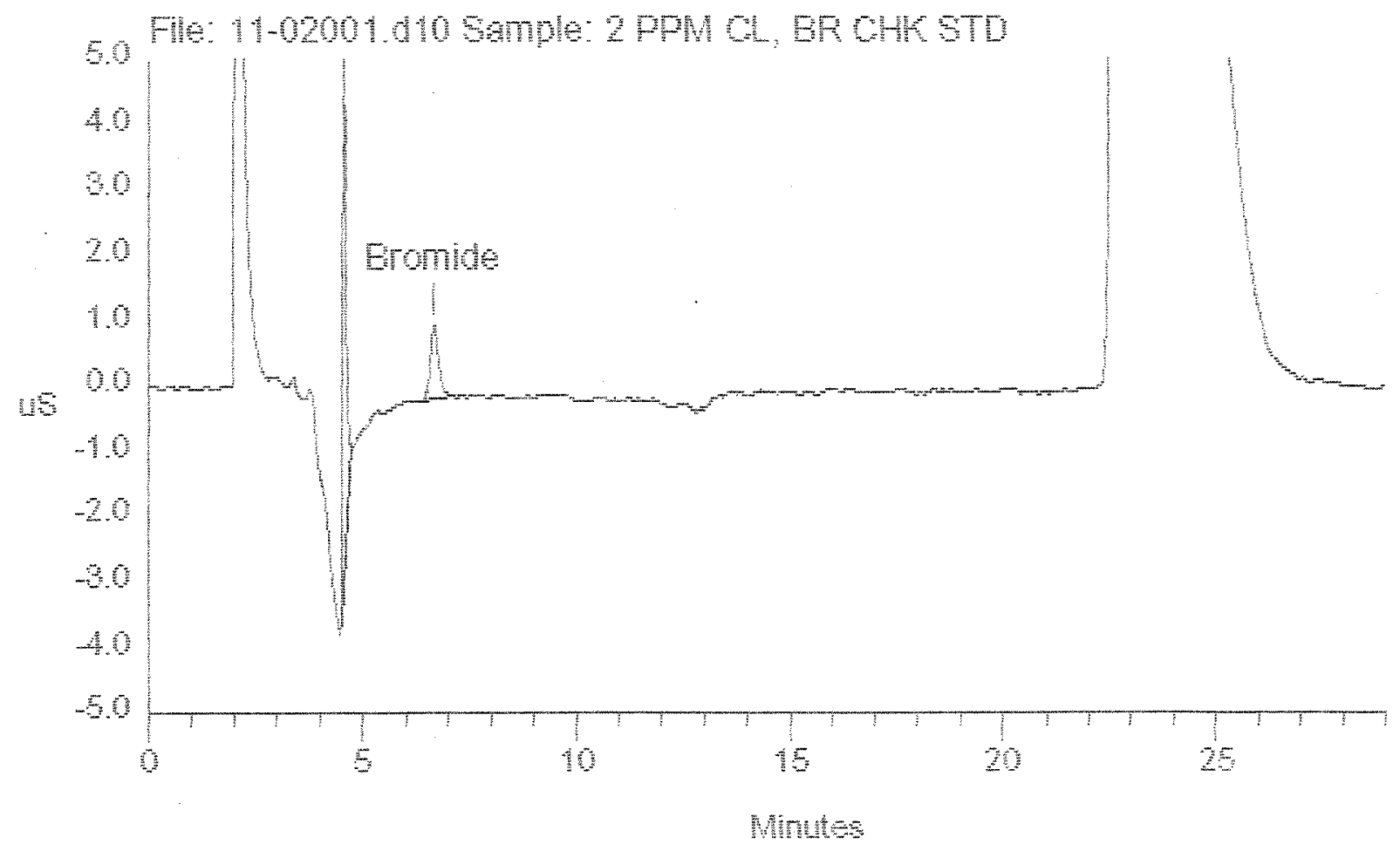


อ̀̀े 284.4

@EJL

\section{METCO ENVIRONMENTAL}

Data Reprocessed On 11/02/2005 16:02:06

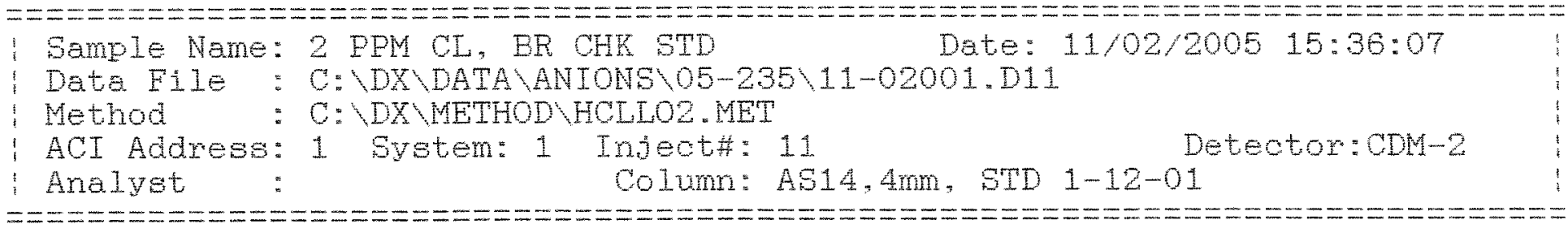

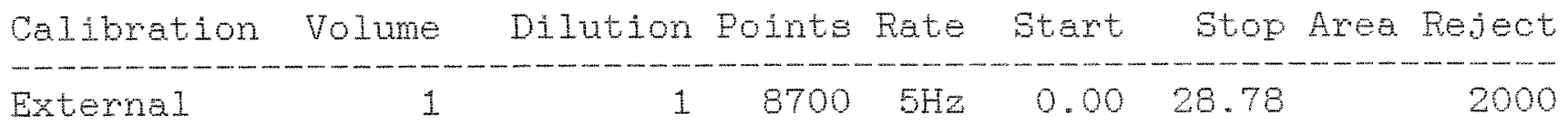

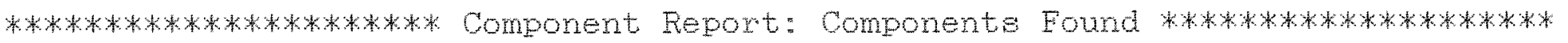

Ret Component Area Height Concentration

Time Name pom

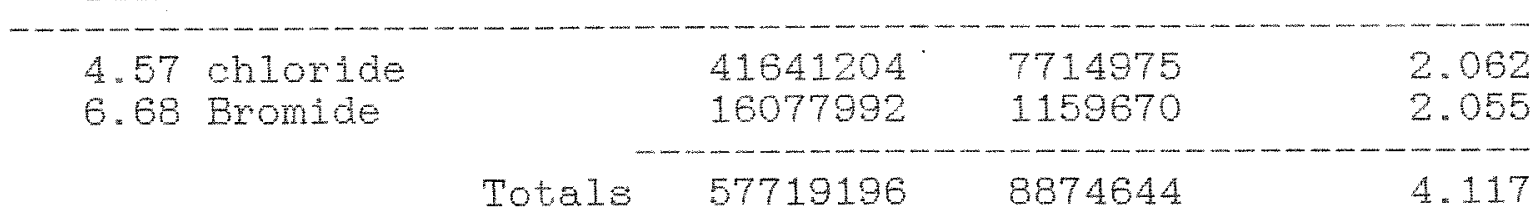

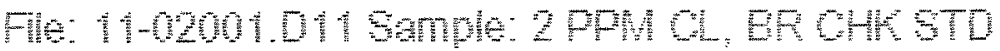

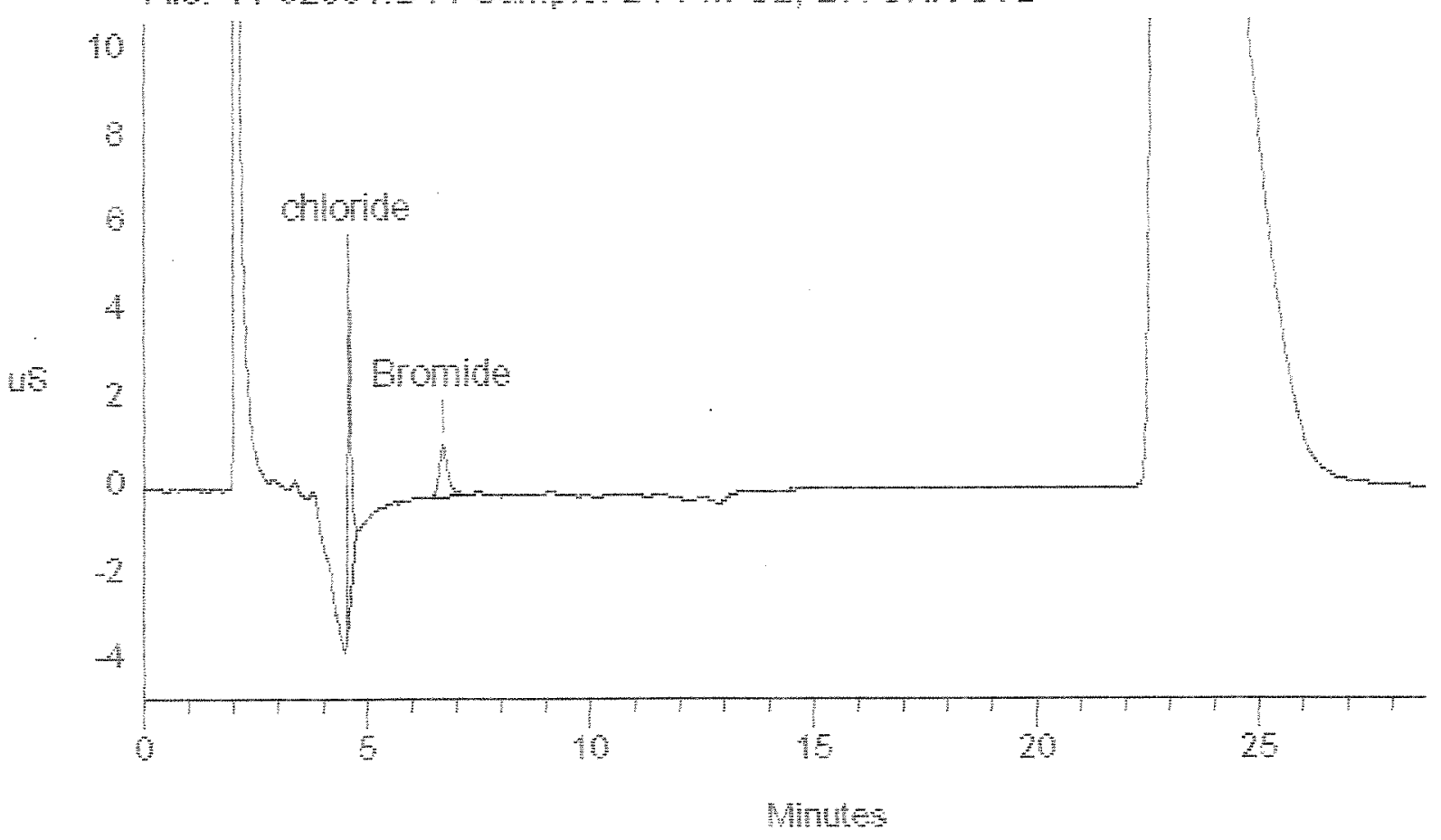




\section{METCO ENVIRONMENTAL}

Data Reprocessed On 11/03/2005 09:20:40

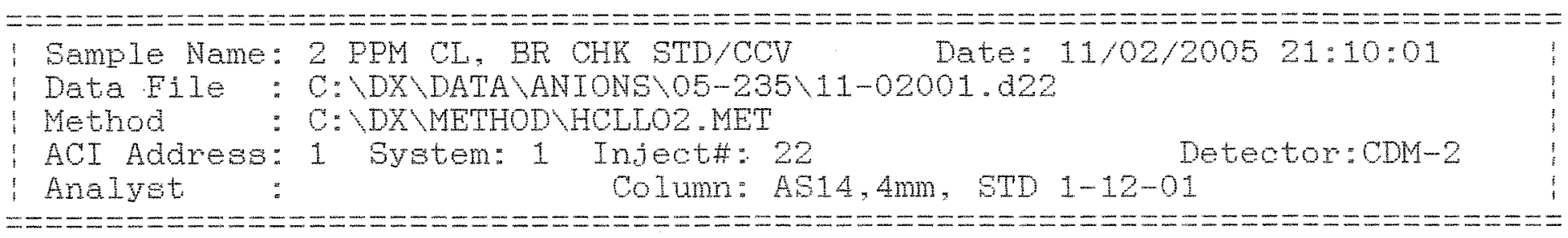

Calibration Volume Dilution Pointe Rate Start Stop Area Reject

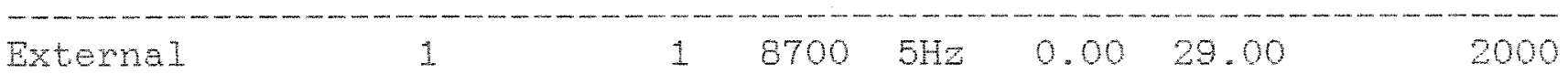

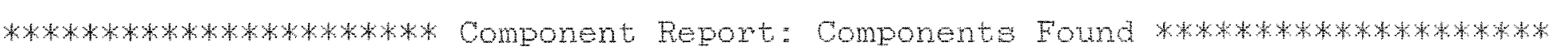

Ret Component Area Height Concentration

Time Name ppm

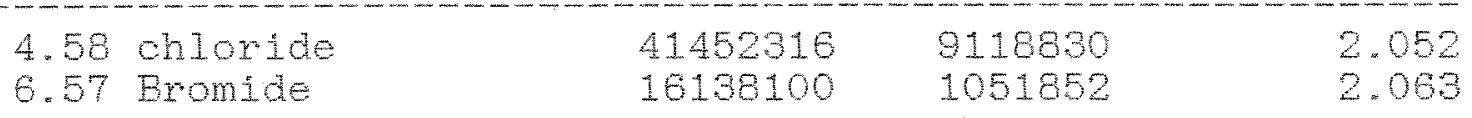

Totals $57590416 \quad 10170682 \quad 4.115$

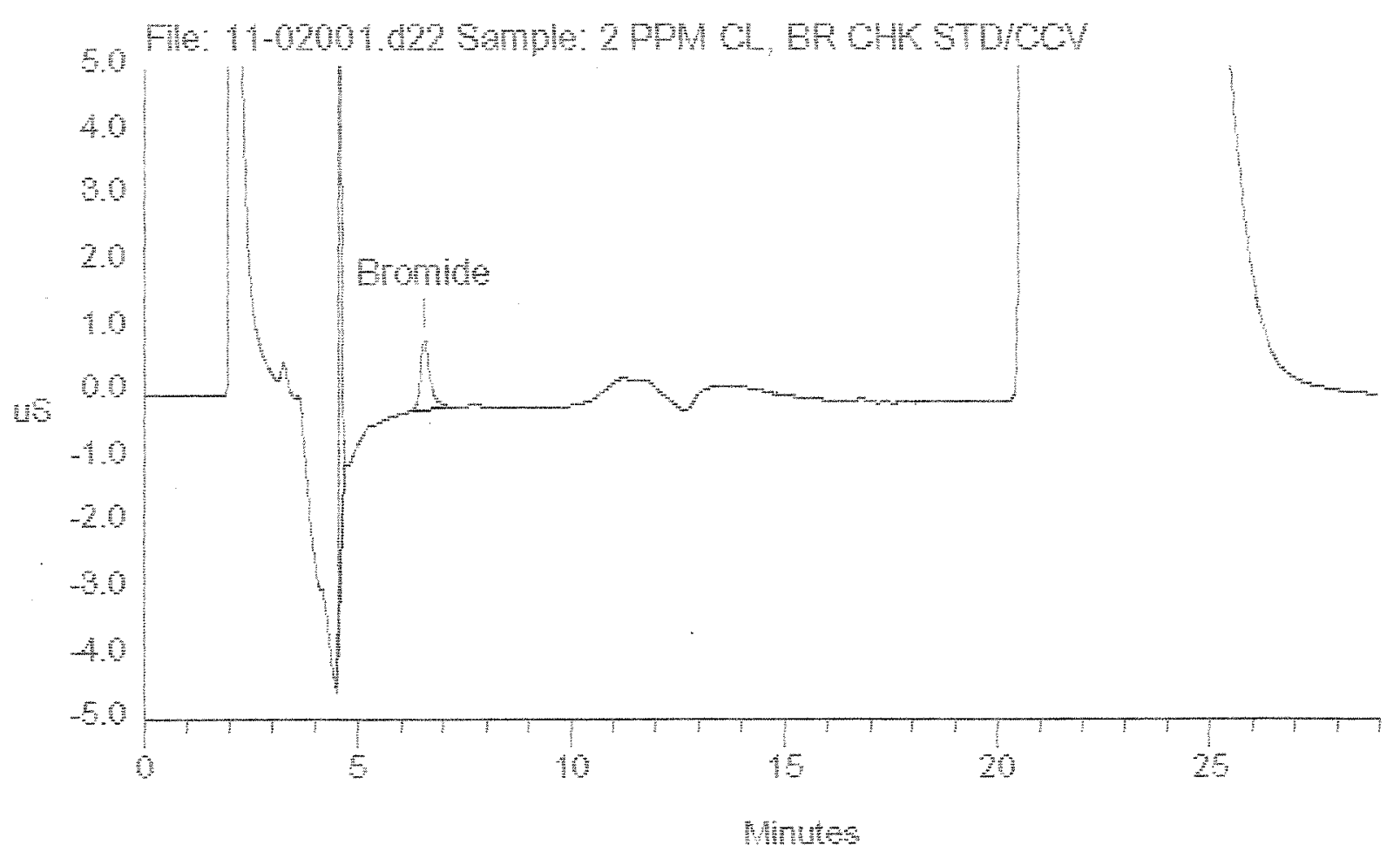




\section{METCO ENVIRONMENTAL}

Data Reprocessed On 11/03/2005 09:20:40

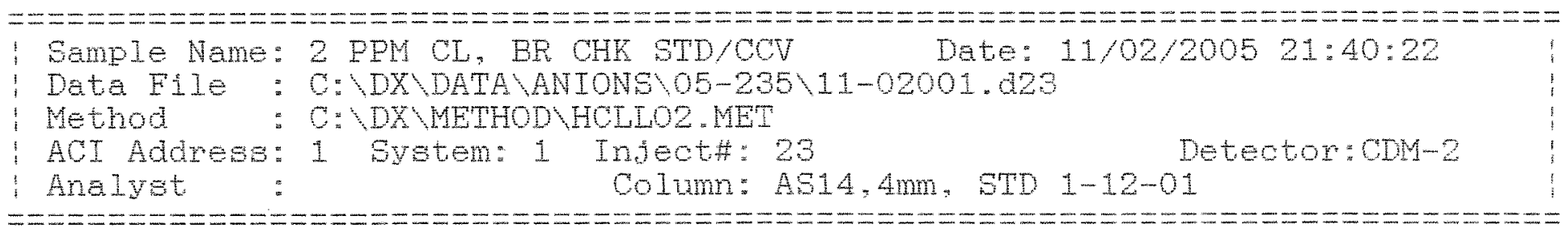

Caifbration Volume Dilution Points Rate Start Stop Area Relect

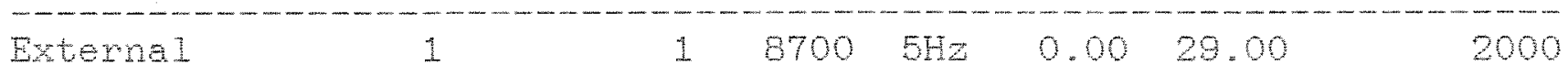

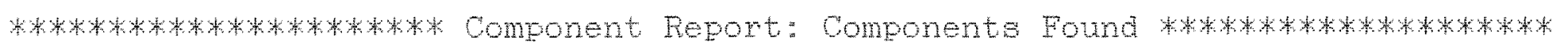

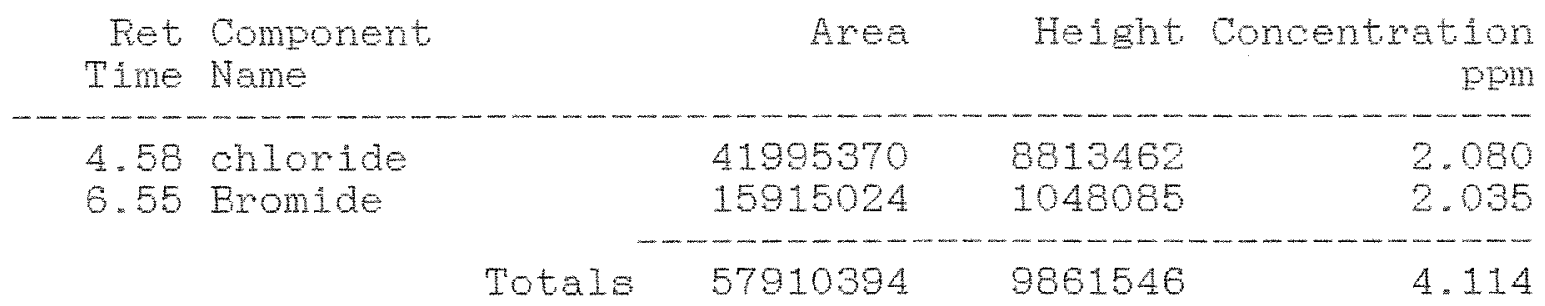

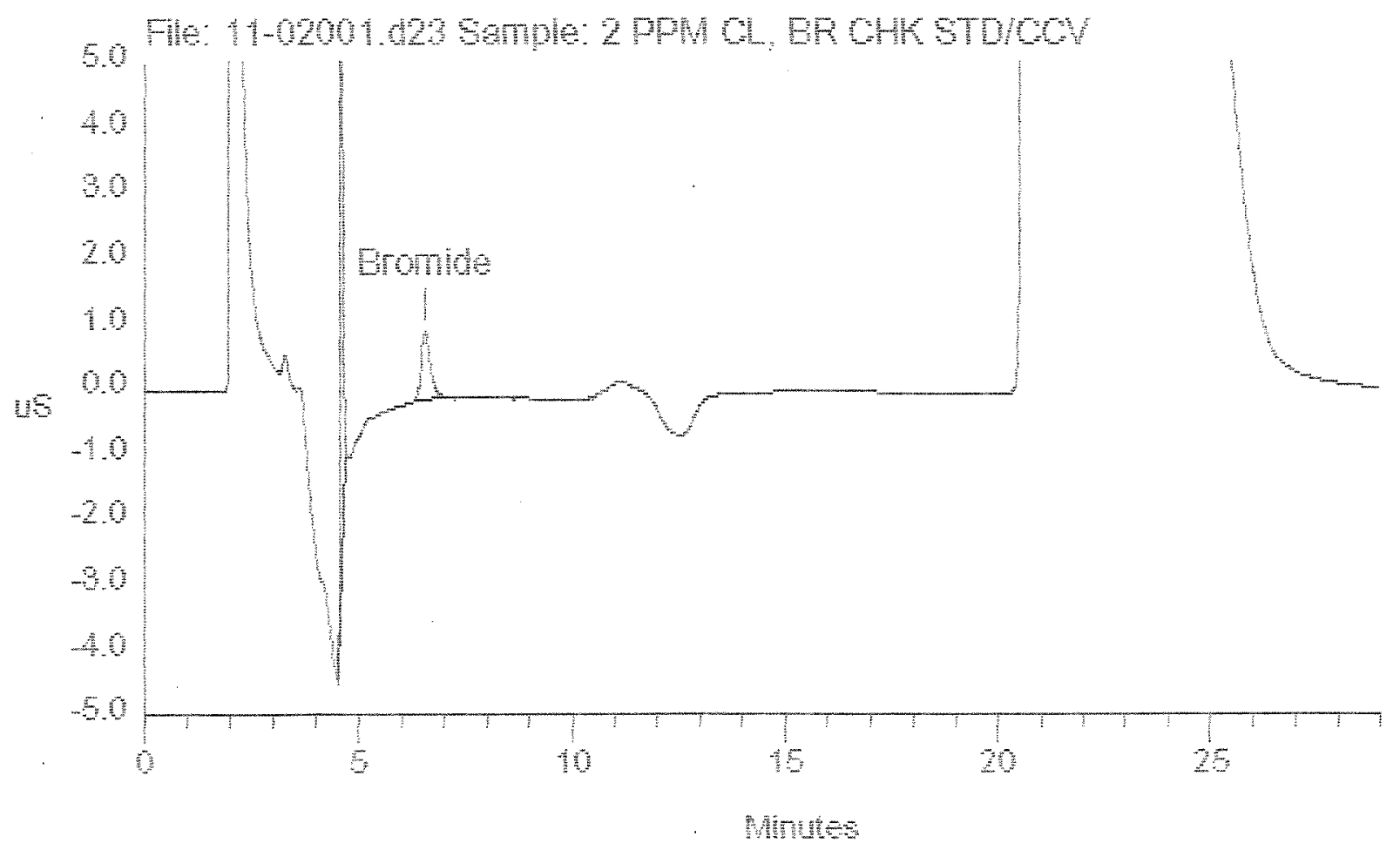




\section{METCO ENVIRONMENTAL}

Data Reproceseed On 11/03/2005 09:20:47

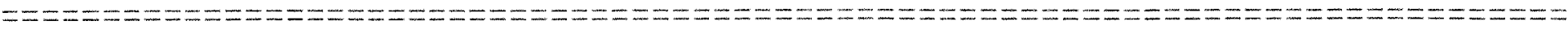

Sample Name: 2 PPM CL, BR CHK STD/COV Date: 11/03/2005 01:12:49

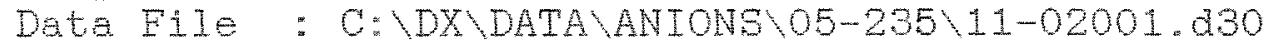

Method :C: DXYMETHODYHCLLO2.MET

ACI Address: 1 System: 1 Inject\#: 30

Analyst: Colum: AS14,4m, STD 1-12-01

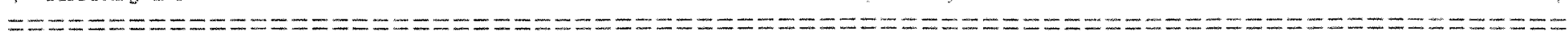

Calibration Volume Dilution Pointe Rate Start Stop Area Reject

- 8700 (1)

$\begin{array}{llllllll}\text { External } & 1 & 1 & 8700 & 5 \mathrm{~Hz} & 0.00 & 29.00 & 2000\end{array}$

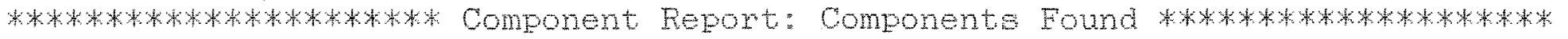

Ret Component Area Height Concentration

Time Name

PDM

4.60 chloride

6.53 Bromide

$41572714 \quad 10073500$

2.058

$16043266 \quad 1060656 \quad 2.051$

Totals $57615980 \quad 11134156 \quad 4.109$

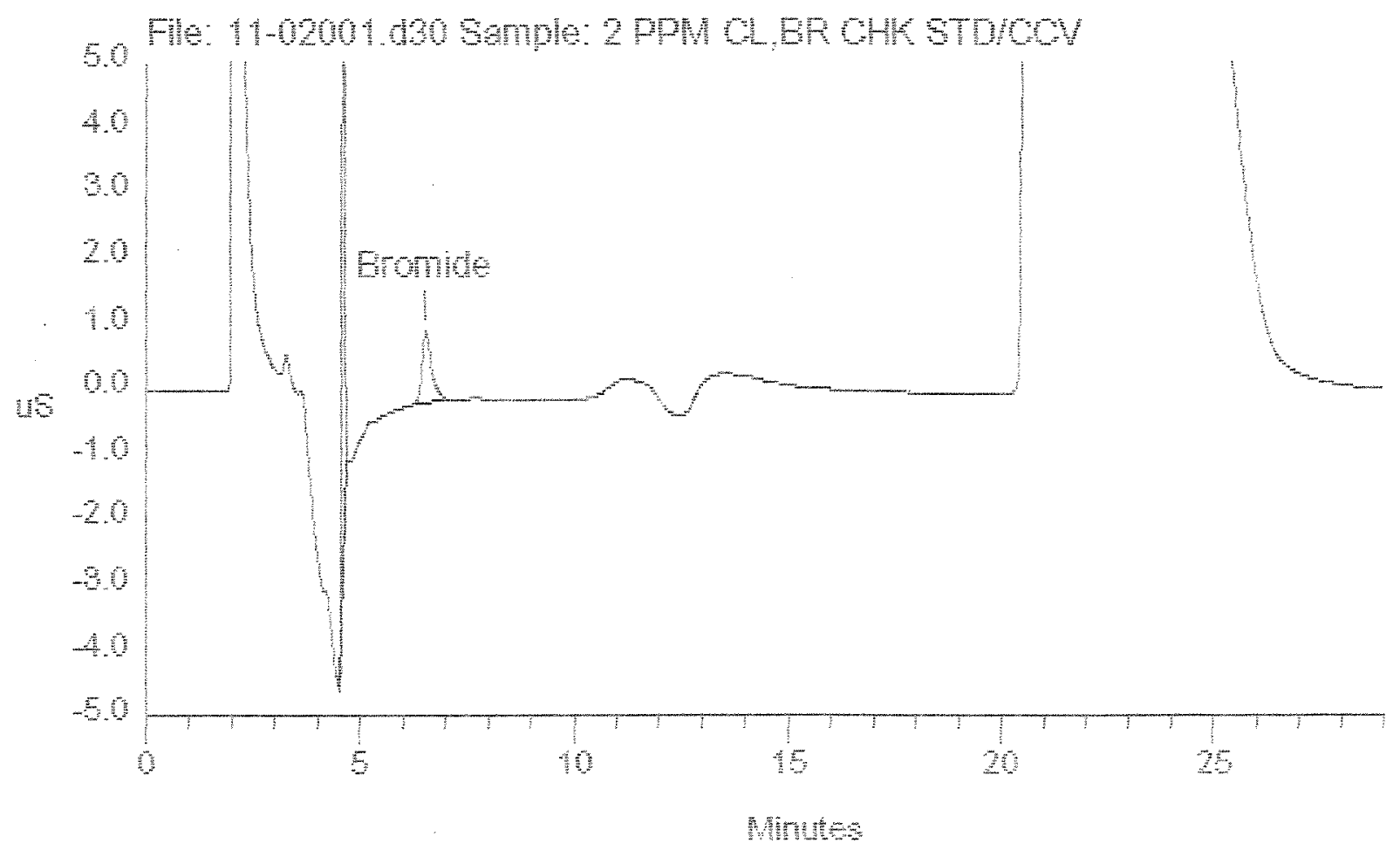


METCO ENVIRONMENTAL

Data Reprocessed on $11 / 03 / 200509: 20: 48$

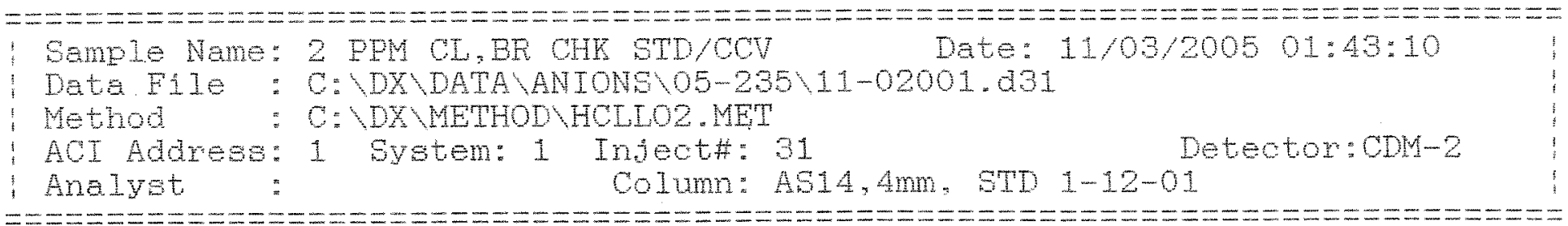

Calibration Volume Dilution Points Rate Start Stop Area Reject

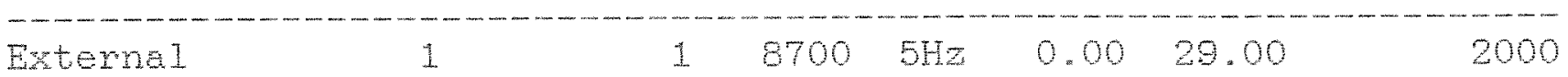

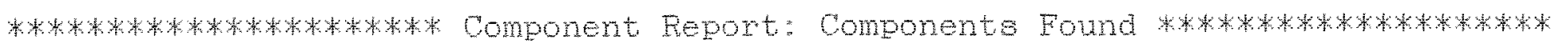

Ret Component Area Height Concentration

Time Name ppm

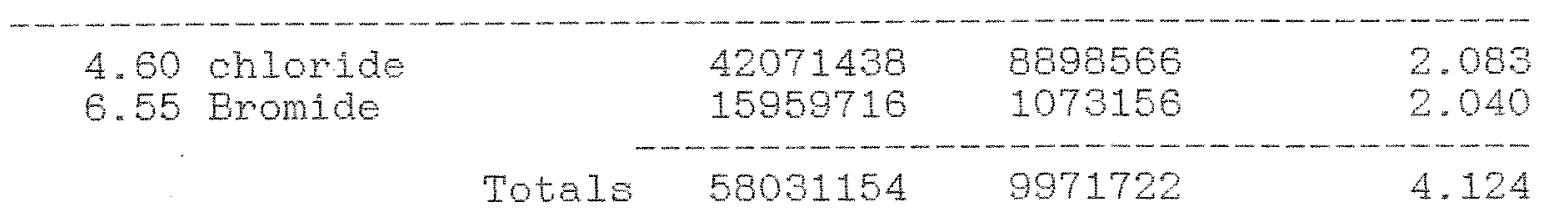

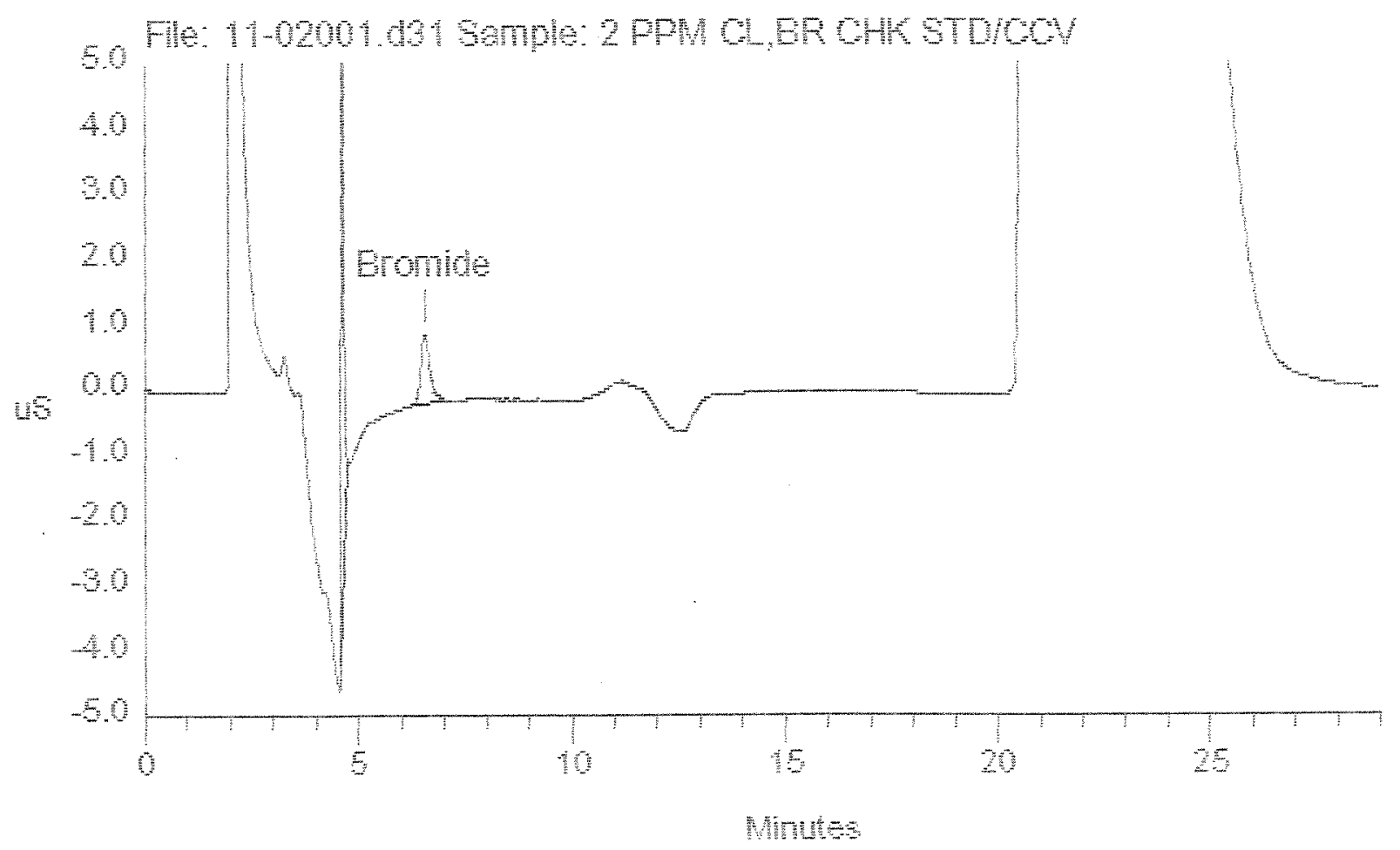




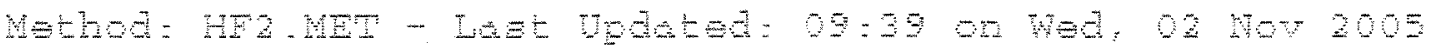
Omponent: Fluoride

Fit Type: Linets

$\mathrm{L}^{\mathrm{B}}=0.99920 \mathrm{~g}$

Ant $=$ FegP * $3.0240-008+0.3974$

Reep = Ant $2.7590+007+-1.069 e+007$

Btanderdiztion: Externel

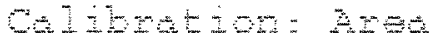

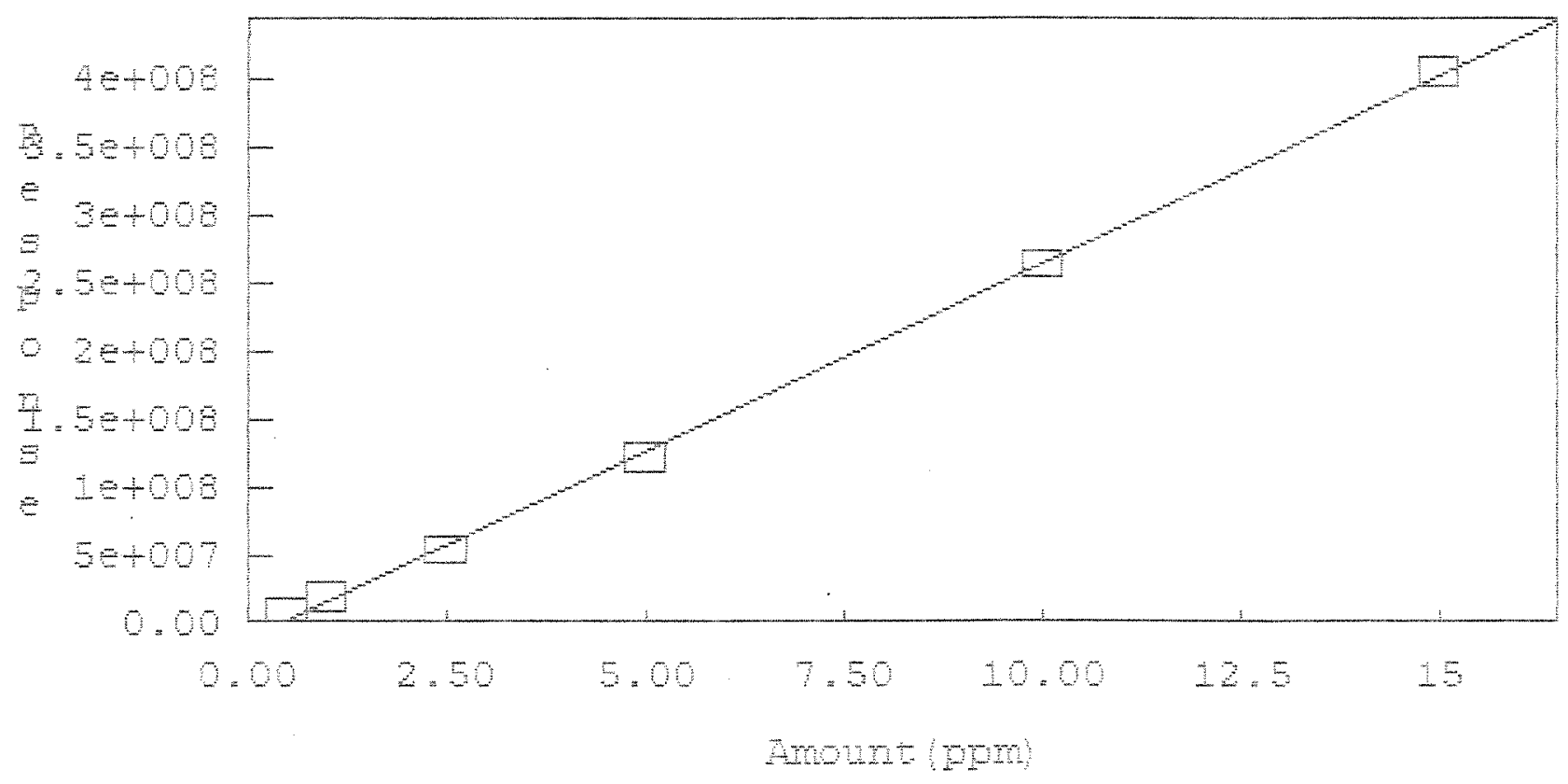


Method: HF2.MET - LeEt Updeted a 099 on Hed, 02 Nov 2005

compont = olonide

Fit Tye: Linedr

$x^{i}=0.906144$

$\mathrm{Bnt}=\mathrm{ReOP} * 5.2790-00 \mathrm{5}+0.25 \mathrm{~s}$

Reep = Ant $1.8949+007 \div-4.826 \theta+000$

etanderizetion: Extemel

calibtion: Bret

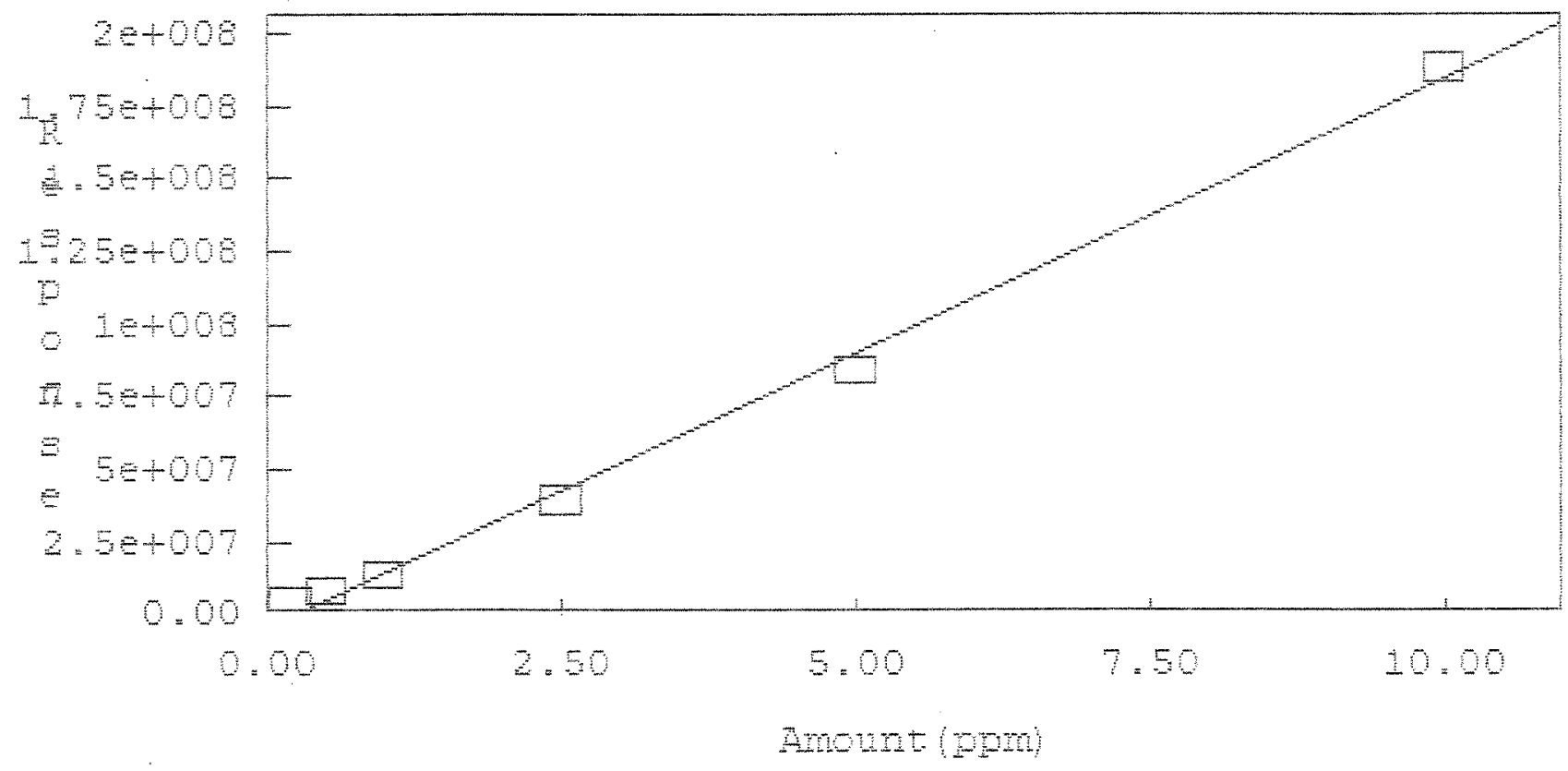




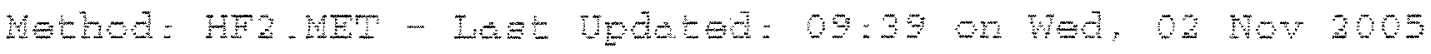

Component: Bronide

Fit Tyes: Linedr

$x^{2}=099078$

Ant $=$ ReEP $* 1.3030-007+0.1650$

Rese = Ant $7.6730+000+-1.2729+006$

Standerdiation: Extend

dibretion: Rreat

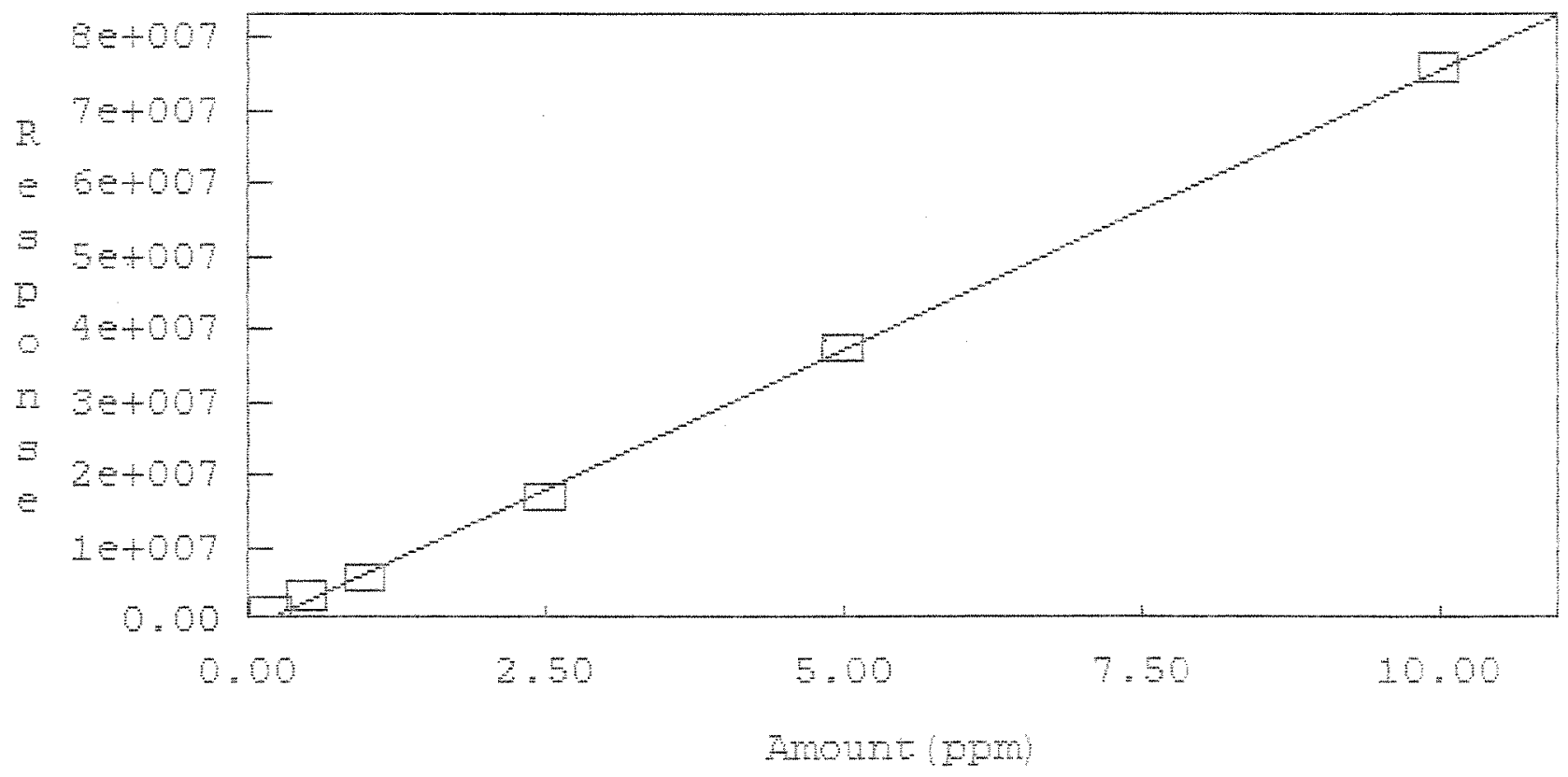




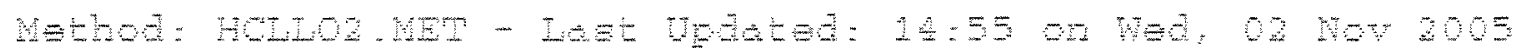

emponent : choride

Fit Tyes : Litied

$x^{*}=0.99827$

Mnt = ReEP * $5.0230-000+-0.02973$

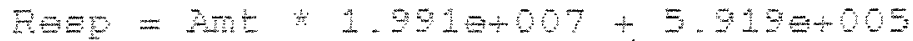

Gtenderdizetion: Extemel

culibreton: $2 \mathrm{rec}$

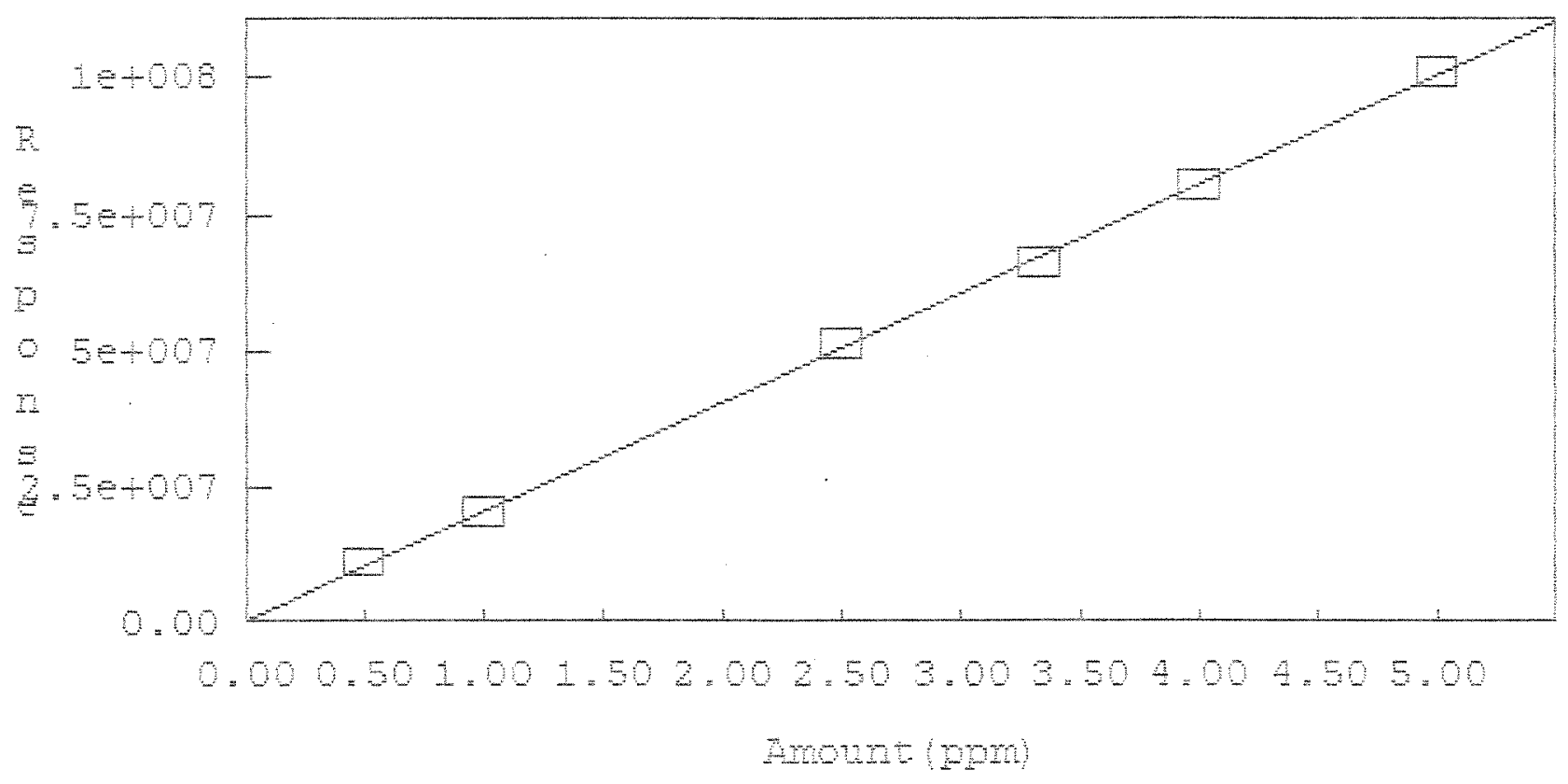

E-81 


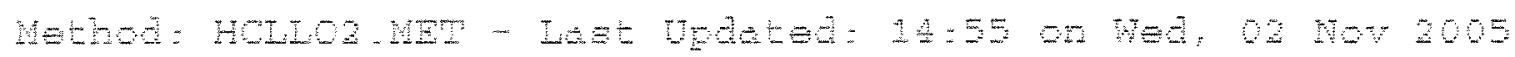

conponent: Eromide

Fit Tre : Linedr

$r^{2}=0.999373$

Mnt = EeEP $1.275=-007+0.00523$

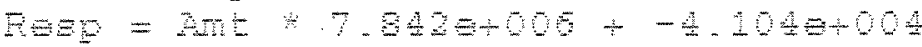

etanderdiation: Ertemel

Gibretion: Ared

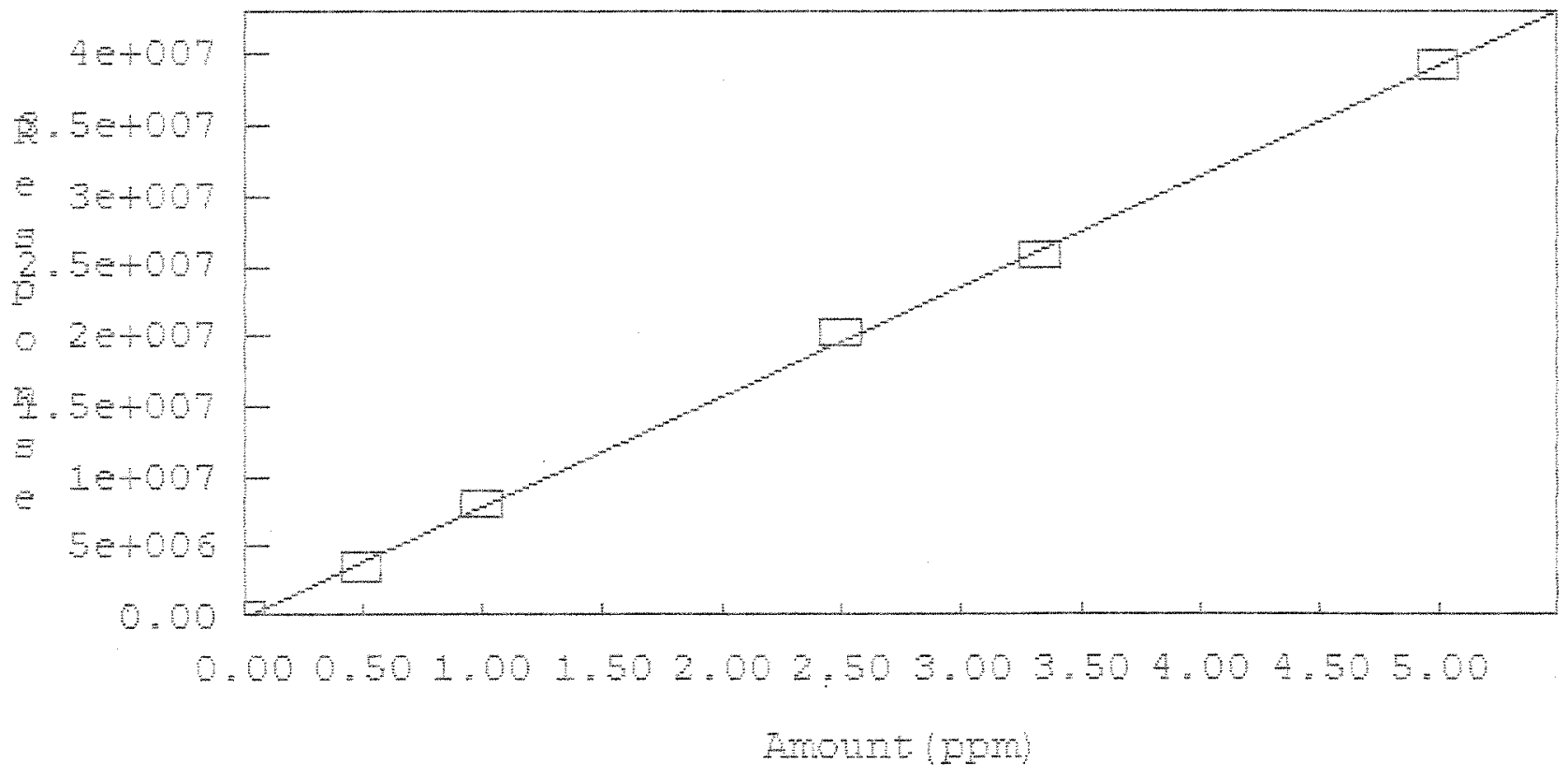

E-82 


\section{RICCA CHEMICAL COMPANY \\ PO Box 13090 Arlington, TX 76094 | http://www.riccachemical.com | 1-888-GO-RICCA | customerservice@riccachemical.com}

Fluoride Standard, $1 \mathrm{~mL}=1 \mathrm{mg} \mathrm{F-,} 1000$ ppm F-

Lot Number: 1411654

Product Number: 3173

ExDiration Date: MAY 2006

Manufacture Date:11/30/2004

The certified value reported is the prepared value based upon the method of preparation of the material. The uncertainty in the prepared value is based upon the volumetric method of preparation.

Contains:

\begin{tabular}{lll}
\hline Name & CAS\# & Grade \\
\hline Sodium Fluoride, NaF & $7681-49-4$ & High Purity \\
Water, Deionized, H2O & $7732-18-5$ & ACS, ASTM D 1193 (Type I), EP, USP
\end{tabular}

\begin{tabular}{lllll}
\hline Test Name & \multicolumn{1}{c}{ Assay Method } & Specification & Result \\
\hline Appearance & Clarity, Color, Odor & Clear, colorless, odorless & Passed Test \\
Certified Concentration & Based on accurate volumetric & $1000 \pm 5$ ppm F- & 1000 ppm F- \\
& preparation & &
\end{tabular}

\begin{tabular}{lll}
\hline Specification & Reference & Method Number \\
\hline Fluoride Solution, Stock $(1.00 \mathrm{~mL}=1.00$ & ASTM & D 5996
\end{tabular}

$\mathrm{mg}$ F)

Fluoride Calibration Stock Solution (1,000 EPA (SW-846)

9214

$\mathrm{mg} / \mathrm{L} \mathrm{F-)}$

Fluoride Stock Solution $(1.00 \mathrm{~mL}=1.00$

EPA (SW-846)

9056

$\mathrm{mg} \mathrm{F-)}$

Fluoride Stock Solution $(1.00 \mathrm{~mL}=1.00$

ASTM

D 4327

$\mathrm{mg} \mathrm{F}$ ?)

Stock Solution, $1.0 \mathrm{~mL}=1.0 \mathrm{mg} \mathrm{F} \quad$ EPA

Fluoride Solution, Stock $(1.00 \mathrm{~mL}=1.00 \quad$ ASTM

340.3

$\mathrm{mg} \mathrm{F}$ )

Fluoride Stock Standard Solution (1 mg ACS

of $\mathrm{F}$ in $1 \mathrm{~mL}$ )

Volumetric glassware complies with Class A tolerance requirements of ASTM E 288 and NIST Circular 434; it is calibrated before first use and recalibrated regularly in accordance with ASTM E 542 and NIST Procedure NBSIR 74-461. Balances are calibrated regularly with weights certified traceable to the NIST national mass standard. Thermometers and temperature probes are calibrated before first use and recalibrated regularly with a thermometer traceable to NIST standards. All products are prepared according to master documents that assure manufacture according to validated methods. Batch records document raw material traceability and production and testing history for each lot manufactured.

Shelf Life (unopened container):

\begin{tabular}{|ll|}
\hline Part Number & Shelf Life \\
\hline $3173-4$ & 18 months \\
$3173-32$ & 18 months \\
$3173-16$ & 18 months \\
Recommended Storage: $15^{\circ} \mathrm{C}-30^{\circ} \mathrm{C}\left(59^{\circ} \mathrm{F}-86^{\circ} \mathrm{F}\right)$ &
\end{tabular}

Recommended Storage: $15^{\circ} \mathrm{C}-30^{\circ} \mathrm{C}\left(59^{\circ} \mathrm{F}-86^{\circ} \mathrm{F}\right)$

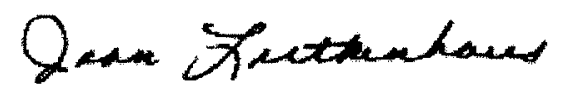

Joan Lutkenhaus

Director of Quality Assurance and Regulatory Affairs

This Certificate of Analysis is designed to comply with ISO Guide 31 "Reference Materials - Contents of Certificates and Labels." To determine manufacturing site using lot number, visit www.riccachemical.com/AboutUs/lot.pdf. 


\section{RICCA CHEMICAL COMPANY \\ PO Box 13090 Arlington, TX 76094 | http://www.riccachemical.com | 1-888-GO-RICCA | customerservice@riccachemical.com}

Chloride Standard, $1 \mathrm{~mL}=1 \mathrm{mg} \mathrm{Cl}-, 1000$ ppm Cl- (0.0282 Normal)

Lot Number: 141200

Product Number: 1955

Expiration Date: MAY 2006

Manufacture Date:12/1/2004

Contains:

\begin{tabular}{lll}
\hline Name & CAS\# & Grade \\
\hline Sodium Chloride, $\mathrm{NaCl}$ & $7647-14-5$ & ACS \\
Water, Deionized, $\mathrm{H} 2 \mathrm{O}$ & $7732-18-5$ & ACS, ASTM D 1193 (Type I), EP, USP
\end{tabular}

\begin{tabular}{lllll}
\hline Test Name & \multicolumn{1}{c}{ Assay Method } & Specification & Result \\
\hline Appearance & Clarity, Color, Odor & Clear, colorless, odorless & Passed Test \\
Assay at $20^{\circ} \mathrm{C}$ (traceable to NIST & Titrimetric vs. Silver Nitrate (Potassium & $1000 \pm 1 \mathrm{ppm} \mathrm{Cl}$ & $999 \mathrm{ppm} \mathrm{Cl}$ \\
SRM 999) & $\cdots$ & Chromate Indicator) & &
\end{tabular}

\begin{tabular}{lll}
\hline Specification & Reference & Method Number \\
\hline Chloride Solution, Stock $(1.00 \mathrm{~mL}=1.00$ & ASTM & D 5996
\end{tabular}

$\mathrm{mg} \mathrm{Cl}$ )

Chloride Calibration Stock Solution

EPA (SW-846)

9212

(1,000 mg/L Cl-)

Chloride Stock Solution $(1.00 \mathrm{~mL}=1.00$

EPA (SW-846)

9056

$\mathrm{mg} \mathrm{Cl}-$ )

Chloride solution (1000 ppm) TAPPI

Chloride solution (1000 ppm) TAPPI

Chloride Stock Solution $(1.00 \mathrm{~mL}=1.00 \quad$ ASTM

T 699 om-87

T 700 om-93

D 4327

$1 \mathrm{mg} \mathrm{Cl}-$ )

Sodium Chloride, $\mathrm{NaCl}$, stock standard

EPA (SW-846)

9057

solution

Sodium Chloride Calibration Standard (1

EPA $(S W-846)$

9021

$\mu \mathrm{g} \mathrm{Cl}-/ \mu \mathrm{L}$

Sodium Chloride Calibration Standắrd (1

EPA (SW-846)

9023

$\mu \mathrm{g} \mathrm{Cl}-/ \mu \mathrm{L})$

Sodium Chloride Solution, $1 \mathrm{~mL}=1 \mathrm{mg}$

ASTM

D 4458

$\mathrm{Cl}$ -

Chloride Solution, Stock $(1000 \mathrm{mg} / \mathrm{L})$

Chloride Solution Stock $(1.00 \mathrm{~mL}=1.00$

ASTM

ASTM

D $512 \mathrm{C}$

mg chloride)

Standard Chloride Solution, $1000 \mathrm{mg} / \mathrm{L}$

Stock Chloride Solution

APHA

APHA

D 5542

$4110 \mathrm{~B}$

4500-Cl- E

Volumetric glassware complies with Class A tolerance requirements of ASTM E 288 and NIST Circular 434; it is calibrated before first use and recalibrated regularly in accordance with ASTM E 542 and NIST Procedure NBSIR 74-461. Balances are calibrated regularly with weights certified traceable to the NIST national mass standard. Thermometers and temperature probes are calibrated before first use and recalibrated regularly with a thermometer traceable to NIST standards. All products are prepared according to master documents that assure manufacture according to validated methods. Batch records document raw material traceability and production and testing history for each lot manufactured.

Shelf Life (unopened container):

\begin{tabular}{|ll|}
\hline Part Number & Shelf Life \\
\hline $1955-4$ & 18 months \\
$1955-8$ & 18 months \\
$1955-16$ & 18 months
\end{tabular}

Recommended Storage: $15^{\circ} \mathrm{C}-30^{\circ} \mathrm{C}\left(59^{\circ} \mathrm{F}-86^{\circ} \mathrm{F}\right)$ 


\section{Quen Tretenchares}

Joan Lutkenhaus

Director of Quality Assurance and Regulatory Affairs

This Certificate of Analysis is designed to comply with ISO Guide 31 "Reference Materials -- Contents of Certificates and Labels." To determine manufacturing site using lot number, visit www.riccachemical.com/AboutUs/lot.pdf. 
PO Box 13090 Anlington, TX 76094 | http://www.riccachemical.com | 1-888-GO-RICCA | customerservice@riccachemical.com

Bromide Standard, $1 \mathrm{~mL}=1 \mathrm{mg} \mathrm{Br}-, 1000 \mathrm{ppm} \mathrm{Br}-$

Lot Number: 1508179

Product Number: 1180

Expiration Date: JAN 2007

Manufacture Date:8/5/2005

The certified value reported is the prepared value based upon the method of preparation of the material. The uncertainty in the prepared value is based upon the volumetric method of preparation.

Contains:

\begin{tabular}{lll}
\hline Name & CASH & Grade \\
\hline Sodium Bromide, NaBr & $7647-15-6$ & High Purity \\
Water, Deionized, H2O & $7732-18-5$ & ACS, ASTM D 1 193 (Type I), EP, USP
\end{tabular}

\begin{tabular}{lllll}
\hline Test Name & \multicolumn{1}{c}{ Assay Method } & Specification & Result & \\
\hline Appearance & Clarity, Color, Odor & Clear, colorless, odorless & Passed Test \\
Certified Concentration & Based on accurate volumetric & $1000 \pm 5 \mathrm{ppm} \mathrm{Br}$ & $1000 \mathrm{ppm} \mathrm{Br}$ \\
& preparation & &
\end{tabular}

\begin{tabular}{llll}
\hline Specification & Reference & Method Number \\
\hline Bromide Solution, Standard $(1 \mathrm{~mL}=1 \mathrm{mg}$ & ASTM &. & D 3869 D
\end{tabular}

Br-)

Bromide Stock Solution $(1.00 \mathrm{~mL}=1.00 \mathrm{mg}$

EPA (SW-846)

9056

$\mathrm{Br}-$ )

Bromide Stock Solution $(1.00 \mathrm{~mL}=1.00 \mathrm{mg} \quad$ ASTM

D 4327

$\mathrm{Br}$ ?)

Sodium Bromide Standard Solution, $1000 \quad$ ASTM

D 1246

$\mathrm{mg} / \mathrm{L}$

Standard Bromide Solution, $1000 \mathrm{mg} / \mathrm{L}$

APHA

$4110 \mathrm{~B}$

Volumetric glassware complies with Class A tolerance requirements of ASTM E 288 and NIST Circular 434; it is calibrated before first use and recalibrated regularly in accordance with ASTM E 542 and NIST Procedure NBSIR 74-461. Balances are calibrated regularly with weights certified traceable to the NIST national mass standard. Thermometers and temperature probes are calibrated before first use and recalibrated regularly with a thermometer traceable to NIST standards. All products are prepared according to master documents that assure manufacture according to validated methods. Batch records document raw material traceability and production and testing history for each lot manufactured.

Shelf Life (unopened container):

\begin{tabular}{ll|}
\hline Part Number & Shelf Life \\
\hline $1180-4$ & 18 months \\
$1180-8$ & 18 months \\
$1180-16$ & 18 months
\end{tabular}

Recommended Storage: $15^{\circ} \mathrm{C}-30^{\circ} \mathrm{C}\left(59^{\circ} \mathrm{F}-86^{\circ} \mathrm{F}\right)$

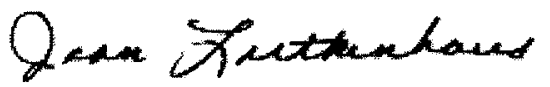

Joan Lutkenhaus

Director of Quality Assurance and Regulatory Affairs

This Certificate of Analysis is designed to comply with ISO Guide 31 "Reference Materials -- Contents of Certificates and Labels." To determine manufacturing site using lot number, visit www.riccachemical.com/AboutUs/lot.pdf. 


\section{Data Analysis Technologies, Inc. \\ 7715 Corporate Blvd. \\ Plain City, OH 43064 \\ NELAP/LELAP Certification 03027}

\section{Sample Analysis Certificate}

$\begin{array}{ll}\text { Client: } & \text { Metco Environmental } \\ \text { Address: } & \text { 3226 Commander Drive } \\ & \text { Carrollton, TX 75006 }\end{array}$

Attn: Steve Bornsen

Your Project: $05-235$ ADA-ES

Sampled By: S.B.

$\begin{array}{ll}\text { Date: } & 11 / 18 / 05 \\ \text { DAT Project \#: } & 1105003 \\ \text { Sample Date: } & 10 / 25-26 / 05 \\ \text { Date Received: } & 11 / 01 / 05 \\ \text { Date Analyzed: } & 11 / 17 / 05 \\ \text { Analyst: } & \text { DE }\end{array}$

\section{Analysis: Mercury \\ Method: Ontario Hydro}

Results: See attached summary tables.

QC: $\quad$ Met all criteria for the method.

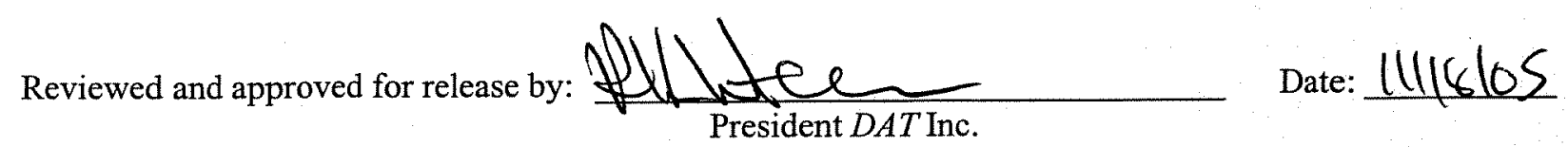




\section{Data Analysis Technologies}

Mercury by Ontario Hydro Method: Data Summary

Project Number:

Client:

Site:

Prep Date:

Analysis Date:
1105003

Metco Environmental

ADA-ES

$11 / 16 / 05$

$11 / 17 / 05$
Client ID

DAT ID

\begin{tabular}{rrrl} 
& Hg & Q & $\begin{array}{l}\text { Dup Result } \\
\text { Total ug }\end{array}$ \\
\hline
\end{tabular}

\section{Blank Train}

Filter/Cont 2

Cont. 3

Cont. 4

Cont. 5

\section{Reagent Blanks}

Cont. 7,12

Cont. 8

Cont. 9

Cont. 10

Cont. 11
1105003- 41,36

1105003- 37

1105003- 38

1105003- 39

1105003- 40
0.02

0.26

0.07
1105003- 31,32

1105003- 33

1105003- 35
0.02

0.01

0.10

0.01

0.02
ND

ND

ND

ND

ND $=$ Not detected at the detection limit shown 


\section{Data Analysis Technologies}

Mercury by Ontario Hydro Method: Data Summary

Project Number: $\quad 1105003$

Client:

Metco Environmental

Site:

ADA-ES

Prep Date:

$11 / 16 / 05$

Analysis Date:

$11 / 17 / 05$

Client ID DAT ID

\begin{tabular}{cccl} 
Hg & Q & $\begin{array}{l}\text { Dup Result } \\
\text { Total ug }\end{array}$ \\
\hline
\end{tabular}

Inlet Run 1

Filter/Cont 2

$1105003-1,2$

0.02

0.19

Cont. 3a

1105003- 3a

0.03

ND

Cont. 3b

$1105003-3 b$

3.30

Cont. 4

1105003- 4

0.07

ND

Cont. 5

1105003- 5

0.07

6.8

Inlet Run 2

Filter/Cont 2

$1105003-6,7$

0.02

0.13

Cont. 3a

1105003- 8a

0.07

3.96

Cont. 3b

$1105003-8 b$

1105003- 9

0.02

ND

Cont. 4

1105003- 10

0.30

ND

Cont. 5

0.07

4.6

Inlet Run 3

Filter/Cont 2

$1105003-11,12$

0.02

0.06

Cont. 3a

1105003- 13a

0.43

Cont. 3b

1105003-13b

0.05

1.42

Cont. 4

1105003- 14

0.04

ND

Cont. 5

1105003- 15

0.25

0.08

6.6

$\mathrm{ND}=$ Not detected at the detection limit shown 


\section{Data Analysis Technologies}

Mercury by Ontario Hydro Method: Data Summary

Project Number:

Client:

Site:

Prep Date:

Analysis Date:
1105003

Metco Environmental

ADA-ES

$11 / 16 / 05$

$11 / 17 / 05$
Client ID

DAT ID
$M D L$

0.02

0.08

0.03

0.26

0.08

0.02

0.08

0.04

0.26

0.08
1105003- 21,22

1105003-23a

1105003- $23 \mathrm{~b}$

1105003- 24

1105003- 25

$1105003-26,27$
$1105003-28 a$
$1105003-28 b$
$1105003-29$
$1105003-30$

1105003-26,27

0.02

0.08

0.05

0.28

0.08

1105003- 30
3.40

0.12

ND

ND

0.5

2.33

0.52

ND

ND

0.9

Outlet Run 3

Cont. $3 \mathrm{a}$

Cont. $3 \mathrm{~b}$

Cont. 4

Cont. 5

$\mathrm{ND}=$ Not detected at the detection limit shown 


\section{Data Analysis Technologies}

Mercury by Ontario Hydro Method: Data Summary

$\begin{array}{ll}\text { Project Number: } & 1105003 \\ \text { Client: } & \text { Metco Envir } \\ \text { Site: } & \text { ADA-ES } \\ & \\ \text { Prep Date: } & 11 / 16 / 05 \\ \text { Analysis Date: } & 11 / 17 / 05\end{array}$

\begin{tabular}{|c|c|c|c|c|}
\hline \multirow[b]{2}{*}{ QC Summary } & & \multirow[t]{2}{*}{$\begin{array}{l}\mathrm{Hg} \\
\mathrm{ug} / \mathrm{L}\end{array}$} & \multirow[t]{2}{*}{$\% \operatorname{Rec}$} & \multirow[t]{2}{*}{$\%$ RPD } \\
\hline & & & & \\
\hline Front Method Blank & 1105003-MB Front & $0.05 \mathrm{ND}$ & & \\
\hline Back Method Blank & 1105003-MB Back & $0.05 \mathrm{ND}$ & & \\
\hline Laboratory Spike & 1105003-LS Front & 12.9 & 129 & \\
\hline Laboratory Spike & 1105003-LS Back & 10.8 & 108 & \\
\hline \multirow[t]{2}{*}{ Matrix Spike } & $1105003-5 \mathrm{MS}$ & 9.83 & 98 & \\
\hline & $1105003-5 \mathrm{MSD}$ & 9.43 & 94 & 4.2 \\
\hline \multirow[t]{2}{*}{ Matrix Spike } & 1105003-13b MS & 9.64 & 96 & \\
\hline & 1105003-13b MSD & 9.39 & 94 & 2.6 \\
\hline \multirow[t]{2}{*}{ Matrix Spike } & $1105003-19 \mathrm{MS}$ & 10.5 & 105 & \\
\hline & 1105003-19 MSD & 10.3 & 103 & 1.9 \\
\hline \multirow[t]{2}{*}{ Duplicate Results } & $1105003-29$ & $0.05 \mathrm{ND}$ & & \\
\hline & $1105003-29 \mathrm{D}$ & $0.05 \mathrm{ND}$ & & \\
\hline
\end{tabular}

$\mathrm{ND}=$ Not detected at the detection limit shown. 


\section{Data Analysis Technologies}

Mercury by Ontario Hydro Method: Data Summary

Project Number: 1105003

Client: Metco Environmental

Site:

ADA-ES

Prep Date:

$11 / 16 / 05$

Analysis Date:

$11 / 17 / 05$

\begin{tabular}{llr} 
Thimble/Filter ID & DAT ID & $\begin{array}{r}\text { Weight } \\
\text { g }\end{array}$ \\
\hline Inlet Run 1 & $1105003-1$ & 0.5158 \\
Inlet Run 2 & $1105003-6$ & 0.5065 \\
Inlet Run 3 & $1105003-11$ & 0.5210 \\
Outlet Run 1 & $1105003-16$ & 0.5195 \\
Outlet Run 2 & $1105003-21$ & 0.5207 \\
Outlet Run 3 & $1105003-26$ & 0.5303 \\
Blank Train & $1105003-31$ & 0.5617 \\
Reagent Blank Cont. 12 & $1105003-41$ & 0.5810 \\
\hline
\end{tabular}




\section{SAMPLE DATA}

Page 7 of 37

\section{E-93}


DAT Labs, Inc. Extraction Form

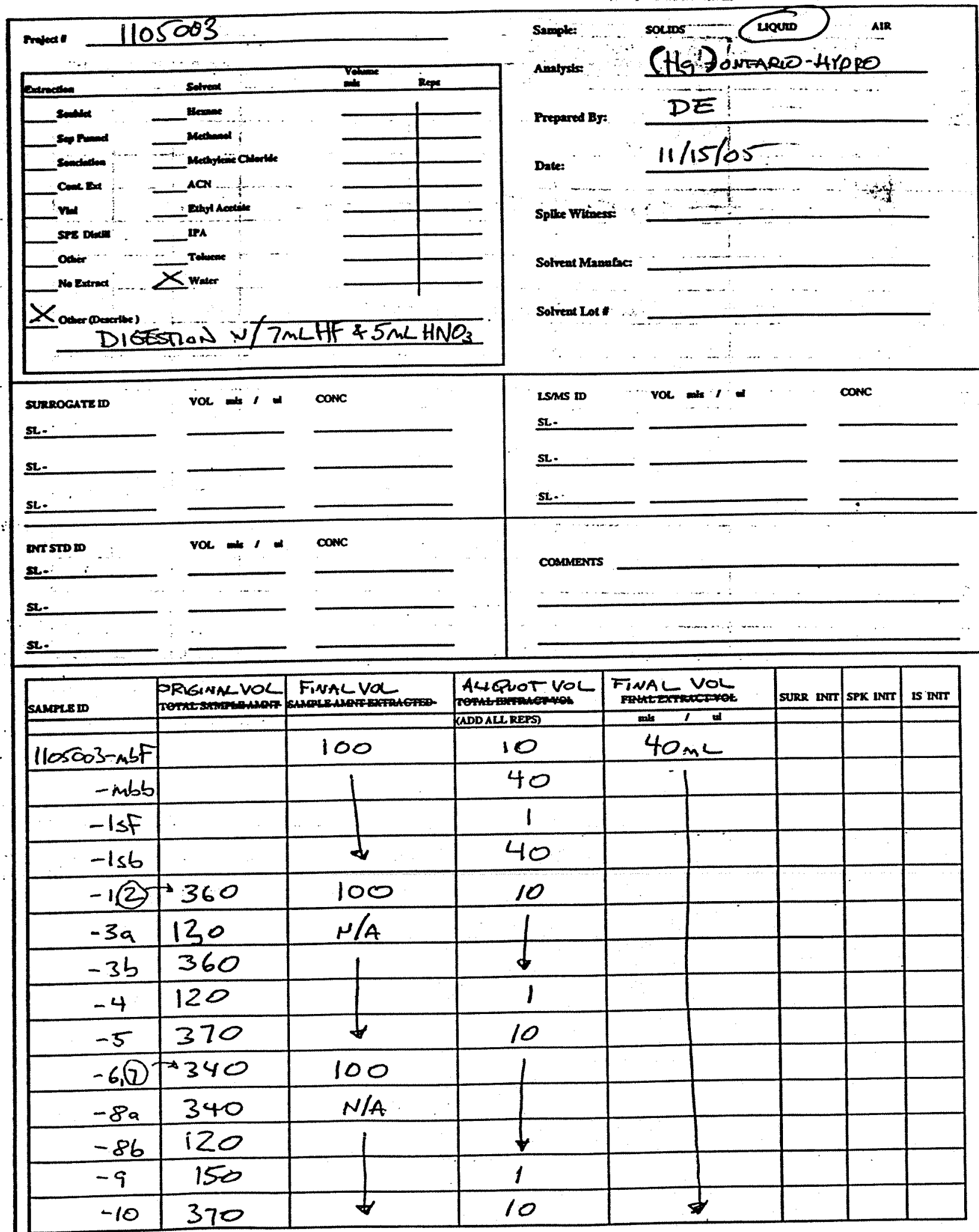

If extract was spilf for additional amalyses, please nole the allquot rolume for this analysis and at what polat the extract was ap ileed.

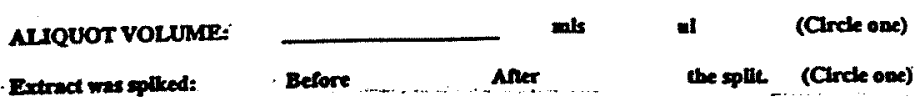









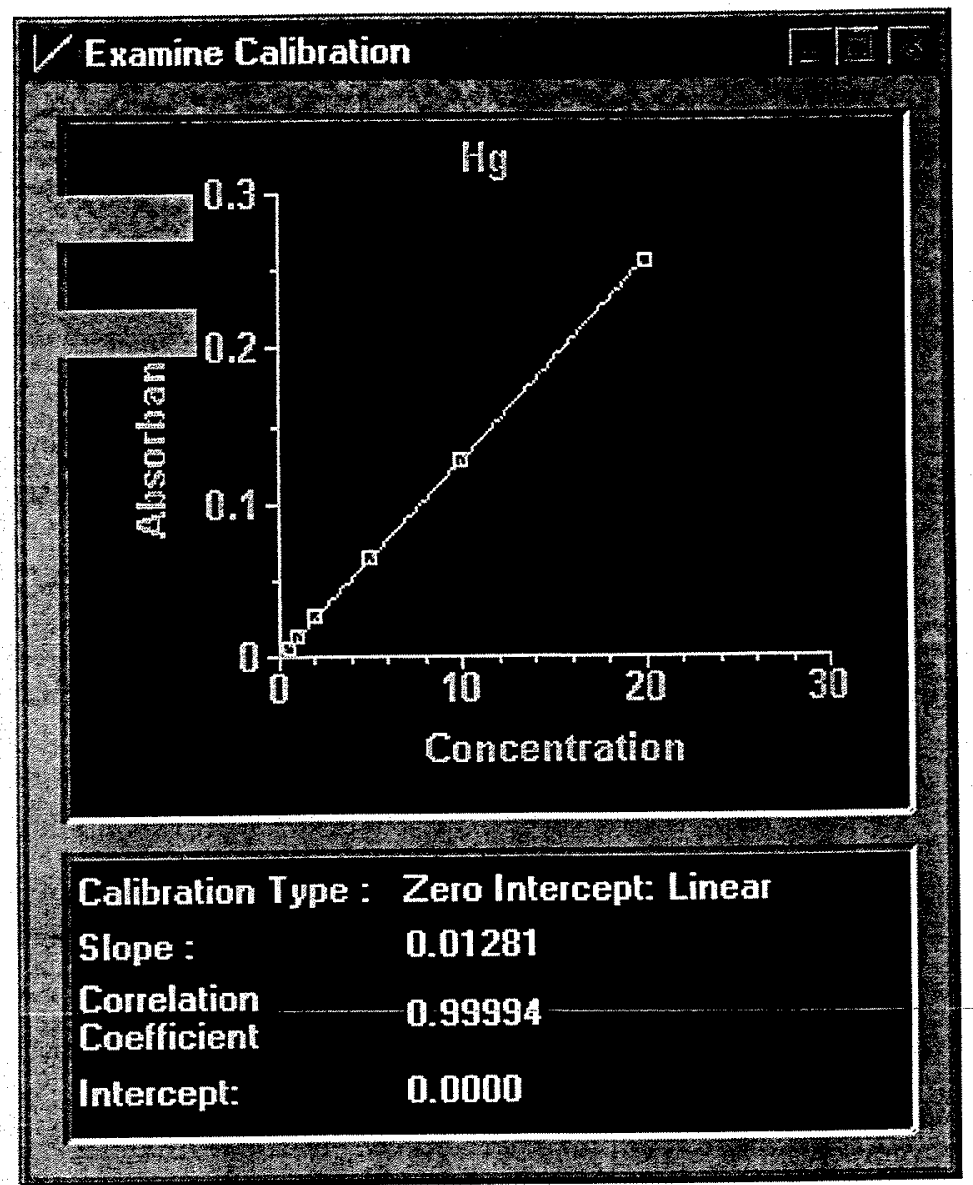

Page 10afe $374-$ 
0.3

0.2

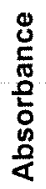

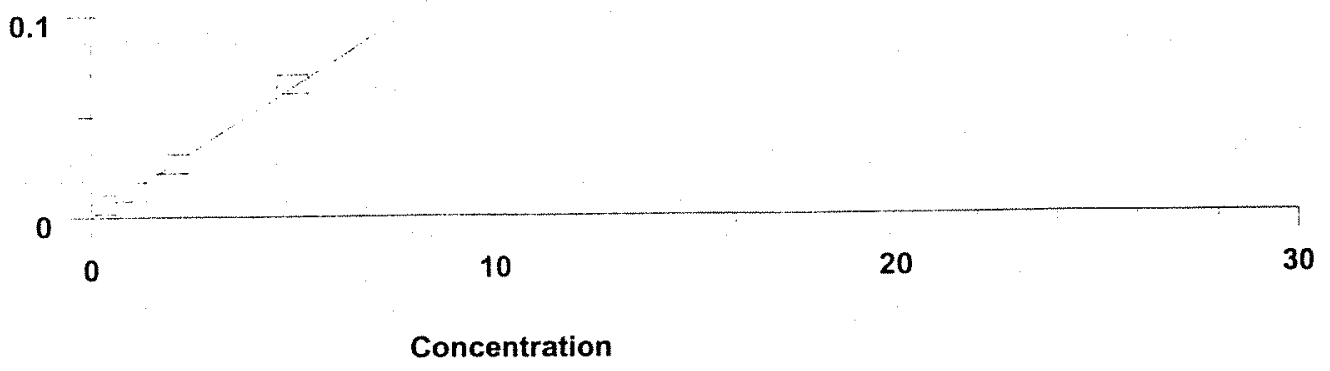

Pagey 11 of 37 


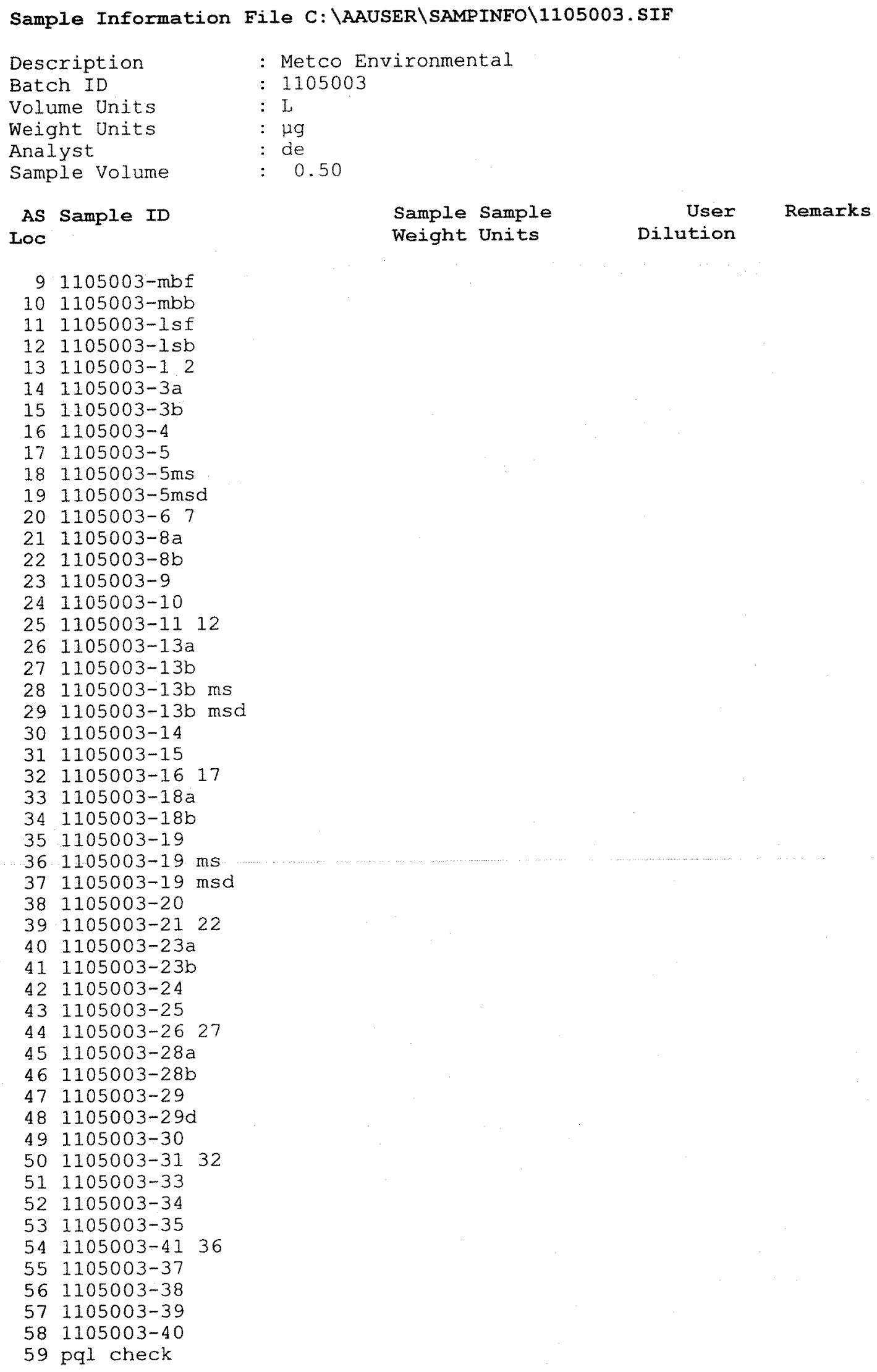

Description

Batch ID

Volume Units

Weight Units

Analyst

Sample Volume

\section{AS Sample ID}

LOC

9 1105003-mbf

$101105003-\mathrm{mbb}$

11 1105003-1sf

$121105003-1 \mathrm{sb}$

$131105003-12$

$141105003-3 a$

$151105003-3 b$

$161105003-4$

17 1105003-5

$18 \quad 1105003-5 \mathrm{~ms}$

$191105003-5 \mathrm{msd}$

$201105003-67$

21 1105003-8a

$221105003-8 \mathrm{~b}$

23 1105003-9

24 1105003-10

25 1105003-11 12

$26 \quad 1105003-13 a$

$271105003-13 \mathrm{~b}$

28 1105003-13b ms

29 1105003-13b msd

$301105003-14$

31 1105003-15

32 1105003-16 17

33 1105003-18a

$341105003-18 \mathrm{~b}$

35 1105003-19

$36 \quad 1105003-19 \mathrm{~ms}$

37 1105003-19 msd

38 1105003-20

39 1105003-21 22

40 1105003-23a

$411105003-23 b$

42 1105003-24

$431105003-25$

$44 \quad 1105003-26 \quad 27$

$451105003-28 a$

$461105003-28 \mathrm{~b}$

47 1105003-29

48 1105003-29d

$491105003-30$

$50 \quad 1105003-31 \quad 32$

51 1105003-33

$521105003-34$

53 1105003-35

54 1105003-41 36

$55 \quad 1105003-37$

$561105003-38$

$571105003-39$

58 1105003-40

59 pql check

: Metco Environmental

: 1105003

L

$\mu g$

: de

: 0.50

Sample Sample

Weight Units

\section{User Remarks}

Dilution 


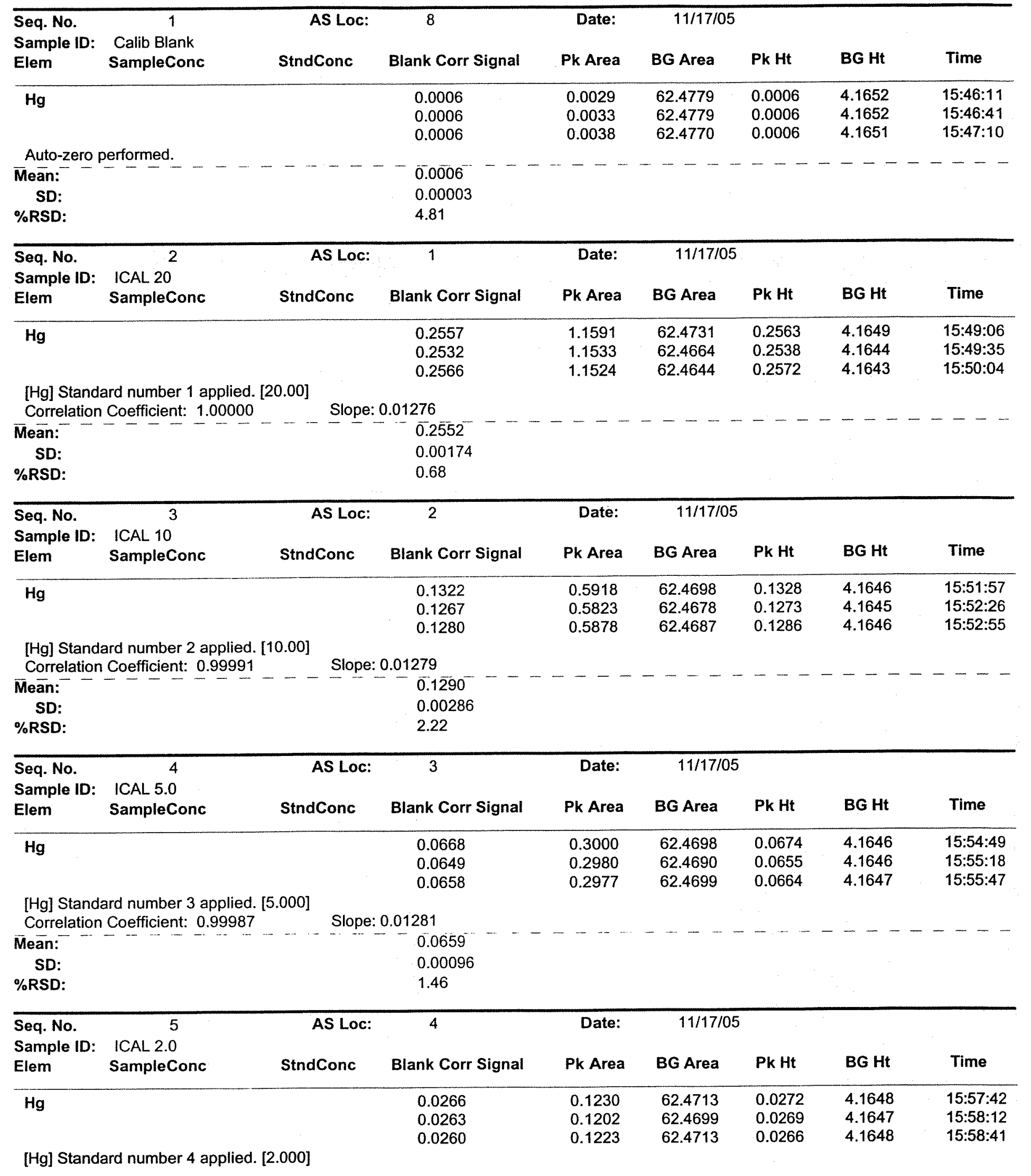




$\begin{array}{ll}\text { Mean: } & 0.0263 \\ \text { SD: } & 0.00028 \\ \text { \%RSD: } & 1.08\end{array}$

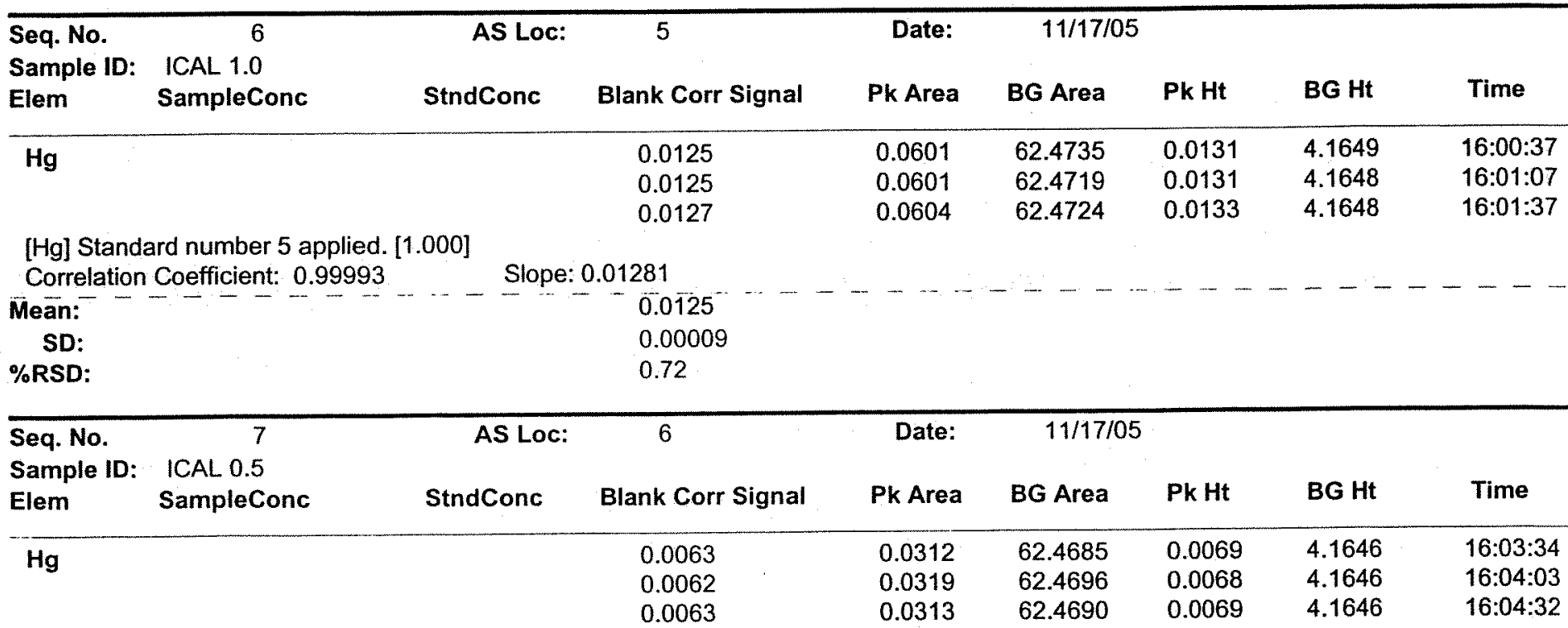

[Hg] Standard number 6 applied. [0.5000]

Correlation Coefficient: $0.99994 \quad$ Slope: 0.01281

$\begin{array}{ll}\text { Mean: } & 0.0063 \\ \text { SD: } & 0.00004 \\ \text { \%RSD: } & 0.62\end{array}$

\begin{tabular}{|c|c|c|c|c|c|c|c|c|}
\hline $\begin{array}{l}\text { Seq. No. } \\
\text { Sample ID: } \\
\text { Elem }\end{array}$ & $\begin{array}{c}8 \\
\text { ICAL } 0.2 \\
\text { SampleConc }\end{array}$ & $\begin{array}{l}\text { AS Loc: } \\
\text { StndConc }\end{array}$ & $\begin{array}{c}7 \\
\text { Blank Corr Signal }\end{array}$ & $\begin{array}{l}\text { Date: } \\
\text { Pk Area }\end{array}$ & BG Area & Pk Ht & BG Ht & Time \\
\hline $\mathrm{Hg}$ & & & $\begin{array}{l}0.0021 \\
0.0022 \\
0.0022\end{array}$ & $\begin{array}{l}0.0119 \\
0.0132 \\
0.0133\end{array}$ & $\begin{array}{l}62.4696 \\
62.4721 \\
62.4710\end{array}$ & $\begin{array}{l}0.0027 \\
0.0028 \\
0.0028\end{array}$ & $\begin{array}{l}4.1646 \\
4.1648 \\
4.1647\end{array}$ & $\begin{array}{l}16: 06: 30 \\
16: 06: 59 \\
16: 07: 29\end{array}$ \\
\hline
\end{tabular}

[Hg] Standard number 7 applied. [0.2000]

Correlation Coefficient: 0.99994 Slope: 0.01281
Mean:
SD:
0.00009
\%RSD:
4.15

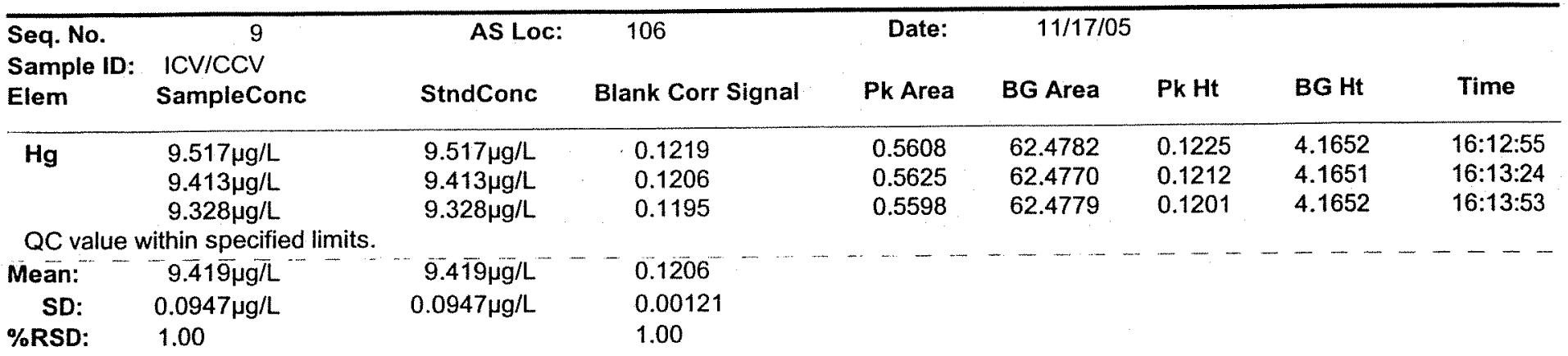




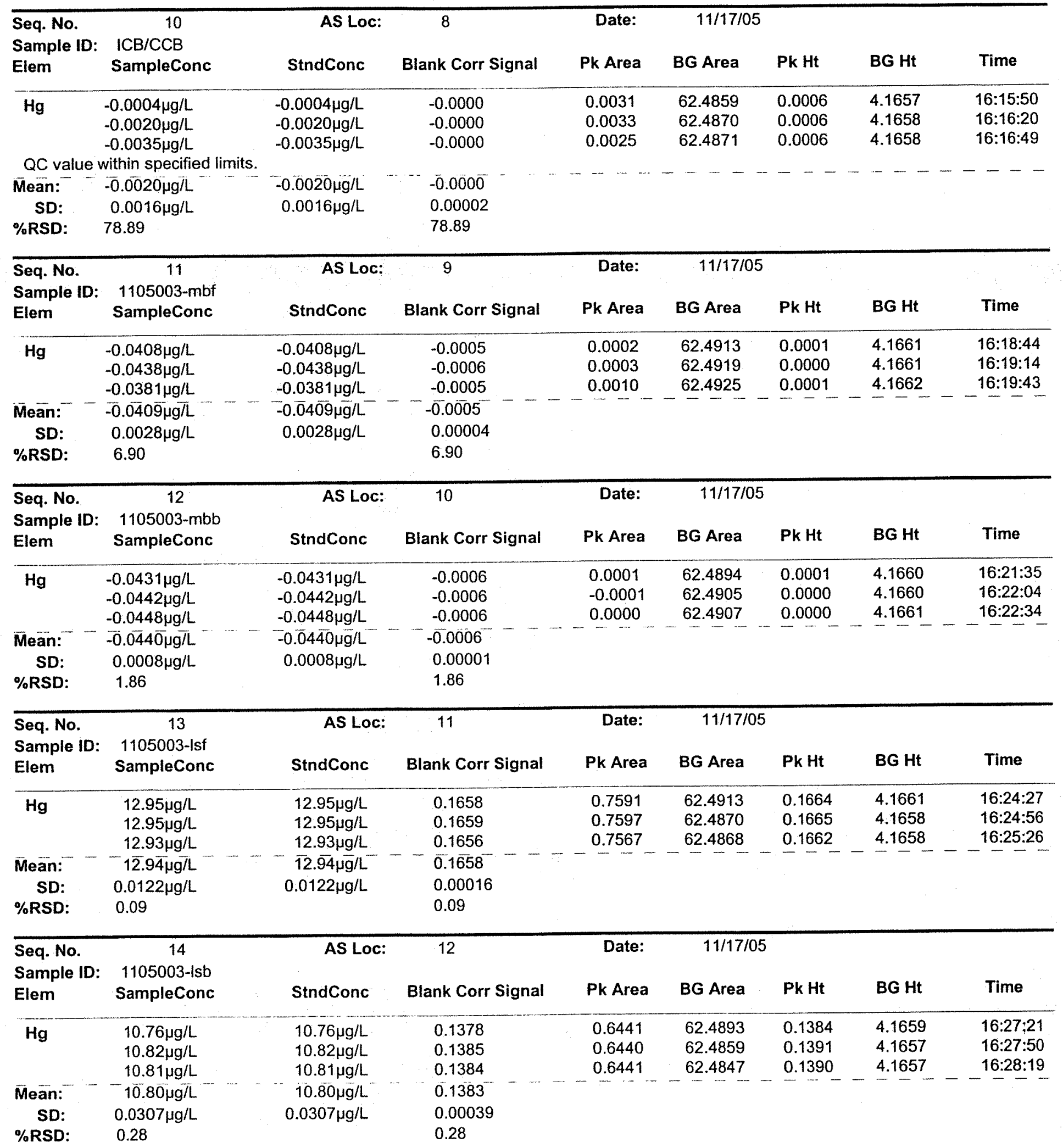




\begin{tabular}{|c|c|c|c|c|c|c|c|c|c|}
\hline $\begin{array}{l}\text { Seq. No. } \\
\text { Sample ID: } \\
\text { Elem }\end{array}$ & $\begin{array}{c}15 \\
1105003-12 \\
\text { SampleConc }\end{array}$ & & $\begin{array}{l}\text { AS Loc: } \\
\text { StndConc }\end{array}$ & $\begin{array}{c}13 \\
\text { Blank Corr Signal }\end{array}$ & $\begin{array}{c}\text { Date: } \\
\text { Pk Area }\end{array}$ & BG Area & Pk Ht & BG Ht & Time \\
\hline $\begin{array}{l}\mathrm{Hg} \\
\text { Mean:- - } \\
\text { SD: } \\
\text { \%RSD: }\end{array}$ & $\begin{array}{l}0.4687 \mu \mathrm{g} / \mathrm{L} \\
0.4646 \mu \mathrm{g} / \mathrm{L} \\
0.4755 \mu \mathrm{g} / \mathrm{L} \\
\overline{0} . \overline{46} \overline{6} \overline{\mathrm{g} / \mathrm{L}} \\
0.0055 \mu \mathrm{g} / \mathrm{L} \\
1.17\end{array}$ & $\cdots$ & $\begin{array}{l}0.4687 \mu \mathrm{g} / \mathrm{L} \\
0.4646 \mu \mathrm{g} / \mathrm{L} \\
0.4755 \mu \mathrm{g} / \mathrm{L} \\
0.4696 \mu \mathrm{g} / \mathrm{L} \\
0.0055 \mu \mathrm{g} / \mathrm{L}\end{array}$ & $\begin{array}{l}0.0060 \\
0.0060 \\
0.0061 \\
-0.0060 \\
0.00007 \\
1.17\end{array}$ & $\begin{array}{l}0.0303 \\
0.0296 \\
0.0309\end{array}$ & $\begin{array}{l}62.4902 \\
62.4902 \\
62.4913 \\
\end{array}$ & $\begin{array}{l}0.0066 \\
0.0066 \\
0.0067 \\
0 .\end{array}$ & $\begin{array}{r}4.1660 \\
4.1660 \\
4.1661 \\
-\end{array}$ & $\begin{array}{r}16: 30: 14 \\
16: 30: 44 \\
16: 31: 13 \\
-\quad-\quad-\end{array}$ \\
\hline $\begin{array}{l}\text { Seq. No. } \\
\text { Sample ID: } \\
\text { Elem }\end{array}$ & $\begin{array}{c}16 \\
1105003-3 a \\
\text { SampleConc }\end{array}$ & & $\begin{array}{l}\text { AS Loc: } \\
\text { StndConc }\end{array}$ & Blank Corr Signal & $\begin{array}{c}\text { Date: } \\
\text { Pk Area }\end{array}$ & $\begin{array}{l}11 / 17 / 05 \\
\text { BG Area }\end{array}$ & Pk Ht & BG Ht & Time \\
\hline $\begin{array}{l}\mathrm{Hg} \\
\text { Mean:- } \\
\text { SD: } \\
\text { \%RSD: }\end{array}$ & $\begin{array}{l}0.0222 \mu \mathrm{g} / \mathrm{L} \\
0.0183 \mu \mathrm{g} / \mathrm{L} \\
0.0168 \mu \mathrm{g} / \mathrm{L} \\
\overline{0.0191 \mu \mathrm{g} / \mathrm{L}} \\
0.0028 \mu \mathrm{g} / \mathrm{L} \\
14.45\end{array}$ & $\ldots$ & $\begin{array}{l}0.0222 \mu \mathrm{g} / \mathrm{L} \\
0.0183 \mu \mathrm{g} / \mathrm{L} \\
0.0168 \mu \mathrm{g} / \mathrm{L} \\
0.0191 \mu \mathrm{g} / \mathrm{L} \\
0.0028 \mu \mathrm{g} / \mathrm{L}\end{array}$ & $\begin{array}{l}0.0003 \\
0.0002 \\
0.0002 \\
0.0002 \\
0.00004 \\
14.45\end{array}$ & $\begin{array}{l}0.0048 \\
0.0044 \\
0.0041\end{array}$ & $\begin{array}{r}62.4962 \\
62.4951 \\
62.4951 \\
\end{array}$ & $\begin{array}{l}0.0009 \\
0.0008 \\
0.0008\end{array}$ & $\begin{array}{r}4.1664 \\
4.1663 \\
4.1663 \\
\end{array}$ & $\begin{array}{r}16: 33: 09 \\
16: 33: 38 \\
-16: 34: 08 \\
\end{array}$ \\
\hline $\begin{array}{l}\text { Seq. No. } \\
\text { Sample ID: } \\
\text { Elem }\end{array}$ & $\begin{array}{c}17 \\
1105003-3 b \\
\text { SampleConc }\end{array}$ & & $\begin{array}{l}\text { AS Loc: } \\
\text { StndConc }\end{array}$ & Blank Corr Signal & $\begin{array}{c}\text { Date: } \\
\text { Pk Area }\end{array}$ & $\begin{array}{l}11 / 17 / 05 \\
\text { BG Area }\end{array}$ & Pk Ht & BG Ht & Time \\
\hline $\begin{array}{l}\text { Hg } \\
\text { Mean:- } \\
\text { SD: } \\
\text { \%RSD: }\end{array}$ & $\begin{array}{l}2.279 \mu \mathrm{g} / \mathrm{L} \\
2.296 \mu \mathrm{g} / \mathrm{L} \\
2.308 \mu \mathrm{g} / \mathrm{L} \\
2.294 \mu \mathrm{g} / \mathrm{L} \\
0.0148 \mu \mathrm{g} / \mathrm{L} \\
0.64\end{array}$ & 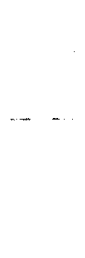 & $\begin{array}{c}2.279 \mu \mathrm{g} / \mathrm{L} \\
2.296 \mu \mathrm{g} / \mathrm{L} \\
2.308 \mu \mathrm{g} / \mathrm{L} \\
2.294 \mu \mathrm{g} / \mathrm{L} \\
0.0148 \mu \mathrm{g} / \mathrm{L}\end{array}$ & $\begin{array}{l}0.0292 \\
0.0294 \\
0.0296 \\
0.0294 \\
0.00019 \\
0.64\end{array}$ & $\begin{array}{l}0.1401 \\
0.1403 \\
0.1401\end{array}$ & $\begin{array}{r}62.4996 \\
62.4984 \\
62.4976 \\
\end{array}$ & $\begin{array}{l}0.0298 \\
0.0300 \\
0.0302\end{array}$ & $\begin{array}{r}4.1666 \\
4.1665 \\
4.1665 \\
-\quad-\quad-\end{array}$ & $\begin{array}{r}16: 36: 04 \\
16: 36: 33 \\
-16: 37: 02 \\
\end{array}$ \\
\hline $\begin{array}{l}\text { Seq. No. } \\
\text { Sample ID: } \\
\text { Elem }\end{array}$ & $\begin{array}{c}18 \\
1105003-4 \\
\text { SampleConc }\end{array}$ & & $\begin{array}{l}\text { AS Loc: } \\
\text { StndConc }\end{array}$ & Blank Corr Signal & $\begin{array}{l}\text { Date: } \\
\text { Pk Area }\end{array}$ & $\begin{array}{l}11 / 17 / 05 \\
\text { BG Area }\end{array}$ & Pk Ht & BG Ht & Time \\
\hline $\begin{array}{l}\mathrm{Hg} \\
\text { Mean: } \\
\text { SD: } \\
\text { \%RSD: }\end{array}$ & $\begin{array}{l}-0.0235 \mu \mathrm{g} / \mathrm{L} \\
-0.0308 \mu \mathrm{g} / \mathrm{L} \\
-0.0213 \mu \mathrm{g} / \mathrm{L} \\
-0.0252 \mu \mathrm{g} / \mathrm{L} \\
0.0050 \mu \mathrm{g} / \mathrm{L} \\
19.80\end{array}$ & - & $\begin{array}{c}-0.0235 \mu \mathrm{g} / \mathrm{L} \\
-0.0308 \mu \mathrm{g} / \mathrm{L} \\
-0.0213 \mu \mathrm{g} / \mathrm{L} \\
-0.0252 \mu \mathrm{g} / \mathrm{L} \\
0.0050 \mu \mathrm{g} / \mathrm{L}\end{array}$ & $\begin{array}{l}-0.0003 \\
-0.0004 \\
-0.0003 \\
-0.0003 \\
0.00006 \\
19.80\end{array}$ & $\begin{array}{l}0.0017 \\
0.0007 \\
0.0017\end{array}$ & $\begin{array}{l}62.5017 \\
62.5028 \\
62.5051\end{array}$ & $\begin{array}{l}0.0003 \\
0.0002 \\
0.0003\end{array}$ & $\begin{array}{r}4.1668 \\
4.1668 \\
-4.1670 \\
-\end{array}$ & $\begin{array}{r}16: 39: 01 \\
16: 39: 30 \\
16: 39: 59 \\
\end{array}$ \\
\hline $\begin{array}{l}\text { Seq. No. } \\
\text { Sample ID: } \\
\text { Elem }\end{array}$ & $\begin{array}{c}19 \\
1105003-5 \\
\text { SampleConc }\end{array}$ & & $\begin{array}{l}\text { AS Loc: } \\
\text { StndConc }\end{array}$ & Blank Corr Signal & $\begin{array}{c}\text { Date: } \\
\text { Pk Area }\end{array}$ & $\begin{array}{l}11 / 17 / 05 \\
\text { BG Area }\end{array}$ & Pk Ht & BG Ht & Time \\
\hline $\begin{array}{l}\text { Mean: } \\
\text { SD: } \\
\text { \%RSD: }\end{array}$ & $\begin{array}{l}4.574 \mu \mathrm{g} / \mathrm{L} \\
4.626 \mu \mathrm{g} / \mathrm{L} \\
4.566 \mu \mathrm{g} / \mathrm{L} \\
4.58 \overline{8} \mu \mathrm{g} / \mathrm{L} \\
0.0324 \mu \mathrm{g} / \mathrm{L} \\
0.71\end{array}$ & 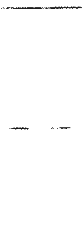 & $\begin{array}{c}4.574 \mu \mathrm{g} / \mathrm{L} \\
4.626 \mu \mathrm{g} / \mathrm{L} \\
4.566 \mu \mathrm{g} / \mathrm{L} \\
-4.5 \overline{8} \mu \mathrm{g} / \mathrm{L} \\
0.0324 \mu \mathrm{g} / \mathrm{L}\end{array}$ & $\begin{array}{l}0.0586 \\
0.0592 \\
0.0585 \\
\overline{0.0588} \\
0.00042 \\
0.71\end{array}$ & $\begin{array}{l}0.2766 \\
0.2781 \\
0.2765\end{array}$ & $\begin{array}{l}62.5098 \\
62.5090 \\
62.5087\end{array}$ & $\begin{array}{l}0.0592 \\
0.0598 \\
0.0591\end{array}$ & $\begin{array}{r}4.1673 \\
4.1673 \\
4.1672 \\
\end{array}$ & $\begin{array}{r}16: 41: 54 \\
16: 42: 23 \\
16: 42: 52 \\
\end{array}$ \\
\hline
\end{tabular}




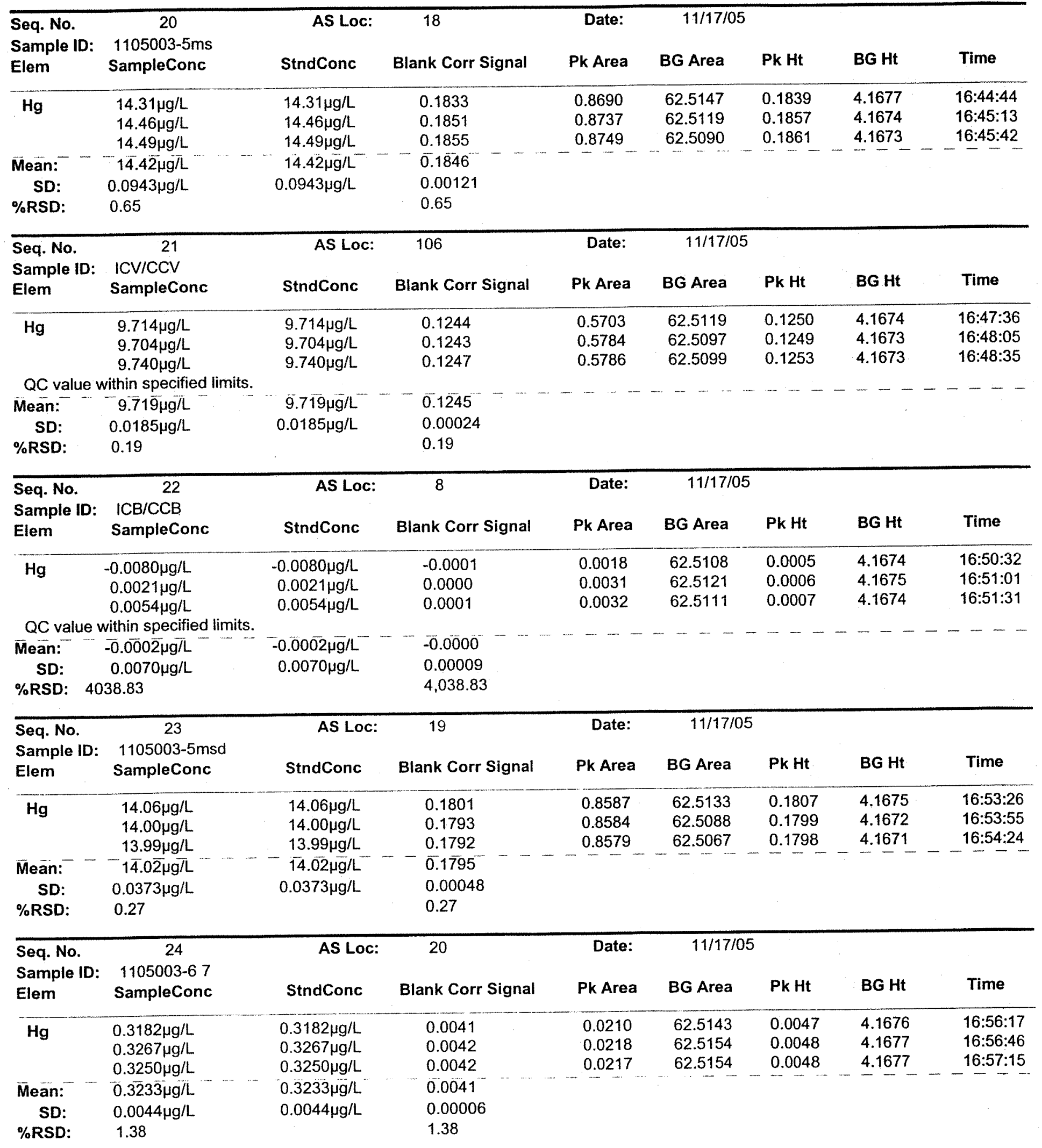

Page 17 of 37 


\begin{tabular}{|c|c|c|c|c|c|c|c|c|}
\hline $\begin{array}{l}\text { Seq. No. } \\
\text { Sample ID: } \\
\text { Elem }\end{array}$ & $\begin{array}{c}25 \\
1105003-8 a \\
\text { SampleConc }\end{array}$ & $\begin{array}{l}\text { AS Loc: } \\
\text { StndConc }\end{array}$ & $\begin{array}{c}21 \\
\text { Blank Corr Signal }\end{array}$ & $\begin{array}{c}\text { Date: } \\
\text { Pk Area }\end{array}$ & BG Area & Pk Ht & BG Ht & Time \\
\hline $\begin{array}{l}\mathrm{Hg} \\
\text { Mean:- - } \\
\text { SD: } \\
\text { \%RSD: }\end{array}$ & $\begin{array}{l}2.917 \mu \mathrm{g} / \mathrm{L} \\
2.917 \mu \mathrm{g} / \mathrm{L} \\
2.909 \mu \mathrm{g} / \mathrm{L} \\
2.915 \mu \mathrm{g} / \mathrm{L} \\
0.0045 \mu \mathrm{g} / \mathrm{L} \\
0.15\end{array}$ & $\begin{array}{r}2.917 \mu \mathrm{g} / \mathrm{L} \\
2.917 \mu \mathrm{g} / \mathrm{L} \\
2.909 \mu \mathrm{g} / \mathrm{L} \\
2.915 \mu \mathrm{g} / \mathrm{L} \\
0.0045 \mu \mathrm{g} / \mathrm{L}\end{array}$ & $\begin{array}{l}0.0374 \\
0.0374 \\
0.0373 \\
0.0373 \\
0.00006 \\
0.15\end{array}$ & $\begin{array}{l}0.1800 \\
0.1796 \\
0.1793 \\
\end{array}$ & $\begin{array}{r}62.5214 \\
62.5216 \\
62.5211 \\
\end{array}$ & $\begin{array}{l}0.0380 \\
0.0380 \\
0.0379\end{array}$ & $\begin{array}{r}4.1681 \\
4.1681 \\
4.1681 \\
-\end{array}$ & $\begin{array}{r}16: 59: 07 \\
16: 59: 37 \\
17: 00: 06 \\
\end{array}$ \\
\hline $\begin{array}{l}\text { Seq. No. } \\
\text { Sample ID: } \\
\text { Elem }\end{array}$ & $\begin{array}{c}26 \\
1105003-8 b \\
\text { SampleConc }\end{array}$ & $\begin{array}{l}\text { AS Loc: } \\
\text { StndConc }\end{array}$ & Blank Corr Signal & $\begin{array}{c}\text { Date: } \\
\text { Pk Area }\end{array}$ & $11 / 17 / 05$ & Pk Ht & BG Ht & Time \\
\hline $\begin{array}{l}\mathrm{Hg} \\
\text { Mean: } \\
\text { SD: } \\
\text { \%RSD: }\end{array}$ & $\begin{array}{l}-0.0042 \mu \mathrm{g} / \mathrm{L} \\
-0.0080 \mu \mathrm{g} / \mathrm{L} \\
-0.0036 \mu \mathrm{g} / \mathrm{L} \\
-0.0053 \mu \mathrm{g} / \mathrm{L} \\
0.0024 \mu \mathrm{g} / \mathrm{L} \\
45.05\end{array}$ & $\begin{array}{c}-0.0042 \mu \mathrm{g} / \mathrm{L} \\
-0.0080 \mu \mathrm{g} / \mathrm{L} \\
-0.0036 \mu \mathrm{g} / \mathrm{L} \\
-0.0053 \mu \mathrm{g} / \mathrm{L} \\
0.0024 \mu \mathrm{g} / \mathrm{L}\end{array}$ & $\begin{array}{l}-0.0001 \\
-0.0001 \\
-0.0000 \\
-0.0001 \\
0.00003 \\
45.05\end{array}$ & $\begin{array}{l}0.0030 \\
0.0021 \\
0.0031 \\
-\end{array}$ & $\begin{array}{r}62.5237 \\
62.5228 \\
62.5250 \\
-\ldots\end{array}$ & $\begin{array}{l}0.0005 \\
0.0005 \\
0.0006\end{array}$ & $\begin{array}{r}4.1682 \\
4.1682 \\
4.1683 \\
\end{array}$ & $\begin{array}{r}17: 01: 59 \\
17: 02: 28 \\
17: 02: 58 \\
-\quad-\quad\end{array}$ \\
\hline $\begin{array}{l}\text { Seq. No. } \\
\text { Sample ID: } \\
\text { Elem }\end{array}$ & $\begin{array}{c}27 \\
1105003-9 \\
\text { SampleConc }\end{array}$ & $\begin{array}{l}\text { AS Loc: } \\
\text { StndConc }\end{array}$ & Blank Corr Signal & $\begin{array}{c}\text { Date: } \\
\text { Pk Area }\end{array}$ & $11 / 17 / 05$ & Pk Ht & BG Ht & Time \\
\hline $\begin{array}{l}\text { Hg } \\
\text { Mean:- - } \\
\text { SD: } \\
\text { \%RSD: }\end{array}$ & $\begin{array}{l}0.0093 \mu \mathrm{g} / \mathrm{L} \\
0.0096 \mu \mathrm{g} / \mathrm{L} \\
0.0156 \mu \mathrm{g} / \mathrm{L} \\
\overline{0.0115 \mu \mathrm{g} / \mathrm{L}}-- \\
0.0035 \mu \mathrm{g} / \mathrm{L} \\
30.81\end{array}$ & $\begin{array}{c}0.0093 \mu \mathrm{g} / \mathrm{L} \\
0.0096 \mu \mathrm{g} / \mathrm{L} \\
0.0156 \mu \mathrm{g} / \mathrm{L} \\
-0.0115 \mu \mathrm{g} / \mathrm{L} \\
0.0035 \mu \mathrm{g} / \mathrm{L}\end{array}$ & $\begin{array}{l}0.0001 \\
0.0001 \\
0.0002 \\
0.0001 \\
0.00005 \\
30.81\end{array}$ & $\begin{array}{l}0.0035 \\
0.0030 \\
0.0044\end{array}$ & $\begin{array}{r}62.5259 \\
62.5259 \\
62.5268 \\
\end{array}$ & $\begin{array}{l}0.0007 \\
0.0007 \\
0.0008\end{array}$ & $\begin{array}{r}4.1684 \\
4.1684 \\
4.1684 \\
\end{array}$ & $\begin{array}{r}17: 04: 52 \\
17: 05: 21 \\
17: 05: 50 \\
-\quad-\quad\end{array}$ \\
\hline $\begin{array}{l}\text { Seq. No. } \\
\text { Sample ID: } \\
\text { Elem }\end{array}$ & $\begin{array}{c}28 \\
1105003-10 \\
\text { SampleConc }\end{array}$ & $\begin{array}{l}\text { AS Loc: } \\
\text { StndConc }\end{array}$ & Blank Corr Signal & $\begin{array}{l}\text { Date: } \\
\text { Pk Area }\end{array}$ & $\begin{array}{l}11 / 17 / 05 \\
\text { BG Area }\end{array}$ & Pk Ht & BG Ht & Time \\
\hline $\begin{array}{l}\text { Mean: } \\
\text { SD: } \\
\text { \%RSD: }\end{array}$ & $\begin{array}{l}3.126 \mu \mathrm{g} / \mathrm{L} \\
3.108 \mu \mathrm{g} / \mathrm{L} \\
3.152 \mu \mathrm{g} / \mathrm{L} \\
3.128 \mu \mathrm{g} / \mathrm{L} \\
0.0224 \mu \mathrm{g} / \mathrm{L} \\
0.72\end{array}$ & $\begin{array}{r}3.126 \mu \mathrm{g} / \mathrm{L} \\
3.108 \mu \mathrm{g} / \mathrm{L} \\
3.152 \mu \mathrm{g} / \mathrm{L} \\
-3.128 \mu \mathrm{g} / \mathrm{L} \\
0.0224 \mu \mathrm{g} / \mathrm{L}\end{array}$ & $\begin{array}{l}0.0400 \\
0.0398 \\
0.0404 \\
\overline{0.040101} \\
0.00029 \\
0.72\end{array}$ & $\begin{array}{l}0.1932 \\
0.1911 \\
0.1933\end{array}$ & $\begin{array}{l}62.5237 \\
62.5225 \\
62.5234 \\
\end{array}$ & $\begin{array}{l}0.0406 \\
0.0404 \\
0.0410 \\
\end{array}$ & $\begin{array}{l}4.1683 \\
4.1682 \\
4.1682 \\
\end{array}$ & $\begin{array}{r}17: 07: 45 \\
17: 08: 14 \\
17: 08: 43 \\
\end{array}$ \\
\hline $\begin{array}{l}\text { Seq. No. } \\
\text { Sample ID: } \\
\text { Elem }\end{array}$ & $\begin{array}{c}29 \\
1105003-1112 \\
\text { SampleConc }\end{array}$ & $\begin{array}{l}\text { AS Loc: } \\
\text { StndConc }\end{array}$ & Blank Corr Signal & $\begin{array}{c}\text { Date: } \\
\text { Pk Area }\end{array}$ & $\begin{array}{l}11 / 17 / 05 \\
\text { BG Area }\end{array}$ & Pk Ht & BG Ht & Time \\
\hline $\begin{array}{l}\text { Mean:- - } \\
\text { SD: } \\
\text { \%RSD: }\end{array}$ & $\begin{array}{l}0.1390 \mu \mathrm{g} / \mathrm{L} \\
0.1417 \mu \mathrm{g} / \mathrm{L} \\
0.1485 \mu \mathrm{g} / \mathrm{L} \\
\overline{0} .1431 \mu \mathrm{g} / \mathrm{L} \\
0.0049 \mu \mathrm{g} / \mathrm{L} \\
3.43\end{array}$ & $\begin{array}{l}0.1390 \mu \mathrm{g} / \mathrm{L} \\
0.1417 \mu \mathrm{g} / \mathrm{L} \\
0.1485 \mu \mathrm{g} / \mathrm{L} \\
0.1431 \mu \mathrm{g} / \mathrm{L} \\
0.0049 \mu \mathrm{g} / \mathrm{L}\end{array}$ & $\begin{array}{l}0.0018 \\
0.0018 \\
0.0019 \\
0.0018 \\
0.00006 \\
3.43\end{array}$ & $\begin{array}{l}0.0110 \\
0.0117 \\
0.0123\end{array}$ & $\begin{array}{l}62.5216 \\
62.5216 \\
62.5214\end{array}$ & $\begin{array}{l}0.0024 \\
0.0024 \\
0.0025\end{array}$ & $\begin{array}{r}4.1681 \\
4.1681 \\
4.1681 \\
\end{array}$ & $\begin{array}{r}17: 10: 38 \\
17: 11: 07 \\
17: 11: 36 \\
\end{array}$ \\
\hline
\end{tabular}









\begin{tabular}{|c|c|c|c|c|c|c|c|c|}
\hline $\begin{array}{l}\text { Seq. No. } \\
\text { Sample ID: } \\
\text { Elem }\end{array}$ & $\begin{array}{c}35 \\
1105003-13 b \mathrm{msd} \\
\text { SampleConc }\end{array}$ & StndConc & Blank Corr Signal & $\begin{array}{l}\text { Date: } \\
\text { Pk Area }\end{array}$ & BG Area & Pk Ht & BG Ht & Time \\
\hline $\begin{array}{l}\mathrm{Hg} \\
\text { Mean:- - } \\
\text { SD: } \\
\text { \%RSD: }\end{array}$ & $\begin{array}{l}10.97 \mu \mathrm{g} / \mathrm{L} \\
11.01 \mu \mathrm{g} / \mathrm{L} \\
11.06 \mu \mathrm{g} / \mathrm{L} \\
11.01 \mu \mathrm{g} / \mathrm{L} \\
0.0437 \mu \mathrm{g} / \mathrm{L} \\
0.40\end{array}$ & $\begin{array}{c}10.97 \mu \mathrm{g} / \mathrm{L} \\
11.01 \mu \mathrm{g} / \mathrm{L} \\
11.06 \mu \mathrm{g} / \mathrm{L} \\
11.01 \mu \mathrm{g} / \mathrm{L} \\
0.0437 \mu \mathrm{g} / \mathrm{L}\end{array}$ & $\begin{array}{l}0.1405 \\
0.1410 \\
0.1416 \\
0.1411 \\
0.00056 \\
0.40\end{array}$ & $\begin{array}{l}0 \\
0 \\
0\end{array}$ & $\begin{array}{l}62 . \\
62 . \\
62 .\end{array}$ & $\begin{array}{l}16 \\
22 \\
\end{array}$ & $\begin{array}{l}77 \\
74 \\
72 \\
\end{array}$ & $\begin{array}{l}08 \\
38 \\
07\end{array}$ \\
\hline $\begin{array}{l}\text { eq. No. } \\
\text { ample ID: } \\
\text { lem }\end{array}$ & $\begin{array}{c}36 \\
1105003-14 \\
\text { SampleConc }\end{array}$ & $\begin{array}{r}\text { AS Loc: } \\
\text { StndConc }\end{array}$ & Blank Corr Signal & Pk Area & BG Area & Pk Ht & BG Ht & Time \\
\hline $\begin{array}{l}\mathrm{Hg} \\
\text { Mean:- } \\
\text { SD: } \\
\text { \%RSD: }\end{array}$ & $\begin{array}{l}-0.0193 \mu \mathrm{g} / \mathrm{L} \\
-0.0117 \mu \mathrm{g} / \mathrm{L} \\
-0.0182 \mu \mathrm{g} / \mathrm{L} \\
-0.016 \overline{4} \mu \mathrm{g} / \mathrm{L} \\
0.0041 \mu \mathrm{g} / \mathrm{L} \\
25.20\end{array}$ & $\begin{array}{c}-0.0193 \mu \mathrm{g} / \mathrm{L} \\
-0.0117 \mu \mathrm{g} / \mathrm{L} \\
-0.0182 \mu \mathrm{g} / \mathrm{L} \\
-0.0164 \mu \mathrm{g} / \mathrm{L} \\
0.0041 \mu \mathrm{g} / \mathrm{L}\end{array}$ & $\begin{array}{c}-0.0002 \\
-0.0001 \\
-0.0002 \\
-0.0002 \\
0.00005 \\
25.20\end{array}$ & $\begin{array}{r}0.0016 \\
0.0028 \\
0.0019 \\
-\quad-\quad\end{array}$ & $\begin{array}{r}62.5086 \\
62.5087 \\
62.5076 \\
\end{array}$ & $\begin{array}{l}0.0004 \\
0.0005 \\
0.0004\end{array}$ & $\begin{array}{r}4.1672 \\
4.1672 \\
4.1672 \\
-\end{array}$ & $\begin{array}{r}17: 31: 03 \\
17: 31: 33 \\
17: 32: 01 \\
-\end{array}$ \\
\hline $\begin{array}{l}\text { Seq. No. } \\
\text { Sample ID: } \\
\text { Elem }\end{array}$ & $\begin{array}{c}37 \\
1105003-15 \\
\text { SampleConc }\end{array}$ & $\begin{array}{l}\text { AS Loc: } \\
\text { StndConc }\end{array}$ & Blank Corr Signal & $\begin{array}{c}\text { Date: } \\
\text { Pk Area }\end{array}$ & BG Area & Pk Ht & BG Ht & Time \\
\hline $\begin{array}{l}\text { Hg } \\
\text { Mean: - } \\
\text { SD: } \\
\text { \%RSD: }\end{array}$ & $\begin{array}{l}4.262 \mu \mathrm{g} / \mathrm{L} \\
4.265 \mu \mathrm{g} / \mathrm{L} \\
4.290 \mu \mathrm{g} / \mathrm{L} \\
4.27 \overline{2} \mu \mathrm{g} / \mathrm{L} \\
0.0155 \mu \mathrm{g} / \mathrm{L} \\
0.36\end{array}$ & $\begin{array}{c}4.262 \mu \mathrm{g} / \mathrm{L} \\
4.265 \mu \mathrm{g} / \mathrm{L} \\
4.290 \mu \mathrm{g} / \mathrm{L} \\
4.272 \mu \mathrm{g} / \mathrm{L} \\
0.0155 \mu \mathrm{g} / \mathrm{L}\end{array}$ & $\begin{array}{l}0.0546 \\
0.0546 \\
0.0549 \\
0.0547 \\
0.00020 \\
0.36\end{array}$ & $\begin{array}{l}0.2616 \\
0.2606 \\
0.2609\end{array}$ & $\begin{array}{l}62.5051 \\
62.5031 \\
62.5039\end{array}$ & & $\begin{array}{r}4.1670 \\
4.1669 \\
4.1669 \\
\end{array}$ & $\begin{array}{r}17: 33: 59 \\
17: 34: 28 \\
17: 34: 57 \\
\end{array}$ \\
\hline $\begin{array}{l}\text { Seq. No. } \\
\text { Sample ID: } \\
\text { Elem }\end{array}$ & $\begin{array}{c}38 \\
1105003-1617 \\
\text { SampleConc }\end{array}$ & $\begin{array}{l}\text { AS Loc: } \\
\text { StndConc }\end{array}$ & Blank Corr Signal & $\begin{array}{l}\text { Date: } \\
\text { Pk Area }\end{array}$ & BG Area & Pk Ht & BG Ht & Time \\
\hline $\begin{array}{l}\text { Hg } \\
\text { Mean:- } \\
\text { SD: } \\
\text { \%RSD: }\end{array}$ & $\begin{array}{l}8.478 \mu \mathrm{g} / \mathrm{L} \\
8.470 \mu \mathrm{g} / \mathrm{L} \\
8.562 \mu \mathrm{g} / \mathrm{L} \\
-8.50 \overline{3 \mu \mathrm{g} / \mathrm{L}} \\
0.0509 \mu \mathrm{g} / \mathrm{L} \\
0.60\end{array}$ & $\begin{array}{c}8.478 \mu \mathrm{g} / \mathrm{L} \\
8.470 \mu \mathrm{g} / \mathrm{L} \\
8.562 \mu \mathrm{g} / \mathrm{L} \\
8.5 \overline{0} \mu \bar{g} / \mathrm{L} \\
0.0509 \mu \mathrm{g} / \mathrm{L}\end{array}$ & $\begin{array}{l}0.1086 \\
0.1085 \\
0.1097 \\
-\overline{0.1089} \\
0.00065 \\
0.60\end{array}$ & $\begin{array}{l}0.5 \\
0.5 \\
0.5\end{array}$ & $\begin{array}{l}62 . \\
62 . \\
62 .\end{array}$ & $\begin{array}{l}092 \\
091 \\
103 \\
\end{array}$ & $\begin{array}{l}668 \\
667 \\
666 \\
-\end{array}$ & $\begin{array}{r}17 \\
17 \\
-\quad 17 \\
-\end{array}$ \\
\hline $\begin{array}{l}\text { Seq. No. } \\
\text { Sample ID: } \\
\text { Elem }\end{array}$ & $\begin{array}{c}39 \\
1105003-18 a \\
\text { SampleConc }\end{array}$ & $\begin{array}{l}\text { AS Loc: } \\
\text { StndConc }\end{array}$ & Blank Corr Signal & $\begin{array}{c}\text { Date: } \\
\text { Pk Area }\end{array}$ & BG Area & Pk Ht & BG Ht & Time \\
\hline $\begin{array}{l}\text { Meān: } \\
\text { SD: } \\
\text { \%RSD: }\end{array}$ & $\begin{array}{l}0.0721 \mu \mathrm{g} / \mathrm{L} \\
0.0736 \mu \mathrm{g} / \mathrm{L} \\
0.0729 \mu \mathrm{g} / \mathrm{L} \\
0.0729 \mu \mathrm{g} / \mathrm{L} \\
0.0008 \mu \mathrm{g} / \mathrm{L} \\
1.09\end{array}$ & $\begin{array}{l}0.0721 \mu \mathrm{g} / \mathrm{L} \\
0.0736 \mu \mathrm{g} / \mathrm{L} \\
0.0729 \mu \mathrm{g} / \mathrm{L} \\
0.0729 \mu \mathrm{g} / \mathrm{L} \\
0.0008 \mu \mathrm{g} / \mathrm{L}\end{array}$ & $\begin{array}{l}0.0009 \\
0.0009 \\
0.0009 \\
0.0009 \\
0.00001 \\
1.09\end{array}$ & $\begin{array}{l}0.0077 \\
0.0076 \\
0.0078\end{array}$ & $\begin{array}{l}62.4993 \\
62.4985 \\
62.5005\end{array}$ & $\begin{array}{l}0.0015 \\
0.0015 \\
0.0015\end{array}$ & $\begin{array}{r}4.1666 \\
4.1666 \\
4.1667 \\
-\quad-\quad-\end{array}$ & $\begin{array}{l}17: 39: 40 \\
17: 40: 09 \\
17: 40: 38\end{array}$ \\
\hline
\end{tabular}




\begin{tabular}{|c|c|c|c|c|c|c|c|c|}
\hline $\begin{array}{l}\text { Seq. No. } \\
\text { Sample ID: } \\
\text { Elem }\end{array}$ & $\begin{array}{c}40 \\
1105003-18 b \\
\text { SampleConc }\end{array}$ & $\begin{array}{l}\text { AS Loc: } \\
\text { StndConc }\end{array}$ & $\begin{array}{l}34 \\
\text { Blank Corr Signal }\end{array}$ & $\begin{array}{c}\text { Date: } \\
\text { Pk Area }\end{array}$ & BG Area & Pk Ht & BG Ht & Time \\
\hline $\begin{array}{l}\text { Hg } \\
\text { Mean: - } \\
\text { SD: } \\
\text { \%RSD: }\end{array}$ & $\begin{array}{l}0.0065 \mu \mathrm{g} / \mathrm{L} \\
0.0049 \mu \mathrm{g} / \mathrm{L} \\
0.0084 \mu \mathrm{g} / \mathrm{L} \\
0.006 \overline{\mathrm{L}} \mu \mathrm{g} / \mathrm{L} \\
0.0017 \mu \mathrm{g} / \mathrm{L} \\
26.39\end{array}$ & $\begin{array}{c}0.0065 \mu \mathrm{g} / \mathrm{L} \\
0.0049 \mu \mathrm{g} / \mathrm{L} \\
0.0084 \mu \mathrm{g} / \mathrm{L} \\
0.0066 \mu \mathrm{g} / \mathrm{L} \\
0.0017 \mu \mathrm{g} / \mathrm{L}\end{array}$ & $\begin{array}{l}0.0001 \\
0.0001 \\
0.0001 \\
0.0001 \\
0.00002 \\
26.39\end{array}$ & $\begin{array}{l}0.0036 \\
0.0034 \\
0.0039\end{array}$ & $\begin{array}{l}62.4996 \\
62.4995 \\
62.4994 \\
\ldots \ldots\end{array}$ & $\begin{array}{l}0.0007 \\
0.0007 \\
0.0007 \\
-\quad-\quad-\end{array}$ & $\begin{array}{l}4.1666 \\
4.1666 \\
4.1666 \\
\end{array}$ & $\begin{array}{r}17: 42: 29 \\
17: 42: 58 \\
17: 43: 27 \\
-\end{array}$ \\
\hline $\begin{array}{l}\text { Seq. No. } \\
\text { Sample ID: } \\
\text { Elem }\end{array}$ & $\begin{array}{c}41 \\
1105003-19 \\
\text { SampleConc }\end{array}$ & $\begin{array}{l}\text { AS Loc: } \\
\text { StndConc }\end{array}$ & $\begin{array}{c}35 \\
\text { Blank Corr Signal }\end{array}$ & $\begin{array}{l}\text { Date: } \\
\text { Pk Area }\end{array}$ & BG Area & Pk Ht & BG Ht & Time \\
\hline $\begin{array}{l}\text { Hg } \\
\text { Mean:- - } \\
\text { SD: } \\
\text { \%RSD: }\end{array}$ & $\begin{array}{l}-0.0426 \mu \mathrm{g} / \mathrm{L} \\
-0.0424 \mu \mathrm{g} / \mathrm{L} \\
-0.0401 \mu \mathrm{g} / \mathrm{L} \\
-0.0417 \mu \mathrm{g} / \mathrm{L} \\
0.0014 \mu \mathrm{g} / \mathrm{L} \\
3.31\end{array}$ & $\begin{array}{c}-0.0426 \mu \mathrm{g} / \mathrm{L} \\
-0.0424 \mu \mathrm{g} / \mathrm{L} \\
-0.0401 \mu \mathrm{g} / \mathrm{L} \\
-0.0417 \mu \mathrm{g} / \mathrm{L} \\
0.0014 \mu \mathrm{g} / \mathrm{L}\end{array}$ & $\begin{array}{l}-0.0005 \\
-0.0005 \\
-0.0005 \\
-\overline{-0.0005}-\ldots \\
0.00002 \\
3.31\end{array}$ & $\begin{array}{l}0.0005 \\
0.0006 \\
0.0007\end{array}$ & $\begin{array}{l}62.4976 \\
62.4973 \\
62.4976 \\
-\end{array}$ & $\begin{array}{l}0.0001 \\
0.0001 \\
0.0001 \\
-+-\end{array}$ & $\begin{array}{l}4.1665 \\
4.1665 \\
4.1665 \\
-\end{array}$ & $\begin{array}{r}17: 45: 20 \\
17: 45: 49 \\
17: 46: 18 \\
\end{array}$ \\
\hline $\begin{array}{l}\text { Seq. No. } \\
\text { Sample ID: } \\
\text { Elem }\end{array}$ & $\begin{array}{c}42 \\
1105003-19 \mathrm{~ms} \\
\text { SampleConc }\end{array}$ & $\begin{array}{l}\text { AS Loc: } \\
\text { StndConc }\end{array}$ & $\begin{array}{c}36 \\
\text { Blank Corr Signal }\end{array}$ & $\begin{array}{c}\text { Date: } \\
\text { Pk Area }\end{array}$ & $11 / 17 / 05$ & Pk Ht & BG Ht & Time \\
\hline $\begin{array}{l}\text { Hg } \\
\text { Mean:- - } \\
\text { SD: } \\
\text { \%RSD: }\end{array}$ & $\begin{array}{l}10.52 \mu \mathrm{g} / \mathrm{L} \\
10.44 \mu \mathrm{g} / \mathrm{L} \\
10.50 \mu \mathrm{g} / \mathrm{L} \\
-1 \overline{0.49 \mu \mathrm{g} / \mathrm{L}}-- \\
0.0401 \mu \mathrm{g} / \mathrm{L} \\
0.38\end{array}$ & $\begin{array}{c}10.52 \mu \mathrm{g} / \mathrm{L} \\
10.44 \mu \mathrm{g} / \mathrm{L} \\
10.50 \mu \mathrm{g} / \mathrm{L} \\
-\overline{10} . \overline{49} \mu \mathrm{g} / \mathrm{L} \\
0.0401 \mu \mathrm{g} / \mathrm{L}\end{array}$ & $\begin{array}{l}0.1347 \\
0.1338 \\
0.1345 \\
\overline{0.1343}-- \\
0.00051 \\
0.38\end{array}$ & $\begin{array}{r}0.6338 \\
0.6305 \\
0.6308 \\
-\end{array}$ & $\begin{array}{l}62.4962 \\
62.4927 \\
62.4930 \\
\end{array}$ & $\begin{array}{l}0.1353 \\
0.1344 \\
0.1351 \\
-\quad-\quad\end{array}$ & $\begin{array}{l}4.1664 \\
4.1662 \\
4.1662 \\
\end{array}$ & $\begin{array}{r}17: 48: 14 \\
17: 48: 44 \\
17: 49: 13 \\
\end{array}$ \\
\hline $\begin{array}{l}\text { Seq. No. } \\
\text { Sample ID: } \\
\text { Elem }\end{array}$ & $\begin{array}{c}43 \\
1105003-19 \mathrm{msd} \\
\text { SampleConc }\end{array}$ & $\begin{array}{l}\text { AS Loc: } \\
\text { StndConc }\end{array}$ & Blank Corr Signal & $\begin{array}{c}\text { Date: } \\
\text { Pk Area }\end{array}$ & $\begin{array}{l}11 / 17 / 05 \\
\text { BG Area }\end{array}$ & Pk Ht & BG Ht & Time \\
\hline $\begin{array}{l}\mathrm{Hg} \\
\text { Mean: } \\
\text { SD: } \\
\text { \%RSD: }\end{array}$ & $\begin{array}{l}10.19 \mu \mathrm{g} / \mathrm{L} \\
10.31 \mu \mathrm{g} / \mathrm{L} \\
10.28 \mu \mathrm{g} / \mathrm{L} \\
10.26 \mu \mathrm{g} / \mathrm{L} \\
0.0630 \mu \mathrm{g} / \mathrm{L} \\
0.61\end{array}$ & $\begin{array}{c}10.19 \mu \mathrm{g} / \mathrm{L} \\
10.31 \mu \mathrm{g} / \mathrm{L} \\
10.28 \mu \mathrm{g} / \mathrm{L} \\
10.26 \mu \mathrm{g} / \mathrm{L} \\
0.0630 \mu \mathrm{g} / \mathrm{L}\end{array}$ & $\begin{array}{l}0.1305 \\
0.1320 \\
0.1317 \\
0.1314 \\
0.00081 \\
0.61\end{array}$ & $\begin{array}{l}0.6140 \\
0.6172 \\
0.6149\end{array}$ & $\begin{array}{l}62.4987 \\
62.4961 \\
62.4965\end{array}$ & $\begin{array}{l}0.1311 \\
0.1327 \\
0.1323\end{array}$ & $\begin{array}{l}4.1666 \\
4.1664 \\
4.1664 \\
-\quad-\quad\end{array}$ & $\begin{array}{r}17: 51: 15 \\
17: 51: 44 \\
17: 52: 13 \\
\end{array}$ \\
\hline $\begin{array}{l}\text { Seq. No. } \\
\text { Sample ID: } \\
\text { Elem }\end{array}$ & $\begin{array}{c}44 \\
1105003-20 \\
\text { SampleConc }\end{array}$ & $\begin{array}{l}\text { AS Loc: } \\
\text { StndConc }\end{array}$ & Blank Corr Signal & $\begin{array}{l}\text { Date: } \\
\text { Pk Area }\end{array}$ & $\begin{array}{l}11 / 17 / 05 \\
\text { BG Area }\end{array}$ & Pk Ht & BG Ht & Time \\
\hline $\begin{array}{l}\text { Mean: - - } \\
\text { SD: } \\
\text { \%RSD: }\end{array}$ & $\begin{array}{l}0.2874 \mu \mathrm{g} / \mathrm{L} \\
0.2886 \mu \mathrm{g} / \mathrm{L} \\
0.2884 \mu \mathrm{g} / \mathrm{L} \\
\overline{0} . \overline{28} 8 \overline{2} \mu \mathrm{g} / \mathrm{L} \\
0.0007 \mu \mathrm{g} / \mathrm{L} \\
0.23\end{array}$ & $\begin{array}{l}0.2874 \mu \mathrm{g} / \mathrm{L} \\
0.2886 \mu \mathrm{g} / \mathrm{L} \\
0.2884 \mu \mathrm{g} / \mathrm{L} \\
0.2882 \mu \mathrm{g} / \mathrm{L} \\
0.0007 \mu \mathrm{g} / \mathrm{L}\end{array}$ & $\begin{array}{l}0.0037 \\
0.0037 \\
0.0037 \\
0.0037 \\
0.00001 \\
0.23\end{array}$ & $\begin{array}{l}0.0203 \\
0.0209 \\
0.0210\end{array}$ & $\begin{array}{l}62.5019 \\
62.5010 \\
62.5018\end{array}$ & $\begin{array}{l}0.0043 \\
0.0043 \\
0.0043\end{array}$ & $\begin{array}{l}4.1668 \\
4.1667 \\
4.1668 \\
\end{array}$ & $\begin{array}{r}17: 54: 06 \\
17: 54: 35 \\
17: 55: 04 \\
-\quad-\quad-\end{array}$ \\
\hline
\end{tabular}




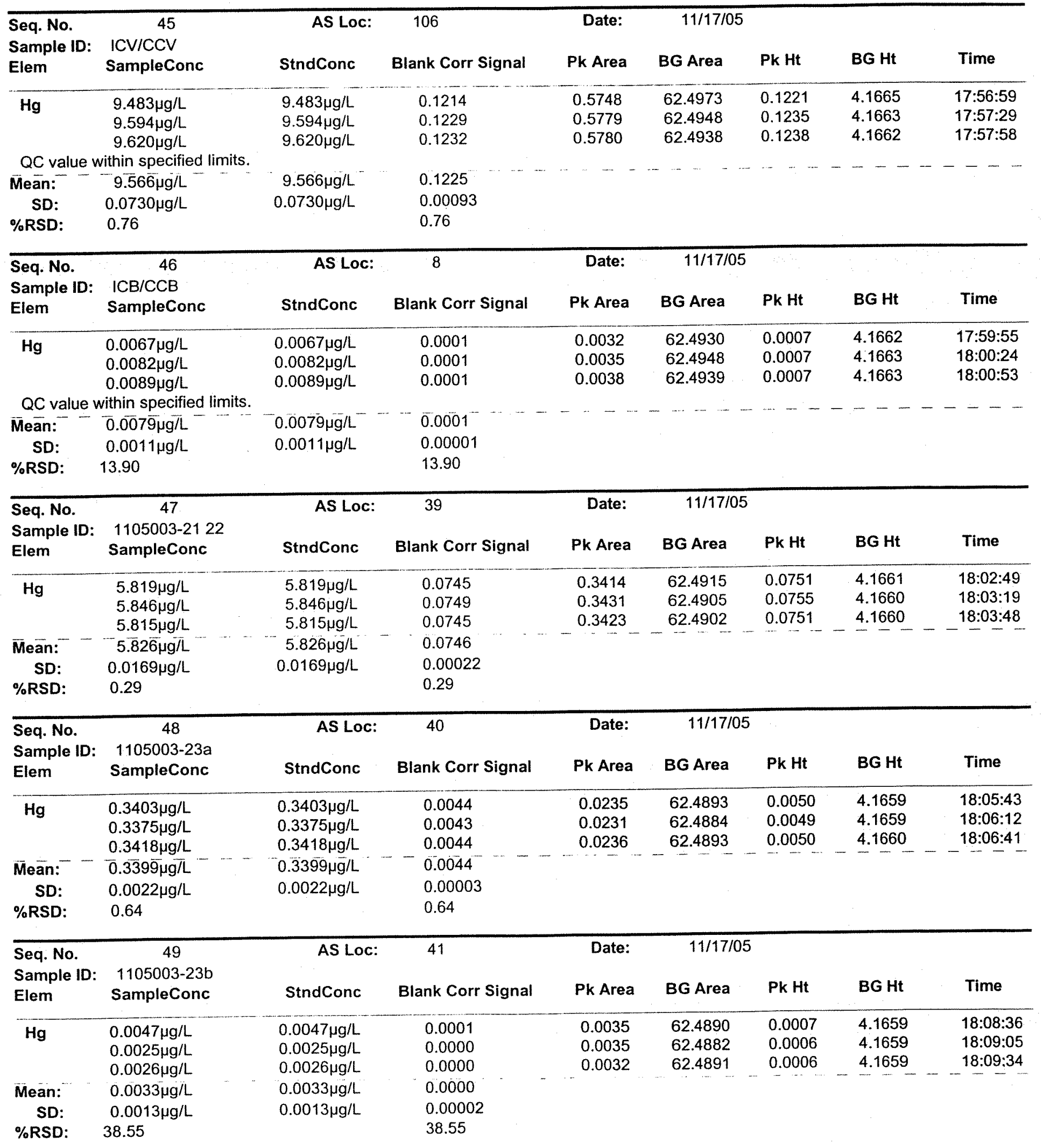




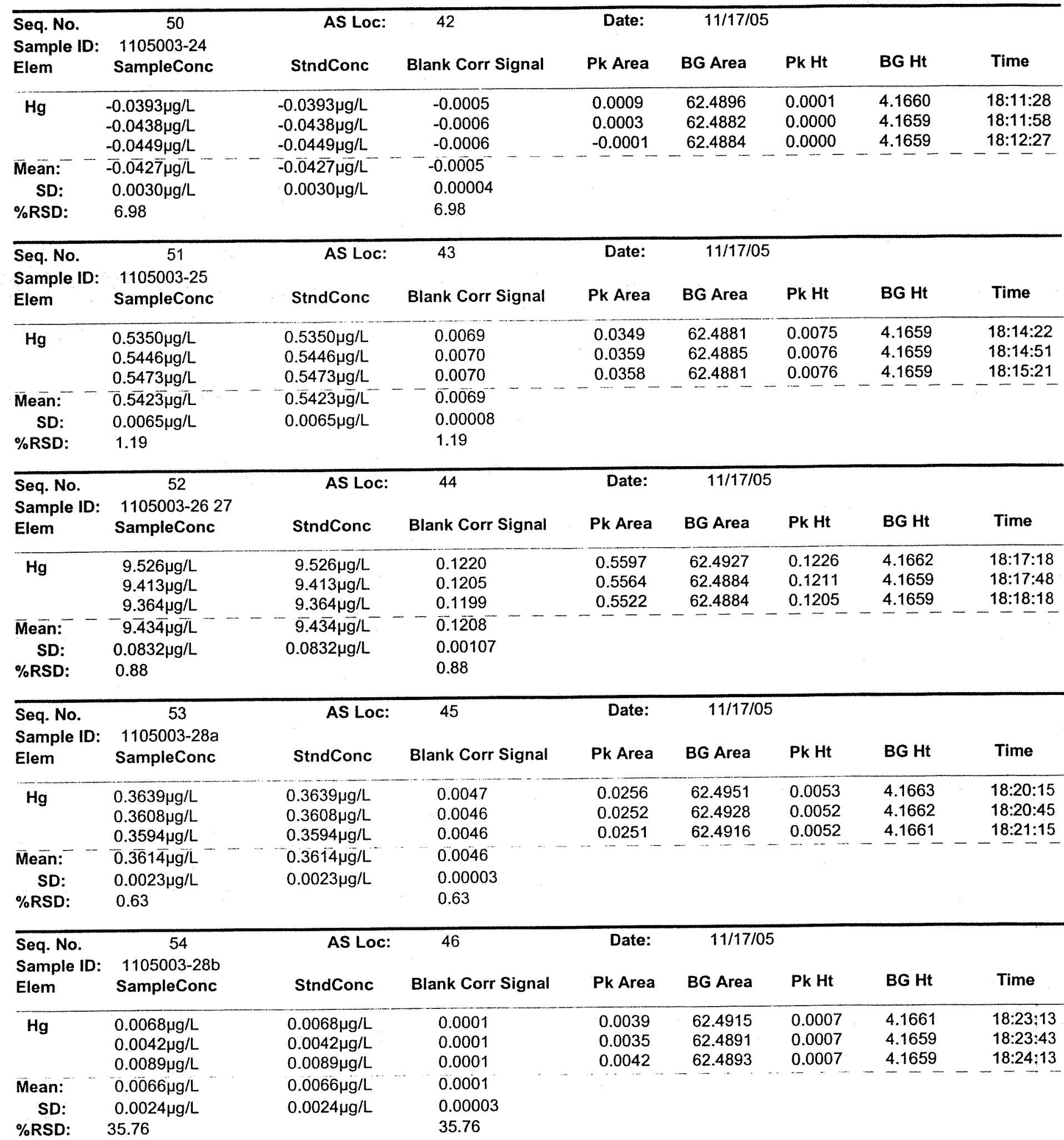




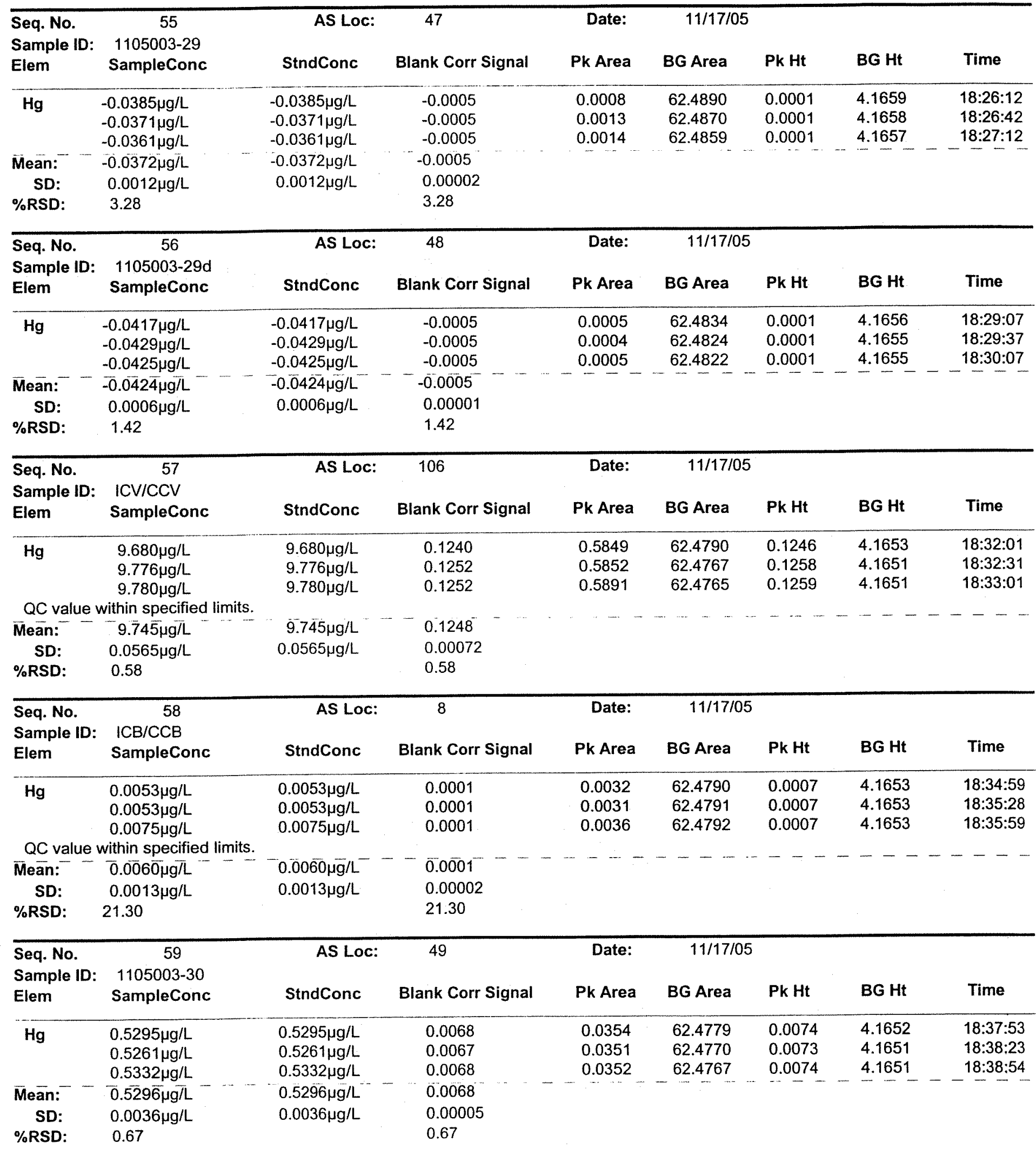




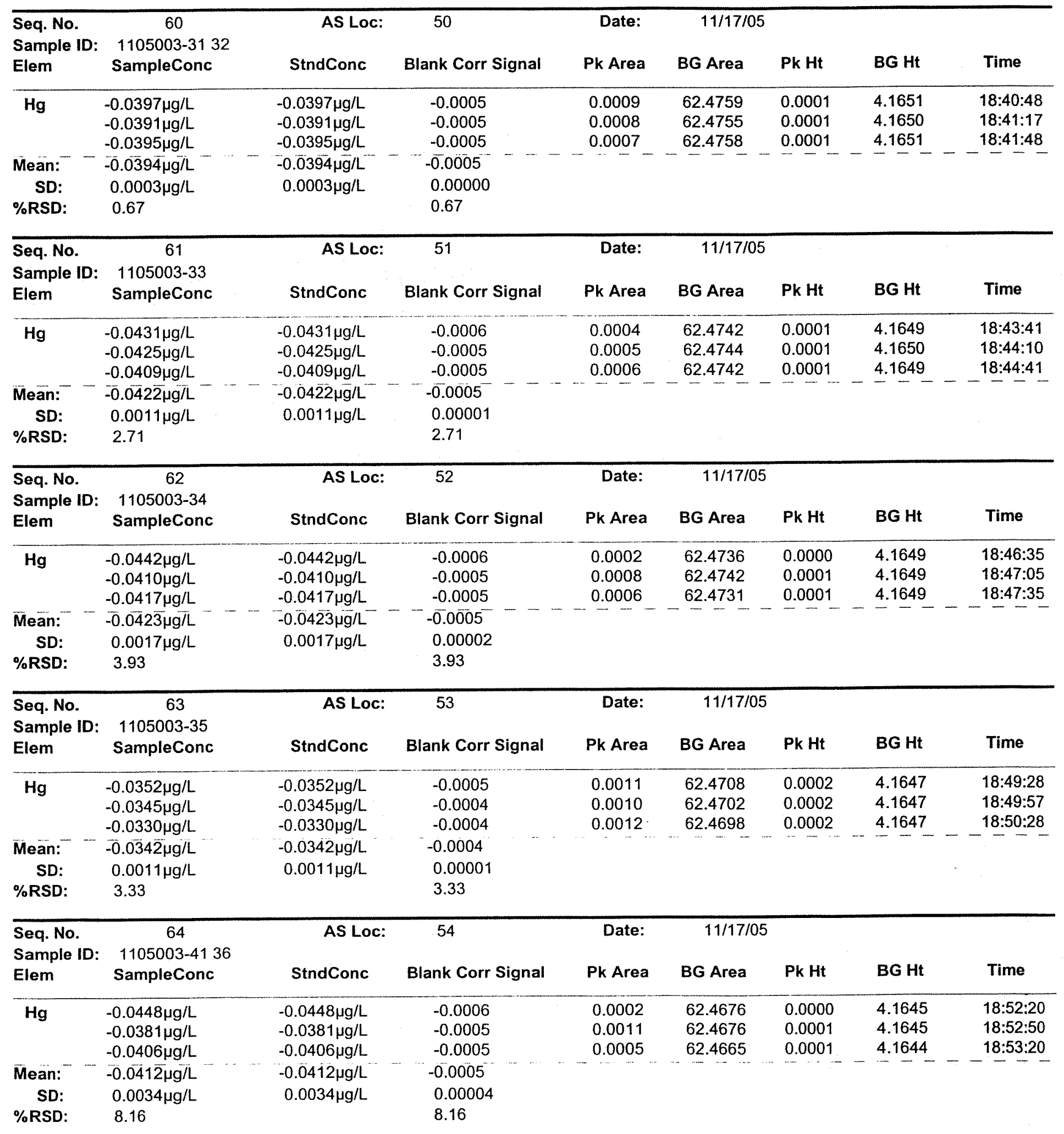




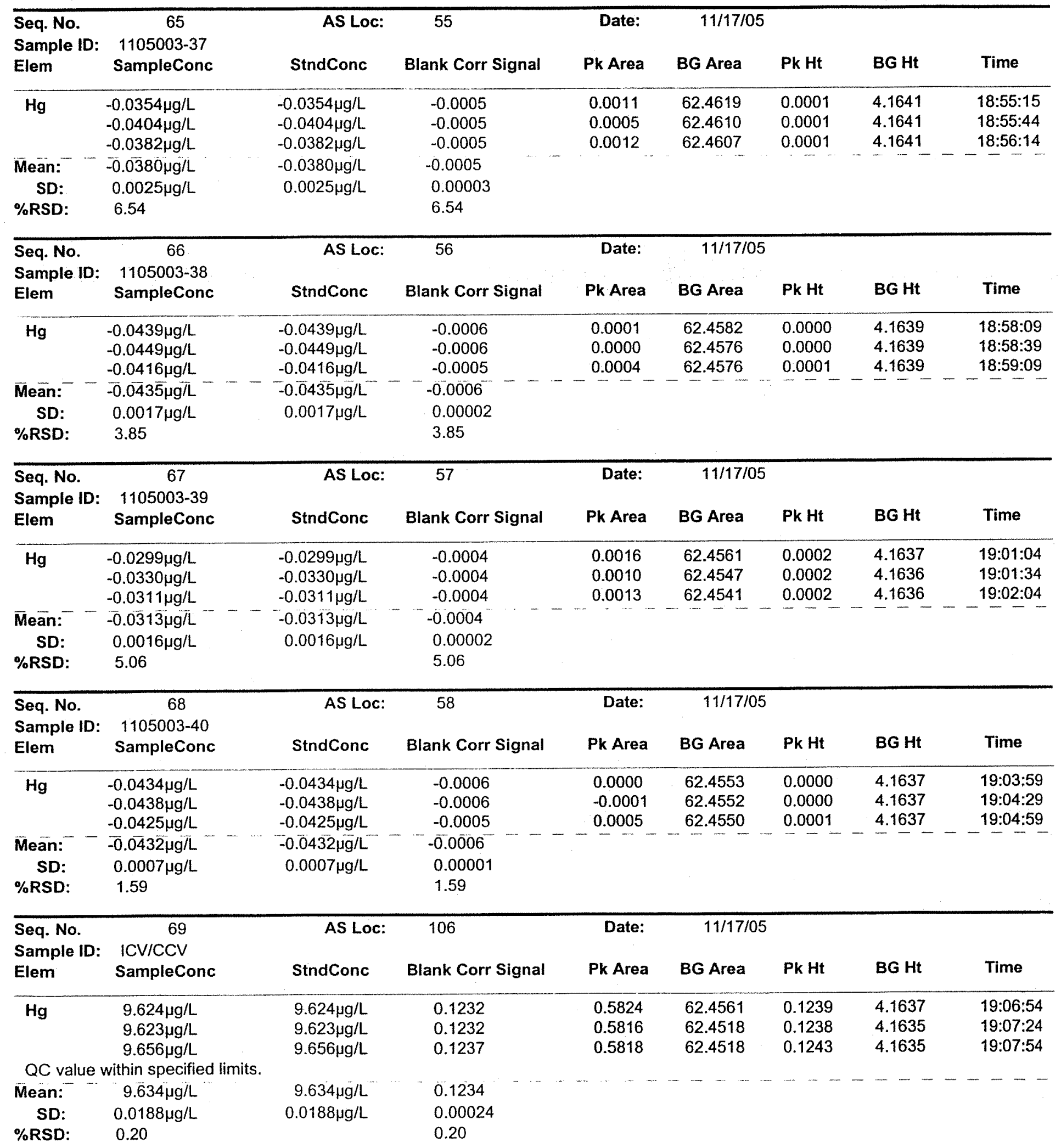




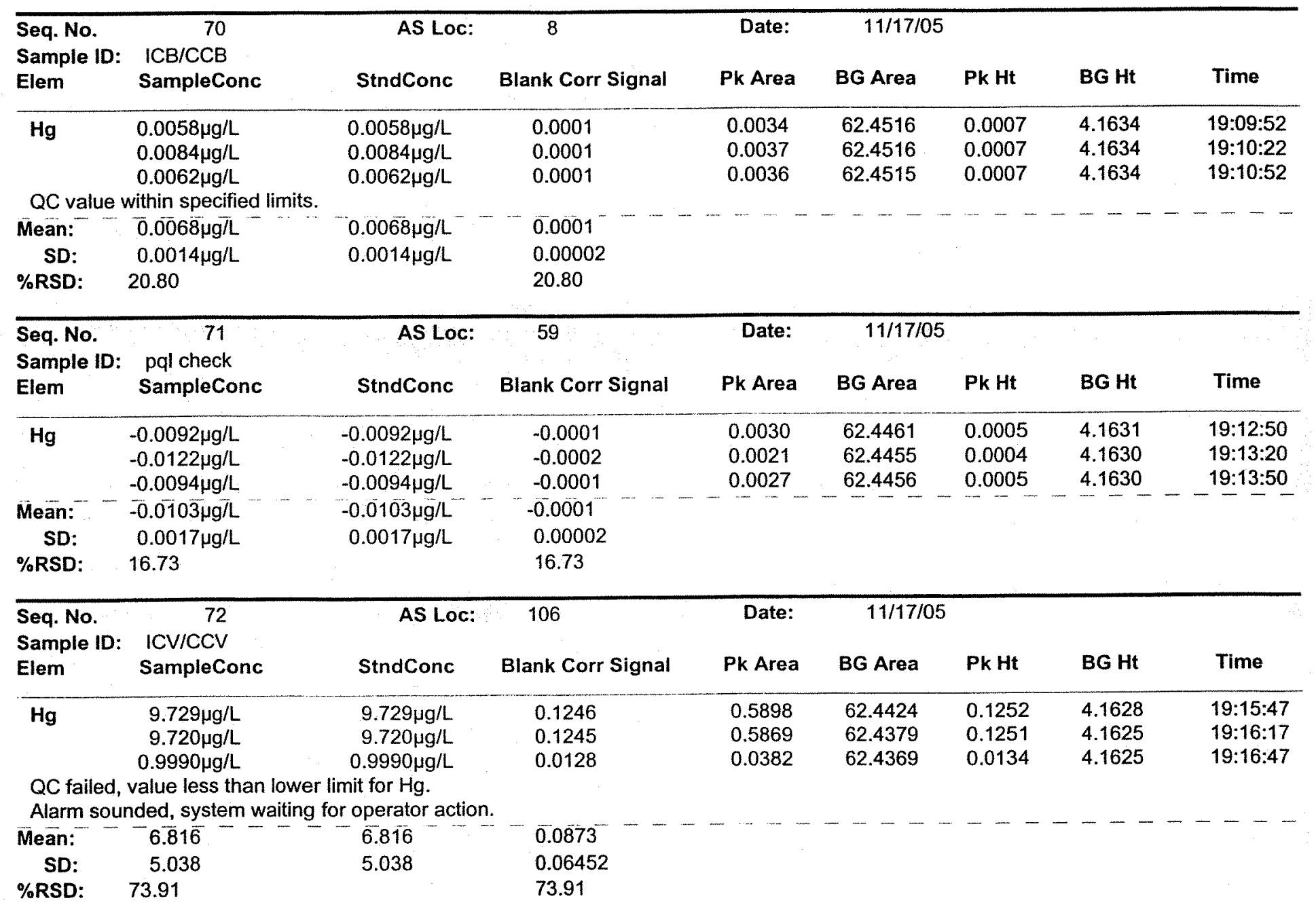




\section{DOCUMENTATION}

Page 28 of 37

E-114 


\section{DAT SAMPLE RECEIVING}

7715 Corporate Blvd. Plain City. $\mathrm{OH} 43064$

Project Number: 1105003

Date Received: $11 / 1 / 2005$

Client Name: Metco Environmental

Tracking number: 602557620651

Custody Seals ?: No
Carrier: FedEx

Analysis: .... Mercury $(\mathrm{Hg}) / O n t a r i o \mathrm{Hy}$

Package Temp: ... ambient

COC: $\quad \forall$ check if COC from client

\section{Sample Information}

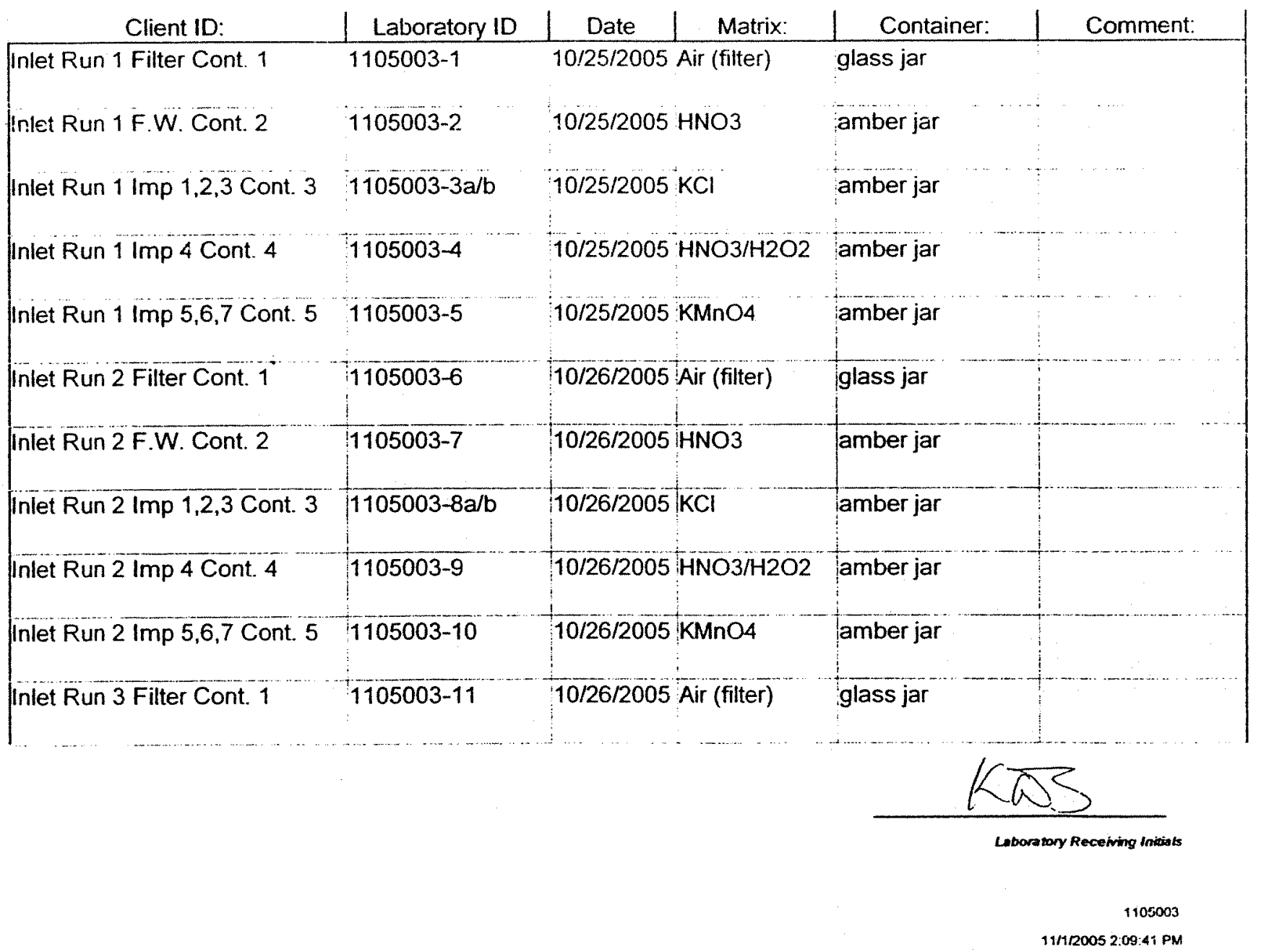

Page 29 of 37 


\section{DAT SAMPLE RECEIVING}

7715 Corporate Blvd. Plain City.OH 43064

Project Number: 1105003

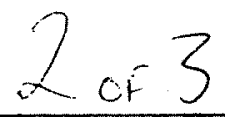

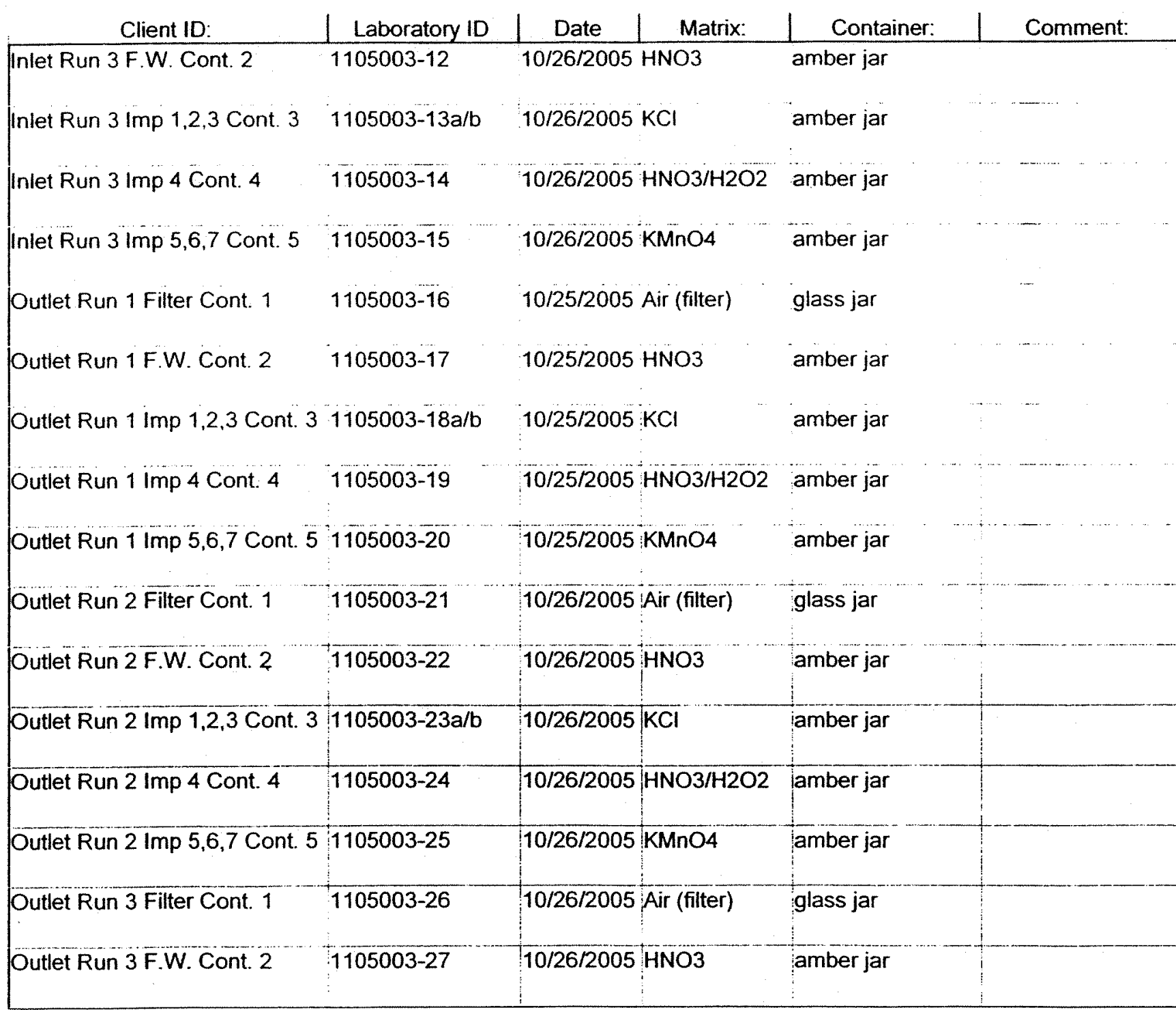

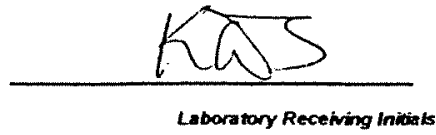




\section{DAT SAMPLE RECEIVING}

7715 Corporate Blvd. Plain City. OH 43064.

Project Number: 1105003

$=05 S$

\begin{tabular}{|c|c|c|c|c|c|}
\hline Client ID: & Laboratory ID & Date & Matrix: & Container: & Comment: \\
\hline Outlet Run 3 Imp 1,2,3 Cont. 3 & $1105003-28 \mathrm{a} / \mathrm{b}$ & $10 / 26 / 2005 r$ & $\mathrm{KCl}$ & amber jar & \\
\hline Outlet Run 3 imp 4 Cont. 4 & $1105003-29$ & $10 / 26 / 2005$ & $\mathrm{HNO} 3 / \mathrm{H} 2 \mathrm{O} 2$ & amber jar & \\
\hline Outlet Run 3 Imp 5,6,7 Cont. 5 & $1105003-30$ & $10 / 26 / 2005$ & $\mathrm{KMnO4}$ & amber jar & \\
\hline BT Filter Cont. 1 & $1105003-31$ & $10 / 26 / 2005$ & Air (filter) & glass jar & \\
\hline BT F.W. Cont. 2 & $1105003-32$ & $10 / 26 / 2005$ & HNO3 & amber jar & \\
\hline BT Imp $1,2,3$ Cont. 3 & $1105003-33$ & $10 / 26 / 2005$ & $\mathrm{KCl}$ & amber jar & \\
\hline BT Imp 4 Cont. 4 & $1105003-34$ & $10 / 26 / 2005$ & $\mathrm{HNO} / \mathrm{H} 2 \mathrm{O} 2$ & amber jar & \\
\hline BT Imp 5,6,7 Cont. 5 & $1105003-35$ & $10 / 26 / 2005$ & $\mathrm{KMnO} 4$ & lamber jar & \\
\hline RB $0.1 \mathrm{~N} \mathrm{HNO} 3$ Cont. 7 & $1105003-36$ & $10 / 26 / 2005$ & $\mathrm{HNO} 3$ & amber jar & \\
\hline RB $1 \mathrm{~N}$ KCl Cont. 8 & $1105003-37$ & $10 / 26 / 2005$ & $\mathrm{KCl}$ & amber jar & \\
\hline RB HNO3/H2O2 Cont. 9. & $1105003-38$ & $10 / 26 / 2005$ & $\mathrm{HNO} / \mathrm{H} 2 \mathrm{O} 2$ & amber jar & \\
\hline RB KMnO4 Cont. 10 & $1105003-39$ & $10 / 26 / 2005$ & $\mathrm{KMnO4}$ & amber jar & \\
\hline RB Hydroxylamine Cont. 11 & $1105003-40$ & $10 / 26 / 2005$ & hydrox.amine & amber jar & \\
\hline RB Filter Cont. 12 & $1105003-41$ & $10 / 26 / 2005$ & Air (filter) & glass jar & \\
\hline
\end{tabular}

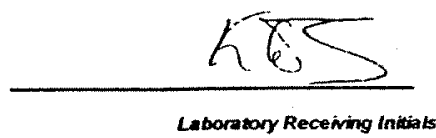




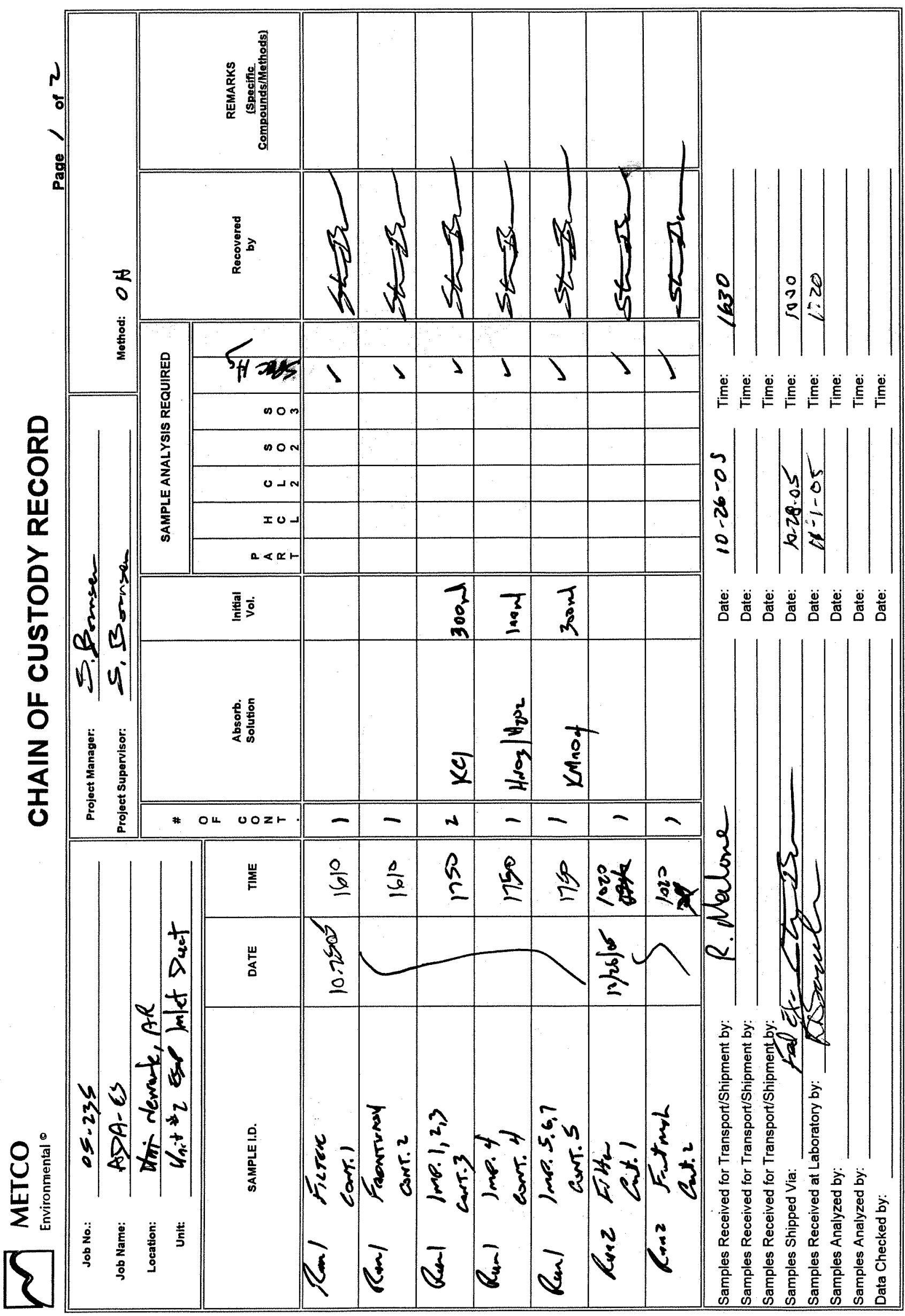

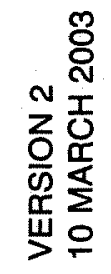

Page 32 of 37 


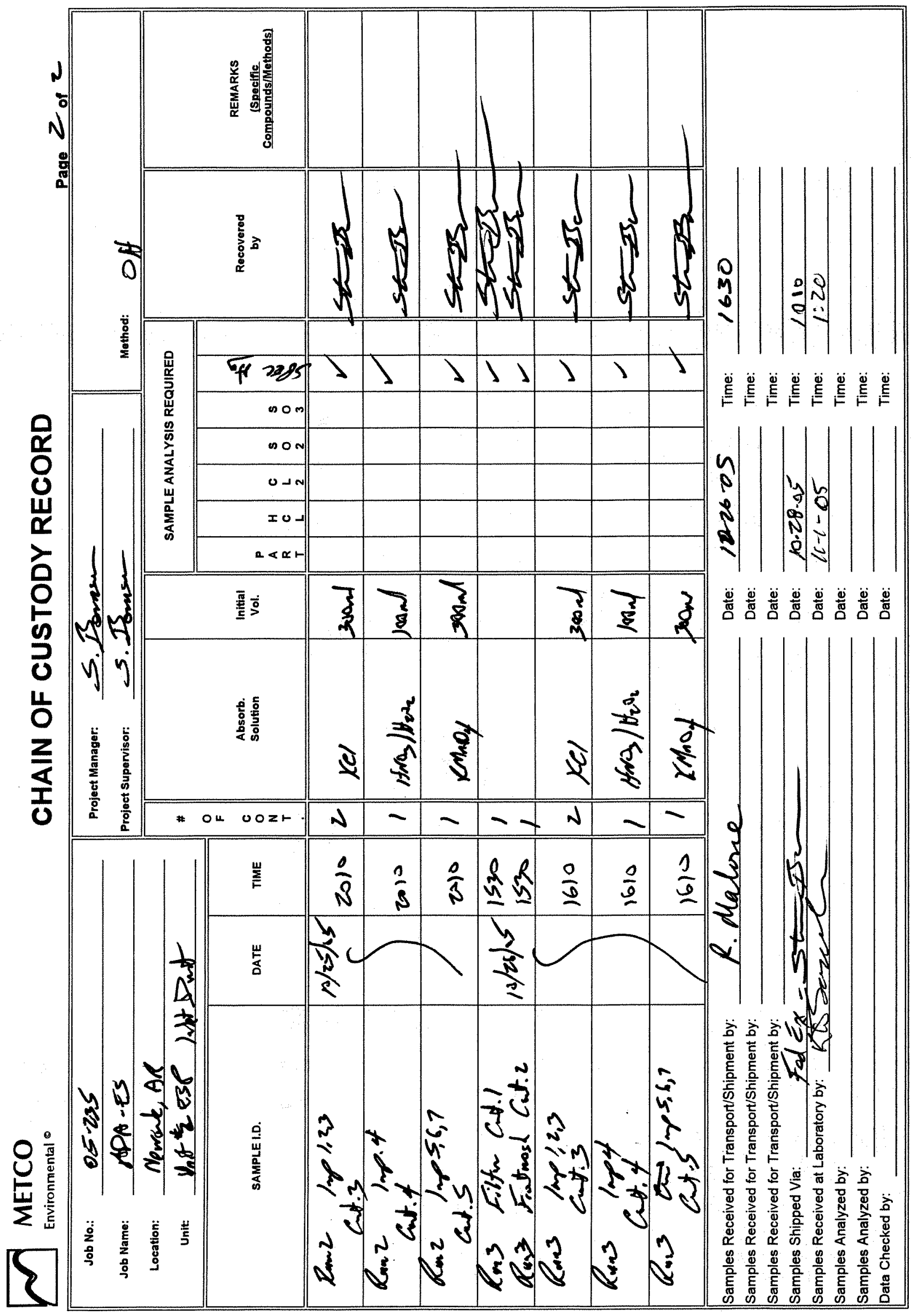

Page 33 of 37 


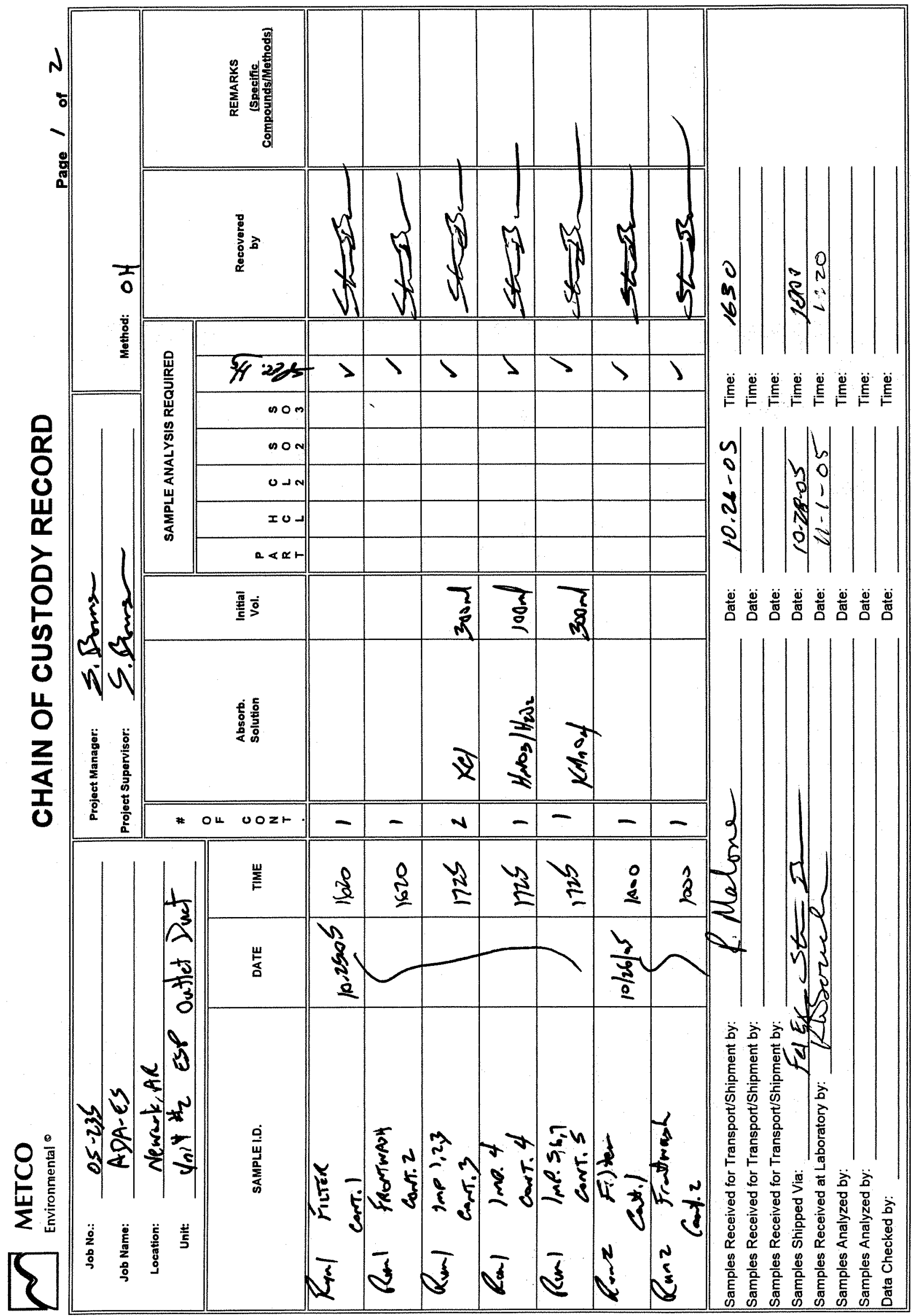

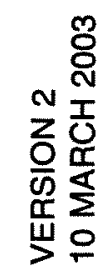

Page 34 of 37 


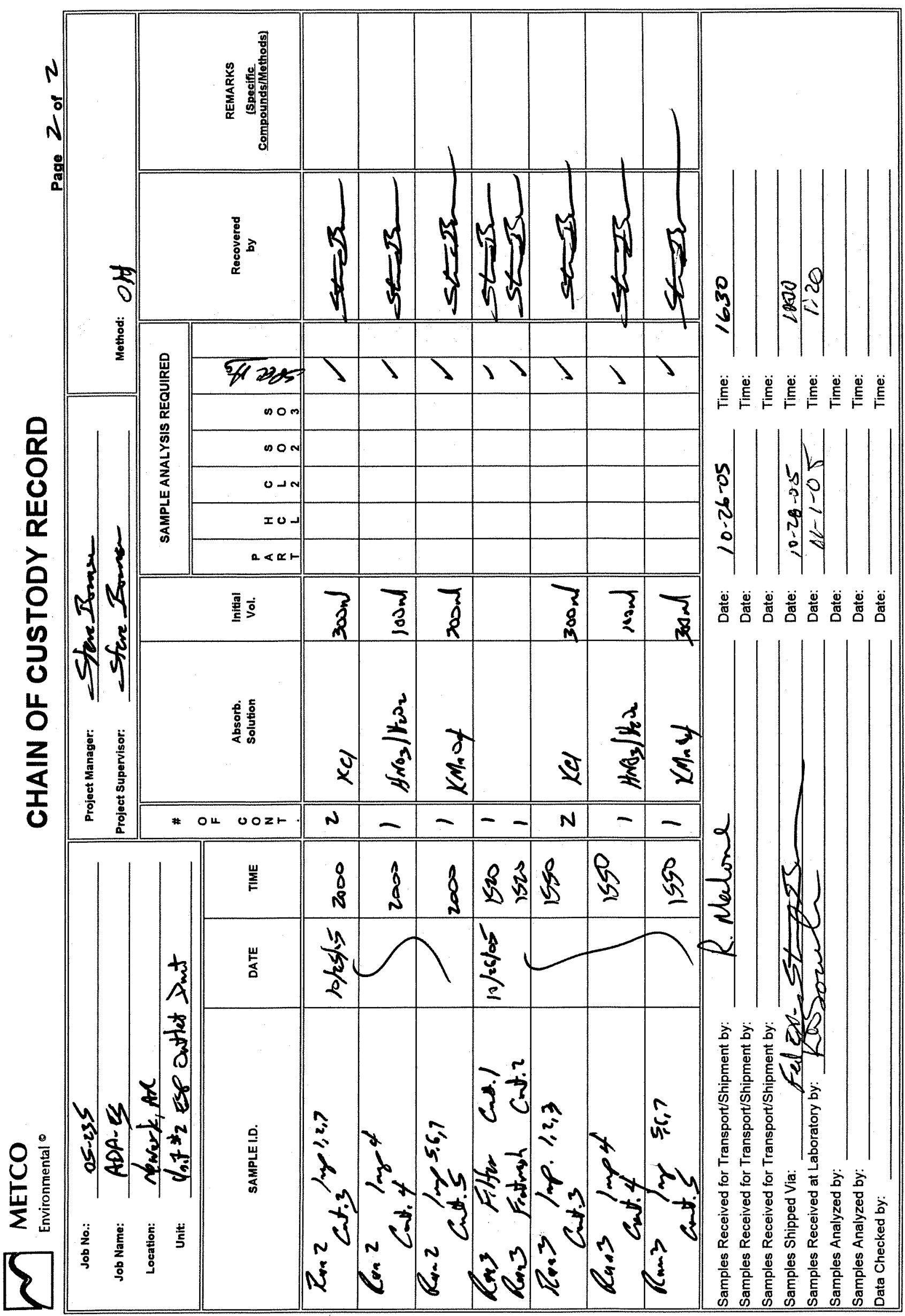

Page 35 of 37 


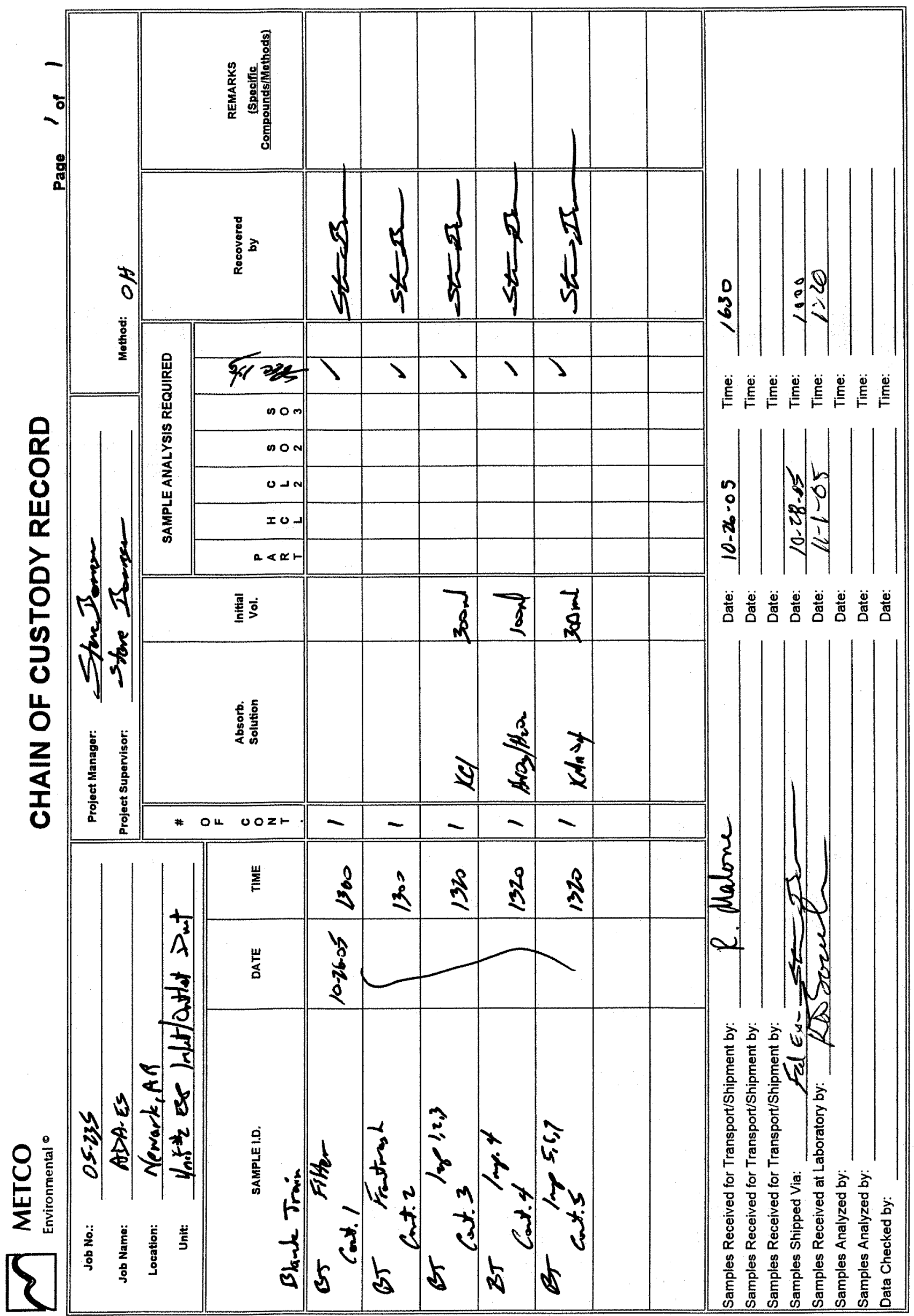

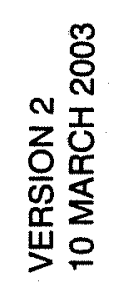

Page 36 of 37 


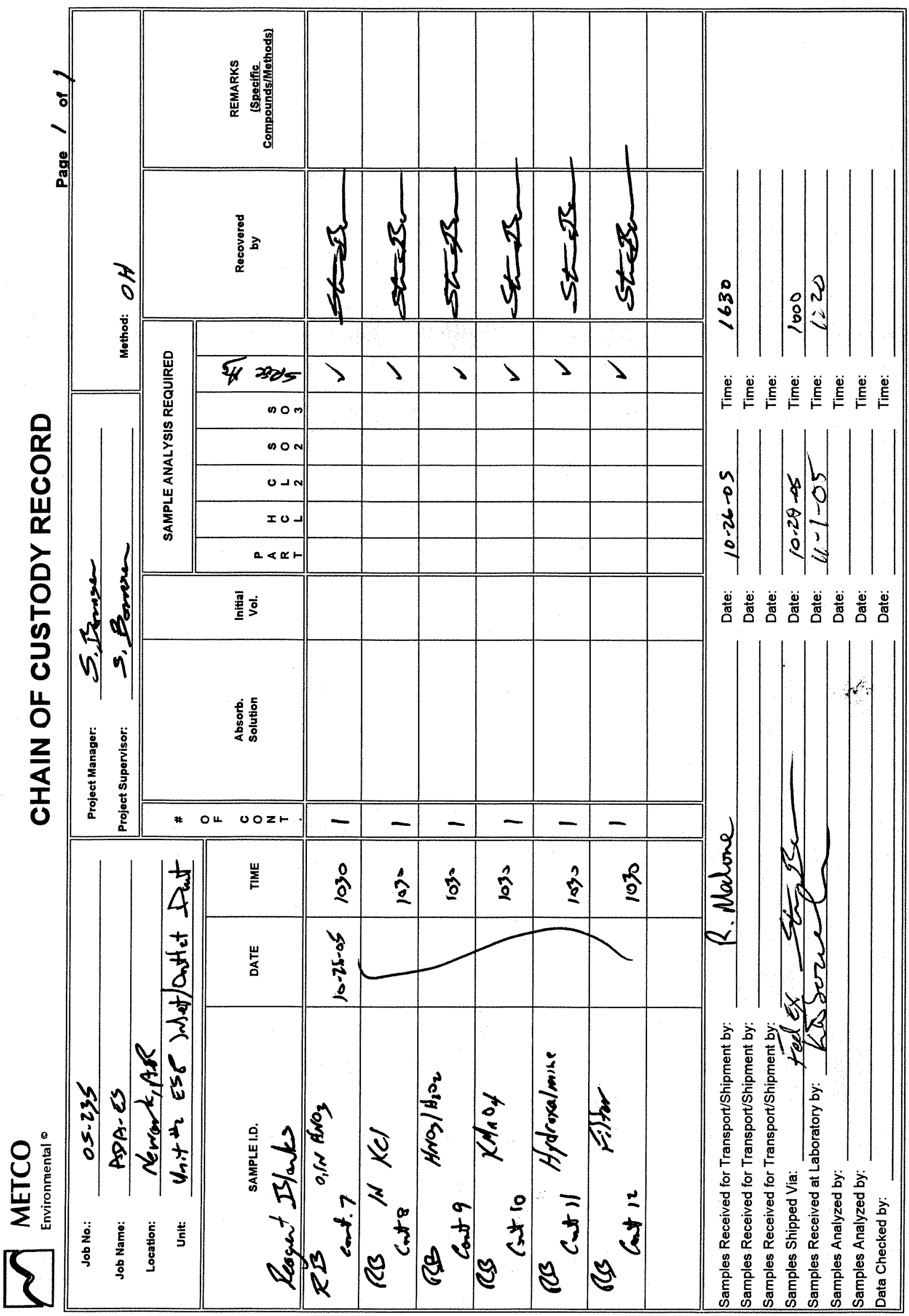

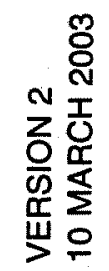

Page 37 of 37 


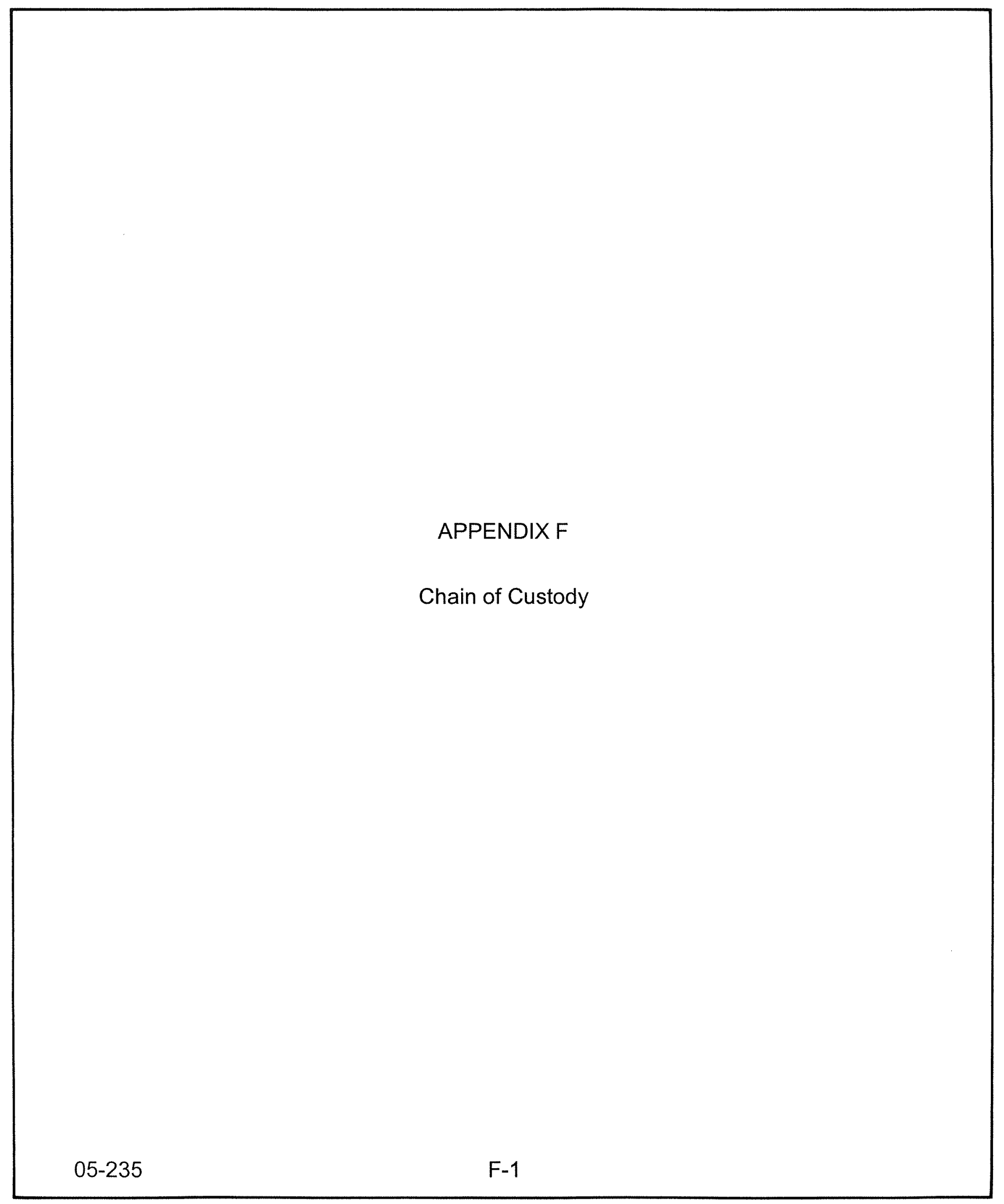




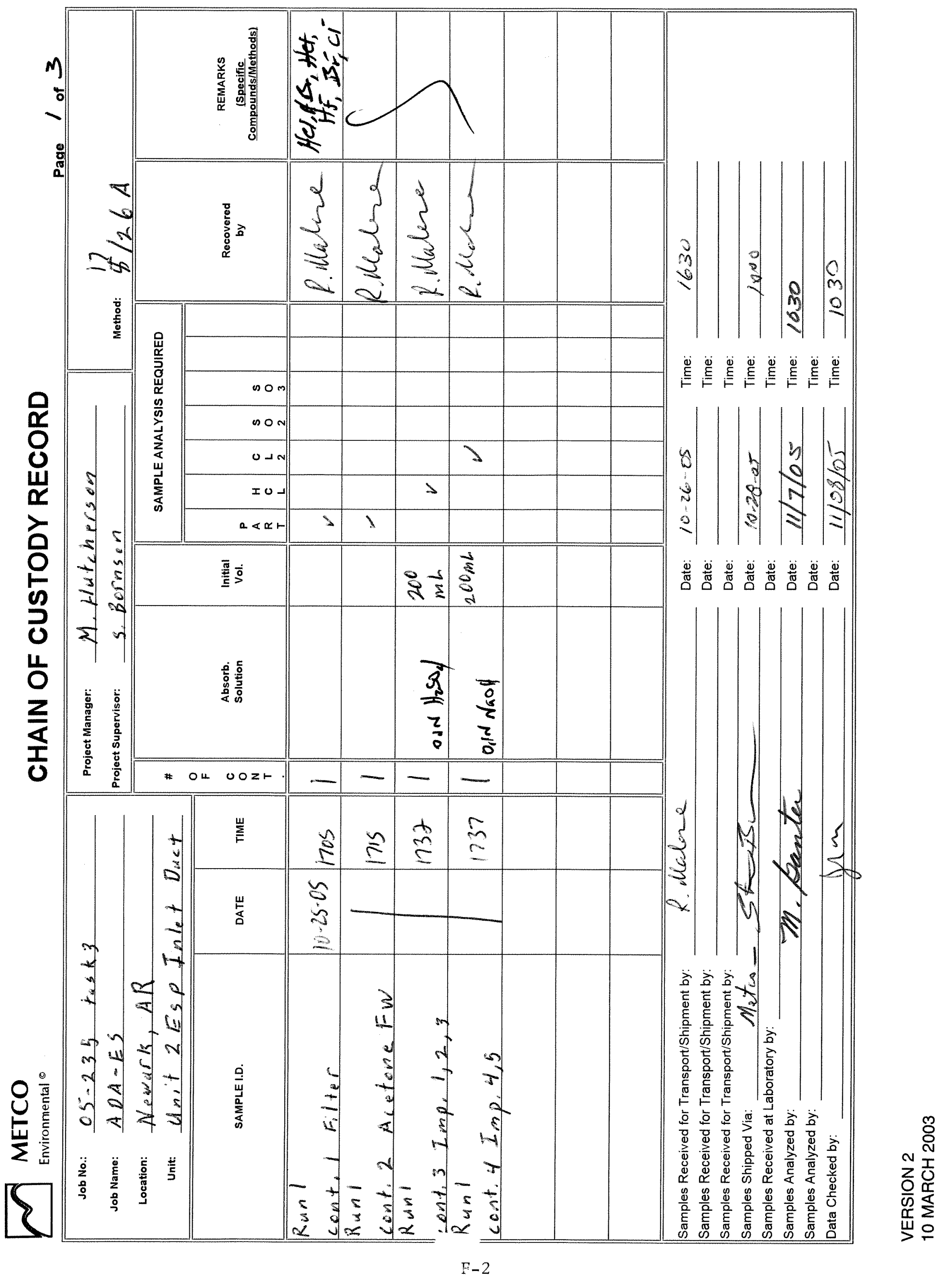




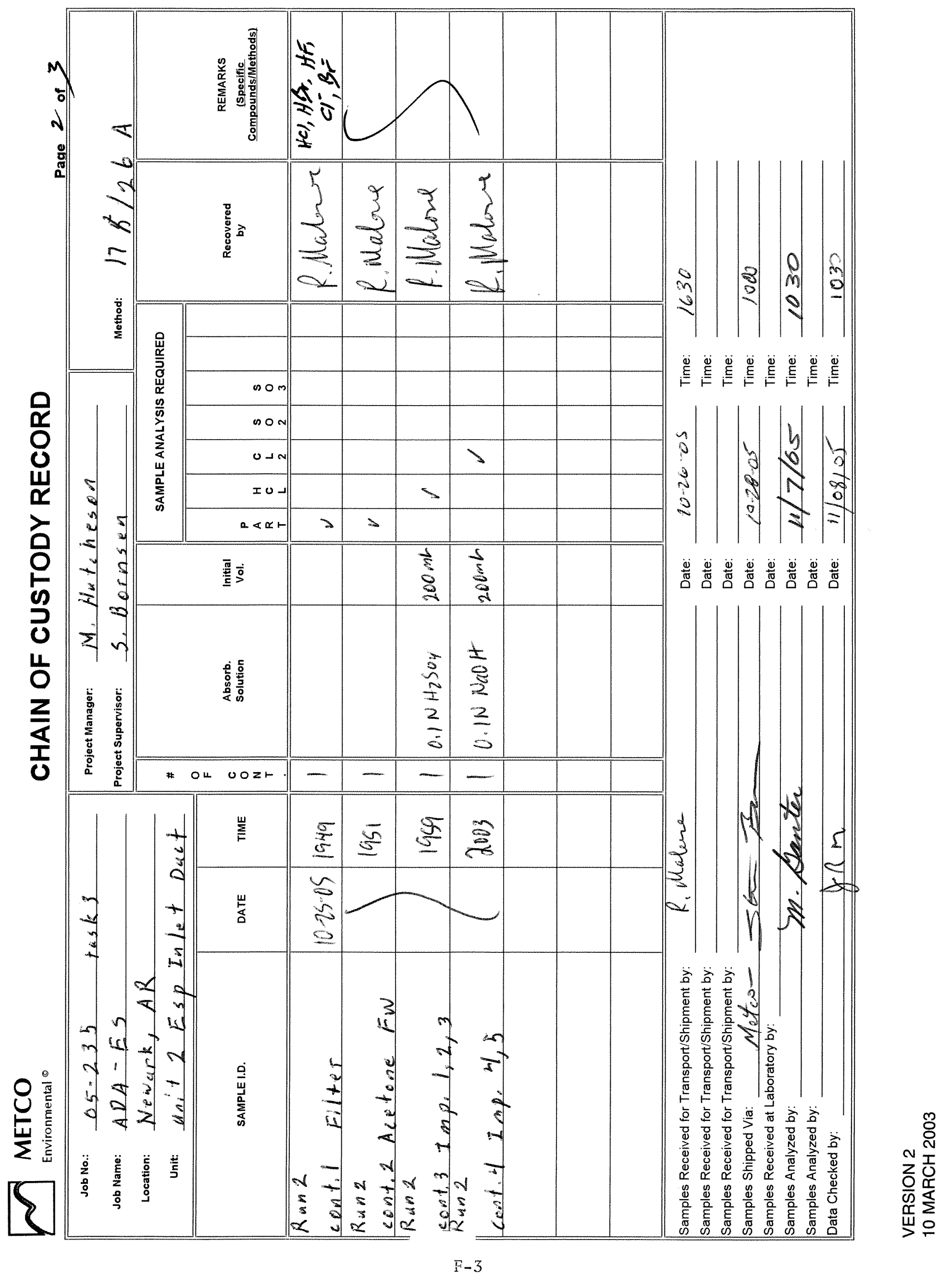




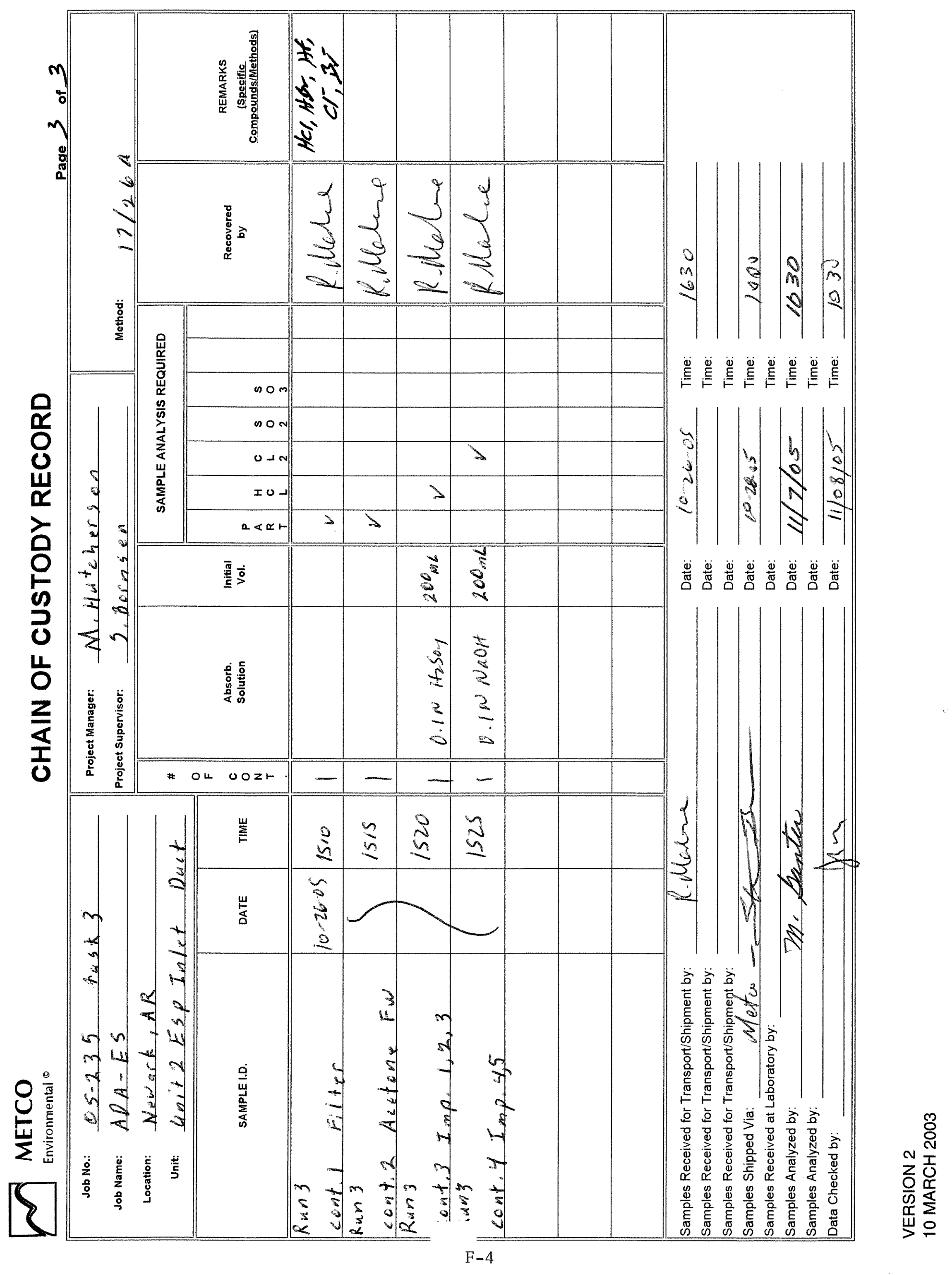




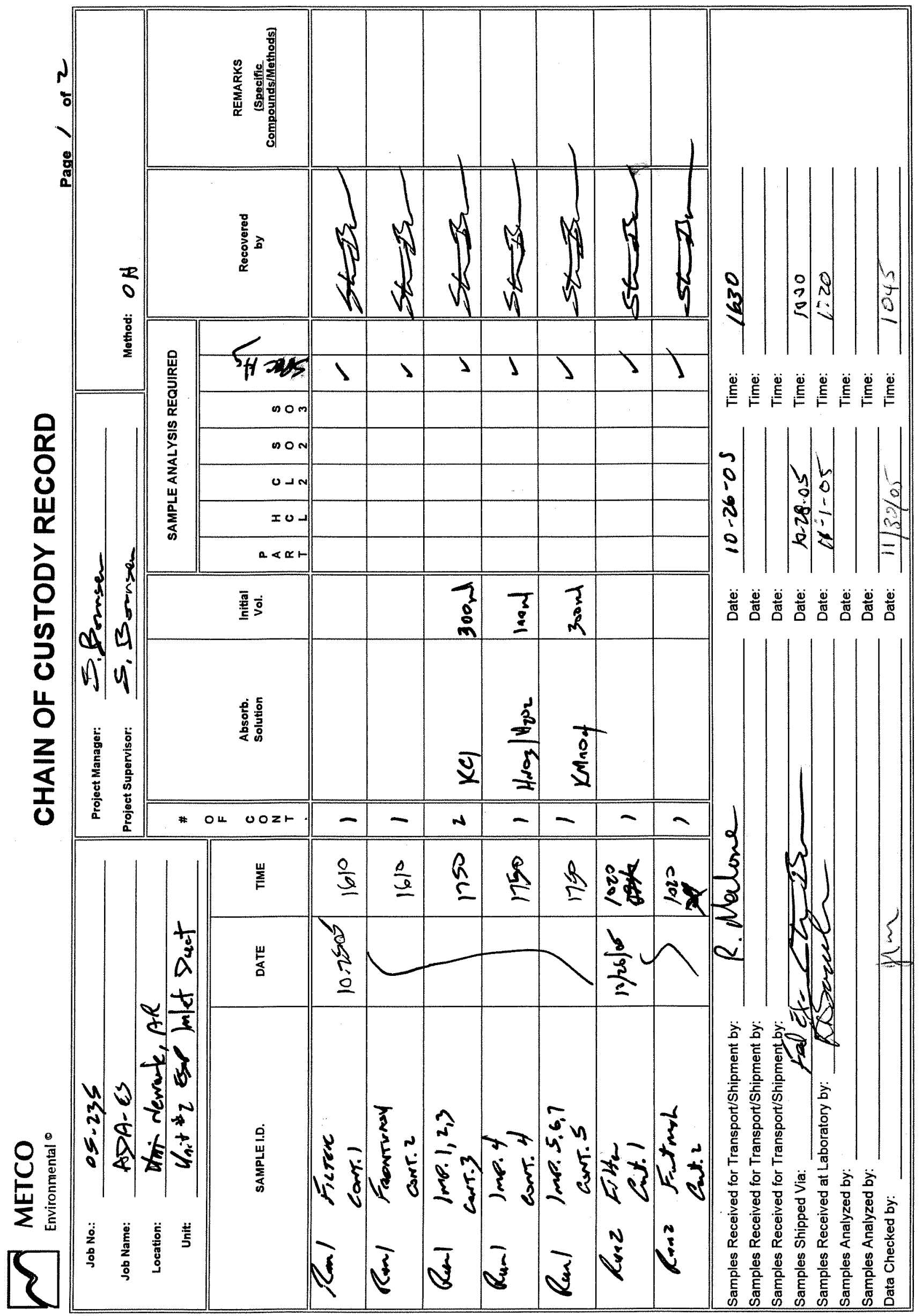

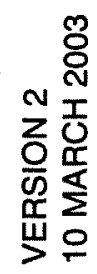

Page 32 of 37 


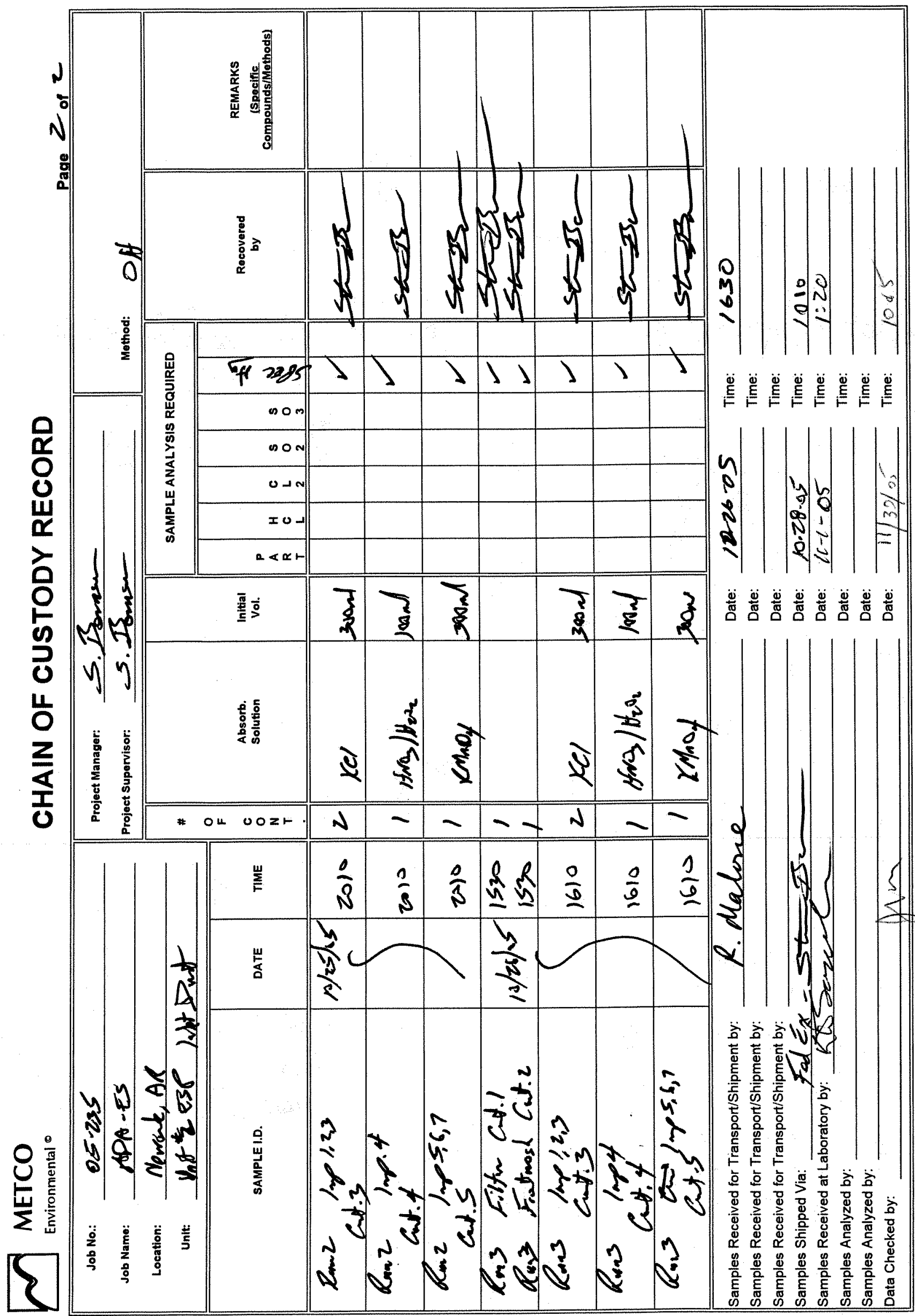

Page 33 of 37 


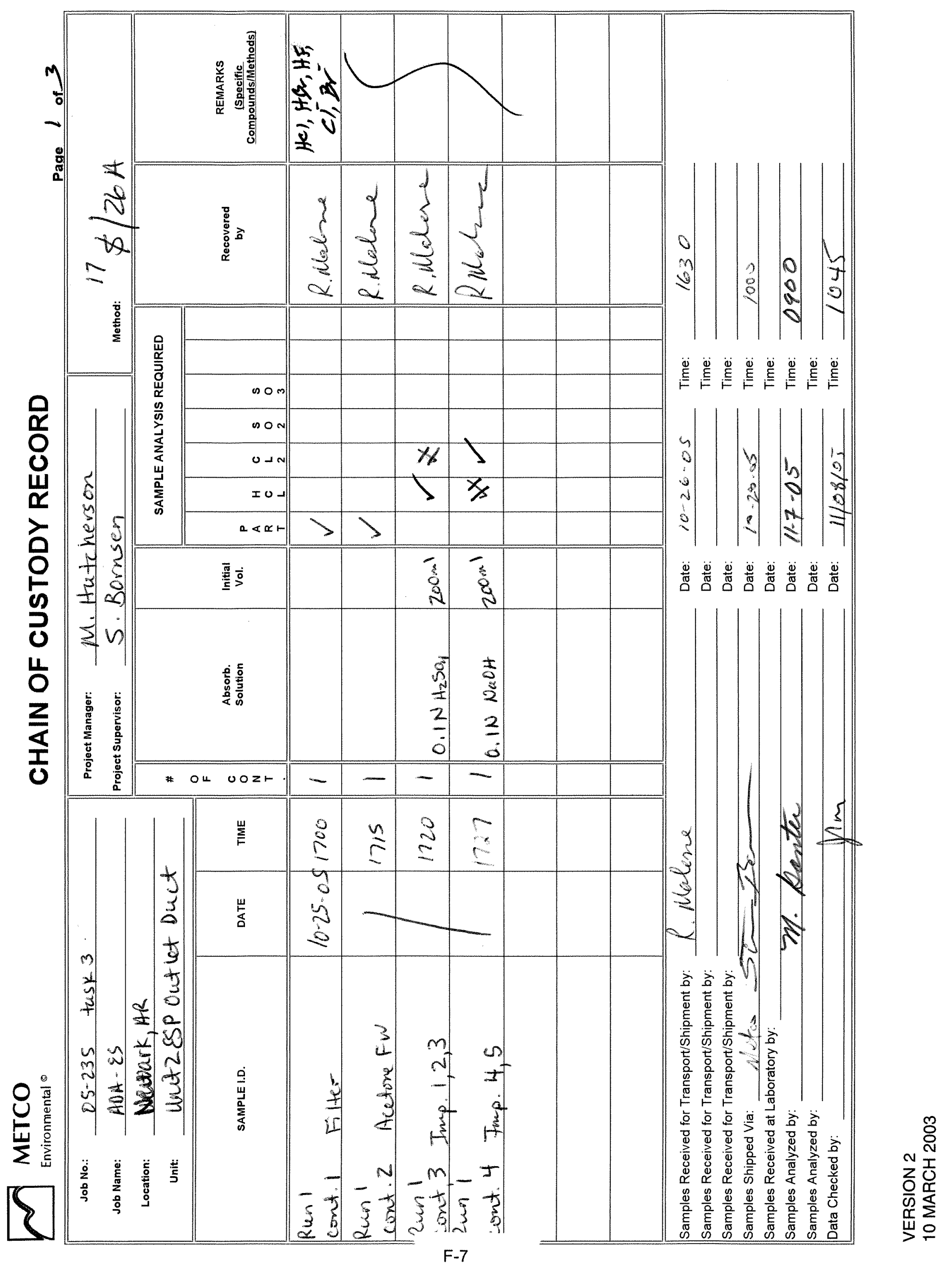




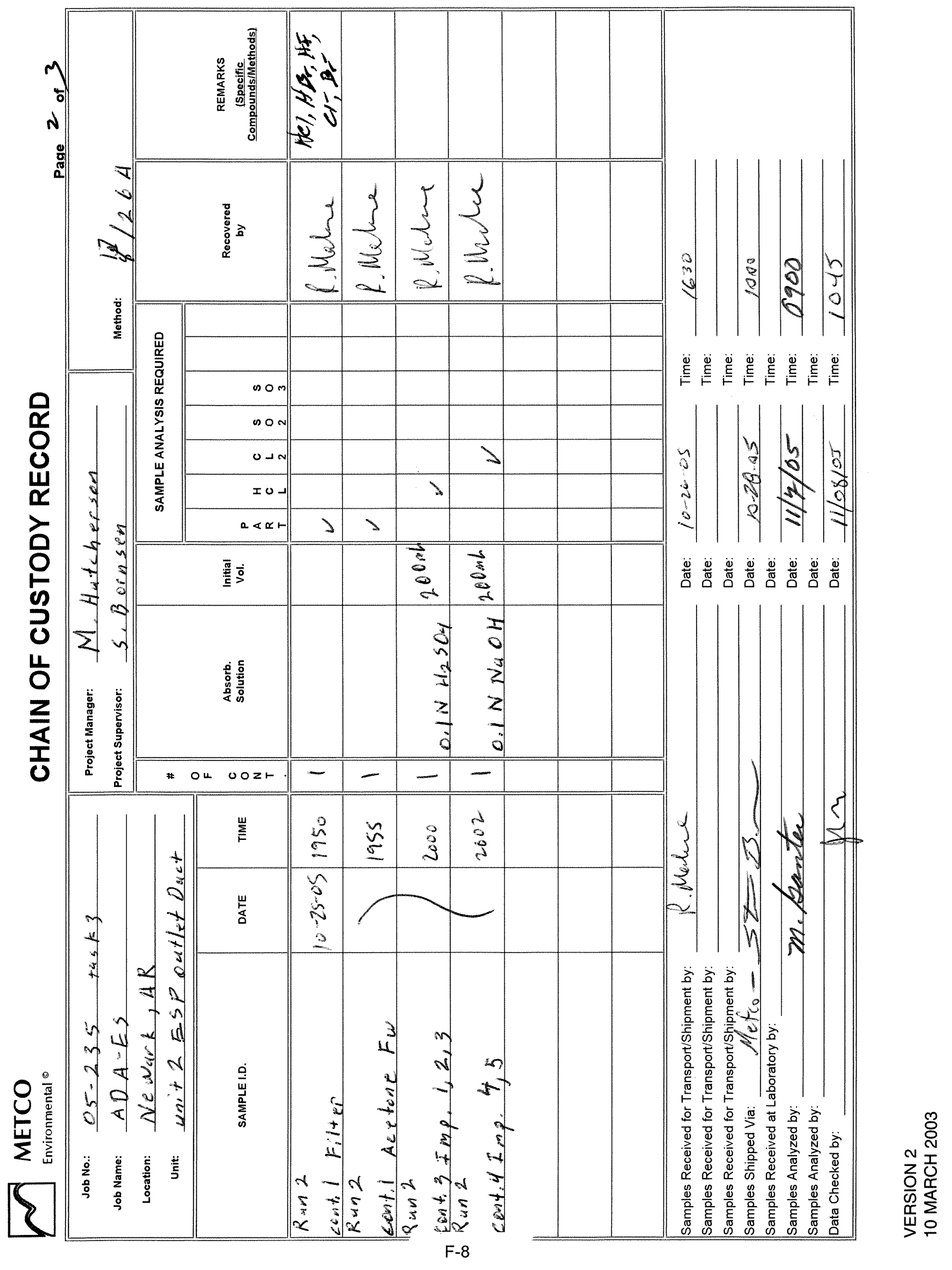




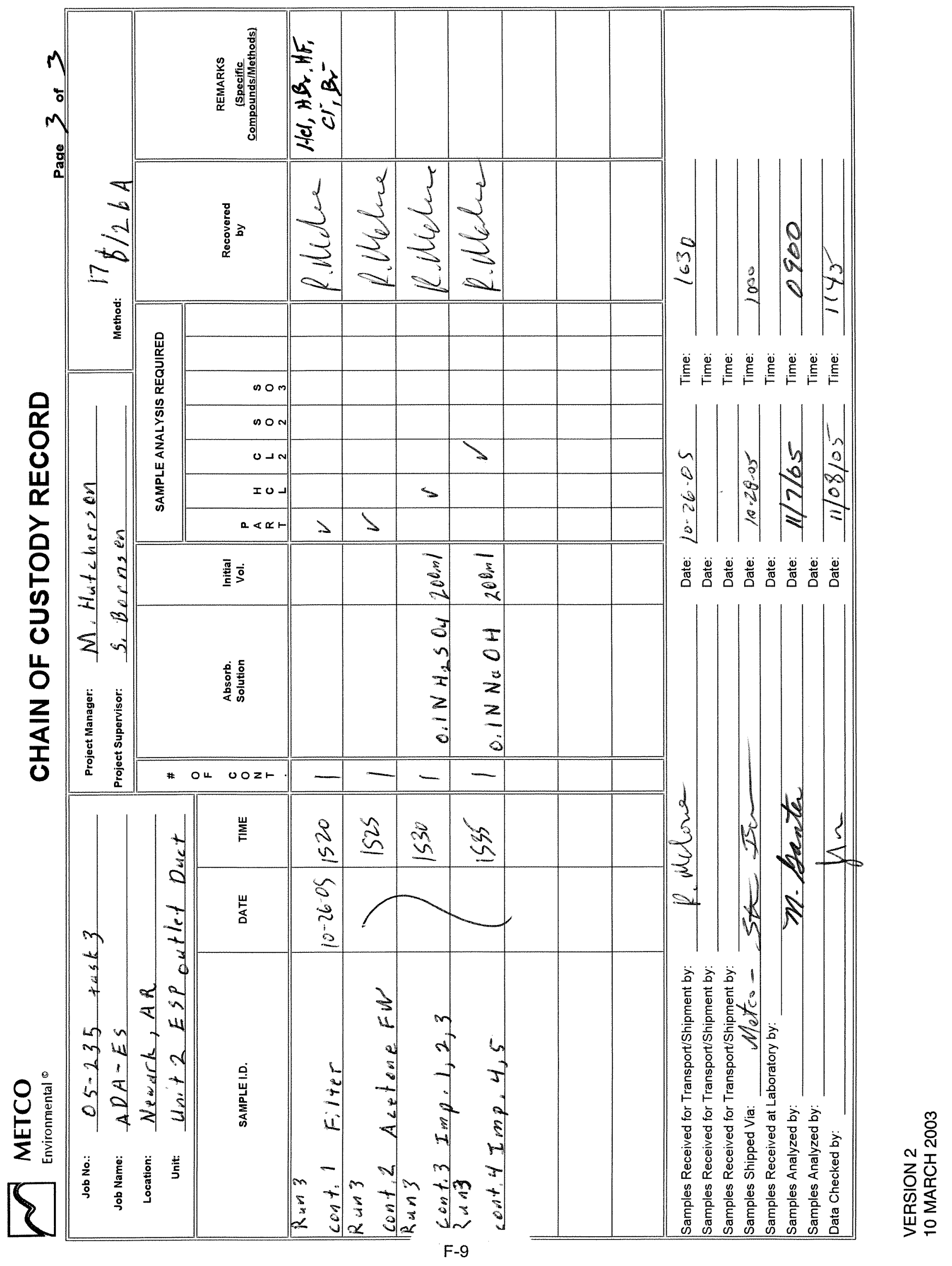




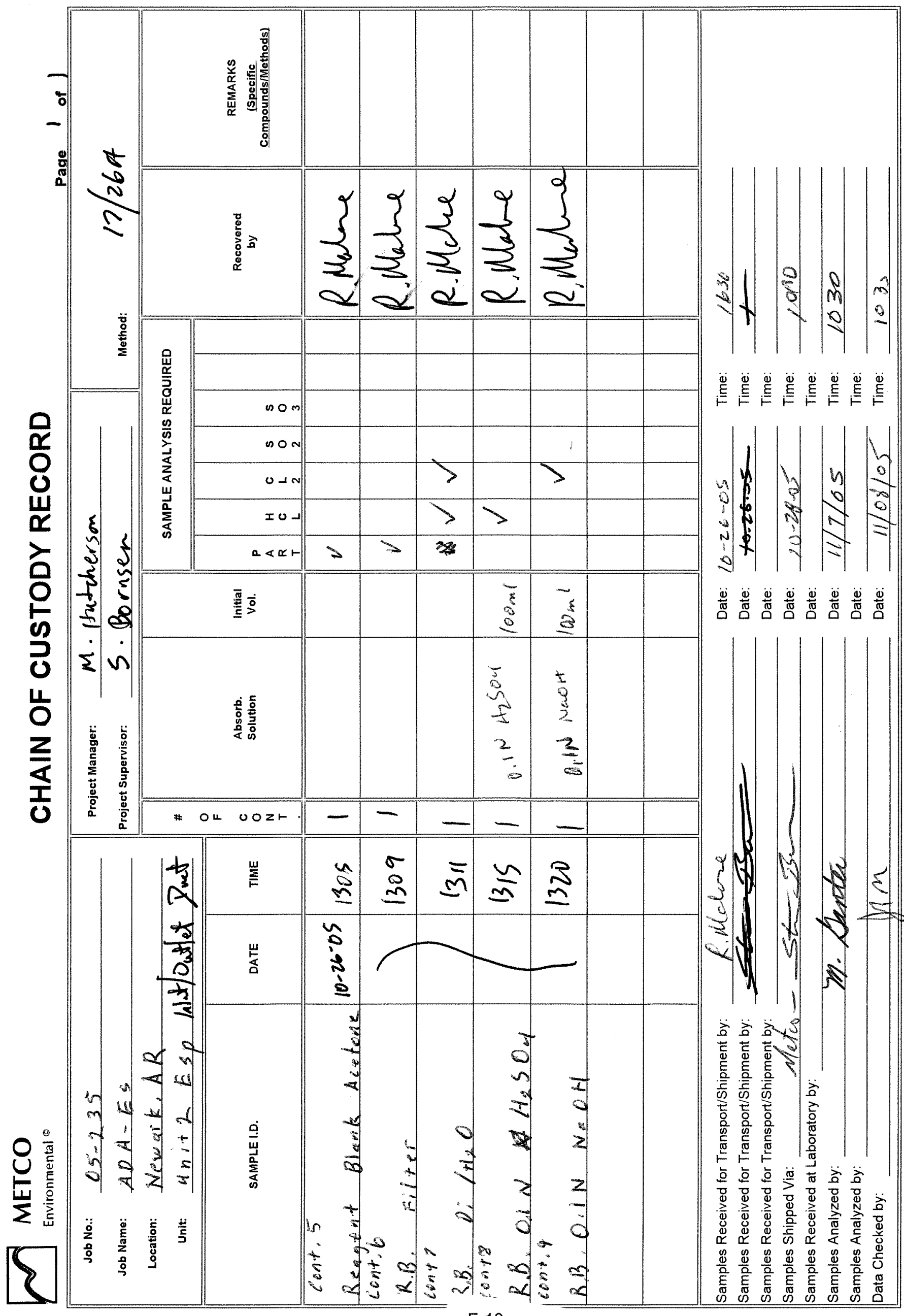

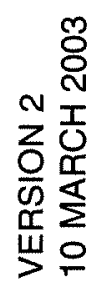
F-10 


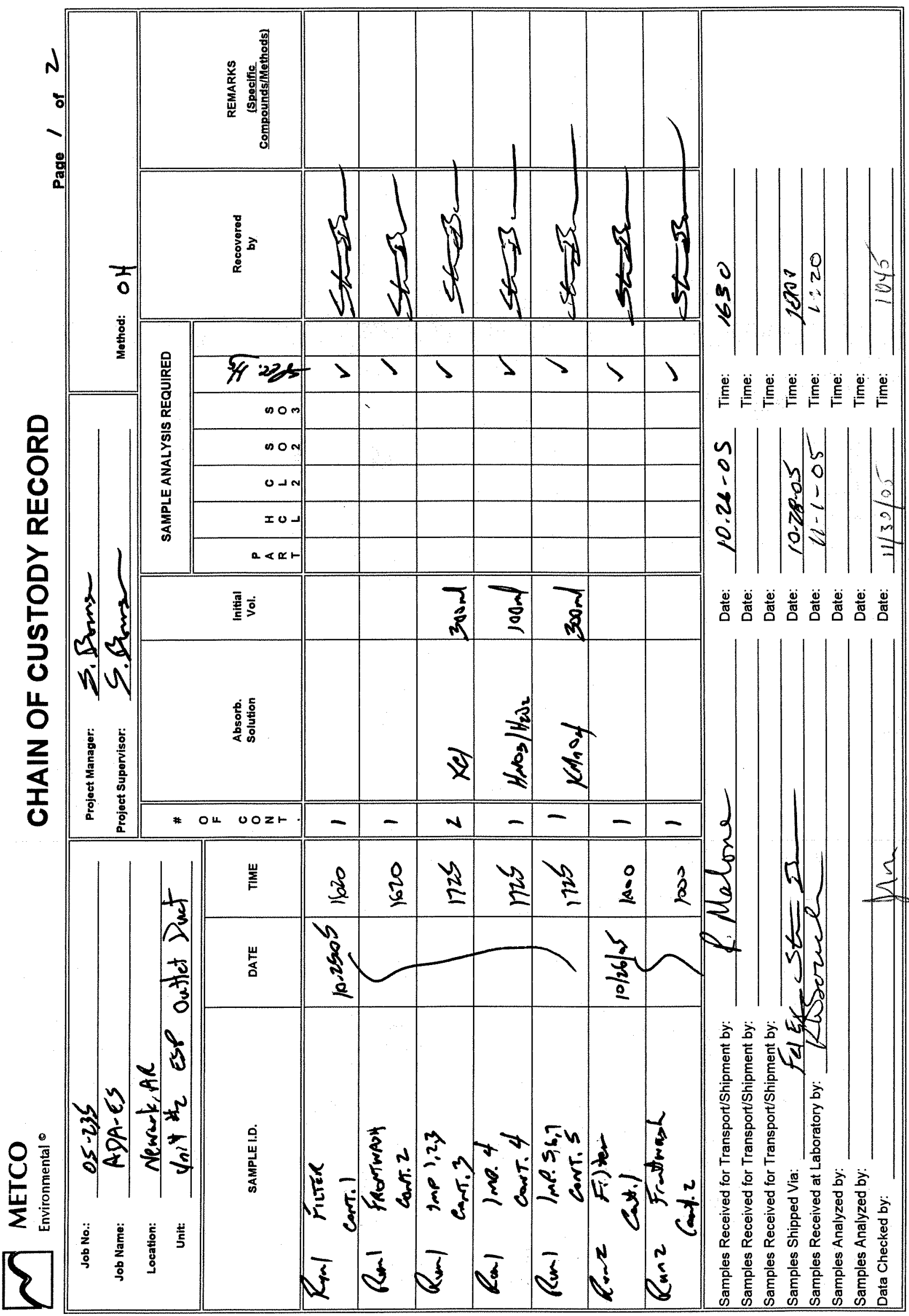

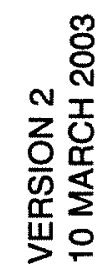

Page 34 of 37 


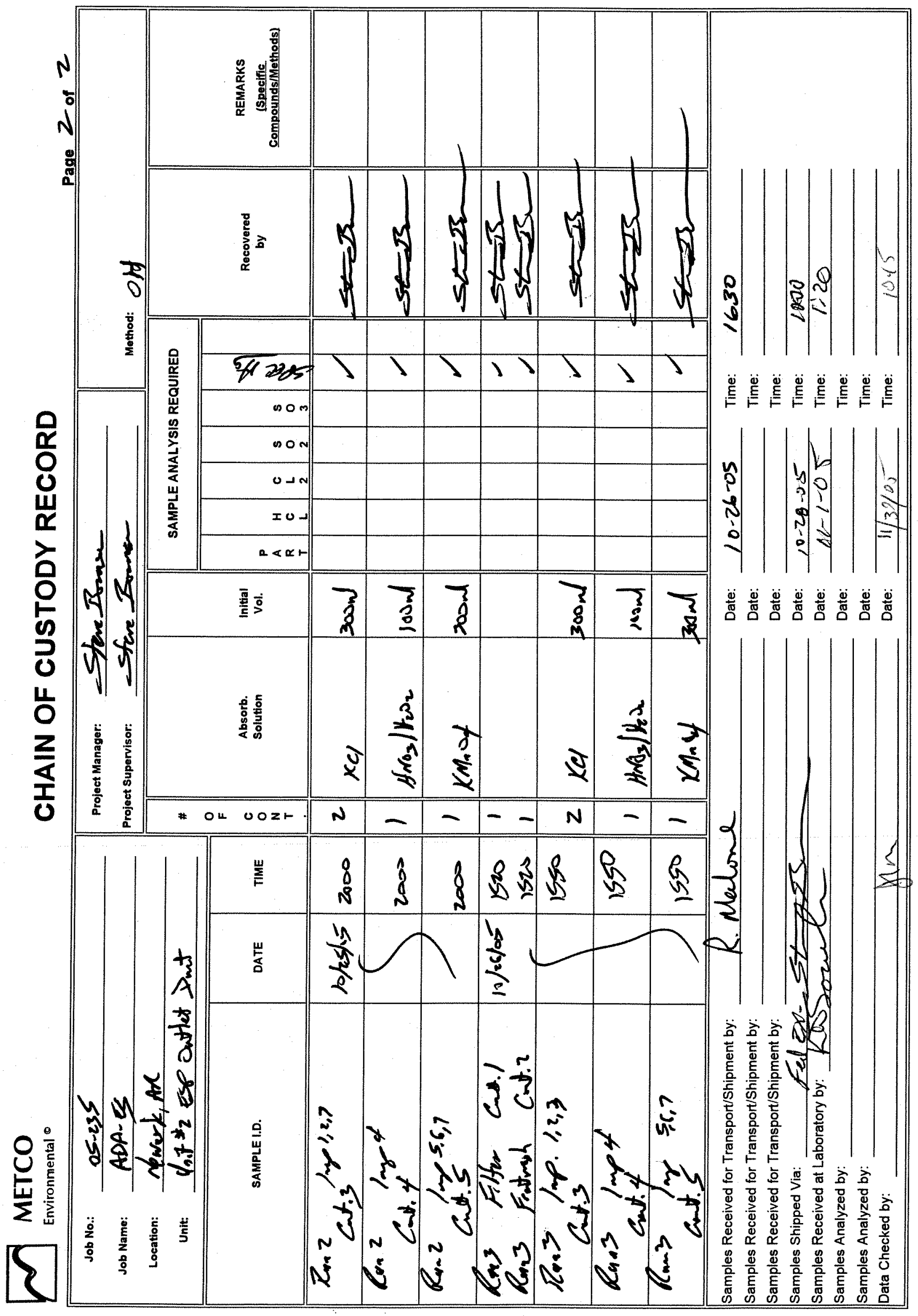

Page 35 of 37 


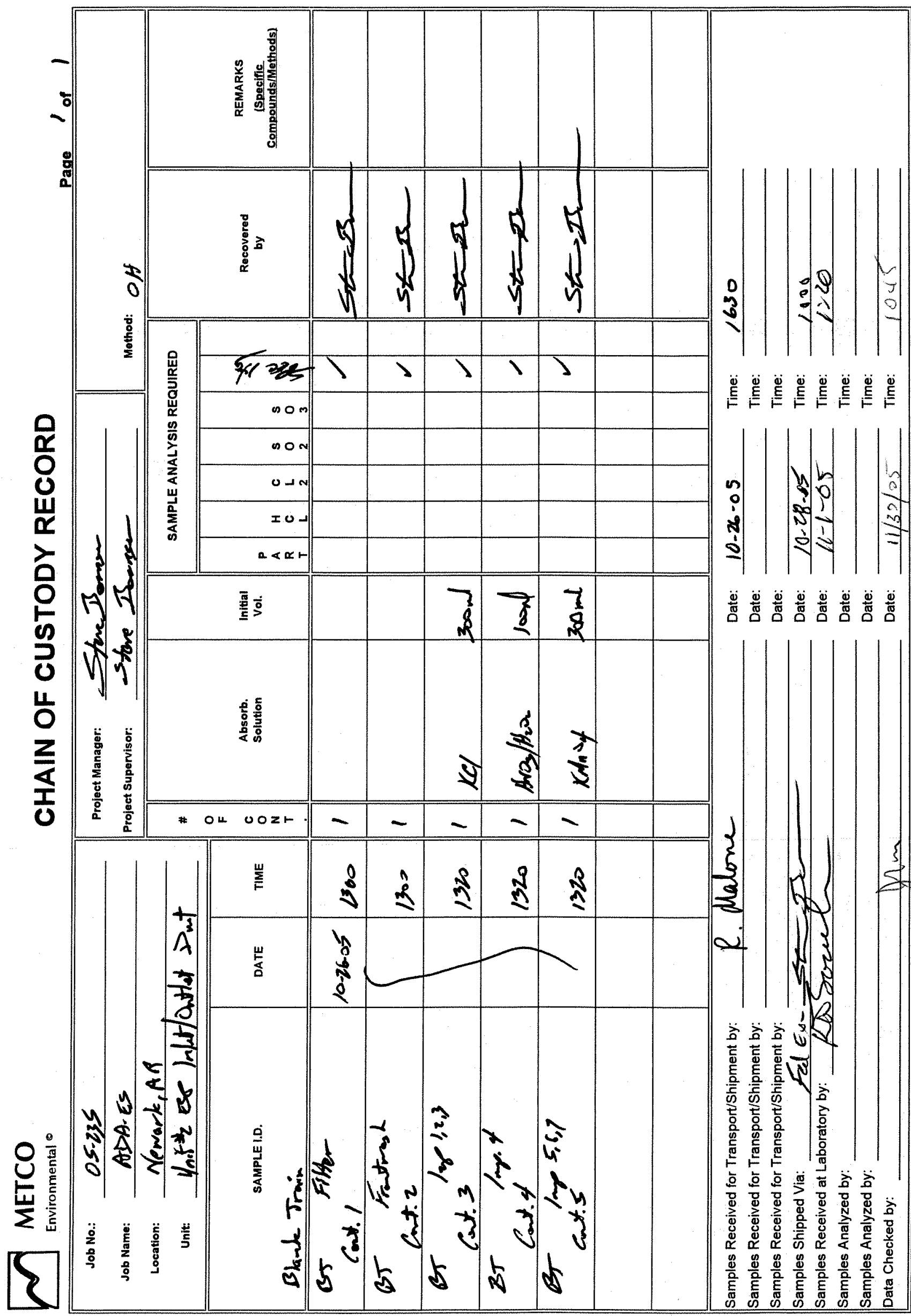

Page 36 of 37 


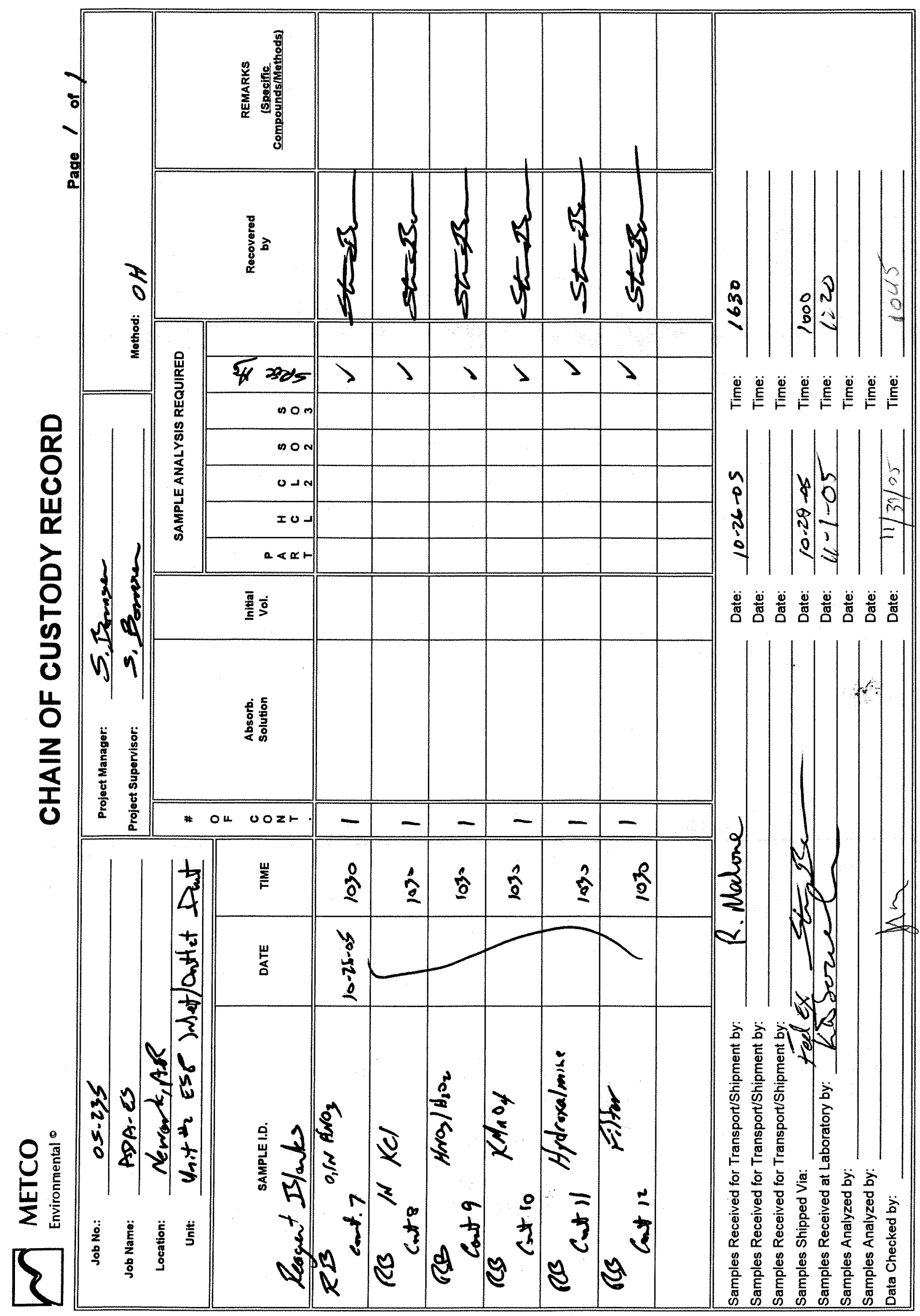

Page 37 of 37

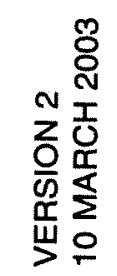




\section{APPENDIX G}

\section{Resumes of Test Personnel}


ROBERT M. PATTERSON; President

Education

Professional

Training

Courses

Certification

Professional

Memberships

Technical Experience
B. S. 1983, Central Michigan University; Mt. Pleasant, Michigan, in Geology and Earth Science-Meteorology.

Attended a two-day short course, "Performing and Observing Source Sampling" in Dallas, Texas.

Attended a one-day short course on basic supervision.

Attended a four-week management course presented by the American Management Association, 1991-1992.

Certified Visible Emissions Evaluator

Certified Cabot Full-Face Respirator Fit Tester

Source Evaluation Society

American Management Association

Participated in the sampling of over 1,000 sources, including several of which were sampled simultaneously using more than one sampling train. Thoroughly trained in all EPA testing procedures, 1986-present.

Over nineteen years experience with EPA and Texas Air Control Board methods of sampling - both stationary sources and ambient air. CFR, Title 40, Chapter I, Part 60, EPA Methods 1 through 25, and 101 through 110. Performance Specifications 1 through 5. CFR, Title 40, Chapter I, Part 50, Appendix A through F. "Sampling Procedures Manual, Texas Air Control Board, January 1983." Parts 1-1 through 14-6, Appendix B through Appendix M.

Experienced with sampling Method 0010, Modified Method 5 Sampling Train; Method 0030, Volatile Organic Sampling Train; and various EPA and "Site Specific" multiple metal and acid gas sampling trains. 
PATTERSON, Rob (cont'd)

Technical

Experience

(cont'd)
Over twenty one years experience with EPA and Texas Air Control Board methods of analysis of both stationary and ambient air samples. Particulate matter, $\mathrm{SO}_{3}, \mathrm{SO}_{2}, \mathrm{H}_{2} \mathrm{SO}_{4}$, $\mathrm{NO}_{\mathrm{x}}, \mathrm{CO}, \mathrm{CO}_{2}, \mathrm{O}_{2}, \mathrm{H}_{2} \mathrm{~S}, \mathrm{~F}$, TRS, $\mathrm{HCl}, \mathrm{Cl}_{2}, \mathrm{NH}_{3}, \mathrm{VOC}, \mathrm{C}_{1}-\mathrm{C}_{7}$, and other organics. Both laboratory and on-site analyses were performed.

Experienced in the sampling and analysis of commercial calibration gas cylinders for sulfur dioxide, oxides of nitrogen, carbon dioxide, oxygen, carbon monoxide, and $\mathrm{C}_{1}-\mathrm{C}_{7}$ hydrocarbons.

Thoroughly trained in the operation and routine maintenance of the following:

- MSA LIRA Model 202S Infrared Analyzer

- Analytical Instrument Development, Inc. Model 340A Calibration System

- Shimadzu GC-Mini 2 Gas Chromatograph

- Thermo Environmental Model 10AR Oxides of Nitrogen Analyzer

- Thermo Oxygen Analyzer

- Teledyne Model 326 Oxygen Analyzer

- Thermo Environmental Model 48 Carbon Monoxide Analyzer

- Thermo Environmental Model 40 Sulfur Dioxide Analyzer

- Ratfisch Model RS 100 Total Hydrocarbon Analyzer

- Western Research Model 721AT Sulfur Dioxide Analyzer

- Horiba Model PIR 2000 Carbon Dioxide Analyzer

- Ratfisch Model RS 55 Total Hydrocarbon Analyzer

- J.U.M. Model VE-7 Total Hydrocarbon Analyzer 
JAMES R. MONFRIES; Senior Quality Assurance Manager

Education

B. S. 1975, University of Texas at Arlington; Arlington, Texas, in Biology with a minor in Chemistry.

Graduate work at the University of Texas at Dallas in the Environmental Science Department.

Professional

Training

$\underline{\text { Courses }}$

Attended a two-day short course, "Performing and Observing Source Sampling" in Dallas, Texas, July 1976.

Certification Certified Visible Emissions Evaluator

Professional

Memberships

Technical

Experience
Air and Waste Management Association

Source Evaluation Society - Past President

Participated in the sampling of over 700 sources, serving in the supervisory capacity on over 500 sources. Many of the sources were sampled simultaneously using more than one sampling train at several points in the flue gas stream, 1976-present.

Has also supervised several ambient air monitoring studies, including a permanent five-station high volume air sampling network in South Texas, a permanent four-station high volume air sampling network in Pennsylvania, and a permanent sevenstation sulfur dioxide sampling network in East Texas.

Was Quality Assurance Manager for several ambient air monitoring studies; including a four-station high volume air sampling network for TSP and PM10 in Midlothian, Texas; a single-station high volume air sampling network for PM10 in South Texas; a two-station high volume air sampling network for TSP in Wichita Falls, Texas; and a four-station continuous air sampling network for TSP and PM10 in Jewett, Texas using Thermo Andersen FH 62 C14 Beta Gauge Dust Monitors. 
MONFRIES, James (cont'd)

Twenty years experience with EPA and Texas Commission on Environmental Quality methods of analysis of both source and ambient air samples for particulates, $\mathrm{SO}_{2}, \mathrm{SO}_{3}, \mathrm{H}_{2} \mathrm{SO}_{4}, \mathrm{H}_{2} \mathrm{~S}$, $\mathrm{HCl}, \mathrm{Cl}_{2}, \mathrm{NO}_{\mathrm{x}}$, Hydrocarbons, and TRS.

Experienced in the analysis of commercial calibration gas cylinders for sulfur dioxide and oxides of nitrogen.

Experienced with VOST and Modified Method 5 Sampling Procedures.

Thoroughly trained in the operation and routine maintenance of the following:

- Lear Siegler, Inc. SM800 Stack Gas Monitor

- Du Pont Model 460/1 Photometric Analyzer System

- Lear Siegler, Inc. SM1000 Ambient SO 2 Monitor

- Calibrated Instruments Ultragas $\mathrm{SO}_{2}$ Monitor

- Meloy 285E SO 2 Analyzer

- Meloy SA-700 Fluorescent $\mathrm{SO}_{2}$ Analyzer

- MSA LIRA Model 202S Infrared Analyzer

- Analytical Instrument Development, Inc. Model 340A Calibration System

- Shimadzu GC-Mini 2 Gas Chromatograph

- Thermo Environmental Model $10 \mathrm{~S} \mathrm{NO}_{x}$ Analyzer

- Thermo Oxygen Analyzer

- Teledyne Model 326 Oxygen Analyzer

- Thermo Environmental Model 48 Carbon Monoxide Analyzer

- Thermo Environmental Model 40 Sulfur Dioxide Analyzer

- Ratfisch Model RS 103 Total Hydrocarbon Analyzer

- Western Research Model 721AT Sulfur Dioxide Analyzer

- Horiba Model PIR 2000 Carbon Dioxide Analyzer

- Ratfisch Model RS 55 Total Hydrocarbon Analyzer

- J.U.M. Model VE-7 Total Hydrocarbon Analyzer

- Thermo Andersen Model FH 62 C14 Dust Monitor 
STEVEN J. BORNSEN; Utilities Associate

Education $\quad$ B. S. in Geology, 1995; University of Texas at Arlington; Arlington, Texas.

Professional

Training

Courses

Attended 40-hour Occupational \& Environmental Training Program on Hazardous Materials (CFR 1910.120); Dallas, Texas, January 1996.

Attended a two-day short course, "Performing and Observing Source Sampling," Dallas, Texas; September 1996.

Certifications Certified Visible Emissions Evaluator

HAZMAT certified

Adult CPR certified

Standard First Aid certified

Technical

Participated in the sampling of over 400 sources, including

Experience several of which were sampled simultaneously using more than one sampling train. Thoroughly trained in all EPA testing procedures, 1995-present.

Experienced with Modified Method 5 Sampling Train (Method 0010).

Experienced with VOST sampling procedure (Method 0030).

Experienced with calibration techniques for all field testing equipment.

Experienced with various multiple metal and acid gas sampling trains.

Experienced with particle size and $\mathrm{PM}_{10}$ sampling procedures.

(continued) 
BORNSEN, Steve (cont'd)

Technical

Experience

(cont'd)

Experienced with on-site gravimetric particulate matter analysis.

Has performed on-site $\mathrm{SO}_{2}$ analysis and $\mathrm{H}_{2} \mathrm{~S}$ fuel gas analysis.

Thoroughly trained in the operation and routine maintenance of the following:

- Thermo Environmental Model $10 \mathrm{~S}$ and $42 \mathrm{C}$ Oxides of Nitrogen Analyzers

- Teledyne Model 326 Oxygen Analyzer

- Western Research Model 721 AT Sulfur Dioxide Analyzer

- Horiba Model PIR 2000 Carbon Dioxide Analyzer

- J.U.M. Model VE-7 Total Hydrocarbon Analyzer

- Thermo Environmental Model 48 Carbon Monoxide Analyzer 
RODNEY MALONE; Project Supervisor II

Education $\quad$ B. S. Forestry, December 2000; Stephen F. Austin State University; Nacogdoches, Texas.

Certifications HAZMAT certified

Adult CPR certified

Standard First Aid certified

Technical

Experience

Participated in the sampling of over 200 sources, including several of which were sampled simultaneously using more than one sampling train.

Thoroughly trained in all EPA testing procedures, 2001-present.

Experienced with Modified Method 5 Sampling Train (Method 0010).

Experienced with VOST Sampling Procedures (Method 0030).

Experienced with calibration techniques for all field testing equipment.

Experienced with various multiple metal and acid gas sampling trains.

Has performed on-site $\mathrm{SO}_{2}$ analysis and $\mathrm{H}_{2} \mathrm{~S}$ fuel gas analysis.

Thoroughly trained in the operation and routine maintenance of the following:

- Thermo Environmental Model $10 \mathrm{~S}, 42 \mathrm{C}$, and $42 \mathrm{CHL}$ Oxides of Nitrogen Analyzers

- Teledyne Model 326 Oxygen Analyzer

- Horiba Model PIR 2000 Carbon Dioxide Analyzer

(continued)

G-8 
MALONE, Rodney (cont'd)

\section{Technical}

Experience (cont'd)

Operation and routine maintenance (cont'd)

- Thermo Environmental Model 48 Carbon Monoxide Analyzer.

- Western Research Model 721M Sulfur Dioxide Analyzer

- J.U.M. VE-7 Hydrocarbon Analyzer

- Shimadzu Gas Chromatograph 14 and 17a 
ERIC STONE; Environmental Scientist II

Education

Technical

Experience
B. S. Environmental Science, December 2004; Stephen F. Austin State University, Nacogdoches, Texas.

Participated in the sampling of over 50 sources, including several of which were sampled simultaneously using more than one sampling train.

Thoroughly trained in all EPA testing procedures, 2005-present. 
JOHN CUTAIA; Environnmental Scientist I

Education Over ten (10) years of experience using Methods 1, 2, 3, 4, 5, $6,7,9,10,11,24,29,201 \mathrm{~A}$.

Analyzers used: Thermo 43A, 51, 48C, 42C, Servomex 1400, 1400C; Fuj. 3300, Horiba VIA-510, Western 721, Rosemont $951 \mathrm{~A}$

Technical

Experience

Participated in the sampling of over 25 sources, including several of which were sampled simultaneously using more than one sampling train.

Thoroughly trained in all EPA testing procedures, 2005-present. 
JOSHUA ORR; Environmental Scientist I

Education

B. S. Environmental Studies, Summer 2002; Southwest Texas State University; San Marcos, Texas.

Technical

Experience

Participated in the sampling of over 25 sources, including several of which were sampled simultaneously using more than one sampling train.

Thoroughly trained in all EPA testing procedures, 2005-present. 
DUSTIN DODSON; Environmental Scientist I

Education

B. S. Industrial Engineering, May 2005; Northwestern State University; Natchitoches, Louisiana.

Technical

Experience

Participated in the sampling of over 25 sources, including several of which were sampled simultaneously using more than one sampling train.

Thoroughly trained in all EPA testing procedures, 2005-present. 
JASON SELLERS; Environmental Scientist I

Education

B. S. Wildlife and Fisheries Sciences, May 2004; Texas A\&M University, College Station, Texas.

Technical

Experience

Participated in the sampling of over 25 sources, including several of which were sampled simultaneously using more than one sampling train.

Thoroughly trained in all EPA testing procedures, 2005-present. 
CHRISTOPHER POSHEK; Environmental Scientist I

Education

B. A. in Geography, May 2001 and B. A. in Economics, August 2003; Southern Illinois University, Carbondale, Illinois.

Technical

Experience

Participated in the sampling of over 25 sources, including several of which were sampled simultaneously using more than one sampling train.

Thoroughly trained in all EPA testing procedures, 2005-present. 
DOUGLAS WARE; Environmental Scientist I

Education

B. S. Criminal Justice, December 10, 2004; Sam Houston State University, Huntsville, Texas.

Technical

Experience

Participated in the sampling of over 25 sources, including several of which were sampled simultaneously using more than one sampling train.

Thoroughly trained in all EPA testing procedures, 2005-present. 


\section{APPENDIX D4: Source Testing-November 1-2, 2005}




\author{
SOURCE EMISSIONS SURVEY \\ OF \\ ARKANSAS POWER \& LIGHT COMPANY \\ INDEPENDENCE STEAM \\ ELECTRIC STATION \\ UNIT NUMBER 2 ESP INLET \\ AND OUTLET DUCTS \\ NEWARK, ARKANSAS \\ FOR \\ ADA-ES
}

NOVEMBER 2005

FILE NUMBER 05-236

" I certify that I have personally checked and am familiar with the information submitted herein, and based on my inquiries of those individuals immediately responsible for obtaining the information, I believe the submitted information is true, accurate, and complete. "

James R. Monfries

Senior Quality Assurance Manager 
TABLE OF CONTENTS

PAGE

INTRODUCTION

1

SUMMARY OF RESULTS

2

DISCUSSION OF RESULTS

7

DESCRIPTION OF SAMPLING LOCATIONS

SAMPLING AND ANALYTICAL PROCEDURES

DESCRIPTION OF TESTS

APPENDICES
A. Location of Sampling Points
B. Source Emissions Calculations
C. Calibration Data
D. Field Testing Data
E. Analytical Data
F. Chain of Custody
G. Resumes of Test Personnel 


\author{
SOURCE EMISSIONS SURVEY \\ ARKANSAS POWER \& LIGHT COMPANY \\ INDEPENDENCE STEAM ELECTRIC STATION \\ UNIT NUMBER 2 ESP INLET AND OUTLET DUCTS \\ NEWARK, ARKANSAS \\ FOR \\ ADA-ES
}

FILE NUMBER 05-236

\title{
INTRODUCTION
}

METCO Environmental, P.O. Box 598, Addison, Texas, conducted a source emissions survey of Arkansas Power \& Light Company, Independence Steam Electric Station, located in Newark, Arkansas, for ADA-ES, on November 1 and 2, 2005. The purpose of these tests was to determine the concentrations of speciated mercury at the Unit Number 2 ESP Inlet and Outlet Ducts.

The sampling was performed by the following METCO personnel: Steve Bornsen Project Supervisor, Dustin Simpson, Steve Trammell, Joshua Orr, and Dustin Dodson.

The sampling followed the procedures set forth in the Code of Federal Regulations, Title 40, Chapter I, Part 60, Appendix A, Methods 1, 2, 3B, 4, 5, and 17; and the Ontario Hydro Method, Revised July 7, 1999 (ASTM D-6784-02). 

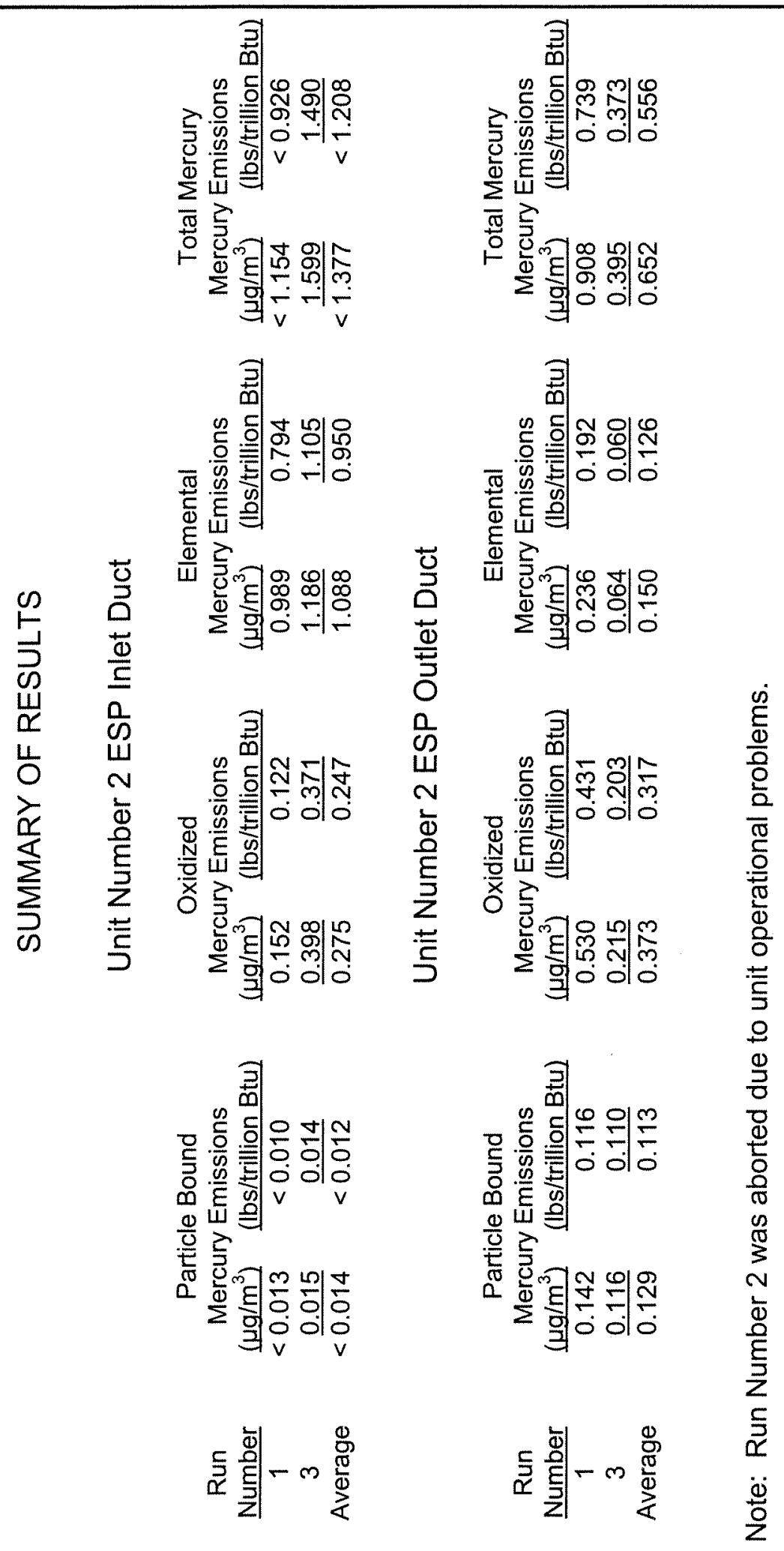


\section{SUMMARY OF RESULTS}

Unit Number 2 ESP Inlet Duct

Ontario Hydro Mercury

\begin{tabular}{||l|c|c||}
\hline \hline Run Number & 1 & 3 \\
\hline Date & $11 / 01 / 05$ & $11 / 02 / 05$ \\
\hline Time & $1340-1603$ & $1415-1636$ \\
\hline Duct Flow Rate - ACFM & 414,707 & 344,005 \\
\hline Duct Flow Rate - DSCFM ${ }^{*}$ & 247,619 & 213,006 \\
\hline$\%$ Water Vapor - \% Vol. & 8.55 & 6.93 \\
\hline$\% \mathrm{CO}_{2}$ - \% Vol. & 13.8 & 11.6 \\
\hline$\% \mathrm{O}_{2}$ - \% Vol. & 5.0 & 7.2 \\
\hline$\%$ Excess Air @ Sampling Point & 30.3 & 50.3 \\
\hline Stack Temperature - ${ }^{\circ} \mathrm{F}$ & 322 & 307 \\
\hline Stack Pressure - "Hg & 28.83 & 28.81 \\
\hline Percent Isokinetic & 101.3 & 99.4 \\
\hline Volume Dry Gas Sampled - DSCF* & 55.706 & 47.042 \\
\hline
\end{tabular}

* $29.92 \mathrm{Hg}, 68^{\circ} \mathrm{F}\left(760 \mathrm{~mm} \mathrm{Hg}, 20^{\circ} \mathrm{C}\right)$

Note: Run Number 2 was aborted due to unit operational problems. 
SUMMARY OF RESULTS

Unit Number 2 ESP Inlet Duct

Ontario Hydro Mercury

\begin{tabular}{||l|c|c||}
\hline \hline Run Number & 1 & 3 \\
\hline Particle Bound Mercury - $\mu \mathrm{g}$ & $<0.02$ & 0.02 \\
\hline Particle Bound Mercury Emissions - $\mu \mathrm{g} / \mathrm{m}^{3^{*}}$ & $<0.013$ & 0.015 \\
\hline Particle Bound Mercury Emissions - $\mathrm{lbs} /$ trillion Btu & $<0.010$ & 0.014 \\
\hline Oxidized Mercury - $\mu \mathrm{g}$ & 0.24 & 0.53 \\
\hline Oxidized Mercury Emissions - $\mu \mathrm{g} / \mathrm{m}^{3^{*}}$ & 0.152 & 0.398 \\
\hline Oxidized Mercury Emissions - $\mathrm{lbs} / \mathrm{trillion} \mathrm{Btu}$ & 0.122 & 0.371 \\
\hline Elemental Mercury - $\mu \mathrm{g}$ & 1.56 & 1.58 \\
\hline Elemental Mercury Emissions - $\mu \mathrm{g} / \mathrm{m}^{3^{*}}$ & 0.989 & 1.186 \\
\hline Elemental Mercury Emissions - $\mathrm{lbs} / \mathrm{trillion} \mathrm{Btu}$ & 0.794 & 1.105 \\
\hline Total Mercury Emissions - $\mu \mathrm{g} / \mathrm{m}^{3^{*}}$ & $<1.154$ & 1.599 \\
\hline Total Mercury Emissions - $\mathrm{lbs} / \mathrm{trillion}$ Btu & $<0.926$ & 1.490 \\
\hline
\end{tabular}

* $29.92 \mathrm{Hg}, 68^{\circ} \mathrm{F}\left(760 \mathrm{~mm} \mathrm{Hg}, 20^{\circ} \mathrm{C}\right)$

Note: Run Number 2 was aborted due to unit operational problems. 
SUMMARY OF RESULTS

Unit Number 2 ESP Outlet Duct

Ontario Hydro Mercury

\begin{tabular}{||l|c|c||}
\hline \hline Run Number & 1 & 3 \\
\hline Date & $11 / 01 / 05$ & $11 / 02 / 05$ \\
\hline Time & $1344-1610$ & $1418-1640$ \\
\hline Duct Flow Rate - ACFM & 437,518 & 372,619 \\
\hline Duct Flow Rate - DSCFM ${ }^{*}$ & 258,122 & 230,337 \\
\hline$\%$ Water Vapor - \% Vol. & 8.85 & 7.20 \\
\hline$\% \mathrm{CO}_{2}$ - \% Vol. & 13.8 & 11.4 \\
\hline$\% \mathrm{O}_{2}$ - \% Vol. & 5.2 & 7.4 \\
\hline$\%$ Excess Air @ Sampling Point & 32.0 & 52.4 \\
\hline Stack Temperature - ${ }^{\circ} \mathrm{F}$ & 326 & 306 \\
\hline Stack Pressure - "Hg & 28.72 & 28.81 \\
\hline Percent Isokinetic & 96.7 & 91.5 \\
\hline Volume Dry Gas Sampled - DSCF* & 71.932 & 60.733 \\
\hline
\end{tabular}

* $29.92 \mathrm{Hg}, 68^{\circ} \mathrm{F}\left(760 \mathrm{~mm} \mathrm{Hg}, 20^{\circ} \mathrm{C}\right)$

Note: Run Number 2 was aborted due to unit operational problems. 


\section{SUMMARY OF RESULTS}

\section{Unit Number 2 ESP Outlet Duct}

Ontario Hydro Mercury

\begin{tabular}{||l|c|c||}
\hline Run Number & 1 & 3 \\
\hline Particle Bound Mercury - $\mu \mathrm{g}$ & 0.29 & 0.20 \\
\hline Particle Bound Mercury Emissions - $\mu \mathrm{g} / \mathrm{m}^{3^{*}}$ & 0.142 & 0.116 \\
\hline Particle Bound Mercury Emissions - $\mathrm{lbs} /$ trillion Btu & 0.116 & 0.110 \\
\hline Oxidized Mercury - $\mu \mathrm{g}$ & 1.08 & 0.37 \\
\hline Oxidized Mercury Emissions - $\mu \mathrm{g} / \mathrm{m}^{3^{*}}$ & 0.530 & 0.215 \\
\hline Oxidized Mercury Emissions - $\mathrm{lbs} / \mathrm{trillion}$ Btu & 0.431 & 0.203 \\
\hline Elemental Mercury - $\mu \mathrm{g}$ & 0.48 & 0.11 \\
\hline Elemental Mercury Emissions - $\mu \mathrm{g} / \mathrm{m}^{3^{*}}$ & 0.236 & 0.064 \\
\hline Elemental Mercury Emissions - $\mathrm{lbs} / \mathrm{trillion} \mathrm{Btu}$ & 0.192 & 0.060 \\
\hline Total Mercury Emissions - $\mu \mathrm{g} / \mathrm{m}^{3^{*}}$ & 0.908 & 0.395 \\
\hline Total Mercury Emissions - $\mathrm{lbs} / \mathrm{trillion}$ Btu & 0.739 & 0.373 \\
\hline
\end{tabular}

* $29.92 \mathrm{Hg}, 68^{\circ} \mathrm{F}\left(760 \mathrm{~mm} \mathrm{Hg}, 20^{\circ} \mathrm{C}\right)$

Note: Run Number 2 was invalid due to unit operational problems. 


\section{DISCUSSION OF RESULTS}

Run Number 2 was aborted due to unit operational problems.

\section{Unit Number 2 ESP Inlet Duct}

The two completed tests for speciated mercury appeared to be valid representations of the actual emissions during the tests. All leak checks performed on the sampling train and the pitot tubes showed no leaks before or after each test. The indicative parameters calculated from the field data were in close agreement. The moisture percentages for the two completed tests were within 10.5 percent of the mean value. The measured flow rates $\left(Q_{s}\right)$ for the tests were within 7.5 percent of the mean value. The rates of sampling for the two completed tests were within the specified limits (90 to 110 percent isokinetic). The greatest deviation from 100 percent isokinetic was

\section{3 percent.}

The calculated emissions (pounds per trillion Btu) of total mercury for the two completed tests showed a range of -23.3 percent to +23.3 percent variation from the mean value.

\section{Unit Number 2 ESP Outlet Duct}

The two completed tests for speciated mercury appeared to be valid representations of the actual emissions during the tests. All leak checks performed on the sampling train and the pitot tubes showed no leaks before or after each test. The indicative parameters calculated from the field data were in close agreement. The moisture percentages for the two completed tests were within 10.3 percent of the mean value. The measured flow rates $\left(Q_{s}\right)$ for the tests were within 5.7 percent of the mean value. The rates of sampling for the two completed tests were within the specified limits (90 to 110 percent isokinetic). The greatest deviation from 100 percent isokinetic was 8.5 percent. 
The calculated emissions (pounds per trillion Btu) of total mercury for the two completed tests showed a range of -32.9 percent to +32.9 percent variation from the mean value. 


\section{DESCRIPTION OF SAMPLING LOCATIONS}

The sampling location on the Unit Number 2 ESP Inlet Duct is 198 feet $61 / 4$ inches above the ground. The sampling ports are located 25 feet 4 inches (2.61 equivalent duct diameters) downstream from a bend in the duct and 6 feet $61 / 4$ inches ( 0.67 equivalent duct diameters) upstream from an expansion in the duct.

The sampling location on the Unit Number 2 ESP Outlet Duct is approximately 200 feet above the ground. The sampling ports are located 6 feet 11 inches $(0.71$ equivalent duct diameters) downstream from a constriction in the duct and 3 feet 2 inches ( 0.33 equivalent duct diameters) upstream from a bend in the duct. 


\section{SAMPLING LOCATION}

Unit Number 2 ESP Inlet Duct

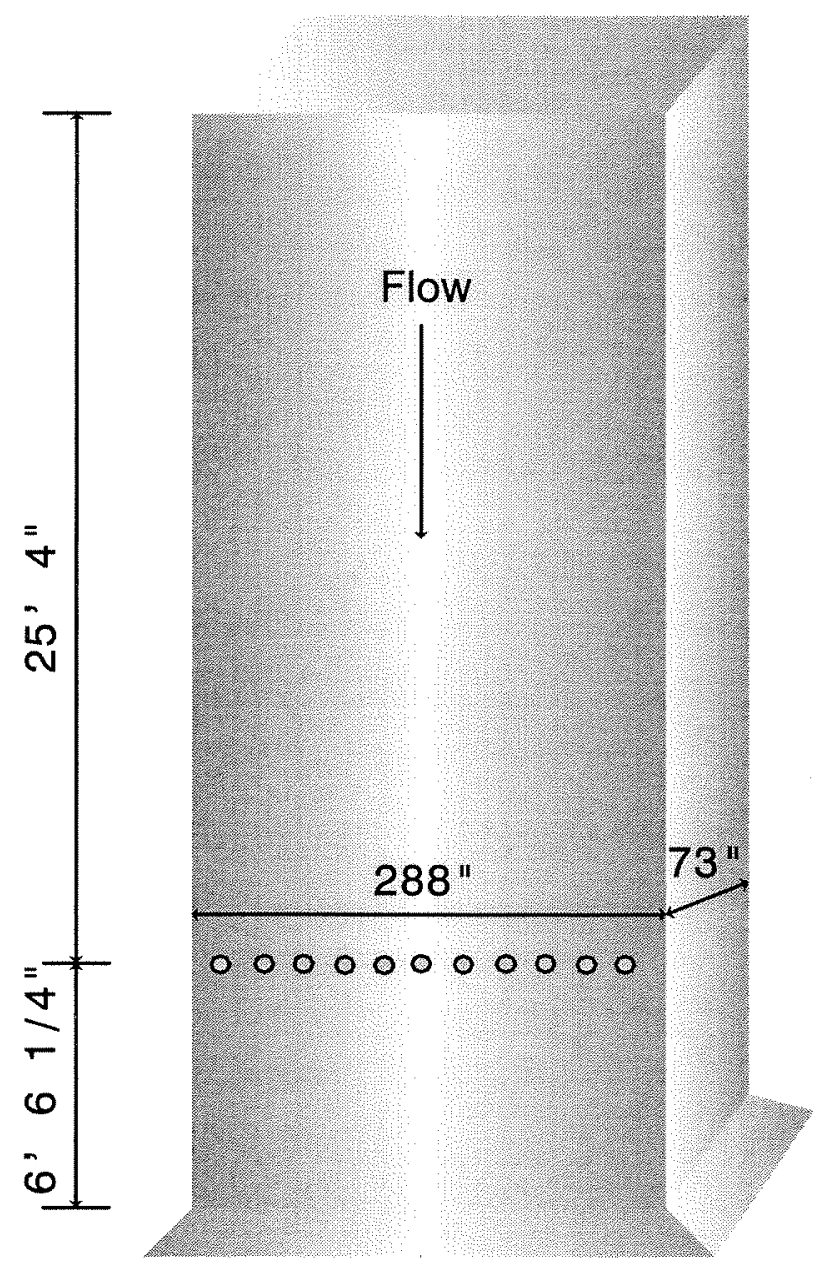




\section{SAMPLING LOCATION}

Unit Number 2 ESP Outlet Duct
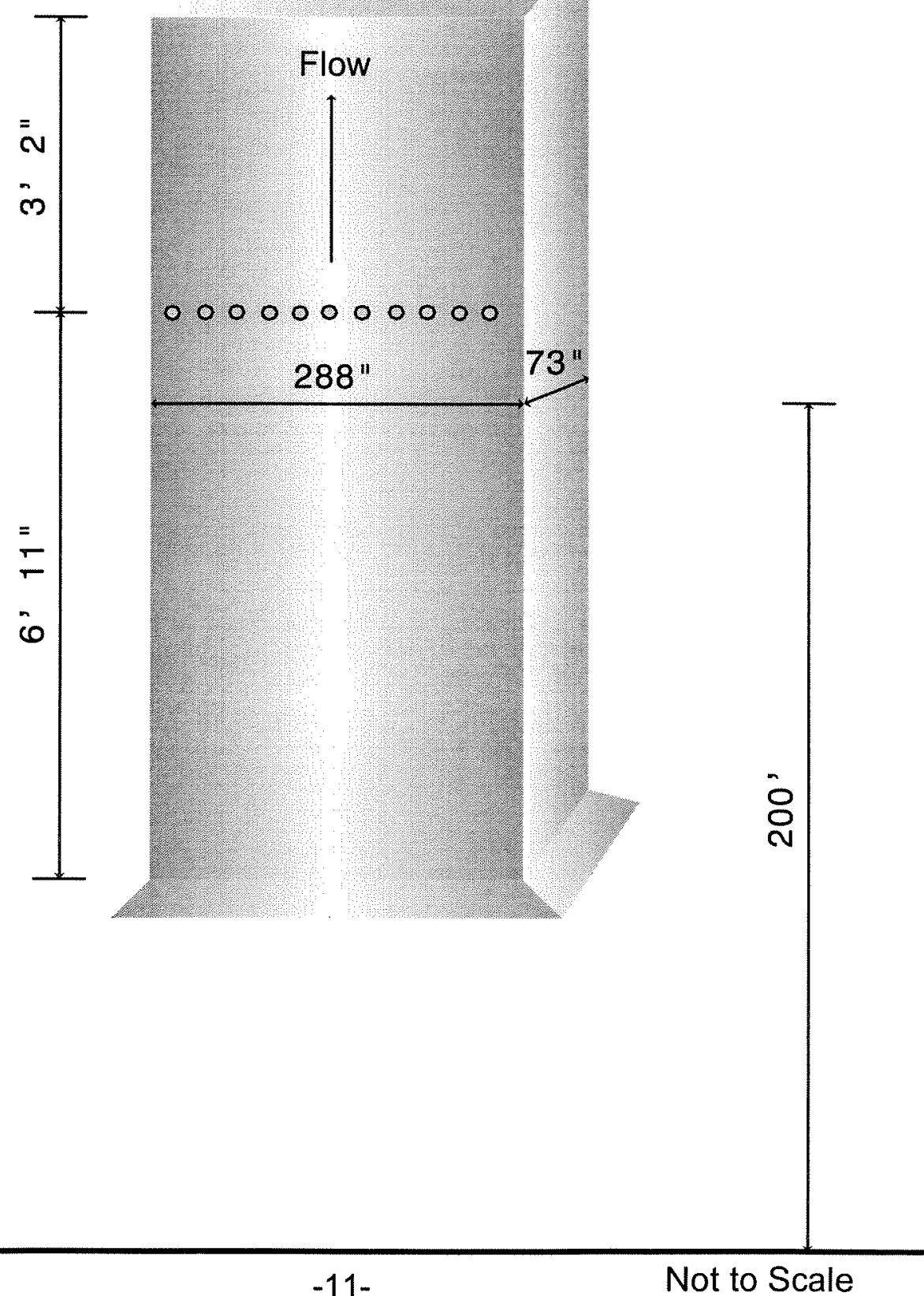


\section{SAMPLING AND ANALYTICAL PROCEDURES}

The sampling followed the procedures set forth in the Code of Federal Regulations, Title 40, Chapter I, Part 60, Appendix A, Methods 1, 2, 3B, 4, 5, and 17; and the Ontario Hydro Method, Revised July 7, 1999 (ASTM D-6784-02).

A preliminary velocity traverse was made at eight of the eleven ports on the Unit Number 2 ESP Inlet Duct, in order to determine the uniformity and magnitude of the flow prior to testing. Several traverse points were checked for cyclonic flow and none was found to be present. Four traverse points were sampled from each of the eight ports for a total of thirty-two traverse points.

A preliminary velocity traverse was made at eight of the twelve ports on the Unit Number 2 ESP Outlet Duct, in order to determine the uniformity and magnitude of the flow prior to testing. Several traverse points were checked for cyclonic flow and none was found to be present. Four traverse points were sampled from each of the eight ports for a total of thirty-two traverse points.

The sampling trains were leak-checked at the nozzle at 15 inches of mercury vacuum before each test, and again after each test at the highest vacuum reading recorded during the test. This was done to predetermine the possibility of a diluted sample.

The pitot tube lines were checked for leaks before and after each test under both a vacuum and a pressure. The lines were also checked for clearance and the manometer was zeroed before each test.

An integrated orsat sample was collected and analyzed according to EPA Method 3B during each test. 


\section{Speciated Mercury}

Duplicate samples for speciated mercury were collected from each source. The samples were taken according to EPA Methods 1, 2, 3B, 4, 5, and 17; and the Ontario Hydro Method, Revised July 7, 1999. For each run, samples of four-minute duration were taken isokinetically at each of the thirty-two traverse points for a total sampling time of 128 minutes. Blank train samples and reagent blanks were submitted.

The " front-half " of the sampling train contained the following components:

Quartz Nozzle

In-Stack Glass Thimble and Filter in a Glass Assembly

Heated Glass Probe @ $248^{\circ} \mathrm{F} \pm 25^{\circ} \mathrm{F}$

Heated Teflon Sample Line @ $248^{\circ} \mathrm{F} \pm 25^{\circ} \mathrm{F}^{*}$

* Only used on the Outlet Duct.

The " back-half " of the sampling train contained the following components:

Impinger

Number

\section{Contents}

1

2

3

4

5

6

7

8
$1 \mathrm{~mol} / \mathrm{L} \mathrm{KCl}$

$1 \mathrm{~mol} / \mathrm{L} \mathrm{KCl}$

$1 \mathrm{~mol} / \mathrm{L} \mathrm{KCl}$

$5 \% \mathrm{HNO}_{3}$ and $10 \% \mathrm{H}_{2} \mathrm{O}_{2}$

$4 \% \mathrm{KMnO}_{4}$ and $10 \% \mathrm{H}_{2} \mathrm{SO}_{4}$

$4 \% \mathrm{KMnO}_{4}$ and $10 \% \mathrm{H}_{2} \mathrm{SO}_{4}$

$4 \% \mathrm{KMnO}_{4}$ and $10 \% \mathrm{H}_{2} \mathrm{SO}_{4}$

Silica Gel

\begin{tabular}{|c|c|}
\hline Amount & Parameter Collected \\
\hline $100 \mathrm{ml}$ & Oxidized Mercury \\
\hline $100 \mathrm{ml}$ & Oxidized Mercury \\
\hline $100 \mathrm{ml}$ & Oxidized Mercury \\
\hline $100 \mathrm{ml}$ & Elemental Mercury \\
\hline $100 \mathrm{ml}$ & Elemental Mercury \\
\hline $100 \mathrm{ml}$ & Elemental Mercury \\
\hline $100 \mathrm{ml}$ & Elemental Mercury \\
\hline $200 \mathrm{~g}$ & Moisture \\
\hline
\end{tabular}

The mercury samples were analyzed by cold vapor atomic absorption spectroscopy. 


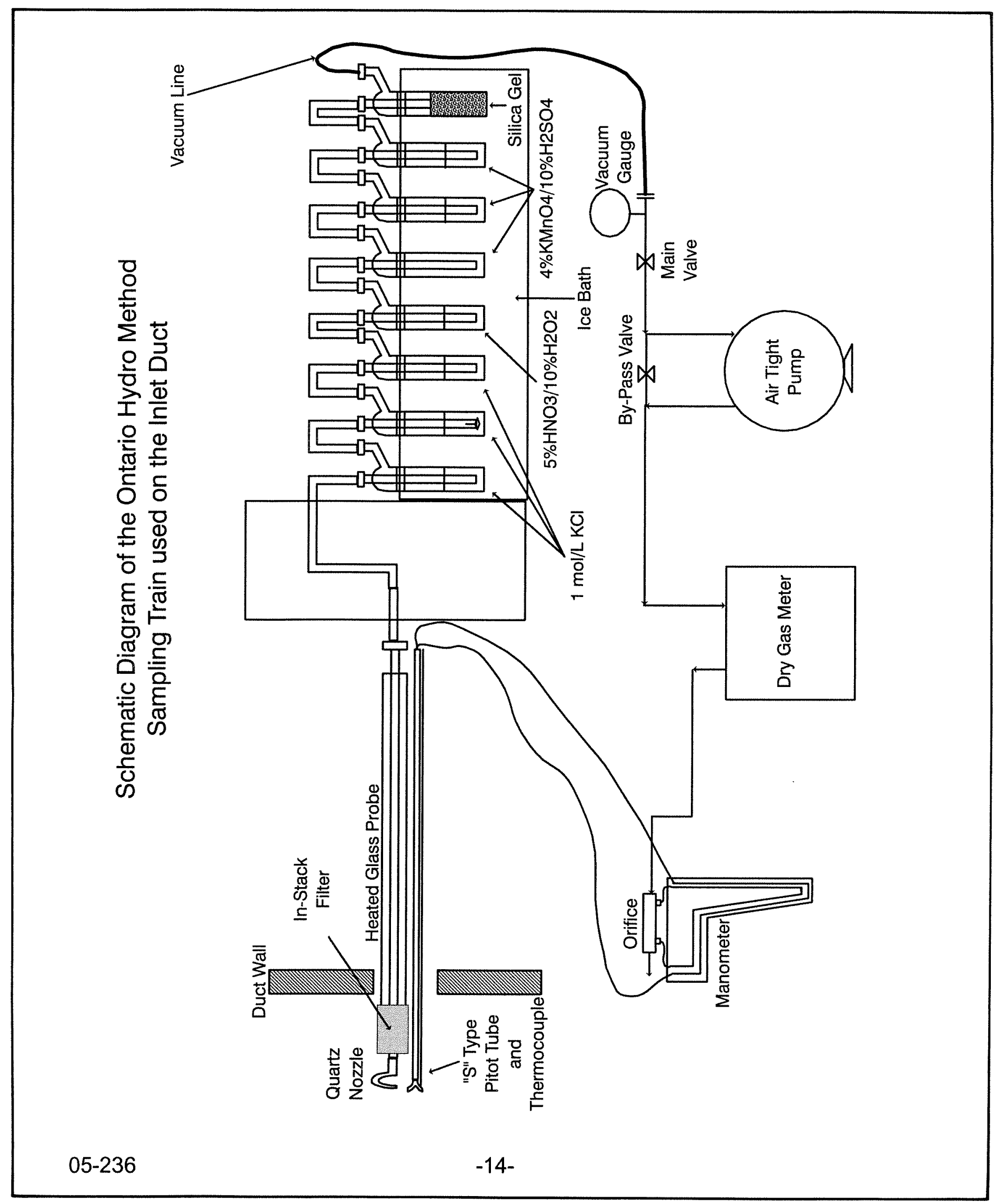




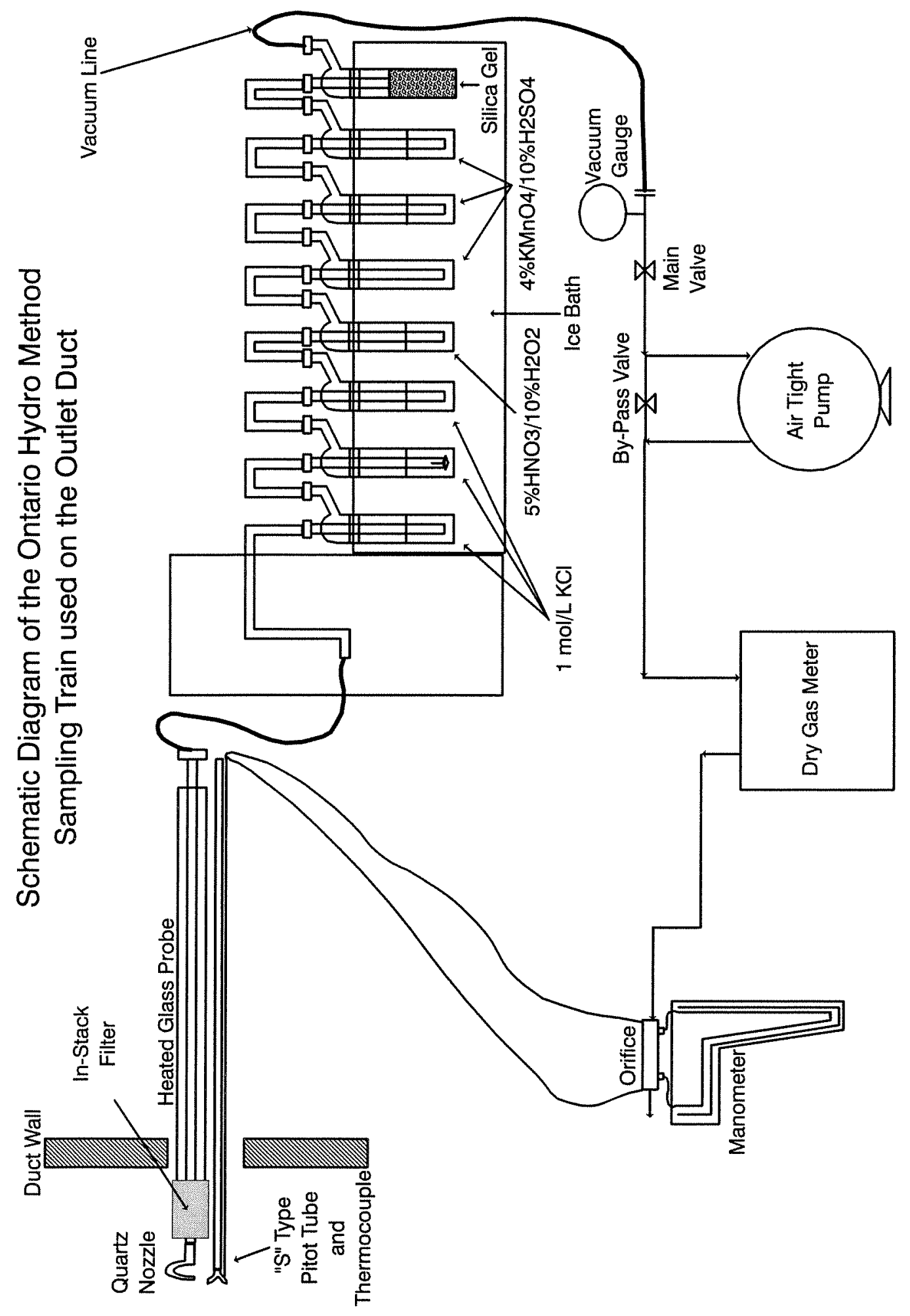




\section{DESCRIPTION OF TESTS}

Personnel from METCO Environmental arrived at the plant at 8:45 a.m. on Tuesday, November 1, 2005. After meeting with plant personnel and attending a brief safety orientation, the equipment was moved onto the Unit Number 2 ESP Inlet and Outlet Ducts and prepared for testing. The first set of tests for speciated mercury began at 1:40 p.m. and was completed at 4:10 p.m. The second set of tests for speciated mercury began at 4:41p.m. and was aborted at 5:52 p.m. due to unit operational problems. The samples were recovered. The equipment was secured for the night and all work was completed at 6:30 p.m.

On Wednesday, November 2, work began at 10:45 a.m. The equipment was prepared for testing. The third set of tests for speciated mercury began at 2:15 p.m. and was completed at 4:40 p.m.

The equipment was moved off of the sampling locations and loaded into the sampling van. The samples were recovered and transported to METCO Environmental's laboratory in Dallas, Texas, for analysis and evaluation.

Operations at Arkansas Power \& Light Company, Independence Steam Electric Station, Unit Number 2 ESP Inlet and Outlet Ducts, located in Newark, Arkansas, for ADA-ES, were completed at 7:15 p.m. on Wednesday, November 2, 2005. 


\section{APPENDICES}
A. Location of Sampling Points
B. Source Emissions Calculations
C. Calibration Data
D. Field Testing Data
E. Analytical Data
F. Chain of Custody
G. Resumes of Test Personnel 


\section{APPENDIX A}

\section{Location of Sampling Points}

Unit Number 2 ESP Inlet Duct

The sampling ports are located 25 feet 4 inches (2.61 equivalent duct diameters) downstream from a bend in the duct and 6 feet $61 / 4$ inches ( 0.67 equivalent duct diameters) upstream from an expansion in the duct. The locations of the sampling points were calculated as follows:

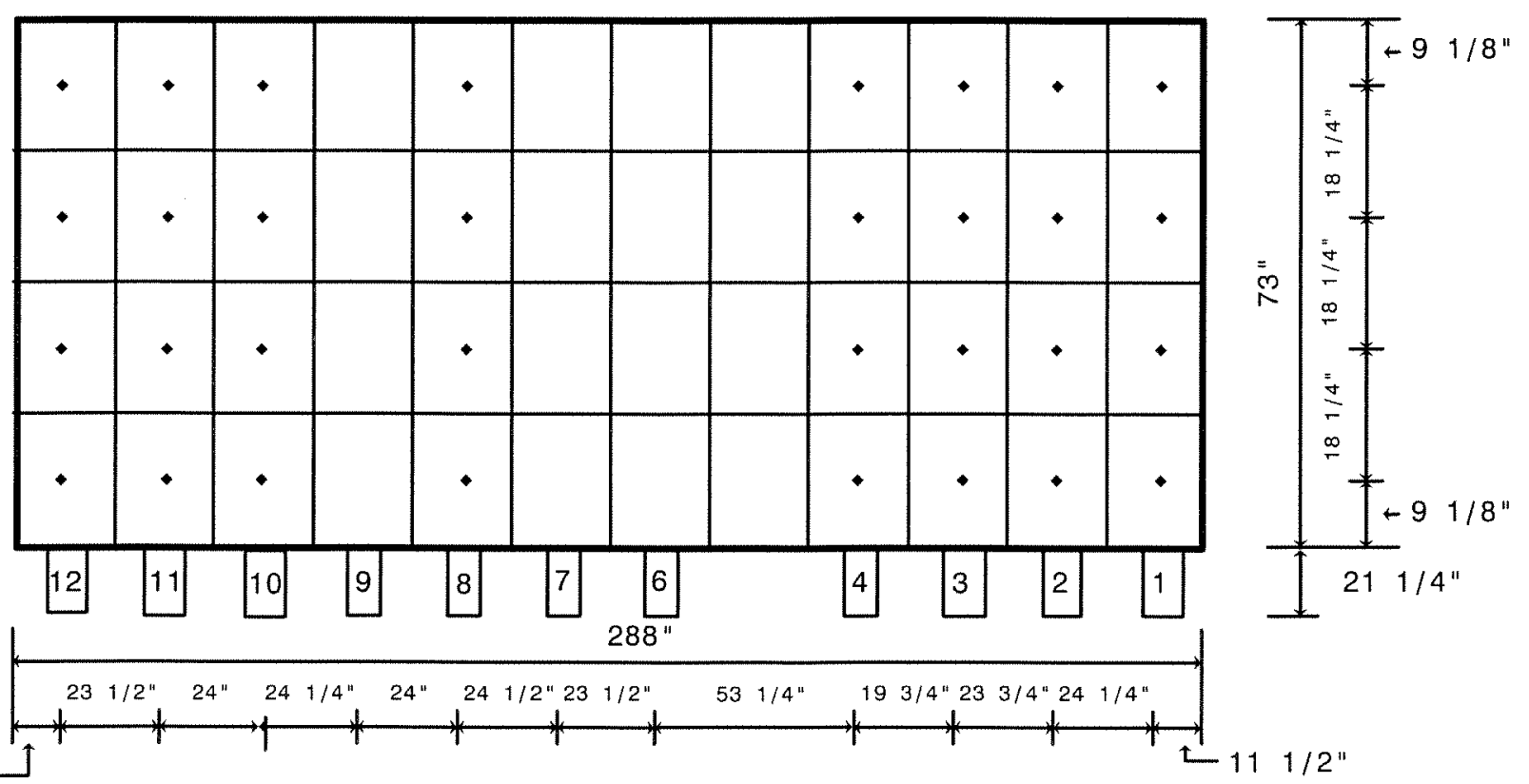

$113 / 4^{\prime \prime}$

Note: The duct has 17 " of insulation. Port 5 does not exist. Ports 6 and 7 are not located in the gas stream and were not sampled. The width of where Ports 6 and 7 are located is 5' 5". This leaves a width of 18' 7" on which the area was calculated. A plant monitor probe is located in Port 9. 


\section{APPENDIX A \\ Location of Sampling Points \\ Unit Number 2 ESP Outlet Duct}

The sampling ports are located 6 feet 11 inches ( 0.71 equivalent duct diameters) downstream from a constriction in the duct and 3 feet 2 inches $(0.33$ equivalent duct diameters) upstream from a bend in the duct. The locations of the sampling points were calculated as follows:
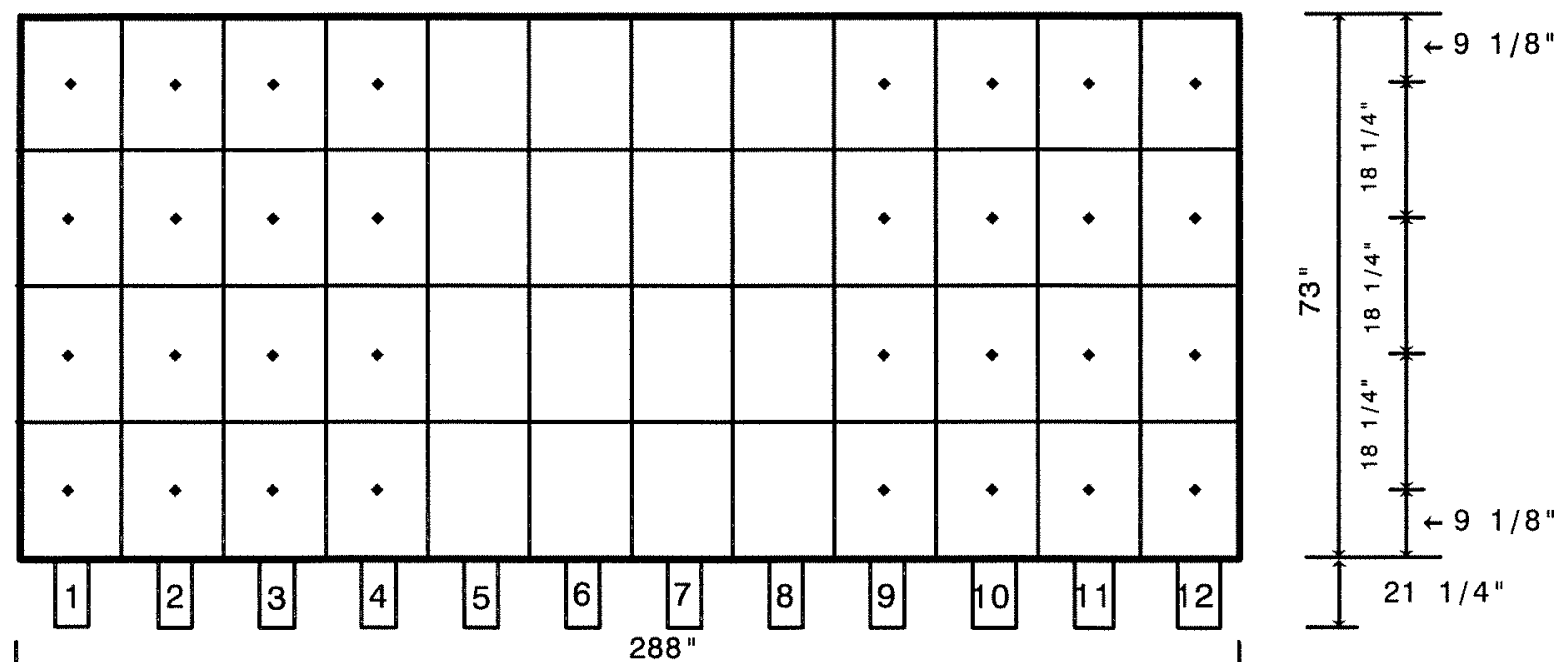

$113 / 4 "-$

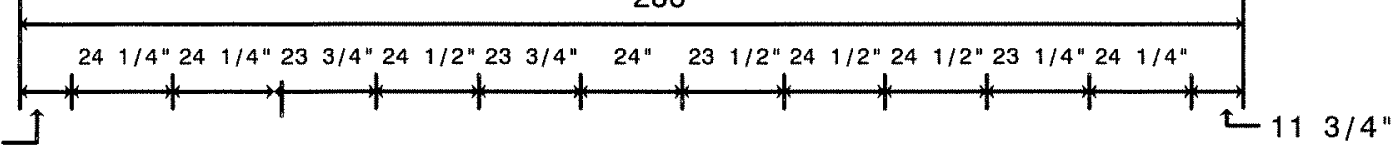

Note: The duct has 17 " of insulation. Ports 5 and 6 were not accessible and could not be sampled. 


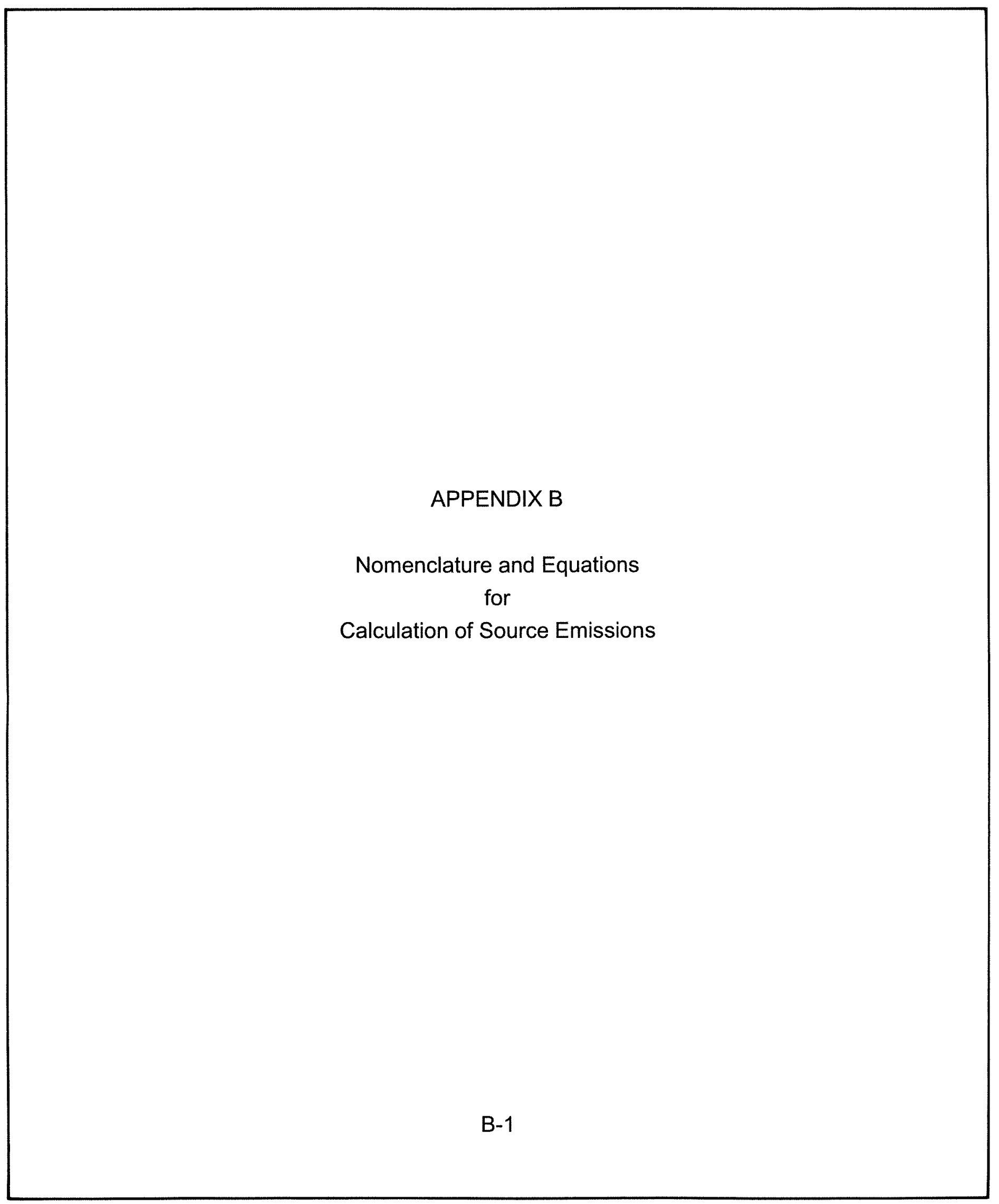




\section{NOMENCLATURE FOR FLOW RATE AND MOISTURE CALCULATIONS}

\begin{tabular}{|c|c|c|c|}
\hline Symbol & $\begin{array}{l}\text { English } \\
\underline{\text { Units }}\end{array}$ & $\begin{array}{l}\text { Metric } \\
\underline{\text { Units }}\end{array}$ & Description \\
\hline$A_{s}$ & in. $^{2}$ & $\mathrm{~m}^{2}$ & Stack Area \\
\hline$C_{p}$ & & & Pitot Tube Calibration Factor \\
\hline$\% E A$ & & & Percent Excess Air at sampling point \\
\hline g & $32.2 \mathrm{ft} / \mathrm{sec}^{2}$ & & Acceleration of Gravity \\
\hline$\%$ & & & Percent Isokinetic \\
\hline$\% M$ & & & $\begin{array}{l}\text { Percent Moisture in the stack gas by } \\
\text { volume }\end{array}$ \\
\hline$M_{d}$ & & & Mole fraction of dry gas \\
\hline$M_{\text {water }}$ & $18 \mathrm{lb} / \mathrm{lb}-\mathrm{mole}$ & & Molecular Weight of water \\
\hline MW & $\mathrm{lb} / \mathrm{lb}$-mole & g/g-mole & Molecular Weight of stack gas \\
\hline $\mathrm{MW}_{\text {air }}$ & $\begin{array}{l}28.96 \mathrm{lb} / \\
\mathrm{lb}-\mathrm{mole}\end{array}$ & & Molecular Weight of air \\
\hline$M W_{d}$ & $\mathrm{lb} / \mathrm{lb}$-mole & g/g-mole & Molecular Weight of dry stack gas \\
\hline$P_{b}$ & $\begin{array}{c}\text { "Hg } \\
\text { Absolute }\end{array}$ & $\mathrm{mm} \mathrm{Hg}$ & Barometric Pressure \\
\hline$P_{m}$ & $" \mathrm{H}_{2} \mathrm{O}$ & $\mathrm{mm} \mathrm{H} \mathrm{H}_{2} \mathrm{O}$ & Orifice Pressure drop \\
\hline
\end{tabular}




\begin{tabular}{|c|c|c|c|}
\hline Symbol & $\begin{array}{l}\text { English } \\
\text { Units }\end{array}$ & $\begin{array}{l}\text { Metric } \\
\text { Units }\end{array}$ & Description \\
\hline$P_{s}$ & $\begin{array}{c}\text { "Hg } \\
\text { Absolute }\end{array}$ & $\mathrm{mm} \mathrm{Hg}$ & Stack Pressure \\
\hline$\Delta \mathrm{P}$ & $" \mathrm{H}_{2} \mathrm{O}$ & $\mathrm{mm} \mathrm{H} \mathrm{H}_{2} \mathrm{O}$ & Velocity Head of stack gas \\
\hline$P_{\text {std }}$ & $29.92 \mathrm{"Hg}$ & $760 \mathrm{~mm} \mathrm{Hg}$ & Standard Barometric Pressure \\
\hline$Q_{a}$ & ACFM & $\mathrm{m}^{3} / \mathrm{hr}$ & $\begin{array}{l}\text { Stack Gas Volume at actual stack } \\
\text { conditions }\end{array}$ \\
\hline$Q_{s}$ & DSCFM $^{*}$ & $\mathrm{dscm} / \mathrm{hr} r^{*}$ & $\begin{array}{l}\text { Stack Gas Volume at } 29.92 \text { "Hg, } \\
528^{\circ} \mathrm{R} \text {, dry }\end{array}$ \\
\hline $\mathrm{R}$ & $\begin{array}{l}21.83 " \mathrm{Hg}- \\
\mathrm{ft}^{3} / \mathrm{lb}-\mathrm{mole}^{\circ} \mathrm{R}\end{array}$ & & Universal Gas Constant \\
\hline$T_{m}$ & ${ }^{\circ} \mathrm{F}$ & ${ }^{\circ} \mathrm{C}$ & Average Gas Meter Temperature \\
\hline$T_{t}$ & $\min$ & $\min$ & Net time of test \\
\hline $\mathrm{T}_{\mathrm{s}}$ & ${ }^{\circ} \mathrm{F}$ & ${ }^{\circ} \mathrm{C}$ & Stack Temperature \\
\hline$T_{\text {std }}$ & $528^{\circ} \mathrm{R}$ & $293^{\circ} \mathrm{K}$ & Standard Temperature \\
\hline$V_{m}$ & $\mathrm{ft}^{3}$ & $\mathrm{~m}^{3}$ & $\begin{array}{l}\text { Volume of dry gas sampled @ meter } \\
\text { conditions }\end{array}$ \\
\hline$V m_{\text {std }}$ & dscf* & dscm* & $\begin{array}{l}\text { Volume of dry gas sampled @ } \\
\text { standard conditions }\end{array}$ \\
\hline $\mathrm{V}_{\mathrm{s}}$ & fpm & $\mathrm{m} / \mathrm{sec}$ & Stack velocity @ stack conditions \\
\hline$V_{w}$ & $\mathrm{ml}$ & $\mathrm{ml}$ & $\begin{array}{l}\text { Total water collected in impingers and } \\
\text { silica gel }\end{array}$ \\
\hline
\end{tabular}

* $29.92 \mathrm{Hg}, 68^{\circ} \mathrm{F}\left(760 \mathrm{~mm} \mathrm{Hg}, 20^{\circ} \mathrm{C}\right) \quad$ B-3 


\begin{tabular}{|c|c|c|c|}
\hline & English & Metric & \\
\hline Symbol & Units & Units & Description \\
\hline$\overline{V_{\text {gas }}}$ & $\overline{s c f^{*}}$ & $\overline{\mathrm{scm}^{*}}$ & $\begin{array}{l}\text { Volume of water vapor collected @ } \\
\text { standard conditions }\end{array}$ \\
\hline Pair & $0.0752 \mathrm{lbs} / \mathrm{ft}^{3}$ & & Density of Air \\
\hline Pwater & $1 \mathrm{~g} / \mathrm{ml}$ & & Density of Water \\
\hline Pman & $62.32 \mathrm{lbs} / \mathrm{ft}^{3}$ & & Density of Manometer Oil \\
\hline
\end{tabular}

Standard Conditions: 29.92 " $\mathrm{Hg}, 68^{\circ} \mathrm{F}\left(760 \mathrm{~mm} \mathrm{Hg}, 20^{\circ} \mathrm{C}\right)$ 


\section{EXAMPLE CALCULATIONS}

1. Volume of dry gas sampled at standard conditions. *

$$
\begin{aligned}
& V m_{\text {std }}=V m\left(\frac{T_{\text {std }}}{T_{m}+460}\right)\left[\frac{P_{b}+\frac{P_{m}}{13.6}}{P_{\text {std }}}\right] \\
& V m_{\text {std }}=17.65 \mathrm{Vm}\left[\frac{P_{b}+\frac{P_{m}}{13.6}}{T_{m}+460}\right]=d s c f \\
& V m_{\text {std }}=d s c f \times 0.028317=d s c m
\end{aligned}
$$

2. Volume of water vapor collected at standard conditions. *

$$
\begin{aligned}
& V w_{\text {gas }}=\frac{\left(V_{w}-g m s \mathrm{SO}_{2}-g m s \mathrm{H}_{2} S\right) \rho_{\text {water }} R T_{\text {std }}}{P_{\text {std }} M_{\text {water }} 453.6} \\
& V w_{\text {gas }}=0.0472\left(V_{w}-g m s \mathrm{SO}_{2}-g m s \mathrm{H}_{2} S\right)=s c f \\
& V w_{\text {gas }}=s c f \times 0.028317=s c m
\end{aligned}
$$

3. Percent moisture in stack gas.

$$
\% M=\frac{V W_{\text {gas }}}{V m_{\text {std }}+V W_{\text {gas }}} \times 100=\%
$$

\footnotetext{
* $29.92 \mathrm{Hg}, 68^{\circ} \mathrm{F}\left(760 \mathrm{~mm} \mathrm{Hg}, 20^{\circ} \mathrm{C}\right) \quad$ B-5
} 
4. Mole fraction of dry gas.

$$
M_{d}=\frac{100-\% M}{100}
$$

5. Average molecular weight of dry stack gas.

$$
\begin{aligned}
M W_{d}=\left[\% \mathrm{CO}_{2} \times \frac{44}{100}\right]+\left[\% \mathrm{O}_{2} \times \frac{32}{100}\right]+\left[\% \mathrm{~N}_{2} \times \frac{28}{100}\right]+\left[\% \mathrm{CO} \times \frac{28}{100}\right] & =\mathrm{lb} / \mathrm{lb}-\text { mole } \\
& =g / \mathrm{g}-\text { mole }
\end{aligned}
$$

6. Molecular weight of stack gas.

$$
M W=M W_{d} \times M_{d}+18\left(1-M_{d}\right)=\frac{l b}{l b-\text { mole }}=g / g-\text { mole }
$$

7 Percent excess air at sampling point.

$$
\% E A=\frac{100\left[\% \mathrm{O}_{2}-(0.5 \% \mathrm{CO})\right]}{0.265\left(\% \mathrm{~N}_{2}\right)-\left[\% \mathrm{O}_{2}-(0.5 \% \mathrm{CO})\right]}
$$

8. Stack Pressure.

$$
P_{s}=P_{b}+\frac{\text { Stack Pressure " } \mathrm{H}_{2} \mathrm{O}}{13.6}=" \mathrm{Hg} \text { Absolute }
$$

$$
P_{s}=" H g \text { Abs. } \times 25.4=m m H g
$$


9. Stack velocity at stack conditions.

$$
\begin{aligned}
& V_{s}=C_{p} 60\left[\frac{2 g \times \rho_{\text {man }} \times P_{\text {std }} \times M W_{\text {air }} \times\left(T_{s}+460\right) \times \Delta P}{12 \times \rho_{\text {air }} \times P_{s} \times M W \times T_{\text {std }}}\right]^{1 / 2} \\
& V_{s}=5,123.8 C_{p}\left[\frac{\left(T_{s}+460\right)}{P_{s} \times M W}\right]^{1 / 2} \sqrt{\Delta P} \text { average }=f p m \\
& V_{s}=f p m \times 0.00508=\mathrm{m} / \mathrm{sec}
\end{aligned}
$$

10. Dry stack gas volume at standard conditions. *

$$
\begin{aligned}
& Q_{s}=\frac{1}{144} V_{s} \times A_{s} \times M_{d} \times \frac{T_{s t d}}{T_{s}+460} \times \frac{P_{s}}{P_{s t d}} \\
& Q_{s}=\frac{0.123 V_{s} \times A_{s} \times M_{d} \times P_{s}}{T_{s}+460}=D S C F M \\
& Q_{s}=D S C F M \times 1.6990=\mathrm{dscm} / \mathrm{hr}
\end{aligned}
$$

11. Actual stack gas volume at stack conditions.

$$
\begin{aligned}
& Q_{a}=\frac{V_{s} \times A_{s}}{144}=A C F M \\
& Q_{a}=A C F M \times 1.6990=m^{3} / h r
\end{aligned}
$$


12. Percent Isokinetic.

$$
\begin{aligned}
& \% I=\frac{V m_{s t d} \times\left(T_{s}+460\right) \times P_{s t d} \times 100 \times 144 \mathrm{in}^{2} / \mathrm{ft}^{2}}{M_{d} \times T_{s t d} \times P_{s} \times T_{t} \times V_{s}\left(\frac{\pi \times D_{n}^{2}}{4}\right)} \\
& \% I=\frac{1039 \times V m_{s t d} \times\left(T_{s}+460\right)}{M_{d} \times P_{s} \times T_{t} \times V_{s} \times D_{n}^{2}}
\end{aligned}
$$

* $29.92 \mathrm{Hg}, 68^{\circ} \mathrm{F}\left(760 \mathrm{~mm} \mathrm{Hg}, 20^{\circ} \mathrm{C}\right)$ 


\title{
EXAMPLE CALCULATIONS
}

\author{
$\mathrm{lbs} /$ trillion Btu $=\underline{\mathrm{lbs} / \mathrm{dscf}^{*} \times \mathrm{F}_{\mathrm{d}} \text { factor } \times 20.9 \% \mathrm{O}_{2}}$ \\ $20.9 \% \mathrm{O}_{2}-\% \mathrm{O}_{2}$ measured \\ $F_{d}=$ Oxygen based $F$ factor \\ Fuel \\ $\underline{E}_{d}$ factor \\ Bituminous Coal \\ $9.780 \times 10^{9} \mathrm{dscf}^{*} /$ trillion Btu
}

* $29.92 \mathrm{Hg}, 68^{\circ} \mathrm{F}\left(760 \mathrm{~mm} \mathrm{Hg}, 20^{\circ} \mathrm{C}\right)$ 
SOURCE EMISSION SURVEY

\author{
JOB NUMBER: $05-236$ \\ JOB NAME: ADA-ES \\ LOCATION: NEWARK, ARKANSAS \\ UNIT TESTED: UNIT NUMBER 2 ESP INLET DUCT
}

SOURCE EMISSION CALCULATIONS

\begin{tabular}{|c|c|c|c|c|c|}
\hline & & & \multicolumn{3}{|c|}{ RUN NUMBER } \\
\hline SYMBOL & DESCRIPTION & UNITS & 1 & 3 & \\
\hline DATE & & & $11 / 01 / 05$ & $11 / 02 / 05$ & \\
\hline BEGIN TIME & & & 1340 & 1415 & \\
\hline END TIME & & & 1603 & 1636 & \\
\hline$P(b)$ & BAROMETRIC PRESSURE & $\begin{array}{l}\text { "Hg Abs. } \\
(\mathrm{mm} \mathrm{Hg})\end{array}$ & $\begin{array}{r}29.90 \\
(759.00)\end{array}$ & $\begin{array}{r}29.88 \\
(759.00)\end{array}$ & \\
\hline$P(m)$ & ORIFICE PRESSURE DROP & $\begin{array}{l}\text { "H2O } \\
(\mathrm{mm} \mathrm{H} 2 \mathrm{O})\end{array}$ & $\begin{array}{c}0.583 \\
(14.800)\end{array}$ & $\begin{array}{r}0.431 \\
(10.900)\end{array}$ & \\
\hline & DGM CALIBRATION FACTOR & & 1.008 & 1.008 & \\
\hline$V(m)$ & $\begin{array}{l}\text { VOLUME DRY GAS SAMPLED } \\
\text { @ METER CONDITIONS } \\
\text { LEAK CHECK VOLUME }\end{array}$ & $\begin{array}{l}\mathrm{ft} .^{\wedge} 3 \\
\left(\mathrm{~m}^{\wedge} 3\right) \\
\mathrm{ft} .^{\wedge} 3\end{array}$ & $\begin{array}{c}55.443 \\
(1.570) \\
0.497 \\
\end{array}$ & $\begin{array}{c}48.295 \\
(1.368) \\
0.491\end{array}$ & \\
\hline$T(m)$ & $\begin{array}{l}\text { AVERAGE GAS METER } \\
\text { TEMPERATURE }\end{array}$ & $\begin{array}{l}\text { DEG.F } \\
\text { (DEG.C) }\end{array}$ & $\begin{array}{c}66 \\
(19) \\
\end{array}$ & $\begin{array}{r}82 \\
(28) \\
\end{array}$ & \\
\hline$V(m[s t d])^{\star}$ & $\begin{array}{l}\text { VOLUME DRY GAS SAMPLED } \\
@ \text { STANDARD CONDITIONS* } \\
\end{array}$ & $\begin{array}{l}\text { DSCF } \\
(\mathrm{DSCM}) \\
\end{array}$ & $\begin{array}{r}55.706 \\
(1.577) \\
\end{array}$ & $\begin{array}{l}47.042 \\
(1.332) \\
\end{array}$ & \\
\hline$V(w)$ & $\begin{array}{l}\text { TOTAL WATER COLLECTED, } \\
\text { IMPINGERS \& SILICA GEL }\end{array}$ & $\mathrm{ml}$ & 110.4 & 74.2 & \\
\hline$V(w[$ gas $])$ & $\begin{array}{l}\text { VOLUME WATER VAPOR } \\
\text { COLLECTED @ STANDARD } \\
\text { CONDITIONS* }\end{array}$ & $\begin{array}{l}\text { SCF } \\
(\mathrm{SCM})\end{array}$ & $\begin{array}{c}5.211 \\
(0.148)\end{array}$ & $\begin{array}{c}3.502 \\
(0.099)\end{array}$ & \\
\hline$\% M$ & $\begin{array}{l}\text { MOISTURE IN STACK GAS } \\
\text { BY VOLUME }\end{array}$ & $\%$ & 8.55 & 6.93 & \\
\hline Md & MOL FRACTION OF DRY GAS & & 0.9145 & 0.9307 & \\
\hline $\mathrm{Tt}$ & NET TIME OF TEST & MINUTES & 128 & 128 & \\
\hline
\end{tabular}

* 68 Deg.F, 29.92 "Hg (20 Deg.C, 760 mm Hg) 
JOB NUMBER: 05-236

\section{SOURCE EMISSION CALCULATIONS}

JOB NAME: ADA-ES

LOCATION: NEWARK, ARKANSAS

UNIT TESTED: UNIT NUMBER 2 ESP INLET DUCT

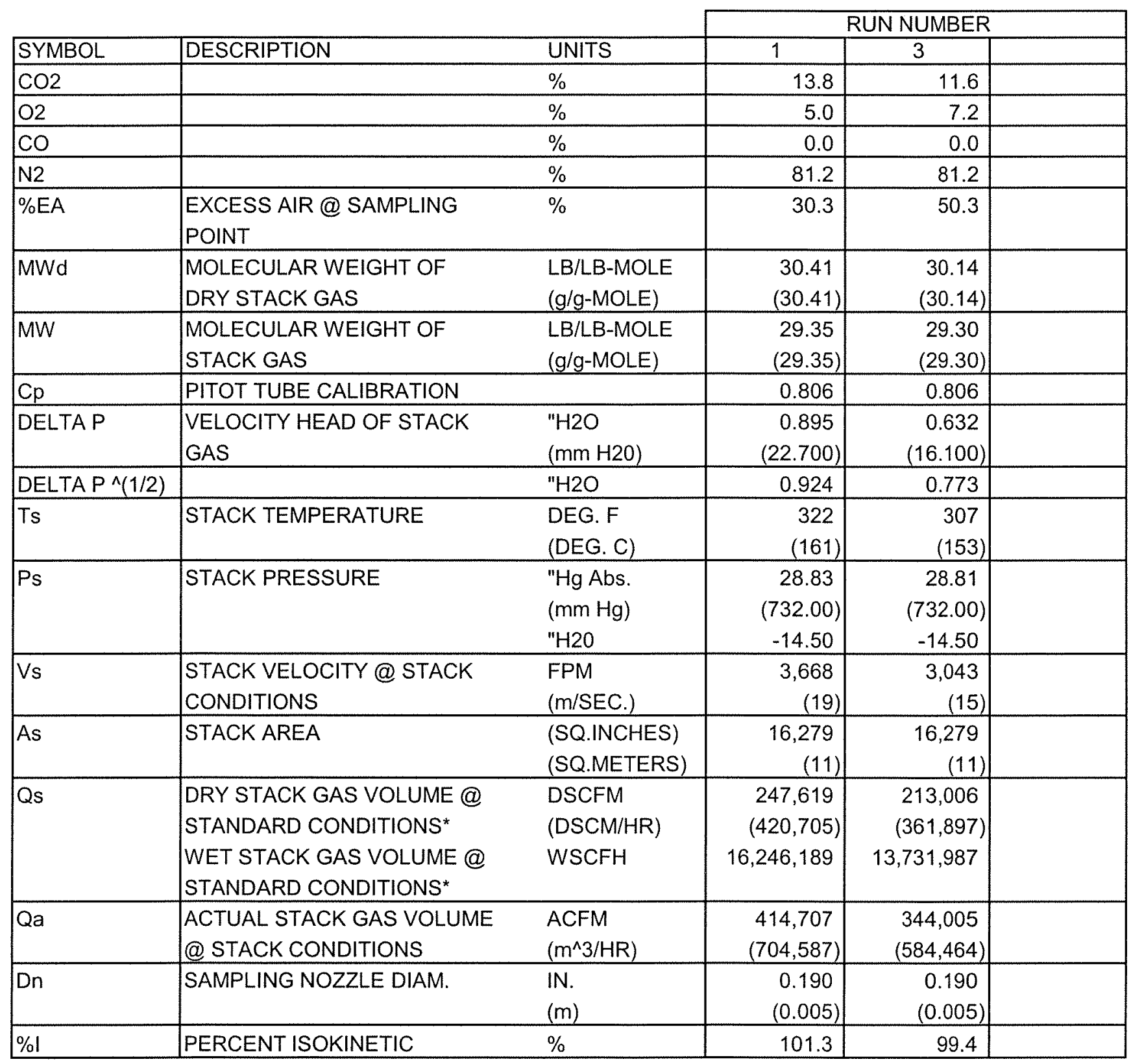

*68 Deg.F, 29.92 "Hg (20 Deg.C, 760 mm Hg) 
SOURCE EMISSION SURVEY

JOB NUMBER: 05-236

JOB NAME: ADA-ES

LOCATION: NEWARK, ARKANSAS

UNIT TESTED: UNIT NUMBER 2 ESP OUTLET DUCT

SOURCE EMISSION CALCULATIONS

\begin{tabular}{|c|c|c|c|c|c|}
\hline \multirow[b]{2}{*}{ SYMBOL } & \multirow[b]{2}{*}{ DESCRIPTION } & \multirow[b]{2}{*}{ UNITS } & \multicolumn{3}{|c|}{ RUN NUMBER } \\
\hline & & & 1 & 3 & \\
\hline DATE & & & $11 / 01 / 05$ & $11 / 02 / 05$ & \\
\hline BEGIN TIME & & & 1344 & 1418 & \\
\hline END TIME & & & 1610 & 1640 & \\
\hline$P(b)$ & BAROMETRIC PRESSURE & $\begin{array}{l}\text { "Hg Abs. } \\
(\mathrm{mm} \mathrm{Hg})\end{array}$ & $\begin{array}{r}29.82 \\
(757.00) \\
\end{array}$ & $\begin{array}{r}29.88 \\
(759.00)\end{array}$ & \\
\hline$P(m)$ & ORIFICE PRESSURE DROP & $\begin{array}{l}\text { "H2O } \\
(\mathrm{mm} \mathrm{H} 2 \mathrm{O}) \\
\end{array}$ & $\begin{array}{r}1.055 \\
(26.800) \\
\end{array}$ & $\begin{array}{r}0.799 \\
(20.300) \\
\end{array}$ & \\
\hline & DGM CALIBRATION FACTOR & & 0.991 & 0.991 & \\
\hline$V(m)$ & $\begin{array}{l}\text { VOLUME DRY GAS SAMPLED } \\
\text { @ METER CONDITIONS } \\
\text { LEAK CHECK VOLUME }\end{array}$ & $\begin{array}{l}\text { ft. }{ }^{\wedge} \\
\left(m^{\wedge} 3\right) \\
\mathrm{ft}^{\wedge} 3\end{array}$ & $\begin{array}{c}71.429 \\
(2.023) \\
0.000\end{array}$ & $\begin{array}{c}61.949 \\
(1.754) \\
0.000\end{array}$ & \\
\hline$T(m)$ & $\begin{array}{l}\text { AVERAGE GAS METER } \\
\text { TEMPERATURE }\end{array}$ & $\begin{array}{l}\text { DEG.F } \\
\text { (DEG.C) }\end{array}$ & $\begin{array}{c}64 \\
(18) \\
\end{array}$ & $\begin{array}{r}79 \\
(26) \\
\end{array}$ & \\
\hline$V(m[s t d])^{*}$ & $\begin{array}{l}\text { VOLUME DRY GAS SAMPLED } \\
\text { @ STANDARD CONDITIONS* }\end{array}$ & $\begin{array}{l}\text { DSCF } \\
\text { (DSCM) }\end{array}$ & $\begin{array}{l}71.932 \\
(2.037) \\
\end{array}$ & $\begin{array}{l}60.733 \\
(1.720) \\
\end{array}$ & \\
\hline$V(w)$ & $\begin{array}{l}\text { TOTAL WATER COLLECTED, } \\
\text { IMPINGERS \& SILICA GEL }\end{array}$ & $\mathrm{ml}$ & 148.0 & 99.9 & \\
\hline$V(w[$ gas $])$ & $\begin{array}{l}\text { VOLUME WATER VAPOR } \\
\text { COLLECTED @ STANDARD } \\
\text { CONDITIONS* }\end{array}$ & $\begin{array}{l}\text { SCF } \\
(\mathrm{SCM})\end{array}$ & $\begin{array}{c}6.986 \\
(0.198)\end{array}$ & $\begin{array}{c}4.715 \\
(0.134)\end{array}$ & \\
\hline$\% M$ & $\begin{array}{l}\text { MOISTURE IN STACK GAS } \\
\text { BY VOLUME }\end{array}$ & $\%$ & 8.85 & 7.20 & \\
\hline Md & MOL FRACTION OF DRY GAS & & 0.9115 & 0.9280 & \\
\hline $\mathrm{Tt}$ & NET TIME OF TEST & MINUTES & 128 & 128 & \\
\hline
\end{tabular}

* 68 Deg.F, 29.92 "Hg (20 Deg.C, 760 mm Hg) 
JOB NUMBER: $\quad 05-236$

SOURCE EMISSION CALCULATIONS

JOB NAME: ADA-ES

LOCATION: NEWARK, ARKANSAS

UNIT TESTED: UNIT NUMBER 2 ESP OUTLET DUCT

\begin{tabular}{|c|c|c|c|c|c|}
\hline \multirow[b]{2}{*}{ SYMBOL } & \multirow[b]{2}{*}{ DESCRIPTION } & \multirow[b]{2}{*}{ UNITS } & \multicolumn{3}{|c|}{ RUN NUMBER } \\
\hline & & & 1 & 3 & \\
\hline $\mathrm{CO} 2$ & & $\%$ & 13.8 & 11.4 & \\
\hline $\mathrm{O} 2$ & & $\%$ & 5.2 & 7.4 & \\
\hline $\mathrm{CO}$ & & $\%$ & 0.0 & 0.0 & \\
\hline N2 & & $\%$ & 81.0 & 81.2 & \\
\hline$\%$ EA & $\begin{array}{l}\text { EXCESS AIR @ SAMPLING } \\
\text { POINT }\end{array}$ & $\%$ & 32.0 & 52.4 & \\
\hline MWd & $\begin{array}{l}\text { MOLECULAR WEIGHT OF } \\
\text { DRY STACK GAS }\end{array}$ & $\begin{array}{l}\text { LB/LB-MOLE } \\
\text { (g/g-MOLE) }\end{array}$ & $\begin{array}{c}30.42 \\
(30.42) \\
\end{array}$ & $\begin{array}{r}30.12 \\
(30.12) \\
\end{array}$ & \\
\hline MW & $\begin{array}{l}\text { MOLECULAR WEIGHT OF } \\
\text { STACK GAS }\end{array}$ & $\begin{array}{l}\text { LB/LB-MOLE } \\
\text { (g/g-MOLE) }\end{array}$ & $\begin{array}{c}29.32 \\
(29.32) \\
\end{array}$ & $\begin{array}{r}29.25 \\
(29.25) \\
\end{array}$ & \\
\hline Cp & PITOT TUBE CALIBRATION & & 0.805 & 0.805 & \\
\hline DELTAP & $\begin{array}{l}\text { VELOCITY HEAD OF STACK } \\
\text { GAS }\end{array}$ & $\begin{array}{l}\mathrm{H} 2 \mathrm{O} \\
(\mathrm{mm} \mathrm{H} 20)\end{array}$ & $\begin{array}{r}0.583 \\
(14.800) \\
\end{array}$ & $\begin{array}{r}0.433 \\
(11.000)\end{array}$ & \\
\hline DELTAP $P^{\wedge}(1 / 2)$ & & "H2O & 0.752 & 0.649 & \\
\hline Ts & STACK TEMPERATURE & $\begin{array}{l}\text { DEG. F } \\
\text { (DEG. C) }\end{array}$ & $\begin{array}{r}326 \\
(163) \\
\end{array}$ & $\begin{array}{r}306 \\
(152) \\
\end{array}$ & \\
\hline Ps & STACK PRESSURE & $\begin{array}{l}\text { "Hg Abs. } \\
(\mathrm{mm} \mathrm{Hg}) \\
\text { "H2O }\end{array}$ & $\begin{array}{c}28.72 \\
(729.00) \\
-15.00 \\
\end{array}$ & $\begin{array}{c}28.81 \\
(732.00) \\
-14.50 \\
\end{array}$ & \\
\hline Vs & $\begin{array}{l}\text { STACK VELOCITY @ STACK } \\
\text { CONDITIONS }\end{array}$ & $\begin{array}{l}\text { FPM } \\
(\mathrm{m} / \mathrm{SEC} .)\end{array}$ & $\begin{array}{r}2,997 \\
(15) \\
\end{array}$ & $\begin{array}{r}2,552 \\
(13) \\
\end{array}$ & \\
\hline As & STACK AREA & $\begin{array}{l}\text { (SQ.INCHES) } \\
\text { (SQ.METERS) }\end{array}$ & $\begin{array}{r}21,024 \\
(14) \\
\end{array}$ & $\begin{array}{r}21,024 \\
(14) \\
\end{array}$ & \\
\hline Qs & $\begin{array}{l}\text { DRY STACK GAS VOLUME @ } \\
\text { STANDARD CONDITIONS* } \\
\text { WET STACK GAS VOLUME @ } \\
\text { STANDARD CONDITIONS* }\end{array}$ & $\begin{array}{l}\text { DSCFM } \\
\text { (DSCM/HR) } \\
\text { WSCFH }\end{array}$ & $\begin{array}{r}258,122 \\
(438,549) \\
16,991,026\end{array}$ & $\begin{array}{r}230,337 \\
(391,343) \\
14,892,478\end{array}$ & \\
\hline Qa & $\begin{array}{l}\text { ACTUAL STACK GAS VOLUME } \\
@ \text { STACK CONDITIONS }\end{array}$ & $\begin{array}{l}\text { ACFM } \\
\left(m^{\wedge} 3 / H R\right)\end{array}$ & $\begin{array}{r}437,518 \\
(743,343) \\
\end{array}$ & $\begin{array}{r}372,619 \\
(633,080) \\
\end{array}$ & \\
\hline Dn & SAMPLING NOZZLE DIAM. & $\begin{array}{l}\text { IN. } \\
(\mathrm{m})\end{array}$ & $\begin{array}{c}0.246 \\
(0.006) \\
\end{array}$ & $\begin{array}{r}0.246 \\
(0.006) \\
\end{array}$ & \\
\hline$\%$ & PERCENT ISOKINETIC & $\%$ & 96.7 & 91.5 & \\
\hline
\end{tabular}

* 68 Deg.F, 29.92 "Hg (20 Deg.C, 760 mm Hg) 


\section{APPENDIX C}

Calibration Data

Equipment

Pitot Tube \#31-5-1 (low side)

Pitot Tube \#31-5-3

Probe Tip \#05-233-2

Probe Tip \#05-233-7

Dry Gas Meter \#27-3

Stack Unit Orifice \#27-3

Digital Temperature Indicator \#27-3

Dry Gas Meter \#31-1

Stack Unit Orifice \#31-1

Digital Temperature Indicator \#31-1

Barometer \#31-1
Calibration Factor

0.806

0.805

0.246

0.190

0.991

1.008

$10 / 03 / 05$

$10 / 04 / 05$

10/03/05

$10 / 12 / 05$ 


\section{PITOT TUBE CALIBRATION}

Date: $10-12-05$

Pitot No.: $31-5-1$

Pitot Dimensions: $5^{\prime} \times 1 / 4^{\prime \prime}$
Time: 1430

$\mathrm{T}_{\mathrm{s}}: 75^{\circ} \mathrm{F}$

$\mathrm{C}_{\text {pstd }}:-0.990$

\begin{tabular}{|c|c|c|c|c|c|c|c|c|c|c|}
\hline \multirow{3}{*}{$\begin{array}{c}\begin{array}{c}\text { Motor } \\
\text { Setting }\end{array} \\
7 \\
\end{array}$} & \multirow{3}{*}{$\begin{array}{c}\begin{array}{c}\text { fps } \\
\text { mark }\end{array} \\
20 \\
\end{array}$} & \multirow{2}{*}{\multicolumn{2}{|c|}{$\begin{array}{c}\text { Calibration } \\
\text { Standard } \\
\text { Start } \quad \text { End } \\
\end{array}$}} & \multirow{3}{*}{$\frac{\sqrt{\text { Standard }}}{\text { Average }}$} & \multirow{3}{*}{$\frac{\text { High }}{0.15}$} & \multirow[b]{2}{*}{$\sqrt{\text { High }}$} & \multirow{2}{*}{$\begin{array}{c}\text { Cal. } \\
\text { Factor }\end{array}$} & \multirow[b]{2}{*}{ Low } & \multirow[b]{2}{*}{$\sqrt{\text { Low }}$} & \multirow{2}{*}{$\begin{array}{l}\text { Cal. } \\
\text { Factor }\end{array}$} \\
\hline & & & & & & & & & & \\
\hline & & 0.10 & 0.10 & & & 0.387 & 0.808 & 0.15 & 0.387 & 0.808 \\
\hline 14 & 30 & 0.22 & 0.22 & 0.469 & 0.33 & 0.575 & 0.808 & 0.33 & 0.575 & 0.808 \\
\hline 20 & 40 & 0.34 & 0.34 & 0.583 & 0.52 & 0.721 & 0.801 & 0.52 & 0.721 & 0.801 \\
\hline 28 & 50 & 0.54 & 0524 & 0.735 & 0.82 & 0.906 & 0.803 & 0.81 & 0.900 & 0.808 \\
\hline 35 & 60 & 0.80 & 0.80 & 0.894 & 1.25 & 1.118 & 0.792 & 1.20 & 1.095 & 0.808 \\
\hline 41 & 70 & 1.00 & 1.00 & 1.000 & 1.55 & 1.245 & 0.795 & 1.50 & 1.225 & $0.80 z$ \\
\hline 50 & 80 & 1.30 & 1.30 & 1.140 & 20 & 1.414 & 0.798 & 2.00 & 1.414 & 0.798 \\
\hline 62 & 90 & 1.60 & 1.60 & 1.265 & 2.45 & 1.565 & 0.800 & 2.40 & 1.549 & 0.808 \\
\hline & & & & & & & & & & \\
\hline & & & & & & & & & & \\
\hline 28 & 50 & 0.54 & 0.54 & 0.735 & 0.82 & 0.906 & 0.803 & 0.82 & 0.906 & 0.803 \\
\hline 28 & 50 & 0.54 & $0.5-4$ & 0.735 & 0.81 & 0.900 & 0.808 & 0.82 & 0.906 & 0.803 \\
\hline & & & & & & & & & & \\
\hline & & & & & & & & & & \\
\hline Ave & & & & & & & $0.80 \mathrm{in}$ & & & 0.806 \\
\hline
\end{tabular}

Summary of Results:

Normal high side calibration factor

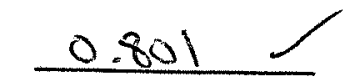

variation $+0.87 /$

variation - $1.12 \mathrm{~V}$

Normal low side calibration factor

0.806

variation $+\frac{0.25 \Omega}{0.99 \Omega}$

Calibrator: DOSH ORR DEKE BRUMMETT

checked By: Tasend Bnown 


\section{PITOT TUBE CALIBRATION}

Date: $10-14-05$

Pitot No.: $31-5-3$

Pitot Dimensions: $5^{\prime} \times 1 / 4^{\prime \prime}$
Time: $\quad 0955$

$\mathrm{T}_{\mathrm{s}}:{ }^{\circ} \mathrm{F}$

$\mathrm{C}_{\mathrm{pstd}}:-0.990$

\begin{tabular}{|c|c|c|c|c|c|c|c|c|c|c|}
\hline $\begin{array}{c}\text { Motor } \\
\text { Setting }\end{array}$ & $\begin{array}{c}\text { fps } \\
\text { mark }\end{array}$ & \multicolumn{2}{|c|}{$\begin{array}{c}\text { Calibration } \\
\text { Standard } \\
\text { Start }\end{array}$} & $\begin{array}{l}\text { Standard } \\
\text { Average }\end{array}$ & High & $\sqrt{\text { High }}$ & $\begin{array}{c}\text { Cal. } \\
\text { Factor }\end{array}$ & Low & $\sqrt{\text { Low }}$ & $\begin{array}{c}\text { Cal. } \\
\text { Factor }\end{array}$ \\
\hline 7 & 20 & 0.10 & 0.10 & 0.316 & 0.15 & 0387 & 0.808 & 0.15 & 0387 & 0.808 \\
\hline 14 & 30 & 0.22 & 0.22 & 0.469 & 0.34 & 0.583 & 0.796 & 0.34 & 0.583 & 0.796 \\
\hline 20 & 40 & 0.34 & 0.34 & 0.583 & 0.51 & 0.714 & 0.808 & 0.51 & 0.714 & 0.808 \\
\hline 28 & 50 & 0.54 & 0.54 & 0.735 & 0.81 & 0.900 & 0.808 & 0.81 & 0.900 & 0.808 \\
\hline 35 & 60 & 0.80 & 0.80 & 0.894 & 1.20 & 1.095 & 0.808 & 1.20 & 1.095 & 0.808 \\
\hline 41 & 70 & 1.00 & 1.00 & 1.000 & 1.50 & 1.225 & 0.808 & 1.50 & 1.225 & 0.808 \\
\hline 50 & 80 & 1.30 & 1.30 & 1.140 & 2.00 & 1.414 & 0.798 & 1.95 & 1.396 & 0.808 \\
\hline 62 & 90 & 1.60 & 1.60 & 1.265 & 2.40 & 1.549 & 0.808 & 240 & 11.549 & 0.808 \\
\hline & & & & & & & & & & \\
\hline & & & & & & & & & & \\
\hline 28 & 50 & 0.52 & 0.54 & 0.735 & 0.81 & 0.900 & 0.808 & 0.82 & 0.906 & 0.803 \\
\hline 28 & 50 & 0.54 & 0.54 & 0.735 & 0.81 & 0.900 & 0.808 & 0.81 & 0.900 & 0.808 \\
\hline & & & & & & & & & & \\
\hline & & & & & & & & & & \\
\hline Average & & & & & & 0.805 & & & 0.807 \\
\hline
\end{tabular}

Summary of Results:

Normal high side calibration factor $\quad 0.805 \checkmark$

variation $+0.37 \swarrow$

variation - $1.12 /$

Normal low side calibration factor $0.807 /$

variation $+0.12 \quad /$

variation - $1.36 \mathrm{~d}$

Calibrator: JOSH ORR

Checked By: Jasod Buan 


\section{NOZZLE CALIBRATION}

Nozzle Set No. 05.233745K/

Date $8-16-05$ $05-233 \div 1 \quad 05-236-2$

Reading 1 $0.247 \quad 0.24$

Reading 2

Reading 3

Reading 4

Reading 5

Reading 6

Reading 7

Reading 8

Reading 9

Réading 10

Average

OS-233- 7 o5.233-8

Reading 1

Reading 2

Reading 3

Reading 4

Reading 5

Reading 6

Reading 7

Rading 8

Reading 9

Reading 10

Average

$$
0.247
$$

0.246

0.247

0.244

0.248

0.248

$0.247 \quad 0.246$

0.247

0.246

0.247

$\frac{0.246}{0.246}$

0.247

$05-233-\underline{3}$

Calibrator:

05-233-4 05-233- $5 \quad$ os-233-6

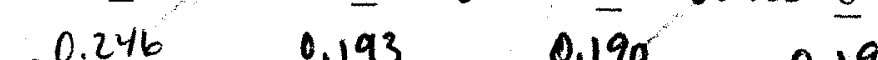




\section{Dry Gas Meter Calibration}

Dry Gas Meter No.: $27-3$

Date: $8 \operatorname{lol}$ los

$\Delta \mathrm{H}$
$\frac{\left(\mathrm{H}_{2} \mathrm{O}\right)}{0.5}$
0.5
1.0
1.5
2.0
3.0
4.0
Average

\begin{tabular}{l}
$\frac{C_{D G}}{0.979}$ \\
$\frac{0.989}{1.008}$ \\
\hline 0.990 \\
\hline 0.988 \\
\hline 0.989 \\
\hline
\end{tabular}

_. 0.991 Variation: + $1.72 \%$

$-$

Calibrator: Ned Shappley

Checked By:

C-5 


\section{DRY GAS METER CALIBRATION}

Meter Number: 27.3

Date: $8 / 12105$

Calibrator: Ned Shppley

$$
\text { Calibration Factor }\left(C_{D G}\right)=\frac{\text { Wet Test Meter } V m_{\text {std }}}{\text { Dry Gas Meter } V m_{\text {std }}}
$$

Run No.: $1 @ 0.5 \mathrm{H}_{2} \mathrm{O}$

$\mathrm{P}_{\mathrm{b}}: \quad 29.40$ "Hg

Control Module Vacuum:

Wet Test Meter (No. 4 )

Meter

Time Reading Temp. $\mathrm{P}_{\mathrm{m}}$

End $1128 \quad 5.170$ of $75^{\circ}{ }^{\circ} \mathrm{F}-0.4 \quad{ }^{\circ} \mathrm{H}_{2} \mathrm{O}$

Start ille 0.000 of $75{ }^{\circ} \mathrm{F}=0.4 \quad \mathrm{H}_{2} \mathrm{O}$

Avg.

$$
5.170 \text { cf } 75 \text { of } \quad 0.4 \quad \text { " } \mathrm{H}_{2} \mathrm{O}
$$

Dry Gas Meter

Meter Temp.

Reading In Out $P_{m}$ $844.539^{\circ}$ cf $70^{\circ} \mathrm{F} 69^{\circ} \mathrm{F} 0.5$ " $\mathrm{H}_{2} \mathrm{O}$ $839.300 \mathrm{cf} 700^{\circ} \mathrm{F} 71^{\circ} \mathrm{F} 0.5 \mathrm{H}_{2} \mathrm{O}$ 5.239 of 70 ${ }^{\circ} \mathrm{F} \quad 0.5 \quad \mathrm{H}_{2} \mathrm{O}$

Wet Test Meter $V m_{s t d}=17.65 \times 5.170\left[\frac{29.40+\frac{-0.4}{13.6}}{75+460}\right] \times 1.004 \quad\left(C_{f}\right)=5.04 d c s f$

Dry Gas Meter $V m_{s t d}=17.65 \times 5.239\left[\frac{29.40+\frac{0.5}{13.6}}{70+460}\right]=5.136 \mathrm{dcsf}$

$$
C_{D G}=\frac{5.029}{5.134 \quad C-6}=0.979
$$




\section{DRY GAS METER CALIBRATION}

Meter Number: $27 \cdot 3$

Date:

Calibrator: Ned Shoppley

Calibration Factor $\left(C_{D G}\right)=\frac{\text { Wet Test Meter } V m_{\text {std }}}{\text { Dry Gas Meter } V m_{s t d}}$

Run No.: 1 @ 1.0" $\mathrm{H}_{2} \mathrm{O}$

$P_{b}: 29.40$ "Hg

Control Module Vacuum: $5.0 \mathrm{Hg}$

Wet Test Meter (No. 4 )

Meter

Time Reading Temp.

End $1141 \quad 5.300$ cf $74 \quad{ }^{\circ} \mathrm{F} \quad-0.4 \quad$ “ $\mathrm{H}_{2} \mathrm{O}$

Start 11310.000 of $74{ }^{\circ} \mathrm{F}=0.4 \quad{ }^{\circ} \mathrm{H}_{2} \mathrm{O}$

Avg.

5.300 of $74 \quad{ }^{\circ} \mathrm{F} \quad-0.40 \quad \mathrm{H}_{2} \mathrm{O}$

\section{Dry Gas Meter}

Meter Temp.

Beading In Qut $\underline{P}_{\mathrm{m}}$ 850.231 of $71^{\circ}{ }^{\circ} \mathrm{F} 73^{\circ} \mathrm{F}, 1.00$ " $\mathrm{H}_{2} \mathrm{O}$ $\$ 44,900$ of $69{ }^{\circ} \mathrm{F}$ 7' ${ }^{\circ} \mathrm{F} 1.00$

5.331 of 71 o $\mathrm{F} \quad 1.00 \quad \mathrm{H}_{2} \mathrm{O}$

Wet Test Meter $V m_{s t d}=17.65 \times 5.300\left[\frac{24.40+\frac{-0.4}{13.6}}{74+460}\right] \times 1.004 \quad\left(C_{f}\right)=\underbrace{5.166} d c s f$

Dry Gas Meter $V m_{s t d}=17.65 \times 5.33 i\left[\frac{29.40+\frac{1.00}{13.6}}{71+460}\right]=5.223 \mathrm{dcsf}$

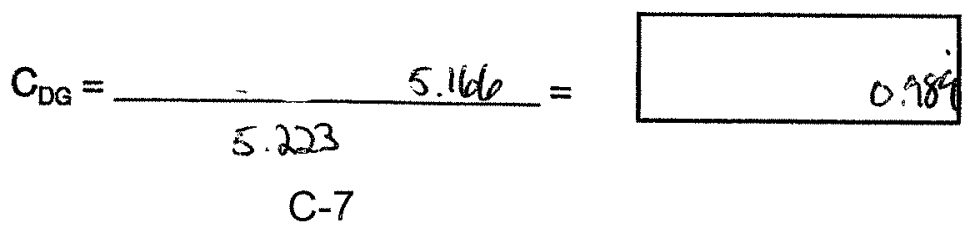




\section{DRY GAS METER CALIBRATION}

Meter Number: $27-3$

Date: $8 / 12 / 05$

Calibrator: Ned Shopley

Calibration Factor $\left(C_{D G}\right)=\frac{\text { Wet Test Meter } V m_{\text {std }}}{\text { Dry Gas Meter } V m_{\text {std }}}$

Run No.: 1 @ 1.5" $\mathrm{H}_{2} \mathrm{O}$

$P_{b}: 29.40$ "Hg

Control Module Vacuum: 5.0 " $\mathrm{Hg}$
Wet Test Meter (No. 4 )

Meter

Time Reading Temp. $\mathrm{P}_{\mathrm{m}}$

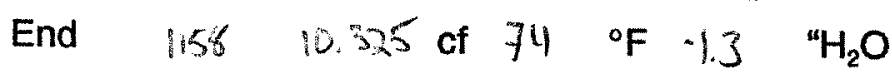

Start $1142 \quad 0.000$ of $74{ }^{\circ} \mathrm{F}=1.3{ }^{\circ} \mathrm{H}_{2} \mathrm{O}$

Avg.
Dry Gas Meter

Meter Temp.

Reading In Out $\mathrm{E}_{\mathrm{m}}$ 360.010 of $78^{\circ} \mathrm{F} \quad 22^{\circ} \mathrm{F}$ is $\quad \mathrm{H}_{2} \mathrm{O}$ 850.398 of $71^{\circ} \mathrm{F} 73{ }^{\circ} \mathrm{F} 1.5{ }^{\circ} \mathrm{H}_{2} \mathrm{O}$ 10.212 of $74 \quad{ }^{\circ} \mathrm{F} \quad 1.5 \quad \mathrm{H}_{2} \mathrm{O}$

Wet Test Meter $V m_{\text {std }}=17.65 \times 10.325\left[\begin{array}{c}29.40+\frac{-1.3}{13.6} \\ -74+460\end{array}\right] \times 1.004 \quad\left(C_{f}\right)=10.041$ dcsf

Dry Gas Meter $V m_{s t d}=17.65 \times 10.212\left[\frac{29.40+\frac{1.5}{13.6}}{79+460}\right]=9.961 \mathrm{dcsf}$

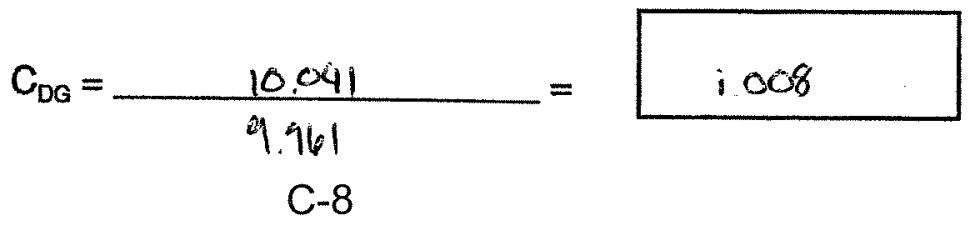




\section{DRY GAS METER CALIBRATION}

Meter Number: 27.3

Date: slizles

Calibrator: Nad Shappley

Calibration Factor $\left(C_{D G}\right)=\frac{\text { Wet Test Meter } V m_{s t d}}{\text { Dry Gas Meter } V m_{\text {std }}}$

Run No.: $1 @ 20^{\prime \prime} H_{2} \mathrm{O}$

$P_{b}: 29.40$

"Hg

Control Module Vacuum: $3.0 \quad \mathrm{Hg}$

Wet Test Meter (No. 4)

Meter

Time Reading Temp. $\mathrm{P}_{\mathrm{m}}$

End $4916 \quad 10.240$ of $74 \quad 4^{\circ} \mathrm{F}-1.5 \quad$ " $\mathrm{H}_{2} \mathrm{O}$

Start $1400 \quad 0000$ cf $74{ }^{\circ} \mathrm{F}-1.5 \mathrm{H}_{2} \mathrm{O}$

Avg.
Dry Gas Meter

Meter Temp.

Beading in Out $\underline{P}_{m}$ 411. 712 cf $87{ }^{\circ} \mathrm{F} 73{ }^{\circ} \mathrm{F} 2.0^{\circ} \quad \mathrm{H}_{2} \mathrm{O}$ $901.343 \mathrm{cf} 80$ o $73{ }^{\circ} \mathrm{F} 2.00$ “ $\mathrm{H}_{2} \mathrm{O}$ 10.369 of $78 \quad{ }^{\circ} \mathrm{F} \quad 2.0 \quad{ }^{\circ} \mathrm{H}_{2} \mathrm{O}$

Wet Test Meter $V m_{s t d}=17.65 \times 10.240\left[\frac{29.40+\frac{-1.5}{13.6}}{74+460}\right] \times 1.004 \quad\left(C_{f}\right)=9.153 \mathrm{dcsf}$

Dry Gas Meter $V m_{s t d}=17.65 \times 10.369\left[\frac{29.40+\frac{2.0}{13.6}}{78+460}\right]={ }^{10.051} d c s f$

$$
C_{D G}=\frac{9.953}{18.051}
$$




\title{
DRY GAS METER CALIBRATION
}

Meter Number: 27.3

Date: S/.2los

\author{
Calibrator: Ded Sheppley
}

Calibration Factor $\left(C_{D G}\right)=\frac{\text { Wet Test Meter } V m_{\text {std }}}{\text { Dry Gas Meter } V m_{\text {std }}}$

Run No.: 1 @ 3.0. $\mathrm{H}_{2} \mathrm{O}$

$P_{b}: 29.40$ "Hg

Control Module Vacuum: 5.0 " $\mathrm{Hg}$

Wet Test Meter (No. 4 )

Meter

Time Reading Temp.

End $1332 \quad 14.780$ cf $74 \quad{ }^{\circ} \mathrm{F} \quad-2.30 \quad \mathrm{H}_{2} \mathrm{O}$

Start $1314 \quad 0.000$ of $74{ }^{\circ} \mathrm{F} .2 .30{ }^{\circ} \mathrm{H}_{2} \mathrm{O}$

Avg.

\section{Dry Gas Meter}

\begin{tabular}{|c|c|c|c|c|}
\hline Meter & Tem & & & \\
\hline Reading & In & Qut & $P_{m}$ & \\
\hline $890.387 \mathrm{cf}$ & $92^{\circ} \mathrm{F}$ & 73 & ${ }^{\circ} \mathrm{F} 3 . \infty$ & " $\mathrm{H}_{2} \mathrm{C}$ \\
\hline $87540.2 \mathrm{cf}$ & $82^{\circ} \mathrm{F}$ & 73 & ${ }^{\circ} \mathrm{F} 3.00$ & $-\mathrm{H}$ \\
\hline & 80 & & & \\
\hline
\end{tabular}

Wet Test Meter $V m_{\text {std }}=17.65 \times 14.780\left[\frac{29.40+\frac{-230}{13.6}}{74+460}\right] \times 1.004 \quad\left(C_{f}\right)=14.337 \mathrm{dcsf}$

Dry Gas Meter $V m_{s t d}=17.65 \times 14.995\left[\frac{29.40+\frac{3.0}{13.6}}{80+460}\right]=14.508 \mathrm{dcsf}$

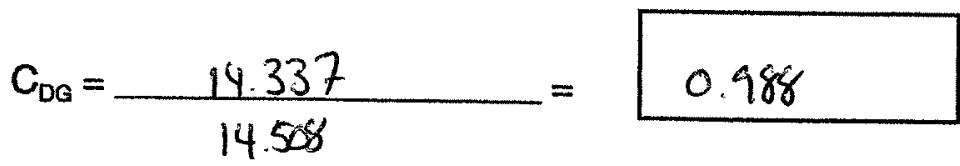

C-10 


\section{DRY GAS METER CALIBRATION}

Meter Number: 273

Date: $8 / 12105$

Calibrator: Ned Shrppley

$$
\text { Calibration Factor }\left(C_{D G}\right)=\frac{\text { Wet Test Meter } V m_{\text {std }}}{\text { Dry Gas Meter } V m_{\text {std }}}
$$

Run No.: 1 @ 40

$P_{b}: 29.40$ "Hg

Control Module Vacuum: 5.0 $\quad$ " $\mathrm{Hg}$

Wet Test Meter (No. i)

Meter

Time Reading Iemp. $\mathrm{E}_{\mathrm{m}}$

End $1343 \quad 10.050$ of $74 \quad{ }^{\circ} \mathrm{F} \quad-3.2 \quad \mathrm{H}_{2} \mathrm{O}$ Start 13.35 0.0es of $74{ }^{\circ} \mathrm{F}-3.2{ }^{\circ} \mathrm{H}_{2} \mathrm{O}$ Avg.
Dry Gas Meter

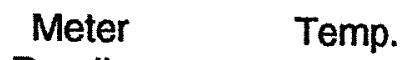

Reading In Out $P_{m}$ 901097 cf $95^{\circ} \mathrm{F} \quad 73^{\circ} \mathrm{F} \% .0 \quad{ }^{\circ} \mathrm{H}_{2} \mathrm{O}$ 890.910 of $90{ }^{\circ} \mathrm{F} 72{ }^{\circ} \mathrm{F} 4.0 \quad{ }^{\circ} \mathrm{H}_{2} \mathrm{O}$ 10.187 cf 83 o $\mathrm{F} 4.0 \quad \mathrm{H}_{2} \mathrm{O}$

Wet Test Meter $V m_{s t d}=17.65 \times 10.050\left[\frac{29.40+\frac{-3.2}{13.6}}{74+460}\right] \times 1.004 \quad\left(C_{t}\right)=9.727 \mathrm{dcsf}$

Dry Gas Meter $V m_{s t d}=17.65 \times 10.187\left[\frac{29.40+\frac{4.0}{13.6}}{83+460}\right]=9.832 \mathrm{dcsf}$

$$
C_{D G}=\frac{9.727}{9.832}=0.989
$$




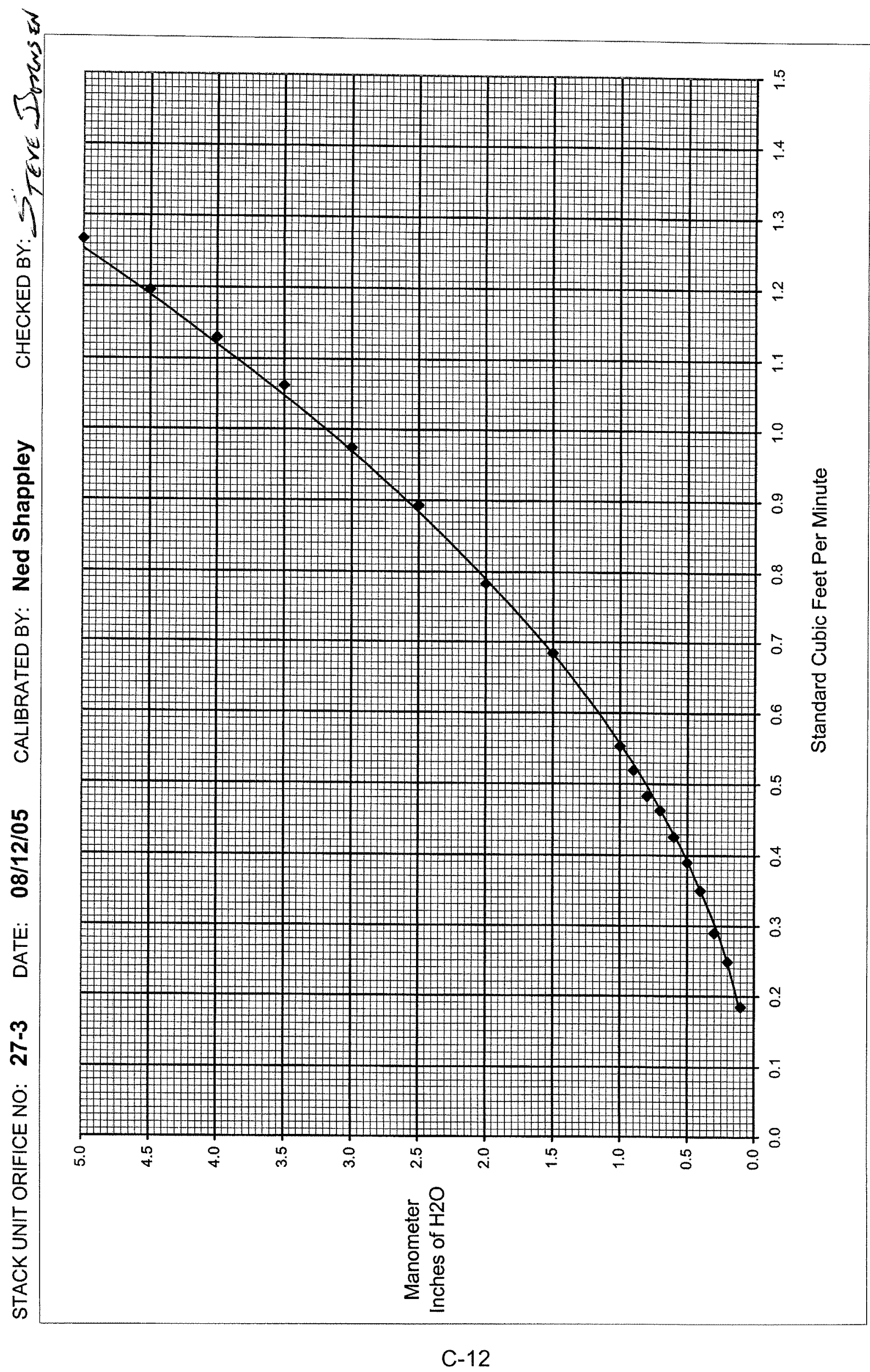




\section{DIGITAL TEMPERATURE INDICATOR NO: $27 \cdot 3$}

\section{CALIBRATION DATA}

DATE: $8 / 12 / 05$

$\underline{\text { Media }}$

Ambient Air

Ice Bath

Boiling Water

Oven

Oven

Oven

Oven

Meter Adjusted?

Reference Thermometer No.
Mercury

Temperature

$\left({ }^{\circ} \mathrm{F}\right)$

\begin{tabular}{ll}
$\frac{77}{32}$ & $\frac{76}{32}$ \\
$\frac{211}{347}$ & $\frac{248}{300}$ \\
$\frac{350}{382}$ & $\frac{349}{380}$ \\
\hline
\end{tabular}

No

DTI

$\left({ }^{\circ} \mathrm{F}\right)$

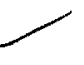

1000

100

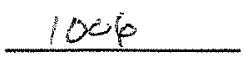

1030

1035

1042

1047

YES

A 


\section{Dry Gas Meter Calibration}

Dry Gas Meter No.: $31-1$

Date: 3 oct 2005

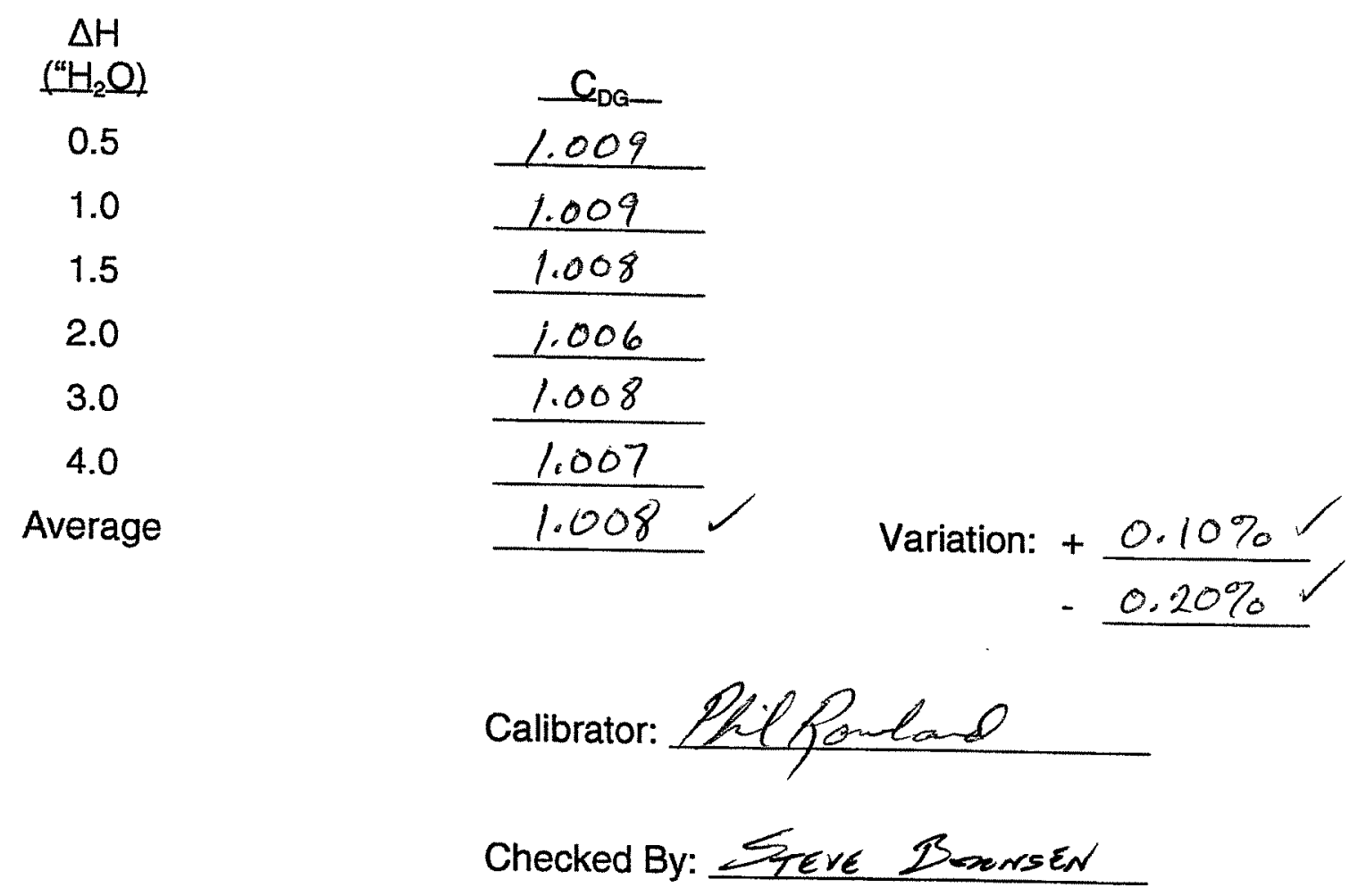

C-14 


\section{DRY GAS METER CALIBRATION}

Meter Number: $3 /-1$

Date: 3 oct 2005

Calibrator:

Calibration Factor $\left(C_{D G}\right)=\frac{\text { Wet Test Meter } V m_{\text {std }}}{\text { Dry Gas Meter } V m_{\text {std }}}$

Run No.: 1 @ 0.50

$P_{b}: 29.58$ "Hg

Control Module Vacuum: $5.0 \quad \mathrm{Hg}$

Wet Test Meter (No. 4 )

Meter

Time Reading Temp.

End $1445 / 1.000$ of $75^{\circ} \mathrm{F}-0.55^{\circ} \mathrm{H}_{2} \mathrm{O}$

Start $1410 \quad 0.000$ of 75 o $=0.60{ }^{\circ} \mathrm{H} \mathrm{H}_{2} \mathrm{O}$

Avg. $35 \quad 1 / .000$ of 75 of $-0.58{ }^{\circ} \mathrm{H}_{2} \mathrm{O}$
Dry Gas Meter

Meter Temp.

Beading In out $\mathrm{P}_{\mathrm{m}}$ $260.949 \mathrm{cf} 86^{\circ} \mathrm{F} 78{ }^{\circ} \mathrm{F} 0.50^{~} \mathrm{H}_{2} \mathrm{O}$ 249.885 of 83 o $79{ }^{\circ}{ }^{\circ} \mathrm{F} 0.50$ " $\mathrm{H}_{2} \mathrm{O}$ 11.064 cf $82 \quad{ }^{\circ} \mathrm{F} \quad 0.50 \quad$ " $\mathrm{H}_{2} \mathrm{O}$

Wet Test Meter $V m_{s t d}=17.65 \times 1 / .000\left[\frac{29.58+\frac{-0.58}{13.6}}{75+460}\right] \times 1.004\left(C_{t}\right)=10.762 \mathrm{dcsf}$

Dry Gas Meter $V m_{s t d}=17.65 \times 1 / .064\left[\frac{29.58+\frac{0.50}{13.6}}{82+460}\right]=10.67 / d c s f$

$$
C_{D G}=\frac{10.762}{10.671}=1.009 \sqrt{ }
$$




\section{DRY GAS METER CALIBRATION}

Meter Number: $31-1$

Calibrator:

Date: $30 c+2005$

$$
\text { Calibration Factor }\left(C_{D G}\right)=\frac{\text { Wet Test Meter } V m_{s t d}}{\text { Dry Gas Meter } V m_{s t d}}
$$

Run No.: 1 @ 1.00

$P_{b}: 29.58$ "Hg

Control Module Vacuum: $5.0 \mathrm{Hg}$

Wet Test Meter (No. 4 )

Meter

Time Reading Temp.

End $1515 \quad 11.000$ cf $75^{\circ} \mathrm{F}-0.90 \quad{ }^{\circ} \mathrm{H}_{2} \mathrm{O}$

Start $1455 \quad 0.000$ of $75{ }^{\circ} \mathrm{F}-\underline{0.85} \times \mathrm{H}_{2} \mathrm{O}$

Avg. $20 \quad 11.000$ cf 75 of $-0.88 \times \mathrm{H}_{2} \mathrm{O}$
Dry Gas Meter

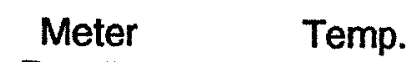

Reading in Out $\mathrm{P}_{\mathrm{m}}$

273.477 cf $91{ }^{\circ} \mathrm{F} 80^{\circ} \mathrm{F} / .00^{\circ} \mathrm{H}_{2} \mathrm{O}$

262.399 of $85^{\circ} \mathrm{F} 78^{\circ} \mathrm{F} \angle .00{ }^{\circ} \mathrm{H}_{2} \mathrm{O}$

11.078 af 84 o $1.00 \quad \mathrm{H}_{2} \mathrm{O}$

Wet Test Meter $V m_{\text {std }}=17.65 \times 1 / .000\left[\frac{29.58+\frac{-0.88}{13.6}}{75+460}\right] \times 1.004\left(C_{f}\right)=10.754 \mathrm{dcsf}$

Dry Gas Meter $V m_{s t d}=17.65 \times 11.078\left[\frac{29.58+\frac{1.00}{13.6}}{84+460}\right]=10.658 \mathrm{dcsf}$

$$
C_{D G}=\frac{10.754}{10.658}=1.009 \quad
$$

C-16 
DRY GAS METER CALIBRATION

Meter Number: $31-1$

Date: 3 oct 2005

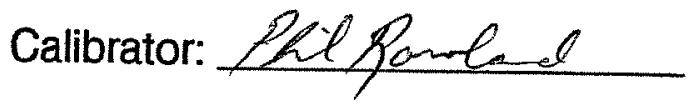

$$
\text { Calibration Factor }\left(C_{D G}\right)=\frac{\text { Wet Test Meter } V m_{\text {std }}}{\text { Dry Gas Meter } V m_{\text {std }}}
$$

Run No.: I @ 1.50

$$
\mathrm{P}_{\mathrm{b}}: 29.58 \quad \mathrm{Hg}
$$

Wet Test Meter (No. 4 )

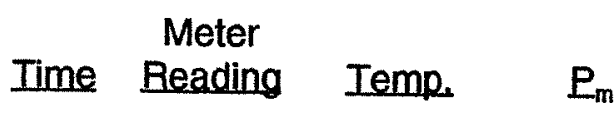

End $1540 \quad 11.020 \mathrm{cf} 75^{\circ} \mathrm{F}-1.20 \quad \mathrm{H}_{2} \mathrm{O}$

Start $1520 \quad 0.000$ of $75{ }^{\circ} \mathrm{F}-1.20{ }^{\circ} \mathrm{H}_{2} \mathrm{O}$

Avg. $20 \quad 1 / .020$ of $75 \quad{ }^{\circ} \mathrm{F}-1.20 \quad{ }^{4} \mathrm{H}_{2} \mathrm{O}$

\section{Dry Gas Meter}

Meter Temp.

Beading In Qut $P_{m}$ $286.256^{\circ f} 94^{\circ} \mathrm{F} \quad 80^{\circ} \mathrm{F} / .50^{\circ} \mathrm{H}_{2} \mathrm{O}$ 275.128 of $89{ }^{\circ} \mathrm{F} 80^{\circ} \mathrm{F} 1.50$ " $\mathrm{H}_{2} \mathrm{O}$ 11.128 of $86 \quad{ }^{\circ} \mathrm{F} / .50 \quad$ " $\mathrm{H}_{2} \mathrm{O}$

Wet Test Meter $V m_{s t d}=17.65 \times 1 / .020\left[\frac{29.58+\frac{-1.20}{13.6}}{75+460}\right] \times 1.004\left(C_{t}\right)=10.765 \mathrm{dcsf}$

Dry Gas Meter $V m_{s t d}=17.65 \times / / .128\left[\frac{29.58+\frac{1.50}{13.6}}{86+460}\right]=10.680 \mathrm{dcsf}$

$$
C_{D G}=\frac{10.765}{10.680}=1.008 \pi
$$




\section{DRY GAS METER CALIBRATION}

Meter Number: $31-1$

Date: $30 c+2005$

Calibrator:

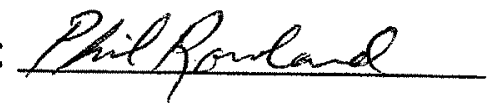

$$
\text { Calibration Factor }\left(C_{D G}\right)=\frac{\text { Wet Test Meter } V m_{s t d}}{\text { Dry Gas Meter } V m_{s t d}}
$$

Run No.: I @2.00

$P_{b}: 29.58 \quad \mathrm{Hg}$

Control Module Vacuum: $5,0 \mathrm{Hg}$

Wet Test Meter (No. 4)

Meter

Time Reading Temp.

End $1555 \quad / 1.000 \mathrm{cf} 75^{\circ} \mathrm{F}-1.50 \quad \mathrm{H}_{2} \mathrm{O}$

Start $1545 \quad 0.000$ of 75 o $\mathrm{F}-1.50$ " $\mathrm{H}_{2} \mathrm{O}$

Avg. $10 \quad 11.000$ of $75 \quad{ }^{\circ} \mathrm{F}-1.50 \quad{ }^{\circ} \mathrm{H}_{2} \mathrm{O}$
Dry Gas Meter

Meter Temp.

Reading In Out $\underline{P}_{\mathrm{m}}$ 298.634 cf $95^{\circ} \mathrm{F} \quad 80^{\circ} \mathrm{F} 2.00{ }^{\prime \prime} \mathrm{H}_{2} \mathrm{O}$ 287.532 of 90 o $\mathrm{F} 800^{\circ} \mathrm{F} 2.00$ " $\mathrm{H}_{2} \mathrm{O}$ 11.102 of $86 \quad{ }^{\circ} \mathrm{F} 2.00 \quad \mathrm{H}_{2} \mathrm{O}$

Wet Test Meter $V m_{s t d}=17.65 \times / / .000\left[\frac{29.58+\frac{-1.50}{13.6}}{75+460}\right] \times 1.004\left(C_{t}\right)=10.737 \mathrm{dcsf}$

Dry Gas Meter $V m_{s t d}=17.65 \times / 1.102\left[\frac{29.58+\frac{2.00}{13.6}}{86+460}\right]=10.669 \mathrm{dcsf}$

$$
C_{D G}=\frac{10.737}{10.669}=1.006 \checkmark
$$

C-18 


\section{DRY GAS METER CALIBRATION}

Meter Number: $31-1$

Date: 3 oct 2005

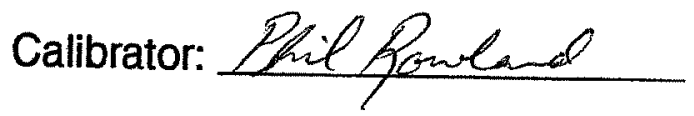

$$
\text { Calibration Factor }\left(C_{D G}\right)=\frac{\text { Wet Test Meter } V m_{\text {std }}}{\text { Dry Gas Meter } V m_{\text {std }}}
$$

Run No.: / @ 3.00

$P_{b}: 29.58$ " $\mathrm{Hg}$

Control Module Vacuum: $5.0 \quad \mathrm{Hg}$
Wet Test Meter (No. 4 )

Meter

Time Beading Temp. $\mathrm{P}_{\mathrm{m}}$

End $16 / 5 \quad 11.000$ of $75 \quad{ }^{\circ} \mathrm{F}-2.25 \quad$ " $\mathrm{H}_{2} \mathrm{O}$

Start 16050.000 cf $75{ }^{\circ} \mathrm{F}-2.20{ }^{\circ} \mathrm{H}_{2} \mathrm{O}$

Avg. $10 \quad 11.000$ of 75 of $-2.23 \quad{ }^{\circ} \mathrm{H}_{2} \mathrm{O}$
Dry Gas Meter

Meter Temp.

Reading In Qut $\underline{P}_{m}$

310.766 of $99{ }^{\circ} \mathrm{F} 81^{\circ} \mathrm{F} 3.00{ }^{\prime \prime} \mathrm{H}_{2} \mathrm{O}$

299.689 of $92{ }^{\circ} \mathrm{F} 81{ }^{\circ} \mathrm{F} 3.00{ }^{\circ} \mathrm{H}_{2} \mathrm{O}$

11.079 of $88{ }^{\circ} \mathrm{F} 3.00 \quad \mathrm{H}_{2} \mathrm{O}$

Wet Test Meter $V m_{s t d}=17.65 \times / 1,000\left[\frac{29.58+\frac{-2.23}{13.6}}{75+460}\right] \times 1.004\left(C_{f}\right)=10.718 \mathrm{dcsf}$

Dry Gas Meter $V m_{s t d}=17.65 \times 11.077\left[\frac{29.58+\frac{3.00}{13.6}}{88+460}\right]=10.632 \mathrm{dcsf}$

$$
C_{D Q}=\frac{10.718}{10.632}=1.008^{\top}
$$

C-19 


\section{DRY GAS METER CALIBRATION}

Meter Number: $31-1$

Date: $30 c+2005$
Calibrator:

Dhil Pow
Meter $V m_{\text {std }}$
Meter $V m_{\text {std }}$

Run No.: $1 @ 4.00$

$P_{b}: 29.58$

Control Module Vacuum: 5,0 "Hg " $\mathrm{Hg}$
Dry Gas Meter

Meter

Time Reading Temp. $\mathrm{P}_{\mathrm{m}}$

End $1630 \quad / 1,000$ of $75^{\circ} \mathrm{F}-3.00 \quad \mathrm{H}_{2} \mathrm{O}$ Start $\angle 420 \quad 0.000$ of 75 \% $-2.90 \quad{ }^{\circ} \mathrm{H}_{2} \mathrm{O}$ Avg. $10 \quad 11,000$ of $75{ }^{\circ} \mathrm{F}-2.95 \quad{ }^{\circ} \mathrm{H}_{2} \mathrm{O}$
Meter Reading $323.057^{\mathrm{cf}} 100{ }^{\circ} \mathrm{F} 82{ }^{\circ} \mathrm{F} 4.00{ }^{\circ} \mathrm{H}_{2} \mathrm{O}$ 311.999 of $94{ }^{\circ} \mathrm{F} 81{ }^{\circ} \mathrm{F} 4.00{ }^{\circ} \mathrm{H}_{2} \mathrm{O}$ 11.058 of $89 \quad{ }^{\circ} \mathrm{F} 4.00 \quad \mathrm{H}_{2} \mathrm{O}$

Wet Test Meter $V m_{\text {std }}=17.65 \times / 1.000\left[\frac{29.58+\frac{-2.95}{13.6}}{75+460}\right] \times 1.004\left(C_{t}\right)=10.698 \mathrm{dcsf}$

Dry Gas Meter $V m_{s t d}=17.65 \times / 1.058\left[\frac{29.58+\frac{4.00}{13.6}}{89+460}\right]=10.620 \mathrm{dcsf}$

$$
C_{D G}=\frac{10.698}{10.620}=1.007 \quad
$$




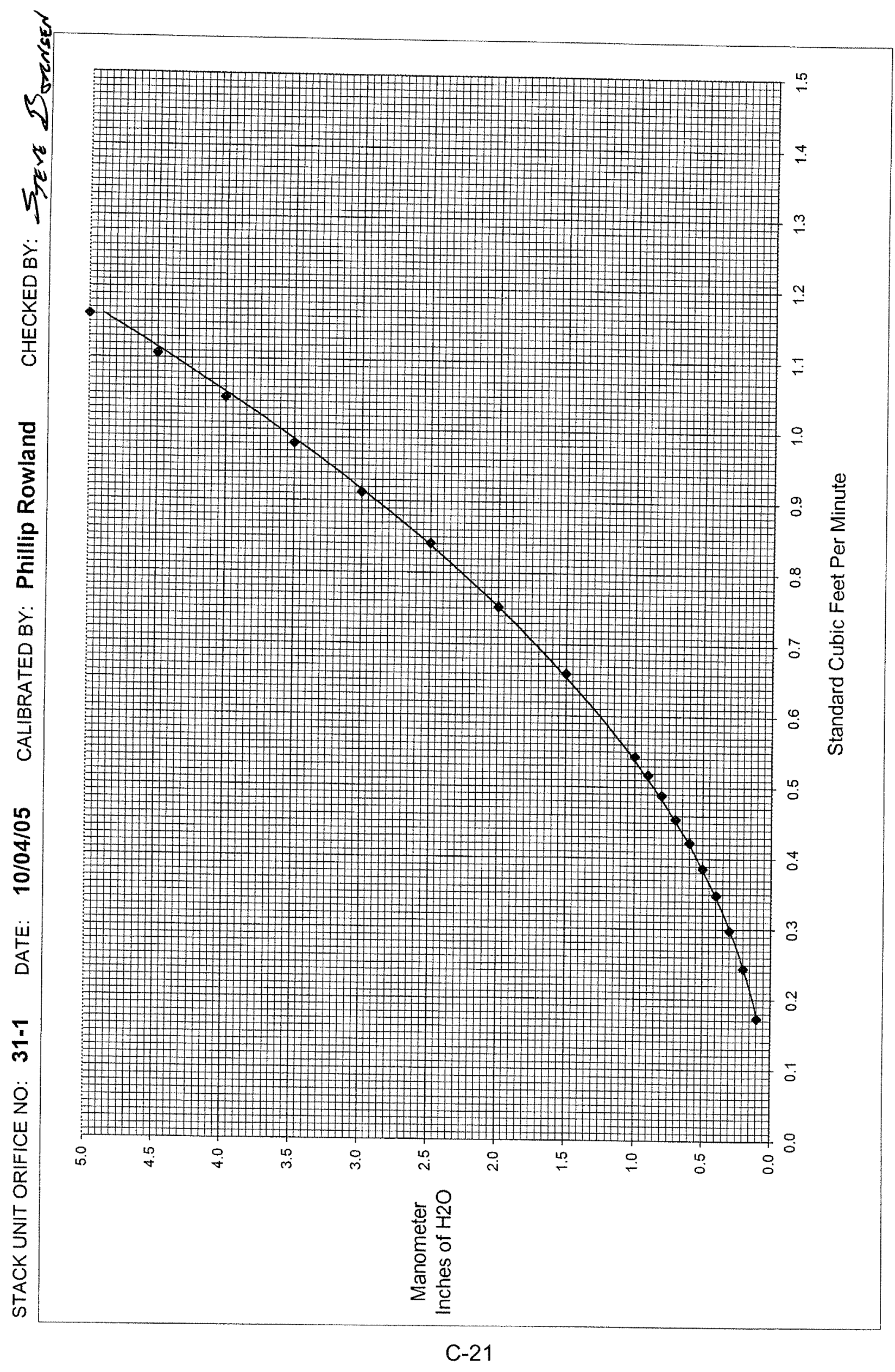




\section{DIGITAL TEMPERATURE INDICATOR NO: $31-1$}

\section{CALIBRATION DATA}

DATE: 3 Oct 2005

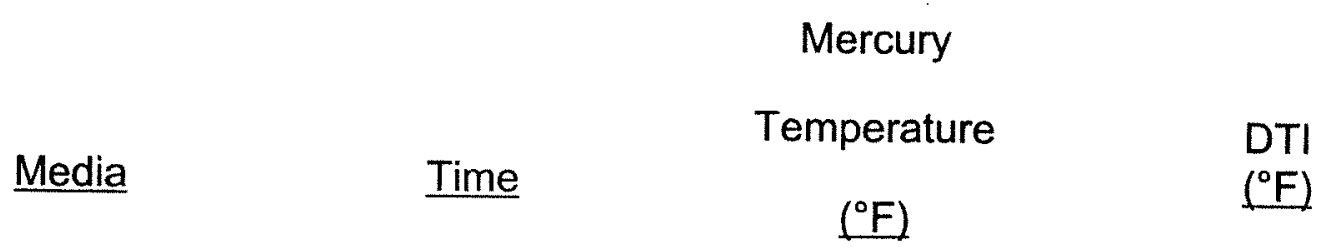

\begin{tabular}{|c|c|c|c|}
\hline Ambient Air & 1306 & 76 & 75 \\
\hline Ice Bath & 1307 & 34 & 34 \\
\hline Boiling Water & 1309 & $2 / 3$ & 211 \\
\hline Oven & 1325 & 376 & 375 \\
\hline Oven & 1332 & 352 & 350 \\
\hline Oven & 1339 & 301 & 300 \\
\hline Oven & 1346 & 251 & 250 \\
\hline
\end{tabular}

Meter Adjusted?

YES

No $\longleftarrow$

Reference Thermometer No.

calibrator PLil Parla

Checked By: 


\section{BAROMETER CALIBRATION}

Barometer No. $30-1$

Date: 8126105

Time: 1500

$\begin{array}{ll}\text { Barometric Pressure @ Baton Rouge Airport @ 70 ft. } & =\frac{29.87}{0.07} \\ \text { Absolute Pressure @ Baton Rouge Airport } & =\frac{29.80}{} \\ \text { Absolute Pressure @ METCO - Baton Rouge @ 26 ft. } & =\frac{29.844}{29.84} \\ \text { Barometer Reading } & =\frac{0.004}{\text { Variation }} \\ \text { Barometer Adjusted? } & =\frac{\text { No }}{\text { Barometer Reading (after adjustment) }}\end{array}$

Barometer Reading (after adjustment)

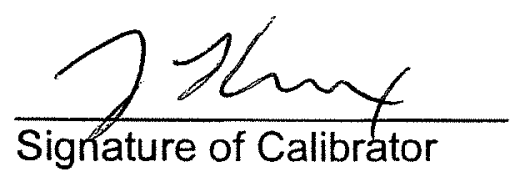

C-23 


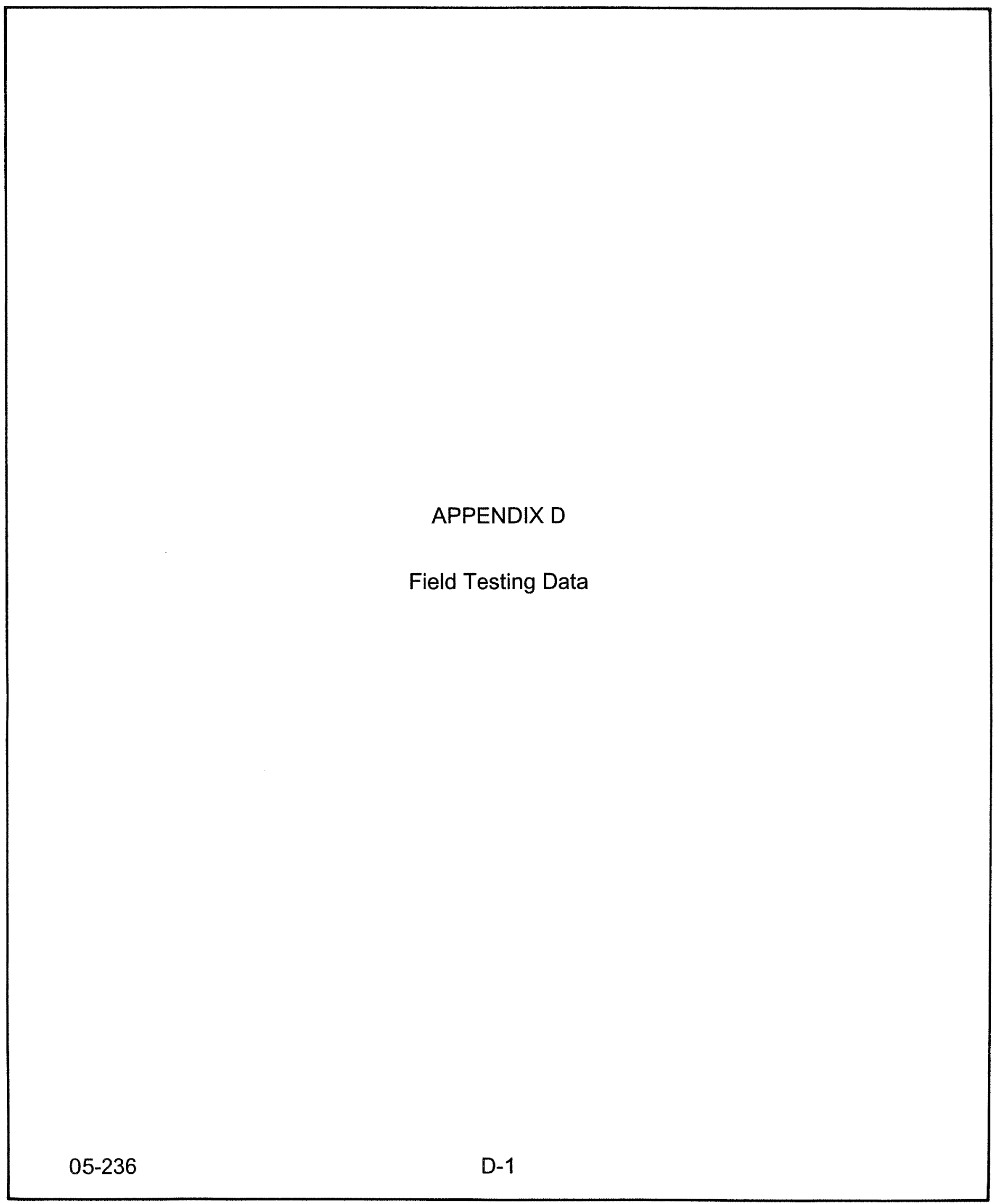


Impinger Box No.

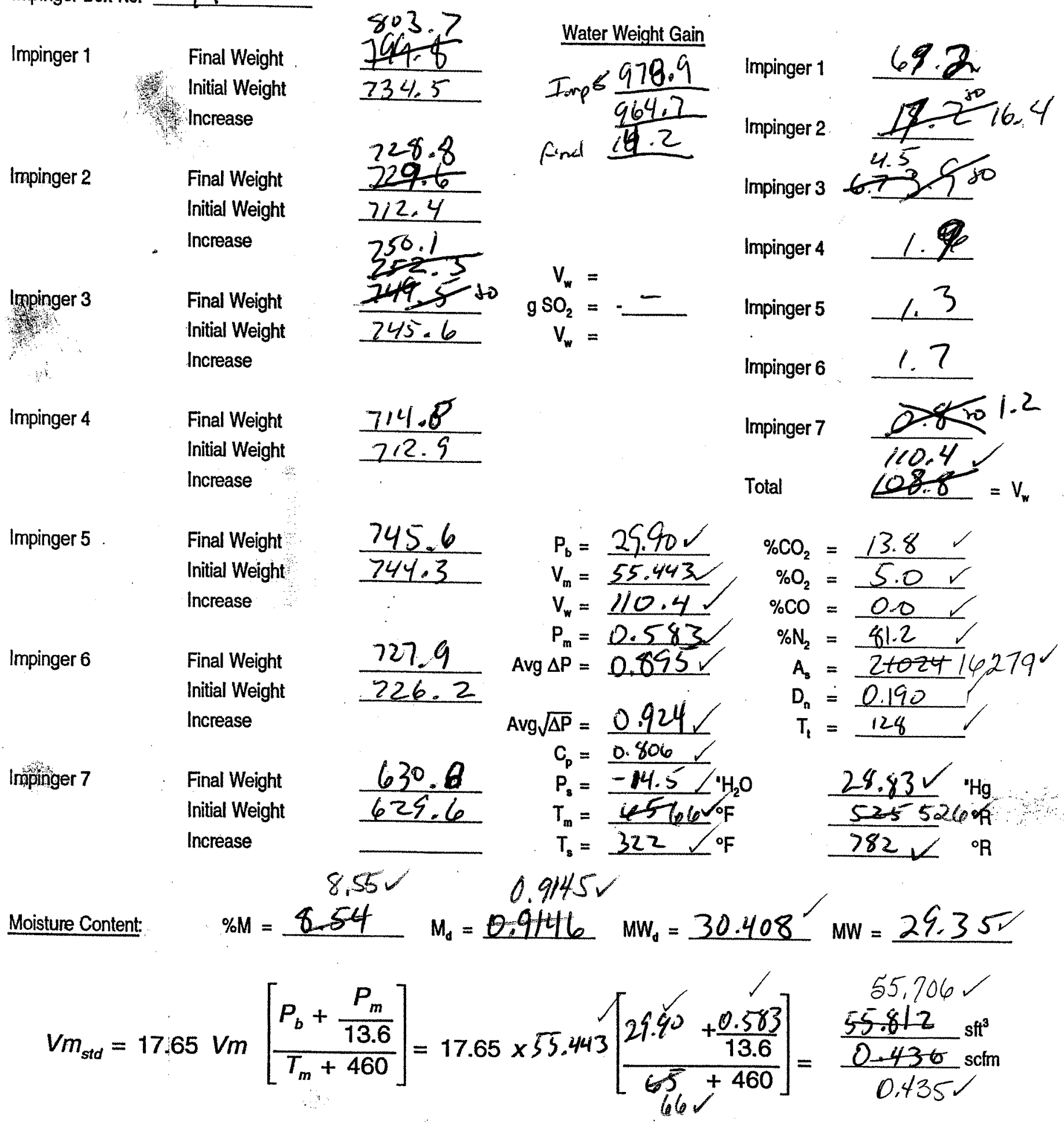

$v_{w_{\text {gas }}}=0.0472 \times v_{w}=0.0472 \times 110.4 \checkmark-5.2 / 1 /$

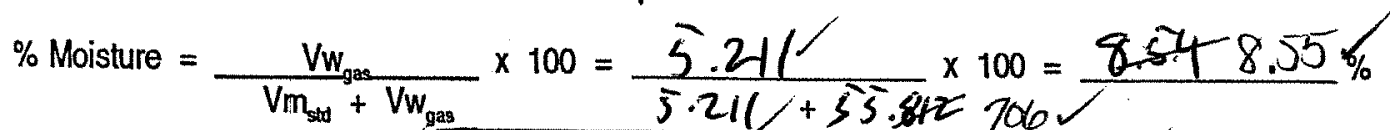
$v_{s}=5123.8 \times \frac{0.806}{\sqrt{28.83} \times 29.35 \checkmark} \times \frac{7824}{706 \checkmark}=\frac{3668 \mathrm{tpm}}{101.3 \mathrm{~V}}$ $\%=\frac{1,039 \times 55.812 \times 782 V}{28.83 \times 0.9146 \times 128 \gamma^{\times} \times 3668 \times(0.190)^{2}}=\frac{101.5}{5 V} \%$

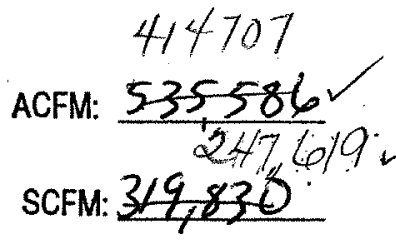

\%EA: 30.3 


$$
\begin{aligned}
& n \\
& 0 \\
& 0 \\
& 0 \\
& j
\end{aligned}
$$

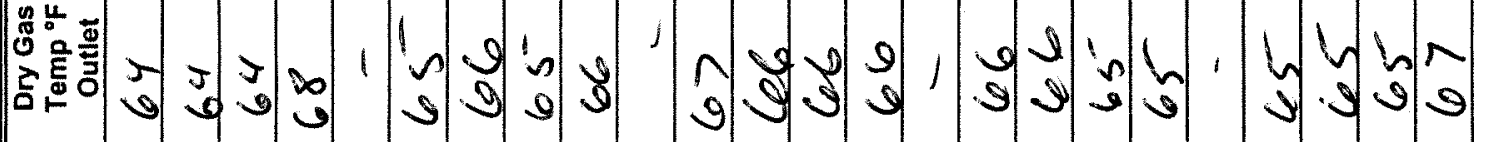
上

gू

造至

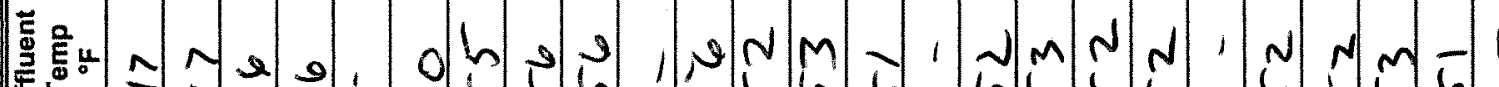

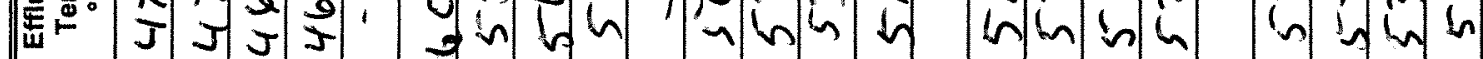

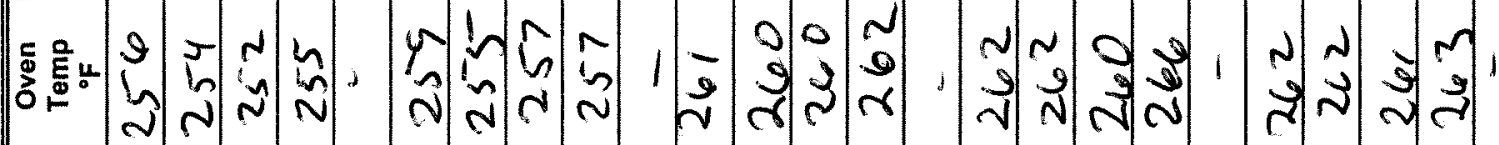

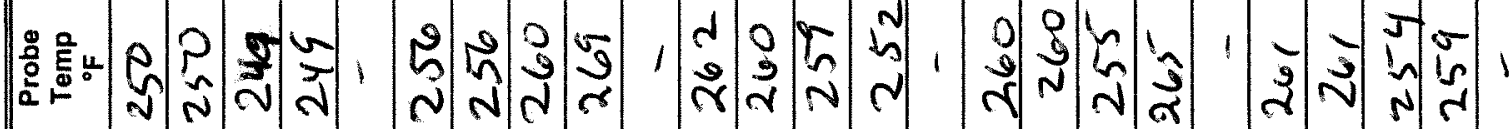

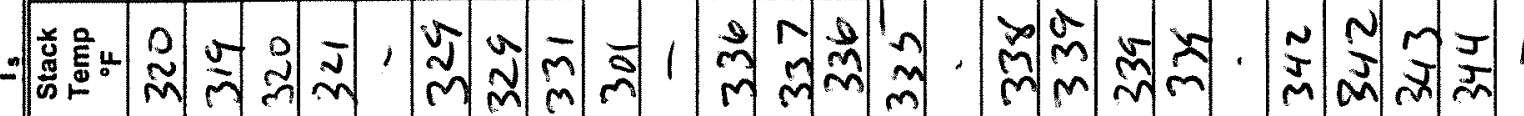

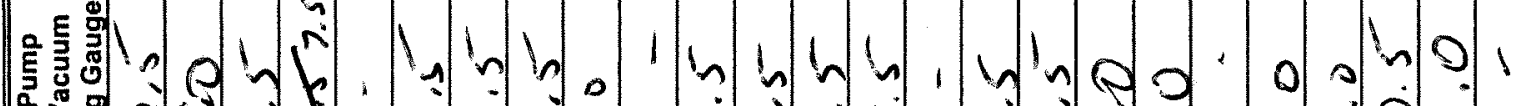

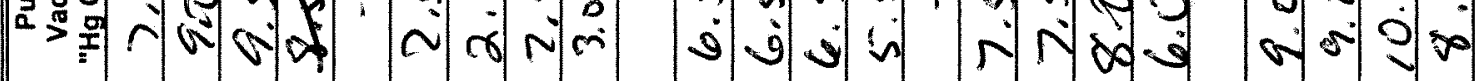

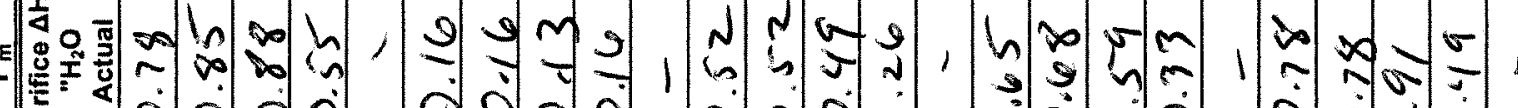

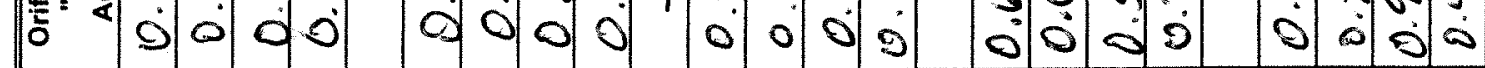

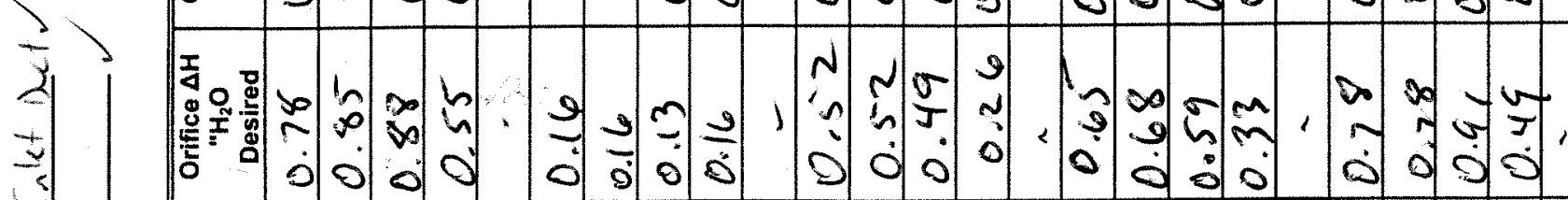

(1) द व

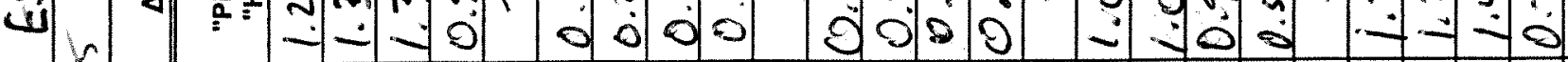

$\sim ?$

$+\frac{1}{1}$

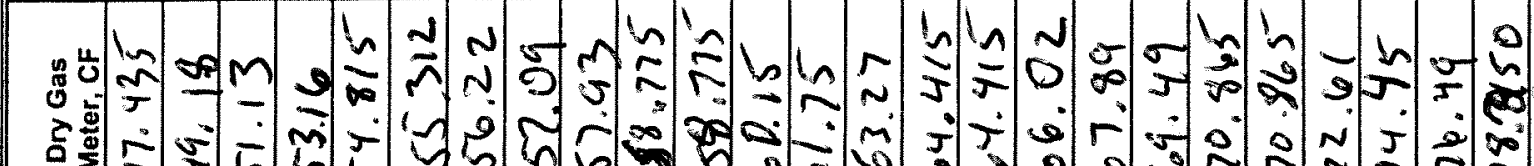

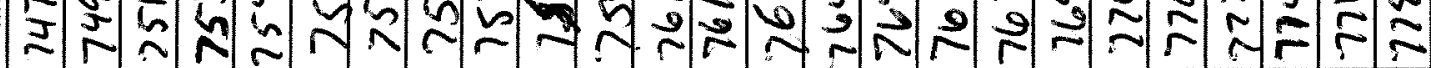

:

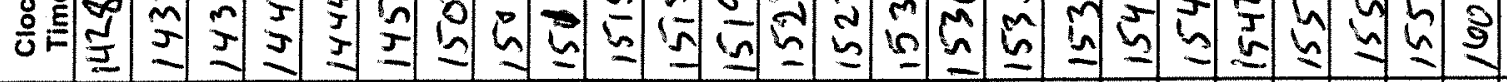

离

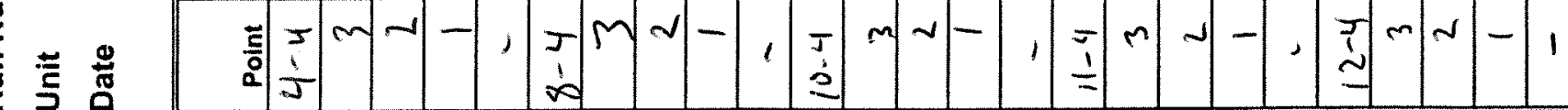




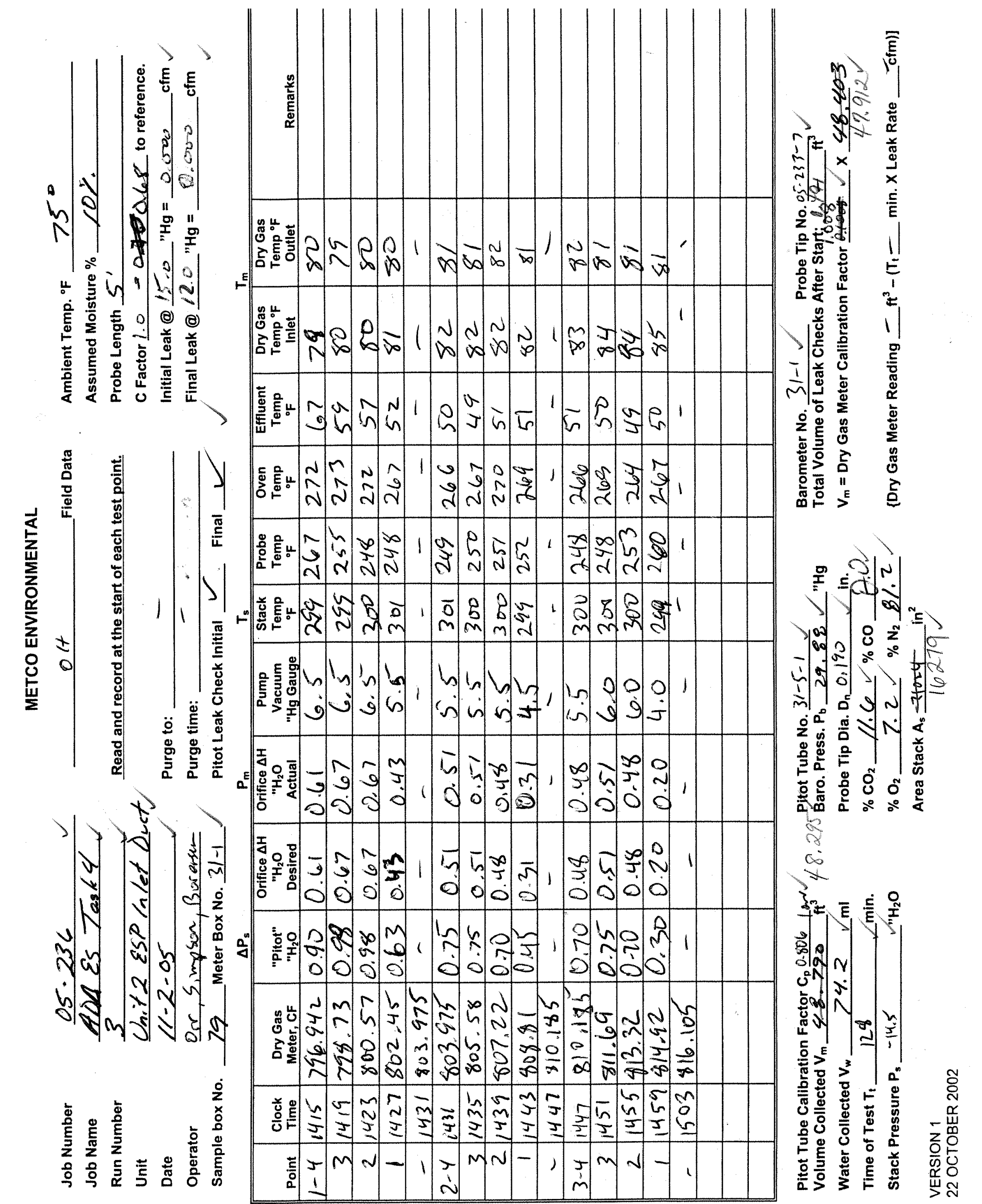


Impinger Box No.

79

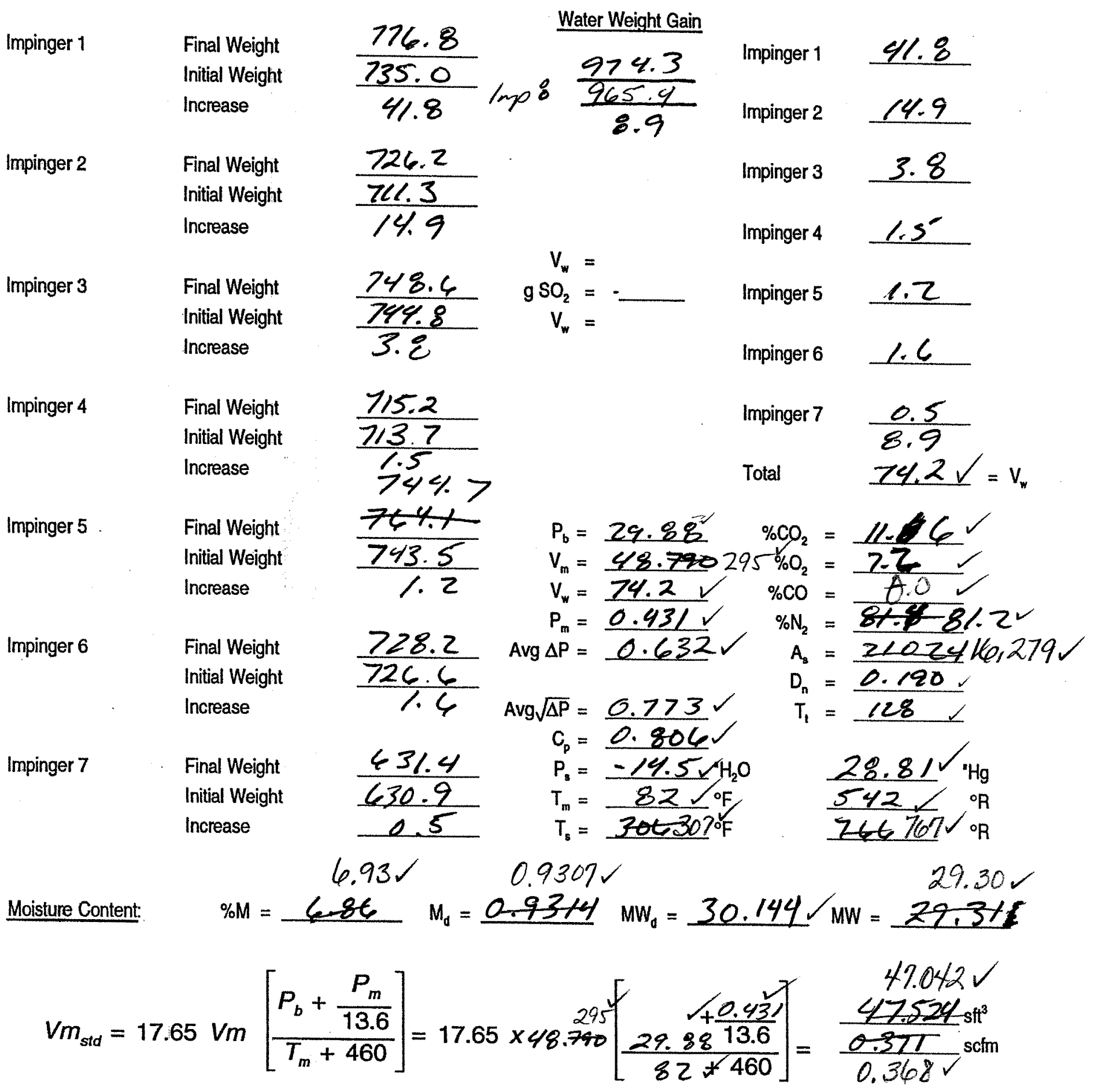

$V w_{g a s}=0.0472 \times V w=0.0472 \times$

$74.2 \%$

$\%$ Moisture $=$ 3.502

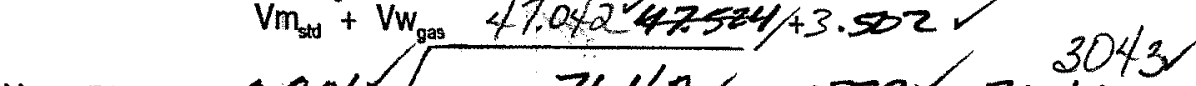

$v_{s}=5123.8 \times \frac{0.806}{\sqrt{28.81 \times 29.3129 .30}} \times \frac{76.773}{3640 \mathrm{fpm}}$

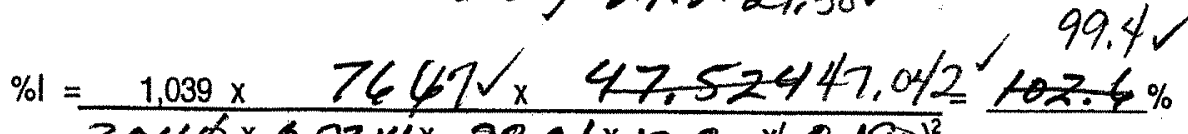
$3049 \times 6.9344 \times 28.81 \times 128 \times(0.190)^{2}$ $\mathrm{sft}^{3}$ 6.93

344,005 ACFM: 443,209 213,006 SCFM: 275,388 \%EA: 50.3 


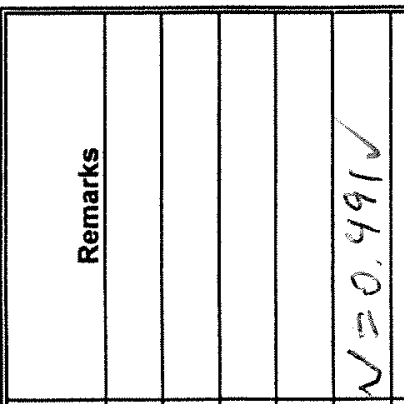

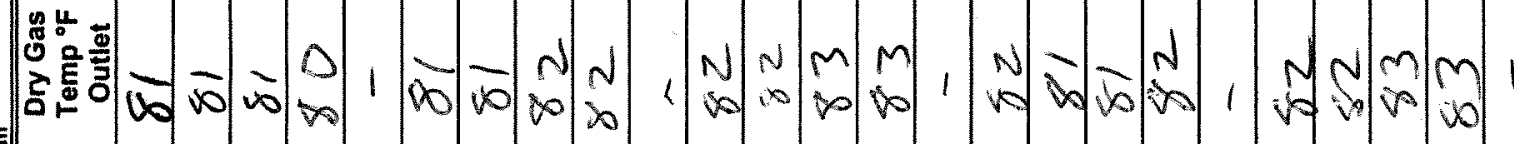
E

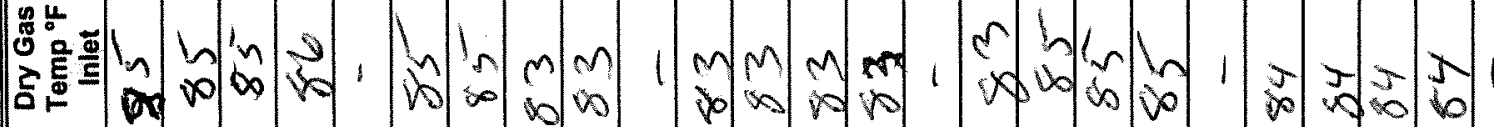

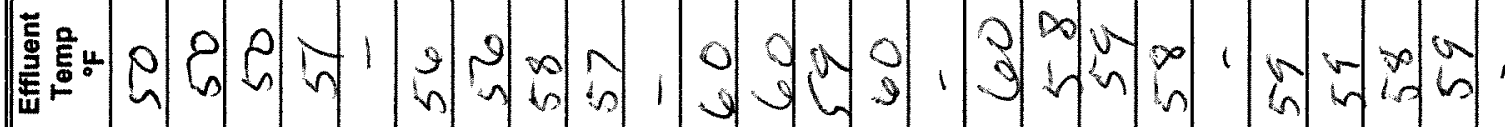

造

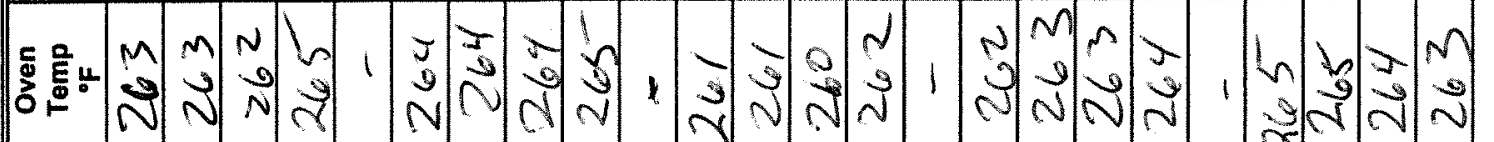

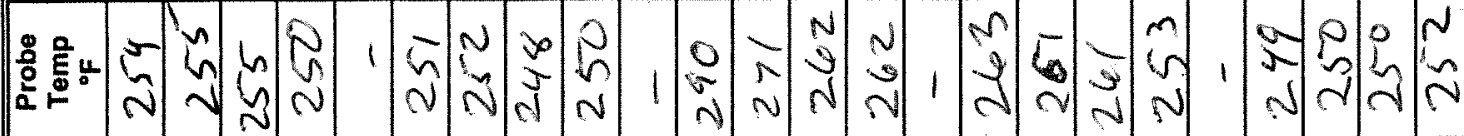

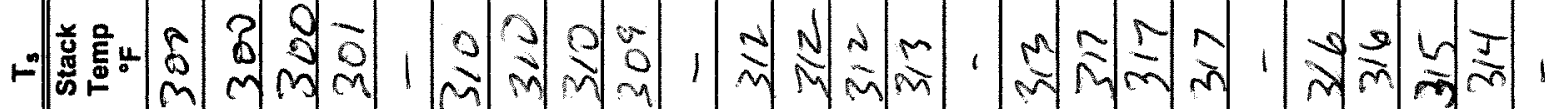

E气 है: के

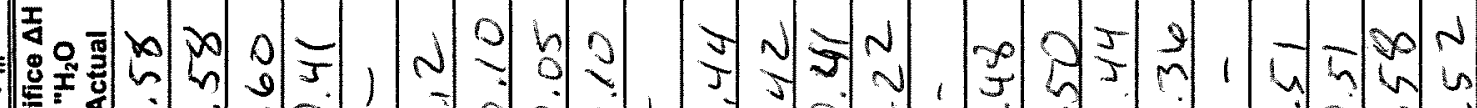

\%

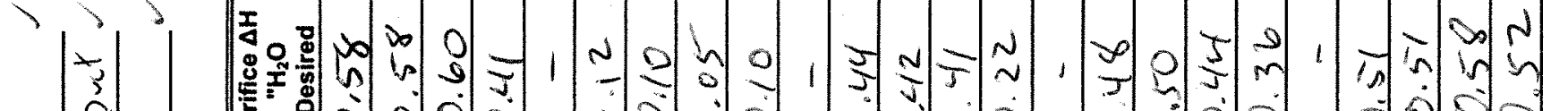

-

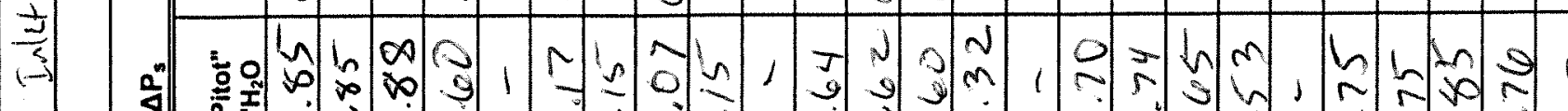

a 4 :

$n$ 站

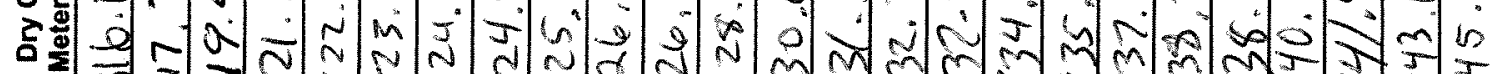

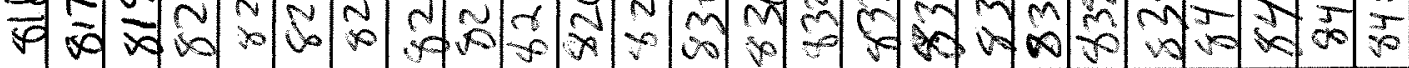

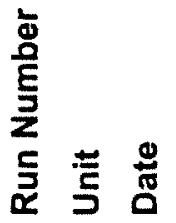

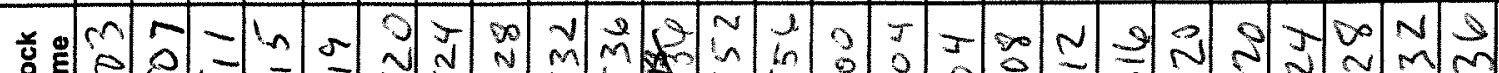

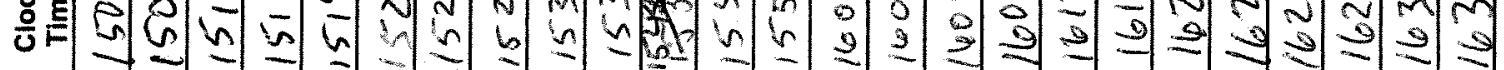

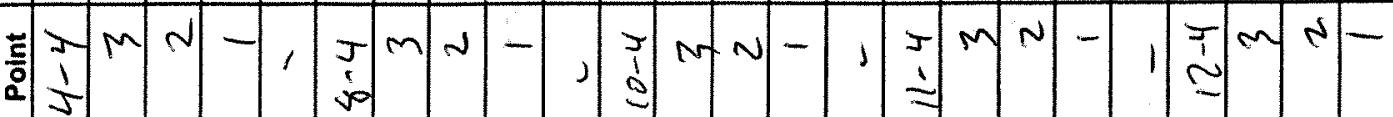




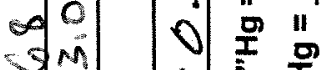

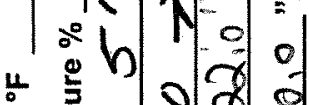

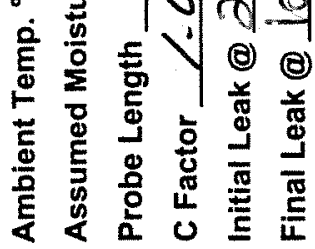

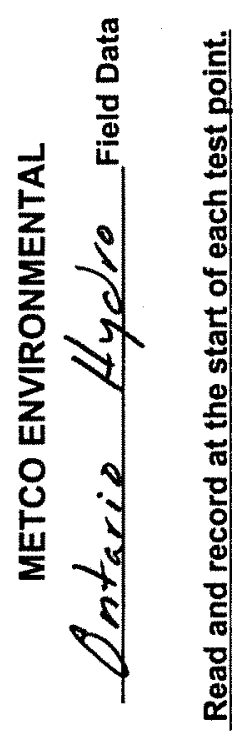

$4 \stackrel{M}{=1}=\frac{1}{2}$

盟告

e्व

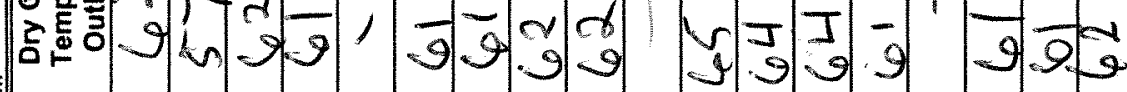
เ

骂:

䃌

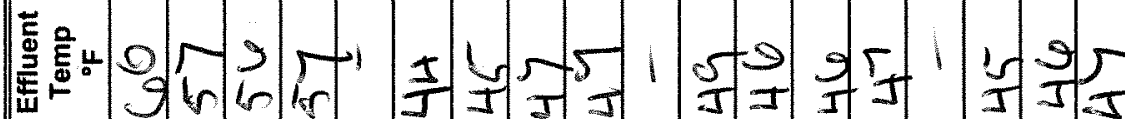
今|

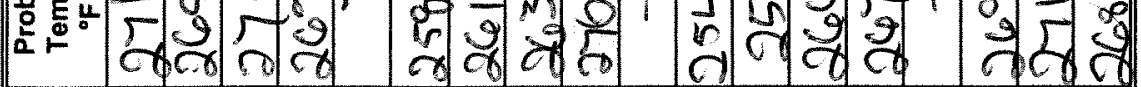
11 ज

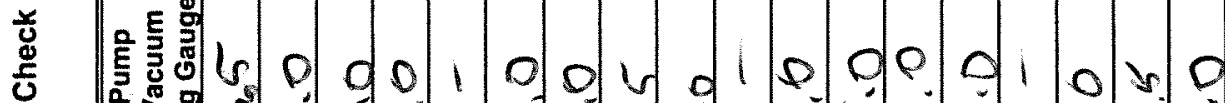

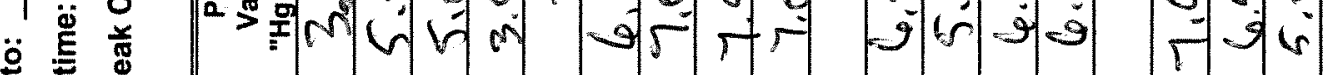

通 总

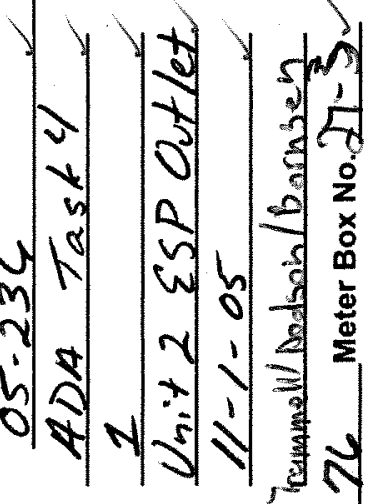

I a) de 0 ? 900 (1) 0 O $-1=$

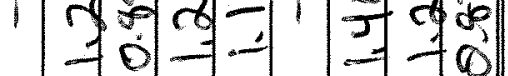
of $\mathrm{g}$ I

这娄

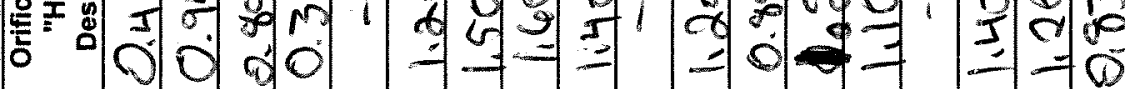

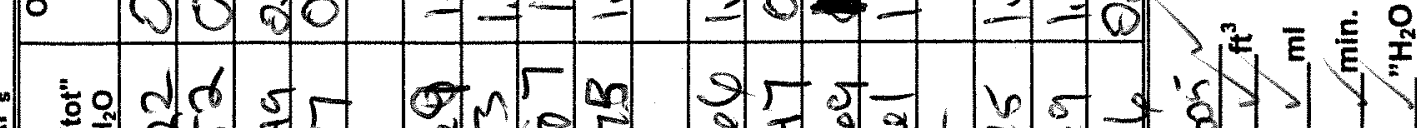

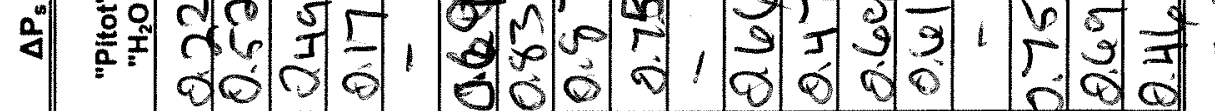
mo न्ग I 00 - 0 जั

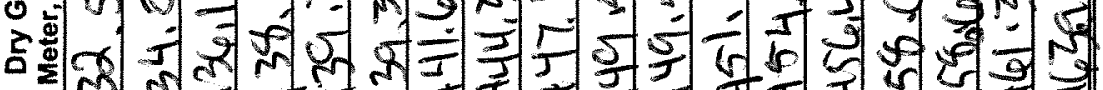

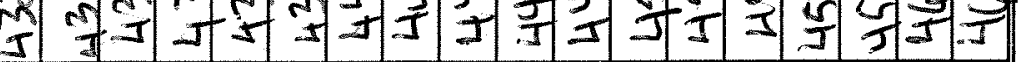

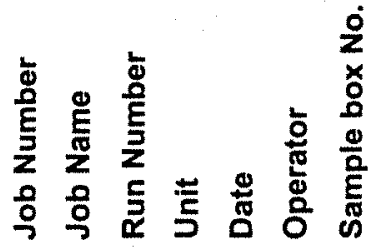

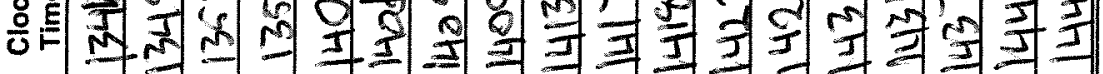

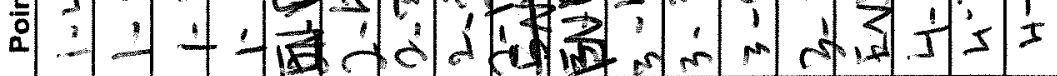

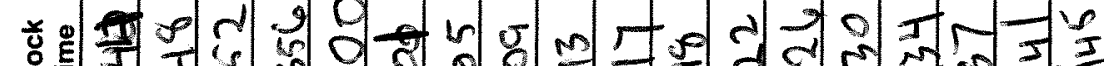

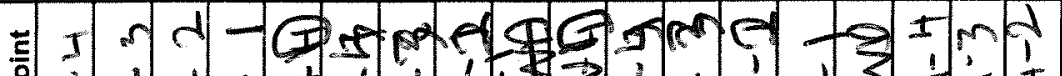

突要
की 을

至

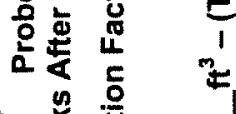

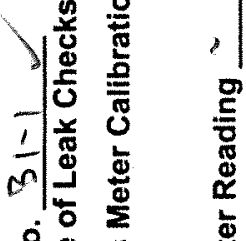

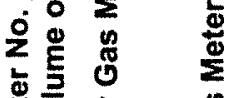

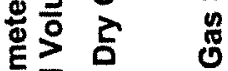

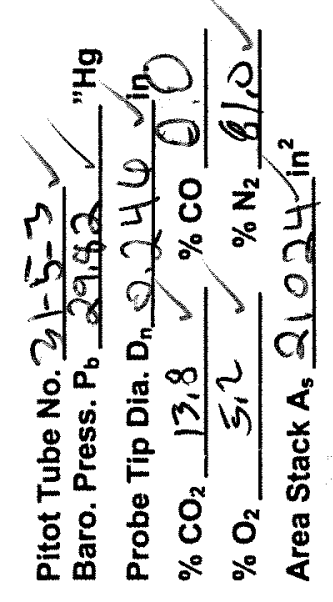


Impinger Box No.

Impinger 1

Impinger 2

Impinger 3

3

Impinger 4

mpinger 5

Impinger 6

Impinger 7

Moisture Content:

$$
\begin{aligned}
& \text { Final Weight } \\
& \text { Initial Weight } \\
& \text { Increase }
\end{aligned}
$$

Final Weight

Initial Weight

Increase

Final Weight

Initial Weight

Increase

Final Weight

Initial Weight

Increase

Final Weight

Initial Weight

Increase

Final Weight

Initial Weight

Increase

Final Weight

Initial Weight

Increase

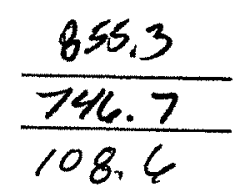

$\frac{690.0}{672.3}$

$\frac{746.0}{\frac{74.4}{3.4}}$

697.6

694.6

3.0

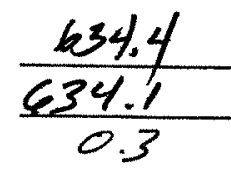

$\frac{736.9}{736.7}$

6690

668.0

L. 0
Water Weight Gain

Impinger $1 \quad 108.5$

Impinger $2 \quad C \mathbb{Z} .7$

Impinger $3 \quad \overline{3} .6$

Impinger $4 \quad 3.0$

Impinger $5 \quad 0.3$

Impinger $6 \quad 0.2$

Impinger $7 \frac{1.0}{13.6}$

Total

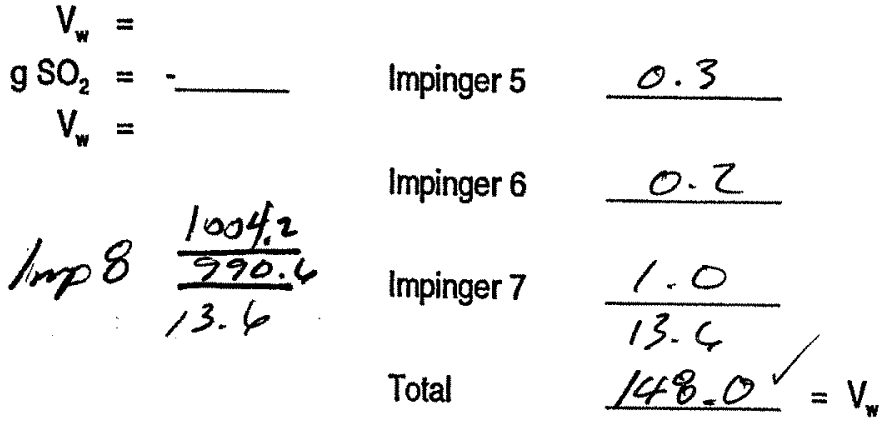

\section{$8.85 \% \%$}

$$
V m_{\text {std }}=17.65 \mathrm{Vm}\left[\frac{P_{b}+\frac{P_{m}}{13.6}}{T_{m}+460}\right]=17.65 \times 71.424\left[\begin{array}{l}
29.82+\frac{1.055}{13.6} \\
64 /+460
\end{array}\right]=\frac{71.931}{0.562} \mathrm{st}^{3}
$$

$V_{w_{\text {gas }}}=0.0472 \times V_{W}=0.0472 \times 146.0 / 7.023-6.986 \mathrm{sft}^{3}$

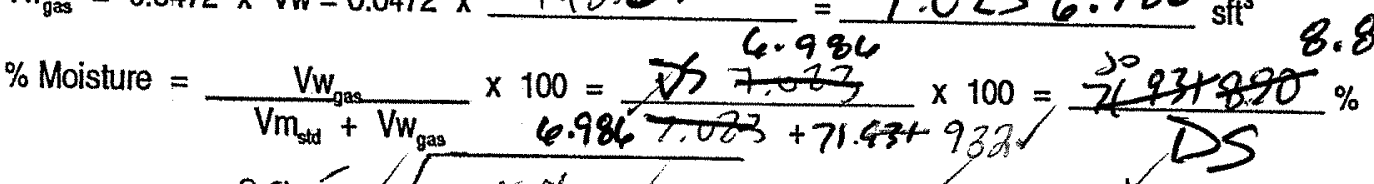

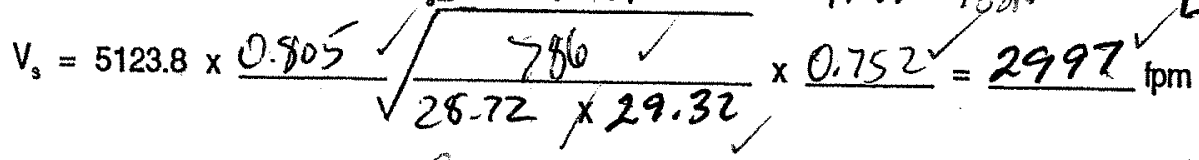

$c_{p}=0.805$

$\begin{aligned} \% \mathrm{CO}_{2} & =\frac{13.8}{5.2} \\ \% \mathrm{O}_{2} & =\frac{5.0}{0.0} \\ \% \mathrm{CO}_{2} & =\frac{51.0}{21024}\end{aligned}$

$A_{s}=21024$

$D_{n}=0.246$

$T_{1}=120$

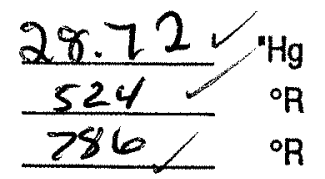

$\% M=\frac{9.90}{1.85} \quad M_{d}=0.9115 / M_{d}=30.416 / M W=29.32$

DS 
㝕 เ

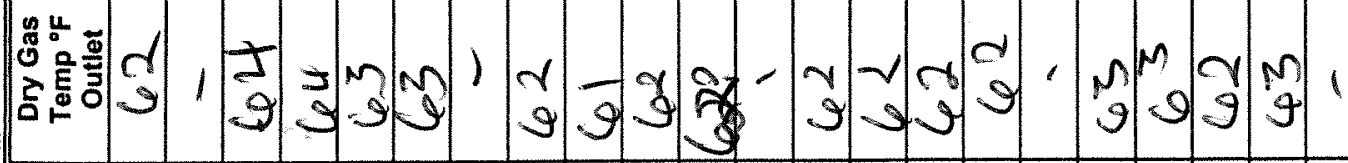

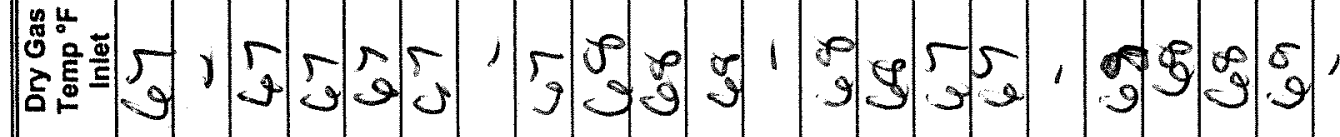

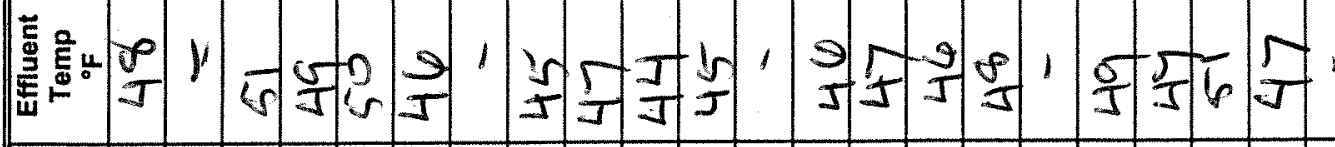

造

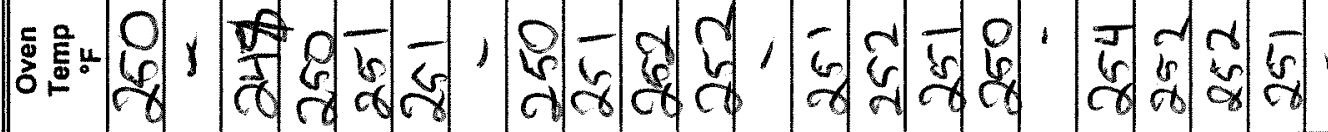

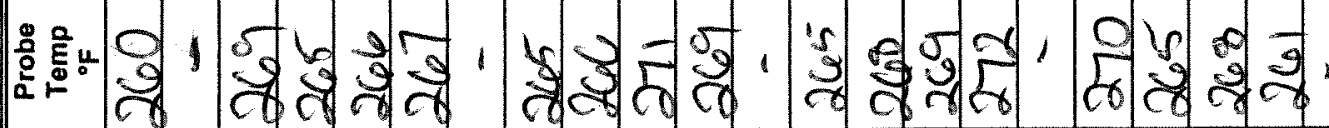

- कूँ की

홍

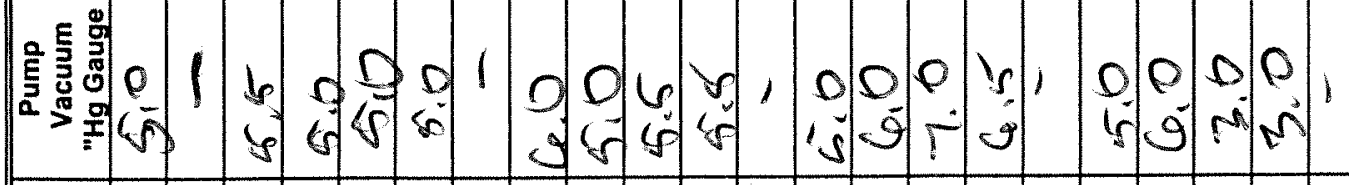

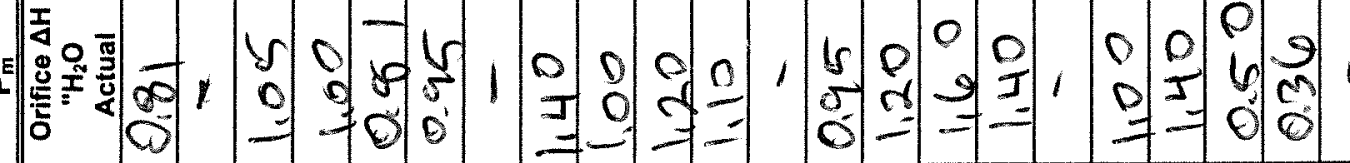

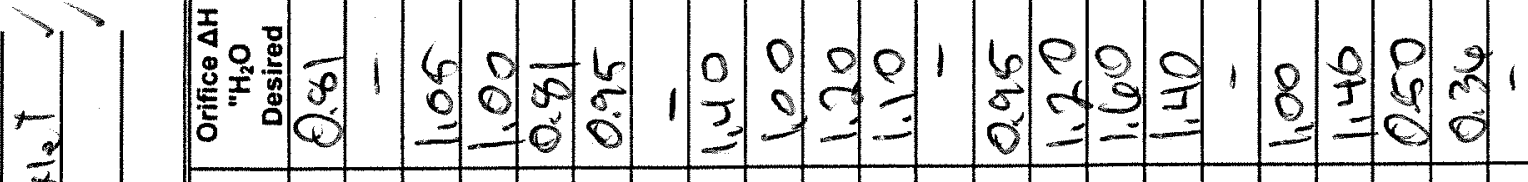

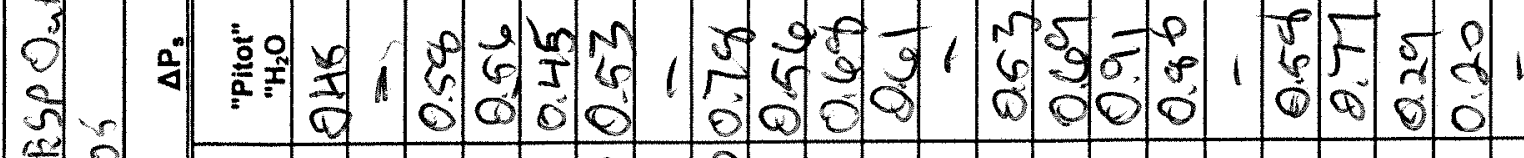

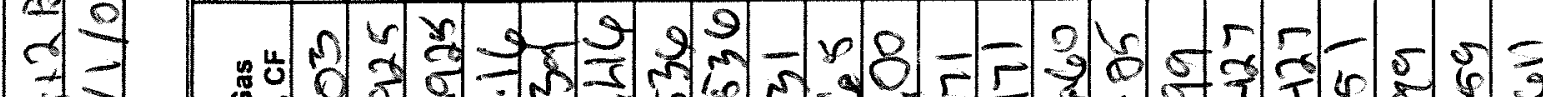

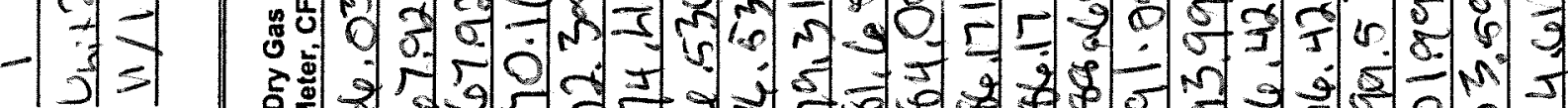

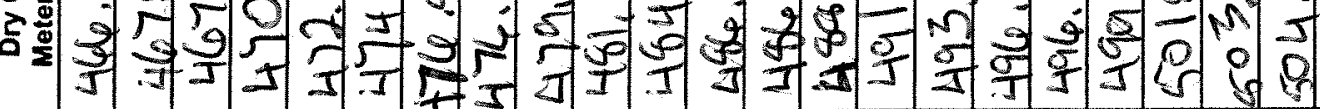

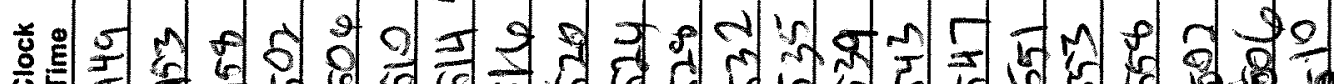

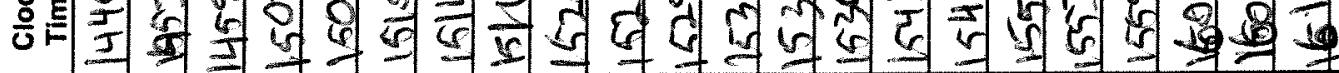

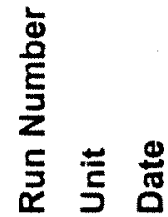

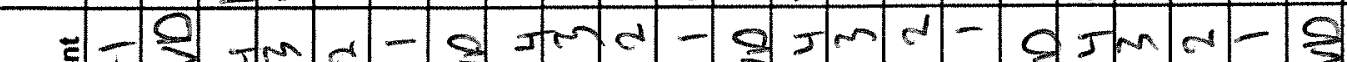

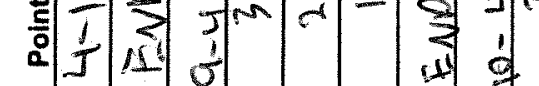

4. 


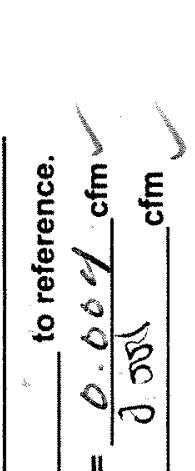

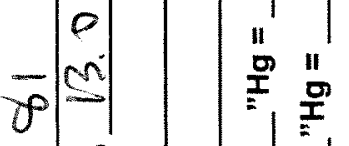

2.

‥

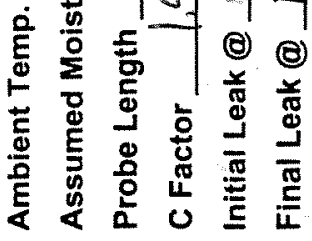

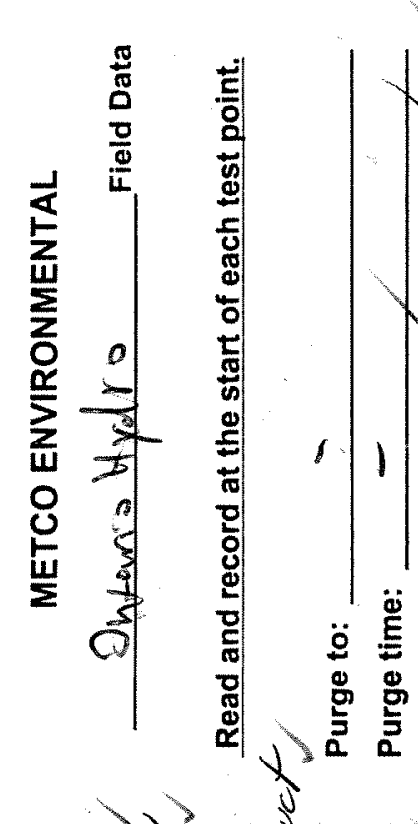

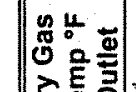

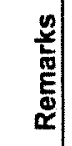
$\vdash$

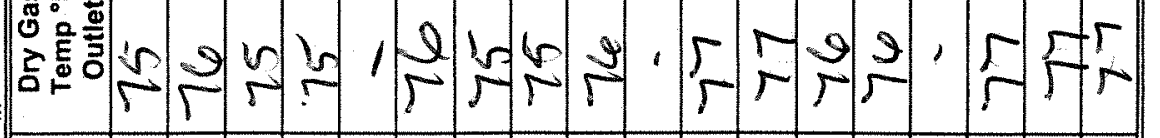

)

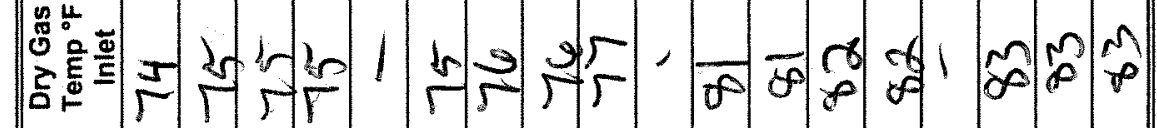

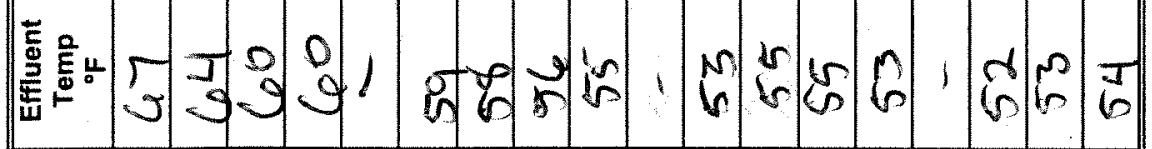

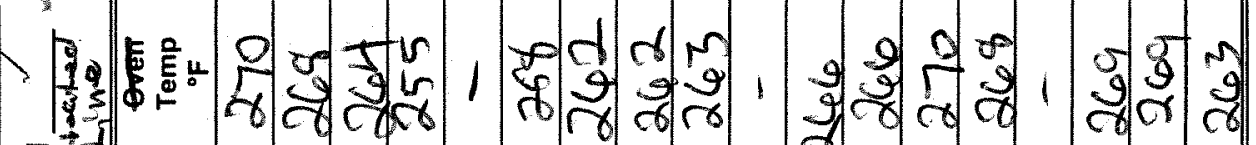
要

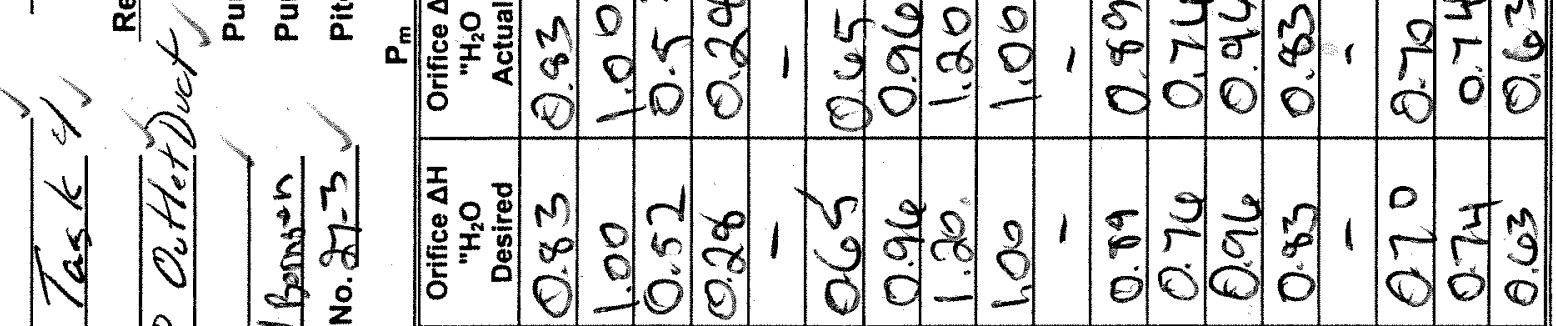

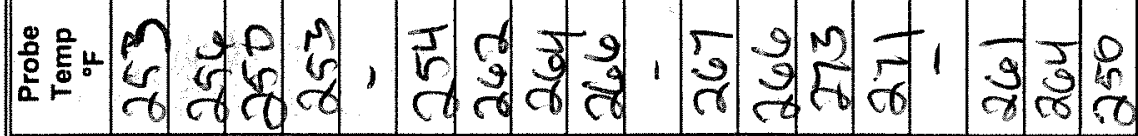

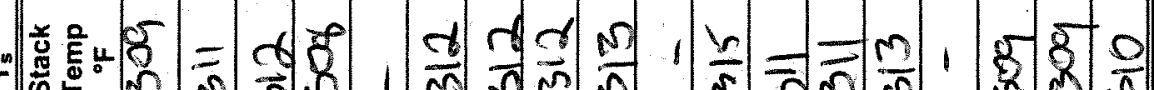

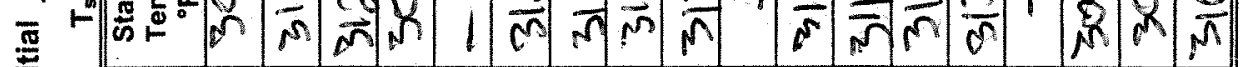

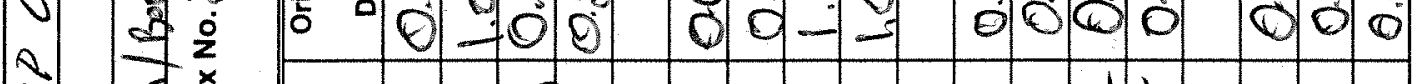

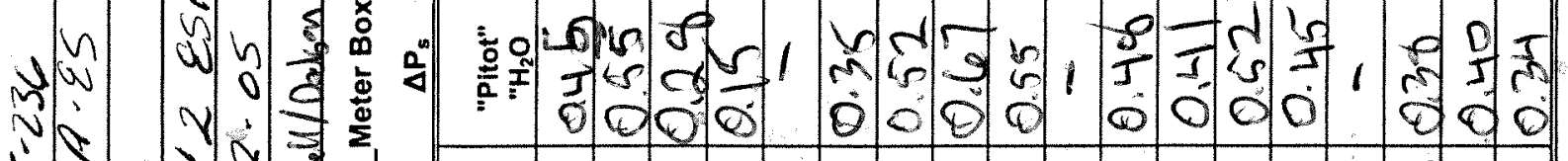
S.

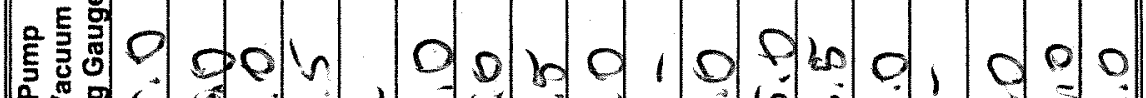

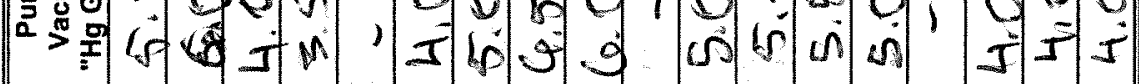

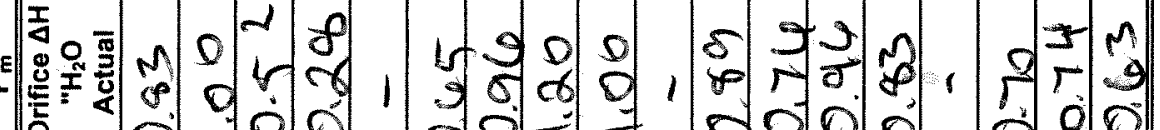
- D 700 - 70000000 กी को in $80>0$ m.t.

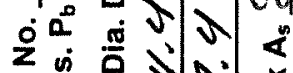
窝

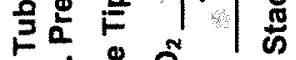
芒若 $0 / M \mid \sqrt{ } \geqslant$ :

过吉

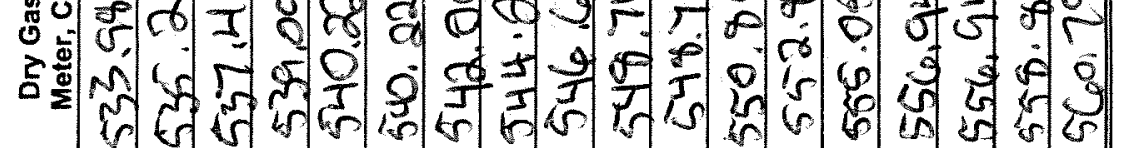

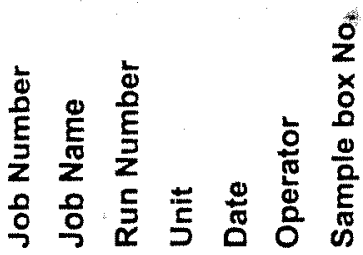

응 है

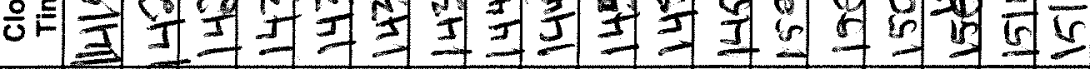

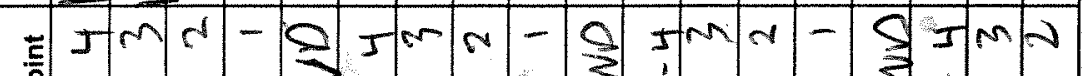
a. (1) 8

C) $\bar{E}$ ह 
Impinger Box No.

Impinger 1

Impinger 2

Final Weight

Initial Weight

Increase

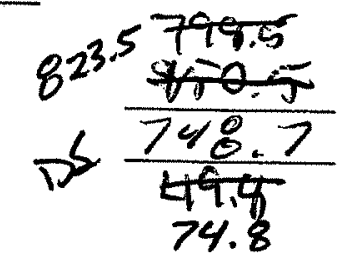

Water Weight Gain

$74.8 \mathrm{~V}$

Impinger 3

(

Finat Weight

Final Weight

Initial Weight

Increase

$$
\frac{681.2}{7.3}
$$

$\lim _{8} \frac{1017.2}{\frac{1003.8}{13.4}}$ Impinger 1

Impinger $3+46$

Impinger $41.9 v$

Final Weight

Initial Weight

Increase

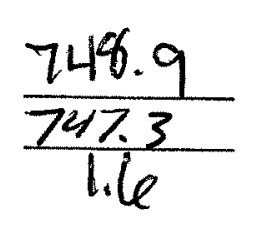

$\begin{aligned} \mathrm{V}_{w} & = \\ \mathrm{gSO}_{2} & =- \\ \mathrm{V}_{\mathrm{w}} & =\end{aligned}$

Impinger

000.3.

Impinger $6 \quad-3.8^{-0.8}$

Impinger 4

Final Weight

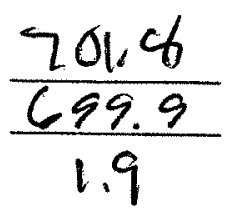

Impinger 7

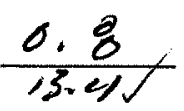

Increase

1.9

Impinger 5

Final Weight

Initial Weight

630.9

Increase

630.6

$000 \cdot 3$

Impinger 6

Final Weight

Initial Weight

73i. $7 \quad P_{m}=\frac{0,779 V}{0.433}$

$P_{b}=29.98$

$v_{\mathrm{m}}=\frac{21.949}{949}$

Total

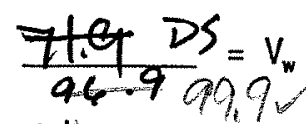

Increase

Impinger 7

$C_{0.1} \quad C_{p}=0.805^{\circ}$

Final Weight

$P_{s}=-14.5-\mathrm{H}_{2} \mathrm{O}$

$\% \mathrm{CO}_{2}=$

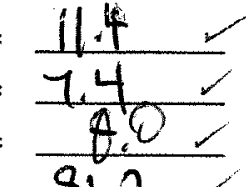

$v_{w}$ DS $7.996 .999 .9 \% \mathrm{CO}=$

$\% N_{2}=81.2 \angle$

Increase

$$
\frac{732.5}{\frac{7.4-0.8}{6.7 .1}} \frac{6}{\frac{66.3}{0.8 .}}
$$

$A_{s}=Q 1024$

$D_{n}=0.246$

$T_{t}=128 V$

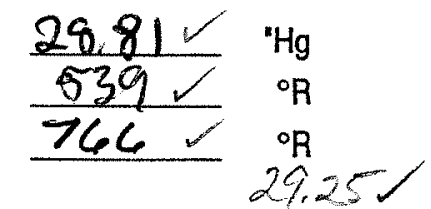

7,201

7.00

Moisture Content:
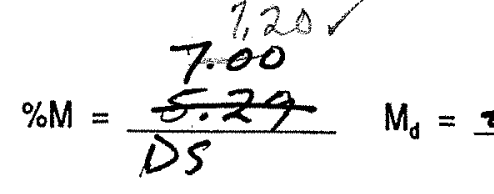

0.93000 .9280 29.25

$120^{\circ} M W=\frac{29.27}{24.48}$

$$
\begin{aligned}
& T_{m}=79 \angle \mathrm{F} \\
& T_{s}=30 \mathrm{C} /{ }^{\circ}
\end{aligned}
$$

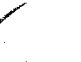

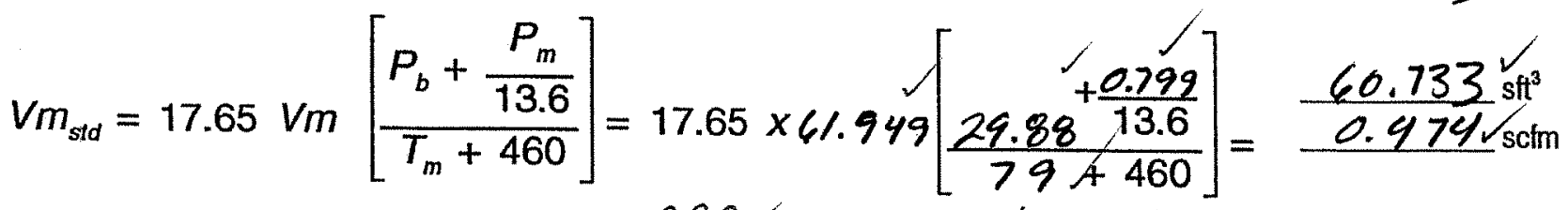

$$
\text { DS } 99.944 .5744 .715
$$

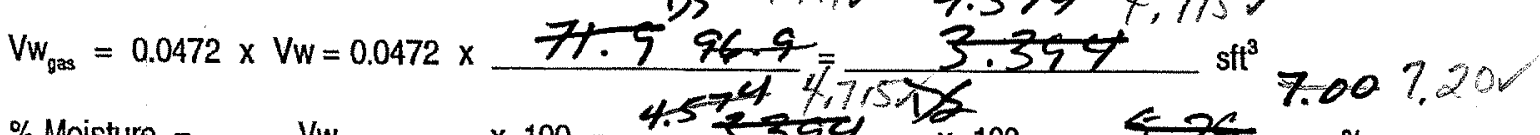

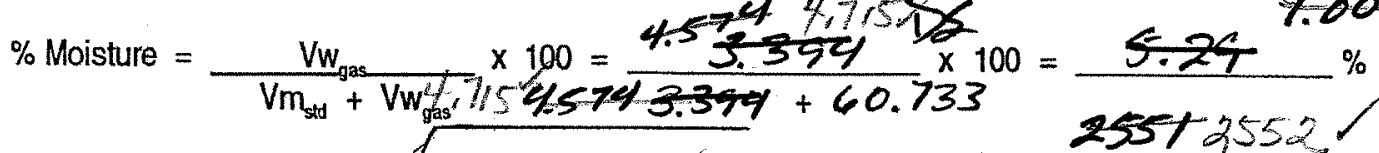

$V_{s}=5123.8 \times \frac{0.805}{D S} \sqrt{\frac{766 V}{25.48 \times 28.81}} \times 0.649 V=\frac{2502}{D S} \mathrm{fm}$

$$
\text { D) } \frac{}{25.48 \times 28.81}
$$

$\% 1=\frac{1.039 \times 764 / \times 6.7336}{91.3 \%} \%$ $2592 \times 0.9971 \times 28.81 \times 128 \times(0.246)^{2}$

DS 372,492

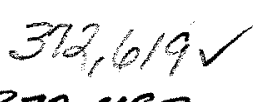

ACFM: $37+62$

SCFM: 230,742 
: 틀

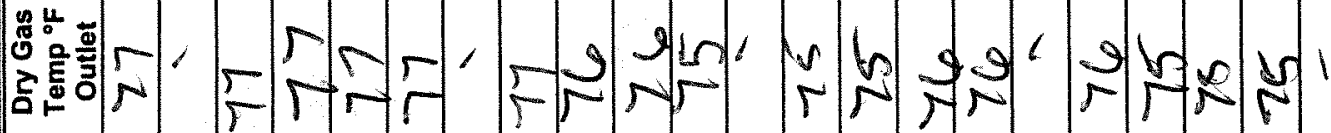

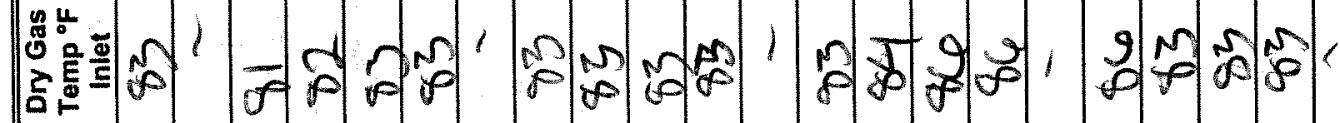

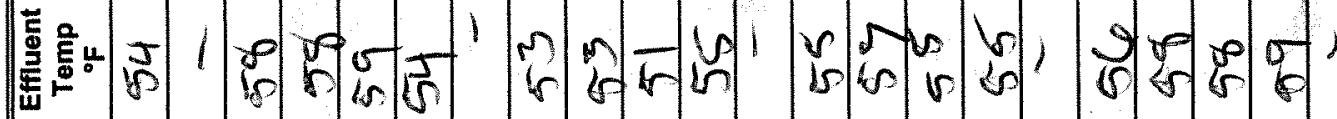

ठิ."

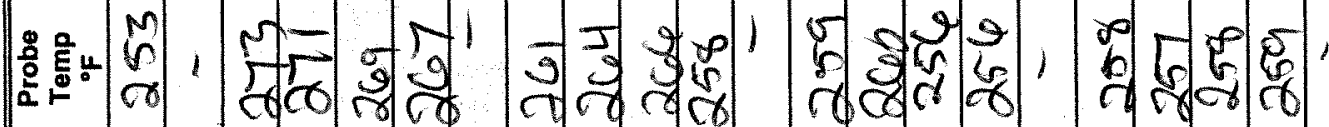

म कूँ

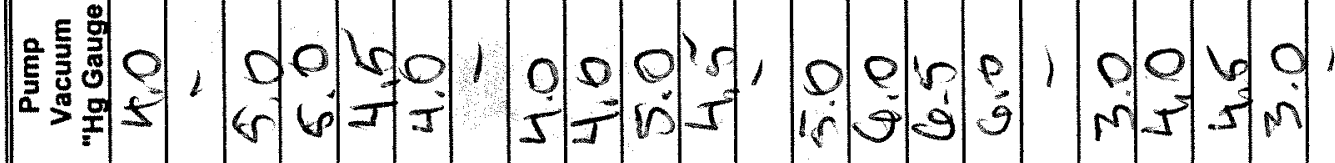

Q.

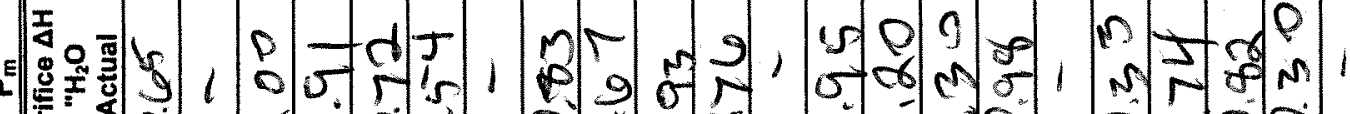

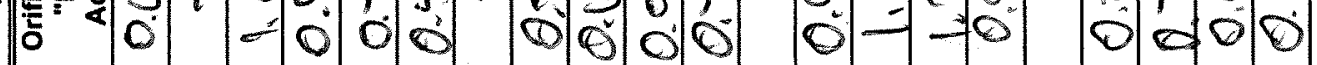

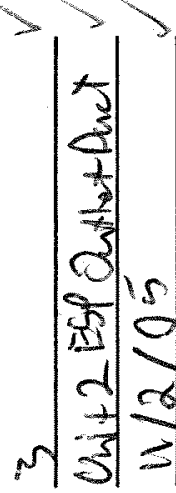

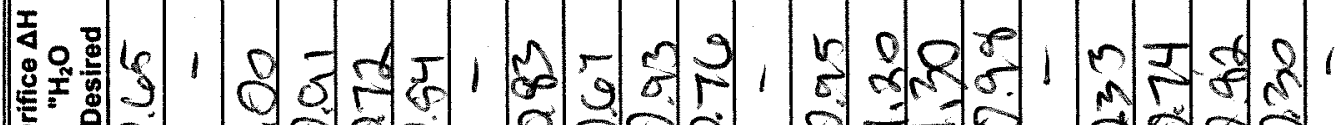

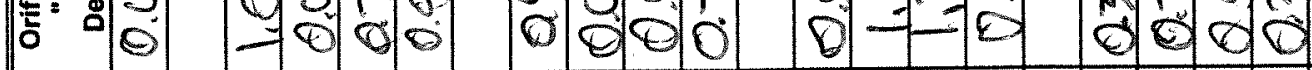

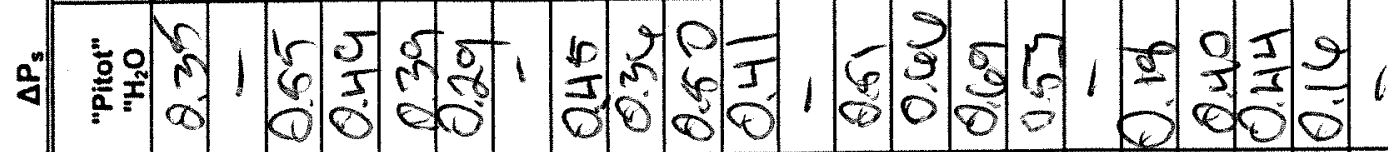

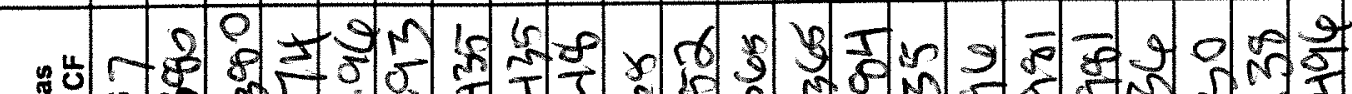

हैँ

टँ

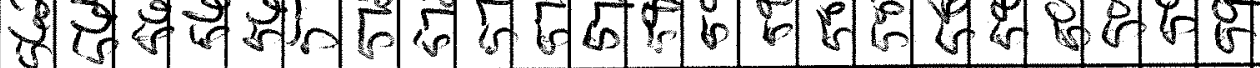

总

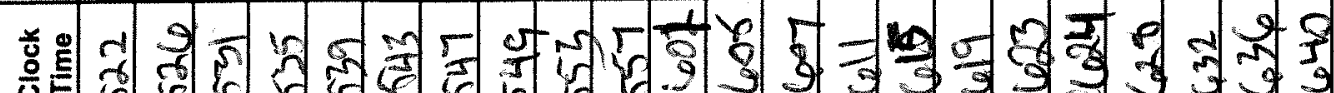

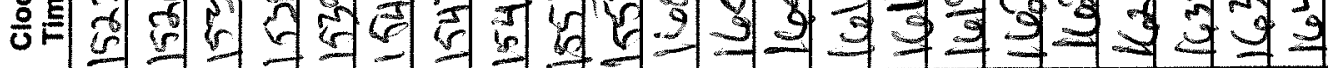

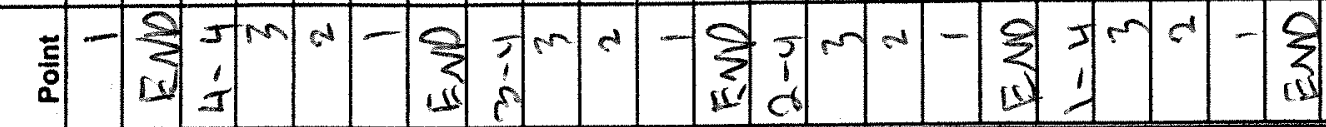




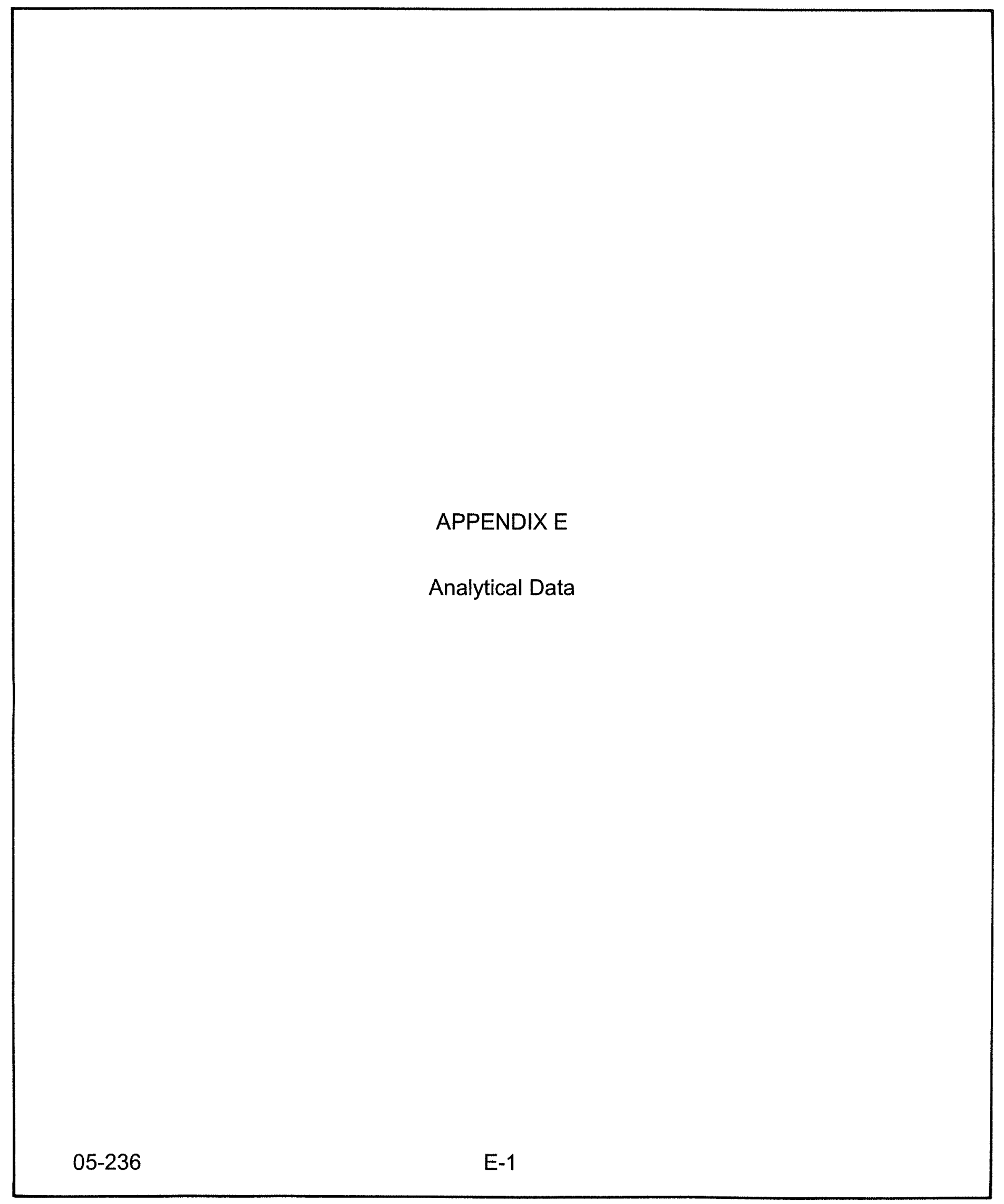




\section{Data Analysis Technologies, Inc.}

7715 Corporate Blvd.

Plain City, $\mathrm{OH} 43064$

NELAP/LELAP Certification 03027

\section{Sample Analysis Certificate}

Client: $\quad$ Metco Environmental

Address: $\quad 3226$ Commander Drive

Carrollton, TX 75006
Date:

DAT Project \#:

Sample Date:

Date Received:

Date Analyzed:

Analyst:
$12 / 8 / 05$

1105021

$11 / 01-02 / 05$

$11 / 15 / 05$

$12 / 08 / 05$

$\mathrm{JL} / \mathrm{DE}$

Attn: $\quad$ Steve Bornsen

Your Project: $05-236$ ADA-ES

Sampled By: S.B.

\section{Analysis: $\quad$ Mercury \\ Method: Ontario Hydro}

Results: $\quad$ See attached summary tables.

QC: $\quad$ Met criteria for the method.

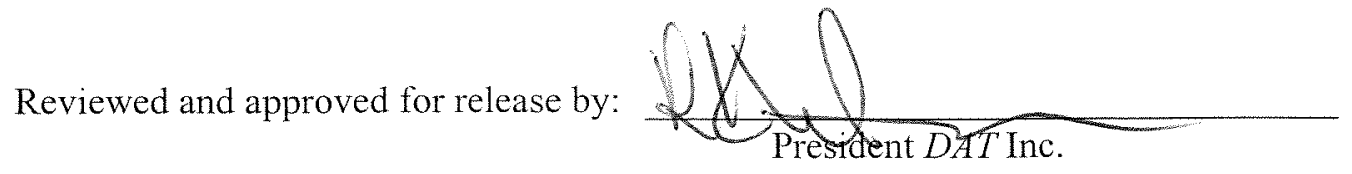

Date: 1215105

\section{E-2}




\section{Data Analysis Technologies}

\section{Mercury by Ontario Hydro Method: Data Summary}

Project Number:

Client:

Site:

Prep Date:

Analysis Date:
1105021

Metco Environmental

ADA-ES

$12 / 7 / 05$

$12 / 8 / 05$
Client ID
DAT ID

$\begin{array}{ll}1105021-1,2 & 0.02 \\ 1105021-3 a & 0.05 \\ 1105021-3 b & 0.04 \\ 1105021-4 & 0.24 \\ 1105021-5 & 0.08\end{array}$

$M D L$

0.02

0.05

.24

0.08

\section{Hg Q Dup Result} Total ug

Total ug

\section{Inlet Run 1}

Filter/F.H. Rinse

B.H. Rinse Imp 1-3

B.H. Rinse Imp 1-3

Imp 4

Imp 5-7

$\left.\begin{array}{l}\mathrm{ND}-\mathrm{PB} \\ \mathrm{ND} \\ 0.24 \\ \mathrm{ND} \\ 1.56\end{array}\right] \mathrm{OY}$

0.02

0.53

ND

ND

1.58

Imp 5-7

1105021-9

0.27

0.08

nlet Run 3

B.H. Rinse Imp 1-3

1105021- 10

0.02

0.07

0.03

$\mathrm{ND}=$ Not detected at the detection limit shown 


\section{Data Analysis Technologies}

Mercury by Ontario Hydro Method: Data Summary

Project Number:

Client:

Site:

Prep Date:

Analysis Date:
1105021

Metco Environmental

ADA-ES

$12 / 7 / 05$

$12 / 8 / 05$
Client ID

DAT ID

$1105021-11,12$

$1105021-13 \mathrm{a}$

$1105021-13 b$

$1105021-14$

$1105021-15$

\section{Outlet Run 1}

Filter/F.H. Rinse

B.H. Rinse Imp 1-3

B.H. Rinse Imp 1-3

Imp 4

Imp 5-7

$\begin{array}{lr}0.02 & 0.29 \\ 0.08 & 1.08 \\ 0.03 & \text { ND } \\ 0.26 & \text { ND } \\ 0.08 & 0.48\end{array}$

Hg Q Dup Result

$M D L$

Total ug

Total ug

\section{Outlet Run 3}

Filter/F.H. Rinse

$1105021-16,17$
$1105021-18 a$
$1105021-18 b$
$1105021-19$
$1105021-20$

0.02

0.20

B.H. Rinse Imp 1-3

0.08

0.37

B.H. Rinse Imp 1-3

0.03

ND

Imp 4

Imp 5-7

$1105021-20$

0.30

ND

0.08

0.11

$\mathrm{ND}=$ Not detected at the detection limit shown

\section{E-4}




\section{Data Analysis Technologies}

Mercury by Ontario Hydro Method: Data Summary

Project Number: $\quad 1105021$

Client: Metco Environmental

Site: ADA-ES

Prep Date: $\quad 12 / 7 / 05$

Analysis Date: $\quad 12 / 8 / 05$

Client ID DAT ID

$M D L \quad$ Total ug Total ug

\section{Field Blank}

Filter/F.H. Rinse

$1105021-21,22$

0.02

ND

B.H. Rinse Imp 1-3

$1105021-23$

0.77

ND

Imp 4

$1105021-24$

0.03

ND

Imp 5-7

$1105021-25$

0.08

ND

\section{Reagent Blanks}

Filter/ 0.1N HNO3

$1105021-31,26$

0.02

ND

$\mathrm{KCL}$

$$
1105021-27
$$

0.01

ND

$5 \% \mathrm{HNO} 3 / 10 \% \mathrm{H} 2 \mathrm{O} 2$

$1105021-28$

$\mathrm{KMnO} 4$

1105021- 29

0.10

ND

$10 \%$ Hydroxylamine

$1105021-30$

0.01

ND

0.02

ND

$\mathrm{ND}=$ Not detected at the detection limit shown 


\section{Data Analysis Technologies}

\section{Mercury by Ontario Hydro Method: Data Summary}

Project Number: $\quad 1105021$

Client: Metco Environmental

Site: $\quad$ ADA-ES

Prep Date: $\quad 12 / 7 / 05$

Analysis Date: $\quad$ 12/8/05

\begin{tabular}{|c|c|c|c|c|}
\hline \multirow[b]{2}{*}{ QC Summary } & & \multirow[t]{2}{*}{$\begin{array}{l}\mathbf{H g} \\
\mathbf{u g} / \mathbf{L}\end{array}$} & \multirow[t]{2}{*}{$\% \operatorname{Rec}$} & \multirow[t]{2}{*}{$\%$ RPD } \\
\hline & & & & \\
\hline Front Method Blank & 1105021-MB Front & $0.05 \mathrm{ND}$ & & \\
\hline Back Method Blank & 1105021-MB Back & $0.05 \mathrm{ND}$ & & \\
\hline Laboratory Spike & 1105021-LS Front & 12.0 & 120 & \\
\hline Laboratory Spike & 1105021-LS Back & 10.4 & 104 & \\
\hline \multirow[t]{2}{*}{ Matrix Spike } & $1105021-5 \mathrm{MS}$ & 9.94 & 99 & \\
\hline & 1105021-5 MSD & 9.73 & 97 & 2.1 \\
\hline \multirow[t]{2}{*}{ Matrix Spike } & $1105021-14 \mathrm{MS}$ & 10.09 & 101 & \\
\hline & 1105021-14 MSD & 9.69 & 97 & 4.0 \\
\hline \multirow[t]{2}{*}{ Matrix Spike } & $1105021-20 \mathrm{MS}$ & 10.03 & 100 & \\
\hline & 1105021-20 MSD & 10.09 & 101 & 0.6 \\
\hline
\end{tabular}

$\mathrm{ND}=$ Not detected at the detection limit shown. 


\section{DOCUMENTATION}

Page 6 of 20 


\section{DAT SAMPLE RECEIVING}

7715 Corporate Blvd. Plain City, $\mathrm{OH} 43064$.

Project Number: 1105021

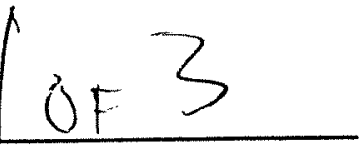

Date Received: $11 / 15 / 2005$

Client Name: Metco Environmental

Tracking number: 602557620798

Custody Seals ?: No
Carrier: FedEx

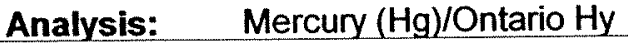

Package Temp: ambient

coc: $\square$ check if $\mathrm{COC}$ from client

\section{Sample Information}

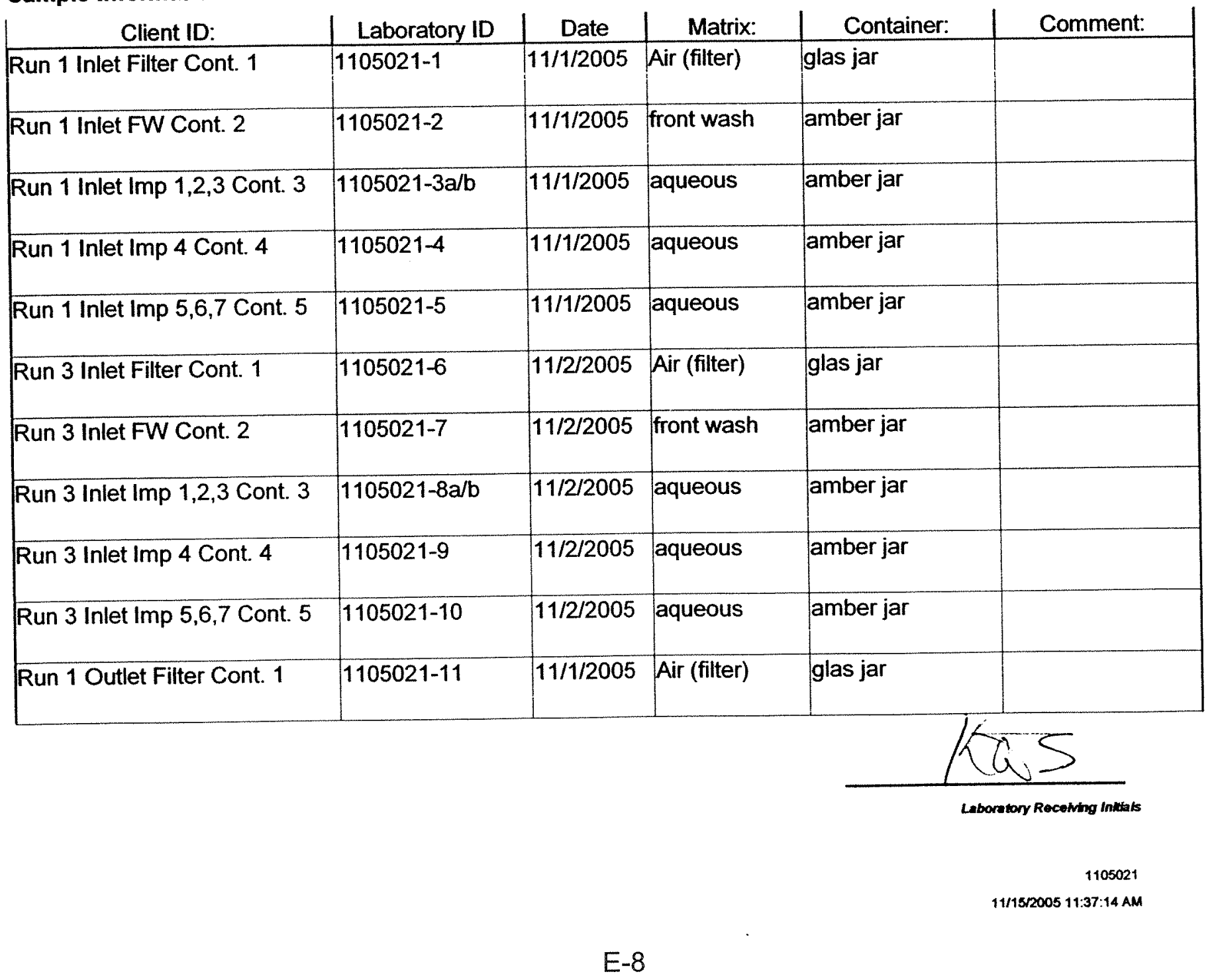




\section{DAT SAMPLE RECEIVING}

7715 Corporate Blvd. Plain City, $\mathrm{OH} 43064$.

Project Number: 1105021

\begin{tabular}{|c|c|c|c|c|c|}
\hline Client ID: & Laboratory ID & Date & Matrix: & Container: & Comment: \\
\hline Run 1 Outlet FW Cont. 2 & $1105021-12$ & $11 / 1 / 2005$ & front wash & amber jar & \\
\hline Run 1 Outlet Imp $1,2,3$ Cont. $3 \mid 1$ & $1105021-13 a / b$ & $11 / 1 / 2005$ & aqueous & amber jar & \\
\hline Run 1 Outlet Imp 4 Cont. 4 & $1105021-14$ & $11 / 1 / 2005$ & aqueous & amber jar & \\
\hline Run 1 Outlet Imp 5,6,7 Cont. 5 & $1105021-15$ & $11 / 1 / 2005$ & aqueous & amber jar & \\
\hline Run 3 Outlet Filter Cont. 1 & $1105021-16$ & $11 / 2 / 2005$ & Air (filter) & glas jar & \\
\hline Run 3 Outlet FW Cont. 2 & $1105021-17$ & $11 / 2 / 2005$ & front wash & amber jar & \\
\hline Run 3 Outlet Imp 1,2,3 Cont. 3 & $1105021-18 \mathrm{a} / \mathrm{b}$ & $11 / 2 / 2005$ & aqueous & amber jar & \\
\hline Run 3 Outlet Imp 4 Cont. 4 & $1105021-19$ & $11 / 2 / 2005$ & aqueous & amber jar & \\
\hline Run 3 Outlet Imp 5,6,7 Cont. 5 & $1105021-20$ & $11 / 2 / 2005$ & aqueous & amber jar & \\
\hline BT Filter Cont. 1 & $1105021-21$ & $11 / 2 / 2005$ & Air (filter) & glas jar & \\
\hline BT FW Cont. 2 & $1105021-22$ & $11 / 2 / 2005$ & front wash & amber jar & \\
\hline BT Imp 1,2,3 Cont. 3 & $1105021-23$ & $11 / 2 / 2005$ & aqueous & amber jar & \\
\hline BT Imp 4 Cont. 4 & $1105021-24$ & $11 / 2 / 2005$ & aqueous & amber jar & \\
\hline BT Imp 5,6,7 Cont. 5 & $1105021-25$ & $11 / 2 / 2005$ & aqueous & amber jar & \\
\hline RB 0.1N HNO3 Cont. 7 & $1105021-26$ & $11 / 2 / 2005$ & HNO3 & amber jar & \\
\hline RB $1 \mathrm{~N} \mathrm{KCl} \mathrm{Cont.} 8$ & $1105021-27$ & $11 / 2 / 2005$ & $\mathrm{KCl}$ & amber jar & \\
\hline
\end{tabular}




\section{DAT SAMPLE RECEIVING}

7715 Corporate Blvd. Plain City, OH 43064.

Project Number: 1105021

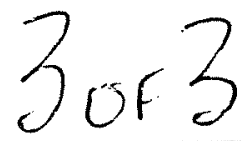

\begin{tabular}{|l|l|c|l|l|l|}
\multicolumn{1}{|c|}{ Client ID: } & Laboratory ID & Date & Matrix: & Container: & Comment: \\
\hline RB HNO3/H2O2 Cont. 9 & $1105021-28$ & $11 / 2 / 2005$ & HNO3/H2O2 & amber jar & \\
\hline RB KMnO4 Cont. 10 & $1105021-29$ & $11 / 2 / 2005$ & HNO3/H2O2 & amber jar & \\
\hline RB Hydroxylamine Cont. 11 & $1105021-30$ & $11 / 2 / 2005$ & hydrox.amine & amber jar & \\
\hline RB Filter Cont. 12 & $1105021-31$ & $11 / 2 / 2005$ & Air (filter0 & glass jar & \\
\hline
\end{tabular}




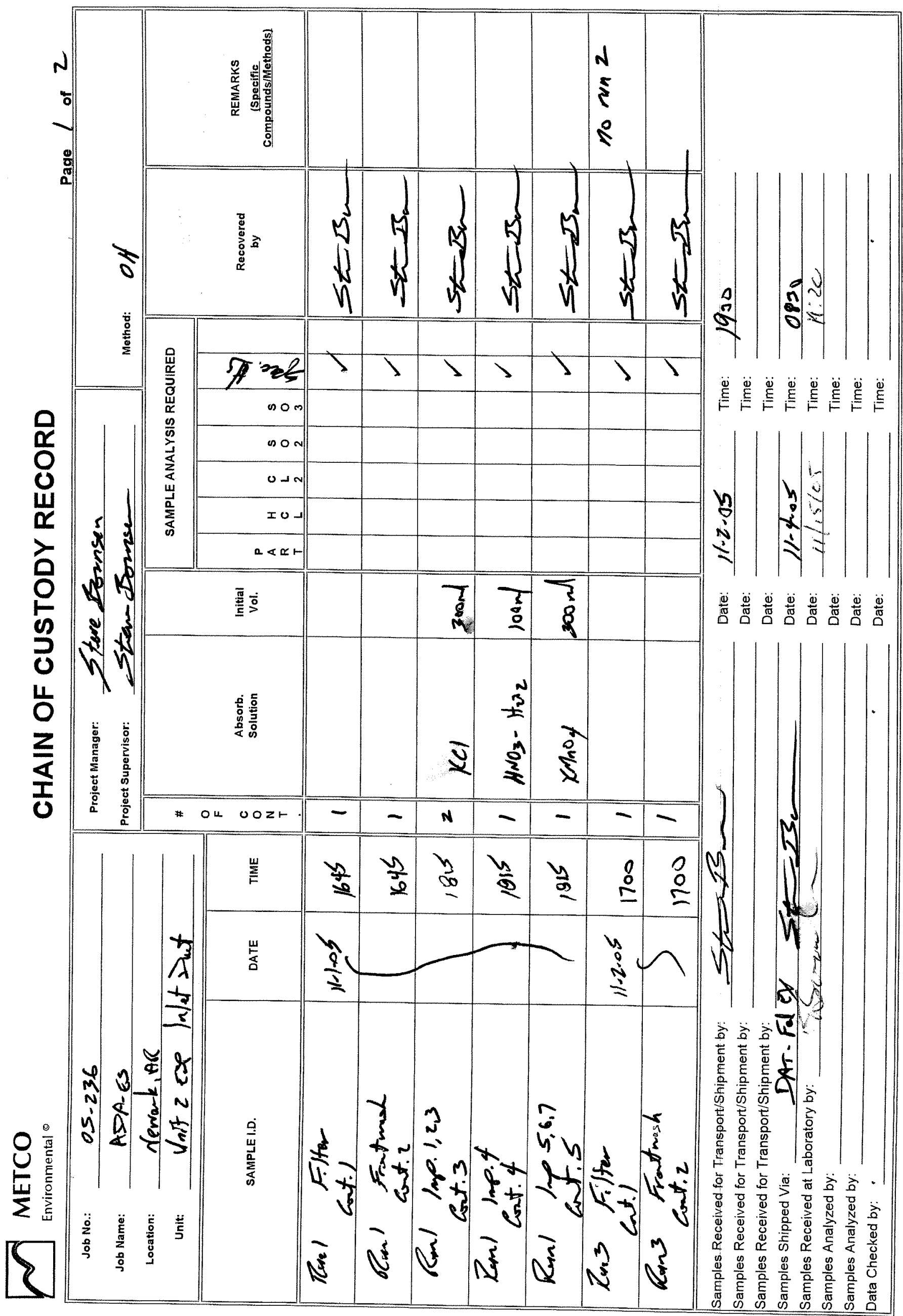




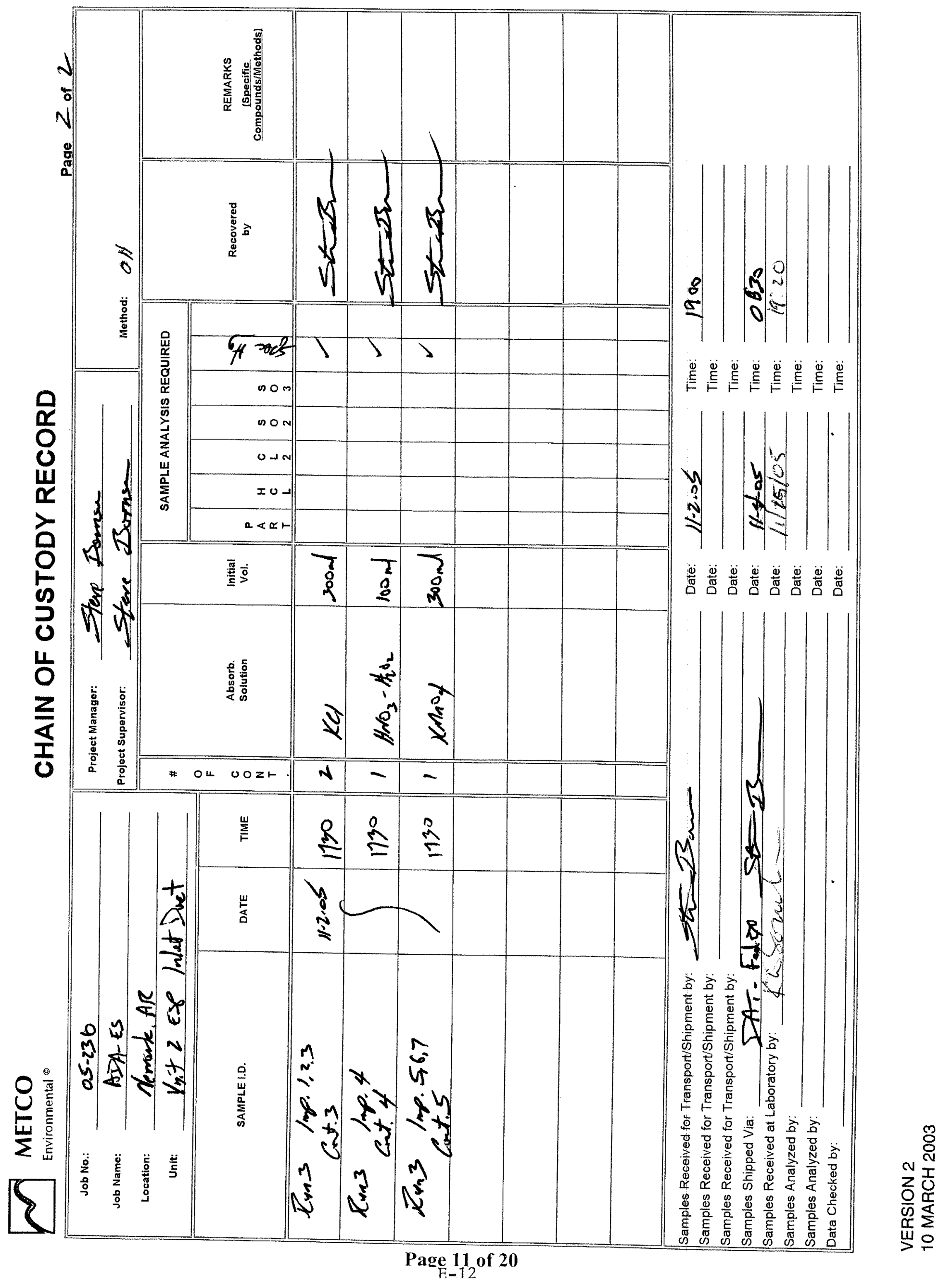




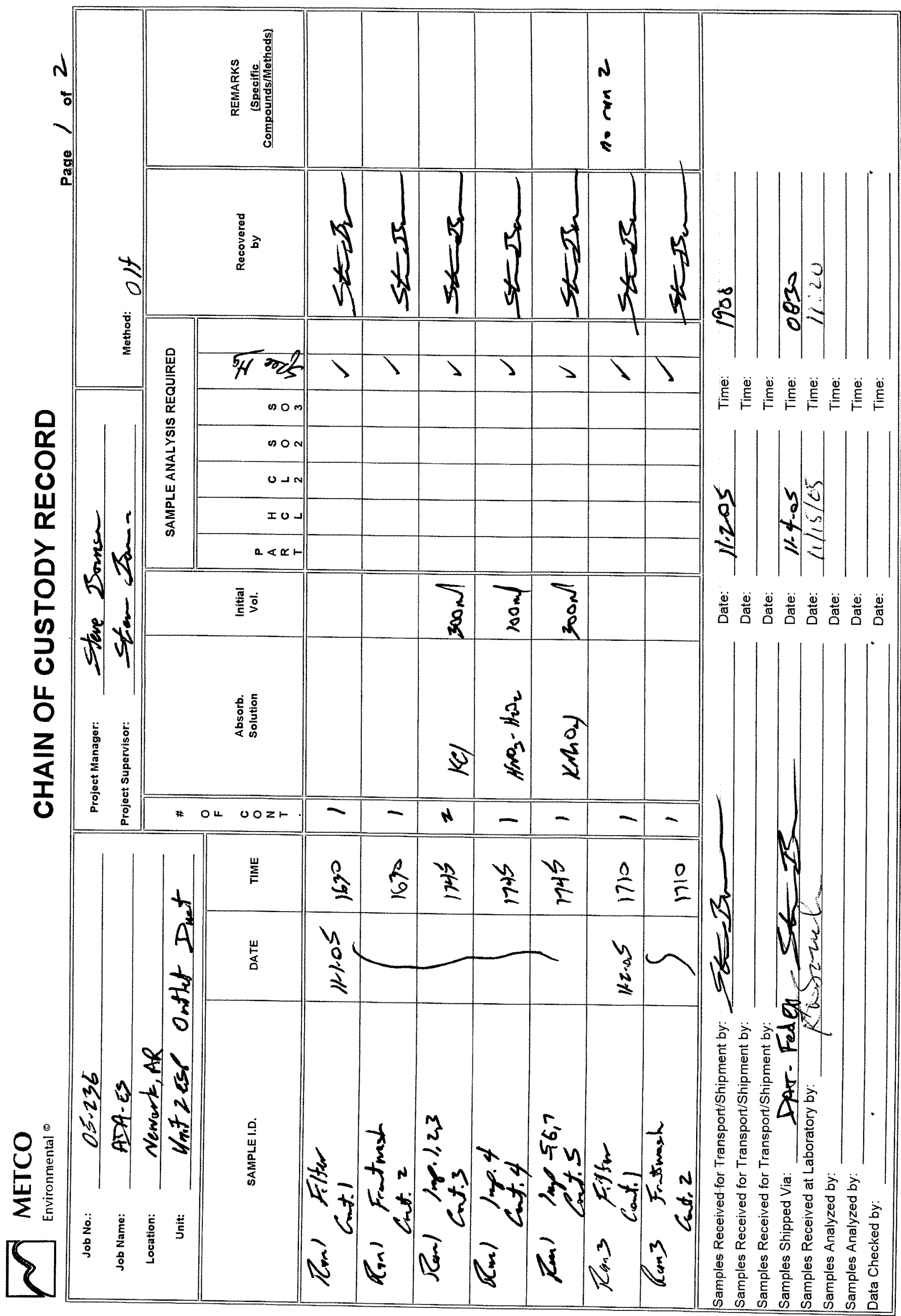




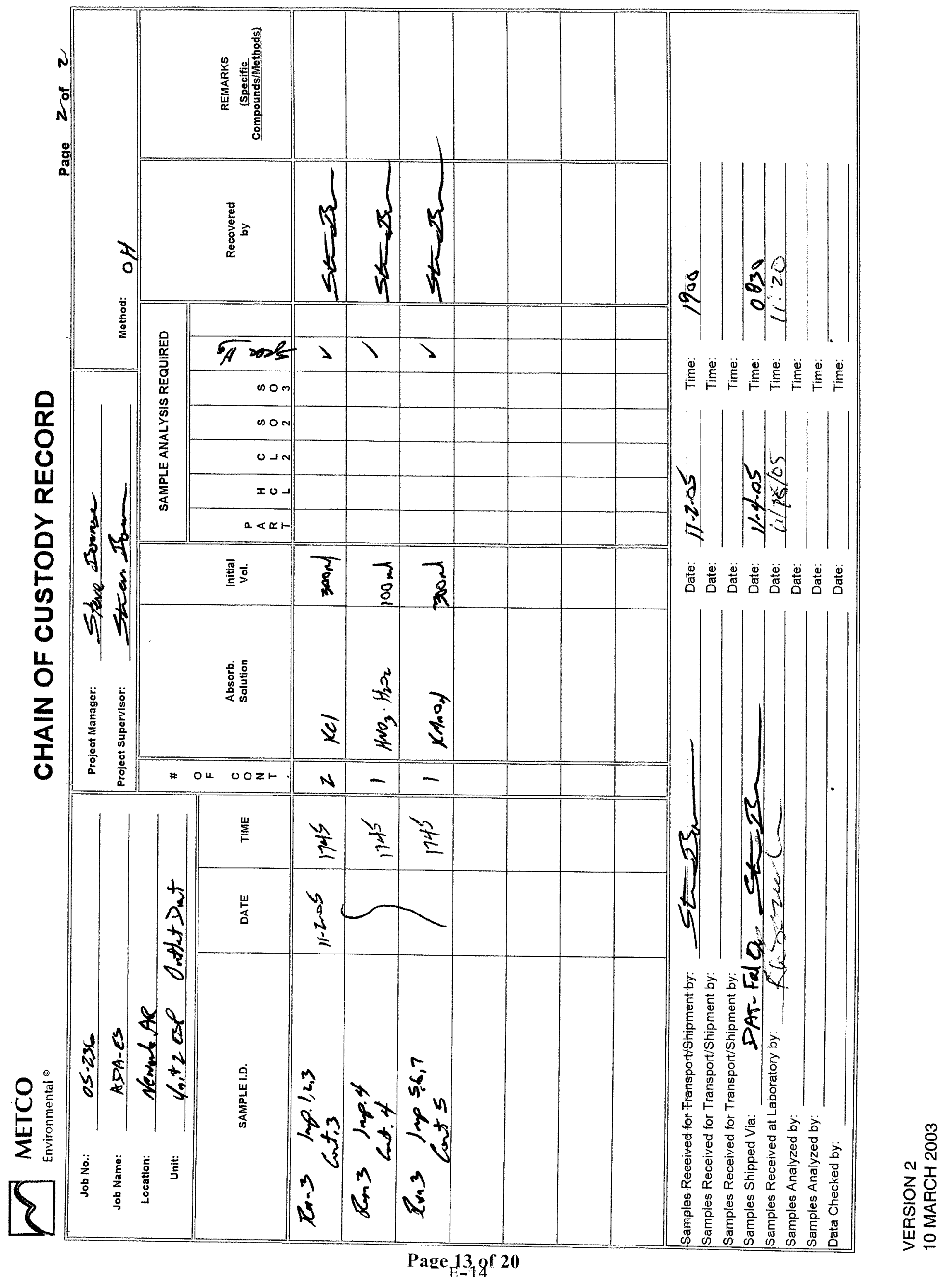




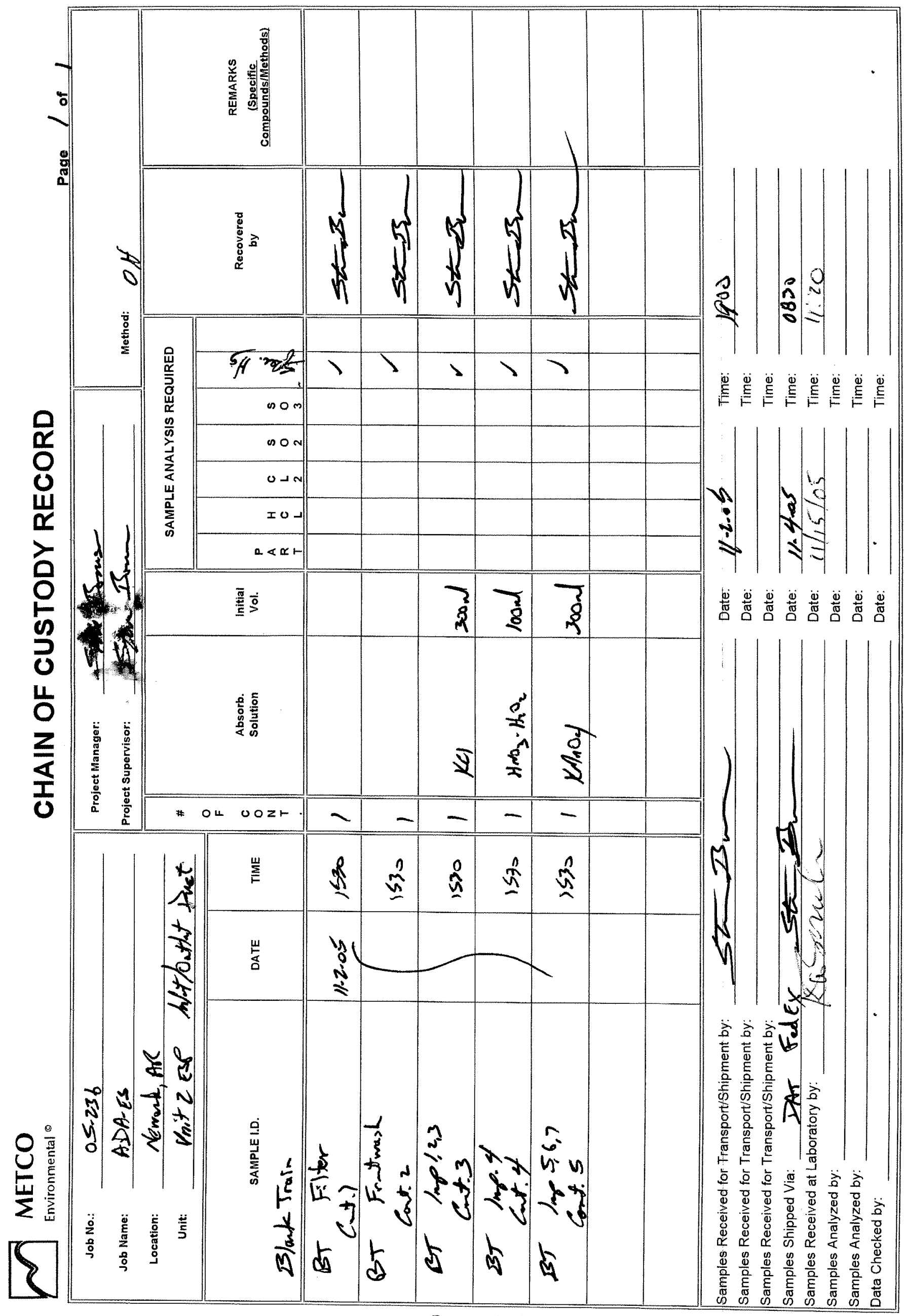




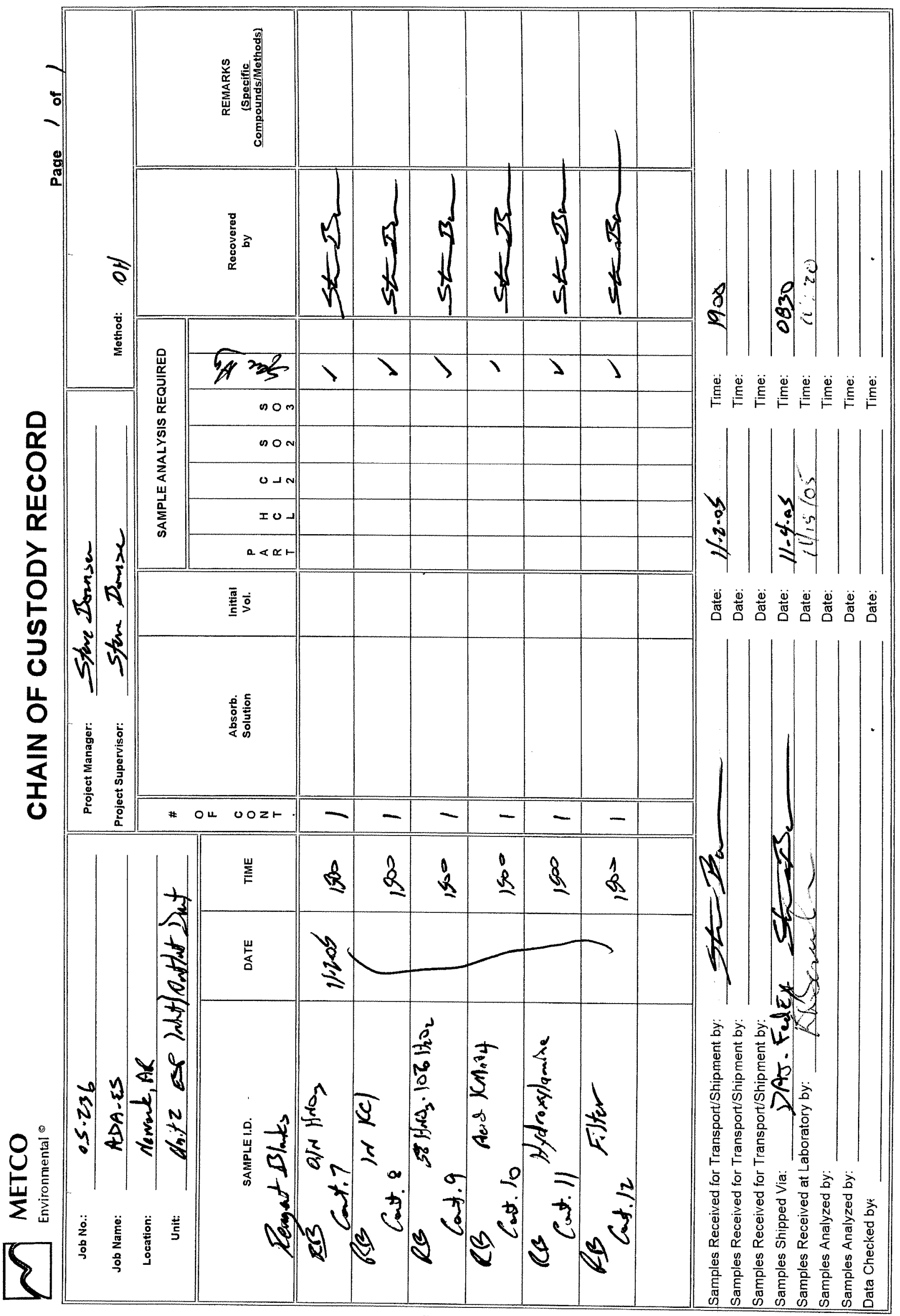


DAT Labs, Inc. Extraction Form

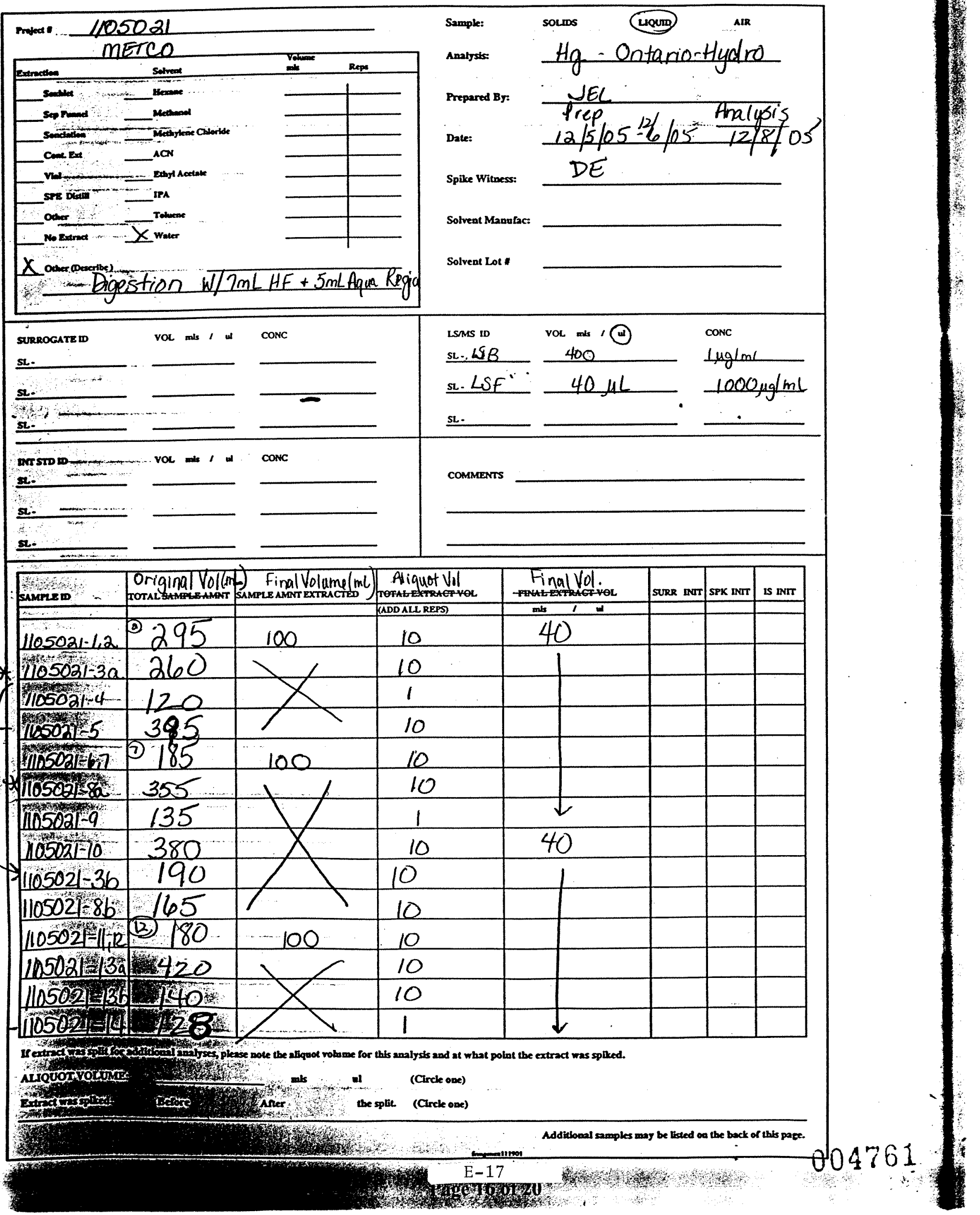




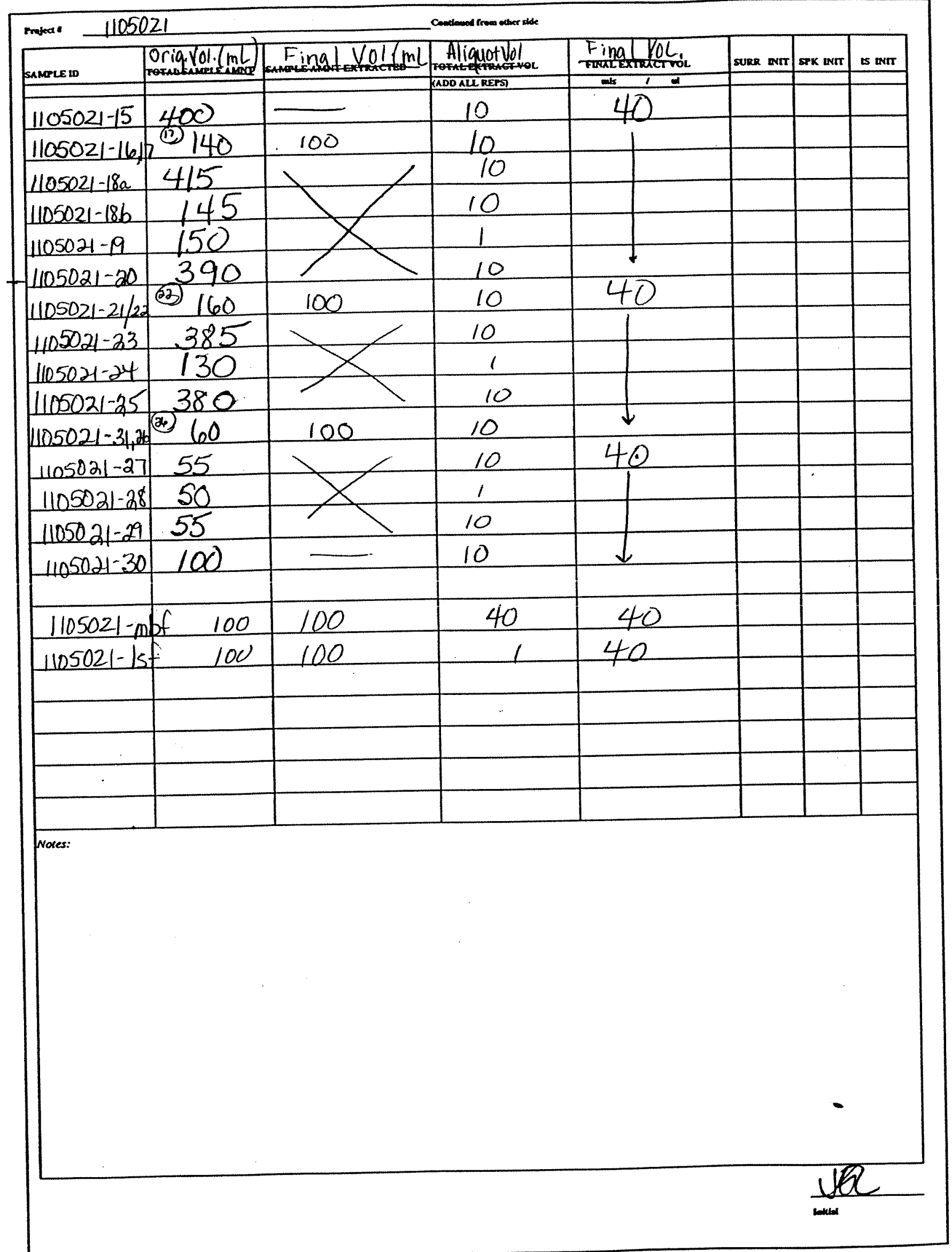




\section{Data Analysis Technologies}

Mercury by Ontario Hydro Method: Data Summary

Project Number:

Client:

Site:

Prep Date:

Analysis Date:
1105021

Metco Environmental

ADA-ES

$12 / 7 / 05$

$12 / 8 / 05$
Thimble Sample \#

$\begin{array}{lc}1105021-1 & 0.5291 \\ 1105021-6 & 0.584 \\ 1105021-11 & 0.5906 \\ 1105021-16 & 0.4958 \\ 1105021-21 & 0.5808 \\ 1105021-26 & 0.5952\end{array}$

Weight Digested (g)

0.5291

.5906

0.5952 


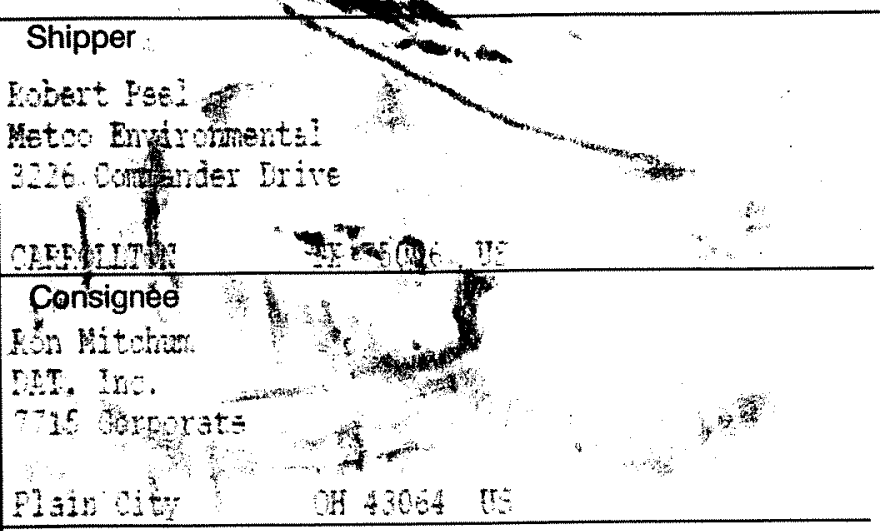

Two completed and signed copies of this Declaration must be handed to the operator

\section{TRANSPORT DETAILS}

This shipment is within the limitations prescribed for:

(delete non applicable)

PASSENGER CARGO

TAND CARGO AIRCRAFT

AIRCRAF, ONLY

Airport of Destination:
Air Waybill No.

Page of Pages

$$
5055720+95 \quad N Z A N H A
$$

Shipper's Reference Number

$$
\text { (optional) }
$$

NATURE AND QUANTITY OF DANGEROUS GOODS

UN Number or Identification Number, Proper Shipping name, Class or Division, Packing Group (if required), and all other required information

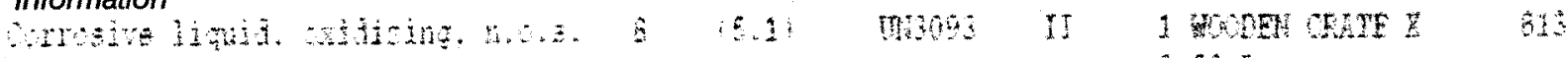

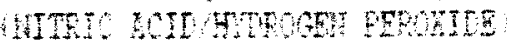

WARNING

Failure to comply with all respects with the applicable Dangerous Goods Regulations may be in breach of the applicable law, subject to legal penalties. This Declaration must not, in any circumstances, be completed and/or signed by a consolidator, a forwarder or an IATA cargo agent.

Shipment type: (delete,non applicable) $f^{4}$

Additional Handing Information

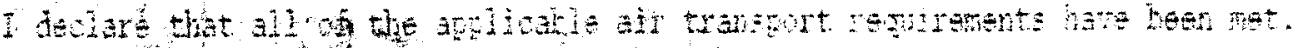

s

I hereby declare that the * accurately described abov by the proper shipping name, and are glassifjed, packaged, mark 19 and labelled/placarded, and are in all respects in proper condition for transport according to applicable interfational and National Governmental Regulations. I declare that all of the applicable air transport requirements have been met.

8002553924 th. Emergency Telephone Number
Name/Title of Signatory

PCEETT DEFI

Place and Date

TALIS

Signature

(see warning above)

FOR RADIOACTIVE MATERIIALOHIPWIENTI ACCEPTABLE FOR PASSENGER AIRCRAFT, THE SHIPMENT CONTAINS RADIOACTIVE MATERIAL INTENDED FOR USEIN DENT TO RESEARCH, MEDICAL DIAGNOSIS, OR TREATMENT.

\section{SHIFEE}

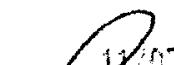

Rotert Fet 


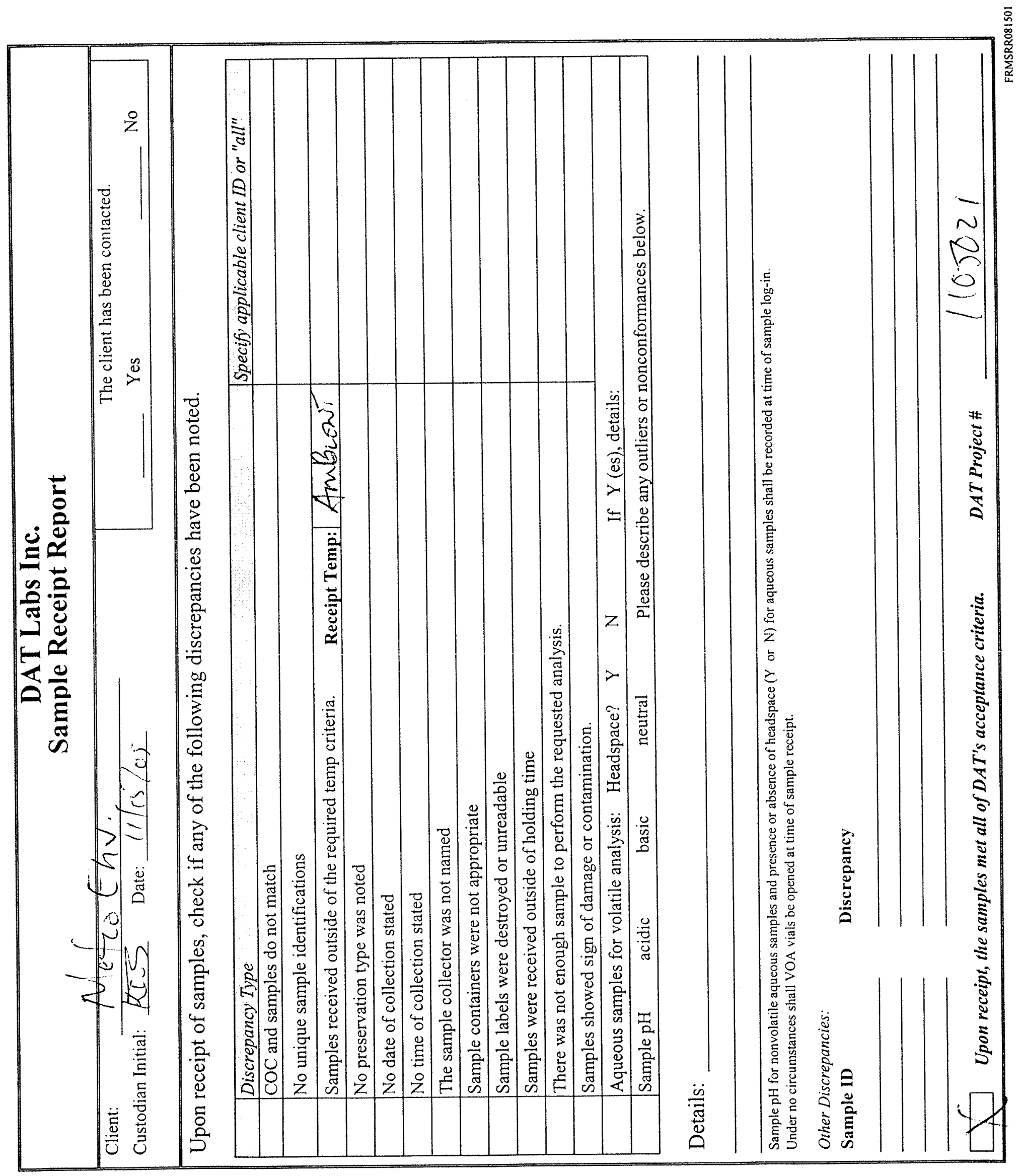




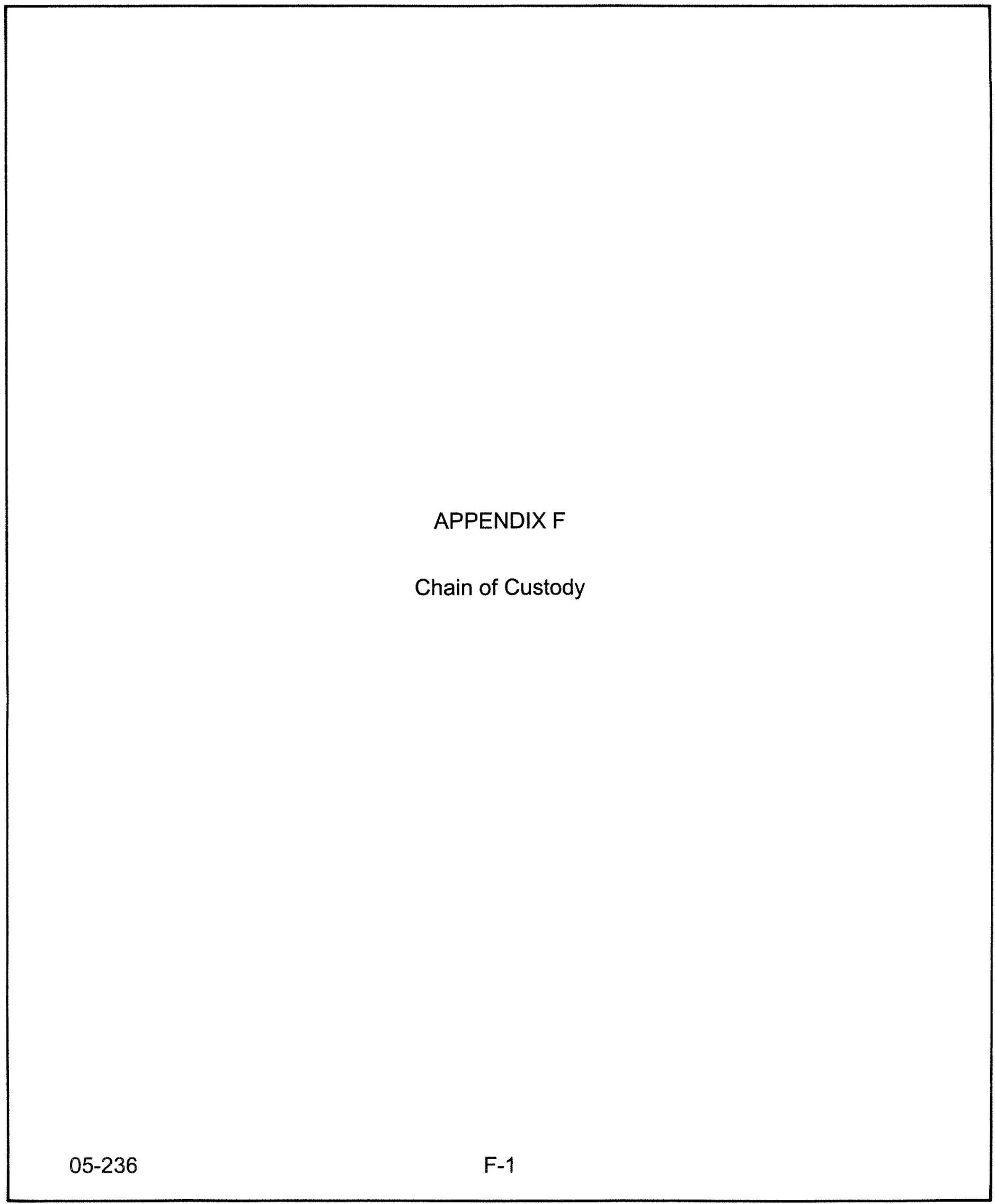




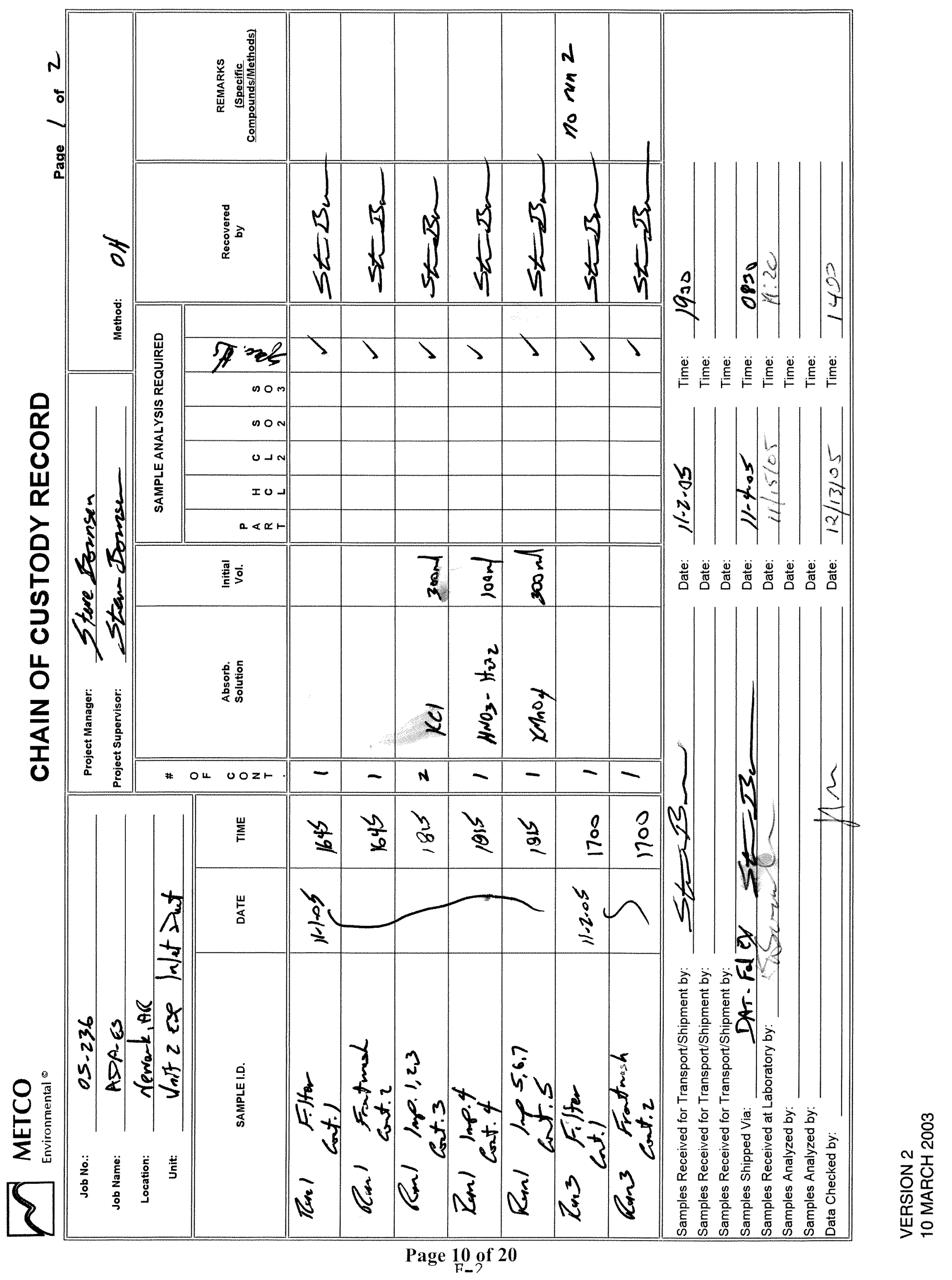




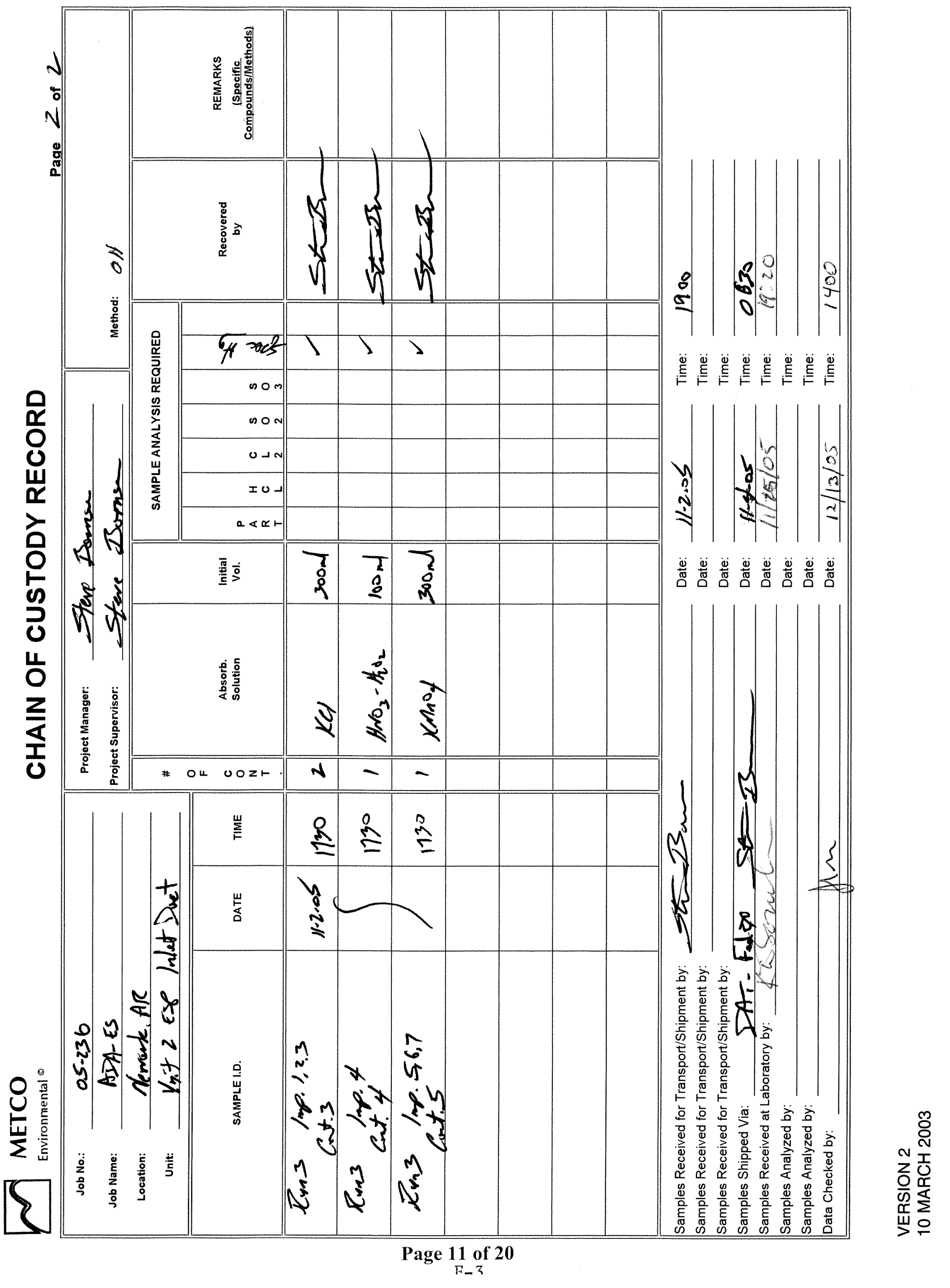






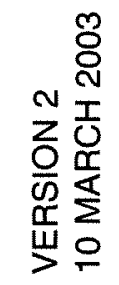




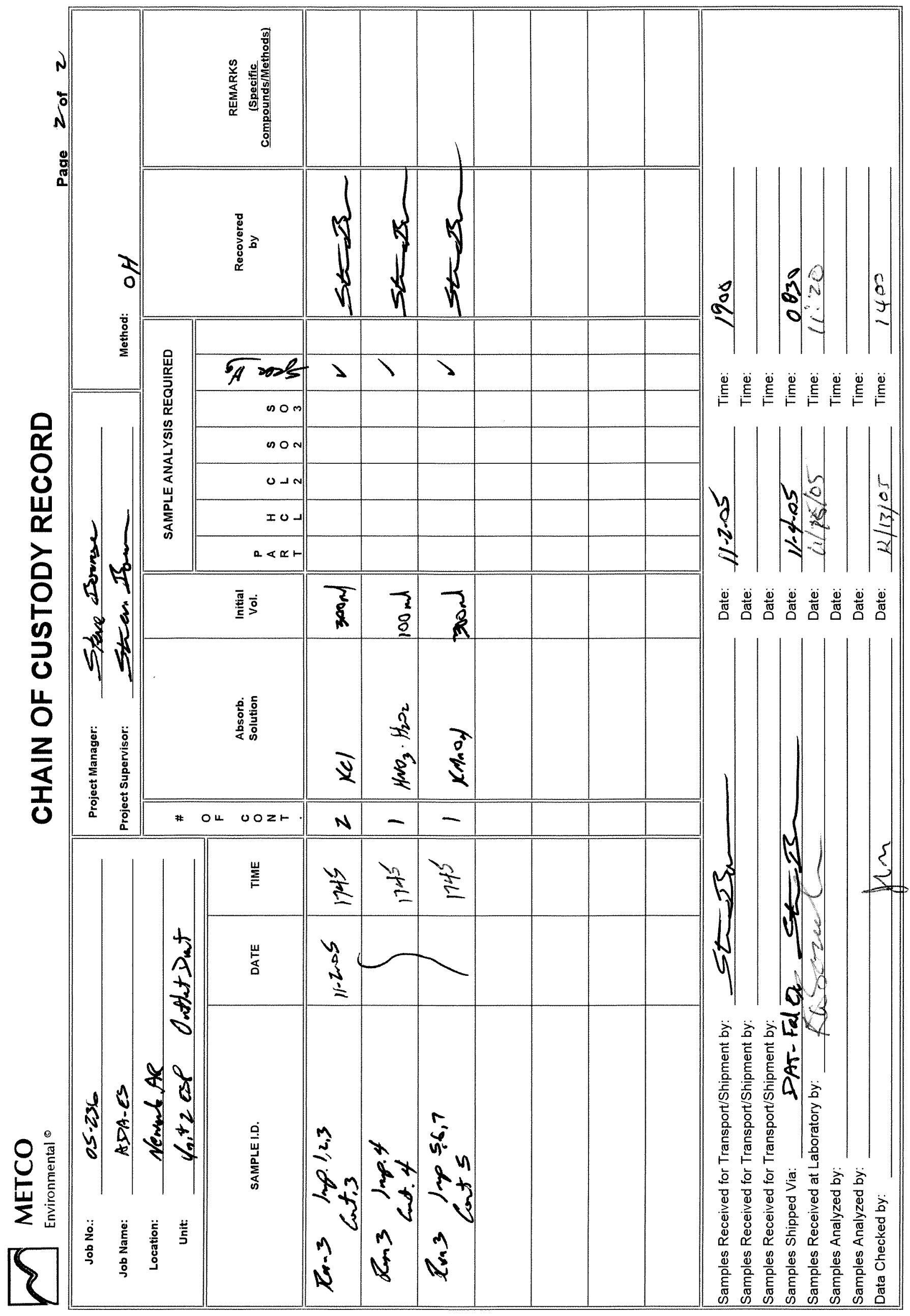

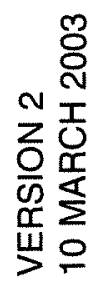




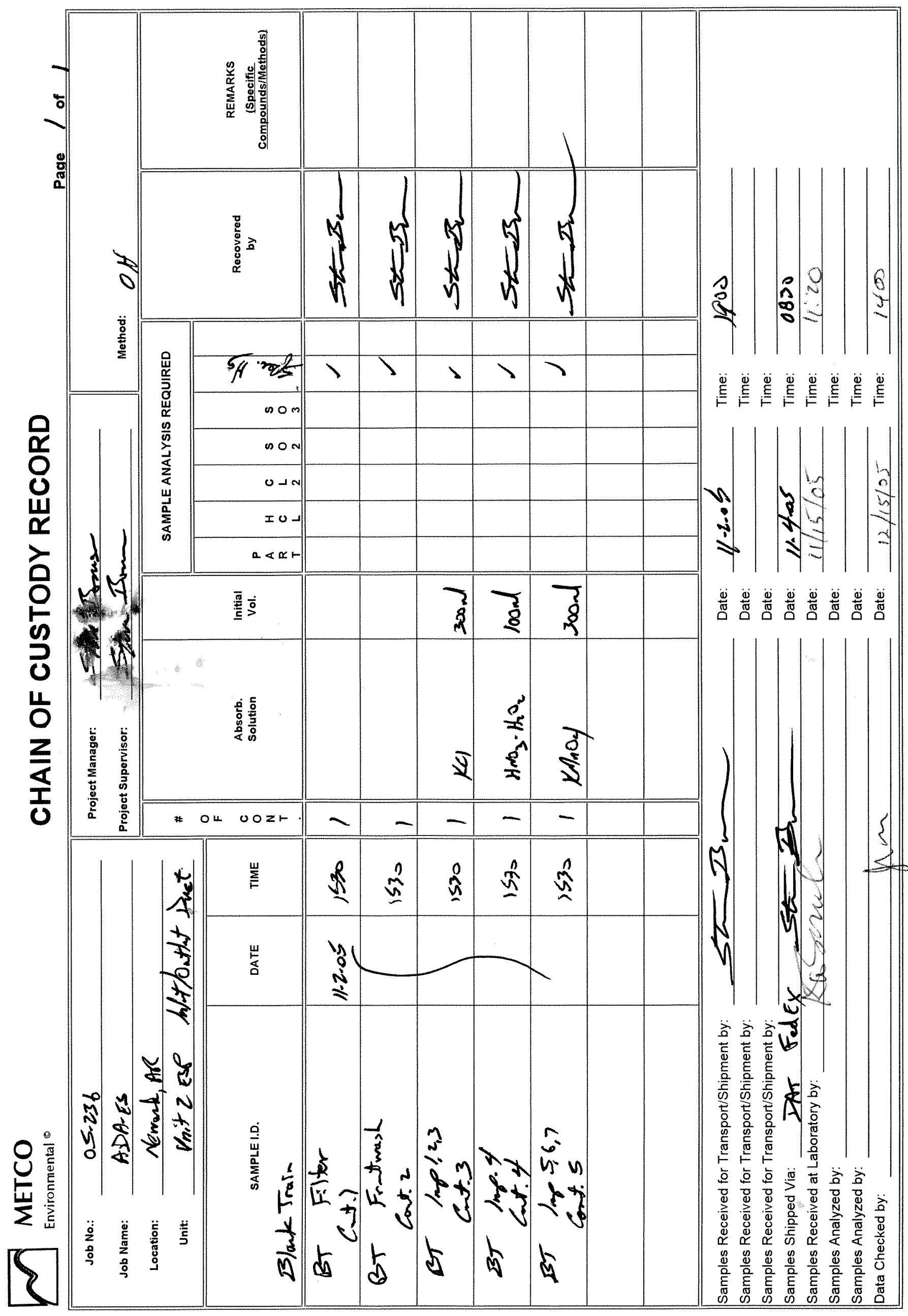




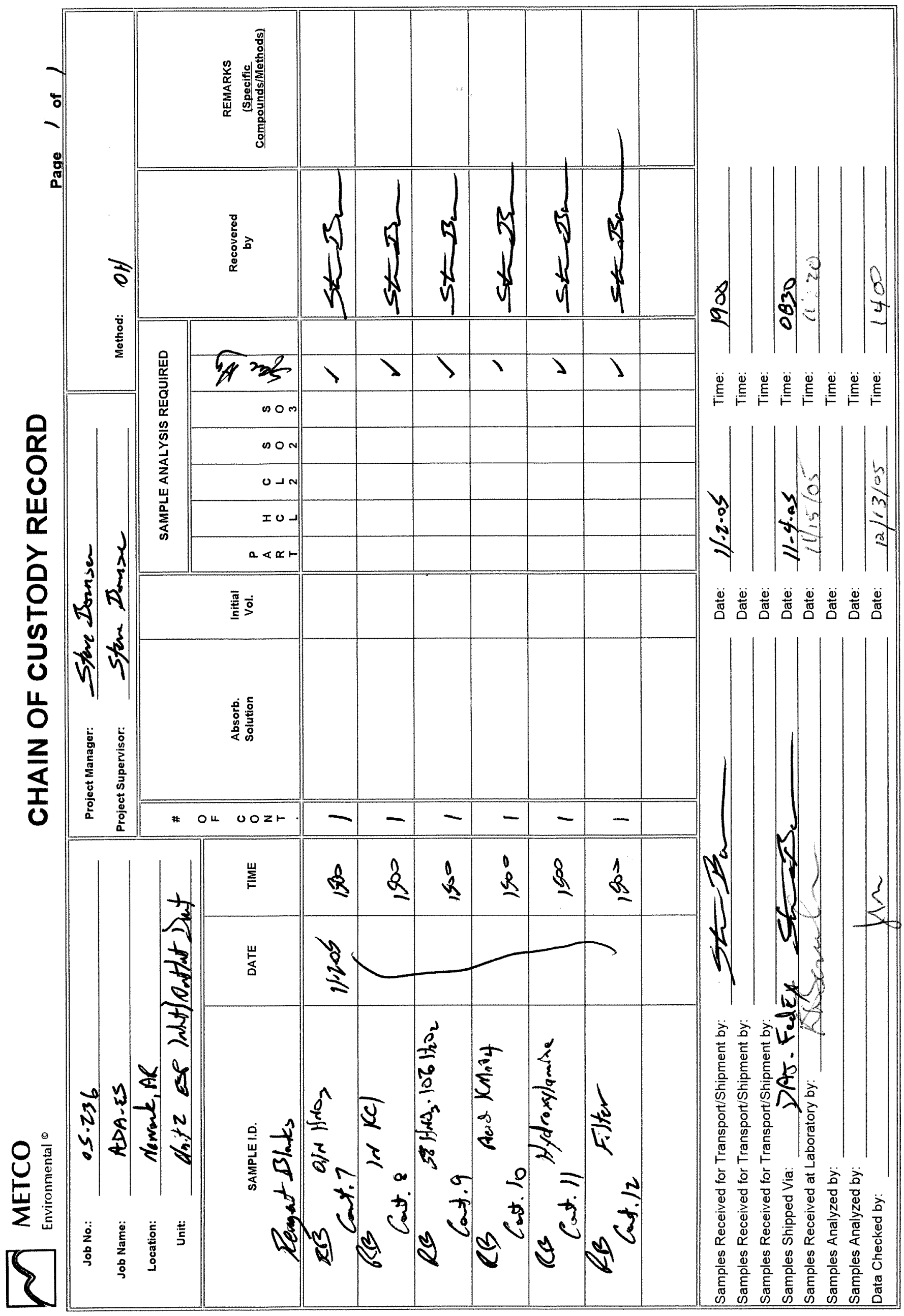

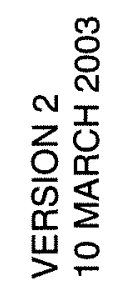




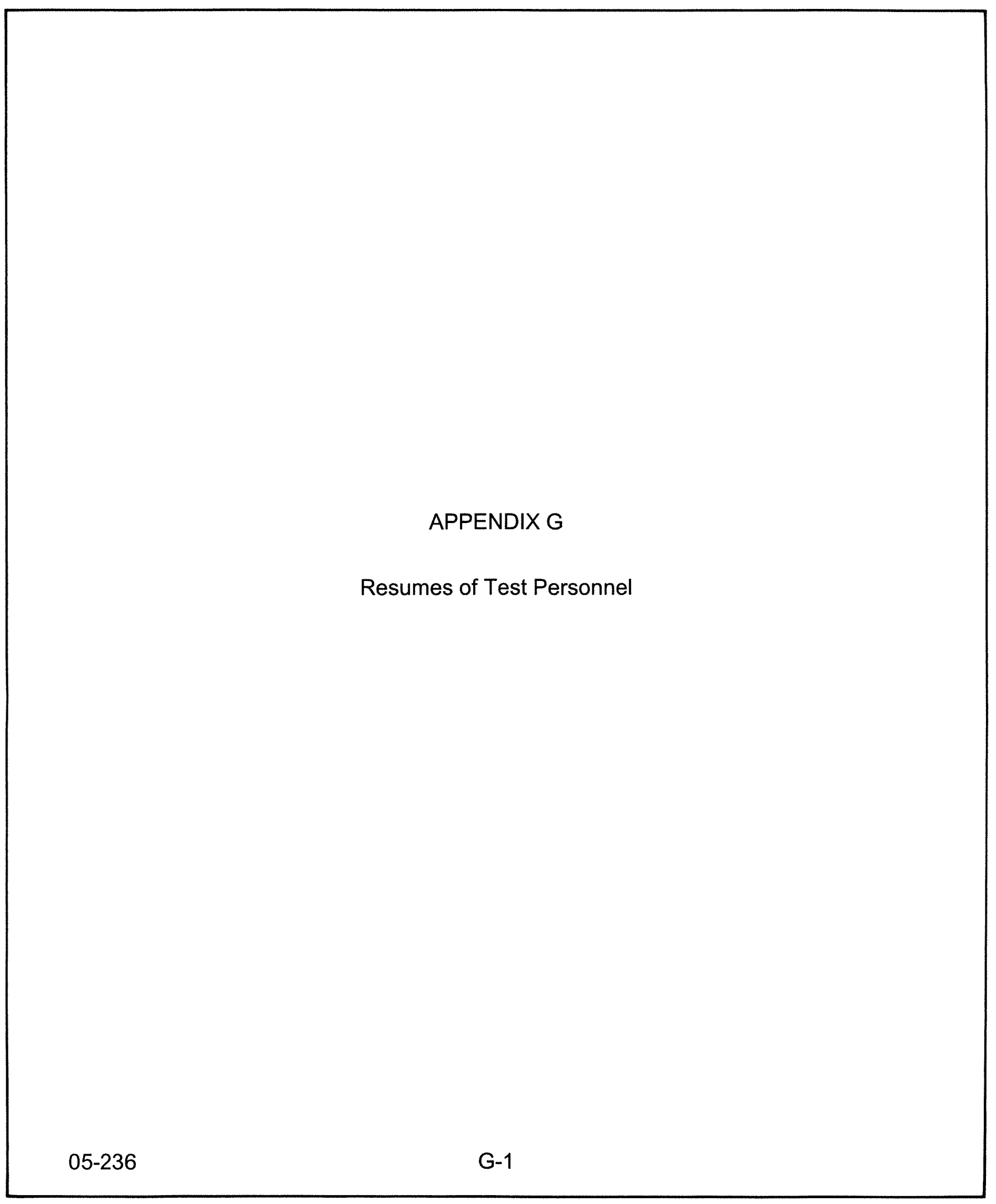


ROBERT M. PATTERSON; President

Education $\quad$ B. S. 1983, Central Michigan University; Mt. Pleasant, Michigan, in Geology and Earth Science-Meteorology.

Professional

Training

Courses

Attended a two-day short course, "Performing and Observing Source Sampling" in Dallas, Texas.

Attended a one-day short course on basic supervision.

Attended a four-week management course presented by the American Management Association, 1991-1992.

Certification

Certified Visible Emissions Evaluator

Certified Cabot Full-Face Respirator Fit Tester

Professional

Memberships

Technical

Experience
Source Evaluation Society

American Management Association

Participated in the sampling of over 1,000 sources, including several of which were sampled simultaneously using more than one sampling train. Thoroughly trained in all EPA testing procedures, 1986-present.

Over nineteen years experience with EPA and Texas Air Control Board methods of sampling - both stationary sources and ambient air. CFR, Title 40, Chapter I, Part 60, EPA Methods 1 through 25, and 101 through 110. Performance Specifications 1 through 5. CFR, Title 40, Chapter I, Part 50, Appendix A through F. "Sampling Procedures Manual, Texas Air Control Board, January 1983." Parts 1-1 through 14-6, Appendix B through Appendix M.

Experienced with sampling Method 0010, Modified Method 5 Sampling Train; Method 0030, Volatile Organic Sampling Train; and various EPA and "Site Specific" multiple metal and acid gas sampling trains.

(continued) 
PATTERSON, Rob (cont'd)

Technical

Experience

(cont'd)
Over twenty one years experience with EPA and Texas Air Control Board methods of analysis of both stationary and ambient air samples. Particulate matter, $\mathrm{SO}_{3}, \mathrm{SO}_{2}, \mathrm{H}_{2} \mathrm{SO}_{4}$, $\mathrm{NO}_{x}, \mathrm{CO}, \mathrm{CO}_{2}, \mathrm{O}_{2}, \mathrm{H}_{2} \mathrm{~S}, \mathrm{~F}, \mathrm{TRS}, \mathrm{HCl}, \mathrm{Cl}_{2}, \mathrm{NH}_{3}, \mathrm{VOC}, \mathrm{C}_{1}-\mathrm{C}_{7}$, and other organics. Both laboratory and on-site analyses were performed.

Experienced in the sampling and analysis of commercial calibration gas cylinders for sulfur dioxide, oxides of nitrogen, carbon dioxide, oxygen, carbon monoxide, and $\mathrm{C}_{1}-\mathrm{C}_{7}$ hydrocarbons.

Thoroughly trained in the operation and routine maintenance of the following:

- MSA LIRA Model 202S Infrared Analyzer

- Analytical Instrument Development, Inc. Model 340A Calibration System

- Shimadzu GC-Mini 2 Gas Chromatograph

- Thermo Environmental Model 10AR Oxides of Nitrogen Analyzer

- Thermo Oxygen Analyzer

- Teledyne Model 326 Oxygen Analyzer

- Thermo Environmental Model 48 Carbon Monoxide Analyzer

- Thermo Environmental Model 40 Sulfur Dioxide Analyzer

- Ratfisch Model RS 100 Total Hydrocarbon Analyzer

- Western Research Model 721AT Sulfur Dioxide Analyzer

- Horiba Model PIR 2000 Carbon Dioxide Analyzer

- Ratfisch Model RS 55 Total Hydrocarbon Analyzer

- J.U.M. Model VE-7 Total Hydrocarbon Analyzer 
JAMES R. MONFRIES; Senior Quality Assurance Manager

Education

B. S. 1975, University of Texas at Arlington; Arlington, Texas, in Biology with a minor in Chemistry.

Graduate work at the University of Texas at Dallas in the Environmental Science Department.

Professional

Training

Courses

Attended a two-day short course, "Performing and Observing Source Sampling" in Dallas, Texas, July 1976.

Certification Certified Visible Emissions Evaluator

Professional

Memberships

Technical Experience
Air and Waste Management Association Source Evaluation Society - Past President

Participated in the sampling of over 700 sources, serving in the supervisory capacity on over 500 sources. Many of the sources were sampled simultaneously using more than one sampling train at several points in the flue gas stream, 1976-present.

Has also supervised several ambient air monitoring studies, including a permanent five-station high volume air sampling network in South Texas, a permanent four-station high volume air sampling network in Pennsylvania, and a permanent sevenstation sulfur dioxide sampling network in East Texas.

Was Quality Assurance Manager for several ambient air monitoring studies; including a four-station high volume air sampling network for TSP and PM10 in Midlothian, Texas; a single-station high volume air sampling network for PM10 in South Texas; a two-station high volume air sampling network for TSP in Wichita Falls, Texas; and a four-station continuous air sampling network for TSP and PM10 in Jewett, Texas using Thermo Andersen FH 62 C14 Beta Gauge Dust Monitors. 
MONFRIES, James (cont'd)

Twenty years experience with EPA and Texas Commission on Environmental Quality methods of analysis of both source and ambient air samples for particulates, $\mathrm{SO}_{2}, \mathrm{SO}_{3}, \mathrm{H}_{2} \mathrm{SO}_{4}, \mathrm{H}_{2} \mathrm{~S}$, $\mathrm{HCl}, \mathrm{Cl}_{2}, \mathrm{NO}_{x}, \mathrm{Hydrocarbons}$, and TRS.

Experienced in the analysis of commercial calibration gas cylinders for sulfur dioxide and oxides of nitrogen.

Experienced with VOST and Modified Method 5 Sampling Procedures.

Thoroughly trained in the operation and routine maintenance of the following:

- Lear Siegler, Inc. SM800 Stack Gas Monitor

- Du Pont Model 460/1 Photometric Analyzer System

- Lear Siegler, Inc. SM1000 Ambient SO2 Monitor

- Calibrated Instruments Ultragas $\mathrm{SO}_{2}$ Monitor

- Meloy 285E $\mathrm{SO}_{2}$ Analyzer

- Meloy SA-700 Fluorescent $\mathrm{SO}_{2}$ Analyzer

- MSA LIRA Model 202S Infrared Analyzer

- Analytical Instrument Development, Inc. Model 340A Calibration System

- Shimadzu GC-Mini 2 Gas Chromatograph

- Thermo Environmental Model 10S NO Analyzer

- Thermo Oxygen Analyzer

- Teledyne Model 326 Oxygen Analyzer

- Thermo Environmental Model 48 Carbon Monoxide Analyzer

- Thermo Environmental Model 40 Sulfur Dioxide Analyzer

- Ratfisch Model RS 103 Total Hydrocarbon Analyzer

- Western Research Model 721AT Sulfur Dioxide Analyzer

- Horiba Model PIR 2000 Carbon Dioxide Analyzer

- Ratfisch Model RS 55 Total Hydrocarbon Analyzer

- J.U.M. Model VE-7 Total Hydrocarbon Analyzer

- Thermo Andersen Model FH 62 C14 Dust Monitor 
STEVEN J. BORNSEN; Utilities Associate

Education $\quad$ B. S. in Geology, 1995; University of Texas at Arlington; Arlington, Texas.

Professional

Training

Courses

Attended 40-hour Occupational \& Environmental Training Program on Hazardous Materials (CFR 1910.120); Dallas, Texas, January 1996.

Attended a two-day short course, "Performing and Observing Source Sampling," Dallas, Texas; September 1996.

Certifications Certified Visible Emissions Evaluator

HAZMAT certified

Adult CPR certified

Standard First Aid certified

Technical

Experience

Participated in the sampling of over 400 sources, including several of which were sampled simultaneously using more than one sampling train. Thoroughly trained in all EPA testing procedures, 1995-present.

Experienced with Modified Method 5 Sampling Train (Method 0010).

Experienced with VOST sampling procedure (Method 0030).

Experienced with calibration techniques for all field testing equipment.

Experienced with various multiple metal and acid gas sampling trains.

Experienced with particle size and $\mathrm{PM}_{10}$ sampling procedures.

(continued) 
BORNSEN, Steve (cont'd)

Technical

Experience

(cont'd)

Experienced with on-site gravimetric particulate matter analysis.

Has performed on-site $\mathrm{SO}_{2}$ analysis and $\mathrm{H}_{2} \mathrm{~S}$ fuel gas analysis.

Thoroughly trained in the operation and routine maintenance of the following:

- Thermo Environmental Model $10 \mathrm{~S}$ and $42 \mathrm{C}$ Oxides of Nitrogen Analyzers

- Teledyne Model 326 Oxygen Analyzer

- Western Research Model 721 AT Sulfur Dioxide Analyzer

- Horiba Model PIR 2000 Carbon Dioxide Analyzer

- J.U.M. Model VE-7 Total Hydrocarbon Analyzer

- Thermo Environmental Model 48 Carbon Monoxide Analyzer 
DUSTIN J. SIMPSON; Environmental Scientist II

Education $\quad$ B. S. in Environmental Science, August 2004; Stephen F. Austin State University, Nacogdoches, Texas.

Certifications Adult CPR certified

Standard First Aid certified

Technical Participated in the sampling of over 50 sources, including

Experience several of which were sampled simultaneously using more than one sampling train.

Thoroughly trained in all EPA testing procedures, 2004-present. 
STEVE TRAMMELL; Environmental Scientist II

Education $\quad$ B. S. Resource and Environmental, August 2003; Southwest Texas State University; San Marcos, Texas.

Certifications HAZMAT certified

Adult CPR certified

Standard First Aid certified

Technical

Experience

Participated in the sampling of over 50 sources, including several of which were sampled simultaneously using more than one sampling train.

Thoroughly trained in all EPA testing procedures, 2004-present. 
JOSHUA ORR; Environmental Scientist I

Education

Technical

Experience
B. S. Environmental Studies, Summer 2002; Southwest Texas State University; San Marcos, Texas.

Participated in the sampling of over 25 sources, including several of which were sampled simultaneously using more than one sampling train.

Thoroughly trained in all EPA testing procedures, 2005-present. 
DUSTIN DODSON; Environmental Scientist I

Education

B. S. Industrial Engineering, May 2005; Northwestern State University; Natchitoches, Louisiana.

Technical

Experience

Participated in the sampling of over 25 sources, including several of which were sampled simultaneously using more than one sampling train.

Thoroughly trained in all EPA testing procedures, 2005-present. 
APPENDIX D5: Source Testing-January 29-February 2, 2007 


\author{
STACK SAMPLING REPORT \\ FOR \\ PARTICULATE MATTER TESTING \\ ON THE
}

ENTERGY SERVICES, INC. INDEPENDENCE POWER PLANT UNIT NO. 2 STACK

NEWARK, ARKANSAS

PROJECT NO. 07-008

JANUARY AND FEBRUARY 2007

PREPARED FOR:

ADA-ES, INC.

8100 SOUTHPARK WAY

LITTLETON, CO 80120

PREPARED BY:

AIR SAMPLING ASSOCIATES, INC.

P.O. BOX 1175

LEWISVILLE, TEXAS 75067

(Total Number of Pages: 117 pages) 


\section{TABLE OF CONTENTS}

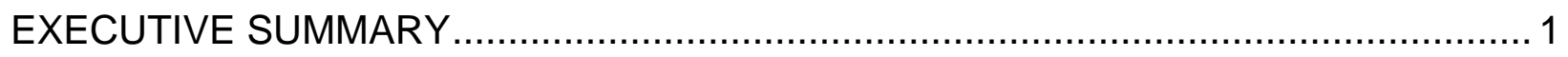

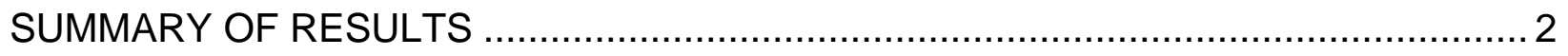

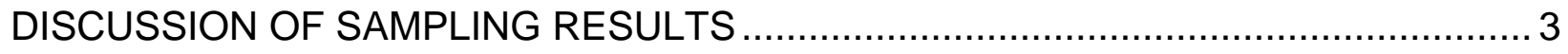

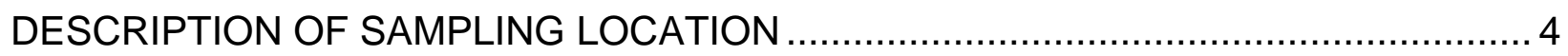

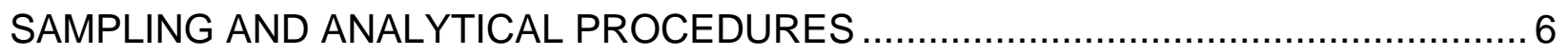

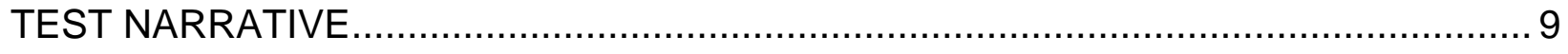

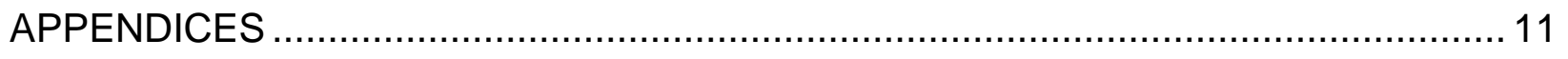

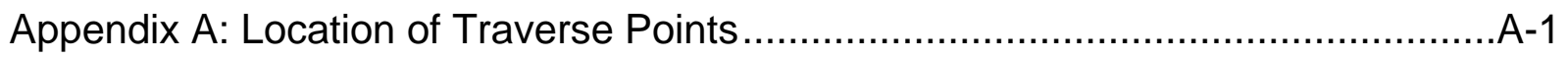

Appendix B: Nomenclature and Equations for Calculation of Source Emissions......B-1

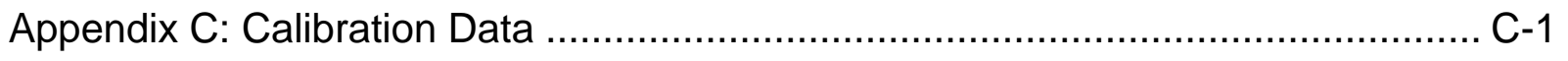

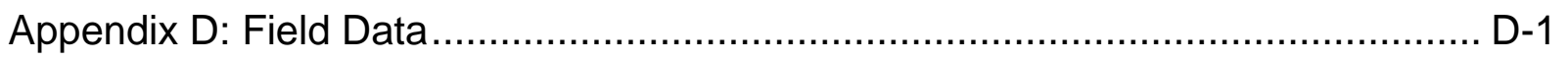

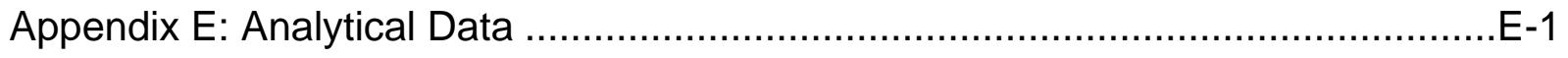

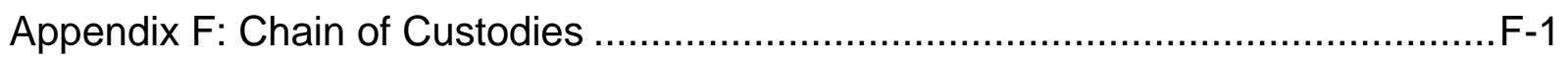

Appendix G: Resumes of Test Personnel .......................................................... G-1

\section{TABLES}

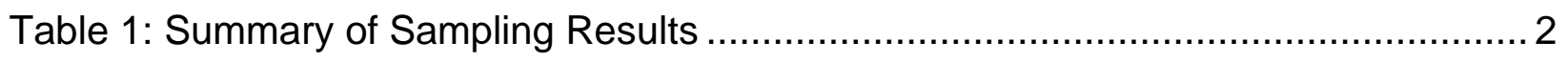

Table 2: Reference Method 5B and 202 Sampling Train.......................................... 7

Table 3: Location of Traverse Points Independence Unit No. 2 Stack ........................A-2

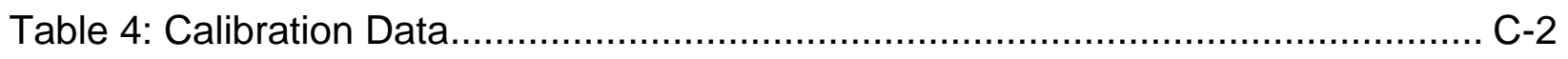

\section{FIGURES}

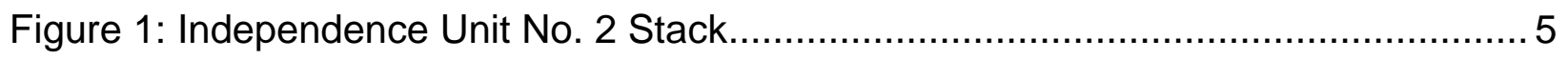

Figure 2: EPA Methods 1, 2, 3, 4, 5B, and 202 Sampling Train ............................... 8

Figure 3: Location of Traverse Points Independence Unit No. 2 Stack ......................A-3 


\section{II)}

\section{EXECUTIVE SUMMARY}

Air Sampling Associates, Inc. of Lewisville, Texas conducted testing on the Entergy Services, Inc., Independence Power Plant, located near Newark, Arkansas, for ADA$\mathrm{ES}$, Inc. The testing was performed to determine the amount of particulate matter being emitted to the atmosphere via the Unit No. 2 Stack. The testing was conducted on January 29 through February 2, 2007.

The sampling team consisted of Mr. Gary Goldman and Mr. Scot Jackson. Mr. Goldman was the test team leader.

The sampling followed the procedures set forth in Title 40 of the Code of Federal Regulations, Part 60 (40CFR60), Appendix A, Test Methods 1, 2, 3, 4, 5B, and 202.

The average emission rate of particulate matter for the five, six-hour tests from the Independence Unit No. 2 Stack was equal to $0.006 \mathrm{lbs} / \mathrm{mmBtu}$ - Front Half. The average emission rate of particulate matter for the five, six-hour tests from the Independence Unit No. 2 Stack was equal to $0.013 \mathrm{lbs} / \mathrm{mmBtu}$ - Total.

$\overline{\text { Billy J. Mullins, Jr. P.E., Q.E.P., D.E.E. }}$ 


\section{AlinMpLNG}

\section{SUMMARY OF RESULTS}

Table 1: Summary of Sampling Results

\begin{tabular}{|c|c|c|c|c|c|c|}
\hline Run No. & 1 & 2 & 3 & 4 & 5 & Average \\
\hline Test Date & $01 / 29 / 07$ & $01 / 30 / 07$ & $01 / 31 / 07$ & $02 / 01 / 07$ & $02 / 02 / 07$ & $\begin{array}{l}---- \\
\end{array}$ \\
\hline Test Time & 0830-1533 & 0645-1336 & $0642-1320$ & 0705-1342 & 0700-1336 & ----- \\
\hline Flow Rate - DSCFM & $2,072,263$ & $2,113,513$ & $2,128,865$ & $2,124,346$ & $2,149,896$ & $2,117,777$ \\
\hline Stack Temperature - ${ }^{\circ} \mathrm{F}$ & 315 & 310 & 309 & 313 & 319 & 313 \\
\hline $\mathrm{O}_{2}-\%$ Volume dry & 6.4 & 6.8 & 6.7 & 6.9 & 6.8 & 6.7 \\
\hline $\mathrm{CO}_{2}-\%$ Volume dry & 13.0 & 13.0 & 13.0 & 12.8 & 13.0 & 13.0 \\
\hline Percent Excess Air & 42.8 & 47.0 & 46.0 & 48.0 & 47.0 & 46.2 \\
\hline Moisture Content - \% & 10.02 & 9.73 & 9.64 & 10.53 & 10.07 & 10.00 \\
\hline Percent Isokinetic & 98.5 & 98.3 & 98.9 & 100.1 & 99.7 & 99.1 \\
\hline $\begin{array}{l}\text { Particulate Matter } \\
\text { - gr/dscf (Front Half) } \\
\text { - Ibs/mmBtu (Front Half)* } \\
\text { - Ibs/hr (Front Half) }\end{array}$ & $\begin{array}{c}0.0032 \\
0.006 \\
57.52\end{array}$ & $\begin{array}{c}0.0032 \\
0.007 \\
58.03\end{array}$ & $\begin{array}{c}0.0030 \\
0.006 \\
55.24\end{array}$ & $\begin{array}{c}0.0031 \\
0.006 \\
55.62\end{array}$ & $\begin{array}{c}0.0033 \\
0.007 \\
60.40\end{array}$ & $\begin{array}{c}0.0032 \\
0.006 \\
57.36\end{array}$ \\
\hline $\begin{array}{l}\text { Particulate Matter } \\
\text { - gr/dscf (Total) } \\
\text { - Ibs/mmBtu (Total)* } \\
\text { - Ibs/hr (Total) }\end{array}$ & $\begin{array}{c}0.0062 \\
0.012 \\
110.24\end{array}$ & $\begin{array}{c}0.0074 \\
0.015 \\
134.70\end{array}$ & $\begin{array}{c}0.0065 \\
0.013 \\
118.43\end{array}$ & $\begin{array}{c}0.0058 \\
0.012 \\
105.90\end{array}$ & $\begin{array}{c}0.0066 \\
0.014 \\
121.03\end{array}$ & $\begin{array}{c}0.0065 \\
0.013 \\
118.06\end{array}$ \\
\hline
\end{tabular}

* Calculated using an $F_{d}$ Factor of 9,780 


\section{II}

\section{DISCUSSION OF SAMPLING RESULTS}

The five tests for particulate matter appeared to be valid representations of the actual emissions during the tests. All leak checks performed on the sampling train and the pitot tubes indicated no leaks before or after each test. The indicative parameters calculated from the field data were in reasonable agreement. The measured moisture contents for the tests were within $5.3 \%$ of the mean value. The measured flow rates (DSCFM) for the tests were within $2.1 \%$ of the mean value. The rates of sampling for the tests were within the specified limits (90 to 110 percent isokinetic). The greatest deviation from 100 percent isokinetic was $1.7 \%$.

The calculated emissions (Ibs/mmBtu-Front Half) of particulate matter for the five tests indicated a range of $-6.3 \%$ to $+9.4 \%$ deviation from the mean value. 


\section{DESCRIPTION OF SAMPLING LOCATION}

The sampling ports on the Independence Unit No. 2 Stack are approximately 500 feet above the ground. The sampling ports are located 250 feet (9.69 stack diameters) downstream from the inlet to the stack and 501 feet (19.42 stack diameters) upstream from the outlet to the stack. 


\section{SAMPLING LOCATION}

Figure 1: Independence Unit No. 2 Stack

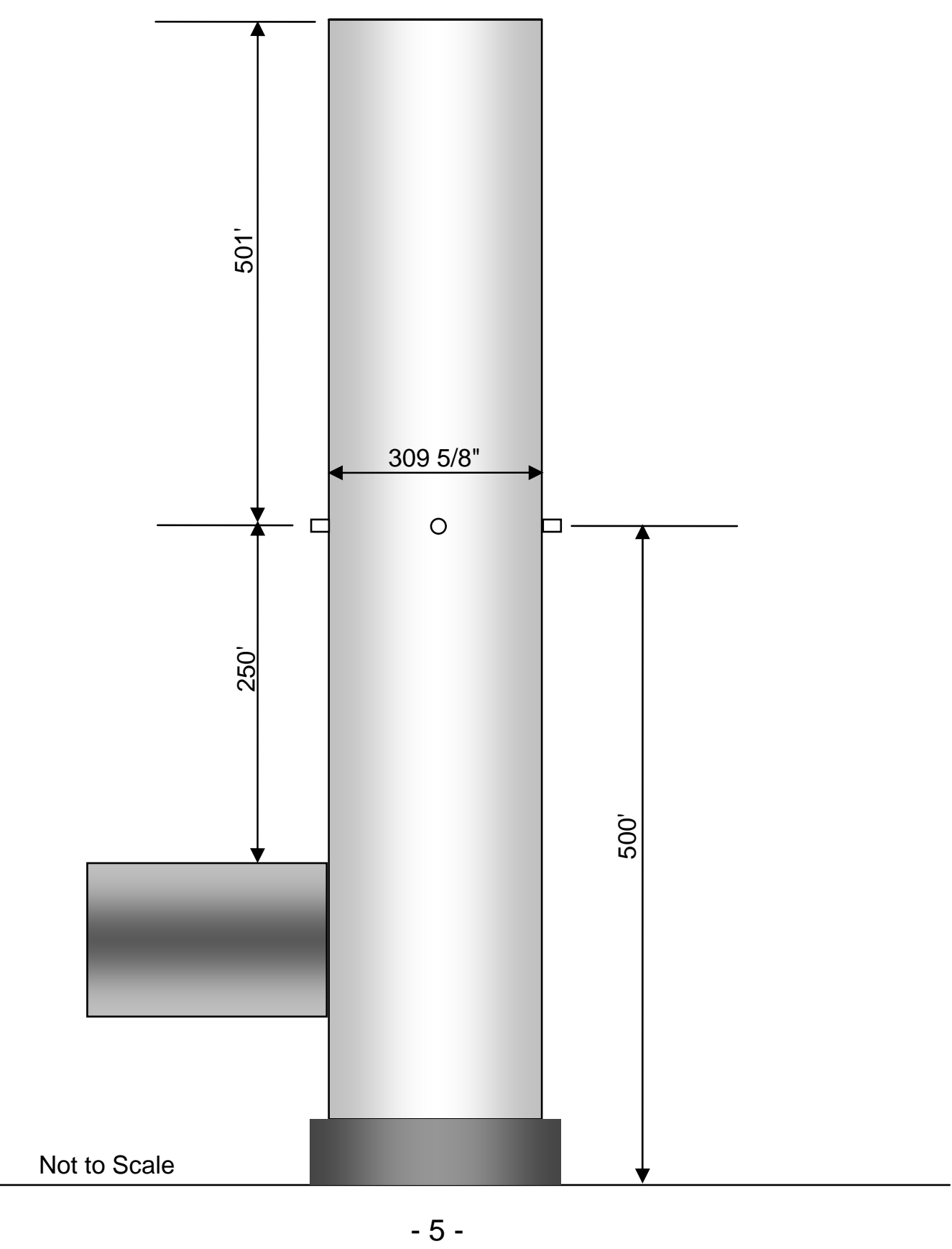




\section{SAMPLING AND ANALYTICAL PROCEDURES}

The sampling followed the procedures set forth in 40CFR60, Appendix A, Test Methods $1,2,3,4,5 B$, and 202.

Three traverse points were sampled from each of the four ports on the stack for a total of twelve traverse points. All traverse points were checked for cyclonic flow and none was found to be present. For each run, samples of five-minute duration were taken at each of the twelve traverse points during each 60 minute period of testing. Each traverse point was sampled for 30 minutes during the total sampling time of 360 minutes. Data was recorded at five-minute intervals.

The pitot tube lines were checked for leaks before and after each test under a vacuum and a pressure. The lines were also checked for clearance and the manometer was zeroed before each test.

The sampling train was leak checked at the end of the sampling probe at 15" of mercury vacuum before each test, and again at the conclusion of each test at the highest vacuum recorded during sampling. This was done to predetermine the possibility of a diluted sample.

The "front-half" of the sampling train contained the following components:

Stainless steel nozzle Heated glass lined probe@ $320^{\circ} \mathrm{F} \pm 25^{\circ} \mathrm{F}$

Heated glass fiber filter @ $320^{\circ} \mathrm{F} \pm 25^{\circ} \mathrm{F}$ 


\section{II}

The "back-half" of the sampling train contained the following components:

Table 2: Reference Method 5B and 202 Sampling Train

\begin{tabular}{|ccccc|}
\hline Impinger No. & $\begin{array}{c}\text { Impinger } \\
\text { Type }\end{array}$ & $\begin{array}{c}\text { Impinger } \\
\text { Contents }\end{array}$ & Amount & $\begin{array}{c}\text { Parameter } \\
\text { Collected }\end{array}$ \\
\hline $\mathbf{1}$ & Modified & H.P.L.C. $\mathrm{H}_{2} \mathrm{O}$ & $100 \mathrm{ml}$ & $\mathrm{H}_{2} \mathrm{O}$ \\
\hline $\mathbf{2}$ & Greenburg-Smith & H.P.L.C. $\mathrm{H}_{2} \mathrm{O}$ & $100 \mathrm{ml}$ & $\mathrm{H}_{2} \mathrm{O}$ \\
\hline $\mathbf{3}$ & Modified & Empty & ----- & $\mathrm{H}_{2} \mathrm{O}$ \\
\hline $\mathbf{4}$ & Modified & $6 \% \mathrm{H}_{2} \mathrm{O}_{2}$ & $200 \mathrm{ml}$ & $\mathrm{H}_{2} \mathrm{O}$ \\
\hline $\mathbf{5}$ & Modified & Silica Gel & $250 \mathrm{~g}$ & $\mathrm{H}_{2} \mathrm{O}$ \\
\hline
\end{tabular}

At the completion of each run, the "back-half" of the sampling train was purged with nitrogen for 60 minutes at a rate of 20 liters per minute. 
Figure 2: EPA Methods 1, 2, 3, 4, 5B, and 202 Sampling Train

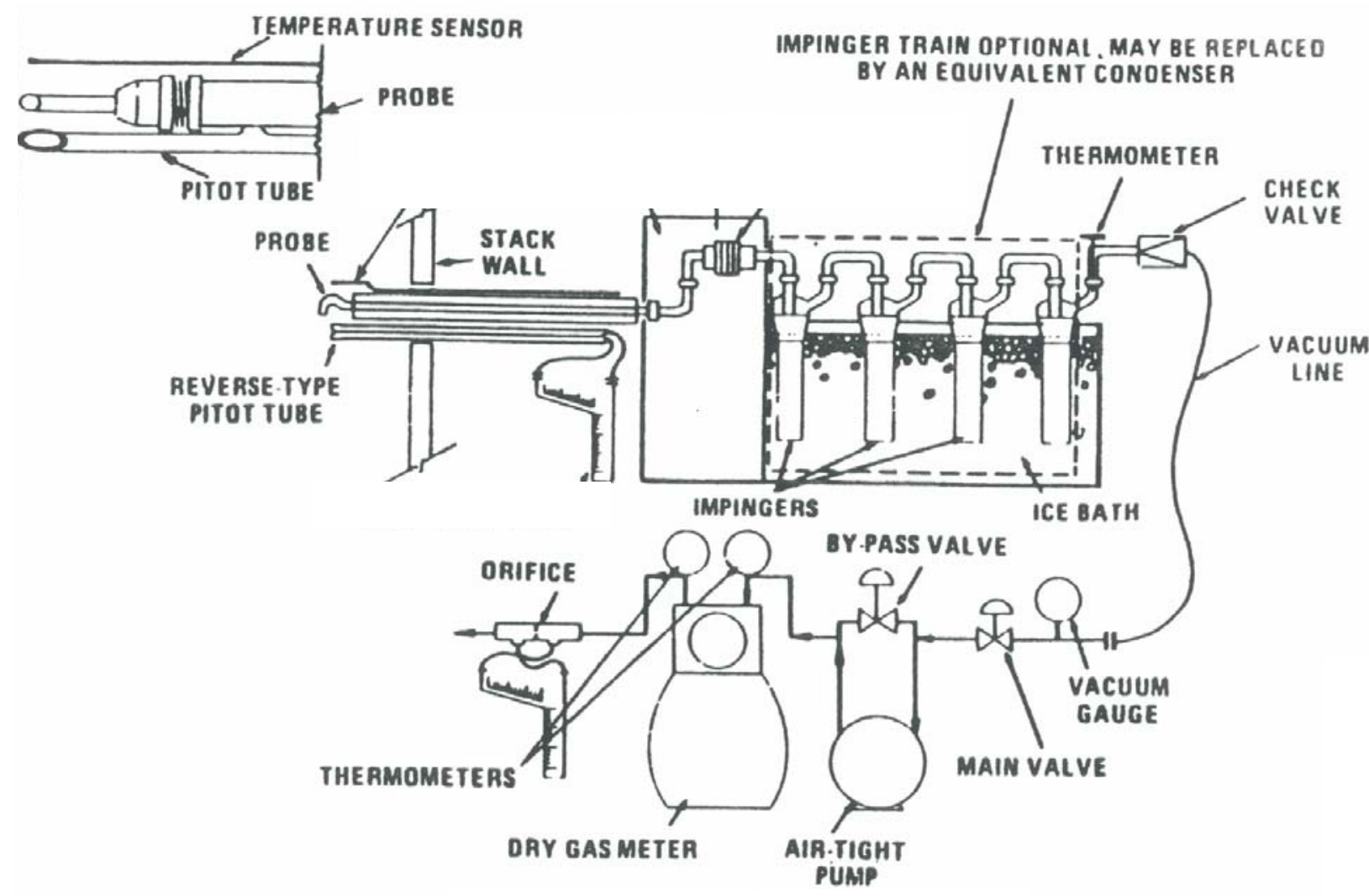




\section{SARMPLNG \\ ASSOCIATES, INC.}

\section{TEST NARRATIVE}

Personnel from Air Sampling Associates, Inc. arrived at the Entergy Services, Inc., Independence Power Plant, located near Newark, Arkansas, at 2:30 p.m. on Sunday, January 28, 2007. The sampling equipment was moved onto the Unit No. 2 Stack and the preliminary data was collected. Personnel departed the plant at 5:30 p.m.

On Monday, January 29, 2007, personnel returned to the plant at 7:00 a.m. The sampling equipment was prepared for testing. The first test for particulate matter began at 8:30 a.m. and was completed at 3:33 p.m. The samples were recovered and the equipment was secured for the night. Personnel departed the plant at 6:30 p.m.

On Tuesday, January 30, 2007, personnel returned to the plant at 6:00 a.m. The sampling equipment was prepared for testing. The second test for particulate matter began at 6:45 a.m. and was completed at 1:36 p.m. The samples were recovered and the equipment was secured for the night. Personnel departed the plant at 4:30 p.m.

On Wednesday, January 31, 2007, personnel returned to the plant at 6:00 a.m. The sampling equipment was prepared for testing. The third test for particulate matter began at 6:42 a.m. and was completed at 1:20 p.m. The samples were recovered and the equipment was secured for the night. Personnel departed the plant at 4:15 p.m.

On Thursday, February 1, 2007, personnel returned to the plant at 5:45 a.m. The sampling equipment was prepared for testing. The fourth test for particulate matter began at 7:05 a.m. and was completed at 1:42 p.m. The samples were recovered and the equipment was secured for the night. Personnel departed the plant at 4:00 p.m. 


\section{II)}

On Friday, February 2, 2007, personnel returned to the plant at 5:30 a.m. The sampling equipment was prepared for testing. The fifth test for particulate matter began at 7:00 a.m. and was completed at 1:36 p.m.

The samples were recovered and the equipment was secured. The data and samples were transported to the Air Sampling Associates, Inc. office in Lewisville, Texas for further review and analysis.

Operations at the Entergy Services, Inc., Independence Power Plant, Unit No. 2 Stack, located near Newark, Arkansas, for ADA-ES, Inc. were completed at 3:30 p.m. on Friday, February 2, 2007. 


\section{APPENDICES}

Appendix A: Location of Traverse Points

Appendix B: Nomenclature and Equations for Calculation of Source Emissions

Appendix C: Calibration Data

Appendix D: Field Data

Appendix E: Analytical Data

Appendix F: Chain of Custodies

Appendix G: Resumes of Test Personnel 
Appendix A:

Location of Traverse Points 


\section{Appendix A:}

\section{Location of Traverse Points Independence Unit No. 2 Stack}

The sampling ports are located 250 feet (9.69 stack diameters) downstream from the inlet to the stack and 501 feet (19.42 stack diameters) upstream from the outlet to the stack. The locations of the traverse points were calculated as follows:

Table 3: Location of Traverse Points Independence Unit No. 2 Stack

\begin{tabular}{|ccc|}
\hline \multicolumn{2}{|c|}{ Port \& Wall Thickness $=411 / 16$ inches } \\
\hline \multicolumn{3}{|l|}{ Inside Stack Diameter $=3095 / 8$ inches } \\
\hline $\begin{array}{c}\text { Point } \\
\text { Number }\end{array}$ & $\begin{array}{c}\text { Percent of } \\
\text { Stack Diameter }\end{array}$ & $\begin{array}{c}\text { Distance } \\
\text { from Wall }\end{array}$ \\
\hline 1 & 4.4 & $135 / 8 "$ \\
\hline 2 & 14.6 & $453 / 16 "$ \\
\hline 3 & 29.6 & $915 / 8 "$ \\
\hline
\end{tabular}

* Calculated as $1 / 2$ of a six point traverse. 


\section{Appendix A:}

Figure 3: Location of Traverse Points Independence Unit No. 2 Stack

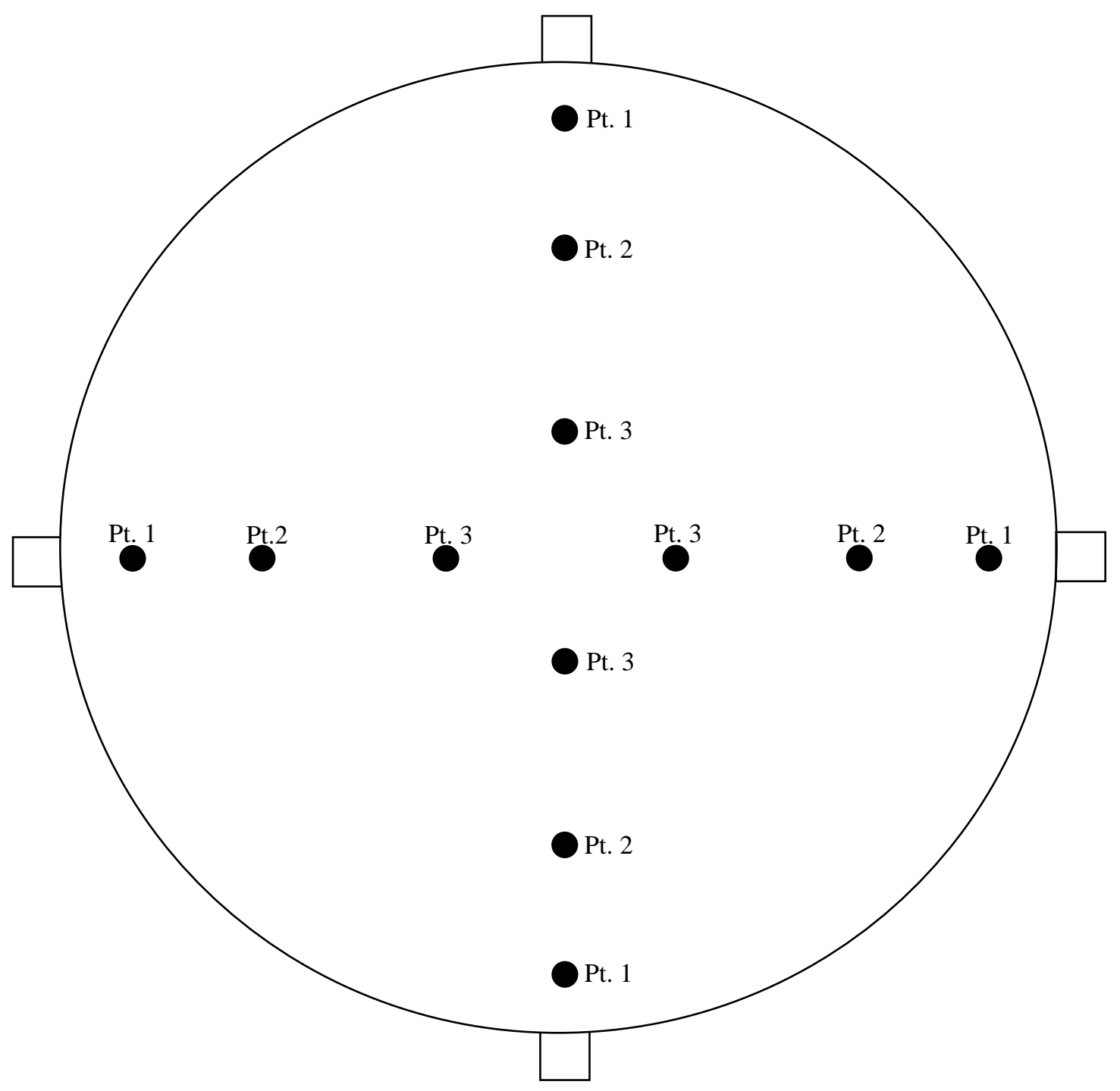

Not to scale. 
Appendix B:

Nomenclature and Equations for Calculation of Source Emissions 


\section{Nomenclature For Flow Rate and Moisture Calculations}

Symbol

$A_{s}$

$\mathrm{C}_{\text {an }}$

$\mathrm{C}_{\mathrm{ao}}$

$\mathrm{C}_{\text {at }}$

$\mathrm{C}_{\mathrm{au}}$

$\mathrm{C}_{\mathrm{aw}}$

$\mathrm{C}_{\mathrm{ax}}$

$C_{p}$

$D_{n}$

$\%$ EA

g

$\% 1$

$\% \mathrm{M}$

$M_{d}$

$\mathrm{m}_{\mathrm{f}}$

$\mathrm{mt}$

$\mathrm{M}_{\text {water }}$

MW

$\mathrm{MW}_{\text {air }}$
English

Units

in. ${ }^{2}$

$\mathrm{gr} / \mathrm{dscf}^{*}$

gr/dscf*

gr/CF @

stack conditions

gr/CF @ stack conditions

$\mathrm{lbs} / \mathrm{hr}$

$\mathrm{lbs} / \mathrm{hr}$

in.

$32.2 \mathrm{ft} / \mathrm{sec}^{2}$

mg

$\mathrm{mg}$

$18 \mathrm{lb} / \mathrm{lb}-$ mole

lb/lb-mole

$28.84 \mathrm{lb} / \mathrm{lb}-\mathrm{mole}$
Metric

$\underline{\text { Units }}$

$\mathrm{m}^{2}$

$\mathrm{g} / \mathrm{dscm}^{*}$

$\mathrm{g} / \mathrm{dscm}$ *

$\mathrm{g} / \mathrm{m} 3$

$\mathrm{g} / \mathrm{m} 3$

$\mathrm{kg} / \mathrm{hr}$

$\mathrm{kg} / \mathrm{hr}$

m

m

Pitot Tube Calibration Factor

Sampling Nozzle Diameter

Percent Excess Air at Sampling Point

Acceleration of gravity

Percent Isokinetic

Percent Moisture in the Stack Gas by Volume

Mole Fraction of Dry Gas

mg

mg

g/g-mole

Particulate - probe, cyclone, and filter

Particulate - total

Molecular Weight of Water

Molecular Weight of Stack

Gas

Molecular Weight of Air 
Symbol

$\mathrm{MW}_{\mathrm{d}}$

$\mathrm{P}_{\mathrm{b}}$

$\mathrm{P}_{\mathrm{m}}$

Ps

$\Delta \mathrm{P}$

$\mathrm{P}_{\text {std }}$

$\mathrm{Q}_{\mathrm{a}}$

$\mathrm{Q}_{\mathrm{s}}$

R

$T_{m}$

$T_{t}$

$\mathrm{T}_{\mathrm{s}}$

$\mathrm{T}_{\text {std }}$

$\mathrm{V}_{\mathrm{m}}$

$\mathrm{Vm}_{\text {std }}$

$\mathrm{V}_{\mathrm{s}}$

$V_{w}$

$V w_{\text {gas }}$

$\rho_{\text {air }}$
English

Units

lb/lb-mole

"Hg Absolute

$" \mathrm{H}_{2} \mathrm{O}$

"Hg Absolute

$" \mathrm{H}_{2} \mathrm{O}$

29.92" Hg

ACFM

DSCFM* $^{*}$

21.83" Hg-

$\mathrm{ft}^{3} / \mathrm{lb}$-mole ${ }^{\circ} \mathrm{R}$

${ }^{\circ} \mathrm{F}$

$\min$

${ }^{\circ} \mathrm{F}$

$528^{\circ} \mathrm{R}$

$\mathrm{ft}^{3}$

dscf*

fpm

$\mathrm{ml}$

scf*

$0.0748 \mathrm{lbs} / \mathrm{ft}^{3}$
Metric

Units

g/g-mole

$\mathrm{mm} \mathrm{Hg}$

$\mathrm{mm} \mathrm{H}_{2} \mathrm{O}$

$\mathrm{mm} \mathrm{Hg}$

$\mathrm{mm} \mathrm{H}_{2} \mathrm{O}$

760 mm Hg

$\mathrm{m}^{3} / \mathrm{hr}$

$\mathrm{dscm} / \mathrm{hr}$ *

${ }^{\circ} \mathrm{C}$

$\min$

${ }^{\circ} \mathrm{C}$

$293^{\circ} \mathrm{K}$

$\mathrm{m}^{3}$

dscm*

$\mathrm{m} / \mathrm{sec}$

$\mathrm{ml}$

$\mathrm{scm}^{\star}$
Description

Molecular Weight of Dry Stack Gas

Barometric Pressure

Orifice Pressure drop

Stack Pressure

Velocity Head of Stack Gas

Standard Barometric Pressure

Stack Gas Volume at Actual

Stack Conditions

Stack Gas Volume at 29.92"

$\mathrm{Hg}, 528^{\circ} \mathrm{R}$, dry

Universal Gas Constant

Average Gas Meter

Temperature

Net Time of Test

Stack Temperature

Standard Temperature

Volume of Dry Gas Sampled @ Meter Conditions

Volume of Dry Gas Sampled

@ Standard Conditions

Stack Velocity @ Stack Conditions

Total Water Collected in Impingers and Silica Gel

Volume of Water Vapor Collected @ Standard Conditions

Density of Air 
Symbol

$$
\begin{gathered}
\rho_{\text {water }} \\
\rho_{\text {man }}
\end{gathered}
$$

$$
\text { English }
$$

$\underline{\text { Units }}$

$1 \mathrm{~g} / \mathrm{ml}$

$62.32 \mathrm{lbs} / \mathrm{ft}^{3}$

\author{
Metric \\ $\underline{\text { Units }}$
}

Description

Density of Water

Density of Manometer Oil

(Inches of Water)

Standard Conditions: 29.92" Hg, 68 F (760 mm Hg, $\left.20^{\circ} \mathrm{C}\right)$ 


\section{Example Calculations}

1. Volume of dry gas sampled at standard conditions. *

$$
\begin{aligned}
& V_{m_{s t d}}=V_{m}\left(\frac{T_{s t d}}{T_{m}+460}\right)\left[\frac{P_{b}+\frac{P_{m}}{13.6}}{P_{s t d}}\right] \\
& V_{m s d}=17.65 V_{m}\left[\frac{P_{b}+\frac{P_{m}}{13.6}}{T_{m}+460}\right]=d s c f \\
& V_{m s t d}=d s c f \times 0.028317=d s c m
\end{aligned}
$$

2. Volume of water vapor collected at standard conditions. *

$$
\begin{aligned}
& V_{\text {was }}=\frac{\left(V_{w}-g m s \mathrm{SO}_{2}-g m s H_{2} S\right) \rho_{\text {water }} R T_{\text {std }}}{P_{\text {std }} M_{\text {water }} 453.6} \\
& V_{\text {was }}=0.0472\left(V_{w}-g m s \mathrm{SO}_{2}-g m s H_{2} S\right)=s c f \\
& V_{w_{\text {gas }}}=s c f \times 0.028317=\mathrm{scm}
\end{aligned}
$$

3. Percent moisture in stack gas.

$$
\% M=\frac{V_{\text {was }}}{V_{m_{\text {std }}}+V_{w_{\text {gas }}}} \times 100=\%
$$

* 29.92" Hg, $68^{\circ} \mathrm{F}\left(760 \mathrm{~mm} \mathrm{Hg}, 20^{\circ} \mathrm{C}\right)$ 
4. Mole fraction of dry gas.

$$
M_{d}=\frac{100-\% M}{100}
$$

5. Average molecular weight of dry stack gas.

$$
\begin{aligned}
M W_{d}=\left[\% \mathrm{CO}_{2} \times \frac{44}{100}\right]+\left[\% \mathrm{O}_{2} \times \frac{32}{100}\right]+\left[\% \mathrm{~N}_{2} \times \frac{28}{100}\right]+\left[\% \mathrm{CO} \times \frac{28}{100}\right] & =\mathrm{lb} / \mathrm{lb}-\text { mole } \\
& =g / \mathrm{g}-\text { mole }
\end{aligned}
$$

6. Molecular weight of stack gas.

$$
M W=M W_{d} \times M_{d}+18\left(1-M_{d}\right)=\frac{l b}{l b-\text { mole }}=g / g-\text { mole }
$$

7. Percent excess air at sampling point.

$$
\% E A=\frac{100\left[\% \mathrm{O}_{2}-(0.5 \% \mathrm{CO})\right]}{0.265\left(\% \mathrm{~N}_{2}\right)-\left[\% \mathrm{O}_{2}-(0.5 \% \mathrm{CO})\right]}
$$

8. Stack Pressure.

$$
P_{s}=P_{b}+\frac{\text { Stack Pressure " } \mathrm{H}_{2} \mathrm{O}}{13.6}=" \mathrm{Hg} \text { Absolute }
$$

$$
P_{s}=" H g \text { Abs. } \times 25.4=m m H g
$$

9. Stack velocity at stack conditions.

$$
\begin{aligned}
& V_{s}=C_{p} 60\left[\frac{2 g \times \rho_{\text {man }} \times P_{\text {std }} \times M_{\text {air }} \times\left(T_{s}+460\right) \times \Delta P}{12 \times \rho_{\text {air }} \times P_{s} \times M W \times T_{\text {std }}}\right]^{1 / 2} \\
& V_{s}=5,123.8 C_{p}\left[\frac{\left(T_{s}+460\right)}{P_{s} \times M W}\right]^{1 / 2} \sqrt{\Delta P} \text { average }=f p m \\
& V_{s}=f p m \times 0.00508=\mathrm{m} / \mathrm{sec}
\end{aligned}
$$


10. Dry stack gas volume at standard conditions. *

$$
\begin{aligned}
& Q_{s}=\frac{1}{144} V_{s} \times A_{s} \times M_{d} \times \frac{T_{s t d}}{T_{s}+460} \times \frac{P_{s}}{P_{s t d}} \\
& Q_{s}=\frac{0.123 V_{s} \times A_{s} \times M_{d} \times P_{s}}{T_{s}+460}=D S C F M \\
& Q_{s}=D S C F M \times 1.6990=d s c m / h r
\end{aligned}
$$

11. Actual stack gas volume at stack conditions.

$$
\begin{aligned}
& Q_{a}=\frac{V_{s} \times A_{s}}{144}=A C F M \\
& Q_{a}=A C F M \times 1.6990=\mathrm{m}^{3} / \mathrm{hr}
\end{aligned}
$$

12. Percent Isokinetic

$$
\begin{aligned}
& \% l=\frac{V_{m_{s t d}} \times\left(T_{s}+460\right) \times P_{s t d} \times 100 \times 144 i .^{2} / f^{2}}{M_{d} \times T_{s t d} \times P_{s} \times T_{t} \times V_{s}\left(\frac{\Pi \times D_{n}{ }^{2}}{4}\right)} \\
& \% l=\frac{1039 \times V_{\text {std }} \times\left(T_{s}+460\right)}{M_{d} \times P_{s} \times T_{t} \times V_{s} \times D_{n}{ }^{2}}
\end{aligned}
$$

*29.92" Hg, $68^{\circ} \mathrm{F}\left(760 \mathrm{~mm} \mathrm{Hg}, 20^{\circ} \mathrm{C}\right)$ 
13. Particulate - Probe, cyclone, and filter.

$$
\begin{aligned}
& C_{\text {an }}=\frac{\mathrm{m}_{\mathrm{f}}}{\mathrm{V}_{\mathrm{mstd}}} \times \frac{1 \mathrm{gr}}{64.8 \mathrm{mg}} \\
& C_{a n}=0.0154 \times \frac{\mathrm{mf}}{\mathrm{V}_{\mathrm{mstd}}} \mathrm{gr} / \mathrm{dscf} \text { * } \\
& C_{\text {an }}=\mathrm{gr} / \mathrm{dscf} \times 2.290=\mathrm{g} / \mathrm{dscm} \text { * }
\end{aligned}
$$

14. Particulate total.

$$
\begin{aligned}
& \mathrm{C}_{\mathrm{ao}}=0.0154 \times \frac{\mathrm{m}_{\mathrm{t}}}{\mathrm{V}_{\mathrm{msd}}}=\mathrm{gr} / \mathrm{dscf} \text { * } \\
& \mathrm{C}_{\mathrm{ao}}=\mathrm{gr} / \mathrm{dscf} \times 2.290=\mathrm{g} / \mathrm{dscm} \text { * }
\end{aligned}
$$

15. Particulate - probe, cyclone, and filter at stack conditions.

$$
\begin{aligned}
& C_{a t}=C_{a n} \times \frac{P_{s}}{P_{s t d}} \times \frac{\left(T_{s t d}\right)}{\left(T_{s}+460\right)} \times M_{d} \\
& C_{a t}=\frac{17.65 \times C_{a n} \times P s \times M d}{T x+460}=g r / C F \\
& C_{a t}=g r / C F \times 2.290=g / m^{3}
\end{aligned}
$$

16. Particulate - total, at stack conditions.

$$
\begin{aligned}
& C_{a u}=\frac{17.65 \times C_{a o} \times P_{s} \times M_{d}}{T_{s}+460}=g r / C F \\
& C_{a u}=g r / C F \times 2.290=g / \mathrm{m}^{3}
\end{aligned}
$$

* 29.92" Hg, $68^{\circ} \mathrm{F}\left(760 \mathrm{~mm} \mathrm{Hg}, 20^{\circ} \mathrm{C}\right)$ 


\title{
Emission Rate Calculations
}

$$
\mathrm{lbs} / \mathrm{mmBtu}=\frac{\frac{\mathrm{gr} / \mathrm{dscf}}{7,000} \times F_{\mathrm{d}} \times 20.9}{\left(20.9-\mathrm{O}_{2} \%\right)}
$$

$$
F_{d}=\text { Oxygen based } F \text { factor }
$$

$\frac{\text { Fuel }}{\text { Coal }}$

\author{
E factor \\ $9,780 \mathrm{dscf} / \mathrm{mmBtu}$
}


SOURCE EMISSION SURVEY

$\begin{aligned} \text { JOB NUMBER: } & 07-008 \\ \text { JOB NAME: } & \text { ADA-ES } \\ \text { LOCATION: } & \text { Newark/AR } \\ \text { UNIT TESTED: } & \text { Unit No. } 2 \text { Stack }\end{aligned}$

SOURCE EMISSION CALCULATIONS

\begin{tabular}{|c|c|c|c|c|c|}
\hline \multirow{2}{*}{ SYMBOL } & \multirow[b]{2}{*}{ DESCRIPTION } & \multirow[b]{2}{*}{ UNITS } & \multicolumn{3}{|c|}{ RUN NUMBER } \\
\hline & & & 1 & 2 & 3 \\
\hline DATE & & & $01 / 29 / 07$ & $01 / 30 / 07$ & $01 / 31 / 07$ \\
\hline BEGIN TIME & & & 0830 & 0645 & 0642 \\
\hline END TIME & & & 1533 & 1336 & 1320 \\
\hline$P(b)$ & BAROMETRIC PRESSURE & $\begin{array}{l}\text { "Hg Abs. } \\
(\mathrm{mm} \mathrm{Hg})\end{array}$ & $\begin{array}{r}29.67 \\
(754.00) \\
\end{array}$ & $\begin{array}{r}29.72 \\
(755.00) \\
\end{array}$ & $\begin{array}{r}29.70 \\
(754.00) \\
\end{array}$ \\
\hline$P(m)$ & ORIFICE PRESSURE DROP & $\begin{array}{l}\text { "H2O } \\
(\mathrm{mm} \mathrm{H} 2 \mathrm{O})\end{array}$ & $\begin{array}{r}1.432 \\
(36.400) \\
\end{array}$ & $\begin{array}{r}1.475 \\
(37.500) \\
\end{array}$ & $\begin{array}{r}1.504 \\
(38.200) \\
\end{array}$ \\
\hline & DGM CALIBRATION FACTOR & & 0.988 & 0.988 & 0.988 \\
\hline$V(m)$ & $\begin{array}{l}\text { VOLUME DRY GAS SAMPLED } \\
@ \text { METER CONDITIONS } \\
\text { LEAK CHECK VOLUME }\end{array}$ & $\begin{array}{l}\mathrm{ft} .^{\wedge} 3 \\
\left(\mathrm{~m}^{\wedge} 3\right) \\
\mathrm{ft} .^{\wedge} 3\end{array}$ & $\begin{array}{c}237.553 \\
(6.727) \\
0.248 \\
\end{array}$ & $\begin{array}{c}239.240 \\
(6.775) \\
0.000 \\
\end{array}$ & $\begin{array}{c}238.480 \\
(6.753) \\
0.000 \\
\end{array}$ \\
\hline$T(m)$ & $\begin{array}{l}\text { AVERAGE GAS METER } \\
\text { TEMPERATURE }\end{array}$ & $\begin{array}{l}\text { DEG.F } \\
\text { (DEG.C) }\end{array}$ & $\begin{array}{r}62 \\
(17) \\
\end{array}$ & $\begin{array}{r}63 \\
(17) \\
\end{array}$ & $\begin{array}{r}54 \\
(12) \\
\end{array}$ \\
\hline$V(m[s t d])^{*}$ & $\begin{array}{l}\text { VOLUME DRY GAS SAMPLED } \\
\text { @ STANDARD CONDITIONS* }\end{array}$ & $\begin{array}{l}\text { DSCF } \\
\text { (DSCM) }\end{array}$ & $\begin{array}{r}239.161 \\
(6.772) \\
\end{array}$ & $\begin{array}{r}240.828 \\
(6.820) \\
\end{array}$ & $\begin{array}{r}244.120 \\
(6.913) \\
\end{array}$ \\
\hline$V(w)$ & $\begin{array}{l}\text { TOTAL WATER COLLECTED, } \\
\text { IMPINGERS \& SILICA GEL }\end{array}$ & $\mathrm{ml}$ & 564.2 & 549.7 & 551.5 \\
\hline $\mathrm{V}(\mathrm{w}[$ gas $])$ & $\begin{array}{l}\text { VOLUME WATER VAPOR } \\
\text { COLLECTED @ STANDARD } \\
\text { CONDITIONS* }\end{array}$ & $\begin{array}{l}\text { SCF } \\
(\mathrm{SCM})\end{array}$ & $\begin{array}{r}26.630 \\
(0.754)\end{array}$ & $\begin{array}{l}25.946 \\
(0.735)\end{array}$ & $\begin{array}{r}26.031 \\
(0.737)\end{array}$ \\
\hline$\% \mathrm{M}$ & $\begin{array}{l}\text { MOISTURE IN STACK GAS } \\
\text { BY VOLUME }\end{array}$ & $\%$ & 10.02 & 9.73 & 9.64 \\
\hline Md & MOL FRACTION OF DRY GAS & & 0.8998 & 0.9027 & 0.9036 \\
\hline $\mathrm{Tt}$ & NET TIME OF TEST & MINUTES & 360 & 360 & 360 \\
\hline
\end{tabular}

* 68 Deg.F, 29.92 "Hg (20 Deg.C, 760 mm Hg) 


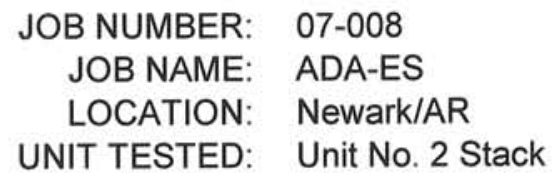

\begin{tabular}{|c|c|c|c|c|c|}
\hline & & & & JN NUMBER & \\
\hline SYMBOL & DESCRIPTION & UNITS & 1 & 2 & 3 \\
\hline $\mathrm{CO} 2$ & & $\%$ & 13.0 & 13.0 & 13.0 \\
\hline $\mathrm{O} 2$ & & $\%$ & 6.4 & 6.8 & 6.7 \\
\hline $\mathrm{CO}$ & & $\%$ & 0.0 & 0.0 & 0.0 \\
\hline N2 & & $\%$ & 80.6 & 80.2 & 80.3 \\
\hline$\%$ EA & $\begin{array}{l}\text { EXCESS AIR @ SAMPLING } \\
\text { POINT }\end{array}$ & $\%$ & 42.8 & 47.0 & 46.0 \\
\hline MWd & $\begin{array}{l}\text { MOLECULAR WEIGHT OF } \\
\text { DRY STACK GAS }\end{array}$ & $\begin{array}{l}\text { LB/LB-MOLE } \\
\text { (g/g-MOLE) }\end{array}$ & $\begin{array}{r}30.34 \\
(30.34) \\
\end{array}$ & $\begin{array}{c}30.35 \\
(30.35) \\
\end{array}$ & $\begin{array}{c}30.35 \\
(30.35) \\
\end{array}$ \\
\hline MW & $\begin{array}{l}\text { MOLECULAR WEIGHT OF } \\
\text { STACK GAS }\end{array}$ & $\begin{array}{l}\text { LB/LB-MOLE } \\
\text { (g/g-MOLE) }\end{array}$ & $\begin{array}{c}29.10 \\
(29.10) \\
\end{array}$ & $\begin{array}{c}29.15 \\
(29.15) \\
\end{array}$ & $\begin{array}{r}29.16 \\
(29.16) \\
\end{array}$ \\
\hline $\mathrm{Cp}$ & PITOT TUBE CALIBRATION & & 0.825 & 0.825 & 0.825 \\
\hline DELTA P & $\begin{array}{l}\text { VELOCITY HEAD OF STACK } \\
\text { GAS }\end{array}$ & $\begin{array}{l}\text { "H2O } \\
(\mathrm{mm} \mathrm{H} 20)\end{array}$ & $\begin{array}{r}2.656 \\
(67.500) \\
\end{array}$ & $\begin{array}{r}2.728 \\
(69.300) \\
\end{array}$ & $\begin{array}{r}2.765 \\
(70.200) \\
\end{array}$ \\
\hline DELTA P ^(1/2) & & "H2O & 1.629 & 1.651 & 1.661 \\
\hline Ts & STACK TEMPERATURE & $\begin{array}{l}\text { DEG. F } \\
\text { (DEG. C) }\end{array}$ & $\begin{array}{r}315 \\
(157) \\
\end{array}$ & $\begin{array}{r}310 \\
(154) \\
\end{array}$ & $\begin{array}{r}309 \\
(154) \\
\end{array}$ \\
\hline Ps & STACK PRESSURE & $\begin{array}{l}\text { "Hg Abs. } \\
\text { (mm Hg) } \\
\text { "H2O }\end{array}$ & $\begin{array}{c}29.41 \\
(747.00) \\
-3.50 \\
\end{array}$ & $\begin{array}{c}29.45 \\
(748.00) \\
-3.70 \\
\end{array}$ & $\begin{array}{c}29.44 \\
(748.00) \\
-3.50 \\
\end{array}$ \\
\hline Vs & $\begin{array}{l}\text { STACK VELOCITY @ STACK } \\
\text { CONDITIONS }\end{array}$ & $\begin{array}{l}\text { FPM } \\
(\mathrm{m} / \mathrm{SEC} .)\end{array}$ & $\begin{array}{r}6,553 \\
(33) \\
\end{array}$ & $\begin{array}{r}6,610 \\
(34) \\
\end{array}$ & $\begin{array}{r}6,645 \\
(34) \\
\end{array}$ \\
\hline As & STACK AREA & $\begin{array}{l}\text { (SQ.INCHES) } \\
\text { (SQ.METERS) }\end{array}$ & $\begin{array}{r}75,294 \\
(49) \\
\end{array}$ & $\begin{array}{r}75,294 \\
(49) \\
\end{array}$ & $\begin{array}{r}75,294 \\
(49) \\
\end{array}$ \\
\hline Qs & $\begin{array}{l}\text { DRY STACK GAS VOLUME @ } \\
\text { STANDARD CONDITIONS* }\end{array}$ & $\begin{array}{l}\text { DSCFM } \\
(\mathrm{DSCM} / \mathrm{HR})\end{array}$ & $\begin{array}{c}2,072,263 \\
(3,520,775)\end{array}$ & $\begin{array}{c}2,113,513 \\
(3,590,859)\end{array}$ & $\begin{array}{c}2,128,865 \\
(3,616,942)\end{array}$ \\
\hline Qa & $\begin{array}{l}\text { ACTUAL STACK GAS VOLUME } \\
\text { @ STACK CONDITIONS }\end{array}$ & $\begin{array}{l}\text { ACFM } \\
\left(m^{\wedge} 3 / H R\right)\end{array}$ & $\begin{array}{c}3,426,273 \\
(5,821,238)\end{array}$ & $\begin{array}{r}3,456,006 \\
(5,871,754) \\
\end{array}$ & $\begin{array}{r}3,474,675 \\
(5,903,473) \\
\end{array}$ \\
\hline $\mathrm{Dn}$ & SAMPLING NOZZLE DIAM. & $\begin{array}{l}\text { IN. } \\
(\mathrm{m})\end{array}$ & $\begin{array}{c}0.177 \\
(0.004) \\
\end{array}$ & $\begin{array}{c}0.176 \\
(0.004) \\
\end{array}$ & $\begin{array}{c}0.176 \\
(0.004) \\
\end{array}$ \\
\hline$\%$ & PERCENT ISOKINETIC & $\%$ & 98.5 & 98.3 & 98.9 \\
\hline
\end{tabular}

*68 Deg.F, 29.92 "Hg (20 Deg.C, 760 mm Hg) 
SOURCE EMISSION CALCULATIONS

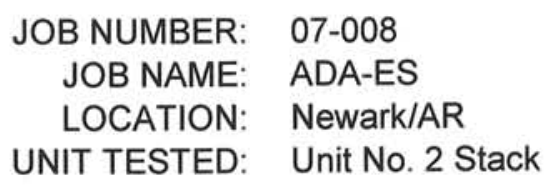

\begin{tabular}{|c|c|c|c|c|c|}
\hline \multirow{2}{*}{ SYMBOL } & \multirow[b]{2}{*}{ DESCRIPTION } & \multirow[b]{2}{*}{ UNITS } & \multicolumn{3}{|c|}{ RUN NUMBER } \\
\hline & & & 1 & 2 & 3 \\
\hline Mf & $\begin{array}{l}\text { PARTICULATE - PROBE, } \\
\text { CYCLONE AND FILTER }\end{array}$ & $\mathrm{mg}$ & 50.3 & 50.1 & 48.0 \\
\hline Mt & PARTICULATE - TOTAL & $\mathrm{mg}$ & 96.4 & 116.3 & 102.9 \\
\hline Can & $\begin{array}{l}\text { PARTICULATE - PROBE, } \\
\text { CYCLONE AND FILTER }\end{array}$ & $\begin{array}{l}\text { gr/DSCF* } \\
\text { (g/DSCM) }\end{array}$ & $\begin{array}{r}0.0032 \\
(0.0074) \\
\end{array}$ & $\begin{array}{r}0.0032 \\
(0.0073) \\
\end{array}$ & $\begin{array}{r}0.0030 \\
(0.0069) \\
\end{array}$ \\
\hline Cao & PARTICULATE - TOTAL & $\begin{array}{l}\text { gr/DSCF* } \\
\text { (g/DSCM) }\end{array}$ & $\begin{array}{r}0.0062 \\
(0.0142) \\
\end{array}$ & $\begin{array}{r}0.0074 \\
(0.0170) \\
\end{array}$ & $\begin{array}{r}0.0065 \\
(0.0149) \\
\end{array}$ \\
\hline Cat & $\begin{array}{l}\text { PARTIC.-PROBE, CYCLONE } \\
\text { AND FILTER @ STACK COND. }\end{array}$ & $\begin{array}{l}\mathrm{gr} / \mathrm{CF} \\
(\mathrm{g} / \mathrm{m} 3)\end{array}$ & $\begin{array}{r}0.0020 \\
(0.0046) \\
\end{array}$ & $\begin{array}{r}0.0020 \\
(0.0046) \\
\end{array}$ & $\begin{array}{r}0.0018 \\
(0.0041) \\
\end{array}$ \\
\hline Cau & $\begin{array}{l}\text { PARTICULATE - TOTAL @ } \\
\text { STACK CONDITIONS }\end{array}$ & $\begin{array}{l}\mathrm{gr} / \mathrm{CF} \\
(\mathrm{g} / \mathrm{m} 3)\end{array}$ & $\begin{array}{c}0.0037 \\
(0.0085) \\
\end{array}$ & $\begin{array}{r}0.0045 \\
(0.0103) \\
\end{array}$ & $\begin{array}{r}0.0040 \\
(0.0092) \\
\end{array}$ \\
\hline Caw & $\begin{array}{l}\text { PARTICULATE - PROBE, } \\
\text { CYCLONE AND FILTER }\end{array}$ & $\begin{array}{l}\text { LBS/HR } \\
(\mathrm{Kg} / \mathrm{HR}) \\
\end{array}$ & $\begin{array}{r}57.52 \\
(26.09) \\
\end{array}$ & $\begin{array}{r}58.03 \\
(26.32) \\
\end{array}$ & $\begin{array}{r}55.24 \\
(25.06) \\
\end{array}$ \\
\hline Cax & PARTICULATE - TOTAL & $\begin{array}{l}\text { LBS/HR } \\
(\mathrm{Kg} / \mathrm{HR})\end{array}$ & $\begin{array}{l}110.24 \\
(50.00) \\
\end{array}$ & $\begin{array}{l}134.70 \\
(61.10)\end{array}$ & $\begin{array}{l}118.43 \\
(53.72) \\
\end{array}$ \\
\hline & & & & & \\
\hline
\end{tabular}

* 68 Deg.F, 29.92 "Hg (20 Deg.C, 760 mm Hg) 
SOURCE EMISSION SURVEY

JOB NUMBER: 07-008

JOB NAME: ADA-ES

LOCATION: Newark/AR

UNIT TESTED: Unit No. 2 Stack

SOURCE EMISSION CALCULATIONS

\begin{tabular}{|c|c|c|c|c|c|}
\hline & & & & NUMBER & \\
\hline SYMBOL & DESCRIPTION & UNITS & 4 & 5 & \\
\hline DATE & & & $02 / 01 / 07$ & $02 / 02 / 07$ & \\
\hline BEGIN TIME & & & 0705 & 0700 & \\
\hline END TIME & & & 1342 & 1336 & \\
\hline$P(b)$ & BAROMETRIC PRESSURE & $\begin{array}{l}\text { "Hg Abs. } \\
(\mathrm{mm} \mathrm{Hg})\end{array}$ & $\begin{array}{r}29.37 \\
(746.00) \\
\end{array}$ & $\begin{array}{r}29.42 \\
(747.00) \\
\end{array}$ & \\
\hline$P(m)$ & ORIFICE PRESSURE DROP & $\begin{array}{l}\text { "H2O } \\
(\mathrm{mm} \mathrm{H} 2 \mathrm{O})\end{array}$ & $\begin{array}{r}1.544 \\
(39.200) \\
\end{array}$ & $\begin{array}{r}1.585 \\
(40.300) \\
\end{array}$ & \\
\hline & DGM CALIBRATION FACTOR & & 0.988 & 0.988 & \\
\hline $\mathrm{V}(\mathrm{m})$ & $\begin{array}{l}\text { VOLUME DRY GAS SAMPLED } \\
@ \text { METER CONDITIONS } \\
\text { LEAK CHECK VOLUME }\end{array}$ & $\begin{array}{l}\text { ft.^3 } \\
\left(\mathrm{m}^{\wedge} 3\right) \\
\mathrm{ft} . \wedge 3\end{array}$ & $\begin{array}{r}245.820 \\
(6.961) \\
0.000 \\
\end{array}$ & $\begin{array}{r}247.868 \\
(7.019) \\
0.000 \\
\end{array}$ & \\
\hline $\mathrm{T}(\mathrm{m})$ & $\begin{array}{l}\text { AVERAGE GAS METER } \\
\text { TEMPERATURE }\end{array}$ & $\begin{array}{l}\text { DEG.F } \\
\text { (DEG.C) }\end{array}$ & $\begin{array}{r}59 \\
(15) \\
\end{array}$ & $\begin{array}{r}60 \\
(16) \\
\end{array}$ & \\
\hline $\mathrm{V}(\mathrm{m}[\mathrm{std}])^{*}$ & $\begin{array}{l}\text { VOLUME DRY GAS SAMPLED } \\
\text { @ STANDARD CONDITIONS* }\end{array}$ & $\begin{array}{l}\text { DSCF } \\
\text { (DSCM) }\end{array}$ & $\begin{array}{r}246.476 \\
(6.979) \\
\end{array}$ & $\begin{array}{r}248.497 \\
(7.037) \\
\end{array}$ & \\
\hline$V(w)$ & $\begin{array}{l}\text { TOTAL WATER COLLECTED, } \\
\text { IMPINGERS \& SILICA GEL }\end{array}$ & $\mathrm{ml}$ & 614.5 & 589.7 & \\
\hline$V(w[$ gas $])$ & $\begin{array}{l}\text { VOLUME WATER VAPOR } \\
\text { COLLECTED @ STANDARD } \\
\text { CONDITIONS* }\end{array}$ & $\begin{array}{l}\text { SCF } \\
(\mathrm{SCM})\end{array}$ & $\begin{array}{c}29.004 \\
(0.821)\end{array}$ & $\begin{array}{r}27.834 \\
(0.788)\end{array}$ & \\
\hline$\% \mathrm{M}$ & $\begin{array}{l}\text { MOISTURE IN STACK GAS } \\
\text { BY VOLUME }\end{array}$ & $\%$ & 10.53 & 10.07 & \\
\hline Md & MOL FRACTION OF DRY GAS & & 0.8947 & 0.8993 & \\
\hline $\mathrm{Tt}$ & NET TIME OF TEST & MINUTES & 360 & 360 & \\
\hline
\end{tabular}

*68 Deg.F, 29.92 "Hg (20 Deg.C, 760 mm Hg) 
JOB NUMBER: 07-008

JOB NAME: ADA-ES

LOCATION: Newark/AR

UNIT TESTED: Unit No. 2 Stack

\begin{tabular}{|c|c|c|c|c|c|}
\hline & & & \multicolumn{3}{|c|}{ RUN NUMBER } \\
\hline SYMBOL & DESCRIPTION & UNITS & 4 & 5 & \\
\hline $\mathrm{CO} 2$ & & $\%$ & 12.8 & 13.0 & \\
\hline $\mathrm{O} 2$ & & $\%$ & 6.9 & 6.8 & \\
\hline $\mathrm{CO}$ & & $\%$ & 0.0 & 0.0 & \\
\hline N2 & & $\%$ & 80.3 & 80.2 & \\
\hline$\% \mathrm{EA}$ & $\begin{array}{l}\text { EXCESS AIR @ SAMPLING } \\
\text { POINT }\end{array}$ & $\%$ & 48.0 & 47.0 & \\
\hline MWd & $\begin{array}{l}\text { MOLECULAR WEIGHT OF } \\
\text { DRY STACK GAS }\end{array}$ & $\begin{array}{l}\text { LB/LB-MOLE } \\
\text { (g/g-MOLE) }\end{array}$ & $\begin{array}{c}30.32 \\
(30.32) \\
\end{array}$ & $\begin{array}{c}30.35 \\
(30.35) \\
\end{array}$ & \\
\hline MW & $\begin{array}{l}\text { MOLECULAR WEIGHT OF } \\
\text { STACK GAS }\end{array}$ & $\begin{array}{l}\text { LB/LB-MOLE } \\
\text { (g/g-MOLE) }\end{array}$ & $\begin{array}{r}29.03 \\
(29.03) \\
\end{array}$ & $\begin{array}{c}29.11 \\
(29.11) \\
\end{array}$ & \\
\hline $\mathrm{Cp}$ & PITOT TUBE CALIBRATION & & 0.825 & 0.825 & \\
\hline DELTAP & $\begin{array}{l}\text { VELOCITY HEAD OF STACK } \\
\text { GAS }\end{array}$ & $\begin{array}{l}\text { "H2O } \\
(\mathrm{mm} \mathrm{H} 20)\end{array}$ & $\begin{array}{r}2.840 \\
(72.100) \\
\end{array}$ & $\begin{array}{r}2.906 \\
(73.800) \\
\end{array}$ & \\
\hline DELTA $P^{\wedge}(1 / 2)$ & & "H2O & 1.684 & 1.703 & \\
\hline Ts & STACK TEMPERATURE & $\begin{array}{l}\text { DEG. F } \\
\text { (DEG. C) }\end{array}$ & $\begin{array}{c}313 \\
(156) \\
\end{array}$ & $\begin{array}{r}319 \\
(159) \\
\end{array}$ & \\
\hline Ps & STACK PRESSURE & $\begin{array}{l}\text { "Hg Abs. } \\
\text { (mm Hg) } \\
\text { "H2O }\end{array}$ & $\begin{array}{c}29.11 \\
(739.00) \\
-3.60 \\
\end{array}$ & $\begin{array}{c}29.16 \\
(741.00) \\
-3.50\end{array}$ & \\
\hline Vs & $\begin{array}{l}\text { STACK VELOCITY @ STACK } \\
\text { CONDITIONS }\end{array}$ & $\begin{array}{l}\text { FPM } \\
\text { (m/SEC.) }\end{array}$ & $\begin{array}{r}6,808 \\
(35) \\
\end{array}$ & $\begin{array}{r}6,896 \\
(35) \\
\end{array}$ & \\
\hline As & STACK AREA & $\begin{array}{l}\text { (SQ.INCHES) } \\
\text { (SQ.METERS) }\end{array}$ & $\begin{array}{r}75,294 \\
(49) \\
\end{array}$ & $\begin{array}{r}75,294 \\
(49) \\
\end{array}$ & \\
\hline Qs & $\begin{array}{l}\text { DRY STACK GAS VOLUME @ } \\
\text { STANDARD CONDITIONS* }\end{array}$ & $\begin{array}{l}\text { DSCFM } \\
\text { (DSCM/HR) }\end{array}$ & $\begin{array}{c}2,124,346 \\
(3,609,264)\end{array}$ & $\begin{array}{c}2,149,896 \\
(3,652,673)\end{array}$ & \\
\hline Qa & $\begin{array}{l}\text { ACTUAL STACK GAS VOLUME } \\
\text { @ STACK CONDITIONS }\end{array}$ & $\begin{array}{l}\text { ACFM } \\
\left(m^{\wedge} 3 / H R\right)\end{array}$ & $\begin{array}{r}3,559,846 \\
(6,048,178) \\
\end{array}$ & $\begin{array}{c}3,605,890 \\
(6,126,407) \\
\end{array}$ & \\
\hline Dn & SAMPLING NOZZLE DIAM. & $\begin{array}{l}\text { IN. } \\
(\mathrm{m})\end{array}$ & $\begin{array}{c}0.176 \\
(0.004) \\
\end{array}$ & $\begin{array}{c}0.176 \\
(0.004) \\
\end{array}$ & \\
\hline$\%$ & PERCENT ISOKINETIC & $\%$ & 100.1 & 99.7 & \\
\hline
\end{tabular}

* 68 Deg.F, 29.92 "Hg (20 Deg.C, 760 mm Hg) 
SOURCE EMISSION CALCULATIONS
JOB NUMBER: 07-008
JOB NAME: ADA-ES
LOCATION: Newark/AR
UNIT TESTED: Unit No. 2 Stack

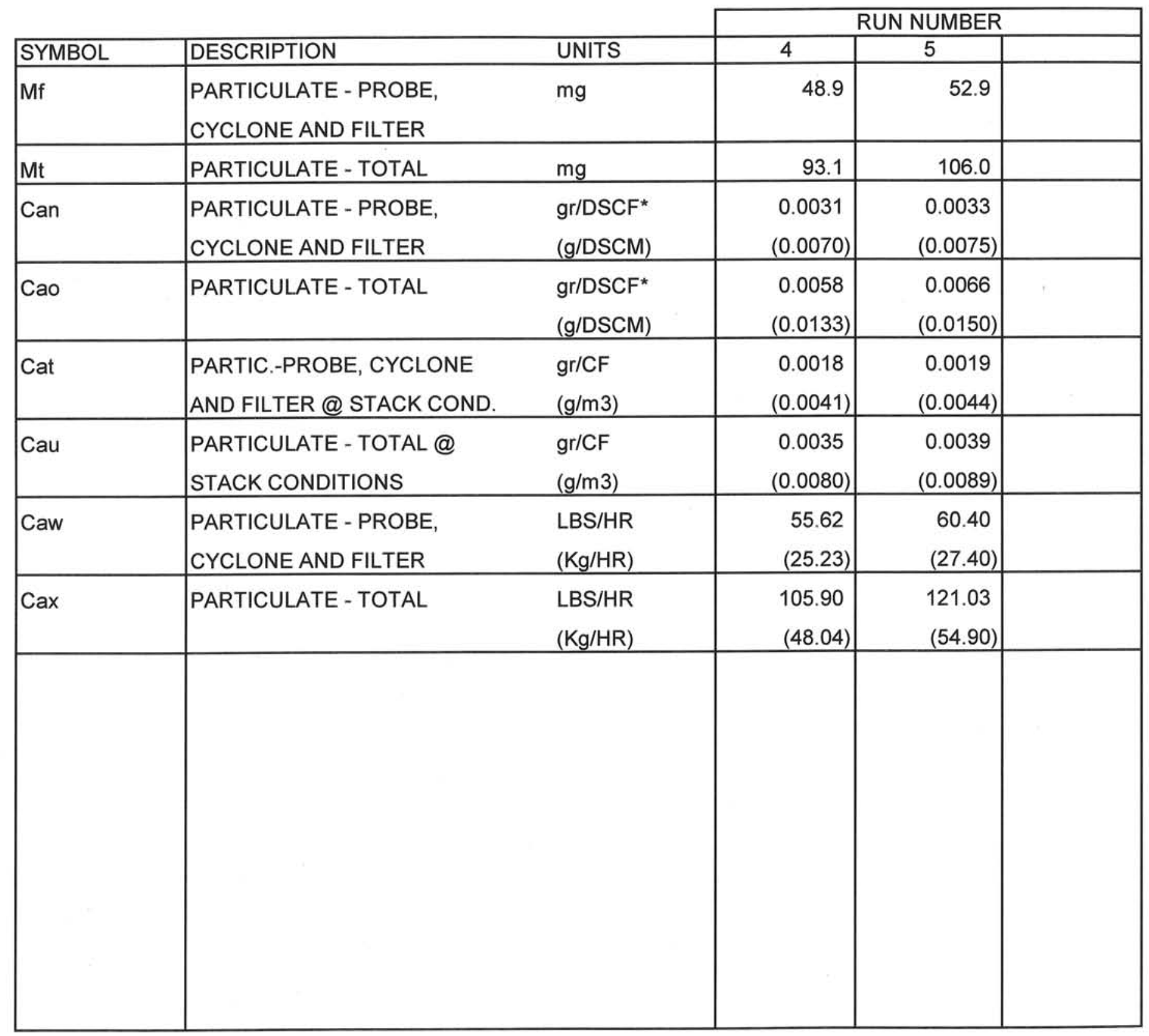

* 68 Deg.F, 29.92 "Hg (20 Deg.C, 760 mm Hg) 


\section{AII SAMPLING ASSOCIATES, INC.}

Appendix C:

Calibration Data 


\section{Appendix C}

\section{Table 4: Calibration Data}

Pre-test Calibrations:

Equipment

Dry Gas Meter 2-2

Digital Temperature Indicator 2-2

Dry Gas Meter 2-2 Orifice

Pitot Tube 1-4

Nozzle 1-2

Nozzle 2-2

Barometer 1

\section{Post-test Calibrations:}

Equipment

Dry Gas Meter 2-2

Pitot Tube 1-4

Nozzle 1-2

Nozzle 2-2

Barometer 1
Calibration Factor

0.988

0.825

0.176

0.177

NIST Traceable

12/19/06

$01 / 02 / 07$

01/02/07

01/03/07

01/02/07

01/05/07

01/05/07

\section{Calibration Date}

Calibration Factor

1.002

03/16/07

0.825

03/28/07

0.174

03/28/07

0.176

03/28/07

NIST Traceable

03/21/07 


\section{Appendix C}

\section{Table 4: Calibration Data}

Pre-test Calibrations:

\section{Equipment}

Dry Gas Meter 2-2

Digital Temperature Indicator 2-2

Dry Gas Meter 2-2 Orifice

Pitot Tube 1-4

Nozzle 1-2

Nozzle 2-2

Barometer 1

\section{Calibration Factor}

0.988

0.825

0.176

0.177

NIST Traceable

\section{Calibration Date}

01/02/07

$01 / 02 / 07$

01/03/07

01/02/07

$01 / 05 / 07$

01/05/07

$12 / 19 / 06$

\section{Post-test Calibrations:}

\section{Equipment}

Dry Gas Meter 2-2

Pitot Tube 1-4

Nozzle 1-2

Nozzle 2-2

Barometer 1

\section{Calibration Factor}

1.002

0.825

0.174

0.176

NIST Traceable
Calibration Date

03/16/07

03/28/07

$03 / 28 / 07$

$03 / 28 / 07$

$03 / 21 / 07$ 


\section{Calibration Data}

Dry Gas Meter Calibration

Meter Console No. ASAI 2-2

Date Calibration Performed:

$01 / 02 / 07$

$\Delta \mathrm{H}$ Setting

\begin{tabular}{c}
$\left(" \mathrm{H}_{2} \mathrm{O}\right)$ \\
\hline 0.50 \\
1.00 \\
1.50 \\
2.00 \\
3.00 \\
4.00 \\
Average
\end{tabular}

\begin{tabular}{c}
$\mathrm{C}_{\mathrm{DG}}$ \\
\hline 0.987 \\
\hline 0.990 \\
\hline 0.987 \\
\hline 0.985 \\
\hline 0.988 \\
\hline 0.989 \\
\hline 0.988 \\
\hline
\end{tabular}

Variation + :

$0.20 \%$

Variation -:

$-0.30 \%$

Certified by:

Soot Uachson 1/2/07

Calibrator (Signature / Date)

Bill Hefley 1/2/07

QA Officer (Signature / Date) 


\author{
Calibration Data \\ Dry Gas Meter Calibration
}

Meter Console No.

Date Calibration Performed:

ASAI 2-2

01/02/07

$$
\begin{aligned}
& \text { Run } 1 \text { at } 0.5 " \Delta \mathrm{H} \\
& \text { Wet Test Meter } \mathrm{C}_{\mathrm{f}}=1.018 \text { Console Pump Vacuum }=-5.0 \mathrm{Hg}
\end{aligned}
$$

\begin{tabular}{|c|c|c|c|c|c|c|c|}
\hline & Time & Reading & & Temp. & & $\underline{P_{m}}$ & \\
\hline nd & $9: 52$ & 5.165 & cf & 62 & ${ }^{\circ} \mathrm{F}$ & -0.3 & $" \mathrm{H}_{2} \mathrm{O}$ \\
\hline art & 9:39 & $\underline{0.000}$ & cf & $\underline{63}$ & ${ }^{\circ} \mathrm{F}$ & $\underline{-0.3}$ & $" \mathrm{H}_{2} \mathrm{O}$ \\
\hline & age & 5.165 & cf & 63 & ${ }^{\circ} \mathrm{F}$ & -0.3 & " $\mathrm{H}_{2} \mathrm{O}$ \\
\hline
\end{tabular}

\begin{tabular}{|c|c|c|c|c|c|}
\hline Reading & $\underline{\ln }$ & & Out & & $\underline{\mathrm{P}_{\mathrm{m}}}$ \\
\hline 305.368 cf & 71 & ${ }^{\circ} \mathrm{F}$ & 71 & ${ }^{\circ} \mathrm{F}$ & 0.50 \\
\hline$\underline{299.952}$ cf & $\underline{72}$ & ${ }^{\circ} \mathrm{F}$ & $\underline{74}$ & ${ }^{\circ} \mathrm{F}$ & $\underline{0.50}$ \\
\hline $5.416 \mathrm{cf}$ & 72 & ${ }^{\circ} \mathrm{F}$ & 73 & ${ }^{\circ} \mathrm{F}$ & 0.50 \\
\hline
\end{tabular}




\author{
Calibration Data \\ Dry Gas Meter Calibration \\ Meter Console No. \\ ASAI 2-2 \\ Date Calibration Performed:
Run 1 at 1.0" $\Delta \mathrm{H} \quad \mathrm{Pb}=30.05 " \mathrm{Hg}$ Wet Test Meter $\mathrm{C}_{\mathrm{f}}=1.018$ Console Pump Vacuum $=-\underline{-5.0}$ " Hg

Wet Test Meter No. ASAl-0 Meter

$\underline{\text { Time }}$ Reading Temp. $\quad \underline{\mathrm{P}_{\mathrm{m}}}$

End 10:08 5.033 cf $61{ }^{\circ} \mathrm{F} \quad-0.3$

Start $\underline{9: 59} \quad \underline{0.000}$ of $\quad \underline{61}{ }^{\circ} \mathrm{F} \quad \underline{\underline{-0.3}}$

$\begin{array}{llllll}\text { Average } & 5.033 \text { cf } & 61 & { }^{\circ} \mathrm{F} & -0.3\end{array}$

$$
\begin{gathered}
\text { Wet Test Meter } V_{m s t d}=17.65 \times V_{m}\left[\frac{P_{b}+\frac{P_{m}}{13.6}}{\left(T_{m}+460\right)}\right] \times C_{f}=\underline{5.212} d c s f \\
\text { Dry Gas Meter } V_{m s d}=17.65 \times \mathrm{Vm}\left[\frac{P_{b}+\frac{P_{m}}{13.6}}{\left(T_{m}+460\right)}\right]=\underline{5.267} \mathrm{dcsf}
\end{gathered}
$$

$$
\text { Calibration Factor }\left(C_{D G}\right)=\frac{\text { Wet Test Meter } V_{\text {mstd }}}{\text { Dry Gas Meter } V_{\text {mstd }}}=\underline{0.990}
$$




\author{
Calibration Data \\ Dry Gas Meter Calibration \\ Meter Console No. \\ ASAI 2-2 \\ Date Calibration Performed: \\ $01 / 02 / 07$
}
Run 1 at $1.5^{\prime \prime} \Delta \mathrm{H}$
Wet Test Meter $\mathrm{C}_{\mathrm{f}}=1.018$ Console Pump Vacuum $=-5.0 \mathrm{Hg}$

Wet Test Meter No. ASAI-0 Meter

Dry Gas Meter

Meter Temperature

$\underline{\text { Time }}$ Reading Temp. $\quad \underline{P_{m}}$

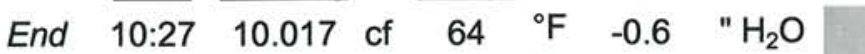

Start $\underline{10: 12} \underline{0.000}$ cf $\underline{62} \quad{ }^{\circ} \mathrm{F} \quad \underline{-0.6}$

$$
\text { " } \mathrm{H}_{2} \mathrm{O}
$$

Reading

In $\quad$ Out

322.946 cf $76 \quad{ }^{\circ} \mathrm{F} \quad 70 \quad{ }^{\circ} \mathrm{F} \quad 1.50 \quad{ }^{\prime} \mathrm{H}_{2} \mathrm{O}$

Average

$10.017 \mathrm{cf}$

$63{ }^{\circ} \mathrm{F} \quad-0.6$

" $\mathrm{H}_{2} \mathrm{O}$

$\underline{312.503}$ ef $\quad \underline{70}{ }^{\circ} \mathrm{F} \quad \underline{69}{ }^{\circ} \mathrm{F} \quad \underline{1.50} \quad " \mathrm{H}_{2} \mathrm{O}$

10.443 cf $73{ }^{\circ} \mathrm{F} \quad 70 \quad{ }^{\circ} \mathrm{F} \quad 1.50 \quad \mathrm{H}_{2} \mathrm{O}$

Wet Test Meter $V_{\text {ssd }}=17.65 \times V_{m}\left[\frac{P b+\frac{P_{m}}{13.6}}{\left(T_{m}+460\right)}\right] \times C_{f}=\underline{10.326} \mathrm{dcsf}$
Dry Gas Meter $V_{\text {mstd }}=17.65 \times \mathrm{Vm}\left[\frac{P_{b}+\frac{P_{m}}{13.6}}{\left(T_{m}+460\right)}\right]=\underline{10.464} \mathrm{dcsf}$

Calibration Factor $\left(C_{D G}\right)=\frac{\text { Wet Test Meter } V_{\text {mstd }}}{\text { Dry Gas Meter } V_{m s t d}}=\underline{0.987}$ 


\author{
Calibration Data \\ Dry Gas Meter Calibration \\ Meter Console No. \\ ASAI 2-2 \\ Date Calibration Performed:

Run 1 at 2.0" $\Delta \mathrm{H}$

$\mathrm{Pb}=30.05 " \mathrm{Hg}$

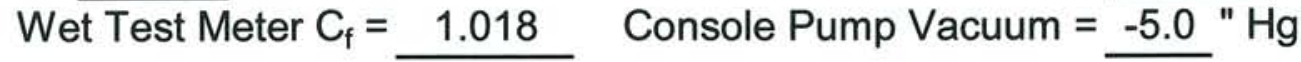

Wet Test Meter No. ASAl-O Meter

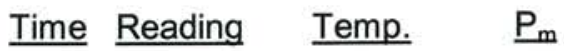

\begin{tabular}{|c|c|c|c|c|c|c|c|}
\hline & $\frac{\text { Time }}{10: 45}$ & $\frac{\text { Reading }}{10.750}$ & & $\frac{\text { Temp. }}{65}$ & ${ }^{\circ} \mathrm{F}$ & $\begin{array}{l}\frac{P_{n}}{-0 .} \\
-0 .\end{array}$ & " $\mathrm{H}_{2} \mathrm{O}$ \\
\hline & $10: 31$ & $\underline{0.000}$ & $f$ & $\underline{65}$ & ${ }^{\circ} \mathrm{F}$ & -0 . & " $\mathrm{H}_{2} \mathrm{O}$ \\
\hline & & 10.750 & & 65 & & -0 . & $\mathrm{H}_{2} \mathrm{O}$ \\
\hline
\end{tabular}

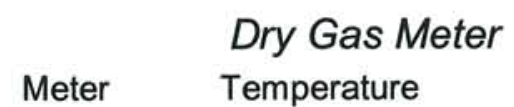
Reading In $\underline{\text { Out }} \quad \underline{P_{m}}$ 334.904 cf $77 \quad{ }^{\circ} \mathrm{F} \quad 71 \quad{ }^{\circ} \mathrm{F} \quad 2.00 \quad " \mathrm{H}_{2} \mathrm{O}$ $\underline{323.701} \mathrm{cf} \quad \underline{74}{ }^{\circ} \mathrm{F} \quad \underline{70}{ }^{\circ} \mathrm{F} \quad \underline{2.00} \quad " \mathrm{H}_{2} \mathrm{O}$ 11.203 cf $76{ }^{\circ} \mathrm{F} \quad 71{ }^{\circ} \mathrm{F} \quad 2.00 \quad{ }^{\prime \prime} \mathrm{H}_{2} \mathrm{O}$

$$
\begin{gathered}
\text { Wet Test Meter } V_{m s t d}=17.65 \times V_{m}\left[\frac{P_{b}+\frac{P_{m}}{13.6}}{\left(T_{m}+460\right)}\right] x C_{f}=\underline{11.037} d c s f \\
\text { Dry Gas Meter } V_{m s t d}=17.65 \times V m\left[\frac{P_{b}+\frac{P_{m}}{13.6}}{\left(T_{m}+460\right)}\right]=\underline{11.203} d c s f \\
\text { Calibration Factor }(C D G)=\frac{\text { Wet Test Meter } V_{m_{s t d}}}{\text { Dry Gas Meter } V_{m_{s t d}}}=\underline{\underline{0.985}}
\end{gathered}
$$




\author{
Calibration Data \\ Dry Gas Meter Calibration \\ Meter Console No. \\ ASAI 2-2 \\ Date Calibration Performed: \\ 01/02/07
}

$\begin{aligned} \text { Run } \frac{1}{\mathrm{~Pb}} & =30.05 \mathrm{Hg} \\ \text { Wet Test Meter } \mathrm{C}_{\mathrm{f}}=1.018 \text { Console Pump Vacuum } & =-5.0 \mathrm{Hg}\end{aligned}$

Wet Test Meter No. ASAI-0

Dry Gas Meter Meter Meter Temperature

\begin{tabular}{|c|c|c|c|c|c|c|c|c|c|c|c|c|c|c|}
\hline & $\underline{\text { Time }}$ & Reading & & Temp. & & $\underline{P_{m}}$ & & Reading & $\underline{\ln }$ & & $\underline{\text { Out }}$ & & $\underline{P_{m}}$ & \\
\hline End & $10: 59$ & 10.389 & cf & 64 & ${ }^{\circ} \mathrm{F}$ & -1.0 & $" \mathrm{H}_{2} \mathrm{O}$ & $346.196 \mathrm{cf}$ & 78 & ${ }^{\circ} \mathrm{F}$ & 71 & ${ }^{\circ} \mathrm{F}$ & 3.00 & " $\mathrm{H}_{2} \mathrm{O}$ \\
\hline a & $10: 48$ & $\underline{0.000}$ & cf & $\underline{65}$ & ${ }^{\circ} \mathrm{F}$ & $\underline{-1.0}$ & $" \mathrm{H}_{2} \mathrm{O}$ & $\underline{335.407} \mathrm{cf}$ & $\underline{75}$ & ${ }^{\circ} \mathrm{F}$ & $\underline{71}$ & ${ }^{\circ} \mathrm{F}$ & $\underline{3.00}$ & $" \mathrm{H}_{2} \mathrm{O}$ \\
\hline & rage & 10.389 & cf & 65 & ${ }^{\circ} \mathrm{F}$ & -1.0 & $" \mathrm{H}_{2} \mathrm{O}$ & 10.789 cf & 77 & ${ }^{\circ} \mathrm{F}$ & 71 & ${ }^{\circ} \mathrm{F}$ & 3.00 & "H \\
\hline
\end{tabular}

$$
\begin{gathered}
\text { Wet Test Meter } V_{m s d}=17.65 \times V_{m}\left[\frac{P_{b}+\frac{P_{m}}{13.6}}{\left(T_{m}+460\right)}\right] \times C_{f}=\underline{10.668} \mathrm{dcsf} \\
\text { Dry Gas Meter } V_{\text {mstd }}=17.65 \times \mathrm{Vm}\left[\frac{P_{b}+\frac{P_{m}}{13.6}}{\left(T_{m}+460\right)}\right]=\underline{10.800} \mathrm{dcsf} \\
\text { Calibration Factor }\left(C_{D G}\right)=\frac{\text { Wet Test Meter } V_{\text {mstd }}}{\text { Dry Gas Meter } V_{m_{s t}}}=\underline{0.988}
\end{gathered}
$$




\author{
Calibration Data \\ Dry Gas Meter Calibration \\ Meter Console No. \\ ASAI 2-2 \\ Date Calibration Performed: \\ 01/02/07
}

$\begin{aligned} \operatorname{Run} \frac{1}{\mathrm{~Pb}} & =30.05 \mathrm{Hg} \\ \text { Wet Test Meter } \mathrm{C}_{\mathrm{f}}=1.018 \text { Console Pump Vacuum } & =-5 . \mathrm{Hg}^{\mathrm{H}} \mathrm{Hg}\end{aligned}$

Wet Test Meter No. ASAI-0

Dry Gas Meter

Meter

Meter Temperature

\begin{tabular}{|c|c|c|c|c|c|c|c|c|c|c|c|c|}
\hline & Time & Reading & Temp. & & $\underline{P_{m}}$ & & Reading & In & & Out & $\underline{P_{m}}$ & \\
\hline End & $11: 12$ & 10.882 & 64 & ${ }^{\circ} \mathrm{F}$ & -0.8 & $" \mathrm{H}_{2} \mathrm{O}$ & 357.874 cf & 78 & ${ }^{\circ} \mathrm{F}$ & 70 & ${ }^{\circ} \mathrm{F} \quad 4.00$ & $" \mathrm{H}_{2} \mathrm{O}$ \\
\hline tart & 11:02 & $\underline{0.000}$ & cf & ${ }^{\circ} \mathrm{F}$ & $\underline{-0.8}$ & $" \mathrm{H}_{2} \mathrm{O}$ & $\underline{346.605} \mathrm{cf}$ & $\underline{75}$ & ${ }^{\circ} \mathrm{F}$ & $\underline{71}$ & ${ }^{\circ} \mathrm{F} \quad \underline{4.00}$ & $" \mathrm{H}_{2}$ \\
\hline & & 10.882 & 64 & ${ }^{\circ} \mathrm{F}$ & -0.8 & $" \mathrm{H}_{2} \mathrm{O}$ & $11.269 \mathrm{cf}$ & 77 & ${ }^{\circ} \mathrm{F}$ & 71 & ${ }^{\circ} \mathrm{F} \quad 4.00$ & " $\mathrm{H}_{2}$ \\
\hline
\end{tabular}

$$
\begin{gathered}
\text { Wet Test Meter } V_{m s t d}=17.65 \times V_{m}\left[\frac{P_{b}+\frac{P_{m}}{13.6}}{\left(T_{m}+460\right)}\right] \times C_{f}=\underline{11.191} \mathrm{dcsf} \\
\text { Dry Gas Meter } V_{m s t d}=17.65 \times \mathrm{Vm}\left[\frac{P_{b}+\frac{P_{m}}{13.6}}{\left(T_{m}+460\right)}\right]=\underline{11.313} \mathrm{dcsf} \\
\text { Calibration Factor }\left(C_{D G}\right)=\frac{\text { Wet Test Meter } V_{\text {mstd }}}{\text { Dry Gas Meter } V_{\text {mstd }}}=\underline{0.989}
\end{gathered}
$$


Calibration Data

Digital Temperature Indicator Calibration

DTI Unit No. ASAI 2-2

Date Calibration Performed:

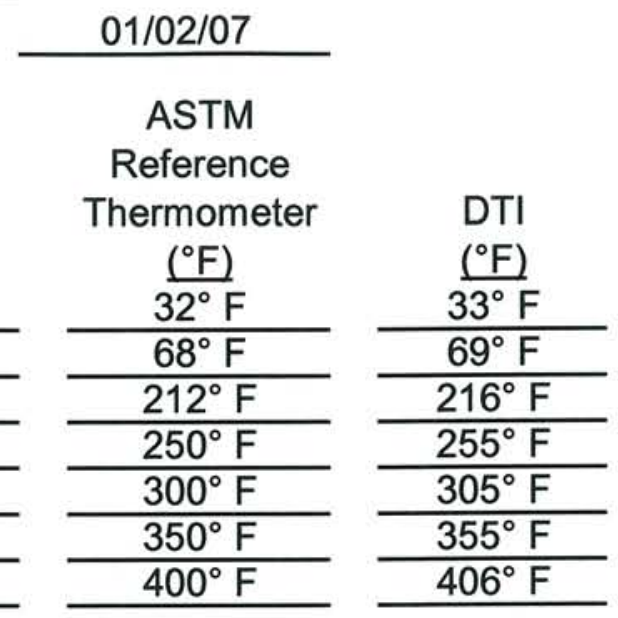

Meter Adjusted?

No

ASTM Reference Thermometer:

SN: 06000047

Range: $\quad-0.1^{\circ} \mathrm{C}$ to $1360.6^{\circ} \mathrm{C}$

Range:

Range:

Certified by:

Scot Jachson 1/02/07

Calibrator (Signature / Date)

Bill Hefley 1/02/07

QA Officer (Signature / Date) 


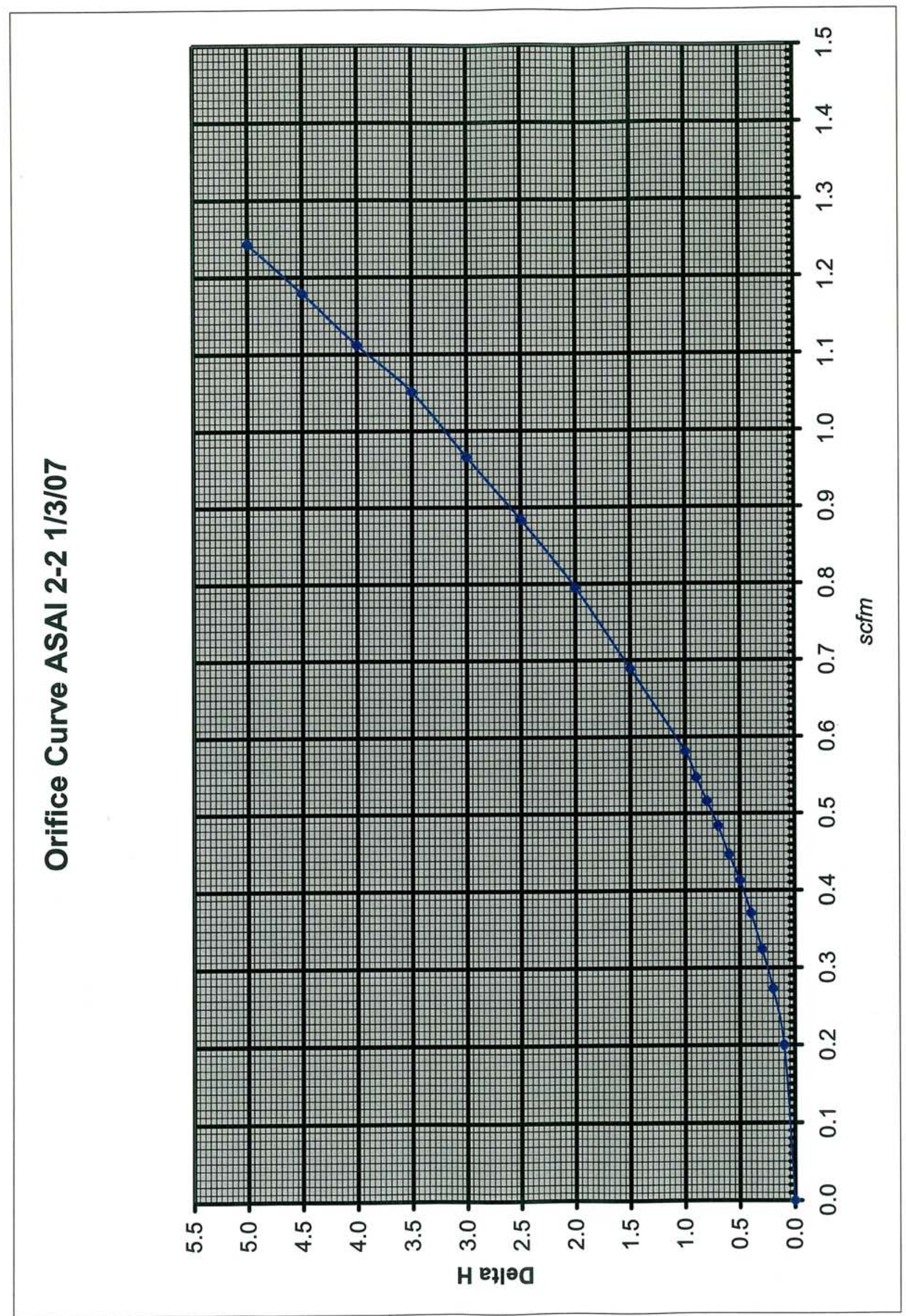




\section{TiR

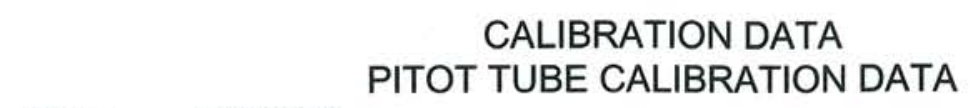

$\begin{array}{lc}\text { Date: } & 01 / 02 / 07 \\ \text { I.D. \# } & \text { ASAI 1-4 } \\ \text { C }_{\text {pstd: }}: \frac{0.990}{}\end{array}$

Time: $13: 00$

$\mathrm{T}_{\mathrm{s}}: \frac{13.00}{{ }^{\circ} \mathrm{F}}$

$\mathrm{Pb}: 29.97 \mathrm{Hg}$

\begin{tabular}{|c|c|c|c|c|c|c|c|c|c|c|}
\hline $\begin{array}{c}\text { fps } \\
\text { Mark }\end{array}$ & $\begin{array}{c}\text { Desired } \\
\text { Calibration } \\
\text { Standard }\end{array}$ & \multicolumn{2}{c|}{$\begin{array}{c}\text { Calibration } \\
\text { Standard } \\
\text { Start }\end{array}$} & $\begin{array}{c}\text { End } \\
\text { v Standard } \\
\text { Average }\end{array}$ & High & v High & $\begin{array}{c}\text { Cal. } \\
\text { Factor }\end{array}$ & Low & v Low & $\begin{array}{c}\text { Cal. } \\
\text { Factor }\end{array}$ \\
\hline 20 & 0.09 & 0.09 & 0.09 & 0.300 & 0.13 & 0.361 & 0.824 & 0.13 & 0.361 & 0.824 \\
\hline 30 & 0.21 & 0.21 & 0.21 & 0.458 & 0.30 & 0.548 & 0.828 & 0.30 & 0.548 & 0.828 \\
\hline 40 & 0.37 & 0.37 & 0.37 & 0.608 & 0.54 & 0.735 & 0.819 & 0.54 & 0.735 & 0.819 \\
\hline 50 & 0.58 & 0.58 & 0.58 & 0.762 & 0.84 & 0.917 & 0.823 & 0.84 & 0.917 & 0.823 \\
\hline 60 & 0.83 & 0.83 & 0.83 & 0.911 & 1.20 & 1.095 & 0.823 & 1.20 & 1.095 & 0.823 \\
\hline 70 & 1.13 & 1.15 & 1.15 & 1.072 & 1.65 & 1.285 & 0.826 & 1.65 & 1.285 & 0.826 \\
\hline 80 & 1.48 & 1.50 & 1.50 & 1.225 & 2.15 & 1.466 & 0.827 & 2.15 & 1.466 & 0.827 \\
\hline 90 & 1.87 & 1.85 & 1.85 & 1.360 & 2.65 & 1.628 & 0.827 & 2.65 & 1.628 & 0.827 \\
\hline & & & & & & & & & & \\
\hline & & & & & & & & & & \\
\hline 50 & 0.58 & 0.58 & 0.58 & 0.762 & 0.84 & 0.917 & 0.823 & 0.84 & 0.917 & 0.823 \\
\hline 50 & 0.58 & 0.58 & 0.58 & 0.762 & 0.84 & 0.917 & 0.823 & 0.84 & 0.917 & 0.823 \\
\hline & & & & & & & & & & \\
\hline & & & & & & & & & & \\
\hline \multicolumn{2}{|l|}{ Average } & & & & & & 0.825 & & & 0.825 \\
\hline
\end{tabular}

Summary of Results:

Normal high side calibraiton factor variation $+\frac{0.36 \%}{-0.73 \%}$

Normal low side calibration factor: variation $-: \frac{-0.73 \%}{0.825}$ variation $+0.36 \%$ variation $-: \frac{-0.73 \%}{-}$

Certification:

I certify that the Type S pitot tube, the standard type pitot tube, and the calibration setup meet or exceed all specifications, criteria and/or applicable design features and hereby assign a pitot tube calibration factor $C_{p}$ of: $\quad \underline{0.825}$

Certified by:

Patrich Selabovich 1/02/07 Calibrator (Signature/Date)
Bill Hefley 1/02/07

QA Officer (Signature/Date) 


\section{SARPLING \\ ASSOCIATES, INC.}

Calibration Data

Nozzle Calibration

Nozzle Set No.

Date Calibration Performed:

ASAI 1-

$01 / 05 / 07$

$\begin{array}{llllllll}-1 & -2 & -3 & -4 & -5 & -6 & -7 & -8\end{array}$

Measurement 1

Measurement 2

Measurement 3

0.119

$0.177 \quad 0.242$

$-4.5$

0.394

0.498

0.235

$\underline{0.117}$

$0.176 \quad 0.242$

$\frac{0.316}{0.315} \frac{0.365}{0.366}$

$\underline{0.395} \quad \underline{0.496}$

0.237

\begin{tabular}{lllllllllll}
0.119 & 0.174 & 0.241 & 0.316 & 0.366 & 0.393 & 0.496 & 0.236 \\
\hline
\end{tabular}

Measurement 4

$\underline{0.119} \stackrel{0.176}{\underline{0.1240}}$

Measurement 5

Measurement 6

Measurement 7

$\begin{array}{llll}0.118 & 0.175 & 0.240\end{array}$

0.120

$\underline{0.176 \quad 0.240}$

$\underline{0.317} \quad 0.368$

$\underline{0.394 \quad 0.496}$

0.234

Measurement 8

0.120

$0.317 \lcm{0.366}$

$\underline{0.394} \quad 0.497$

0.235

$\underline{0.120} \underline{0.177} \underline{0.241}$

Measurement 9

$\begin{array}{lll}0.119 & 0.176 & 0.243\end{array}$

$0.316 \quad 0.367$

$\underline{0.394} \quad 0.497$

0.235

Measurement 16

Average

$\begin{array}{lll}\underline{0.119} & 0.175 & \frac{0.242}{0.119} \\ 0.176 & 0.241\end{array}$

\begin{tabular}{lll}
$\frac{0.315}{0.317}$ & $\frac{0.365}{0.368}$ \\
$\frac{0.316}{0.316}$ & $\frac{0.366}{0.366}$ \\
\hline 0.316 & $\frac{0.366}{0.316}$ & 0
\end{tabular}

0.39

0.236

$0.394 \quad 0.495$

0.236

0.236

0.235

Patrick Selabovich 1/05/07

Calibrator (Signature / Date)

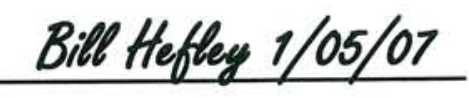

QA Officer (Signature / Date) 


\section{SIR SAMPLNNG \\ ASSOCIATES, INC.}

Calibration Data

Nozzle Calibration

$\begin{array}{rr}\text { Nozzle Set No. } & \text { ASAl - } 2 \\ \text { Date Calibration Performed: } & 01 / 05 / 07 \\ \end{array}$

$\begin{array}{llllllll}-1 & -2 & -3 & -4 & -5 & -6 & -7 & -8\end{array}$

\begin{tabular}{|c|c|c|c|c|c|c|c|c|}
\hline Measurement 1 & 0.119 & 0.178 & 0.241 & 0.316 & 0.367 & 0.387 & 0.499 & 0.237 \\
\hline Measurement 2 & 0.117 & 0.175 & 0.242 & 0.316 & 0.366 & 0.387 & 0.497 & 0.237 \\
\hline Measurement 3 & 0.119 & 0.179 & 0.242 & 0.316 & 0.366 & 0.388 & 0.497 & 0.238 \\
\hline Measurement 4 & 0.118 & 0.177 & 0.239 & 0.318 & 0.365 & 0.386 & 0.496 & 0.238 \\
\hline Measurement 5 & 0.118 & 0.175 & 0.239 & 0.315 & 0.365 & 0.385 & 0.496 & 0.235 \\
\hline Measurement 6 & 0.119 & 0.176 & 0.240 & 0.316 & 0.367 & 0.385 & 0.497 & 0.237 \\
\hline Measurement 7 & 0.120 & 0.176 & 0.240 & 0.317 & 0.365 & 0.385 & 0.497 & 0.238 \\
\hline Measurement 8 & 0.120 & 0.178 & 0.242 & 0.318 & 0.365 & 0.387 & 0.498 & 0.239 \\
\hline Measurement 9 & 0.119 & 0.178 & 0.242 & 0.318 & 0.367 & 0.387 & 0.499 & 0.239 \\
\hline Measurement 10 & 0.118 & 0.177 & 0.242 & 0.319 & 0.368 & 0.387 & 0.497 & 0.237 \\
\hline Average & 0.119 & 0.177 & 0.241 & 0.317 & 0.366 & 0.386 & 0.497 & 0.238 \\
\hline
\end{tabular}

Patrich Selahovich 1/05/07

Calibrator (Signature / Date)

$\frac{\text { Bill Hefley 1/05/07 }}{\text { QA Officer (Signature I Date) }}$ 


\section{Calibration Data \\ Barometer Calibration Data}

\begin{abstract}
Date: 12/19/2006
I.D. \#: ASAI -1
\end{abstract}

Mercury Barometer ASAI - 0 Reading:

Aneroid Barometer Reading:

Barometer Adjusted?
Difference:

Time:

Temperature:

29.92" $\mathrm{Hg}$

29.97" $\mathrm{Hg}$

0.05 " $\mathrm{Hg}$

YES

Certified by: 


\section{AII SAMPLING ASSOCIATES, INC.}

\section{Post-Test Calibration Data}




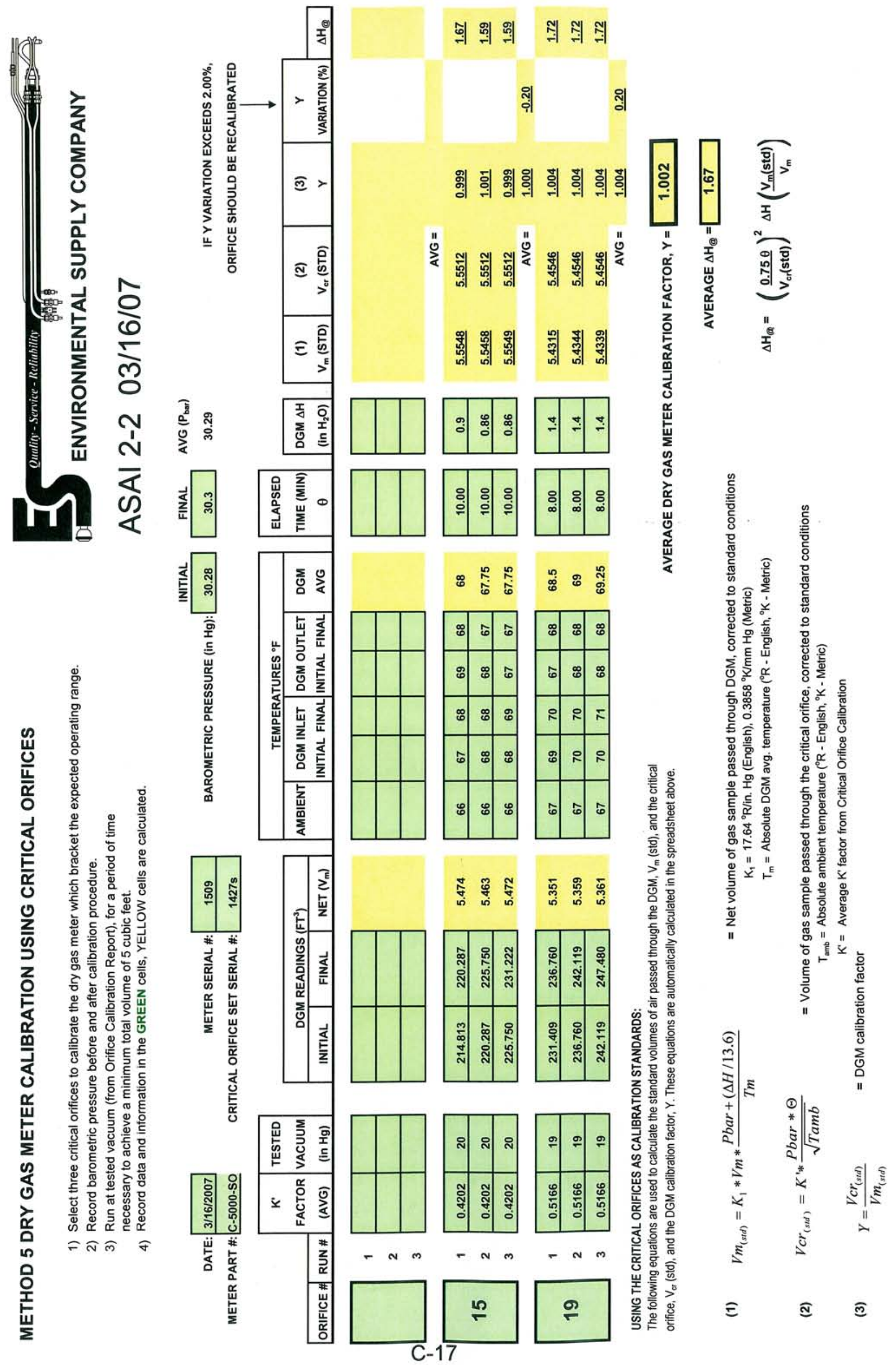


Calibration Data

Digital Temperature Indicator Calibration

DTI Unit No.

ASAI 2-2

Date Calibration Performed:

$03 / 16 / 07$

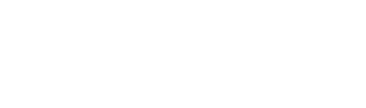

Reference Point

\begin{tabular}{c}
$\frac{\frac{\text { Time }}{9: 23}}{9: 24}$ \\
\hline $9: 25$ \\
\hline $9: 26$ \\
\hline $9: 27$ \\
\hline $9: 28$ \\
\hline $9: 29$ \\
\hline
\end{tabular}

Thermometer

Ice Bath

Ambient Air

Boiling Water

Oven

Oven

Oven

Oven

ASTM
Reference
Thermometer

Meter Adjusted?

No

\begin{tabular}{c}
$\frac{\left({ }^{\circ} \mathrm{F}\right)}{32^{\circ} \mathrm{F}}$ \\
\hline $68^{\circ} \mathrm{F}$ \\
\hline $212^{\circ} \mathrm{F}$ \\
\hline $250^{\circ} \mathrm{F}$ \\
\hline $300^{\circ} \mathrm{F}$ \\
\hline $350^{\circ} \mathrm{F}$ \\
\hline $400^{\circ} \mathrm{F}$ \\
\hline
\end{tabular}

$\frac{\frac{\left({ }^{\circ} \mathrm{F}\right)}{32^{\circ} \mathrm{F}}}{\frac{68^{\circ} \mathrm{F}}{215^{\circ} \mathrm{F}}} \frac{\frac{254^{\circ} \mathrm{F}}{304^{\circ} \mathrm{F}}}{\frac{354^{\circ} \mathrm{F}}{405^{\circ} \mathrm{F}}}$

ASTM Reference Thermometer:

SN: 06000047

Range: $\quad \underline{-0.1^{\circ} \mathrm{C} \text { to } 1,360.6^{\mathrm{c}}}$

Range:

Range:

Certified by:

Patrich Selabovich 3/16/07

Calibrator (Signature / Date)

Bill Hefley 3/16/07

QA Officer (Signature / Date) 


\section{SAMPPLING \\ ASSOCIATES, INC.}

\section{CALIBRATION DATA \\ PITOT TUBE CALIBRATION DATA}

\begin{tabular}{cc} 
Date: & $03 / 28 / 07$ \\
\hline I.D. \# & ASAl 1-4 \\
C $_{\text {pstd }}:$ & 0.990
\end{tabular}
Time: $14: 15$

$\mathrm{T}_{\mathrm{s}}: \frac{14: 15}{}{ }^{\circ} \mathrm{F}$

$\mathrm{Pb}: \overline{29.59} " \mathrm{Hg}$

\begin{tabular}{|c|c|c|c|c|c|c|c|c|c|c|}
\hline $\begin{array}{c}\text { fps } \\
\text { Mark }\end{array}$ & $\begin{array}{c}\text { Desired } \\
\text { Calibration } \\
\text { Standard }\end{array}$ & \multicolumn{2}{c|}{$\begin{array}{c}\text { Calibration } \\
\text { Standard } \\
\text { Start }\end{array}$} & $\begin{array}{c}\text { V Standard } \\
\text { Average }\end{array}$ & High & v High & $\begin{array}{c}\text { Cal. } \\
\text { Factor }\end{array}$ & Low & v Low & $\begin{array}{c}\text { Cal. } \\
\text { Factor }\end{array}$ \\
\hline 20 & 0.09 & 0.09 & 0.09 & 0.300 & 0.13 & 0.361 & 0.824 & 0.13 & 0.361 & 0.824 \\
\hline 30 & 0.20 & 0.21 & 0.21 & 0.458 & 0.30 & 0.548 & 0.828 & 0.30 & 0.548 & 0.828 \\
\hline 40 & 0.36 & 0.37 & 0.37 & 0.608 & 0.54 & 0.735 & 0.819 & 0.54 & 0.735 & 0.819 \\
\hline 50 & 0.56 & 0.58 & 0.58 & 0.762 & 0.84 & 0.917 & 0.823 & 0.84 & 0.917 & 0.823 \\
\hline 60 & 0.80 & 0.83 & 0.83 & 0.911 & 1.20 & 1.095 & 0.823 & 1.20 & 1.095 & 0.823 \\
\hline 70 & 1.09 & 1.15 & 1.15 & 1.072 & 1.65 & 1.285 & 0.826 & 1.65 & 1.285 & 0.826 \\
\hline 80 & 1.43 & 1.50 & 1.50 & 1.225 & 2.15 & 1.466 & 0.827 & 2.15 & 1.466 & 0.827 \\
\hline 90 & 1.80 & 1.85 & 1.85 & 1.360 & 2.65 & 1.628 & 0.827 & 2.65 & 1.628 & 0.827 \\
\hline & & & & & & & & & & \\
\hline & & & & & & & & & & \\
\hline 50 & 0.56 & 0.58 & 0.58 & 0.762 & 0.84 & 0.917 & 0.823 & 0.84 & 0.917 & 0.823 \\
\hline 50 & 0.56 & 0.58 & 0.58 & 0.762 & 0.84 & 0.917 & 0.823 & 0.84 & 0.917 & 0.823 \\
\hline \multicolumn{2}{|r|}{} & & & & & & & & & \\
\hline & & & & & & & & & & \\
\hline \multicolumn{2}{|l|}{ Average } & & & & & & 0.825 & & & 0.825 \\
\hline
\end{tabular}

Summary of Results:

Normal high side calibraiton factor: 0.825

variation +

variation -:

Normal low side calibration factor:

$0.36 \%$

variation +

$-0.73 \%$

variation

0.825

$0.36 \%$

$-0.73 \%$

Certification:

I certify that the Type S pitot tube, the standard type pitot tube, and the calibration setup meet or exceed all specifications, criteria and/or applicable design features and hereby assign a pitot tube calibration factor $C_{p}$ of: $\quad \underline{0.825}$

Certified by:

\section{Seot Jackson 3/28/07}

Calibrator (Signature/Date)
Bill Hefley 3/28/07

QA Officer (Signature/Date) 
Calibration Data
Nozzle Calibration

Calibration Data
Nozzle Calibration

Nozzle Set No.

Date Calibration Performed:

ASAI 1-

$03 / 28 / 07$

$\begin{array}{llllllll}-1 & -2 & -3 & -4 & -5 & -6 & -7 & -8\end{array}$

Measurement 1

Measurement 2

\begin{tabular}{lllllllll}
0.119 & $\frac{0.175}{0.117}$ & $\frac{0.242}{0.173}$ & $\frac{0.316}{0.242}$ & $\frac{0.365}{0.315}$ & $\frac{0.394}{0.366}$ & $\frac{0.498}{0.395}$ & $\frac{0.496}{0}$ & $\frac{0.235}{0.237}$ \\
\hline
\end{tabular}

Measurement 3

$\begin{array}{lllllllllllllll}0.119 & 0.173 & 0.241 & 0.316 & 0.366 & 0.393 & 0.496 & 0.236\end{array}$

Measurement 4

Measurement 5

Measurement 6

Measurement 7

\begin{tabular}{ll}
0.119 & 0.173 \\
\hline 0.118 & 0.174
\end{tabular}

0.240

0.317

.

\begin{tabular}{|c|c|c|c|c|c|c|c|}
\hline 0.120 & 0.171 & 0.240 & 0.316 & 0.367 & 0.394 & 0.497 & 0.235 \\
\hline 0.120 & 0.172 & 0.243 & 0.315 & 0.365 & 0.394 & 0.497 & 0.236 \\
\hline 0.120 & 0.175 & 0.241 & 0.317 & 0.368 & 0.394 & 0.497 & 0.236 \\
\hline 0.119 & 0.174 & 0.2 & 0.3 & 0. & 0. & 0.495 & 0.236 \\
\hline 0.119 & 0.175 & 0.242 & 0.316 & 0.366 & 0.395 & 0.498 & 0.235 \\
\hline 0.11 & 0.174 & 0.241 & 0.316 & 0.366 & 0.394 & 0.497 & 0.236 \\
\hline
\end{tabular}

Average

Measurement 8

Measurement 9

Measurement 16

0.119 
Calibration Data

Nozzle Calibration

Nozzle Set No.

Date Calibration Performed:

$\frac{A S A I-2}{03 / 28 / 07}$

$\begin{array}{llllllll}-1 & -2 & -3 & -4 & -5 & -6 & -7 & -8\end{array}$

$\begin{array}{llllllllllllll}\text { Measurement } 1 & 0.119 & 0.178 & 0.241 & 0.316 & 0.367 & 0.387 & 0.499 & 0.237\end{array}$

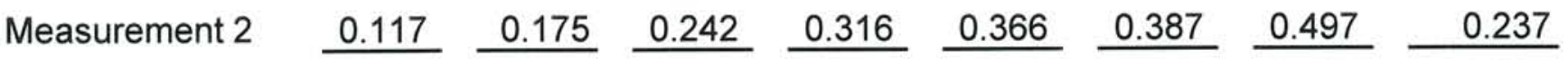

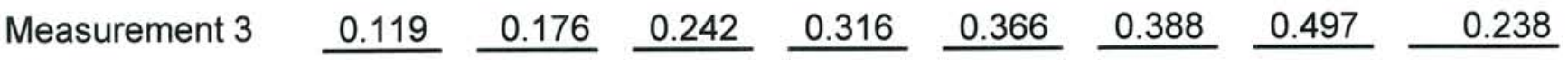

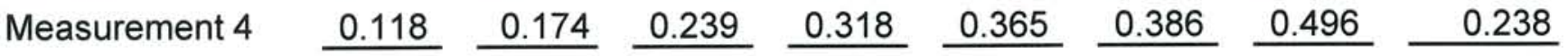

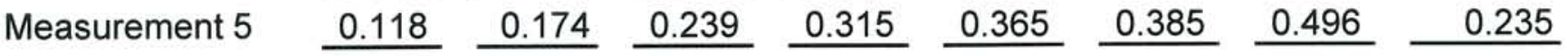

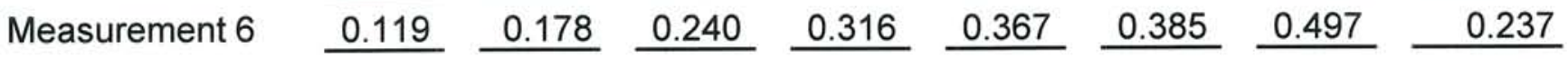

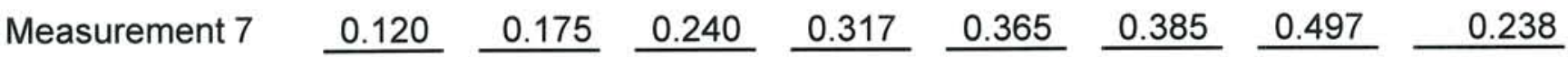

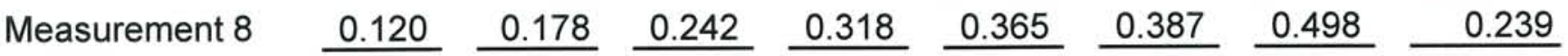

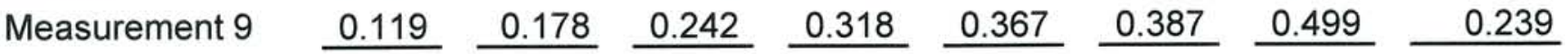

Measurement 10

Average

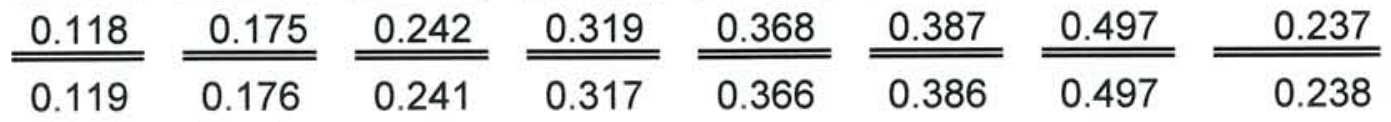

Patrick Selalovick 3/28/07

Calibrator (Signature / Date)

Bill Hefley 3/28/07

QA Officer (Signature / Date) 


\section{Calibration Data \\ Barometer Calibration Data}

\begin{tabular}{|c|c|c|c|}
\hline Date: & $3 / 21 / 2007$ & Time: & $13: 30$ \\
\hline I.D. \# & ASAI - 1 & Temperature: & 74 \\
\hline
\end{tabular}

$\begin{array}{rc}\text { Mercury Barometer ASAI - O Reading: } & 29.74 " \mathrm{Hg} \\ \text { Aneroid Barometer Reading: } & 29.84 " \mathrm{Hg} \\ \text { Difference: } & 0.10^{\prime \prime ~ H g} \\ \text { Barometer Adjusted? } & \text { YES }\end{array}$

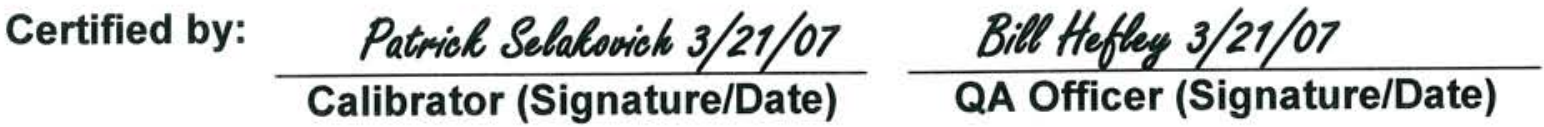




\section{AII SAMPLING ASSOCIATES, INC.}

Appendix D:

Field Data 


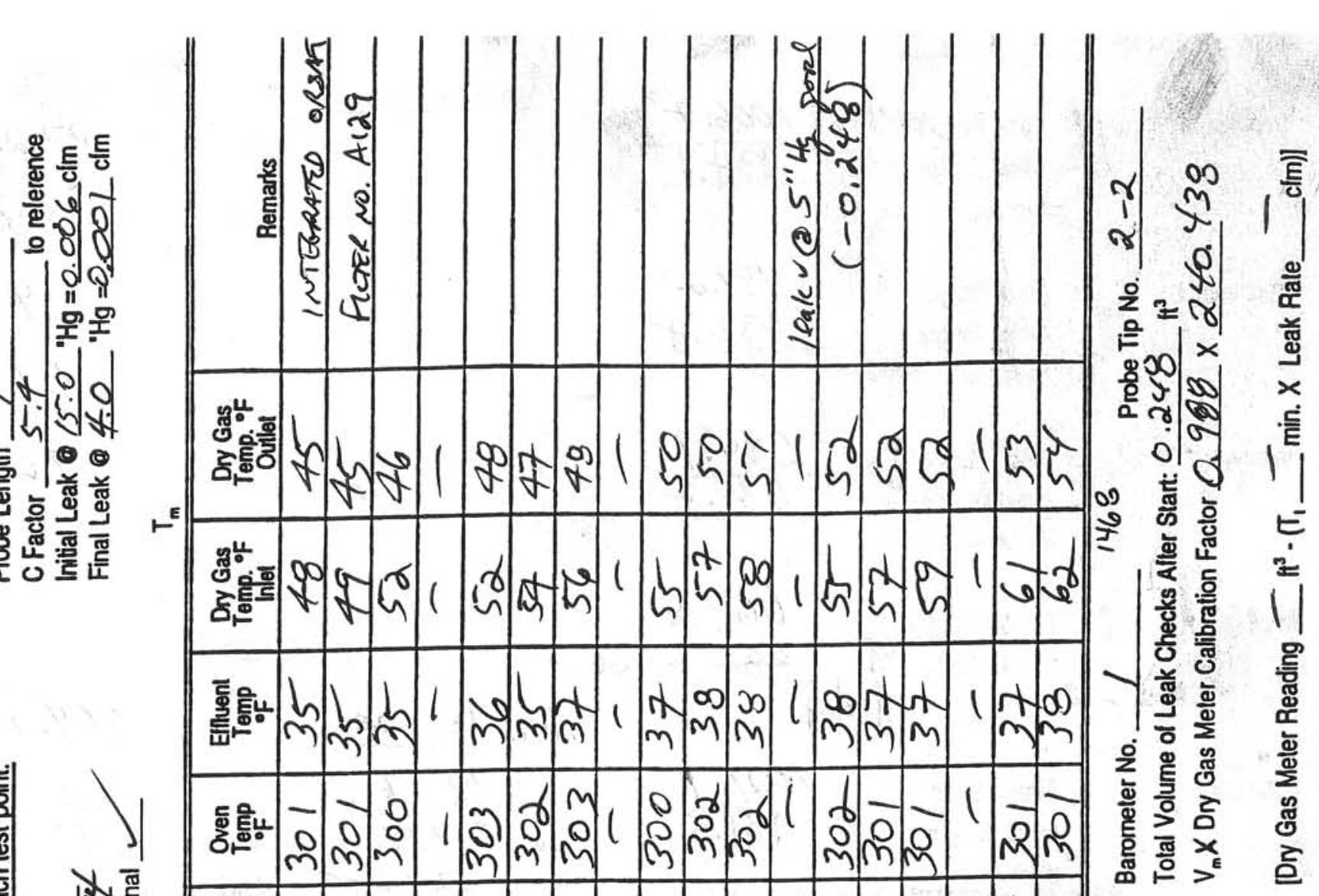

2 운오

F०a

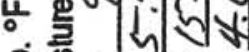

릉옹 듬

명혀

产

टृ:

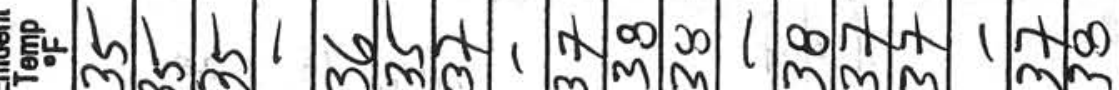

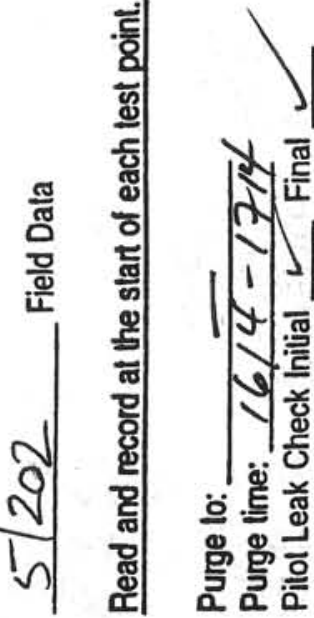

: ชัँ. r $\mathrm{g}$ m

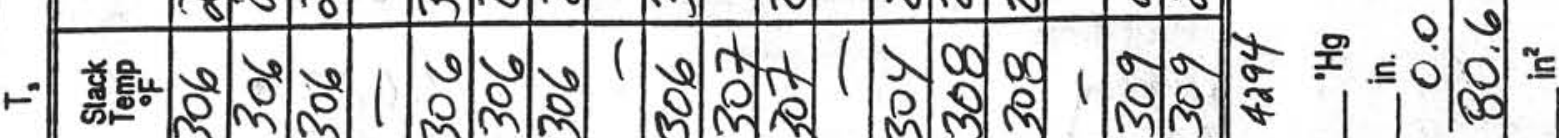

(1)

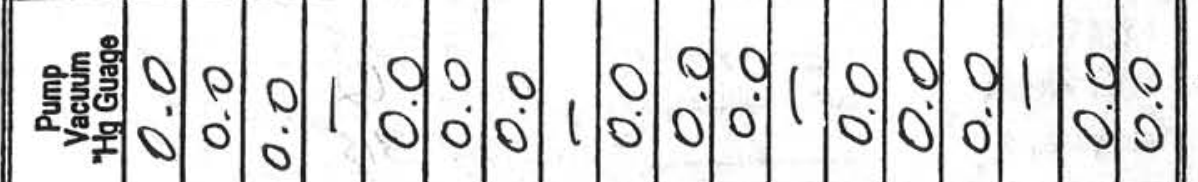
Int 8 o $z^{2}=$

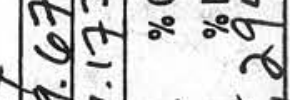

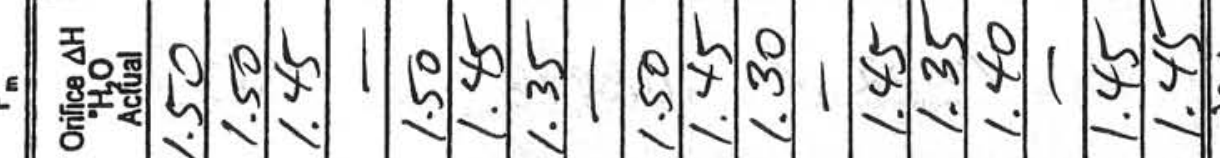
121 th

$1+1+a$ a 0.0 ช⿱乛龰己

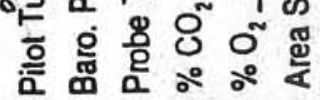

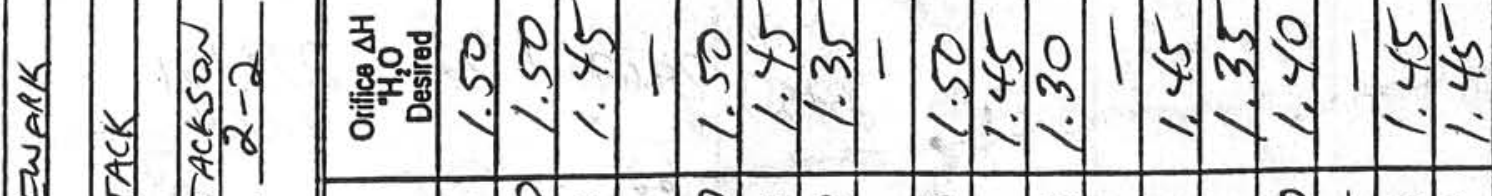

ว 5 h

of क 20 0. 1 र

4. $0+15 \frac{2}{2}=0$

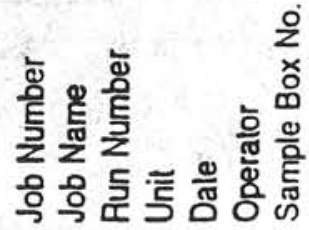

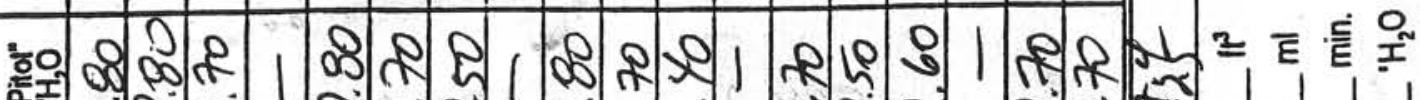

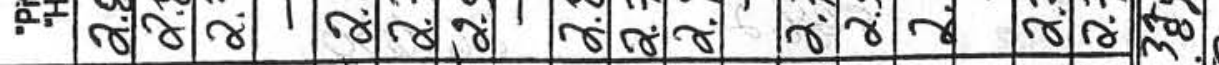

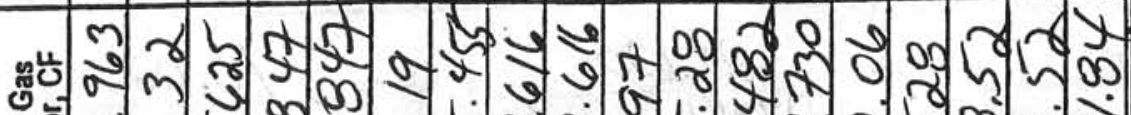

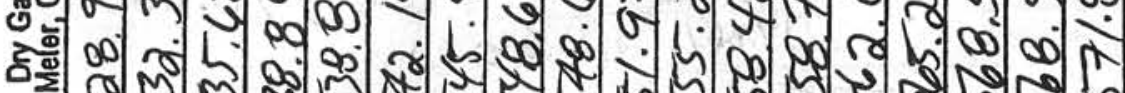

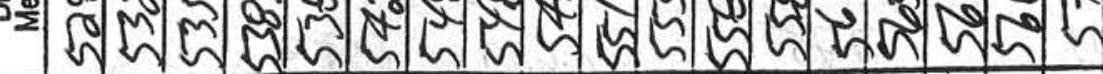

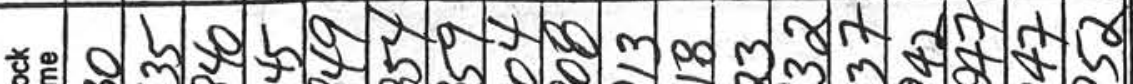

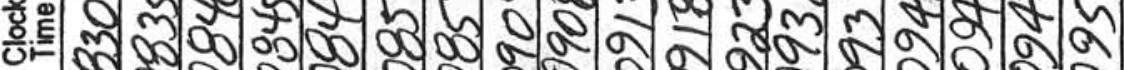

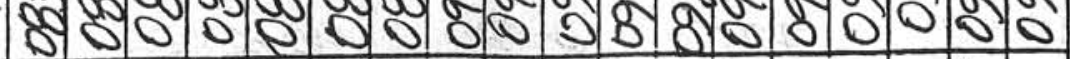

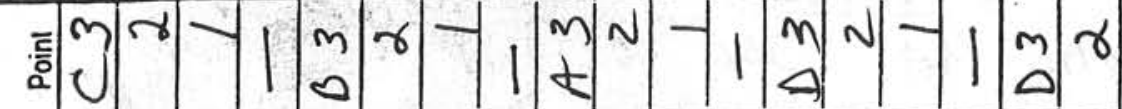

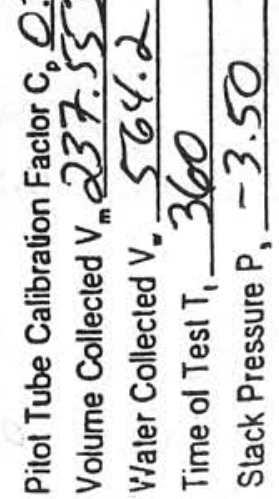


Impinger Box No.

$1^{-1}$

Impinger 1

Final Weight
Initial Weight
Increase

Water Weight Gain

Impinger 1 Initial Weight

Final Weight $\quad \frac{981.2}{732.7}$

Impinger 2

Increase

Impinger 3

Final Weight

Initial Weight

$$
\frac{634.1}{631.7}
$$

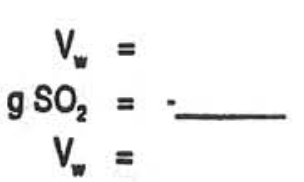

Increase

Impinger $1 \quad 272.6$

Impinger $2 \quad 248.5$

Impinger $3 \quad 2.4$

impinger 4

Impinger $5 \quad 39.6$

Impinger 6

Impinger 4

Final Weight

Initial Weight

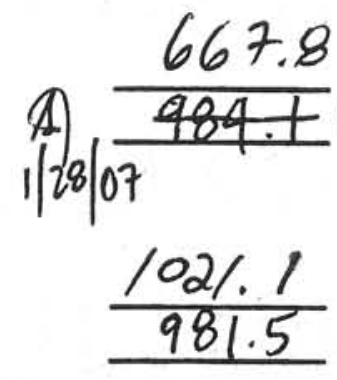

Increase

666.7

Impinger 7

$\begin{array}{ll}\text { Final Weight } & \frac{102 \% 1}{981.5} \\ \text { Initial Weight } & \end{array}$

filter 16. A129

Total

$564.2=v_{w}$

Impinger 5

Increase

Impinger 6

Final Weight

$P_{b}=\frac{29.67}{237.553}$
$v_{m}=2$

$v_{w}=564.2$

$P_{m}=1.432$

Initial Weight

Increase

Avg $\Delta P=2.656$

$\begin{aligned} \% \mathrm{CO}_{2} & =13.0 \\ \% \mathrm{O}_{2} & =\frac{0.4}{0.0} \\ \% \mathrm{CO}_{2} & =\frac{30.6}{75294}\end{aligned}$

$A_{1}=\frac{75294}{0.179}$

$D_{n}=0.177$

$A v g \sqrt{\Delta \mathrm{P}}=1.629$

$T_{1}=360$

$c_{p}=0.925$

Impinger 7

Final Weight

Initial Weight

Increase

$\mathrm{P}_{\mathrm{s}}=-3.5$
$\mathrm{~T}_{\mathrm{m}}=\frac{02}{615}{ }^{\circ} \mathrm{H} \mathrm{O}$
$\mathrm{T}_{\mathrm{s}}={ }^{\circ} \mathrm{F} \mathrm{V}$

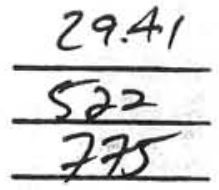

'Hg
OR

Moisture Content: $\quad \% \mathrm{M}=10.02 \checkmark \mathrm{M}_{\mathrm{d}}=\underline{0.8998} \mathrm{MW}_{\mathrm{c}}=30.336 \mathrm{MW}=29.10 \mathrm{~V}$

$$
V m_{s t d}=17.65 \mathrm{Vm}\left[\frac{P_{b}+\frac{P_{m}}{13.6}}{T_{m}+460}\right]=17.65 \times 237.553\left[\frac{29.67+\frac{1.432}{13.6}}{62+460}\right]=\frac{239161}{0.664} \mathrm{stt}^{3} \mathrm{scm}
$$

$V_{w_{0 x}}=0.0472 \times V_{w}=0.0472 \times-564.2=\mathrm{stt}^{3}$

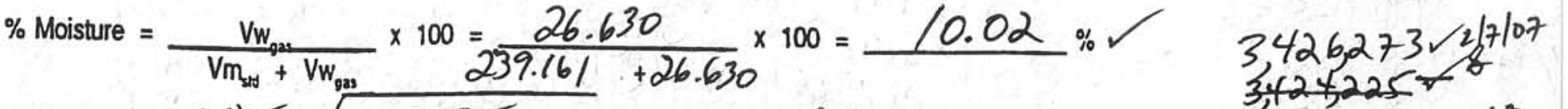

$$
\begin{aligned}
& v_{3}=5123.8 \times \frac{0.825}{\sqrt{29.41 \times 29.10}} \times 1.629=6553 \mathrm{~mm} \\
& \%=\frac{1.039 \times 239.161 \times 775}{6553 \times 0.8998^{\times} 29.41 \times 360 \times(0.177)^{2}}=98.5 \% 1
\end{aligned}
$$




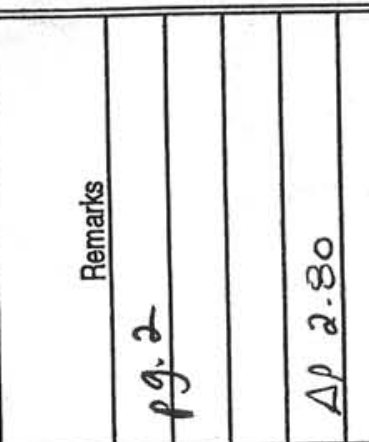

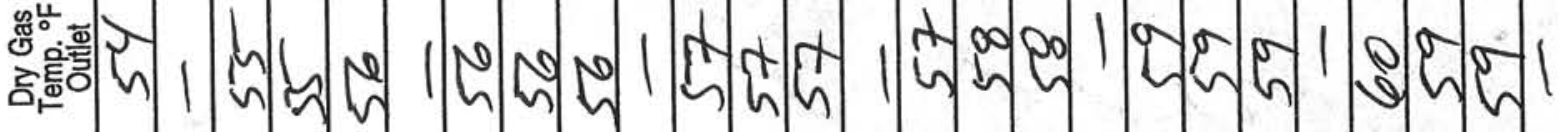
$\vdash^{E}$

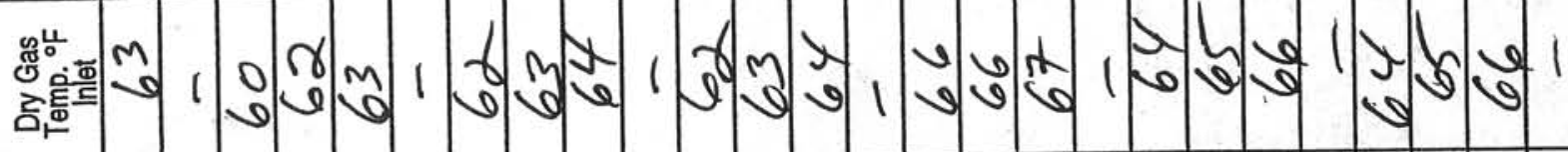

$\frac{m}{\frac{d}{d}}$

吕

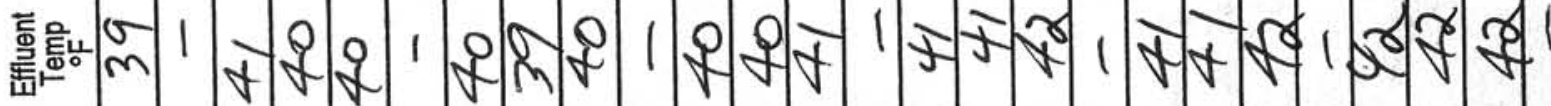
ठ․․

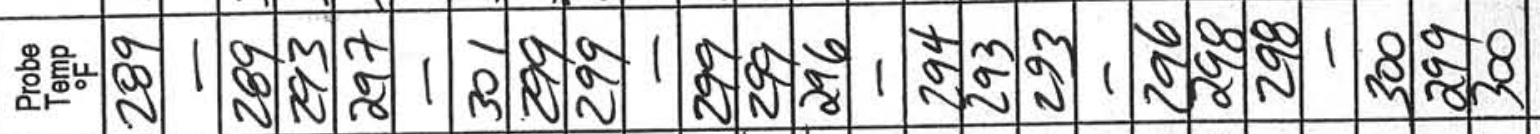
-

$\frac{N}{n}$

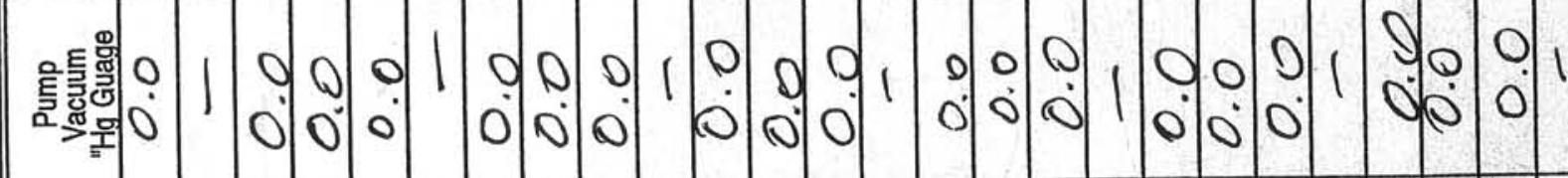

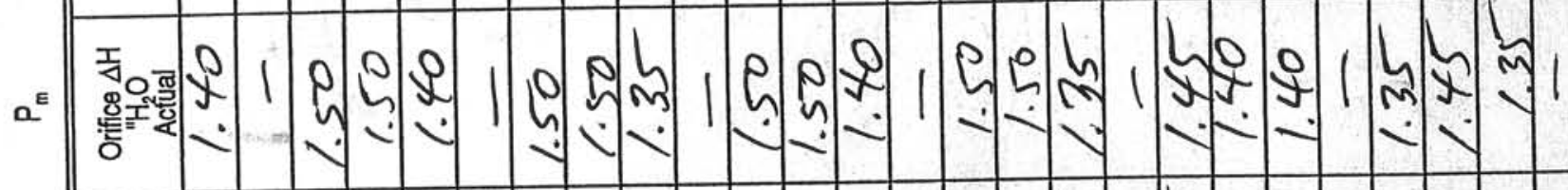

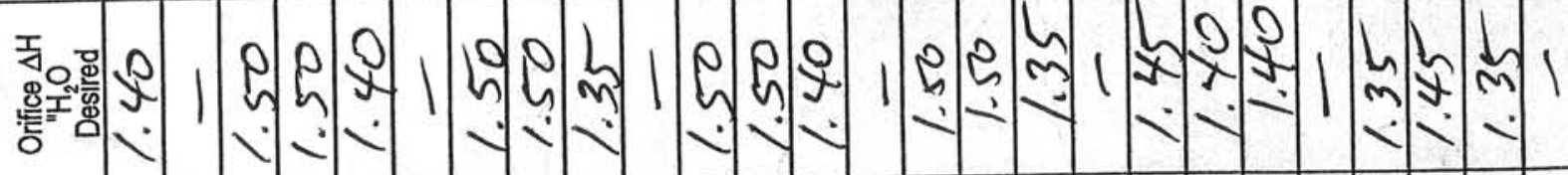

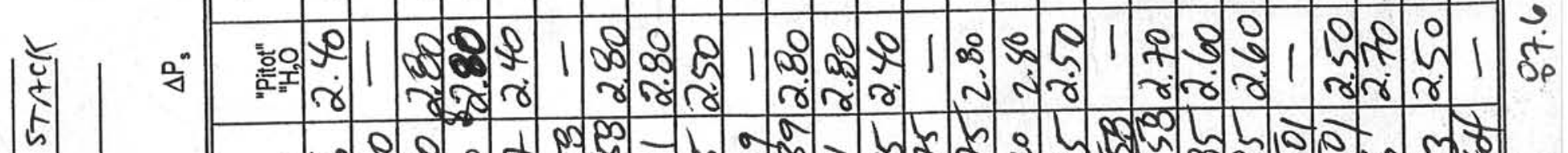
ช

0.4

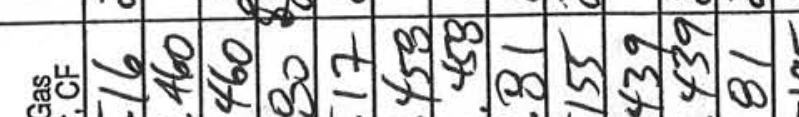
कका Wh 喜 突

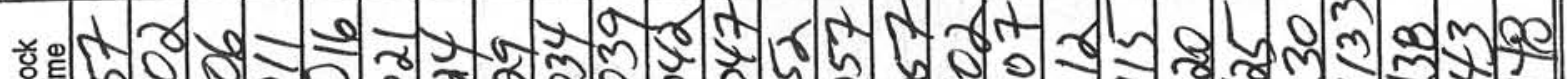

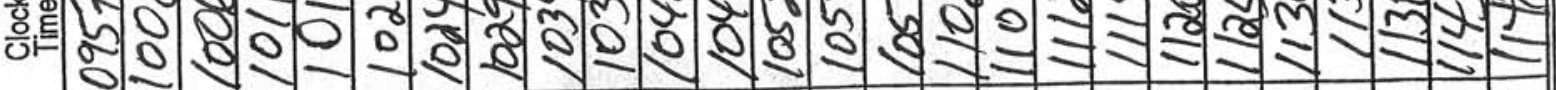

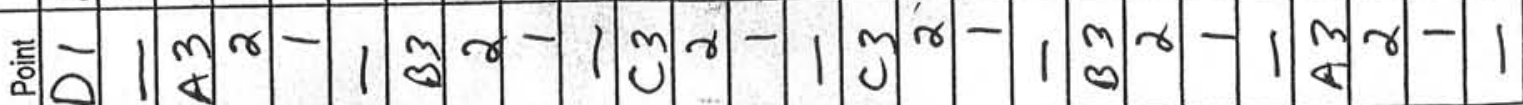









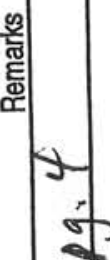

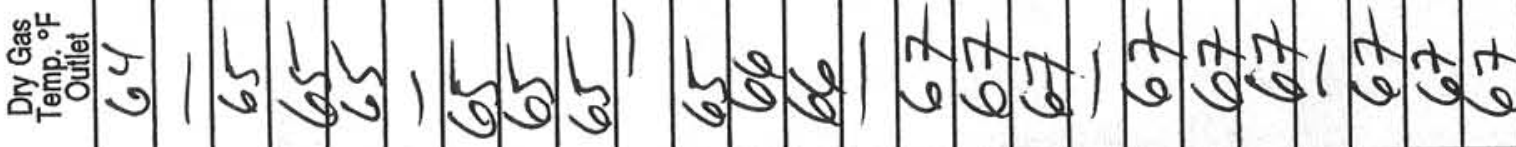
$\vdash^{E}$

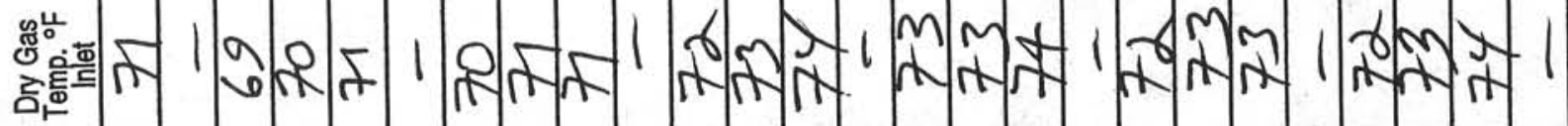

造

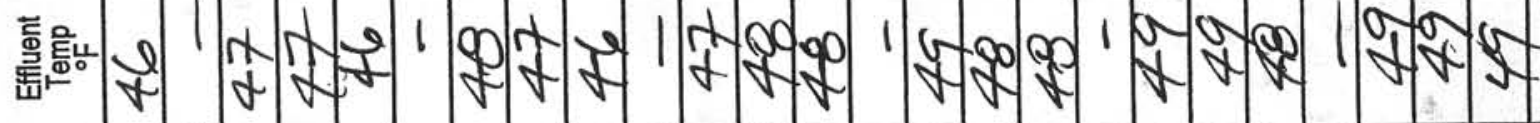

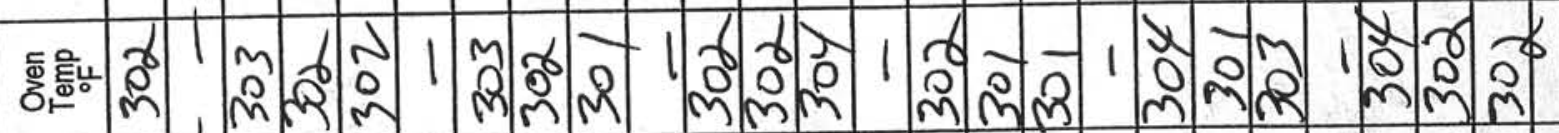

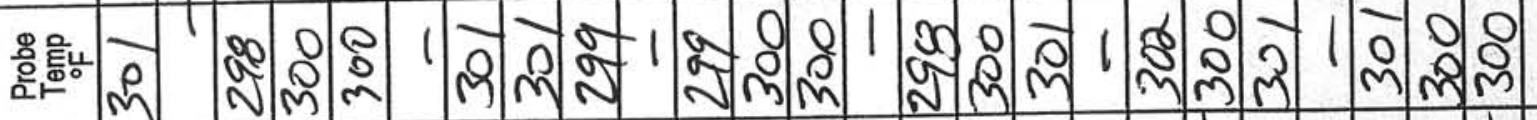

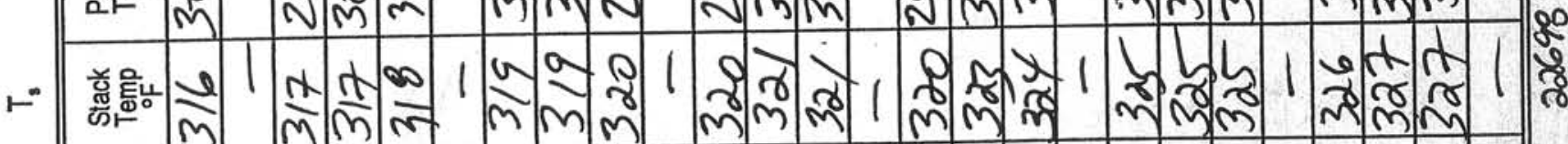

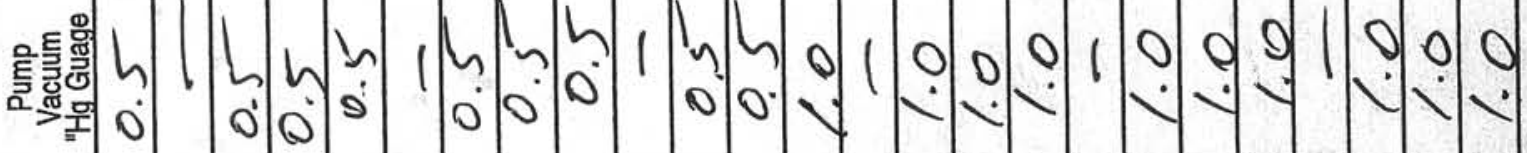

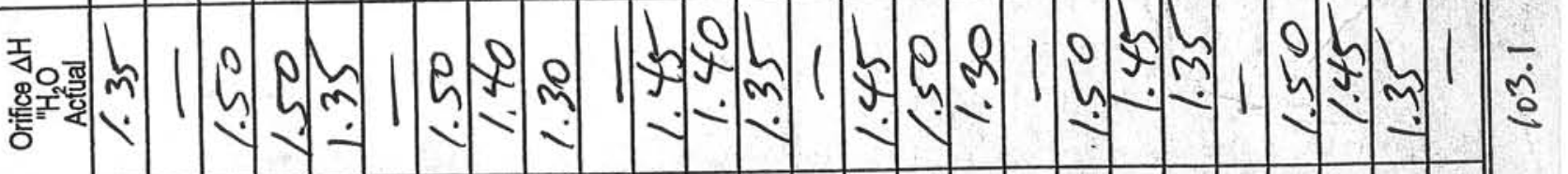

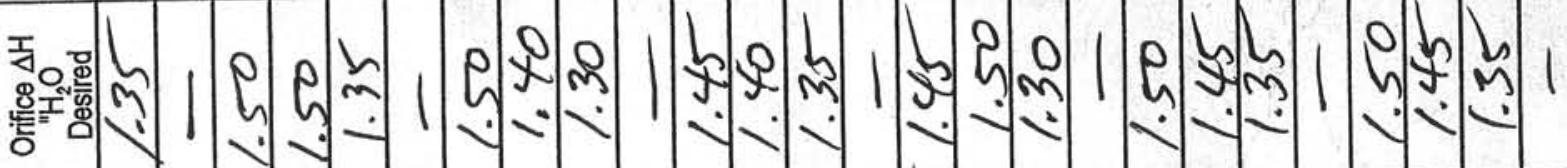

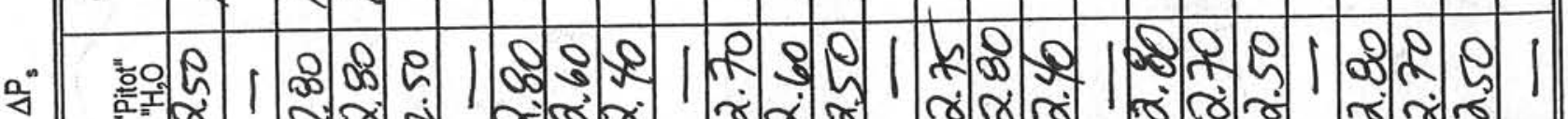
"ᄑ घँ

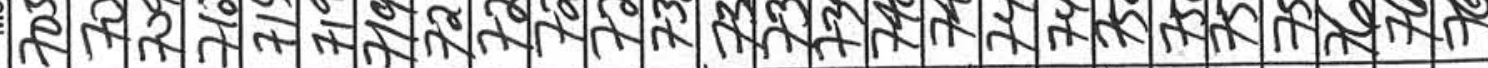

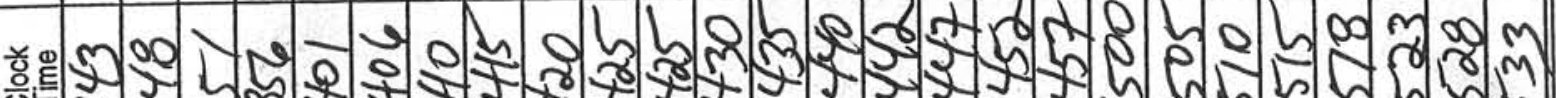

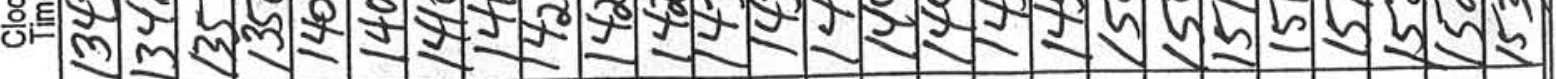

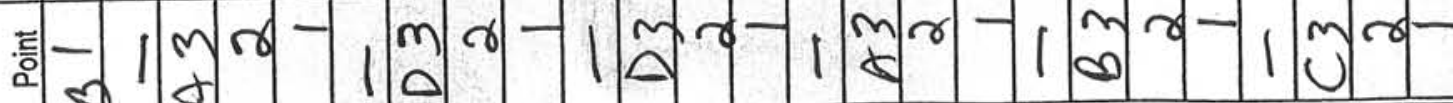





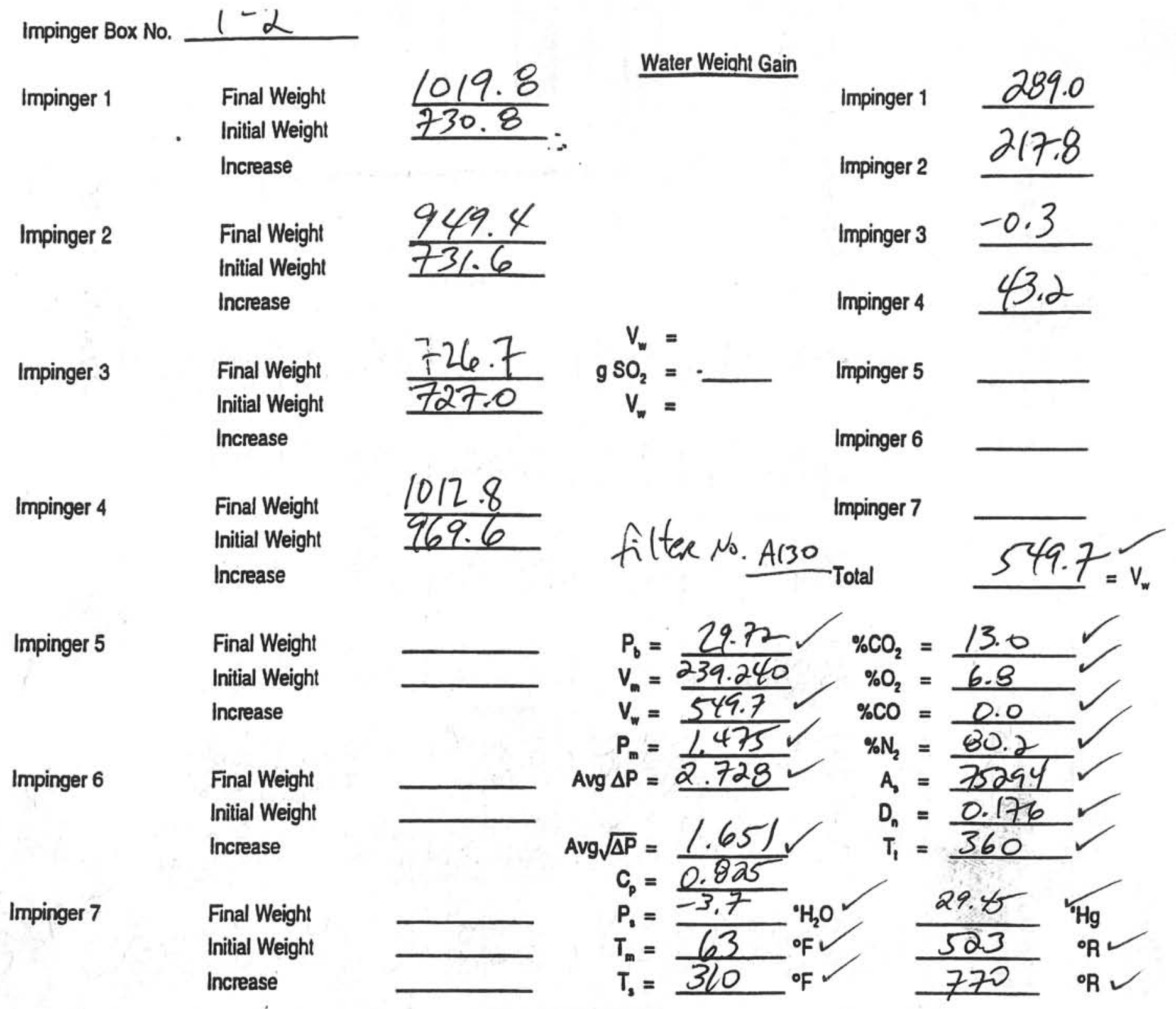

Moisture Content:

$$
\begin{aligned}
& \% \mathrm{M}=9.73 \sigma_{\mathrm{M}_{\mathrm{d}}}=0.9027 \mathrm{MW}_{\mathrm{c}}=30.352 / \mathrm{MW}=2 \mathrm{~g} .15 \\
& V m_{\text {std }}=17.65 \mathrm{Vm}\left[\frac{P_{b}+\frac{P_{m}}{13.6}}{T_{m}+460}\right]=17.65 \times 239.240\left[\frac{29.72+\frac{1.475}{13.6}}{63+460}\right]=\frac{240.828 \mathrm{sth}^{3}}{0.669} \mathrm{sctm}
\end{aligned}
$$

$$
\begin{aligned}
& V_{W_{0 s}}=0.0472 \times V_{W}=0.0472 \times \frac{549.7}{25.946} \mathrm{stt}^{3} \\
& \% \text { Moisture }=\frac{V_{w_{02}}}{V_{m_{21}}+V_{w_{023}}} \times 100=\frac{25.946}{25.946+} \times 100=9.73
\end{aligned}
$$

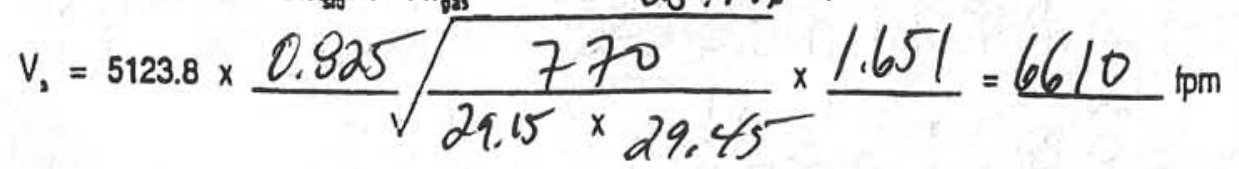

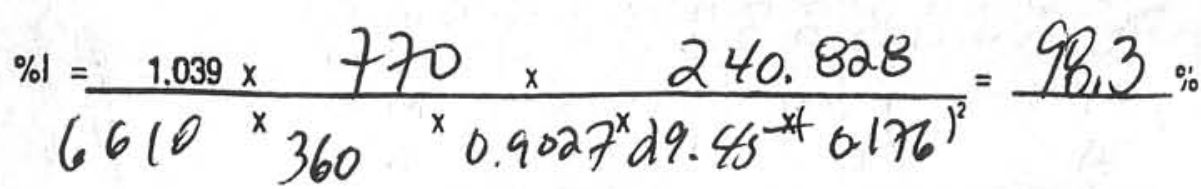

D-8

checked by: $\frac{\&}{1+1.2}$ 


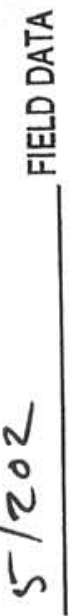

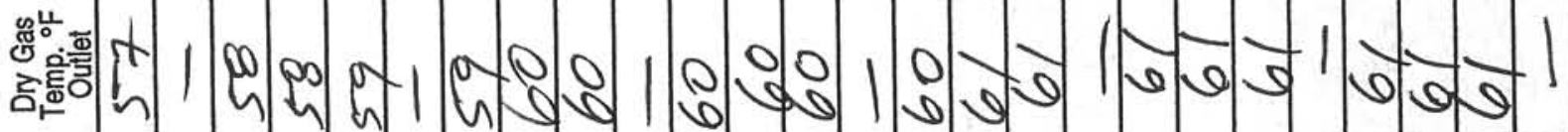

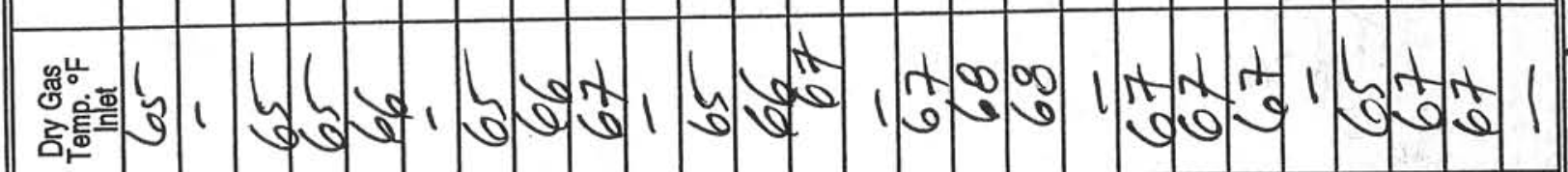

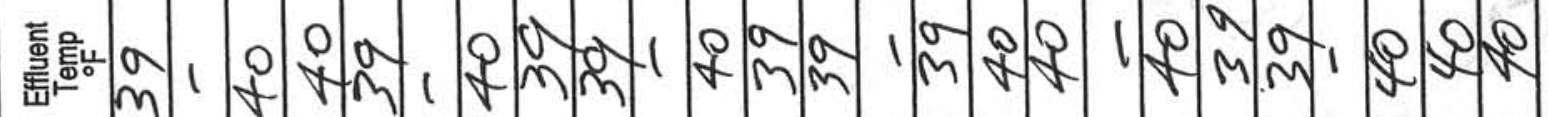

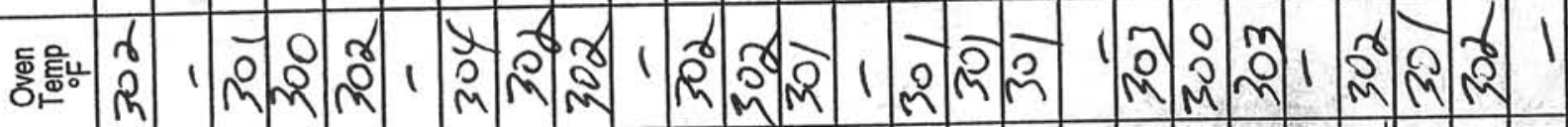

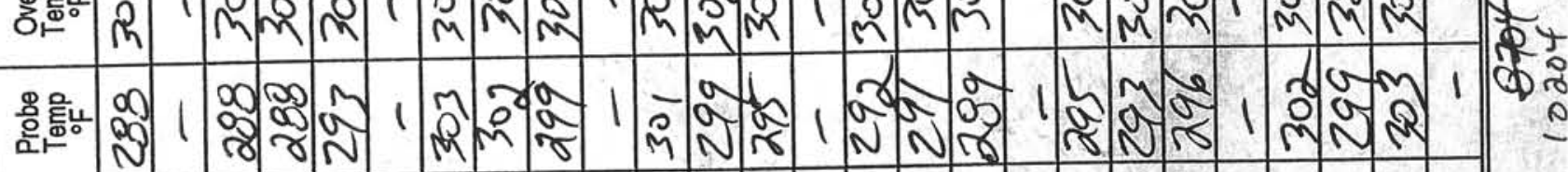

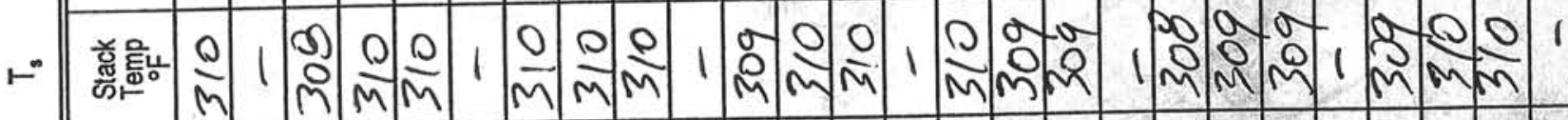

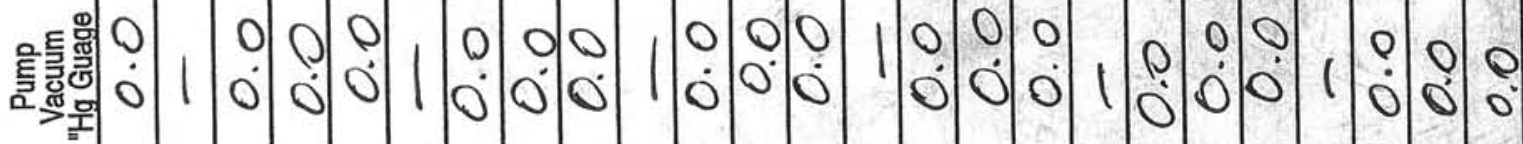
E

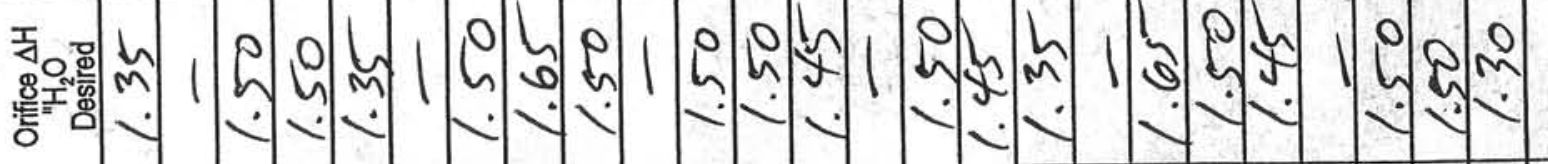

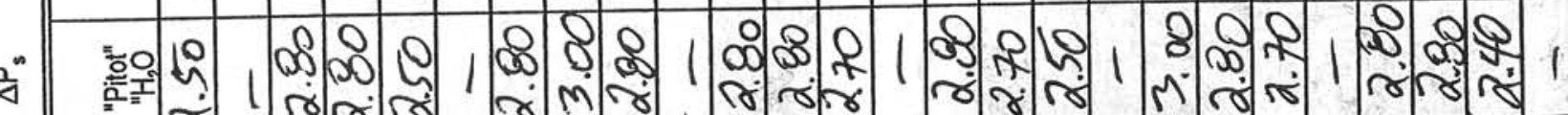

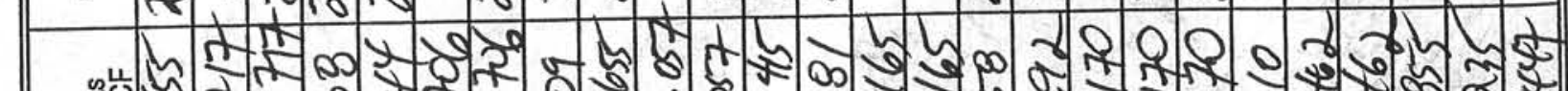

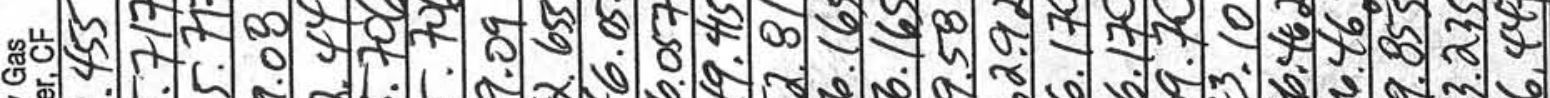

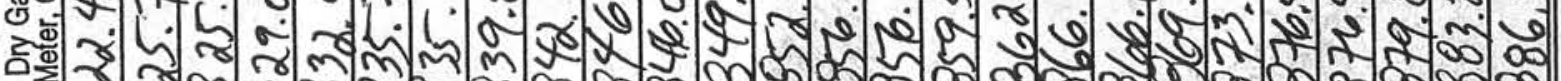

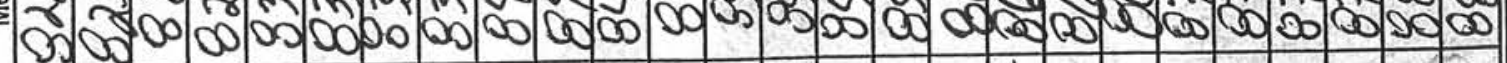

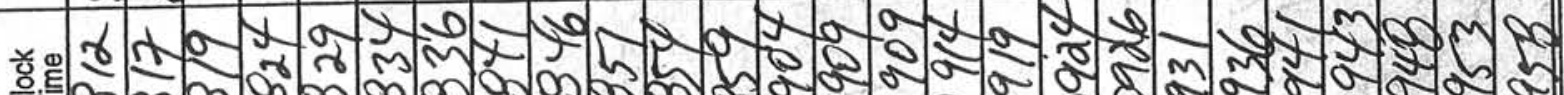
0.

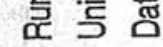

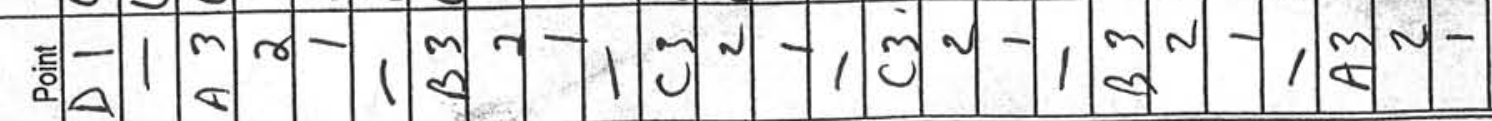


홍

of

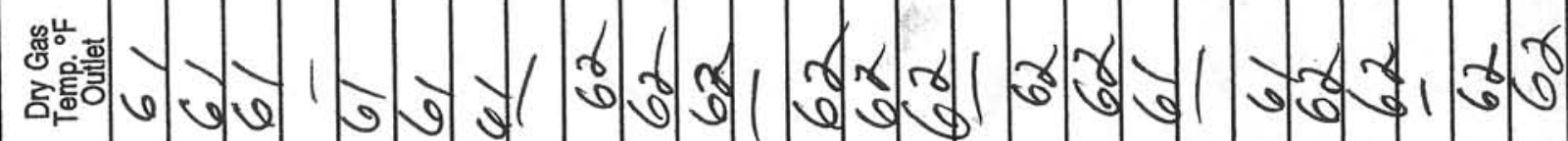
$\vdash$

"ूँ

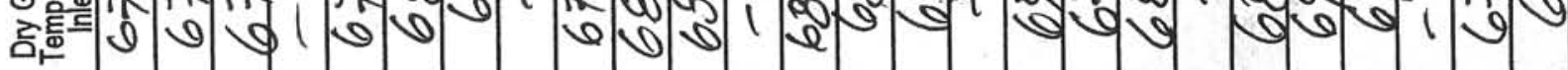

吕

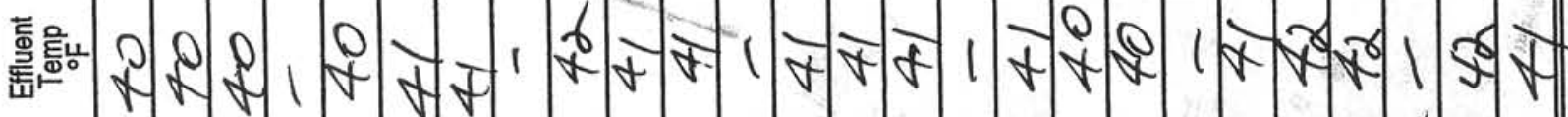
б... 윤.

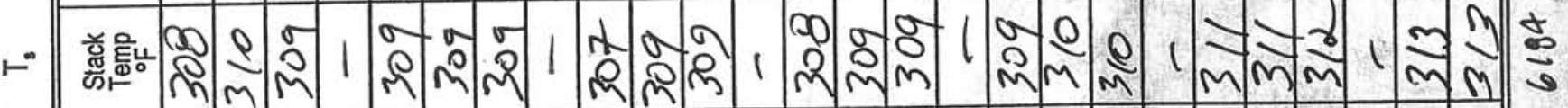

ñ
n

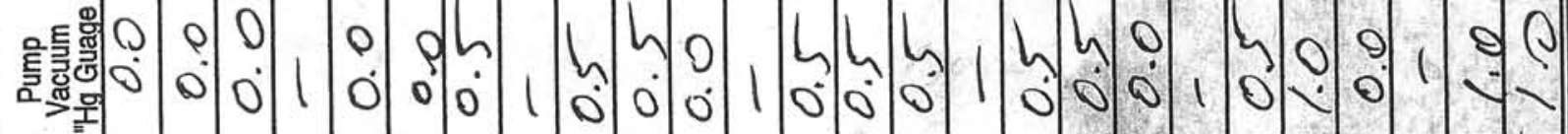

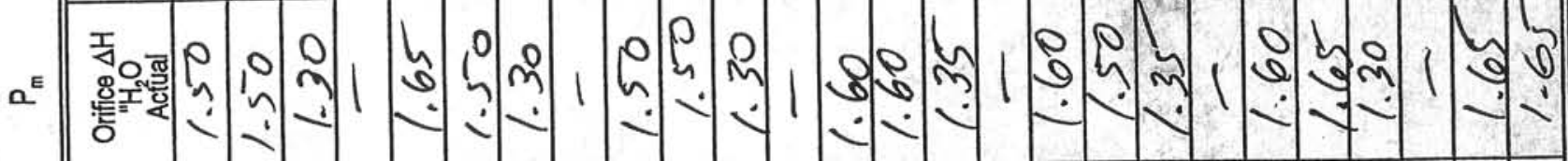

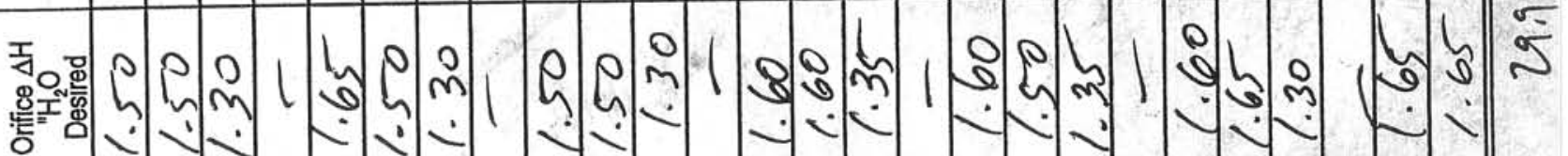

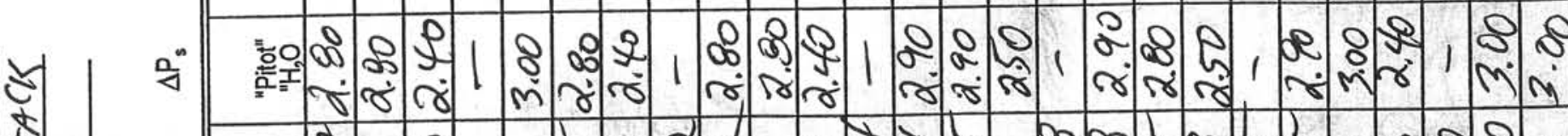

(t)

द्यु

:

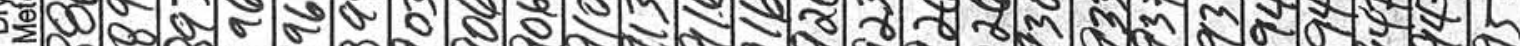

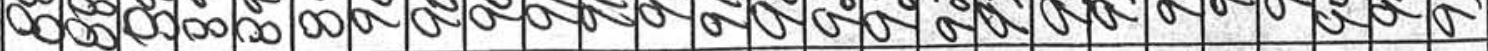

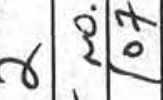

150

을

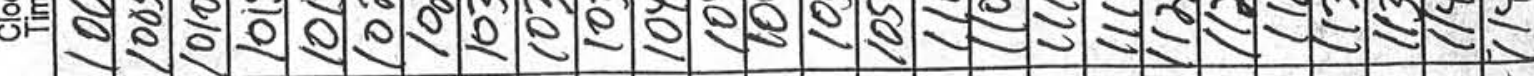

号芒

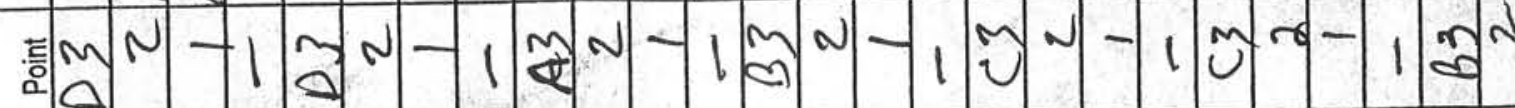




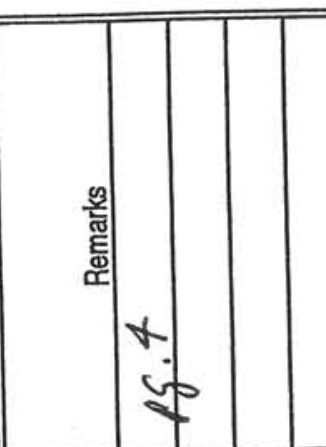

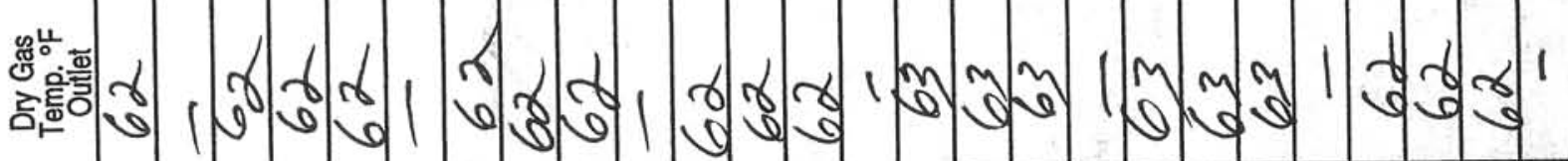
$\vdash$

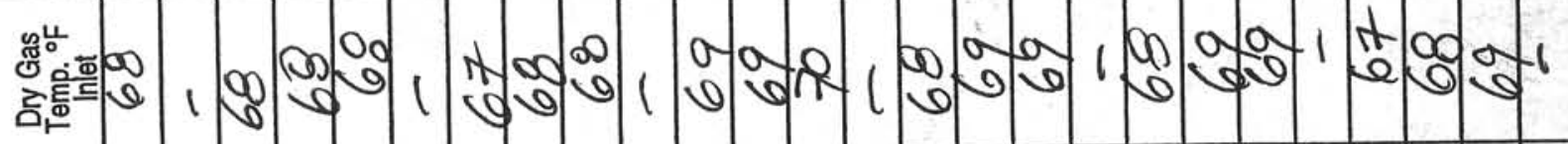

造

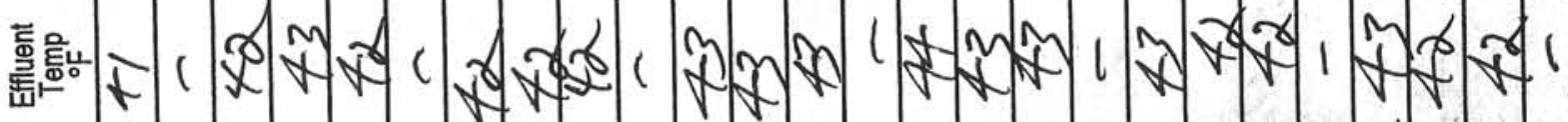

$\frac{1}{n}$

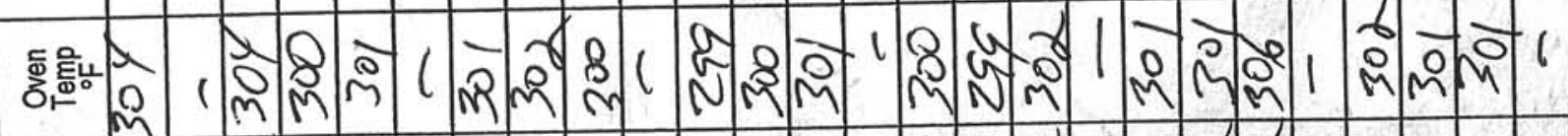

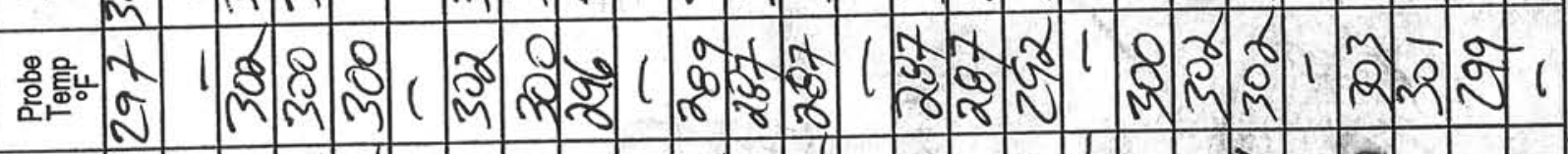

-

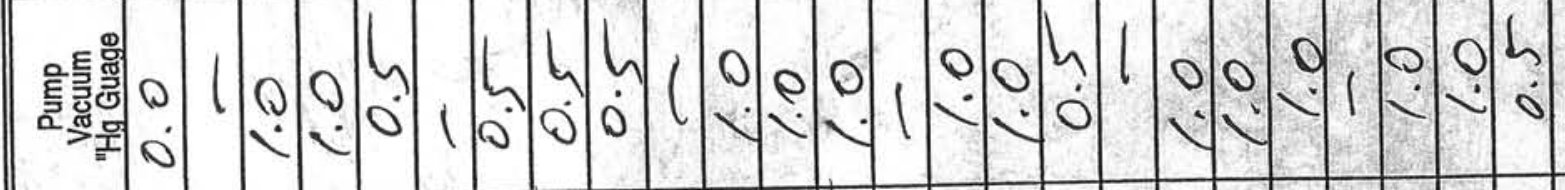

a

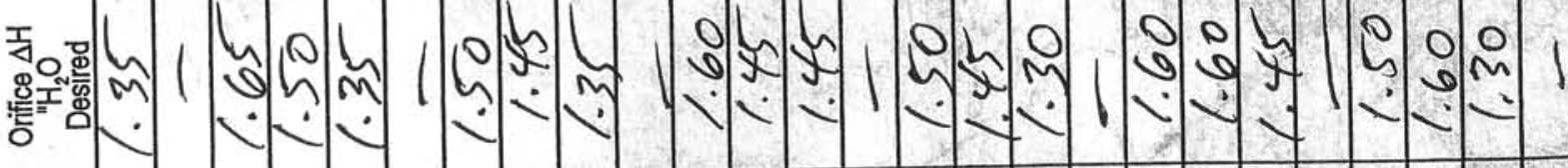

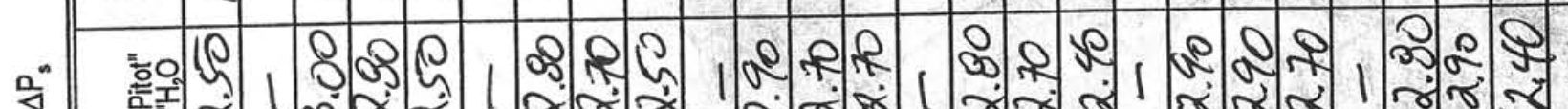

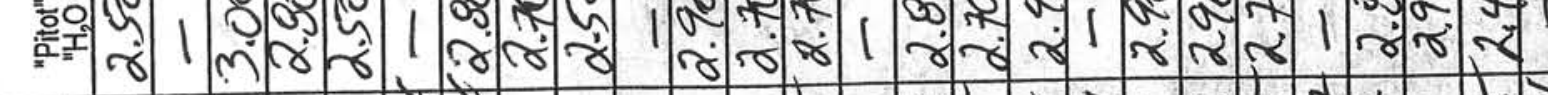
gu gु०

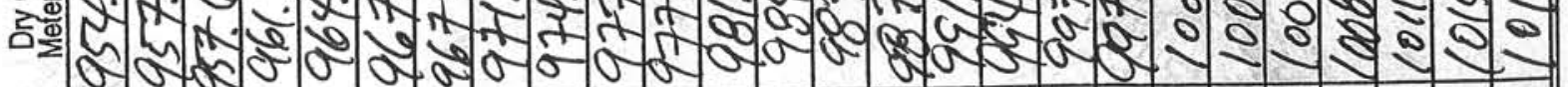

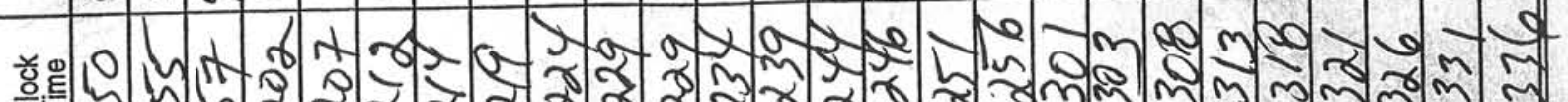

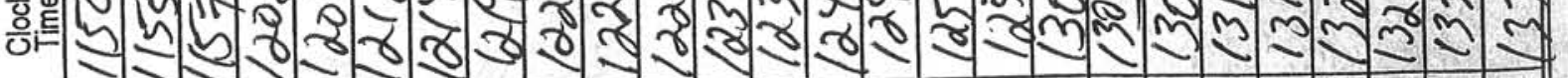

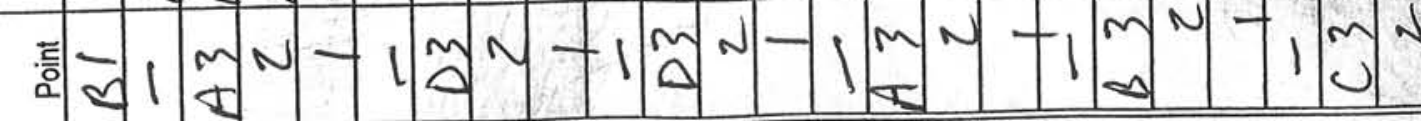




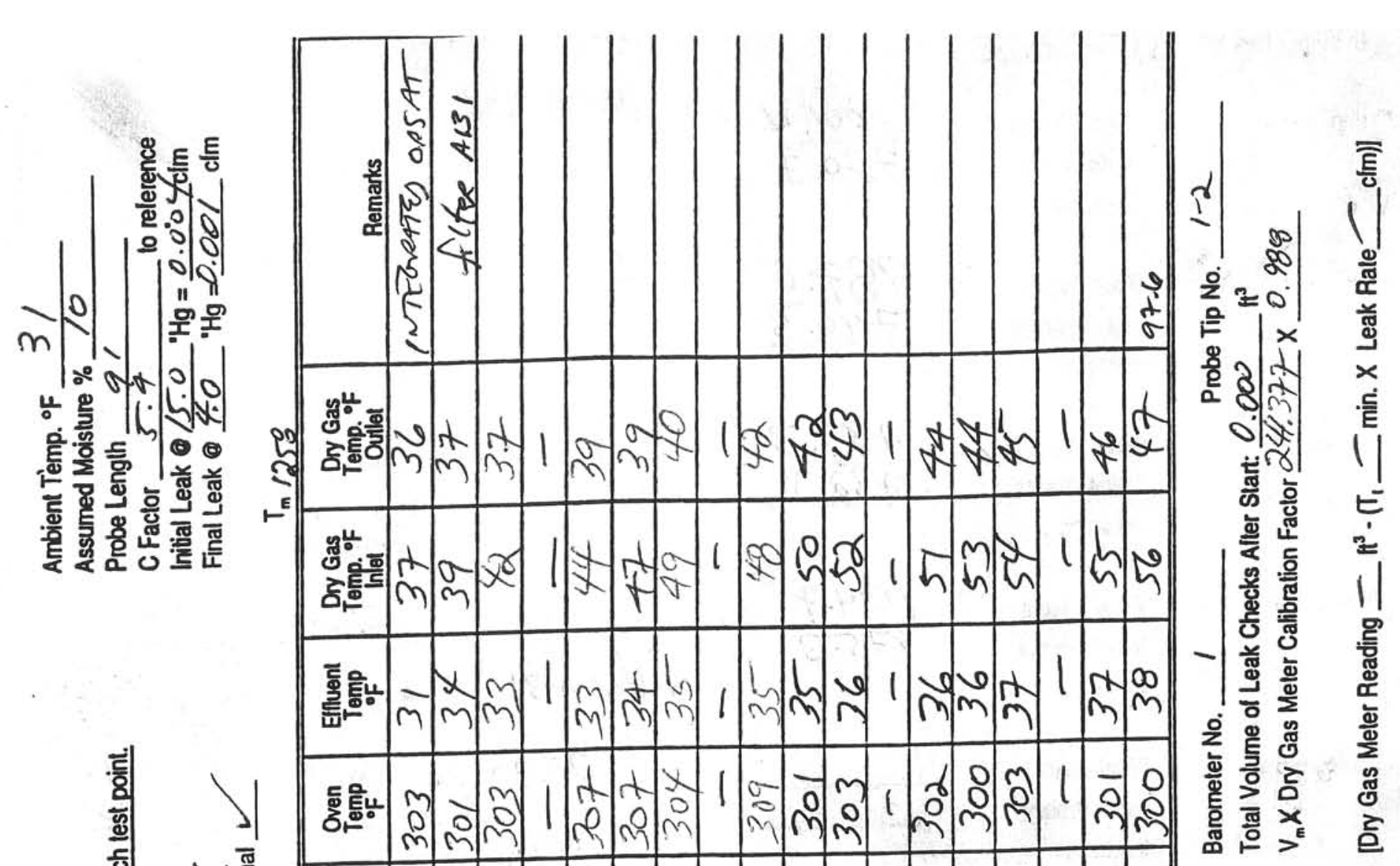


Impinger Box No.

$1-3$

Impinger 1 $\begin{aligned} & \text { Final Weight } \\ & \text { Initial Weight } \\ & \text { Increase }\end{aligned} \quad \frac{1001.4}{730,7}$

Water Weight Gain

Impinger 2

Final Weight

$\frac{987.0}{746.8}$

Initial Weight

increase

Impinger 3

Final Weight

Initial Weight

Increase

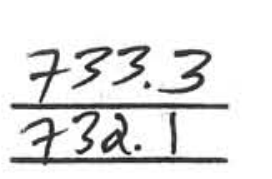

$\begin{array}{ll}\text { Final Weight } & \frac{10 / 4.7}{975.3} \\ \text { Initial Weight } & 975\end{array}$

Impinger 4

Increase

filter $A(3)$

impinger $1 \quad 270.7$

Impinger $2 \quad 240.2$

Impinger 3

1.2

Impinger 4

39.4

Impinger 5

Impinger 6

Impinger 7

Total

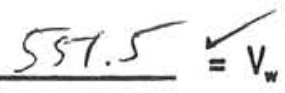

Impinger 5

Final Weight

Initial Weight

Increase

Impinger 6

Final Weight

$P_{b}=\frac{29.70}{239.480}$
$V_{m}=\frac{551.5}{1.504}$
$V_{w}=\frac{2.765}{P_{m}}$
$\Delta P=\frac{1}{25}$

Initial Weight

Increase

Avg $\triangle P=0.765$

$\operatorname{Avg} \sqrt{\Delta \mathrm{P}}=1.661$

$c_{p}=\frac{0.825}{3.55}$

$\begin{aligned} \% \mathrm{CO}_{2} & =13.0 \\ \% \mathrm{O}_{2} & =6.7 \\ \% \mathrm{CO} & =0.0 \\ \% \mathrm{~N}_{2} & =803\end{aligned}$

$A_{1}=75294$

$D_{n}=0.176$

$T_{1}=360$

$\mathrm{P}_{1}=-3.50{ }^{\circ} \mathrm{H}_{2} \mathrm{O}$

$T_{m}=\frac{54}{309}^{\circ} \mathrm{FV}$

$\frac{29.44}{7.4}{ }^{\circ} \cdot{ }^{\circ}$

Impinger 7

Final Weight

Initial Weight

Increase

$\mathrm{T}_{3}=309{ }^{\circ} \mathrm{F}$

$$
\begin{aligned}
& \% M=9.64{ }_{M}=0.9036{ }_{M w}=30.340 \quad M W=29.16 \\
& V m_{s t d}=17.65 \mathrm{Vm}\left[\frac{P_{b}+\frac{P_{m}}{13.6}}{T_{m}+460}\right]=17.65 \times 239.480\left[\frac{29.70+\frac{1.504}{13.6}}{54+460}\right]=\frac{244.120 \mathrm{stt}^{3}}{0.678}
\end{aligned}
$$

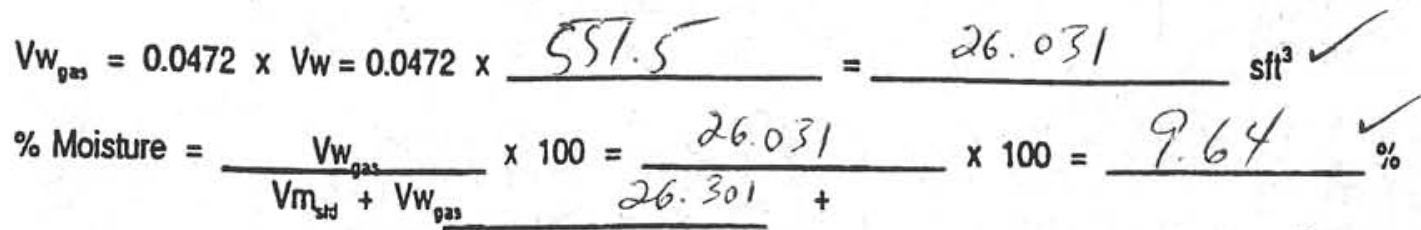

$v_{2}=5123.8 \times \frac{0.825}{\frac{769}{29.44 \times 29.16}} \times 1.661=6645 \mathrm{~mm}$

$\% 1=\frac{1.039 \times 769 \times 244.120}{6645 \times 0.9036 \times 29.44 \times 360 \times(0.176)^{2}}=18,9 \%$

ACFM: 3474,675 SCFM: 2128,865 


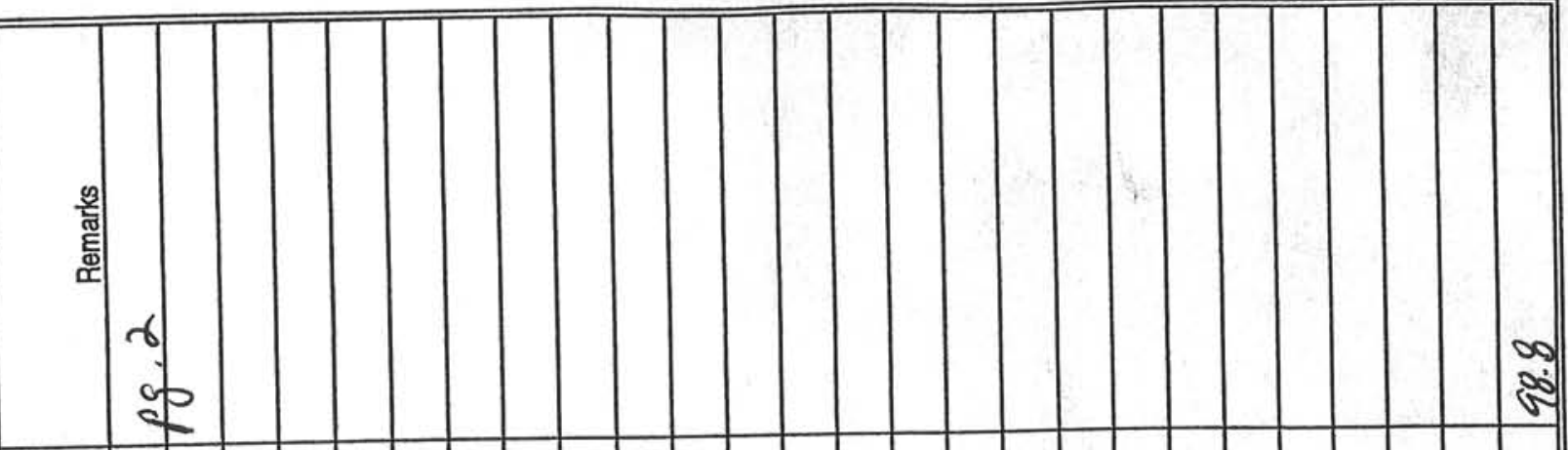

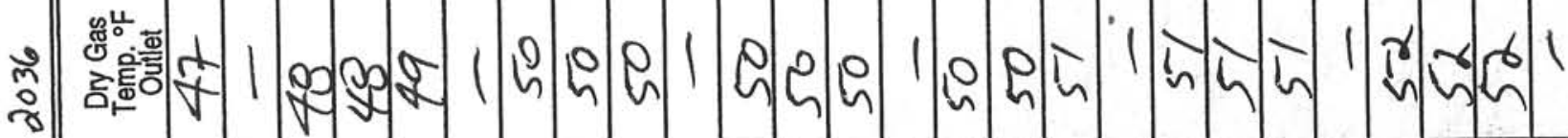
$\vdash^{E}$

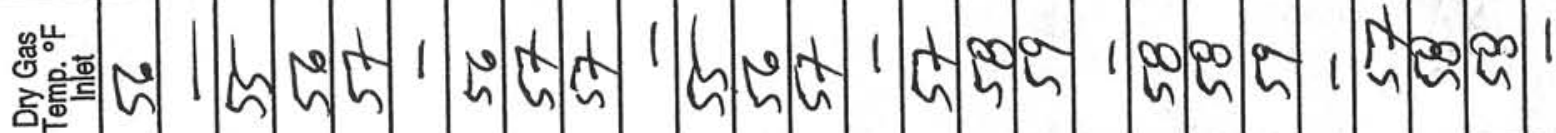

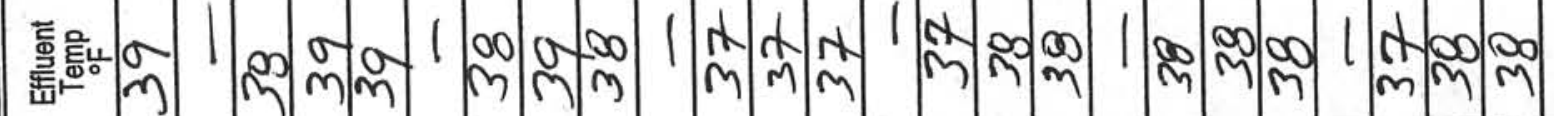

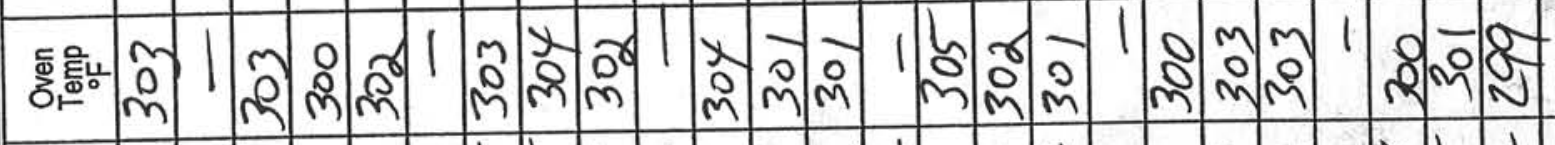

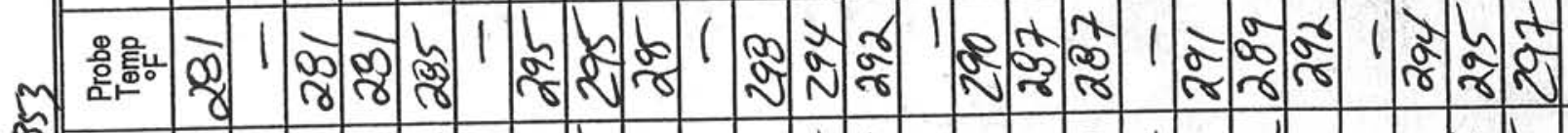

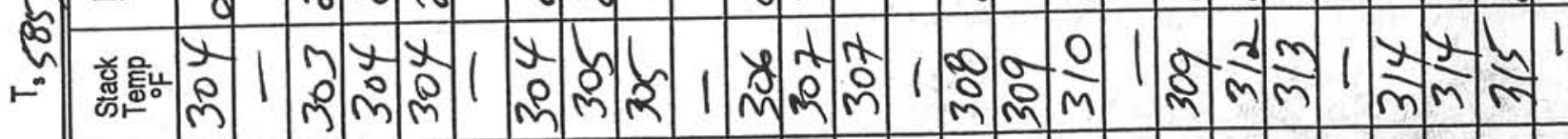

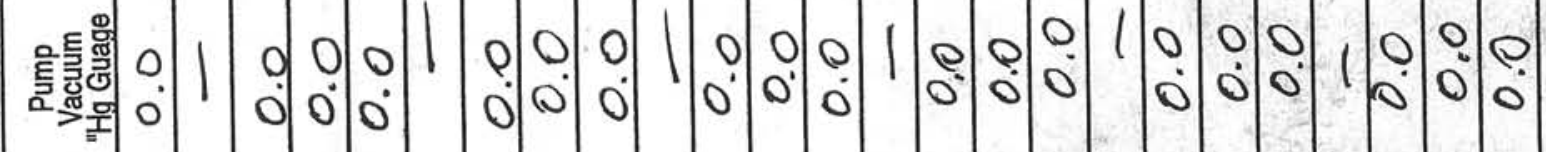
年

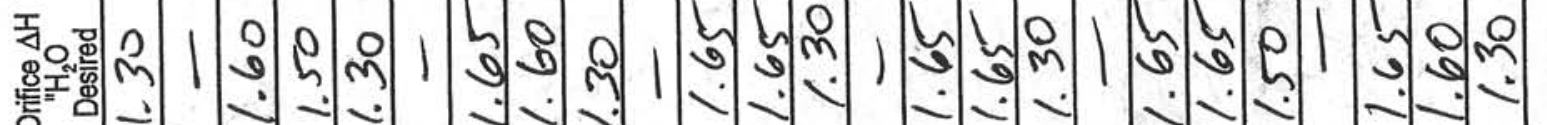
(1)

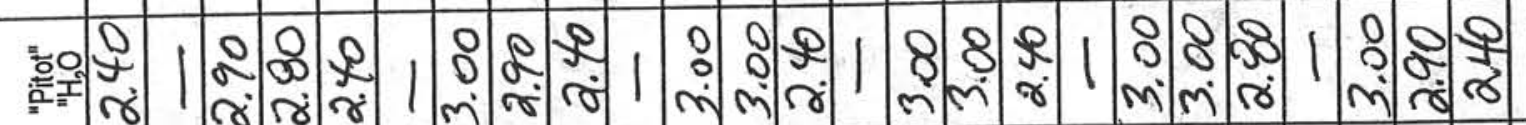

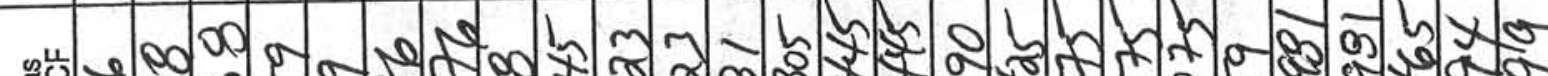
a ร.

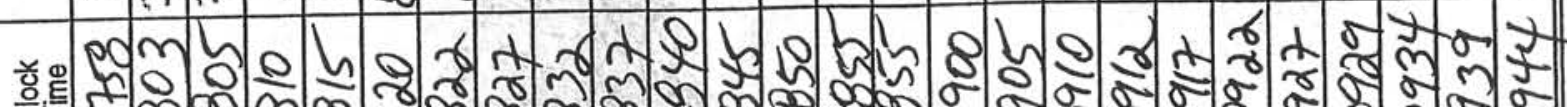
on 0 \% : 


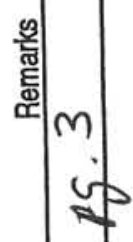

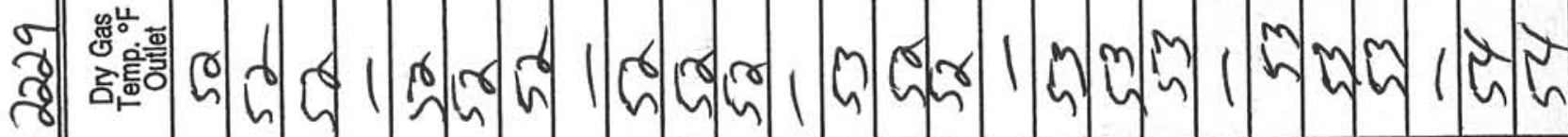

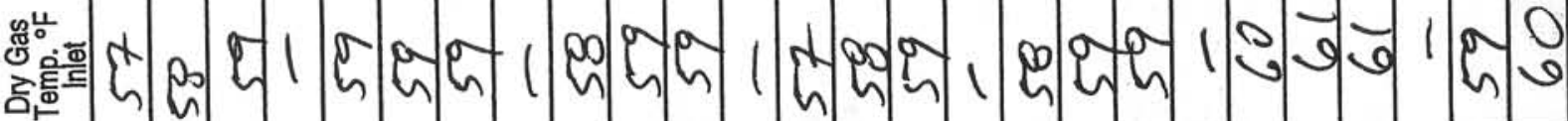

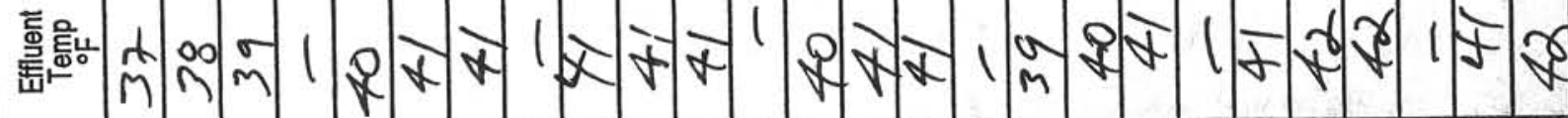

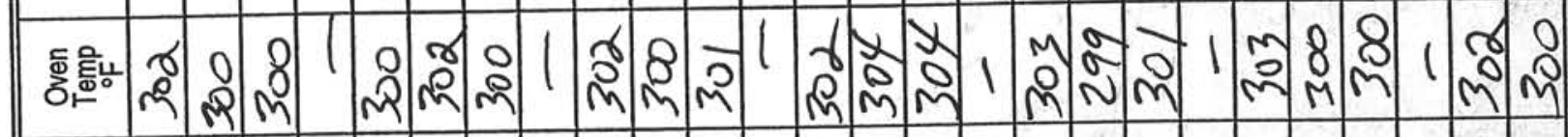

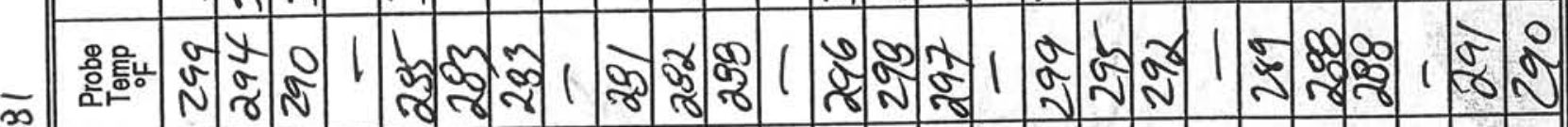

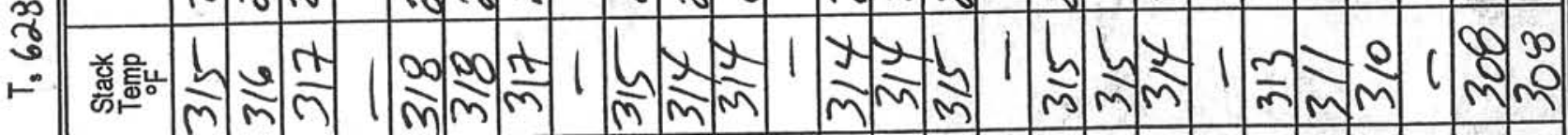

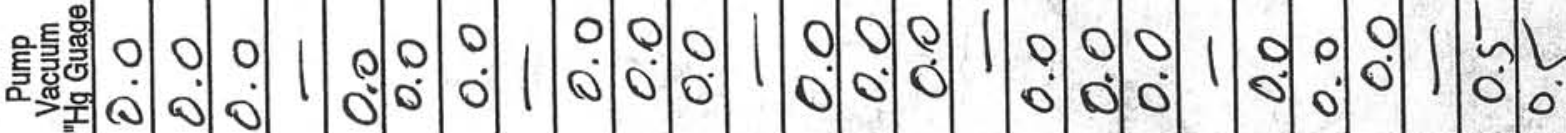

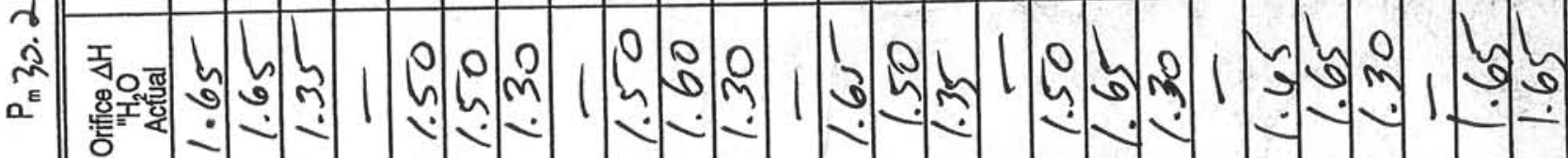

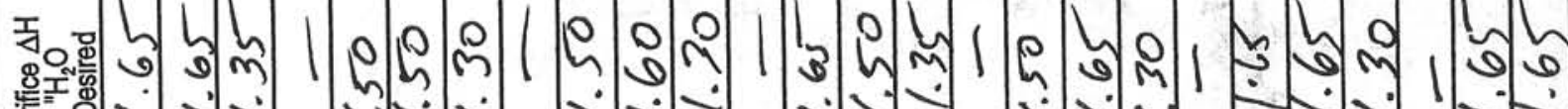
万ह

एक.

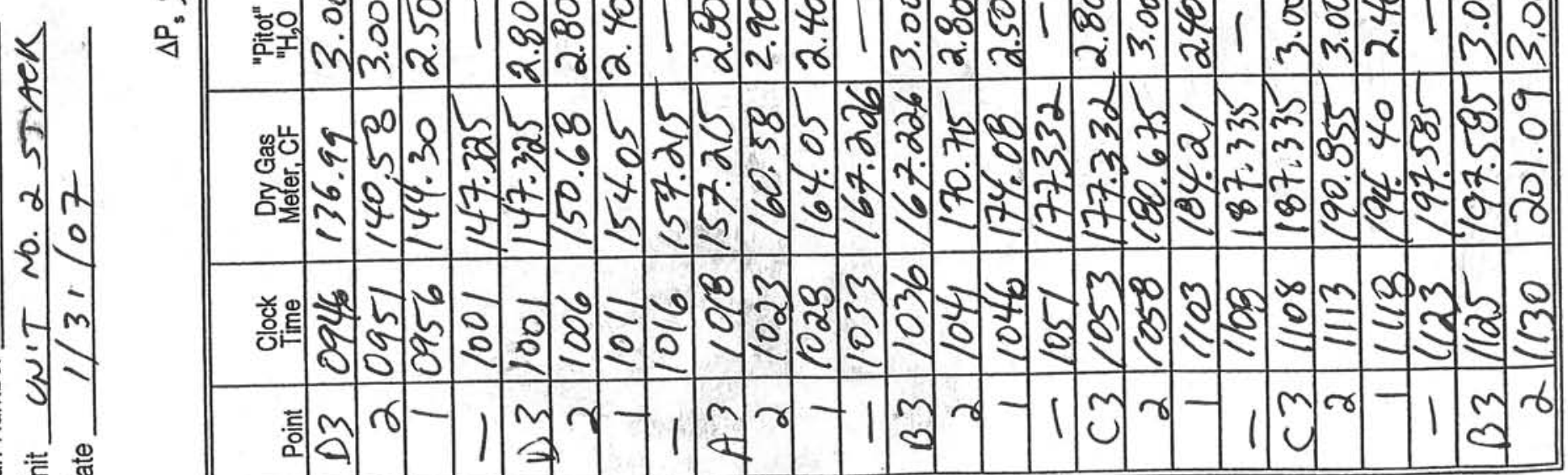
喜容带 


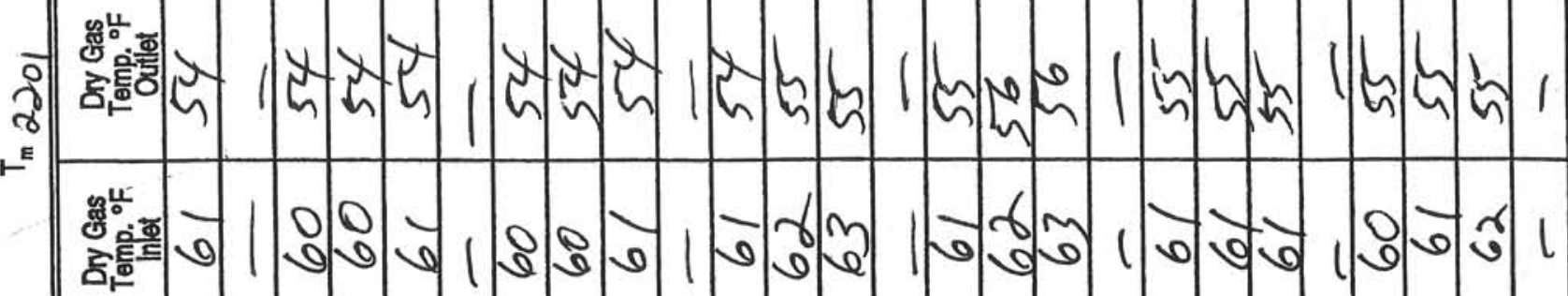

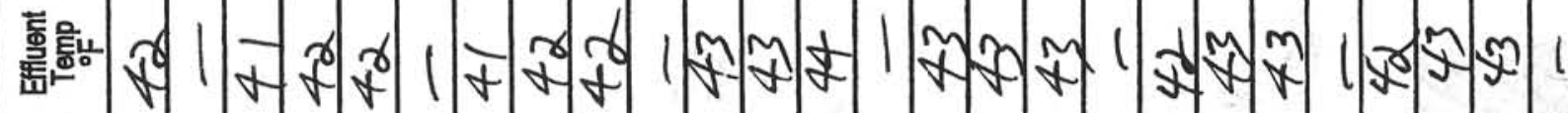

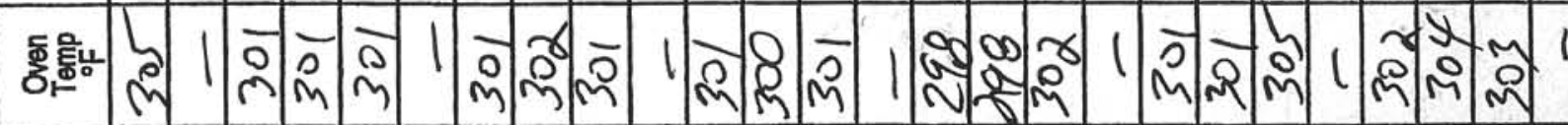
8. ํㅜㄴ

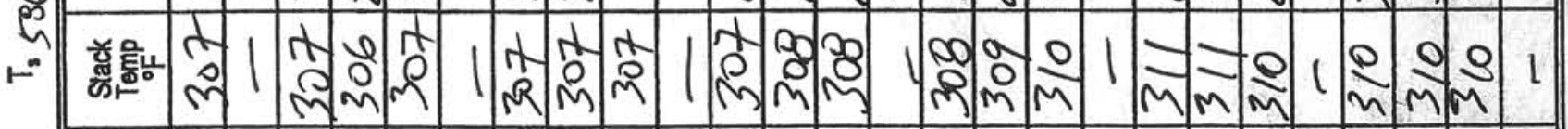

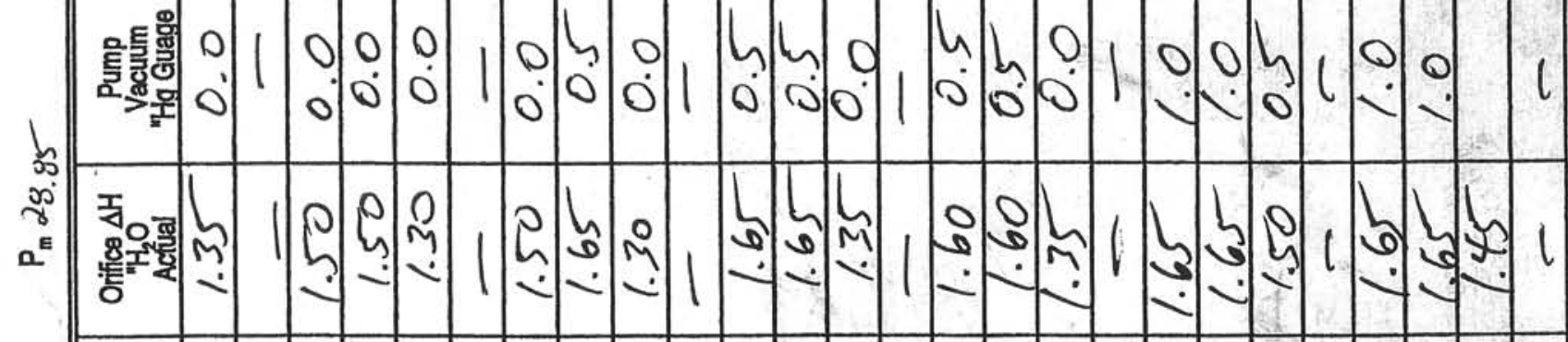

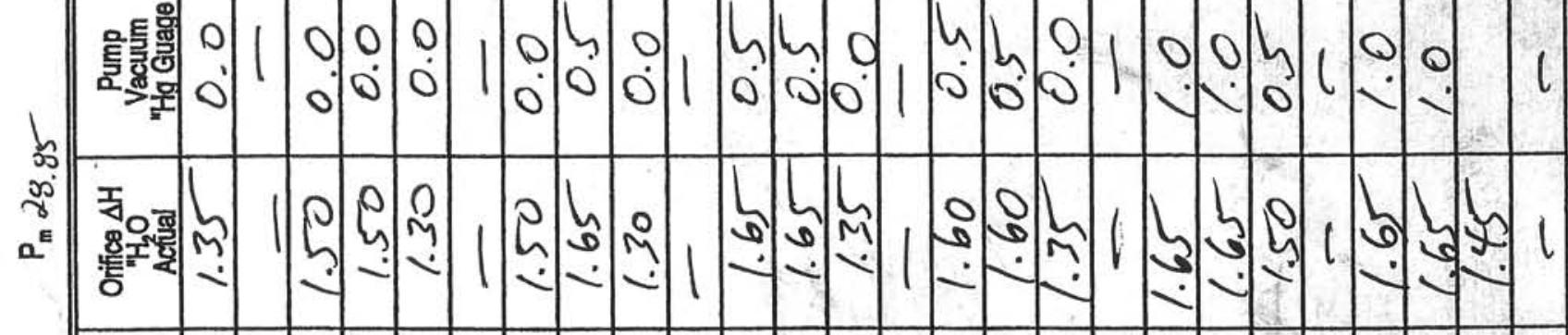

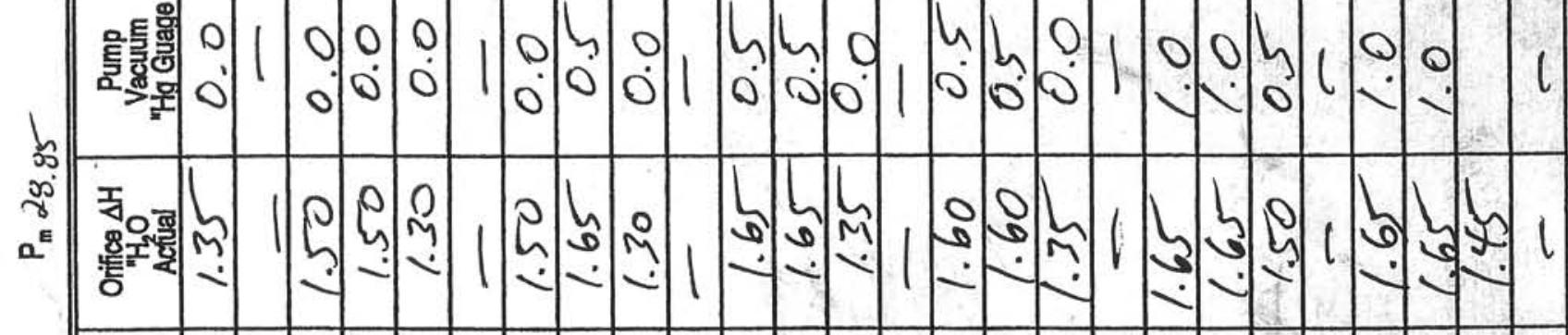

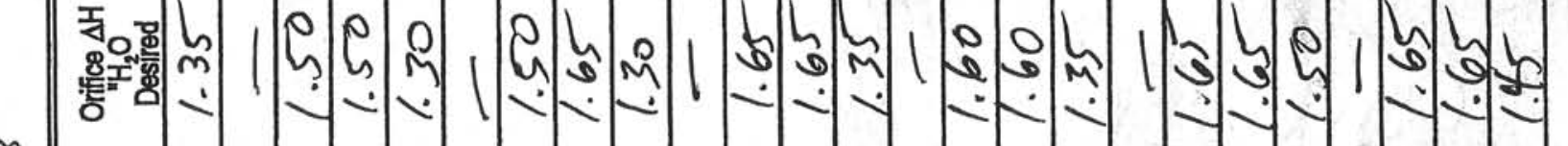

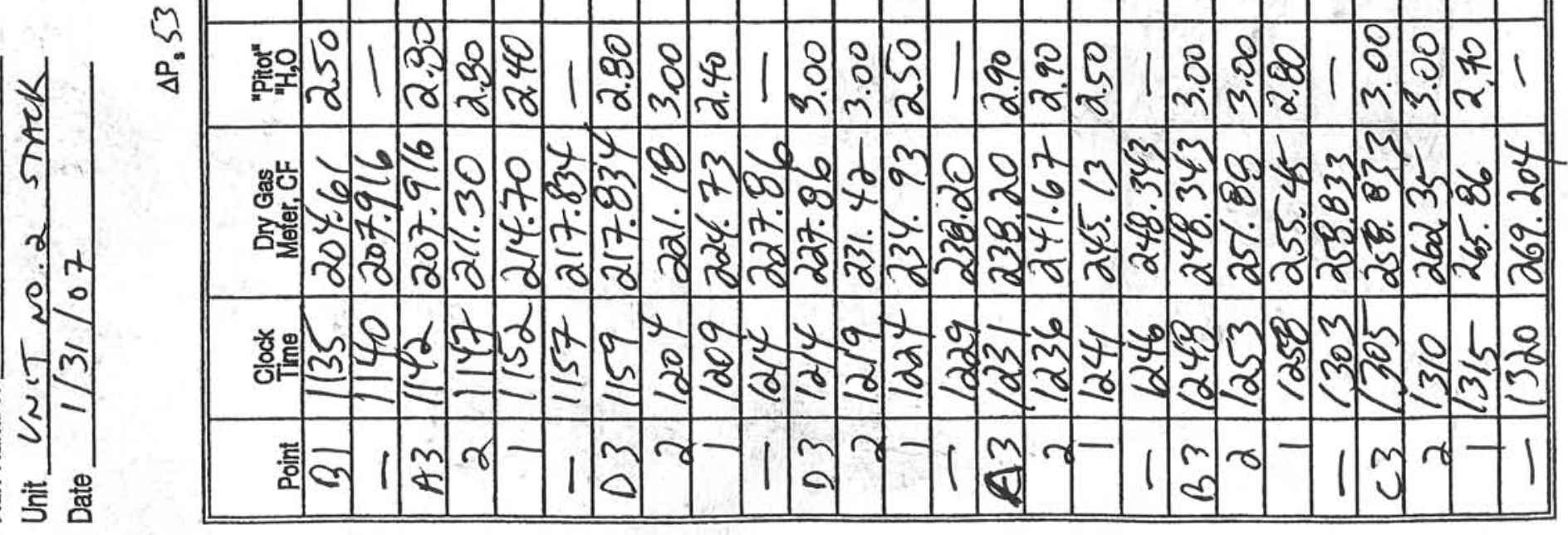
0
0
0
0

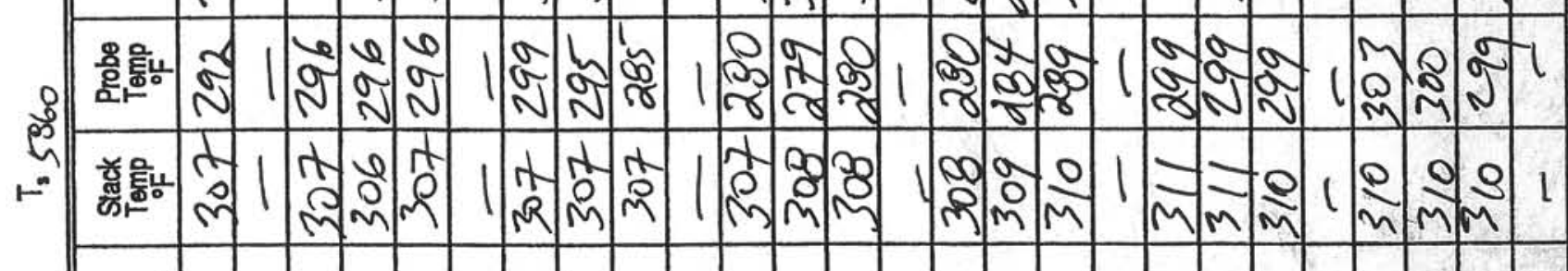

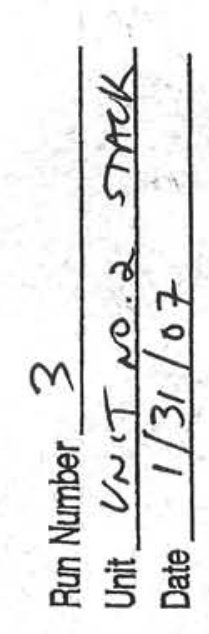





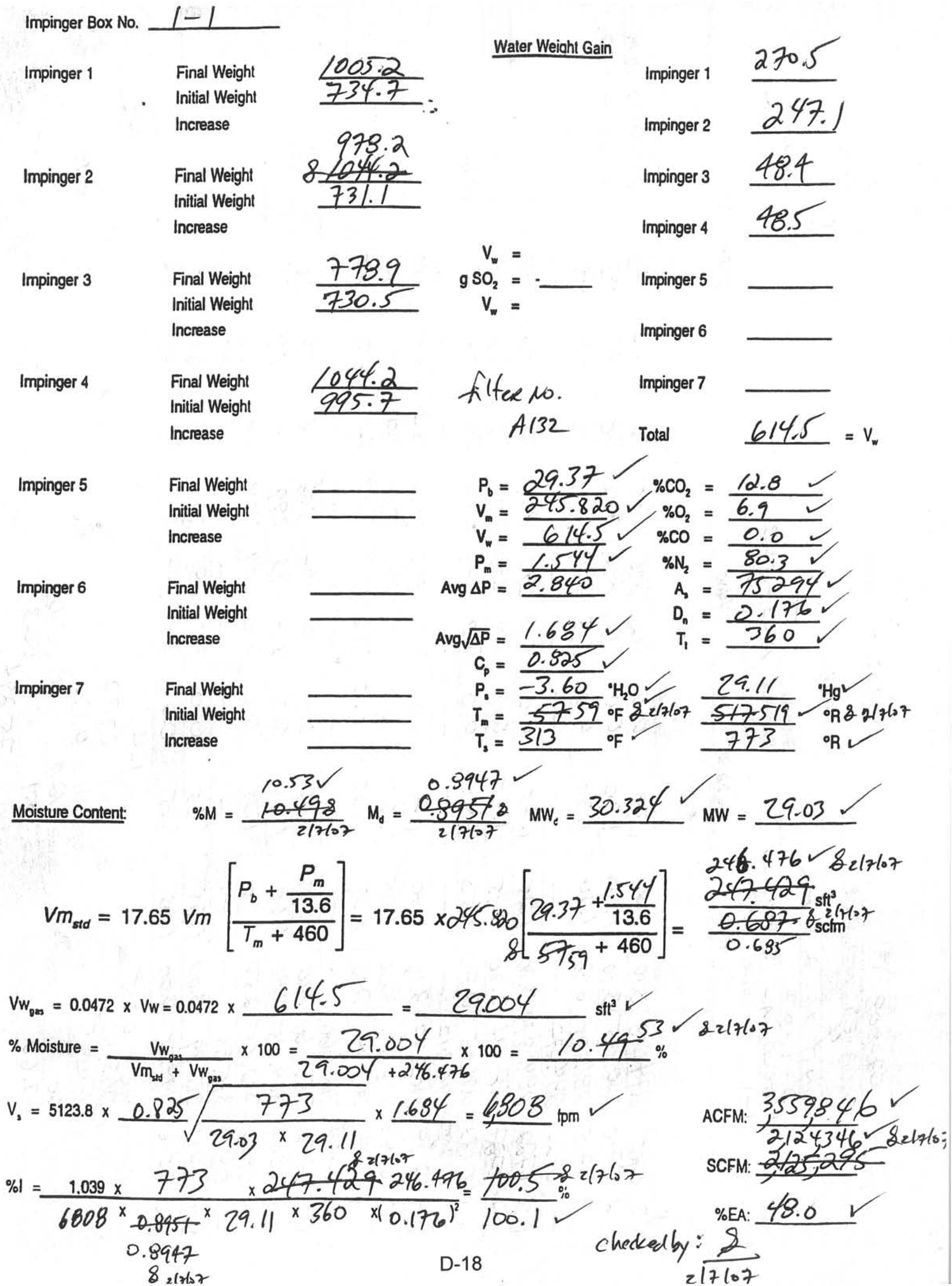


旡
畐

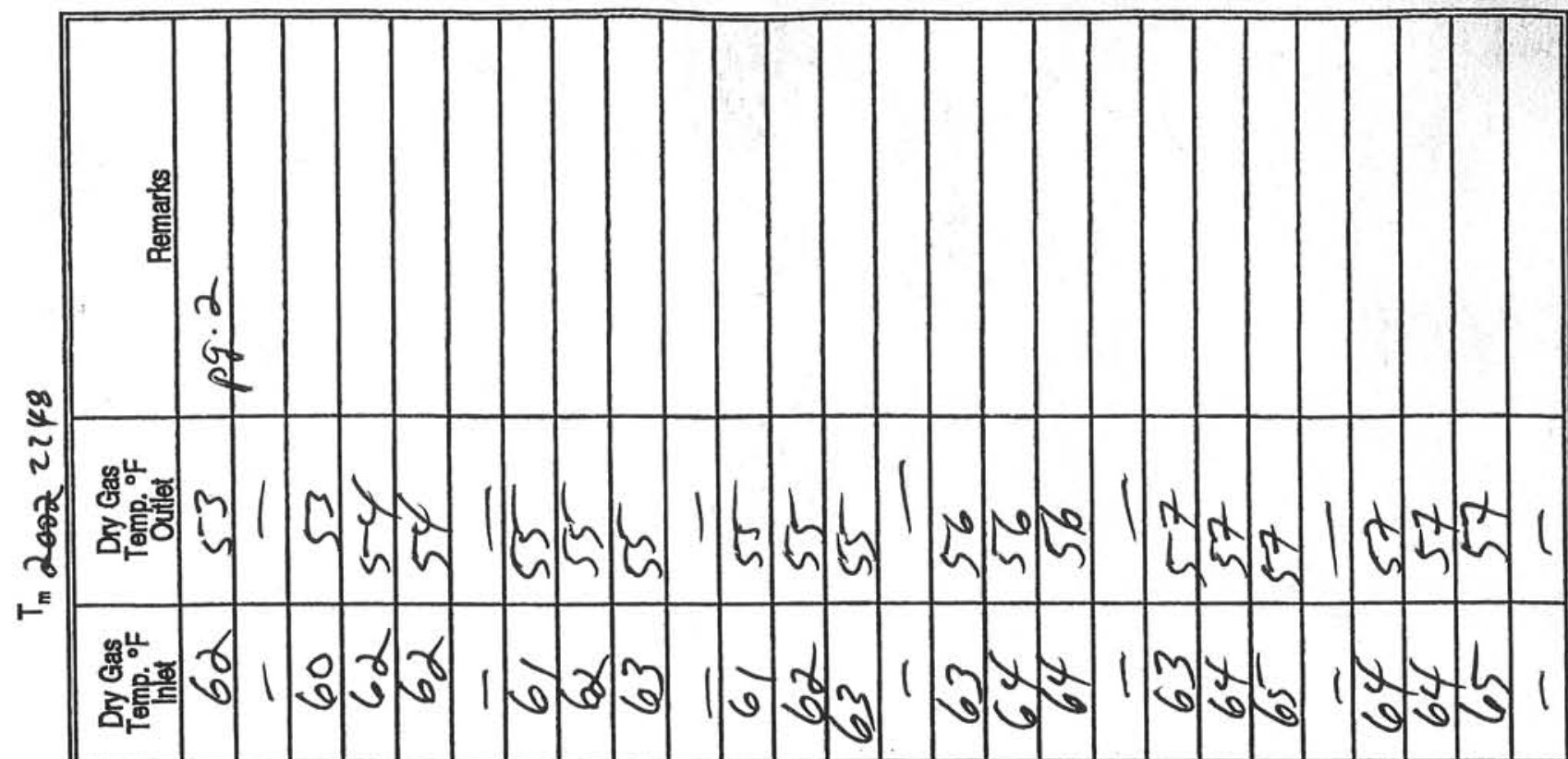

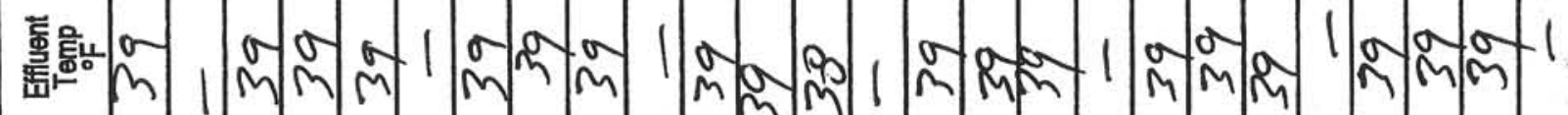

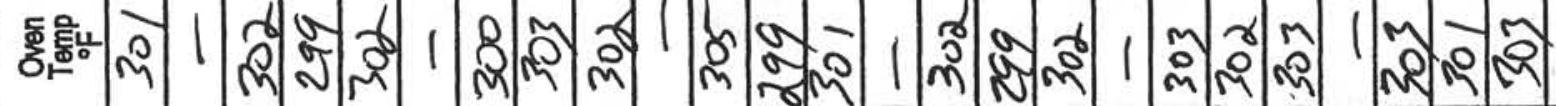
N

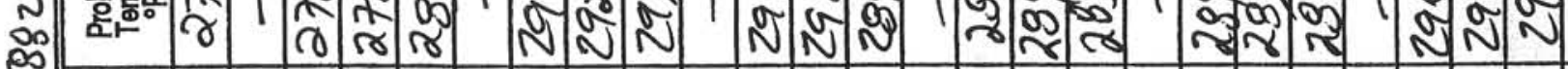

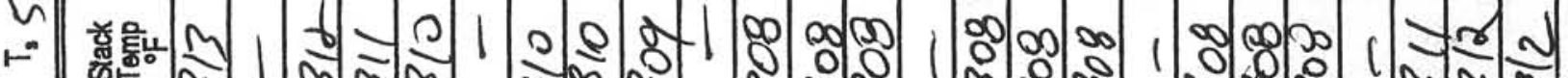
ॠँ

$\frac{n}{n}$

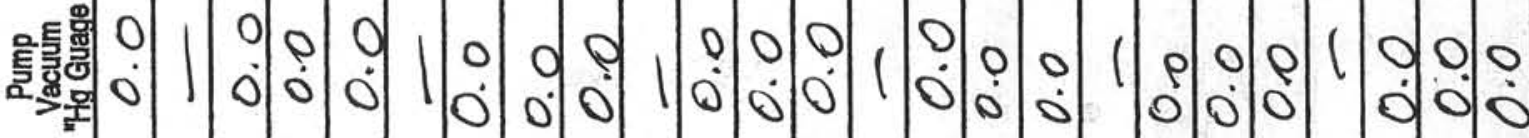

d

م

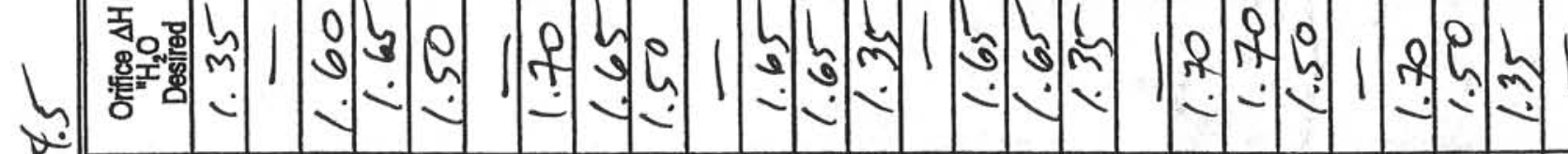

2.

h "원

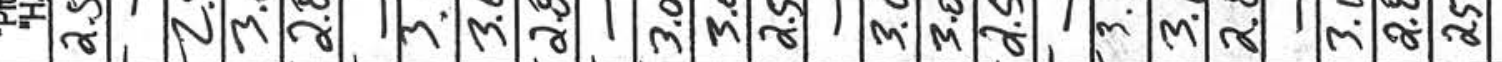
हु० नी व: $7 \leq \frac{1}{1}$

点完要

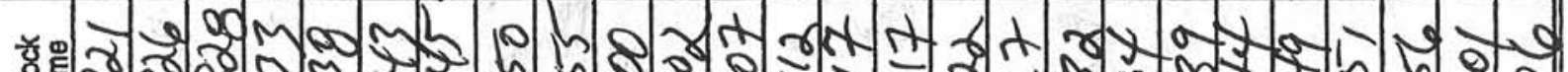
ठำ

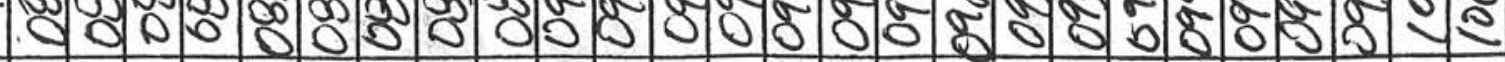

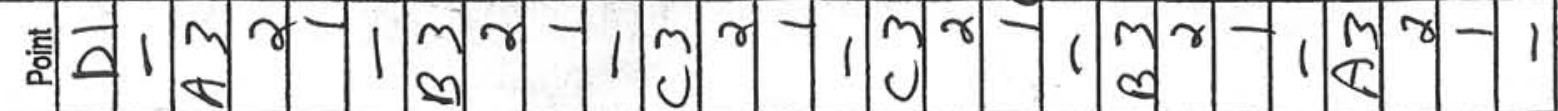




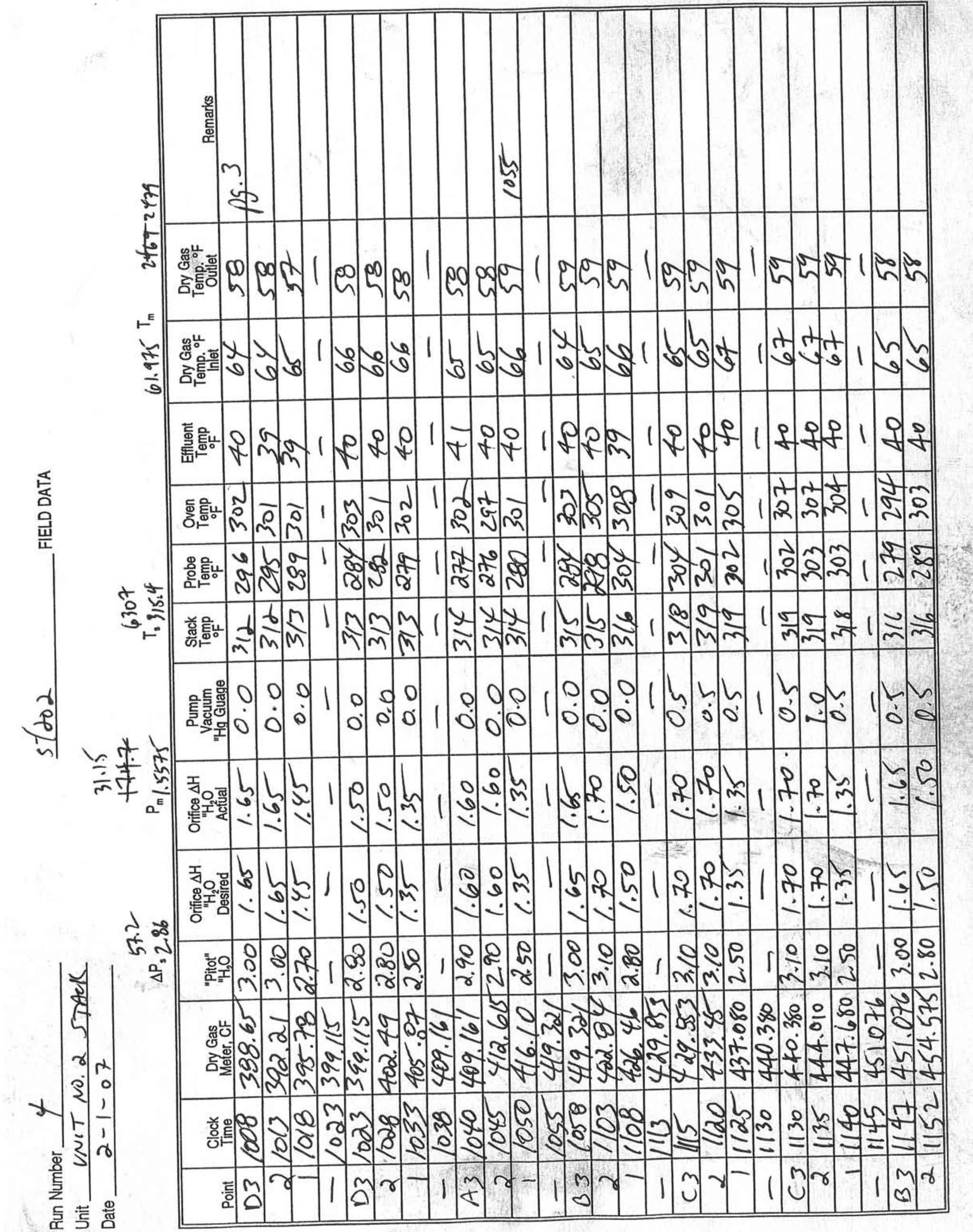




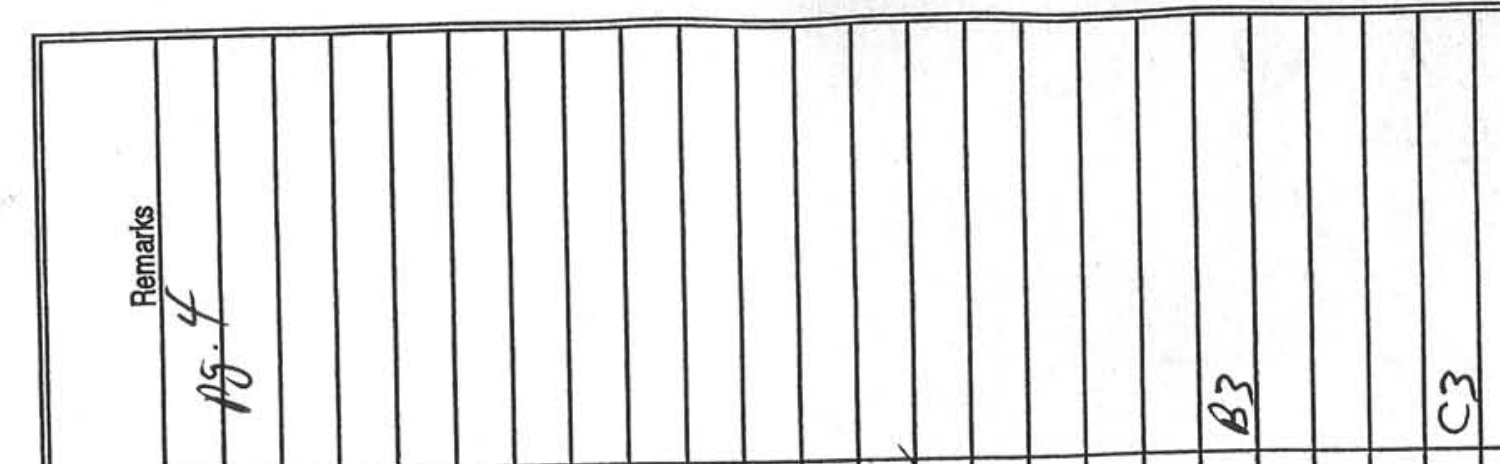

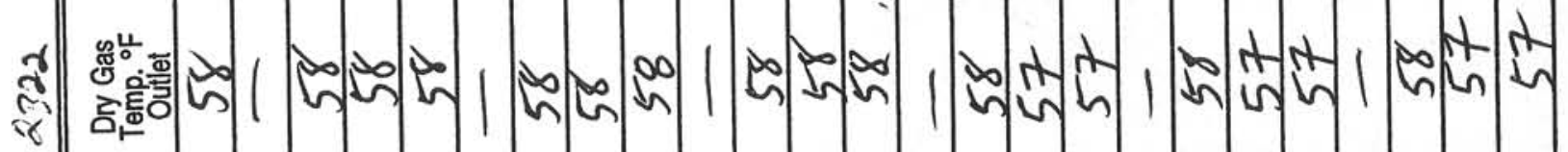
$r^{\mathrm{E}}$

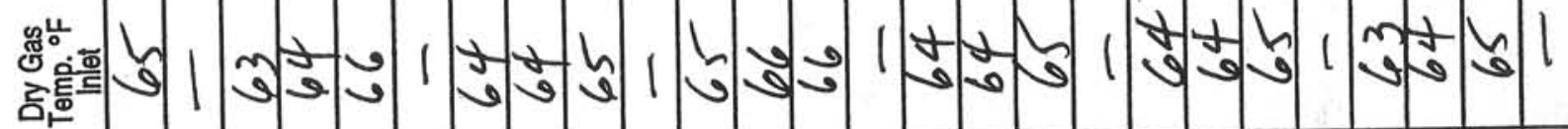

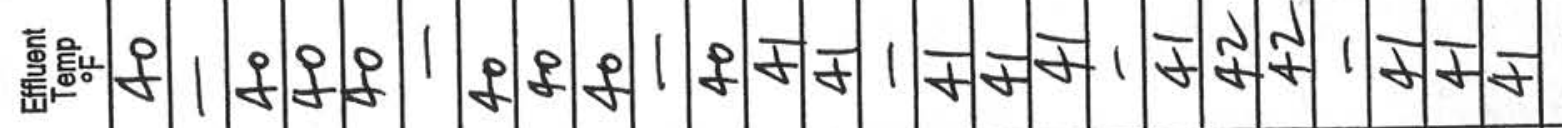

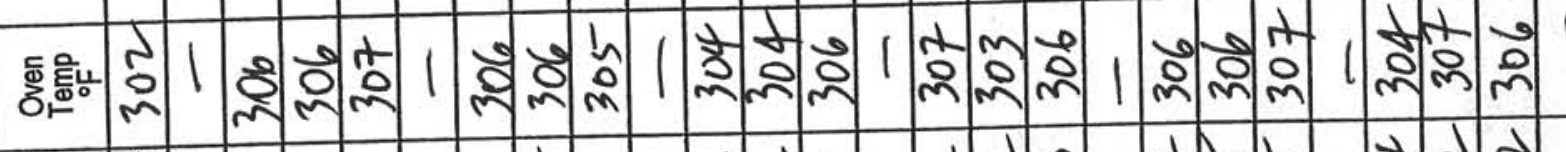

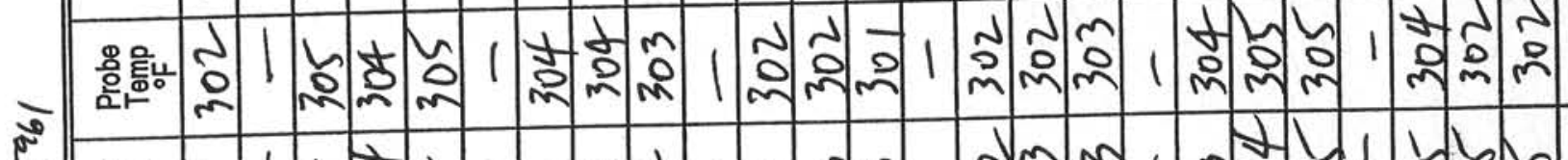

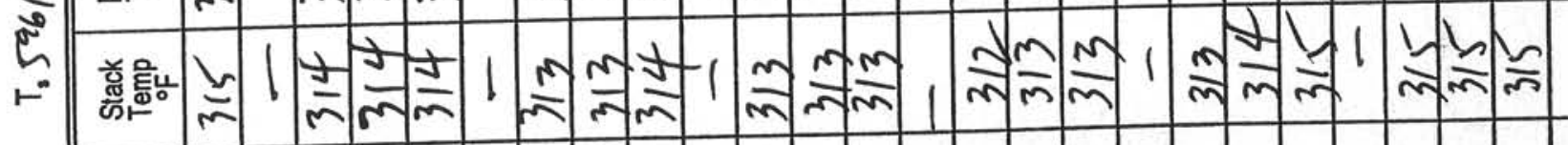
4

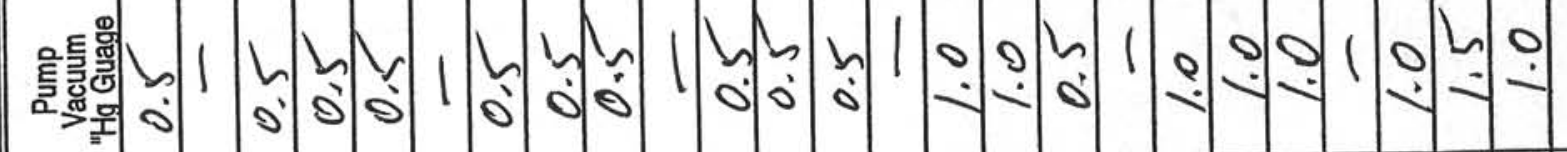

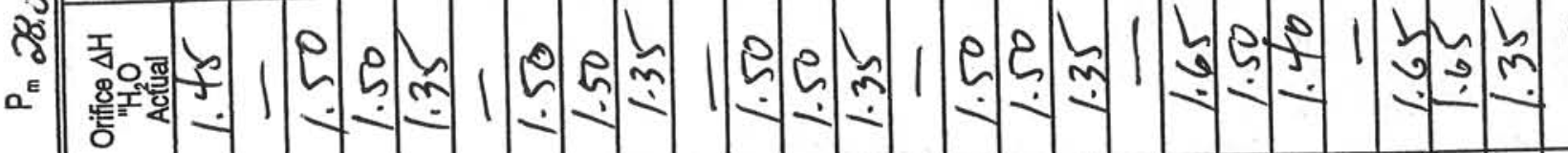

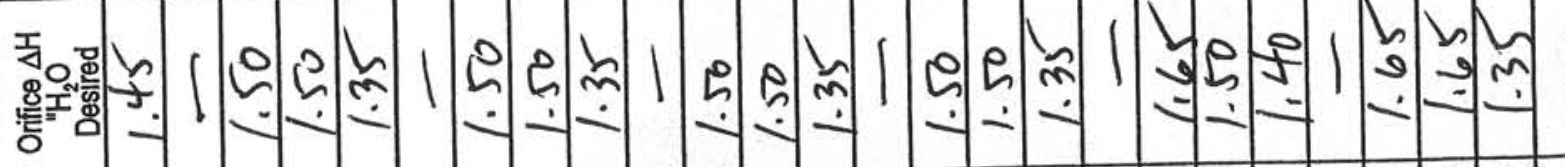
苦| 作

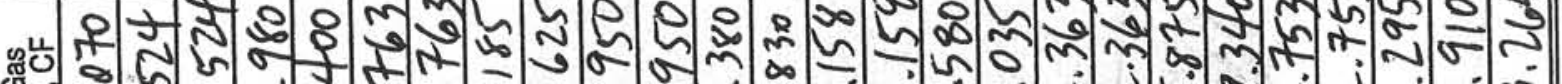

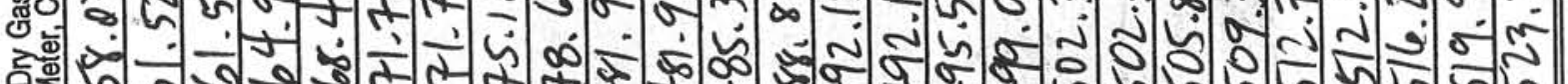

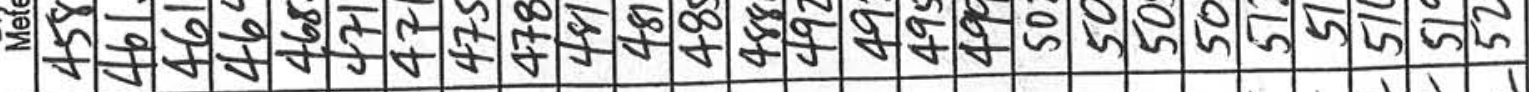

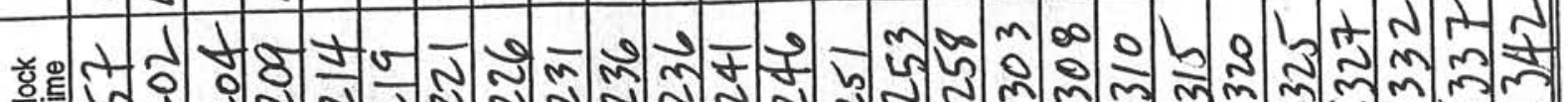

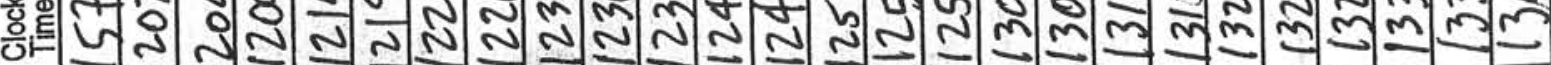
흠 2 l 

Impinger Box No. $1-L$

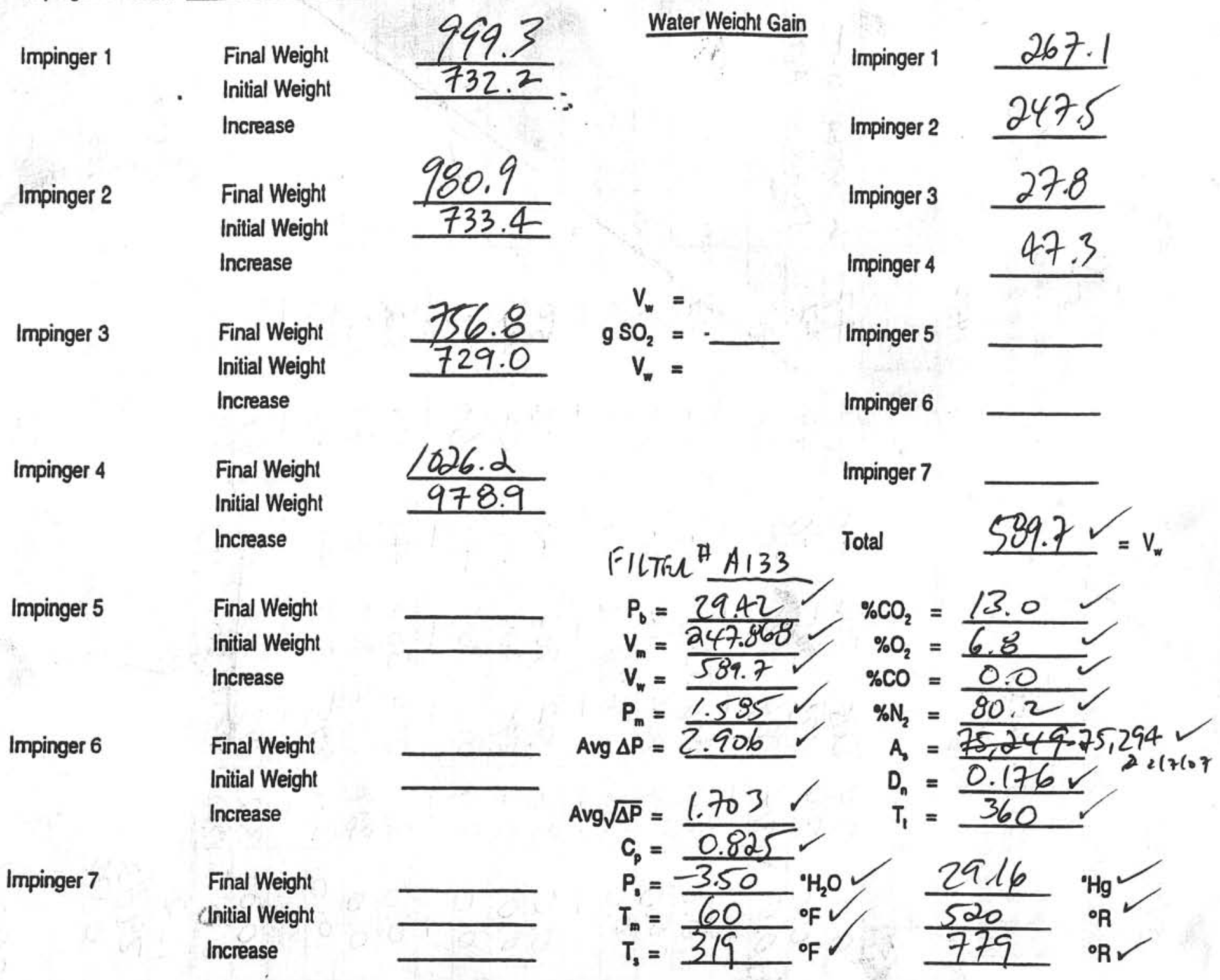

Moisture Content: $\quad \% \mathrm{M}=10.07 \mathrm{M}_{\mathrm{d}}=0.0993 \checkmark \mathrm{MW}_{\mathrm{c}}=30.352 \mathrm{MW}=29.11$

$$
V m_{\text {std }}=17.65 \mathrm{Vm}\left[\frac{P_{b}+\frac{P_{m}}{13.6}}{T_{m}+460}\right]=17.65 \times 247.368\left[\frac{29.42+\frac{1.585}{13.6}}{60+460}\right]=\frac{248.497}{0.690} \mathrm{stt}^{3}
$$

$v_{w_{03}}=0.0472 \times v_{w}=0.0472 \times \quad 539.7=27.834$

$\%$ Moisture $=\frac{V_{w_{02}}}{V_{m_{3+1}}+V_{w_{033}}} \times 100=\frac{27.034}{27.934+248.497} \times 100=\frac{10.07}{0}$

$v_{1}=5123.8 \times \frac{0.025}{\frac{779}{29.11 \times 29.16}} \times 1.703=6,896 \mathrm{pm}$

$\%=\frac{1.039 \times 779 \times 240.497}{6896 \times 0.3993^{\times} \times 19.16 \times 360 \times(76)^{2}}=99.7 \%$ 


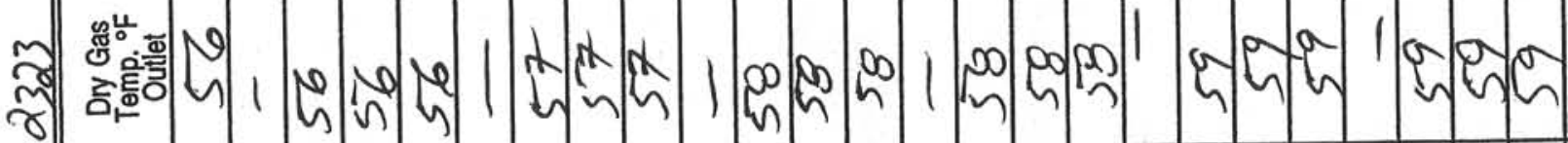
$\vdash$ 皱到

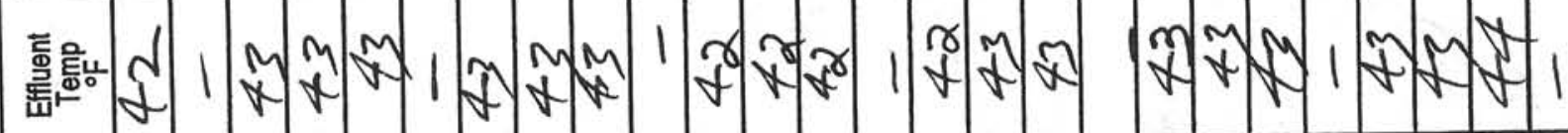

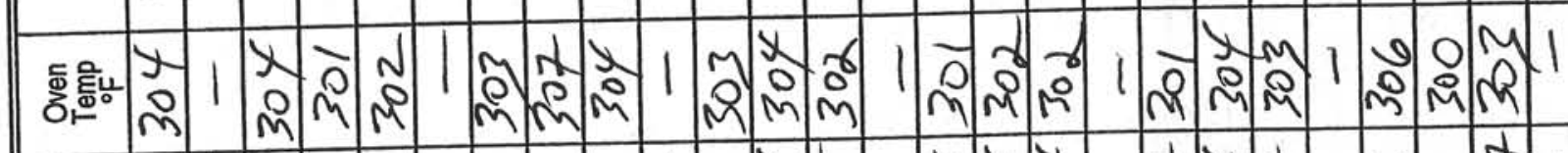
6

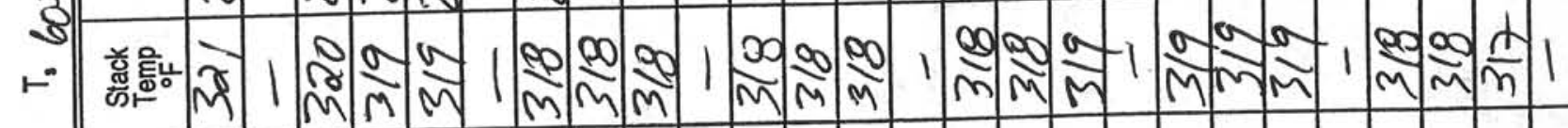

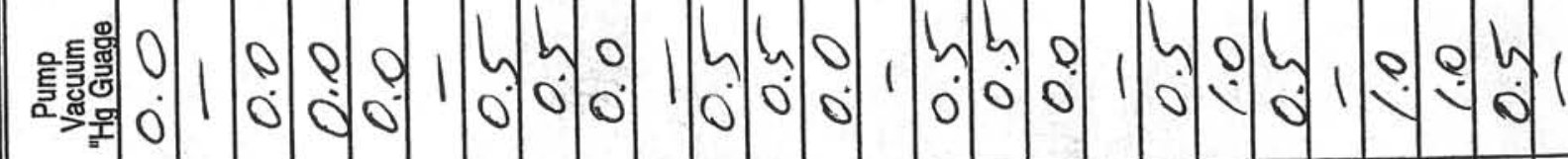

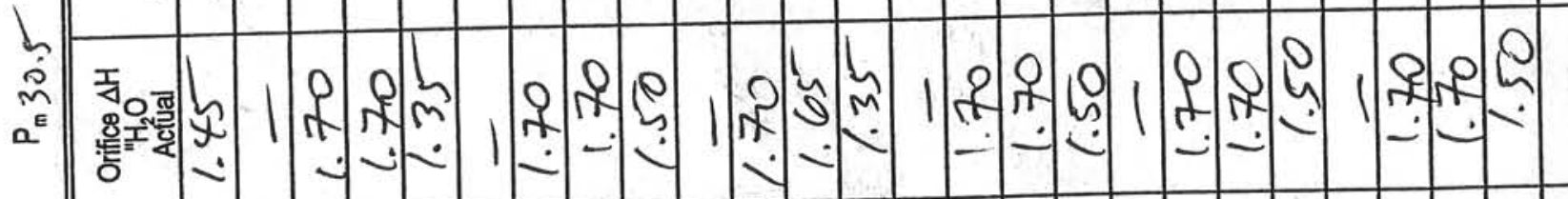

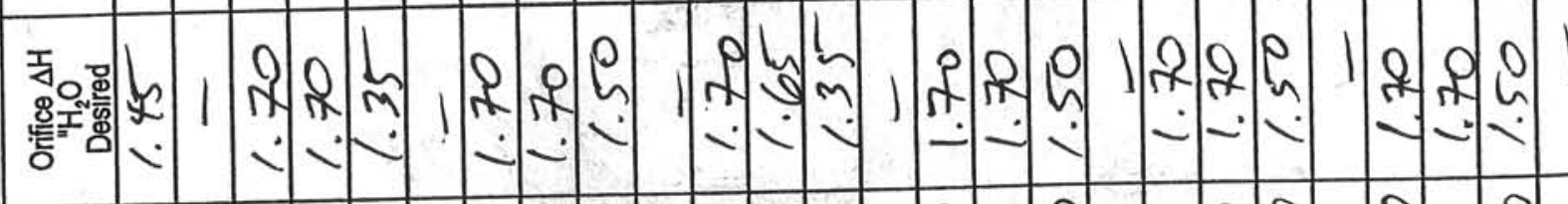

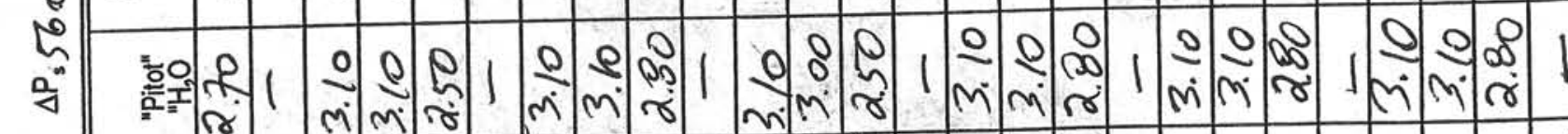

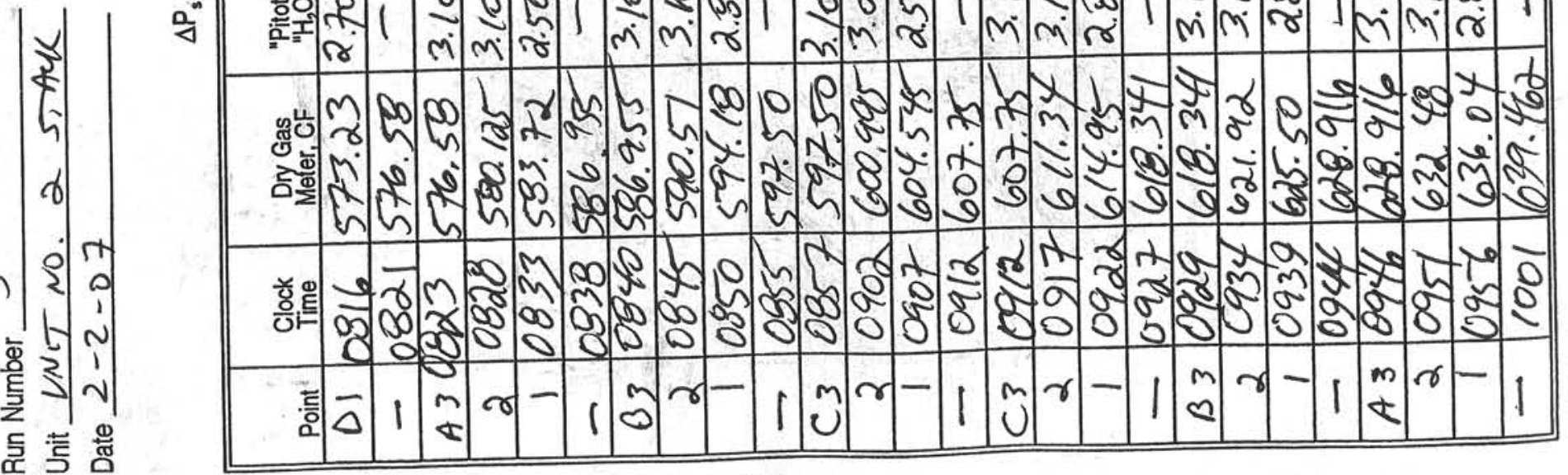




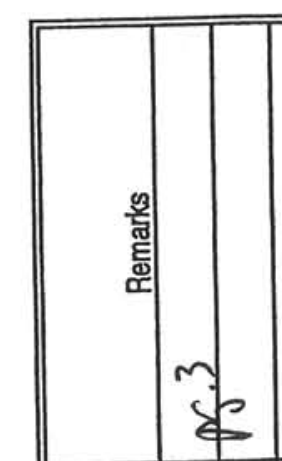

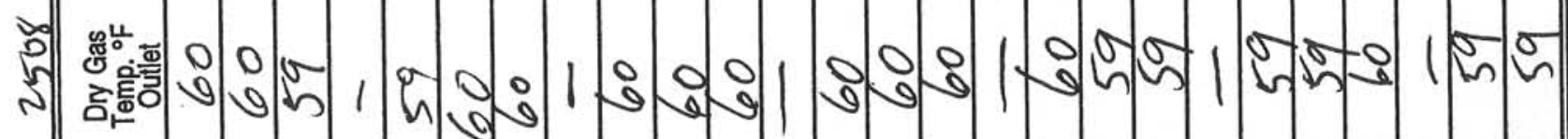
.

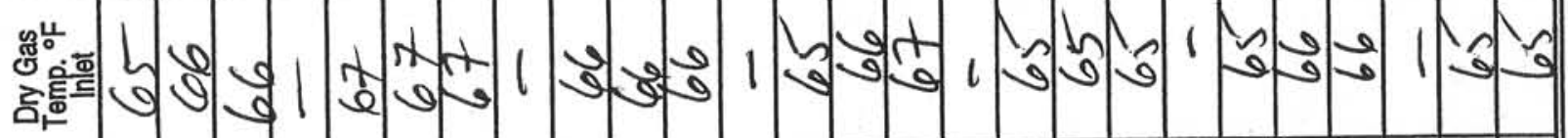

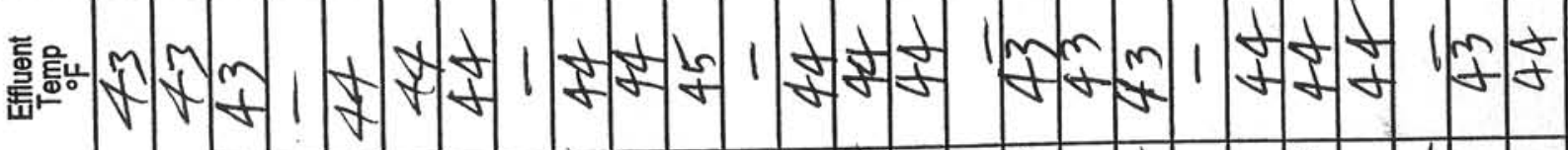

(3) i.

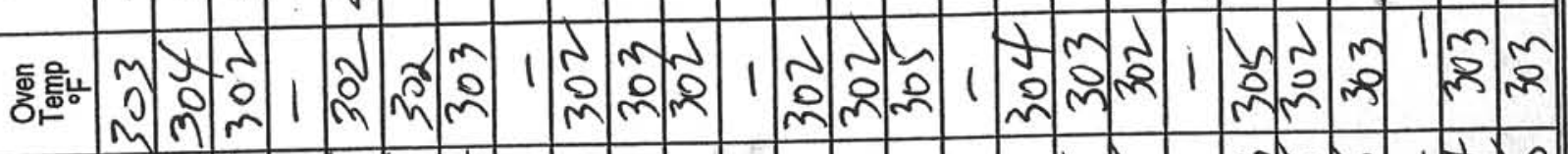

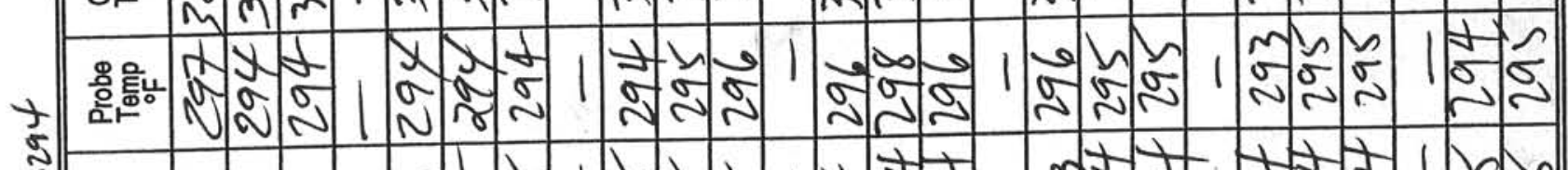
(3)

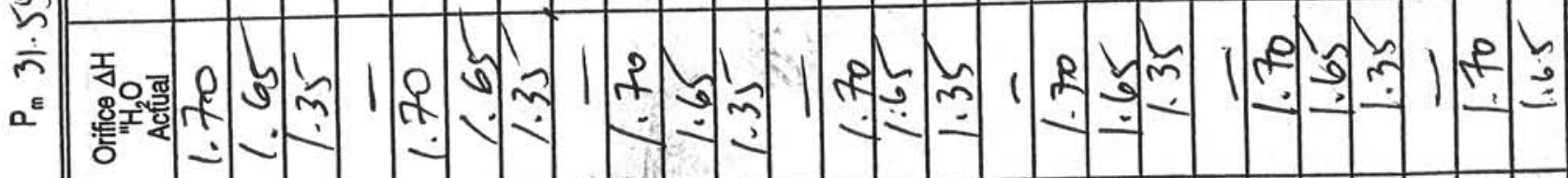

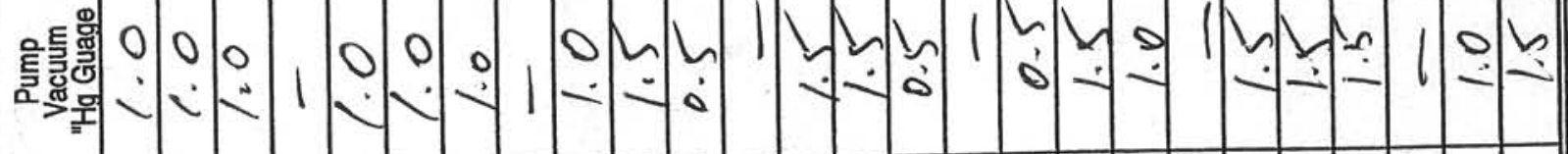

药 n 4

$\ln 8 ?$

-N

है

空

壳壱蹗

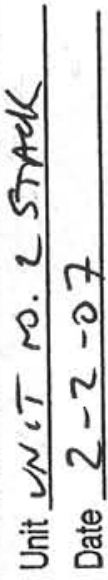

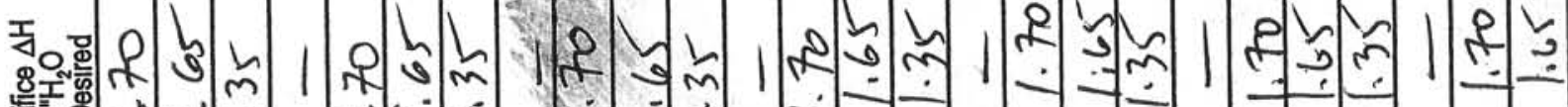
t.

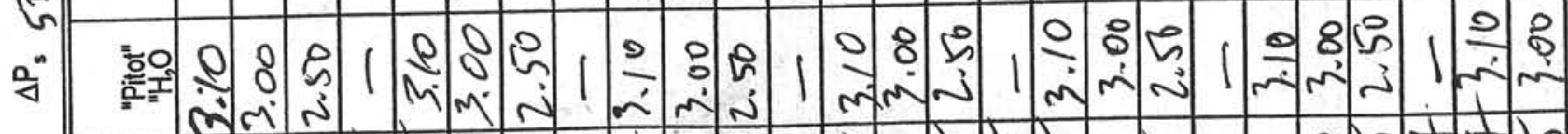

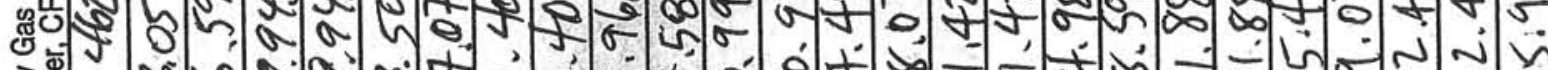

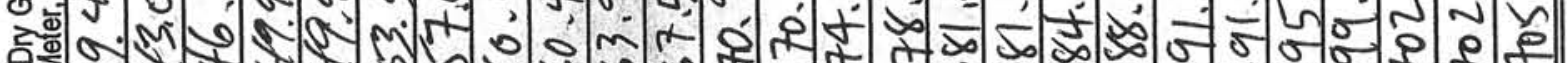

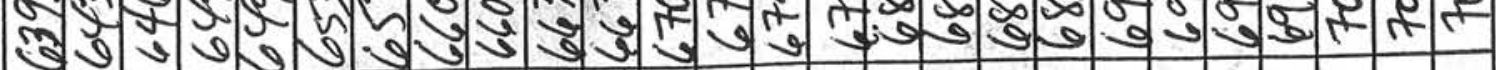

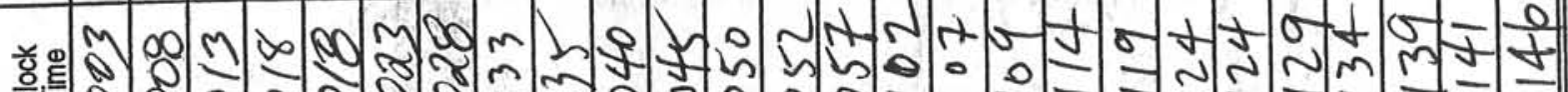

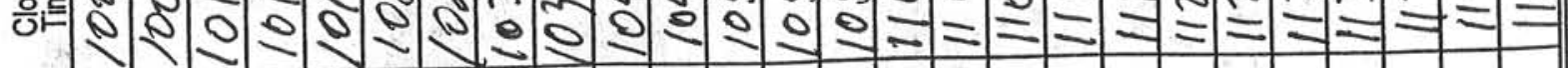

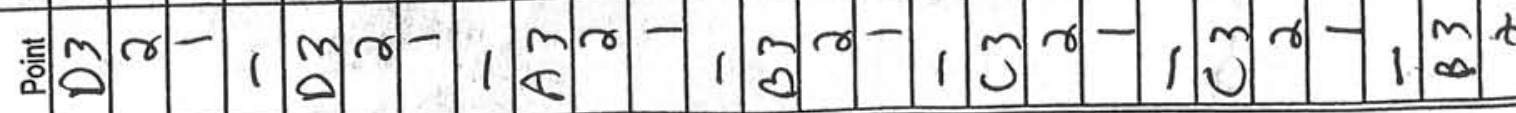


을 $\vdash$

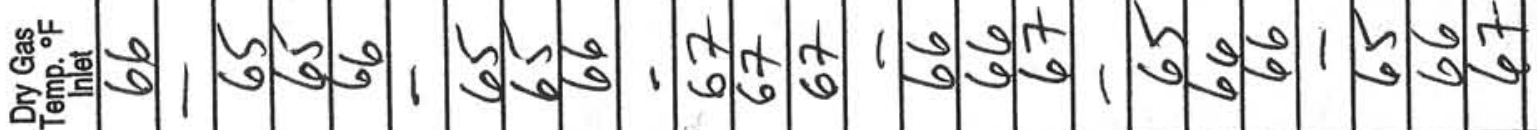

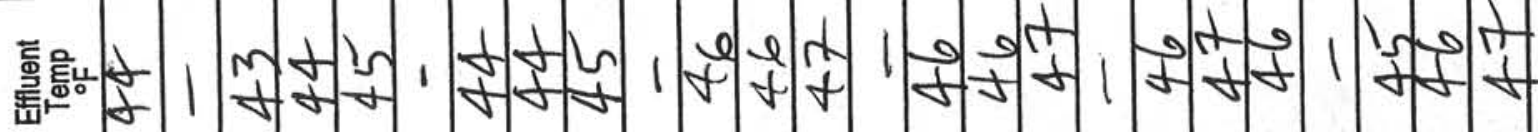

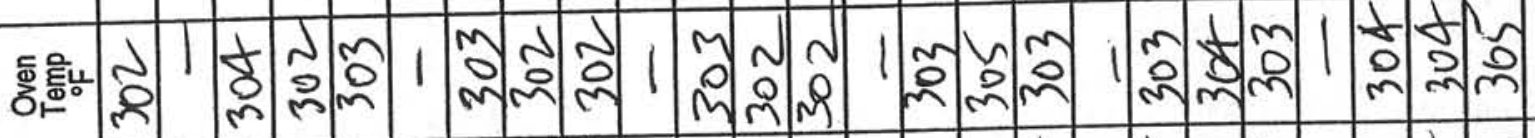
응.

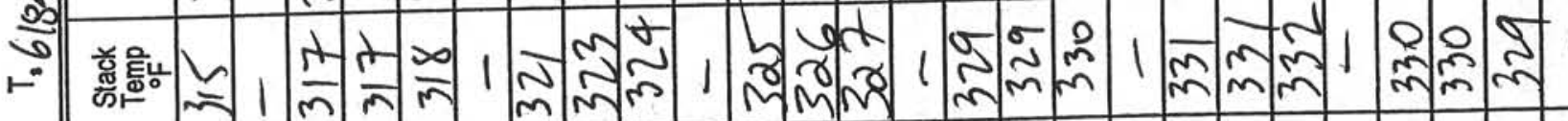

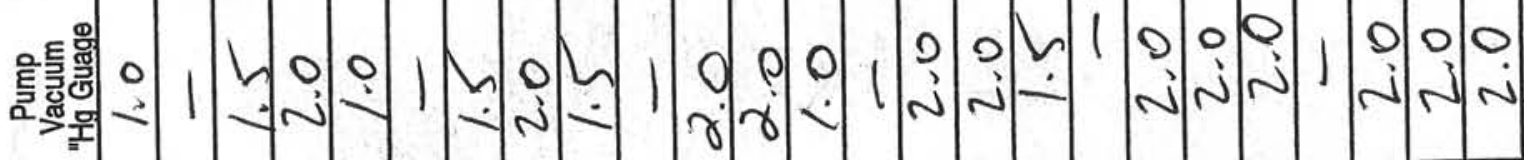
ह5

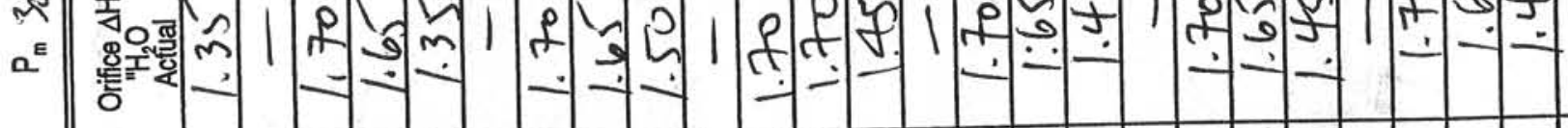

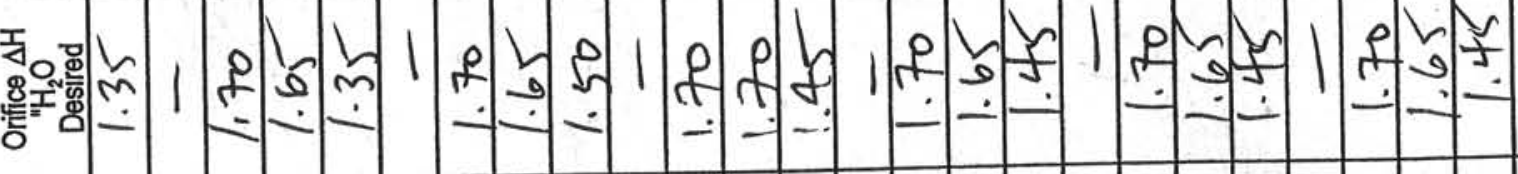

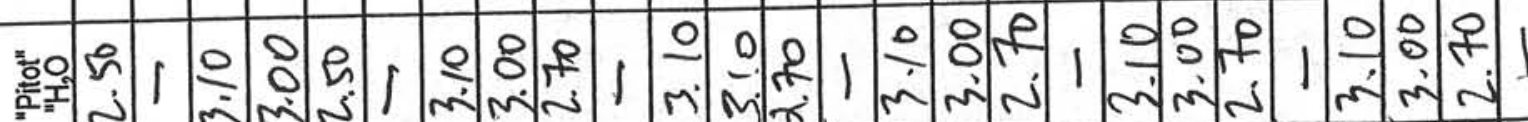
a mm gु०

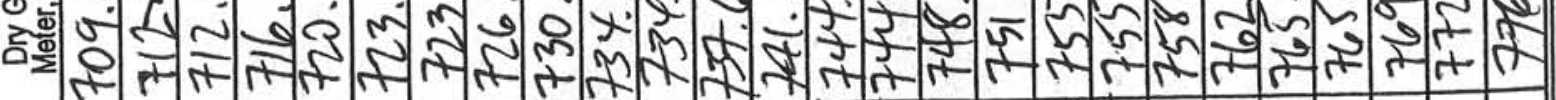

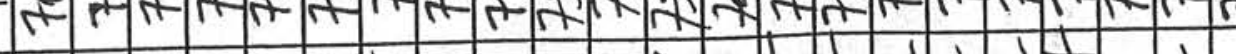

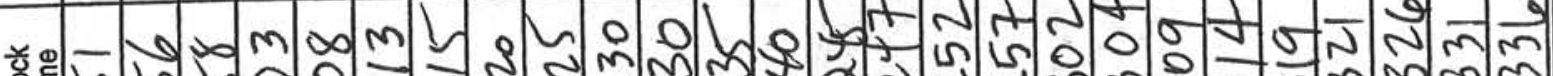

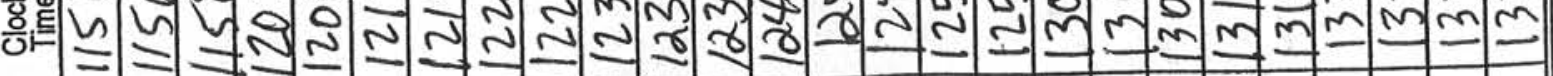

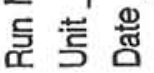

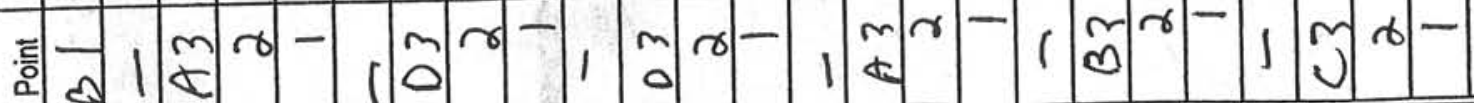


PRElIMINARY VELOCITY TRAVERSE DATA

SAMPLING LOCATION DATA

Job Number $07-008$

Job Name ADA-ES, NEWARK

Sampling Location UNIT NO. 2 STR

Stack Height ft.

Date

Time

Sampling Port Height Above Ground

ft.

Port \& Inside Diameter (in.)

Port \& Wall Thickness (in.)

Inside Stack Diameter (in.) 247

Sampling Ports are

225 . $8 \frac{3}{4}$ in.

Sampling Ports are $5 \overline{\mathrm{ft}} \mathrm{C} \mathrm{C}$ in.

Port B

Port C

$4 \frac{3}{4}$

$47 / 8$

Port D

$9.58 \frac{8.72}{0}$ stack diameters) downstream from disturbance $\left(25.7^{1}\right)$

[info, constriction, bend, expansion)

Peng. dean

(9.42 outlet constriction, bend, expansion)

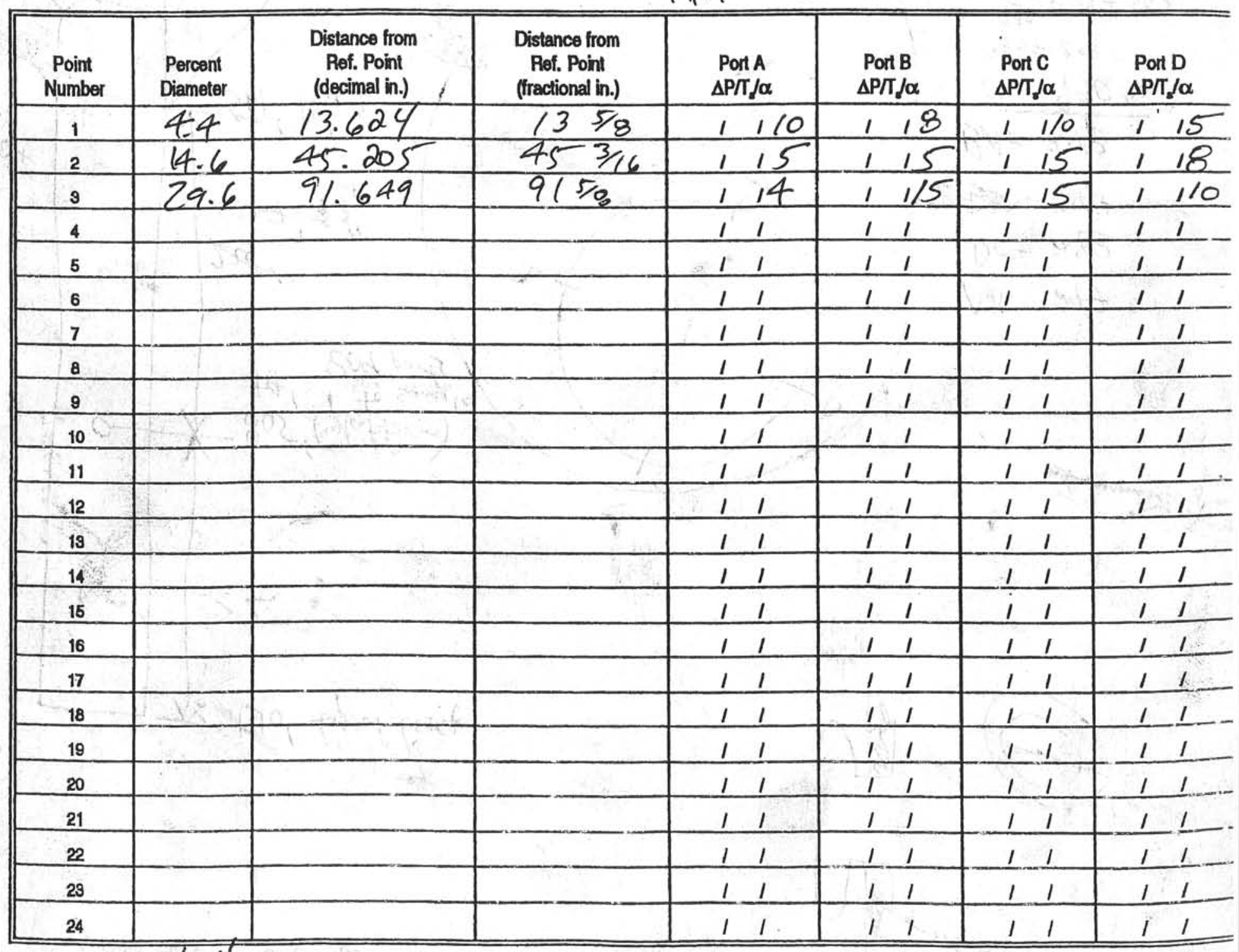

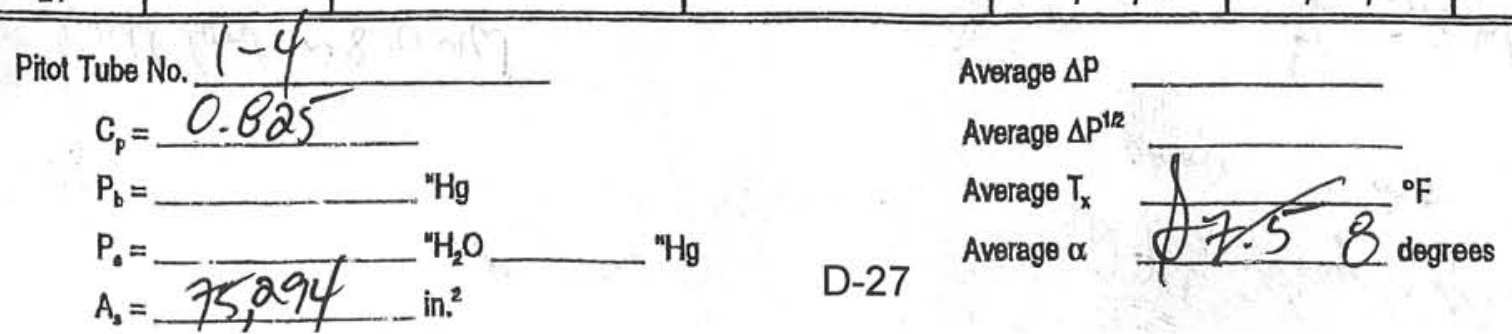

Average $\Delta \mathrm{P}$

$$
\begin{aligned}
& \mathrm{c}_{\mathrm{p}}=0.825 \\
& P_{b}= \\
& \mathrm{P}_{0}= \\
& A_{3}=75,294 \quad \text { in. }^{2}
\end{aligned}
$$

D-27 
$27 \frac{1}{2}$ flange to fearge

fhunge $2.5 \quad 27.5-2(1.714$

$320.56^{2} \mathrm{r}$

Guel hord * $890 \mathrm{~mW}$

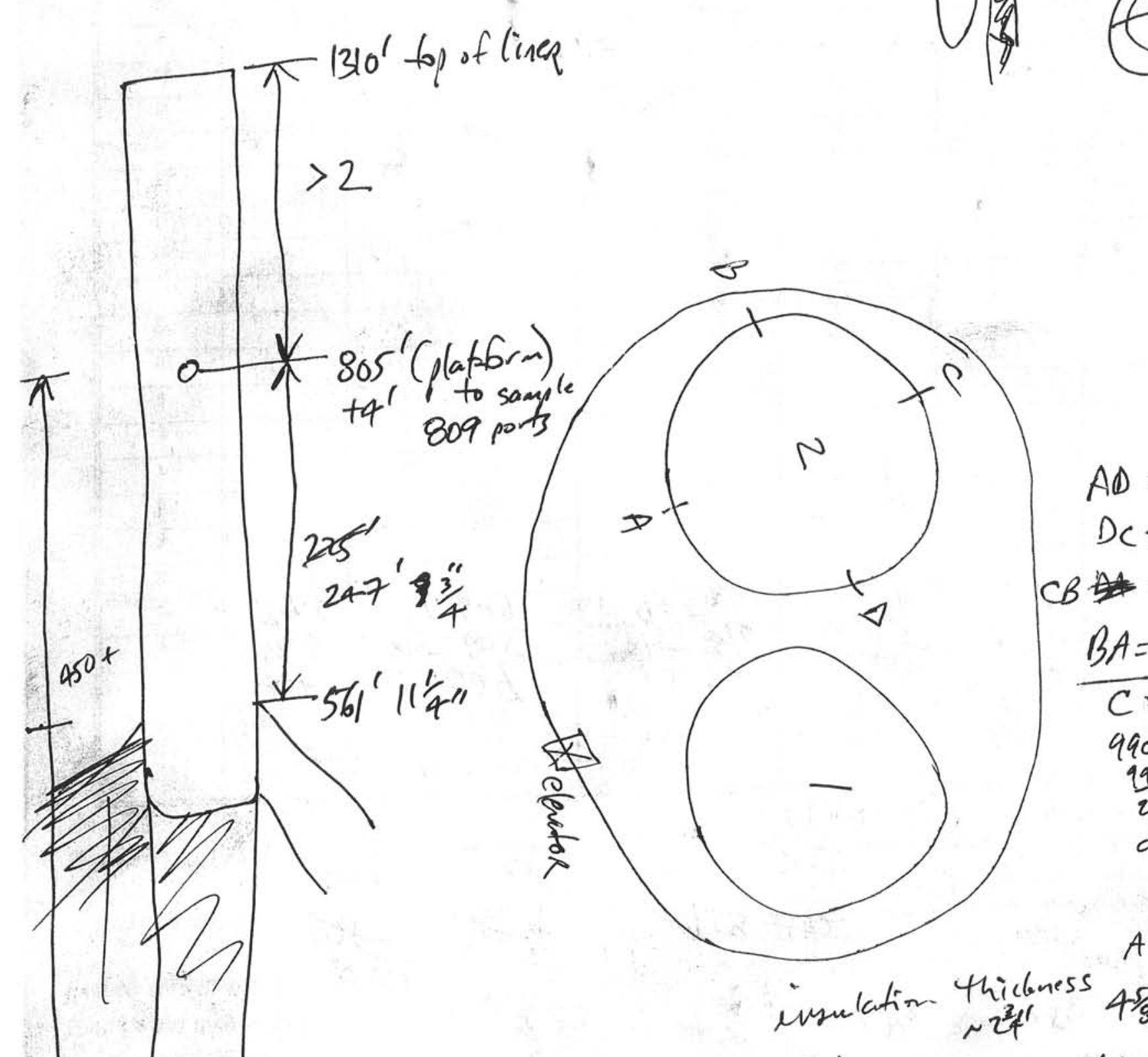

páw
ID $309 \frac{5}{8}$

$$
\begin{aligned}
A D & =247 \\
D C & =249 \\
C B & =247 \\
B A & =248 \\
\hline C & =990^{11} \\
990 & =2 \pi r \\
\frac{990}{2 \pi} & =157.563 \\
d & =315.1268- \\
2(2.75) & =309.6
\end{aligned}
$$$$
A B C D
$$

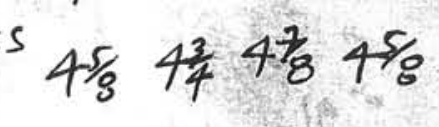
$4.6254 .25 \quad 4.8254 .62$

$$
\bar{x}=4.718
$$

(307) eleunto $\frac{150^{\prime}}{\mathrm{min}}$

D-28 
UNIT NO. 2 STR
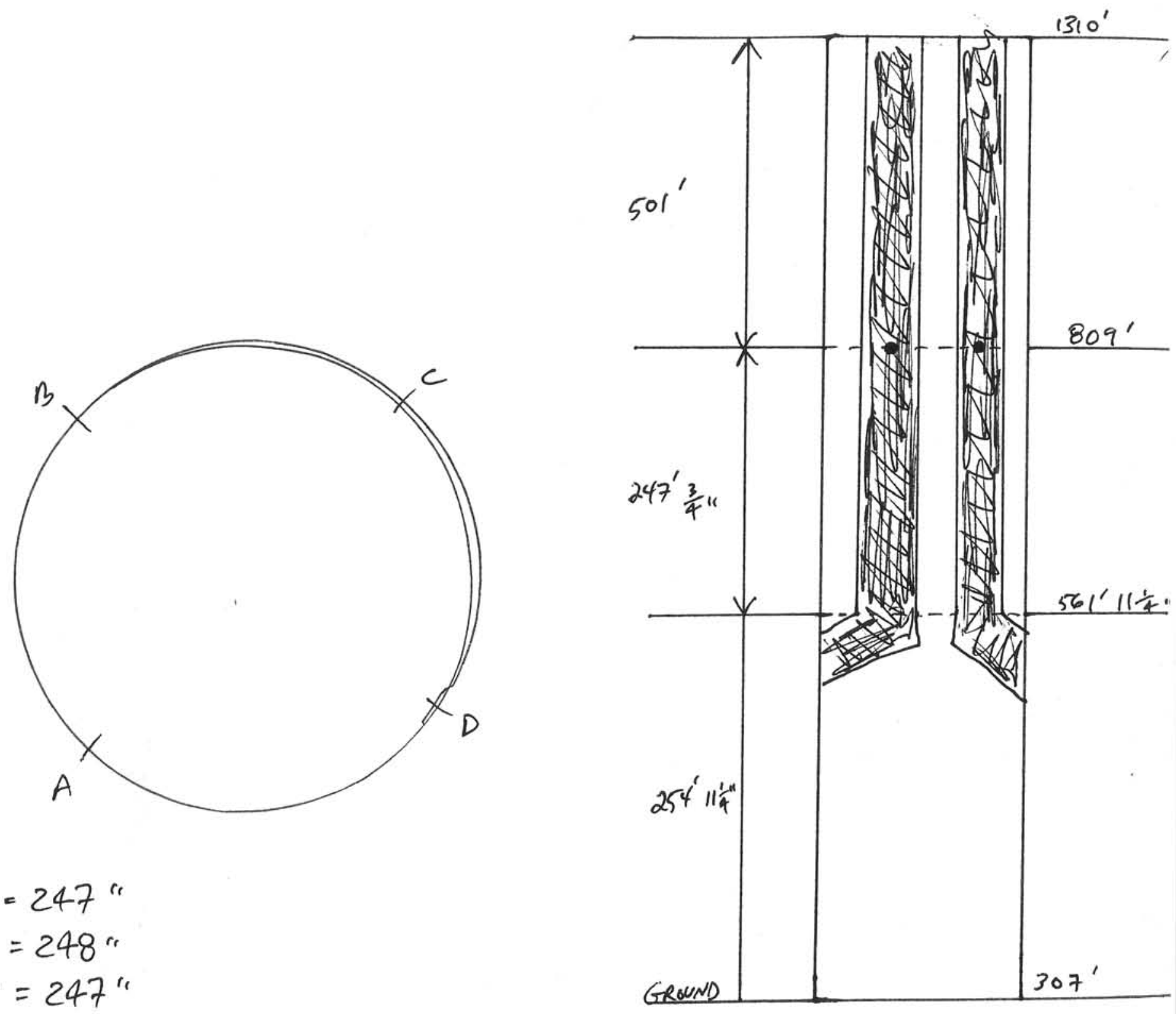

$$
\begin{aligned}
& A D=247^{\prime \prime} \\
& D C=248^{\prime \prime} \\
& C B=247^{\prime \prime} \\
& B A=248^{\prime \prime} \\
& C=990^{\prime \prime} \\
& r=157.563^{\prime \prime} \\
& d=315.1268^{\prime \prime}
\end{aligned}
$$

- twice insulation thickness

$$
\begin{aligned}
& (2 \times 2.75) \\
& =309.627
\end{aligned}
$$$$
=309 \frac{5}{8}
$$

$$
A_{r}=7 r 994^{112}
$$


pg. 1 of 2

ORSAT ANALYSIS DATA FORM

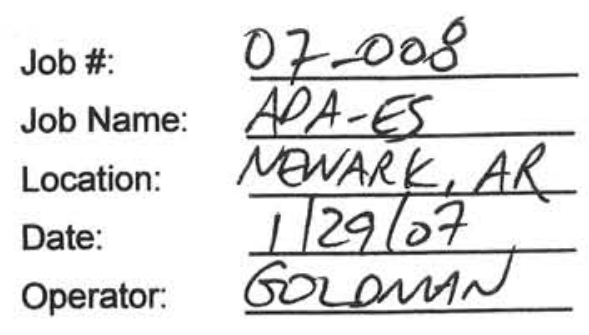

Runt: $\frac{1}{(1 / 29 / 07)}$
Run Time: $0830-1533$
Sample Location: UNIT NO. 2 ST AK

Analytical Method: 3

Sample Type: Single Point (Multi Point Grab Integrated

Leak Check: Time: $>4 \mathrm{~min}$ ( $\geq 4$ minutes) Rate:

Ambient Air Check:

\begin{tabular}{|l|l|}
\hline $\mathrm{CO}_{2}-\%$ Vol. & 0.0 \\
\hline $\mathrm{O}_{2}-\%$ Vol. & 20.9 \\
\hline $\mathrm{N}_{2}-\%$ Vol. & 79.1 \\
\hline
\end{tabular}

\begin{tabular}{|l|l|l|l|l|}
\hline $\mathrm{CO}_{2}-\%$ Vol. & 12.9 & 13.0 & 13.1 & 13.0 \\
\hline $\mathrm{O}_{2}-\%$ Vol. & 6.5 & 6.4 & 6.4 & 6.4 \\
\hline $\mathrm{CO}-\%$ Vol. & 0.0 & 0.0 & 0.0 & 0.0 \\
\hline $\mathrm{N}_{2}-\%$ Vol. & 80.6 & 80.6 & 80.5 & 80.6 \\
\hline
\end{tabular}

Run \#: $\frac{2}{2}(1 / 30 / 07)$
Run Time: $0645-1336$
\begin{tabular}{|l|c|c|c|c|}
\hline $\mathrm{CO}_{2}-\%$ Vol. & 12.9 & 13.0 & 13.1 & 13.0 \\
\hline $\mathrm{O}_{2}-\%$ Vol. & 6.9 & 6.8 & 6.7 & 6.8 \\
\hline $\mathrm{CO}-\%$ Vol. & 0.0 & 0.0 & 0.0 & 0.0 \\
\hline $\mathrm{N}_{2}-\%$ Vol. & 80.2 & 80.2 & 80.2 & 80.2 \\
\hline
\end{tabular}

Run \#: $3 \quad(1 / 31 / 07)$

Run Time: $0642-1320$ Analysis \#1 Analysis \#2 Analysis \#3

Average - \% Volume

\begin{tabular}{|l|l|l|l|l|}
\hline $\mathrm{CO}_{2}-\%$ Vol. & 13.0 & 13.1 & 13.0 & 13.0 \\
\hline $\mathrm{O}_{2}-\%$ Vol. & 6.7 & 6.7 & 6.7 & 6.7 \\
\hline $\mathrm{Co}-\%$ Vol. & 0.0 & 0.0 & 0.0 & 0.0 \\
\hline $\mathrm{N}_{2}-\%$ Vol. & 80.3 & 80.2 & 80.3 & 80.3 \\
\hline
\end{tabular}

D-30 
$p g^{2}$ of 2

ORSAT ANALYSIS DATA FORM

$\begin{array}{ll}\text { Job \#: } & \frac{O 7-008}{\text { Job Name: }} \\ \text { Location: } & \frac{A \text { ADA -ES }}{2-1-07, A R} \\ \text { Date: } & \frac{\text { N ER }}{\text { Operator: }} \\ \text { GILMAN }\end{array}$

Run \#: $4 \quad(2-1-07)$
Sample Location: $\frac{\text { UNIT NO. } 2 \text { STACK }}{3}$

Sample Type: Single Point / Multi Point Grab Integrated

Leak Check: Time: $Z 4 m$ ( 24 minutes) Rate: $\varnothing$

Ambient Air Check:

\begin{tabular}{|l|l|}
\hline $\mathrm{CO}_{2}-\%$ Vol. & 0.0 \\
\hline $\mathrm{O}_{2}-\%$ Vol. & 21.0 \\
\hline $\mathrm{N}_{2}-\%$ Vol. & 79.0 \\
\hline
\end{tabular}

Run Time: $0705-1342$

\begin{tabular}{|l|c|c|c|c|}
\hline $\mathrm{CO}_{2}-\%$ Vol. & 12.8 & 12.8 & 12.7 & 19.8 \\
\hline $\mathrm{O}_{2}-\%$ Vol. & 6.9 & 6.9 & 7.0 & 6.9 \\
\hline $\mathrm{CO}-\%$ Vol. & 0.0 & 0.0 & 0.0 & 0.0 \\
\hline $\mathrm{N}_{2}-\%$ Vol. & 80.3 & 80.3 & 80.3 & 80.3 \\
\hline
\end{tabular}

Run \#: $5(2-2-07)$
Run Time: $0700-1336_{\text {Analysis \#1 }}$ Analysis \#2
\begin{tabular}{|l|c|c|c|c|}
\hline $\mathrm{CO}_{2}-\%$ Vol. & 13.0 & 13.0 & 12.9 & 13.0 \\
\hline $\mathrm{O}_{2}-\%$ Vol. & 6.8 & 6.8 & 6.9 & 6.8 \\
\hline $\mathrm{CO}-\%$ Vol. & 0.0 & 0.0 & 0.0 & 0.0 \\
\hline $\mathrm{N}_{2}-\%$ Vol. & 80.2 & 80.2 & 80.2 & 80.2 \\
\hline
\end{tabular}

Run \#:

Run Time:

Analysis \#1 Analysis \#2

Analysis \#3

Average - $\%$ Volume

\begin{tabular}{|l|l|l|l|l||}
\hline $\mathrm{CO}_{2}-\%$ Vol. & & & & \\
\hline $\mathrm{O}_{2}-\%$ Vol. & & & & \\
\hline $\mathrm{CO}-\%$ Vol. & & & & \\
\hline $\mathrm{N}_{2}-\%$ Vol. & & & & \\
\hline
\end{tabular}

D-31 
Appendix E:

Analytical Data 
Hi l RIPPLing

ASSOCIATES, INC.

Air Sampling Associates, Inc.

Particulate Analysis Summary

Project Number: $07-008$

Project Name: $A D A-E S$
Date Analysis Completed: $2 / 13 / 07$

Unit Tested: Unit No .2 stack

Project Location: Newark, $A R$

Run Number:

Particulate Matter on Filter (mg):

Particulate Matter in Front Wash (mg):

Total Particulate Matter in "Front-Half" - MF (mg):

Particulate Matter in "Back-Half” (mg):

Total Particulate Matter in Sample - MT (mg):

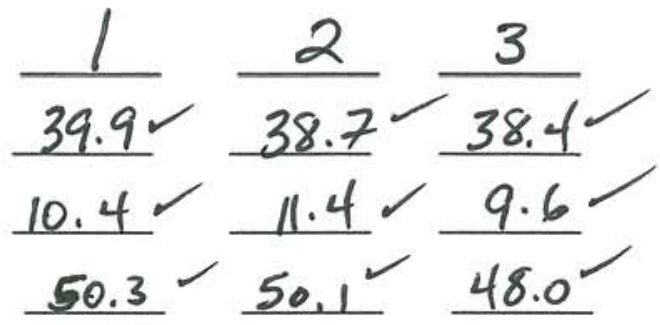

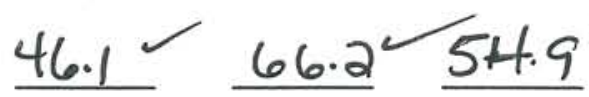

$96.4^{116.3^{\prime}} 102.9$
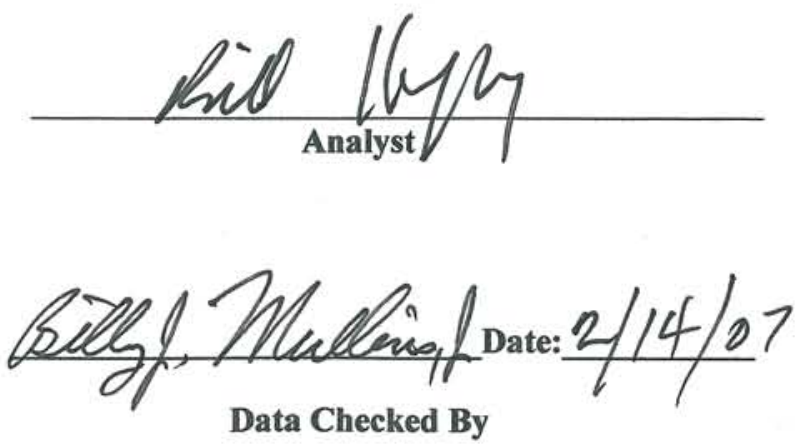

Version No. 1 5/30/06

E-2 


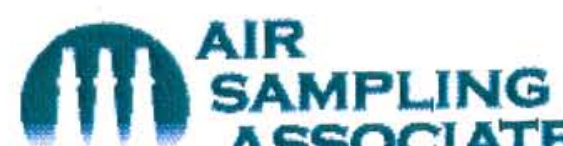 ASSOCIATES, INC.}

Air Sampling Associates, Inc.

\section{Particulate Analysis Summary}

Project Number: $07-008$

Project Name: ADA-ES

Date Analysis Completed: $2 / 13 / 07$

Unit Tested: Unit No. 2 Stack

Project Location: Newark, AR

Run Number:

Particulate Matter on Filter (mg):

Particulate Matter in Front Wash (mg):

Total Particulate Matter in "Front-Half" - MF (mg):

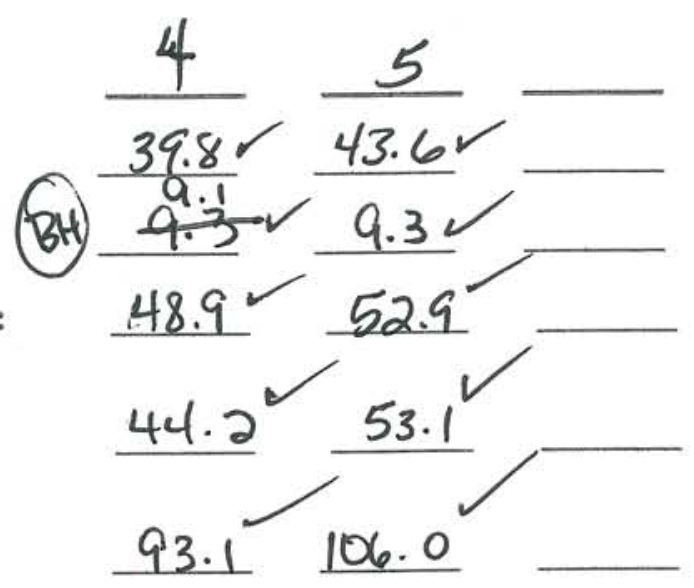

Total Particulate Matter in Sample - MT (mg):
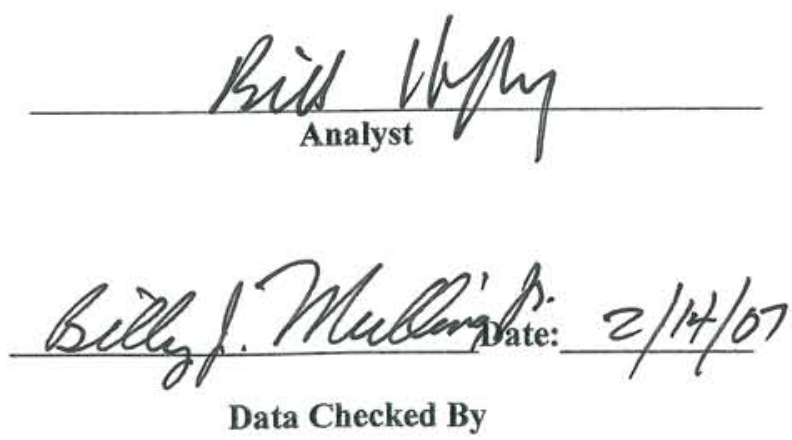

Version No. 1 5/30/06 


\section{II}

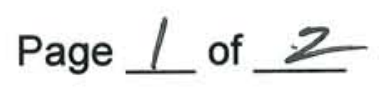

\section{Particulate Analysis EPA Method 5}

\section{Stack Filters}

Project No. $07-008$

Project Name ADA-ES
Location Newark, AR Unit Tested Unit No. 2 Stack

Desiccator Time In 0830 2/5/07 $0855^{2 / 12 / 07}$

Desiccator Time Out $0845 \quad 2 / 12 / 07 / 0830 \quad 2 / 13 / 07$

\begin{tabular}{|c|c|c|c|}
\hline Run No. & & Filter No. A 129 & Sample I.D. \\
\hline Filter \& Particulate (g) & 0.4244 & $0.4244^{\circ}$ & \\
\hline
\end{tabular}

Filter \& Particulate Average (g) 0.4244 Initial Filter Weight $(\mathrm{g}) 0.3845$ Total Particulate $(\mathrm{mg})$ 39.9

\begin{tabular}{|c|c|c|c|}
\hline Run No. 2 & & Filter No. $A_{1} 30$ & Sample I.D. \\
\hline Filter \& Particulate $(\mathrm{g})$ & 0.4204 & $0.4205^{2}$ & \\
\hline
\end{tabular}

Filter \& Particulate Average (g) 0.4205 Initial Filter Weight $(\mathrm{g}) 0.3818$

Total Particulate $(\mathrm{mg})$ 38.7

Run No. 3

Filter \& Particulate (g)
Filter No. A 131 0.4239

\section{Sample I.D.}

Filter \& Particulate Average (g) 0.4239 Initial Filter Weight $(\mathrm{g}) 0.3855$ Total Particulate (mg) 38.4

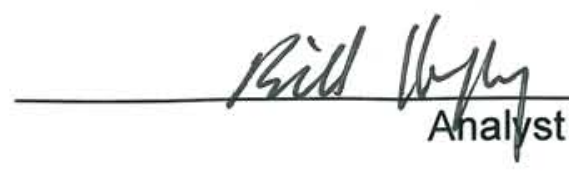




\section{II \\ ASSOCIATES, INC.}

\section{Page 2 of 2}

\section{Particulate Analysis EPA Method 5}

\section{Stack Filters}

Project No. $07-008$

Project Name ADA - ES

Location Newark, AR Unit Tested Linit No. a Stack

Desiccator Time In $08302 / 5 / 07 \quad 0855$

Desiccator Time Out $08452 / 12 / 070835$

\begin{tabular}{|l|l|l|l|l|l|}
\hline \multicolumn{2}{|c|}{ Run No. 4} & Silter No. A132 & & \\
\hline Filter \& Particulate (g) & 0.4234 & $0.4236^{2}$ & & & \\
\hline
\end{tabular}

Filter \& Particulate Average (g) Initial Filter Weight (g)

Total Particulate $(\mathrm{mg})$

$\frac{0.4236 /}{0.3838}$

\begin{tabular}{|c|c|c|c|}
\hline Run No. 5 & & Filter No. $\mathrm{A}_{133}$ & Sample I.D. \\
\hline Filter \& Particulate $(\mathrm{g})$ & 0.4237 & 0.4236 & \\
\hline
\end{tabular}

Filter \& Particulate Average (g) 0.4236 Initial Filter Weight $(\mathrm{g})$ 0. 3800 Total Particulate $(\mathrm{mg})$ 43.6

\begin{tabular}{|c|l|l|l|l|l|}
\hline \multicolumn{2}{|c|}{ Run No. } & \multicolumn{3}{c|}{ Sample I.D. No. } \\
\hline Filter \& Particulate (g) & & & & & \\
\hline
\end{tabular}

Filter \& Particulate Average ( $\mathrm{g}$ ) Initial Filter Weight (g) Total Particulate $(\mathrm{mg})$

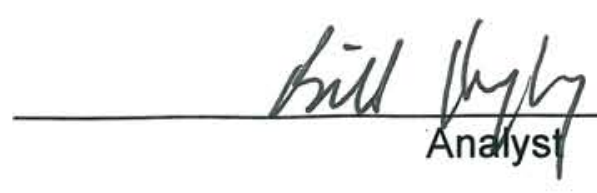


ASSOCIATES, INC.

Filter Weight Log

EPA Method 5

\begin{tabular}{|c|c|c|c|c|c|c|c|}
\hline \multirow[b]{3}{*}{ Filter No. } & Into Desicator & Weight & Weight & Weight & Weight & Weight & \multirow{3}{*}{$\begin{array}{l}\text { Weight } \\
\text { Used }\end{array}$} \\
\hline & Date & Date & Date & Date & Date & Date & \\
\hline & Time & Time & Time & Time & Time & Time & \\
\hline \multirow{3}{*}{$A|2|$} & & 0.3612 & 0.3612 & & & & $0.36 / 2$ \\
\hline & $10 / 2 / 06$ & II/20/06 & $11 / 21 / 06$ & & & & \\
\hline & 0800 & 1251 & 0810 & & & & \\
\hline \multirow{2}{*}{$A 122$} & & 0,3608 & 0.3608 & & & & 0.3608 \\
\hline & $\frac{10 / 2 / 06}{0800}$ & $1 / 20 / 06$ & $\frac{712106}{0811}$ & & & & \\
\hline \multirow{3}{*}{4123} & & 0,3830 & 0.3827 & & & & 0,3827 \\
\hline & $10 / 2106$ & $11 / 20 / 06$ & $11 / 21 / 06$ & & & & \\
\hline & 10800 & 1253 & $0 8 \longdiv { 2 }$ & & & & \\
\hline \multirow{3}{*}{ A124 } & 1012106 & 0.3763 & 0.3762 & & & & 0.3762 \\
\hline & 0800 & $\frac{11 / 20 / 06}{1254}$ & $11 / 21 / 06$ & & & & \\
\hline & $\downarrow$ & 0.379 & 0.3792 & & & & 0.3792 \\
\hline \multirow{2}{*}{$A 125$} & 12115106 & $12 / 15 / 06$ & $12116 / 06$ & & & & \\
\hline & 81703 & $\frac{1703}{0.3803}$ & $\frac{1044}{0.3804}$ & & & & 0.3804 \\
\hline \multirow{2}{*}{ A126 } & $10 / 2 / 06$ & 12115106 & 12116126 & & & & \\
\hline & 0800 & 1703 & 1044 & & & & \\
\hline \multirow{3}{*}{ Al27 } & & 0.3795 & 0.3794 & & & & 0.1794 \\
\hline & $10 / 2106$ & b) & $10 / 16 / 26$ & & & & \\
\hline & 0800 & $\frac{1704}{0.3812}$ & $\frac{1045}{0.3811}$ & & & & 0.3811 \\
\hline \multirow{2}{*}{$A 128$} & $10 / 02106$ & 12115106 & 10116106 & & & & \\
\hline & 0800 & 1704 & 1045 & & & & \\
\hline \multirow{3}{*}{ A 129} & & 0.3843 & 0.3845 & & & & 0.3845 \\
\hline & $10 / 210^{6}$ & $18 / 0106$ & 12116106 & & & & \\
\hline & 0800 & $\frac{1705}{03817}$ & \begin{tabular}{|l|l|}
1045 \\
0.3818
\end{tabular} & & & & 0.3818 \\
\hline \multirow{3}{*}{$A 130$} & 1012106 & $12 / 15106$ & 1416106 & & & & \\
\hline & 0800 & 1705 & 1046 & & & & \\
\hline & & 0.3857 & 0.3855 & & & & 0.3855 \\
\hline \multirow{2}{*}{$A \mid 31$} & $10 / 2 / 06$ & $12 / 15106$ & $18 / 16 / 06$ & & & & \\
\hline & 0800 & 1706 & 1046 & & & & 0.3838 \\
\hline \multirow{2}{*}{$A 132$} & $10 / 2106$ & 0.3840 & 0.3838 & & & & \\
\hline & 0800 & 1706 & 1047 & & & & \\
\hline \multirow{3}{*}{$A 133$} & & 0.3799 & 0.3800 & & & & 0.3800 \\
\hline & 1012106 & collostoce & $18 / 16106$ & & & & \\
\hline & 0800 & 1707 & 1047 & & & & \\
\hline \multirow{3}{*}{$A 134$} & 1125107 & 0.3772 & $\frac{0.3772}{1(27.07}$ & & & & 0.3772 \\
\hline & 0900 & 1000 & $\frac{1124+107}{0631}$ & & & & \\
\hline & 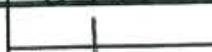 & 0.3780 & 0,3781 & & & & 0,3781 \\
\hline \multirow{2}{*}{ A135 } & & 1126107 & 1127103 & & & & \\
\hline & i & 1007 & $0632^{\circ}$ & & & & \\
\hline
\end{tabular}


Particulate Analysis EPA Method 5

\section{Front Wash}

Project No. $07-008$

Project Name ADA-ES

Location Newark, nte

Unit Tested Unit No.2 Stack

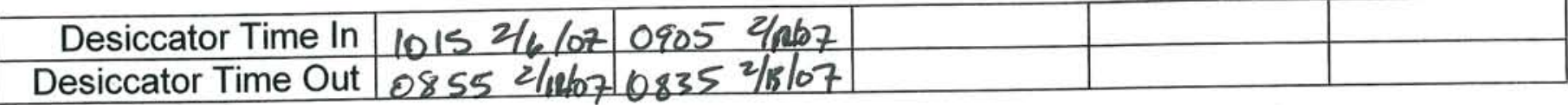

\begin{tabular}{|c|l|l|l|l|l|}
\hline \multicolumn{2}{|c|}{ Run No. / $/ 13.8991$} & $1 / 3.8995$ & & & \\
\hline Final Weight $(\mathrm{g})$ & 113.89 & & \\
\hline Initial Weight $(\mathrm{g})$ & 113.8889 & 113.8889 & & & \\
\hline Particulate Weight $(\mathrm{g})$ & & 0.0106 & & & \\
\hline
\end{tabular}

Particulate Average (mg) Less Acetone Blank (mg)

Total Particulate $(\mathrm{mg}) \quad 10$. 4

10. 6

0.2

\begin{tabular}{|c|c|c|c|c|c|}
\hline \multicolumn{2}{|c|}{ Volume (ml) $66 \quad$ Sample I.D. } \\
\hline Final Weight (g) & 91.4990 & $91.4993^{2}$ & & & \\
\hline Initial Weight (g) & 91.4877 & $91.4877 \checkmark$ & & & \\
\hline Particulate Weight (g) & & 0.0116 & & & \\
\hline
\end{tabular}

Particulate Average (mg)

Less Acetone Blank (mg)

Total Particulate $(\mathrm{mg})$

$\frac{11.6 \Omega}{0.25}$

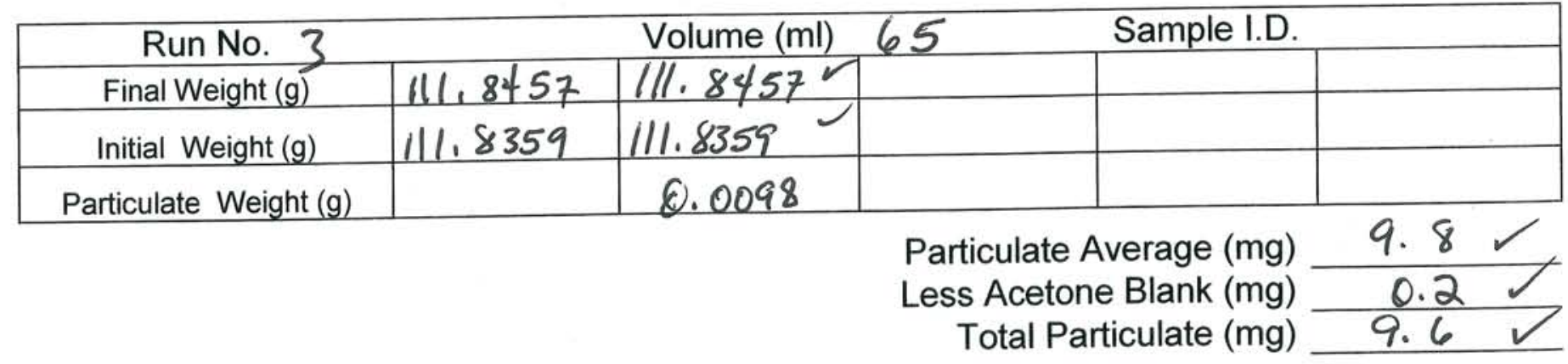

\begin{tabular}{|c|l|l|l|l|l|}
\hline Acetone Blank & & & & \\
\hline Final Weight (g) & & & & \\
\hline Initial Weight (g) & & & & & \\
\hline Particulate Weight (g) & & & Average (mg) \\
\hline
\end{tabular}

* Note: if greater than $7.9 \mathrm{mg} / \mathrm{l}$, use $7.9 \mathrm{mg} / \mathrm{l}$

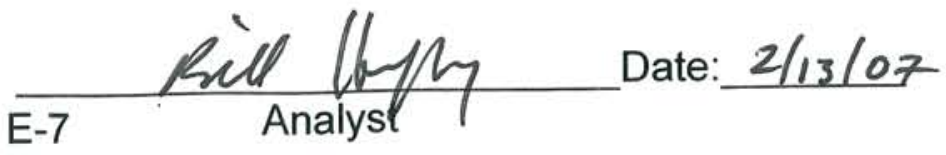


Front Wash

Project No. $07-008$

Project Name AOH -ES
Location Newark, AR Unit Tested Llnit No. 2 stack
Desiccator Time In $10152 / 6 / 07$

Desiccator Time Out $0855^{2 / 1 / 407}$

\begin{tabular}{|c|l|l|l|l|l|}
\hline Run No. $\quad 4$ & Volume (ml) 75 & & \\
\hline Final Weight $(\mathrm{g})$ & 113.8321 & $113.8323^{2}$ & & & \\
\hline Initial Weight $(\mathrm{g})$ & 113.8230 & 113.8230 & & & \\
\hline Particulate Weight $(\mathrm{g})$ & & 0.0093 & & & \\
\hline
\end{tabular}

Particulate Average (mg)

Less Acetone Blank (mg)

Total Particulate $(\mathrm{mg})$

$9.3 \%$
$0.2 \%$

\begin{tabular}{|c|l|l|l|l|l|}
\hline \multicolumn{3}{|c|}{ Run No. 5olume $(\mathrm{ml})$} & 59 & & \\
\hline Final Weight $(\mathrm{g})$ & 114.1803 & 114.1807 & & \\
\hline Initial Weight $(\mathrm{g})$ & 114.1713 & 114.1713 & & & \\
\hline Particulate Weight $(\mathrm{g})$ & & 0.0094 & & & \\
\hline
\end{tabular}

Particulate Average (mg)

Less Acetone Blank (mg)

Total Particulate (mg)

$\frac{9.4 \Omega}{0.1 /}$

\begin{tabular}{|c|l|l|l|l|l|}
\hline Run No. & & & \\
\hline Final Weight (g) & & & & & \\
\hline Initial Weight (g) & & & & & \\
\hline Particulate Weight (g) & & & & & \\
\hline
\end{tabular}

Particulate Average (mg)

Less Acetone Blank (mg)

Total Particulate $(\mathrm{mg})$

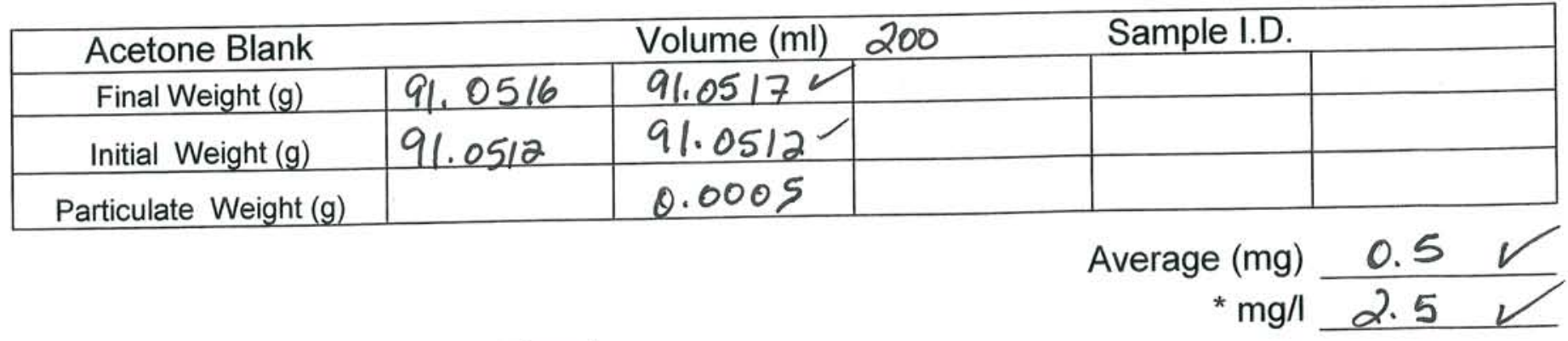

* Note: if greater than $7.9 \mathrm{mg} / \mathrm{l}$, use $7.9 \mathrm{mg} / \mathrm{l}$ 
AIR

SAMPLING

ASSOCIATES, INC.

Particulate Analysis EPA Method 5

Front Half Tare Weights

Project No. $07-008$

Project Name ADA -ES
Location Newark, AR

Unit Tested Unit No. 2 Stack

\begin{tabular}{|r|cc|c|c|c|}
\hline Desiccator Time In & $09302 / 107$ & $1105 \% / 2 / 07$ & & & \\
\hline Desiccator Time Out & $1100 \quad 2 / 21070650 \quad 2 / 407$ & & & \\
\hline
\end{tabular}

\begin{tabular}{|l|l|l|l|l|}
\hline Run No. I & \multicolumn{3}{|c|}{ Sample I.D. } \\
\hline Weight (g) & $/ 13.8893$ & 113.8889 & & \\
\hline
\end{tabular}

\begin{tabular}{|l|l|l|l|l|}
\hline Run No. Z Sample I.D. \\
\hline Weight (g) & 91.4877 & $91.4877^{4}$ & & \\
\hline
\end{tabular}

\begin{tabular}{|l|l|l|l|l|}
\hline Run No. 3 Sample I.D. \\
\hline Weight (g) & 111.8364 & $/ 168359$ & & \\
\hline
\end{tabular}

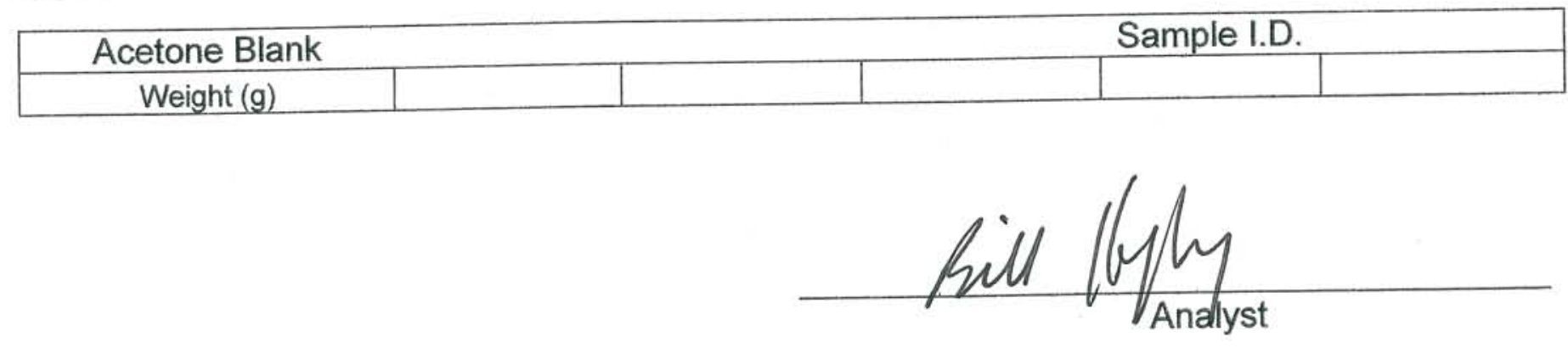

Version No. 1 07/06/06

E-9 


\section{II) AIRMPLNG \\ ASSOCIATES, INC.}

\section{Particulate Analysis EPA Method 5}

\section{Front Half Tare Weights}

Project No. of -008

Project Name ADA-ES

Location Navark, AR Unit Tested Unit No. 2 Stack

\begin{tabular}{|c|l|l|l|l|}
\hline Desiccator Time In $1000^{2} / 1 / 071110^{2} / 2 / 07$ & & & \\
\hline Desiccator Time Out $1105^{2 / 2} / 0706502 / 4107$ & & & \\
\hline
\end{tabular}

\begin{tabular}{|l|l|l|l|l|}
\hline Run No. 4 & \multicolumn{3}{c|}{ Sample I.D. } \\
\hline Weight $(\mathrm{g})$ & $1 / 3.8228$ & 113.8230 & & \\
\hline
\end{tabular}

\begin{tabular}{|l|l|l|l|l|}
\hline Run No. 5 & \multicolumn{3}{c|}{ Sample I.D. } \\
\hline Weight (g) & $/ 1 / 4,17 / 4$ & $/ 14.17 / 3^{d}$ & & \\
\hline
\end{tabular}

\begin{tabular}{|l|l|l|l|l|}
\hline Run No. 6 & \multicolumn{3}{c|}{ Sample I.D. } \\
\hline Weight (g) & 110.4922 & 110.49264 & & \\
\hline
\end{tabular}

Acetone Blank Weight (g)

\section{Sample I.D.}

$91.0508|91.05 / 2-|$

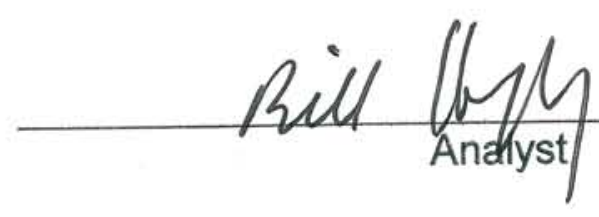




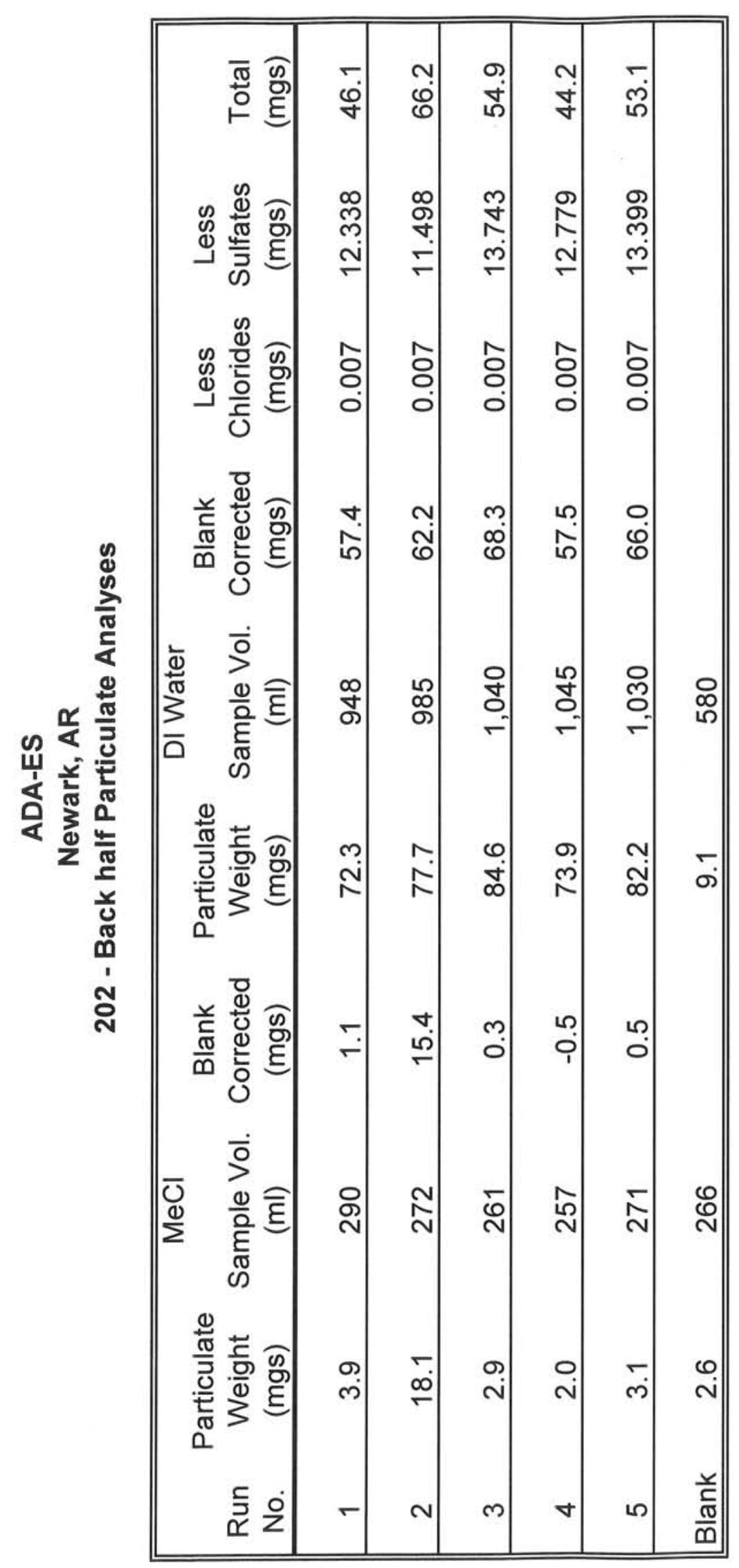




\section{Methylene Chloride Rinse}

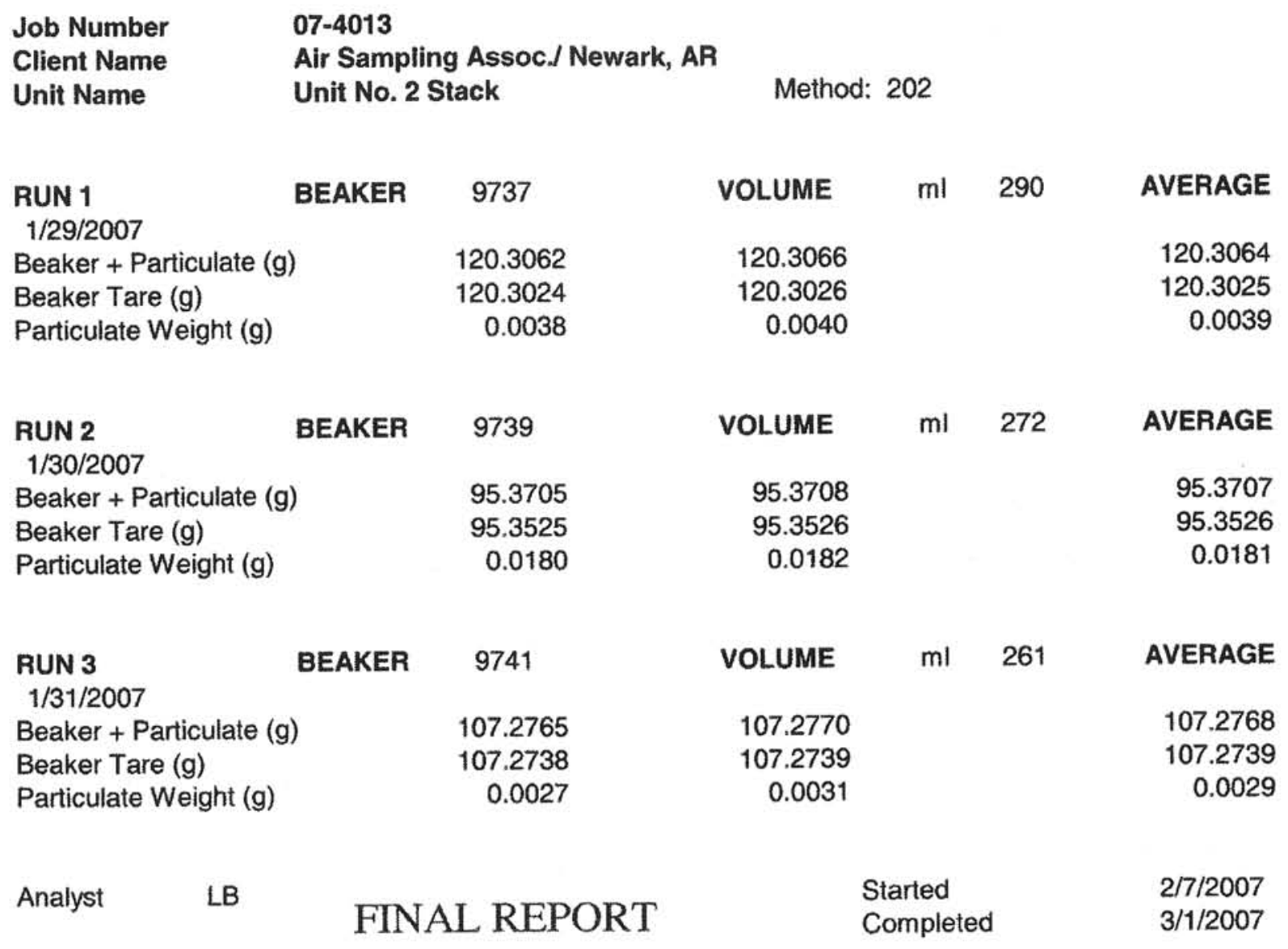




\section{Methylene Chloride Rinse}

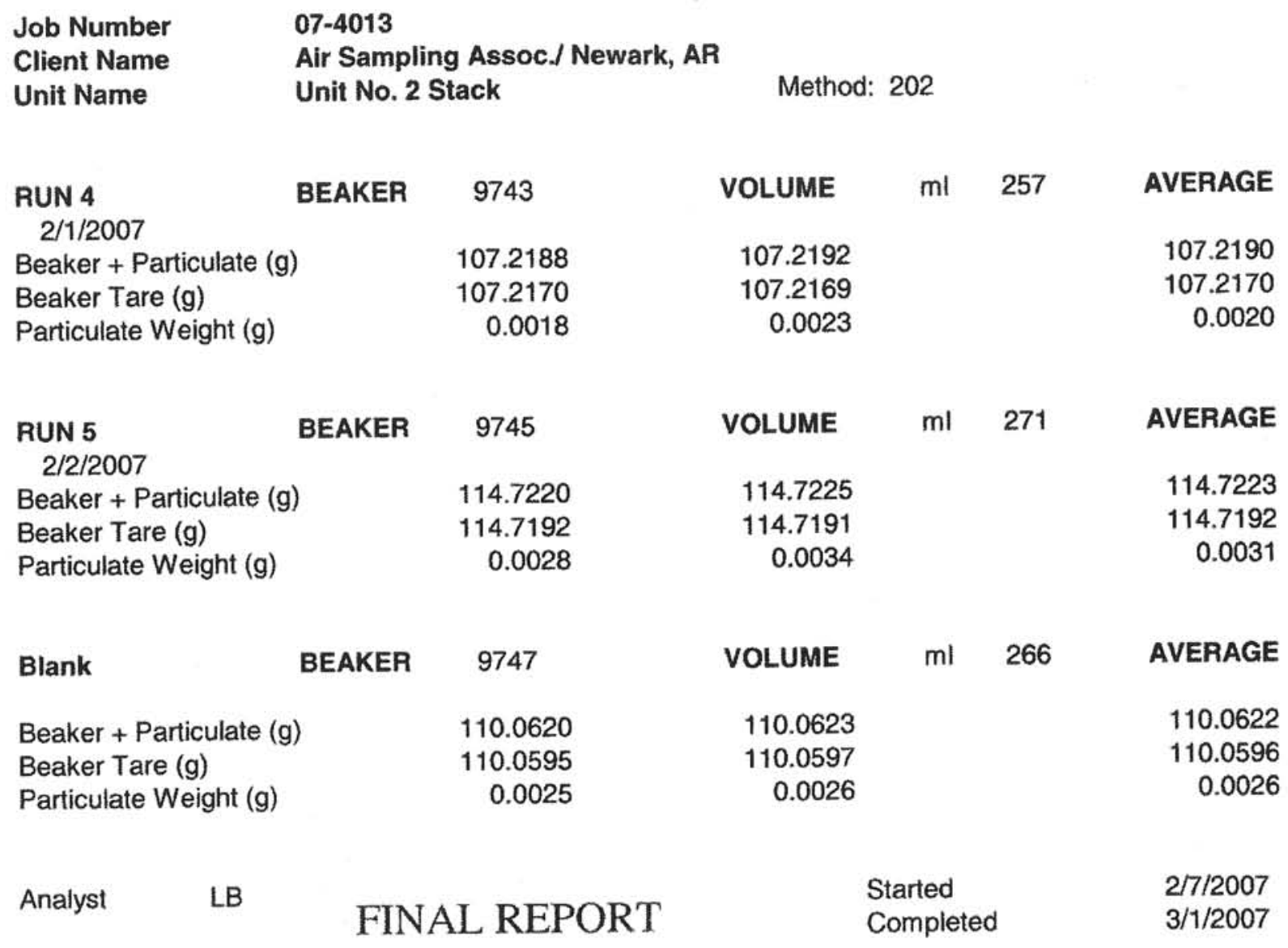




\section{Water Analysis}

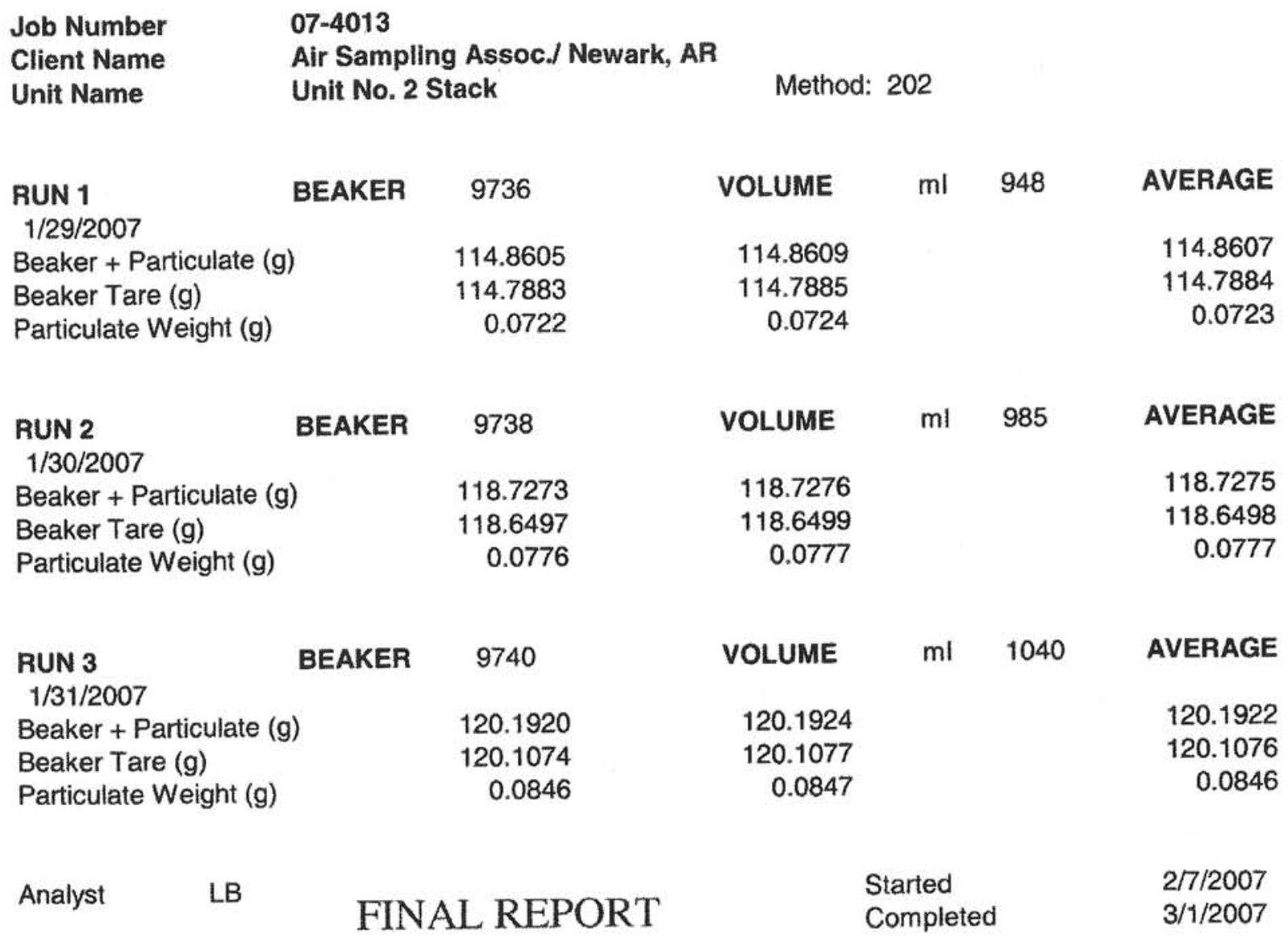


DI Water Analysis

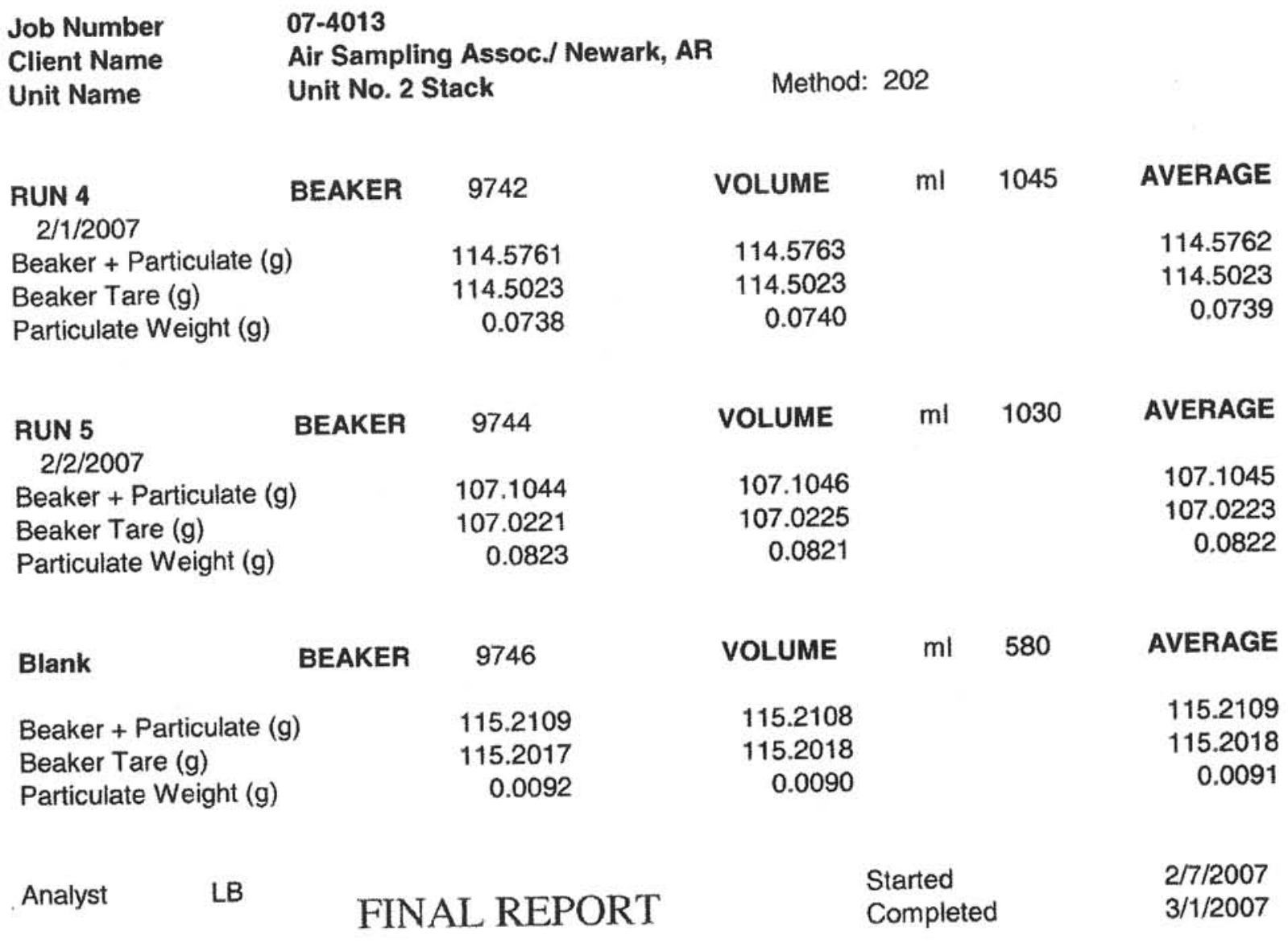




$\begin{array}{lcccccc}\text { 07-4013 } & \begin{array}{c}\text { Sample } \\ \text { Volume, } \\ \mathrm{ml}\end{array} & \begin{array}{c}\text { Lab } \\ \text { Volume, } \\ \mathrm{ml}\end{array} & \begin{array}{c}\text { Lab } \\ \text { Results, } \\ \mathrm{ug} / \mathrm{ml}\end{array} & \begin{array}{c}\text { Blank, } \\ \mathrm{ug} / \mathrm{ml}\end{array} & \begin{array}{c}\text { Final } \\ \text { Results, } \\ \mathrm{mg}\end{array} \\ \text { Run 1 } & \begin{array}{c}\text { Sample No. } \\ \text { 4013-1 }\end{array} & 848 & 20 & 41.1 & 0 & 12.338 \\ \text { Run 2 } & 4013-2 & 885 & 20 & 36.7 & 0 & 11.498 \\ \text { Run 3 } & 4013-3 & 940 & 20 & 41.3 & 0 & 13.743 \\ \text { Run 4 } & 4013-4 & 945 & 20 & 38.2 & 0 & 12.779 \\ \text { Run 5 } & 4013-5 & 930 & 20 & 40.7 & 0 & 13.399 \\ \text { Blank } & 4013-6 & 580 & 20 & 0 & & \end{array}$


Final

Results,

$\begin{array}{cc}\text { Lab } & \mathrm{mg} \\ \text { Results, } & \text { Corrected }\end{array}$

ug Blank, ug to $\mathrm{NH} 4 \mathrm{Cl}$

\begin{tabular}{lcccc}
\multicolumn{5}{c}{ Sample No. } \\
Run 1 & 9736 & $<1.0$ & 5.9 & -0.007 \\
Run 2 & 9738 & $<1.0$ & 5.9 & -0.007 \\
Run 3 & 9740 & $<1.0$ & 5.9 & -0.007 \\
Run 3 & 9742 & $<1.0$ & 5.9 & -0.007 \\
Run 3 & 9744 & $<1.0$ & 5.9 & -0.007 \\
& & & & \\
Blank & 9746 & 5.9 & &
\end{tabular}


Appendix F:

Chain of Custodies 


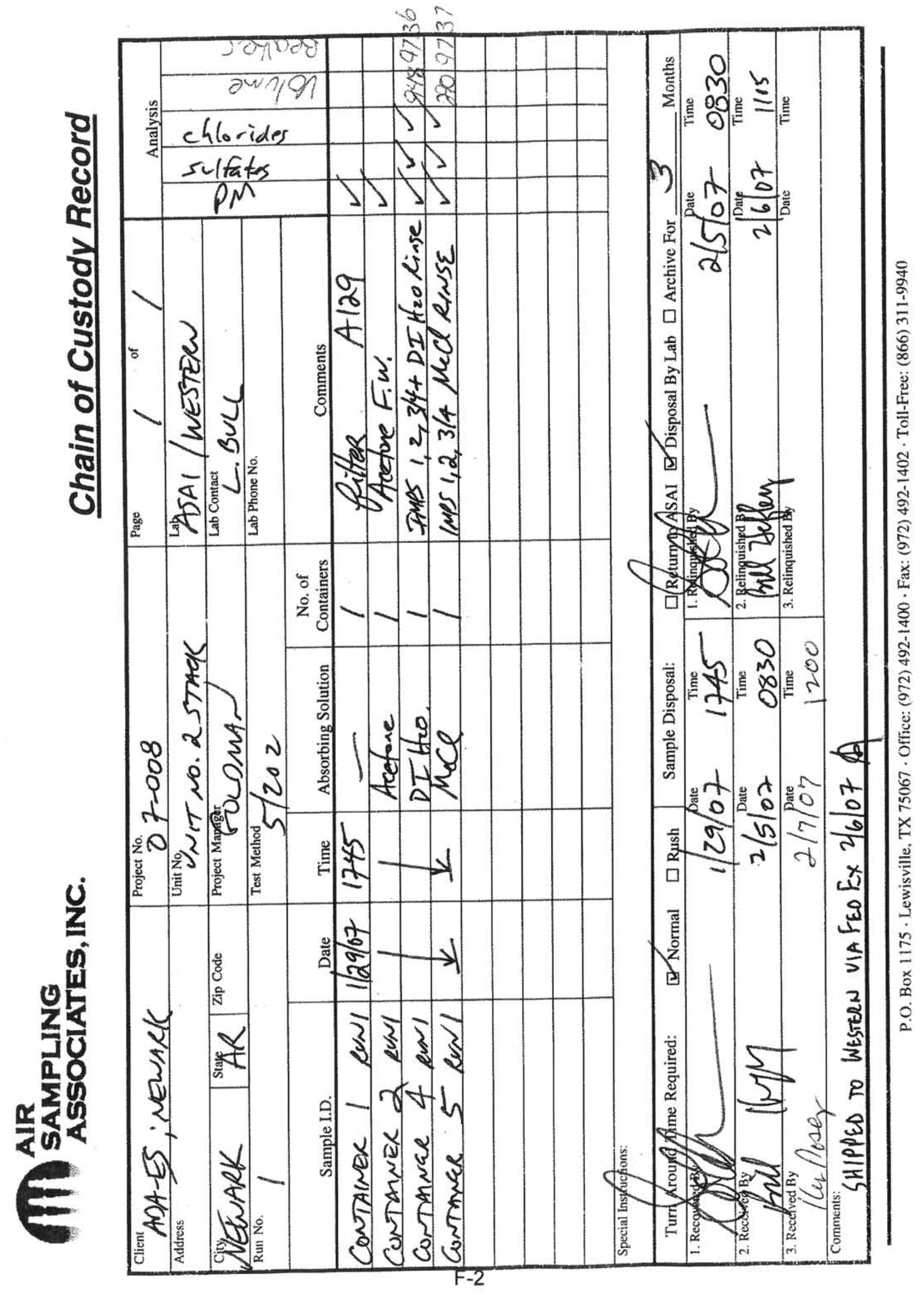




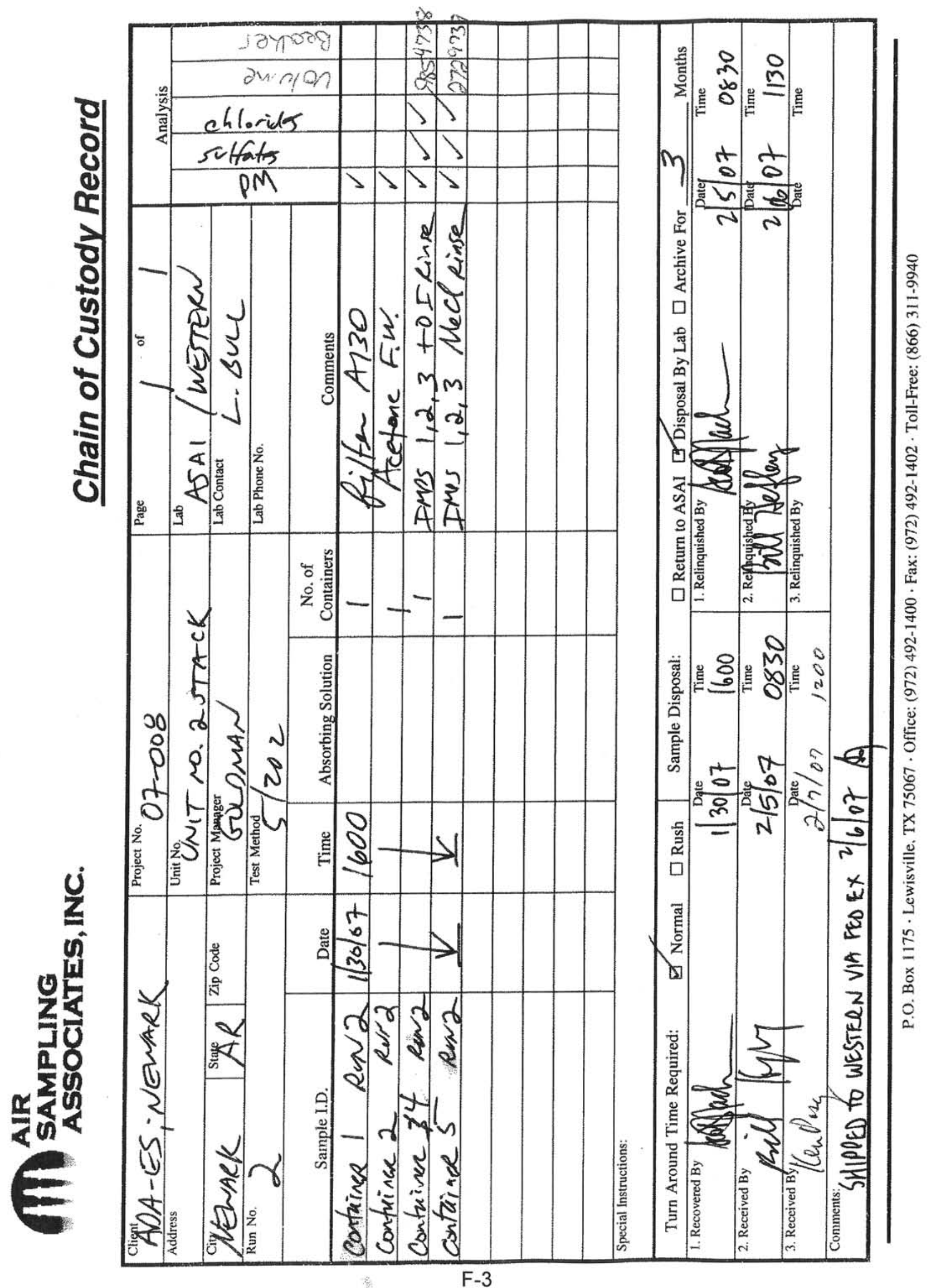




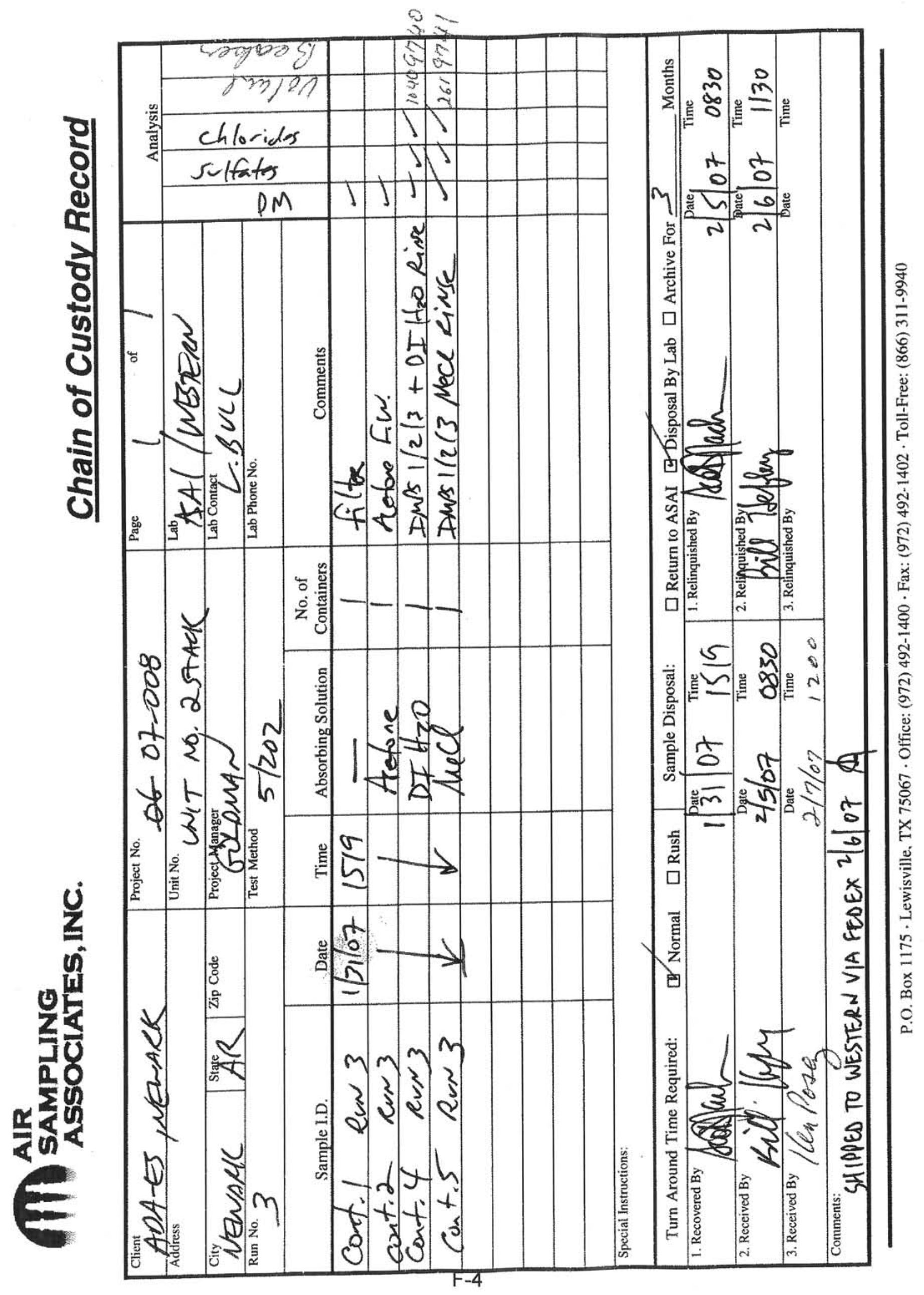




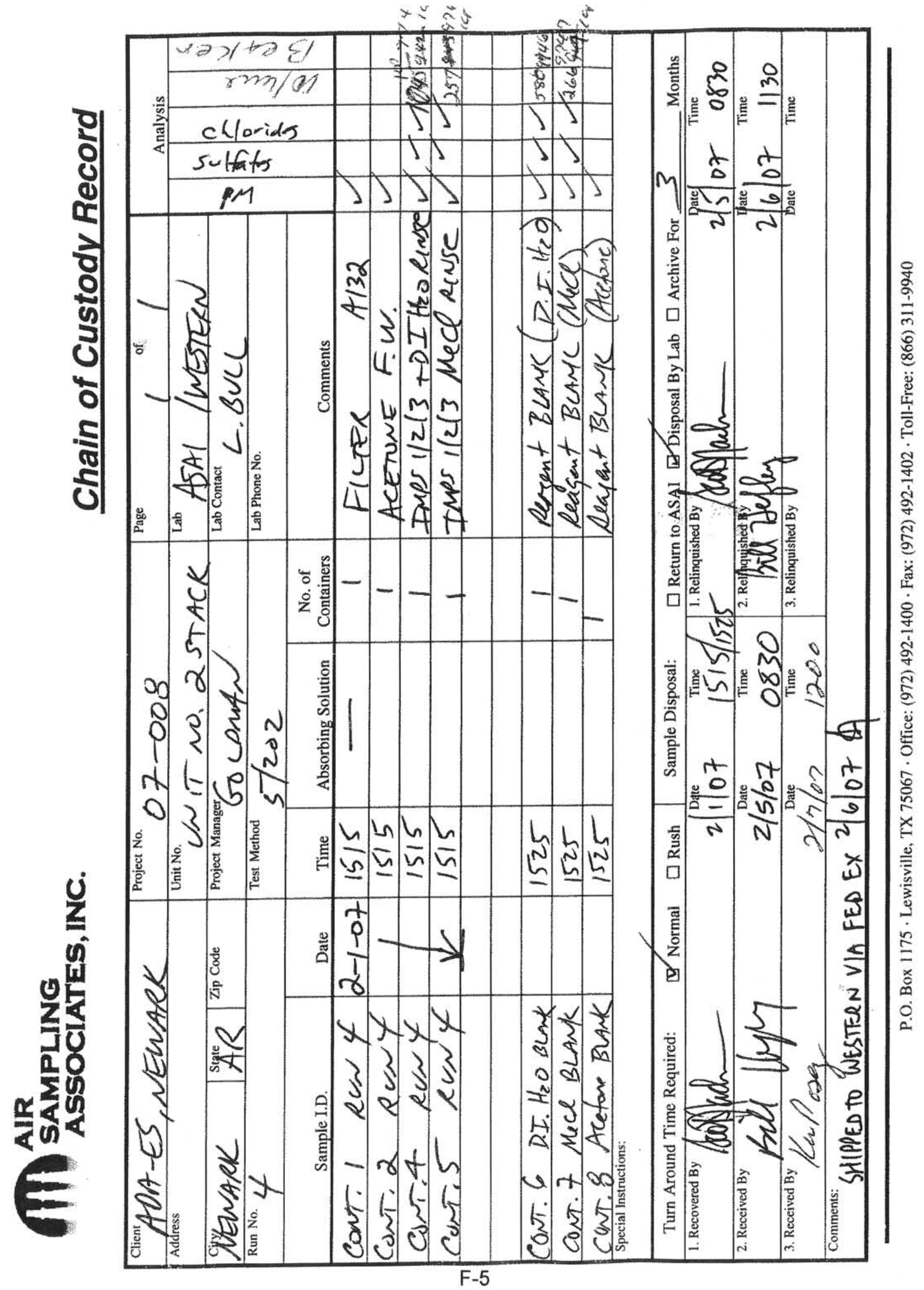




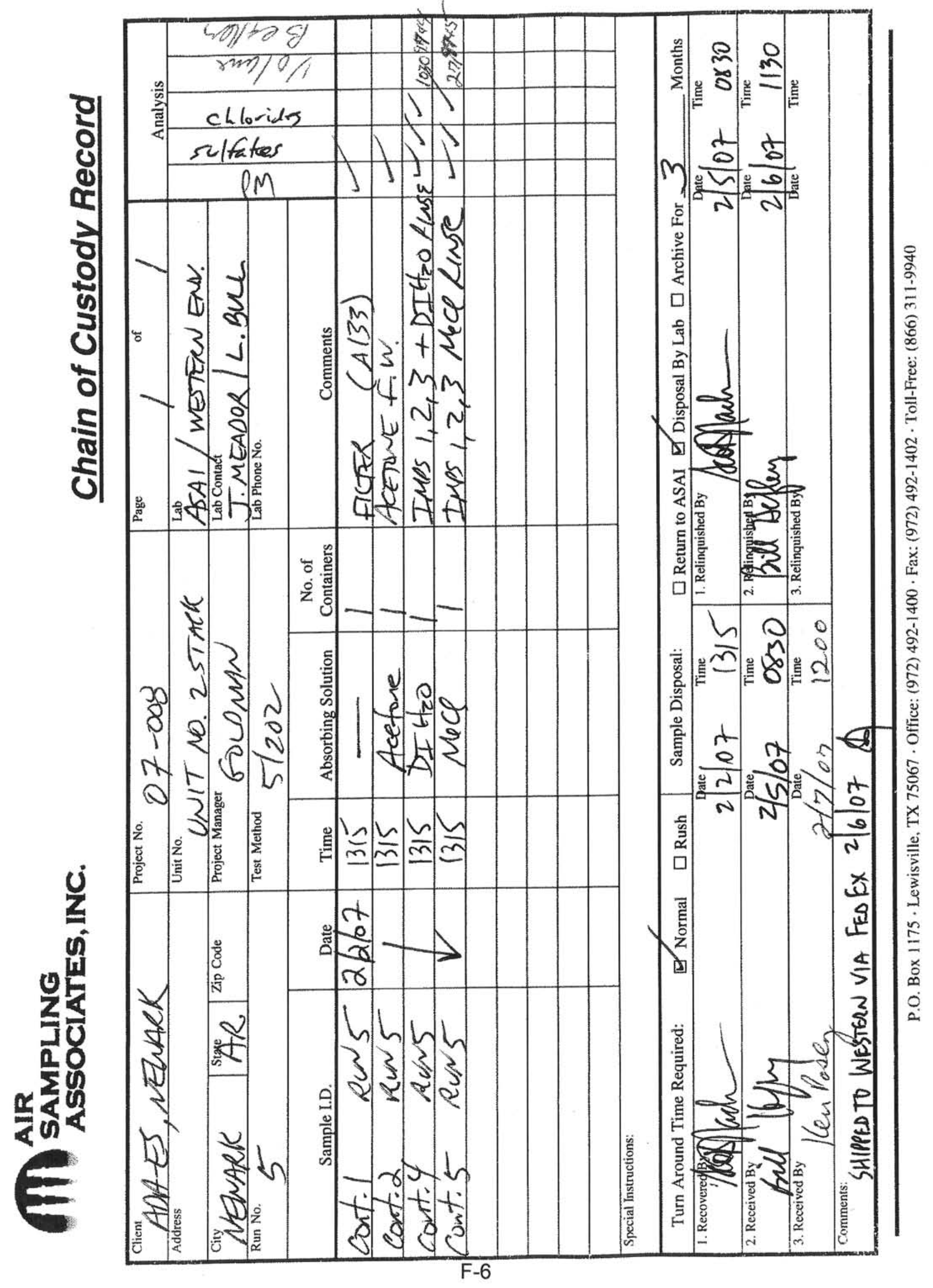




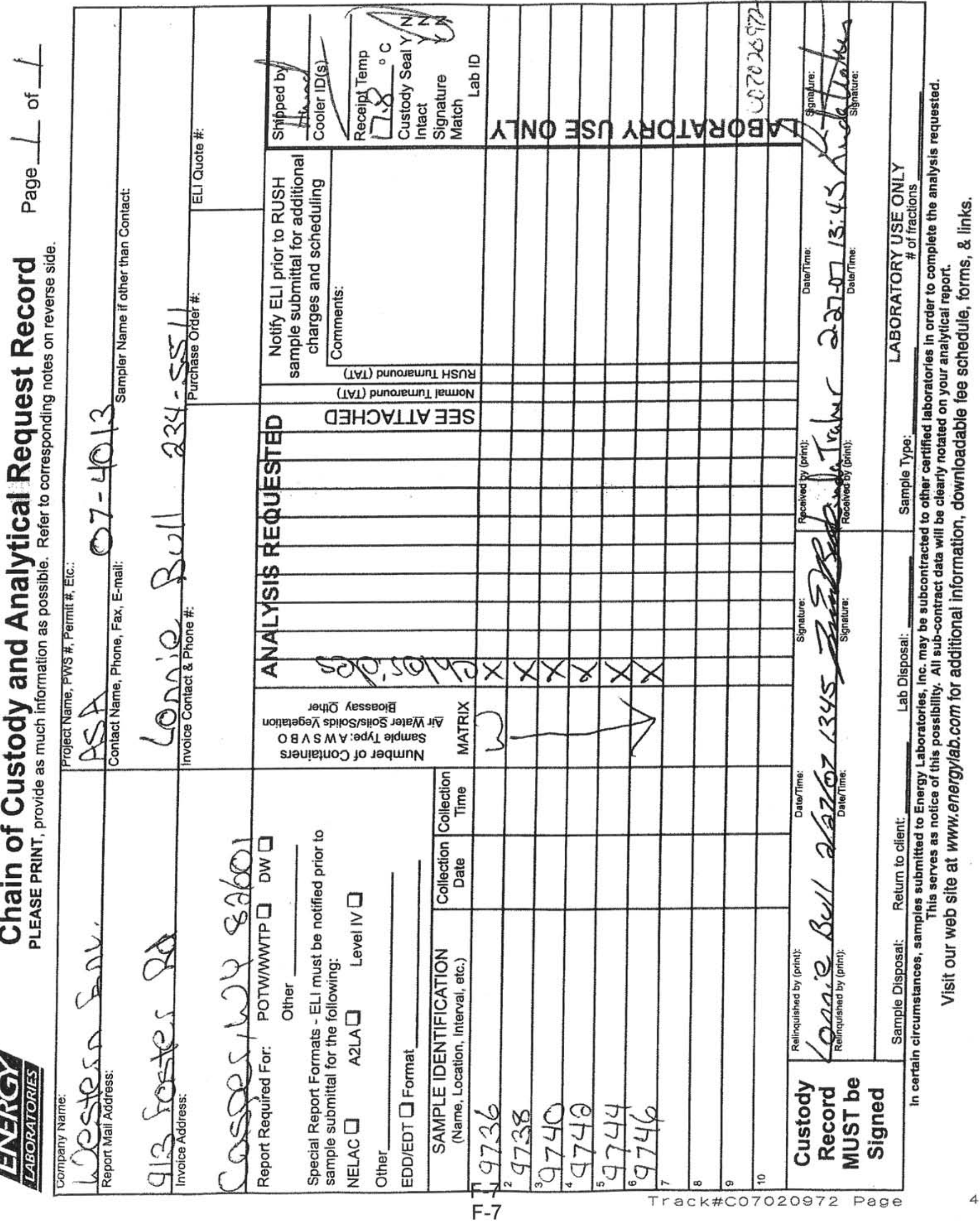




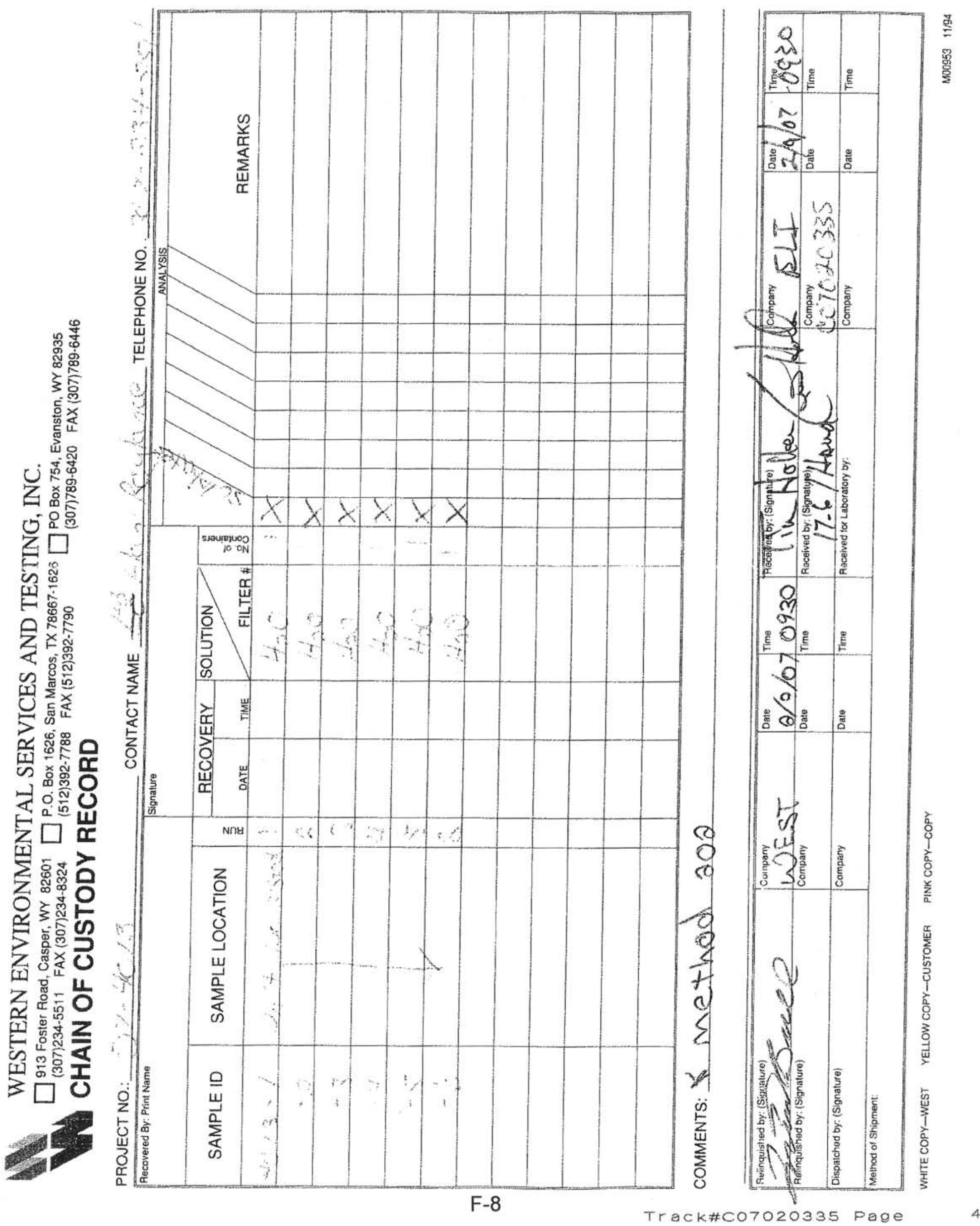


Appendix G:

Resumes of Test Personnel 
BILLY J. MULLINS, JR.; President

Education

Post Graduate Study Environmental Engineering at Southern Methodist University; Dallas, Texas 1970.

M.S. 1969, New York University; New York, New York, in Civil Engineering (Air Resources).

B.S. 1968, Texas Tech University; Lubbock, Texas, in Civil Engineering (Water Resources). Studies in Engineering at the U.S. Naval Academy; Annapolis, Maryland, 1963-1964

Professional Attended Short Course on Air Pollution Engineering at the University Training of Texas at Austin, February 1970.

$\underline{\text { Courses }}$

Attended four-week management course presented by the American Management Association, 1976.

Certification Registered Professional Engineer

Certified Visible Emissions Evaluator

Licensed Private Pilot (Multi-Engine-Land, Instrument)

Diplomat in the American Academy of Environmental Engineers

Inductee into the Stack Sampling Hall of Fame

Certified as Qualified Environmental Professional (QEP)

Professional Air \& Waste Management Association - Past Chairman, Past Vice Memberships Chairman, and Past Board of Directors of North Texas Chapter and Southwest Section; Past Chairman, Consultants Committee; Past Chairman, Source Measurement Committee

Source Evaluations Society - Past President, Past Board of Directors

\section{American Management Association}


MULLINS (p. 2)

Publications Authored "Real World Experience with USEPA's New Sampling and Analytical Methods for Conducting Risk Burn," May 1998.

Co-authored "Sulfur Compound Emissions of the Petroleum Production Industry," December 1974.

Co-authored "Field Procedure for Stabilizing Hydrogen Sulfide Samples to be Analyzed Using Modified Methylene Blue Technique," presented at the Conference on Ambient Air Quality Measurements, Austin, Texas, March 1975.

Co-authored "Atmospheric Emissions Survey of the Sour Gas Industry," October 1975.

Co-authored "Technique for Insuring the Validity of Samples for High Concentrations of Sulfur Dioxide Using the EPA Method 5 Sampling Train," presented at the Third National Conference on Energy and the Environment, College Corner, Ohio, September 1975.

Teaching

Conducted training seminars on sampling methods periodically since Experience 1974 to present.

Conducted a one-day seminar on Part 75 Testing over ten times in 1993 and 1994.

Served as a lecturer in the Environmental Protection Agency's (EPA) training course number 450, "Source Sampling for Particulate Pollutants," for two years from January 1974 to October 1975 and March, 1992.

Conducted a two-day training course entitled "technical Assistance in Source Sampling" at lowa State University, Ames, lowa, for the Environmental Protection Agency (EPA), October 1974.

Conducted Environmental Protection Agency's (EPA) training course number 450, "Source Sampling for Particulate Pollutants," at Research Triangle Park, North Carolina, September 1975. 
MULLINS (p. 3)

Teaching

Experience

(Cont'd)
Technical

Experience
Conducted a two-day short course entitled "Performing and Observing Source Sampling," Dallas, Texas, July 1976, May 1977, October 1977, November 1987 and November 1988; Lake Charles, Louisiana, May 1977; Casper Wyoming, May 1977; Point Comfort, Texas, November 1992.

Served as a lecturer in the Environmental Protection Agency's twoday seminar entitled "Asphalt Industry Environmental Solutions," presented in Dallas, Texas, March 21-22, 1979.

Conducted a two-day short course entitled "Performing and Observing Source Sampling," Phoenix, Arizona, August, 1990, for the State of Arizona, Department of Environmental Quality; Lincoln, Nebraska, March 1980, for the State of Nebraska, Air Quality Control Division.

Directed and performed stack sampling on over 2000 sources of which over 500 were sampled simultaneously using more than one sampling train at several points in the flue gas stream; 1972-present.

Directed and performed over 200 short-term ambient air studies using mobile sampling vans and various ambient air sampling equipment; 1972-present.

Designed, directed and operated over 20 permanent ambient air networks of various size and duration for a variety of parameters; 1972-present.

Designed surface and underground drainage systems for residential subdivisions, public works projects, and shopping centers; 19691972.

Designed several residential subdivisions including lot layout, street design, drainage design, and utility design; 1969-1972. 
MULLINS (p. 4)

$\underline{\text { Research }}$

Projects

Related

Projects
Supervised and conducted a study made by the Hawaiian Sugar Planters' Association to characterize the emissions for several bagasse-fired boilers, April-May 1976.

Supervised and conducted a study made by the Rio Grande Valley Sugar Growers, Inc. to determine the area affected by the burning of sugarcane fields prior to harvesting, November 1974-April 1975.

Supervised and conducted a study by a lightweight aggregate manufacturer to develop a material balance around the process through sampling and analysis of several parameters, November 1973.

Conducted a study in New York City to attempt to develop a correlation in the ambient air between carbon dioxide and sulfur dioxide to provide a tool for predicting air pollution predicting air pollution episodes, January-May 1969.

Served as Chairman of the Engineering Foundation Conference on Stack Sampling and Source Evaluation in Destin, Florida, 2002, and Santa Barbara, California, 1985.

Served as Co-Chairman of the Engineering Foundation Conference on Stack Sampling and Source Evaluation in Destin, Florida, 2001.

Served as Session Chairman at the Engineering Foundation Conference on Stack Sampling and Source Evaluation in Hershey, Pennsylvania, 1984; San Diego, California, 1993; and in Palm Coast, Florida, 1994. 
GARY B. GOLDMAN; Associate

Education

Certification

Professional

Memberships

Technical

Experience
B.S. 1993, University of Texas at Arlington; Arlington, Texas, in Geology.

Certified Visible Emissions Evaluator

\section{Source Evaluation Society}

Senior Emissions Evaluator with the Texas Commission on Environmental Quality, responsible for oversight of all source testing activities within the State of Texas, Region 4, which encompasses 19 counties in the North Texas region, 1999-2005.

Participated in the sampling of over 750 sources, including several of which were sampled simultaneously utilizing more than one sampling train, 1994-1999.

Thoroughly trained in all EPA testing procedures specified in Title 40 of the Code of Federal Regulations, Part 60.

Thoroughly trained in Test Methods for Evaluating Solid Waste, Physical/Chemical Methods, 0010 Through 0100 Series.

Participated in EPA's 3-D probe study. May - August 1997.

Experienced in the analysis of commercial calibration gas cylinders for $\mathrm{NO}_{x}, \mathrm{SO}_{2}, \mathrm{CO}_{2}$, and $\mathrm{O}_{2}$.

Experienced with calibration techniques for all field testing equipment.

Thoroughly trained in the operation and routine maintenance of the following:

Anarad Model AR50-C Carbon Dioxide Analyzer

Anarad Model AR880 Oxides of Nitrogen Analyzer

Anarad Model AR23 Oxygen Analyzer

Anarad Model AR30C2 Sulfur Dioxide Analyzer 
Professional Training
California Analytical Model 300-HFID Total Hydrocarbon Analyzer Servomex Model 1440 Carbon Dioxide Analyzer Servomex Model 1440 Oxygen Analyzer

Teledyne Model 326 Oxygen Analyzer Thermo Environmental Model 10AR/S Oxides of Nitrogen Analyzer Thermo Electron Model 46C Oxides of Nitrogen Analyzer Thermo Electron Model 48C Oxides of Nitrogen Analyzer Western Research Model 721A Sulfur Dioxide Analyzer

Attended 40-hour Occupational and Environmental Training Program on Hazardous Materials (CFR 1910.120) Dallas, Texas, April 1993.

Attended HAZWOPER 8-hour refresher course, 1994-2005.

Attended the following Environmental Protection Agency (EPA) Courses:

Course 345, Emission Capture and Gas Handling System Inspection

Course 380, Fugitive Source Inspection

Course 400, Introduction to Hazardous Air Pollutants Course 413, Control of Particulate Emissions

Course 415, Control of Gaseous Emissions Course 418, Control of Nitrogen Oxide Emissions Course 427, Combustion Evaluation Course 444, Air Pollution Field Enforcement Course 445, Inspection of Particle Control Devices Course 446, Inspection Safety Procedures Course 450, Source Sampling for Particulate Pollutants Course 452, Principles and Practice of Air Pollution Control Course 455, Inspection of Gas Control Devices and Selected Industries

Course 474, Continuous Emission Monitoring Course 482, Sources and Control of Volatile Organic Air Pollutants

Attended the following California Air Resources Board (CARB) Courses

Course 221, Continuous Emission Monitoring Course 233, Solvent Cleaning: Degreasing Operations Course 242, Hot Mix Asphalt Facilities

Course 245, Cement Plants

Course 270, Incinerators 
SCOT JACKSON; Associate

Education

Professional

Training

Certification

Technical

Experience
B.S.B.A. May 1978, Mountain View Jr. College, in General Business.

Purchasing Supervisor for METCO Environmental, Inc. in charge of inventory and supplies. January 1995 - April 2005.

Attended 40-hour Occupational and Environmental Training Program on Hazardous Materials (CFR 1910.120), Dallas, Texas, May 2000.

Attended Fed-Ex Hazardous Goods Shipping Training, June 2004.

Certified Visible Emissions Evaluator

Participated in the sampling of over 100 sources, including several of which were sampled simultaneously using more than one sampling train. Thoroughly trained in all EPA testing procedures, 1995present.

Experience with calibration techniques for all field testing equipment.

Thoroughly trained in the operation and routine maintenance of the following:

California Analytical Model 300-HFID Total Hydrocarbon Analyzer Servomex Model 1440 Carbon Dioxide Analyzer

Servomex Model 1440 Oxygen Analyzer

Thermo Electron Model 42C Oxides of Nitrogen Analyzer

Thermo Electron Model 48C Oxides of Nitrogen Analyzer

Western Research Model 721A Sulfur Dioxide Analyzer 


\section{APPENDIX E:}

Consulting Reports 
APPENDIX E1: REI, Lance Modeling-May 31, 2006 


\section{CFD Modeling of TOXECON II at Independence}

\section{Lance Simulation Results}

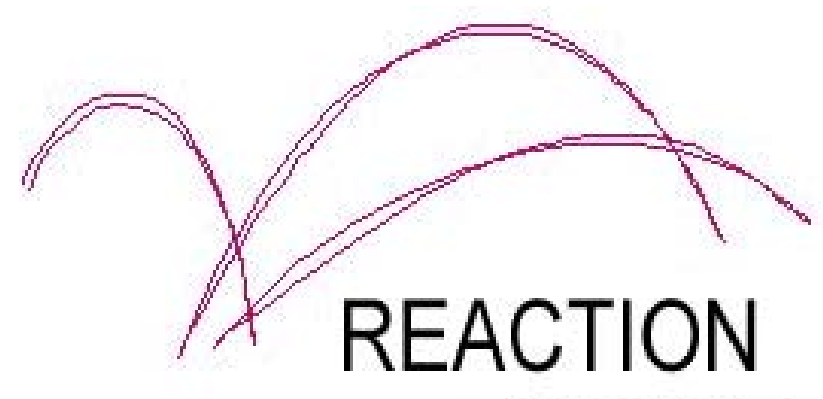

ENGINEERING

INTERNATIONAL

May 31, 2006 


\section{Overview of Project}

- Objective: Estimate the distribution of injected activated carbon between the $2^{\text {nd }}$ and $3^{\text {rd }}$ ESP fields

- Approach:

» Build a CFD model of the multi-nozzle lance arrangement to predict the distribution of $A C$ exiting the lance

» Build a CFD model of the ESP to estimate the resulting distribution of $A C$ entering the third ESP field

- Status:

» A CFD model of one-half of the injection lance has been developed and AC distribution out of the lance has been predicted - results are presented here

» The ESP model is being developed 


\section{Lance Dimensions}

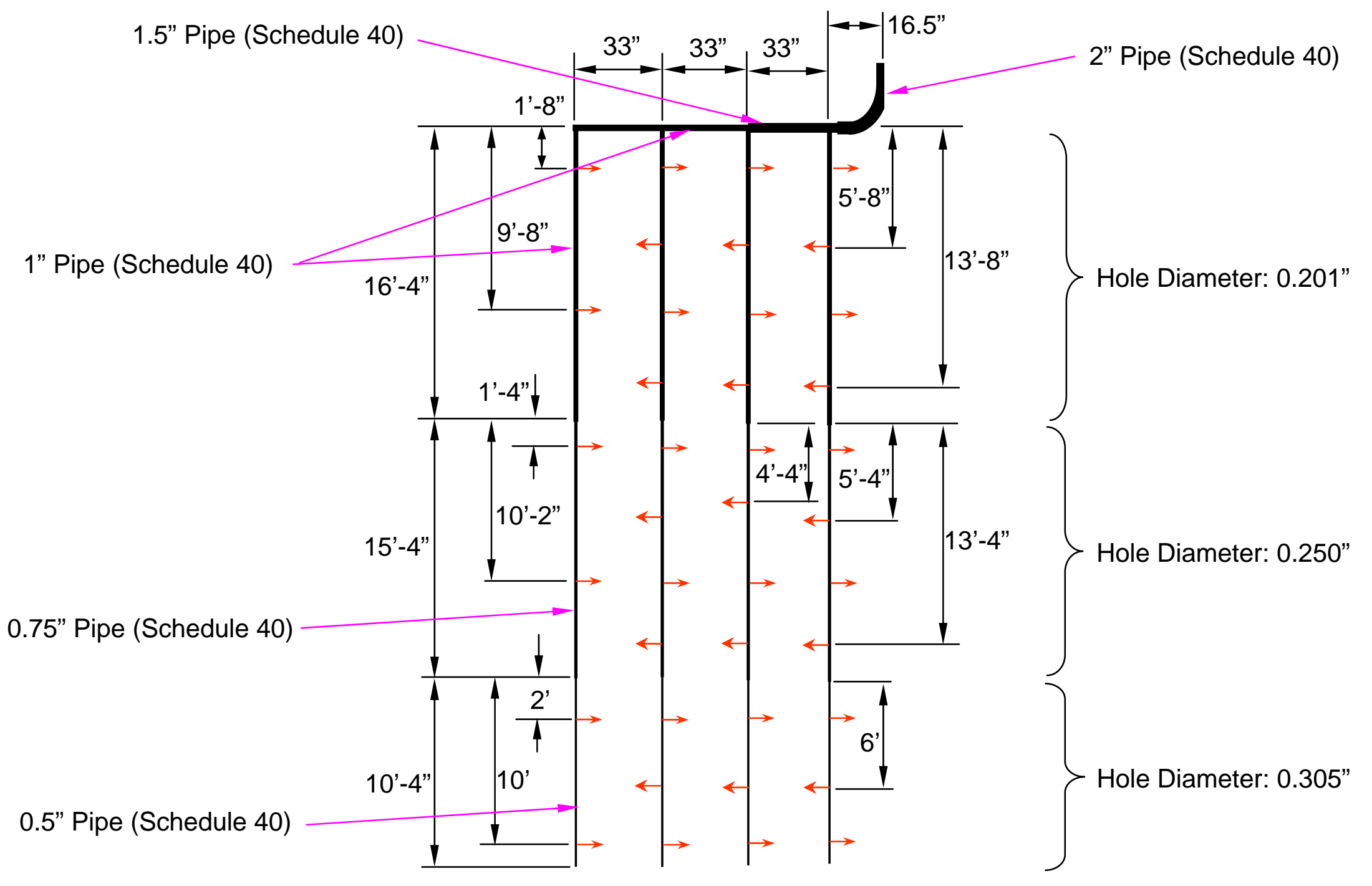

Lance near left side wall was modeled (1/4 of injection grid) 


\section{Lance and Hole Identifiers}

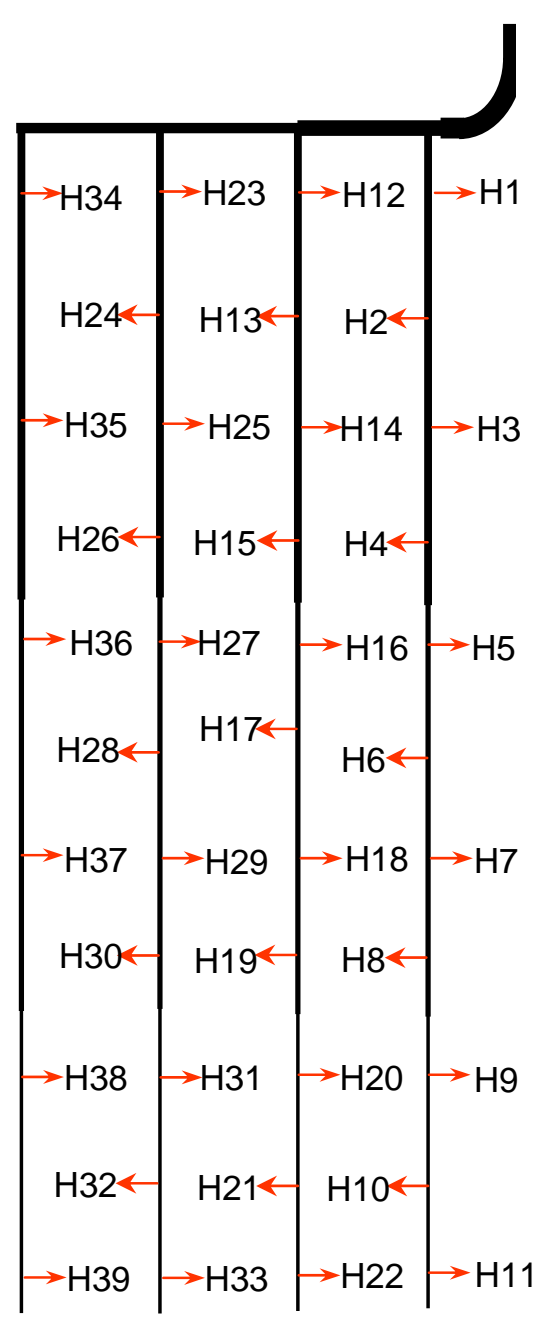

Lance 4 Lance 3 Lance 2 Lance 1 


\section{Lance Model Inputs}

\begin{tabular}{|c|c|}
\hline${\text { Carrier Air Flow (acfm) }{ }^{1}}^{1}$ & 28.1 \\
\hline Carrier Air Flow (lb/hr) & 116.4 \\
\hline Activated Carbon Flow (lb/hr) ${ }^{2}$ & 32.5 \\
\hline Average Carbon Concentration (Ib/mmacf) ${ }^{3}$ & 5 \\
\hline Mass Flow Ratio of Carrier Air to Carbon & 3.58 \\
\hline Carrier Air Temperature $\left({ }^{\circ} \mathrm{F}\right)$ & 110 \\
\hline
\end{tabular}

1. Based on $112.5 \mathrm{acfm}$ for entire injection grid

2. Based on $65 \mathrm{lb} / \mathrm{hr}$ for one half of injection grid

3. Approximately matches the carbon flow rate

based on $3.35 \mathrm{ft} / \mathrm{s}$ flue gas velocity inside ESP 


\section{Activated Carbon Particle Size Distribution}

\begin{tabular}{|c|c|c|}
\hline Size Bin & Diameter $(\mu \mathrm{m})$ & Weight $\%$ \\
\hline 1 & 1.5 & $2 \%$ \\
\hline 2 & 3.3 & $4 \%$ \\
\hline 3 & 4.8 & $6 \%$ \\
\hline 4 & 6.7 & $9 \%$ \\
\hline 5 & 9.2 & $12.5 \%$ \\
\hline 6 & 13.1 & $16.5 \%$ \\
\hline 7 & 19.0 & $16.5 \%$ \\
\hline 8 & 27.2 & $12.5 \%$ \\
\hline 9 & 37.7 & $9 \%$ \\
\hline 10 & 52.3 & $6 \%$ \\
\hline 11 & 74.9 & $4 \%$ \\
\hline 12 & 122.5 & $2 \%$ \\
\hline
\end{tabular}

Based on the lab report provided by ADA-ES 


\section{Computational Grid}

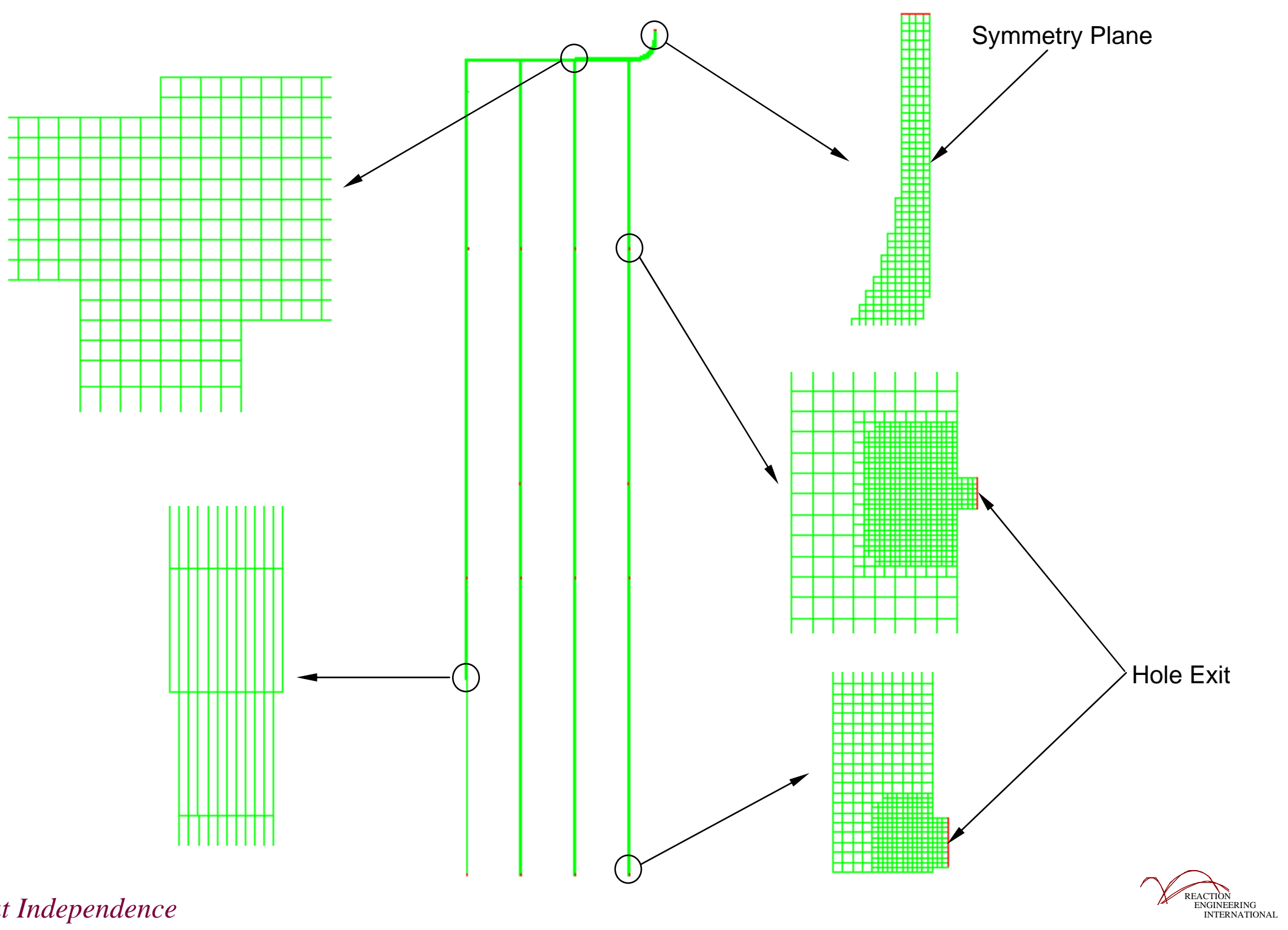




\section{Pressure Distribution \\ Inside Multiple Nozzle Lance}

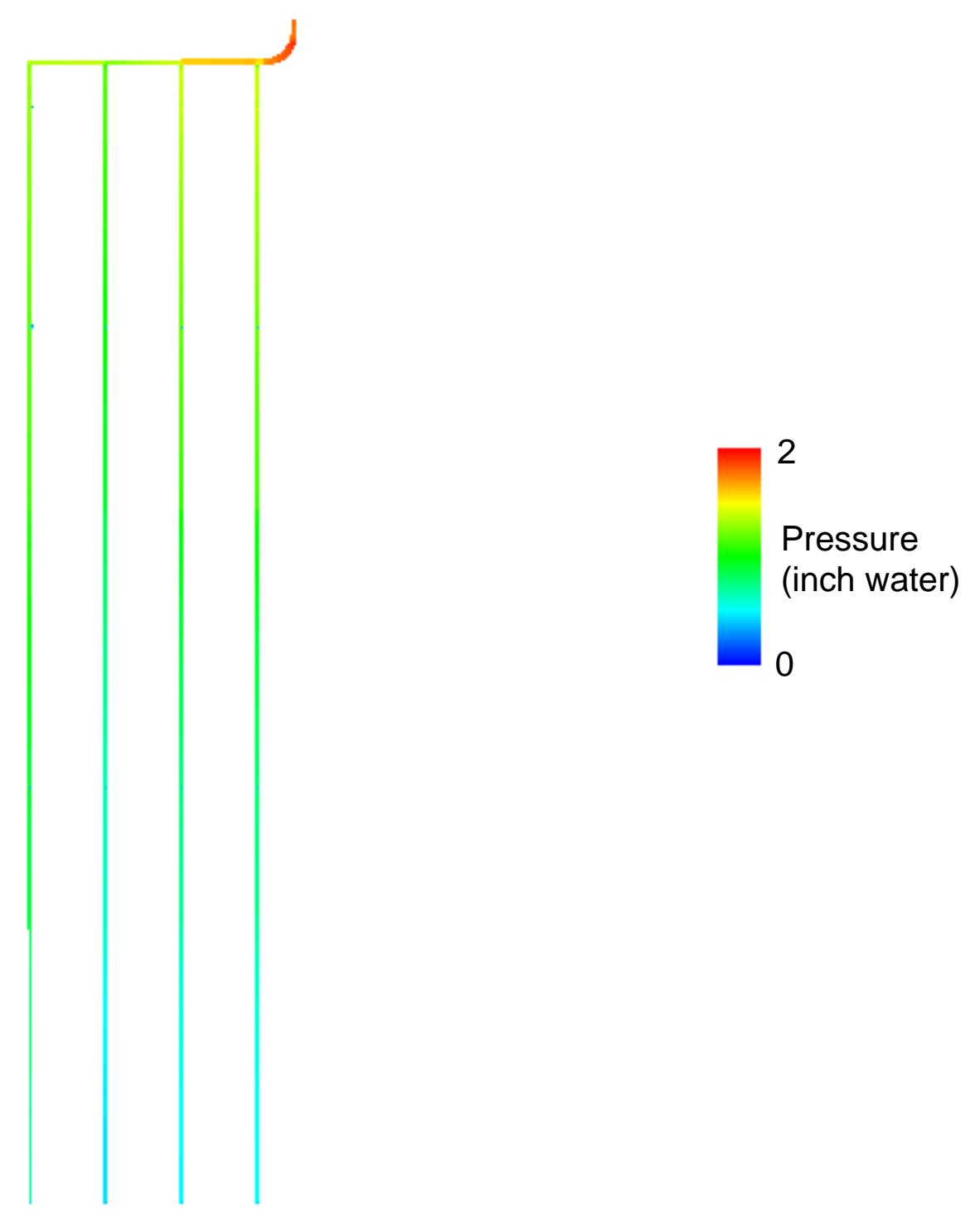




\section{Particle Residence Time}
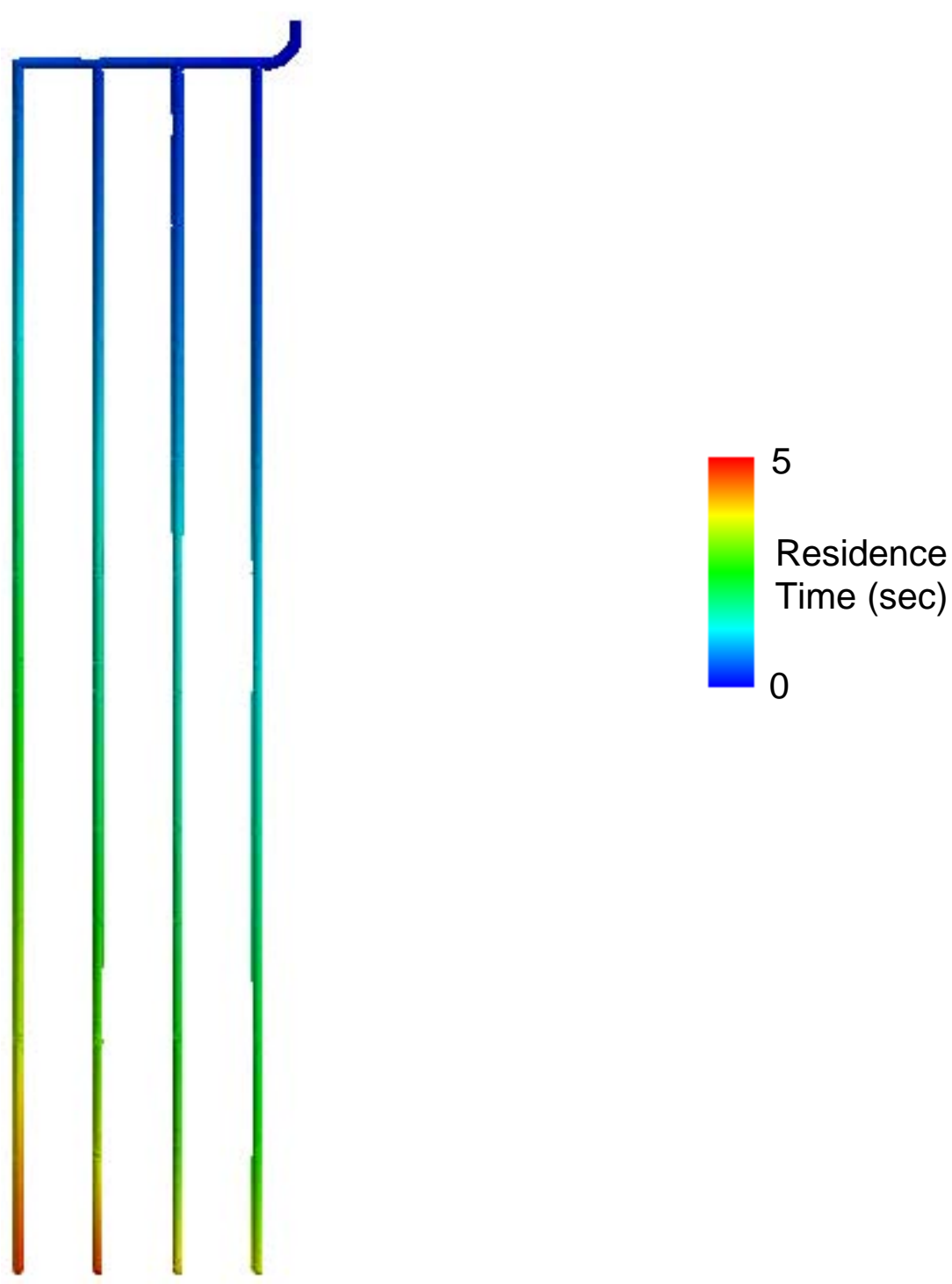


\section{Air Flow Distribution}

\begin{tabular}{|c|c|c|c|c|c|}
\hline Hole ID & $\begin{array}{c}\text { Mass Flow } \\
(\mathbf{l b / h r})\end{array}$ & $\begin{array}{c}\text { Deviation From } \\
\text { Average (\%) }\end{array}$ & Hole ID & $\begin{array}{c}\text { Mass Flow } \\
\text { (lb/hr) }\end{array}$ & $\begin{array}{c}\text { Deviation From } \\
\text { Average (\%) }\end{array}$ \\
\hline $\mathbf{1}$ & 3.050 & $\mathbf{2}$ & $\mathbf{2 1}$ & 2.487 & -15 \\
\hline $\mathbf{2}$ & 2.984 & $\mathbf{2}$ & $\mathbf{2 2}$ & 2.358 & -20 \\
\hline $\mathbf{3}$ & 2.882 & -2 & $\mathbf{2 3}$ & 2.745 & -7 \\
\hline $\mathbf{4}$ & 2.796 & -5 & $\mathbf{2 4}$ & 2.614 & -11 \\
\hline $\mathbf{5}$ & 3.655 & 24 & $\mathbf{2 5}$ & 2.504 & -15 \\
\hline $\mathbf{6}$ & 3.366 & 15 & $\mathbf{2 6}$ & 2.411 & -18 \\
\hline $\mathbf{7}$ & 3.075 & 5 & $\mathbf{2 7}$ & 3.161 & 8 \\
\hline $\mathbf{8}$ & 2.964 & 1 & $\mathbf{2 8}$ & 2.855 & -3 \\
\hline $\mathbf{9}$ & 2.956 & 1 & $\mathbf{2 9}$ & 2.538 & -14 \\
\hline $\mathbf{1 0}$ & 2.606 & -11 & $\mathbf{3 0}$ & 2.535 & -14 \\
\hline $\mathbf{1 1}$ & 2.495 & -15 & $\mathbf{3 1}$ & 2.429 & -17 \\
\hline $\mathbf{1 2}$ & 3.093 & 5 & $\mathbf{3 2}$ & 1.998 & -32 \\
\hline $\mathbf{1 3}$ & 2.955 & 1 & $\mathbf{3 3}$ & 1.844 & -37 \\
\hline $\mathbf{1 4}$ & 2.844 & -3 & $\mathbf{3 4}$ & 3.057 & 4 \\
\hline $\mathbf{1 5}$ & 2.750 & -6 & $\mathbf{3 5}$ & 2.902 & -1 \\
\hline $\mathbf{1 6}$ & 3.583 & 22 & $\mathbf{3 6}$ & 4.010 & 36 \\
\hline $\mathbf{1 7}$ & 3.411 & 16 & $\mathbf{3 7}$ & 3.924 & 34 \\
\hline $\mathbf{1 8}$ & 3.007 & 2 & $\mathbf{3 8}$ & 4.102 & 40 \\
\hline $\mathbf{1 9}$ & 2.904 & -1 & $\mathbf{3 9}$ & 3.855 & 31 \\
\hline $\mathbf{2 0}$ & 2.874 & -2 & & & \\
\hline
\end{tabular}




\section{Air Flow Through Individual Holes}

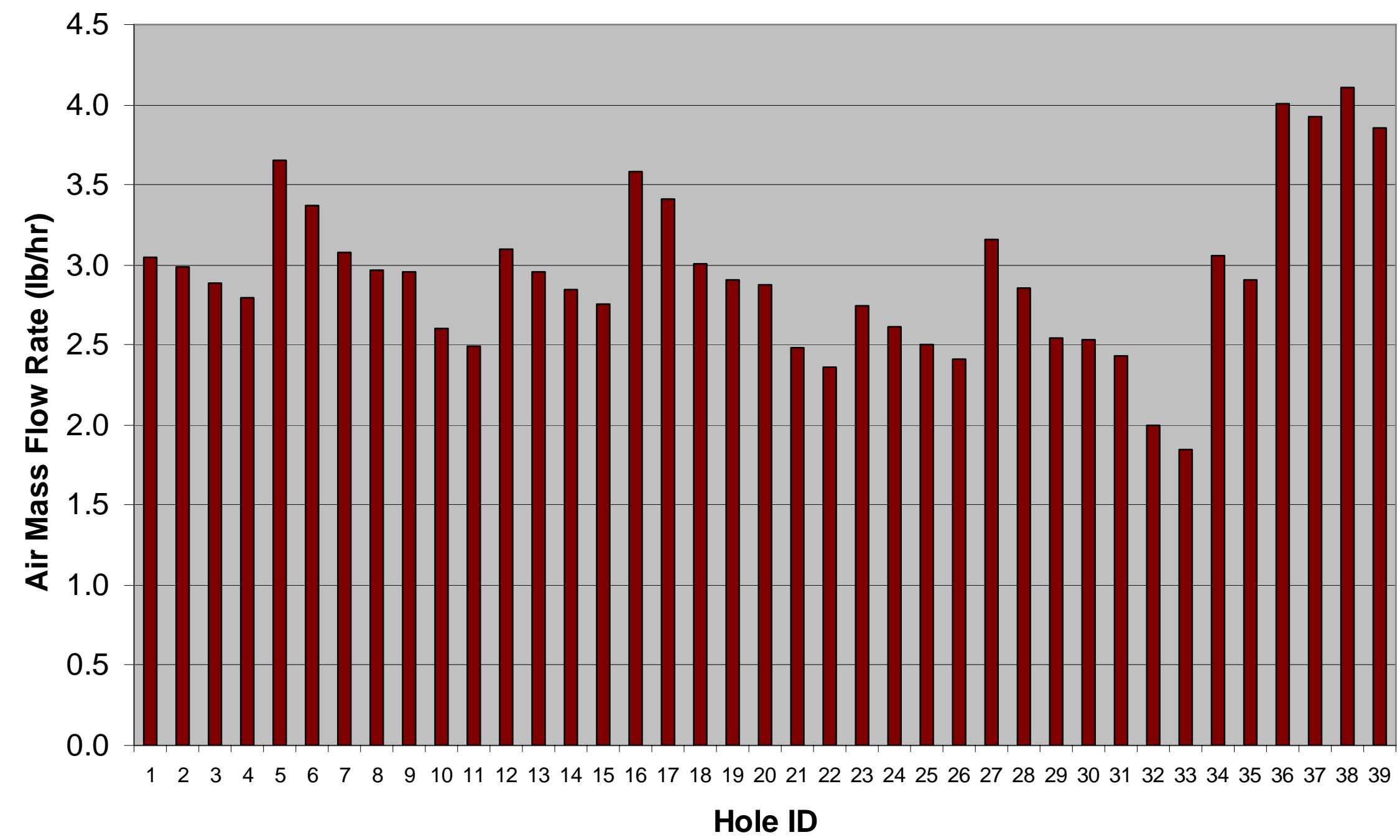




\section{Air Flow Through Individual Lances}

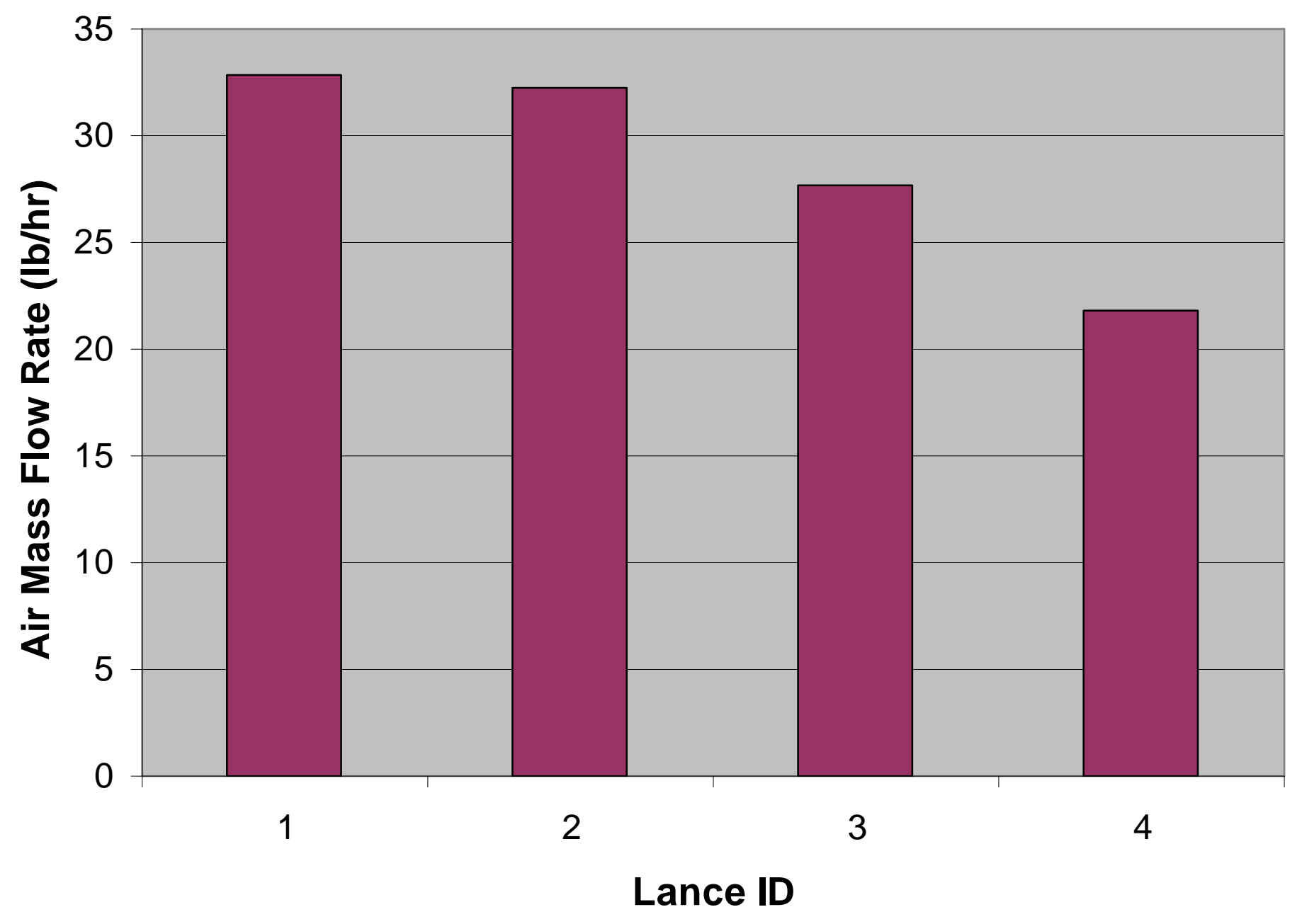




\section{Particle Flow Distribution (All Sizes)}

\begin{tabular}{|c|c|c|c|c|c|}
\hline Hole ID & $\begin{array}{c}\text { Mass Flow } \\
\text { (lb/hr) }\end{array}$ & $\begin{array}{c}\text { Deviation From } \\
\text { Average (\%) }\end{array}$ & Hole ID & $\begin{array}{c}\text { Mass Flow } \\
\text { (lb/hr) }\end{array}$ & $\begin{array}{c}\text { Deviation From } \\
\text { Average (\%) }\end{array}$ \\
\hline $\mathbf{1}$ & 0.125 & -85 & $\mathbf{2 1}$ & 0.270 & -68 \\
\hline $\mathbf{2}$ & 0.075 & -91 & $\mathbf{2 2}$ & 6.771 & 712 \\
\hline $\mathbf{3}$ & 0.031 & -96 & $\mathbf{2 3}$ & 0.300 & -64 \\
\hline $\mathbf{4}$ & 0.469 & -44 & $\mathbf{2 4}$ & 0.000 & -100 \\
\hline $\mathbf{5}$ & 0.038 & -96 & $\mathbf{2 5}$ & 0.148 & -82 \\
\hline $\mathbf{6}$ & 0.028 & -97 & $\mathbf{2 6}$ & 0.697 & -16 \\
\hline $\mathbf{7}$ & 0.112 & -87 & $\mathbf{2 7}$ & 0.181 & -78 \\
\hline $\mathbf{8}$ & 0.144 & -83 & $\mathbf{2 8}$ & 0.000 & -100 \\
\hline $\mathbf{9}$ & 0.144 & -83 & $\mathbf{2 9}$ & 0.919 & 10 \\
\hline $\mathbf{1 0}$ & 0.769 & -8 & $\mathbf{3 0}$ & 0.495 & -41 \\
\hline $\mathbf{1 1}$ & 1.897 & 128 & $\mathbf{3 1}$ & 1.026 & 23 \\
\hline $\mathbf{1 2}$ & 0.194 & -77 & $\mathbf{3 2}$ & 0.856 & 3 \\
\hline $\mathbf{1 3}$ & 0.161 & -81 & $\mathbf{3 3}$ & 5.187 & 522 \\
\hline $\mathbf{1 4}$ & 0.733 & -12 & $\mathbf{3 4}$ & 1.252 & 50 \\
\hline $\mathbf{1 5}$ & 0.772 & -7 & $\mathbf{3 5}$ & 1.406 & 69 \\
\hline $\mathbf{1 6}$ & 0.300 & -64 & $\mathbf{3 6}$ & 0.103 & -88 \\
\hline $\mathbf{1 7}$ & 0.000 & -100 & $\mathbf{3 7}$ & 0.750 & -10 \\
\hline $\mathbf{1 8}$ & 0.559 & -33 & $\mathbf{3 8}$ & 0.534 & -36 \\
\hline $\mathbf{1 9}$ & 0.219 & -74 & $\mathbf{3 9}$ & 4.055 & 387 \\
\hline $\mathbf{2 0}$ & 0.780 & -6 & & & \\
\hline
\end{tabular}




\section{Particle Flow Through Individual Holes}

\section{All Sizes}

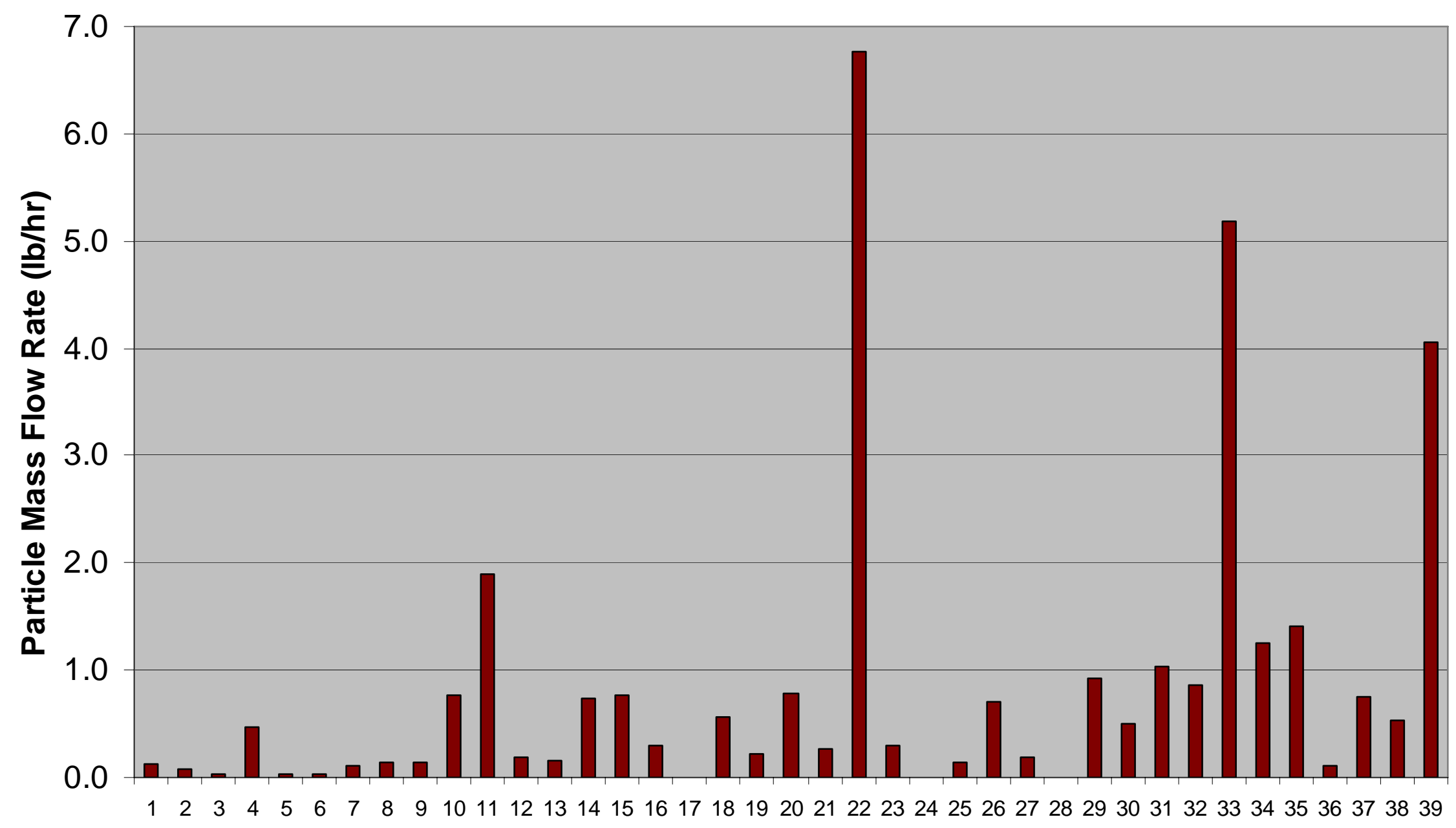

Hole ID 


\section{Particle Flow Through Individual Holes}

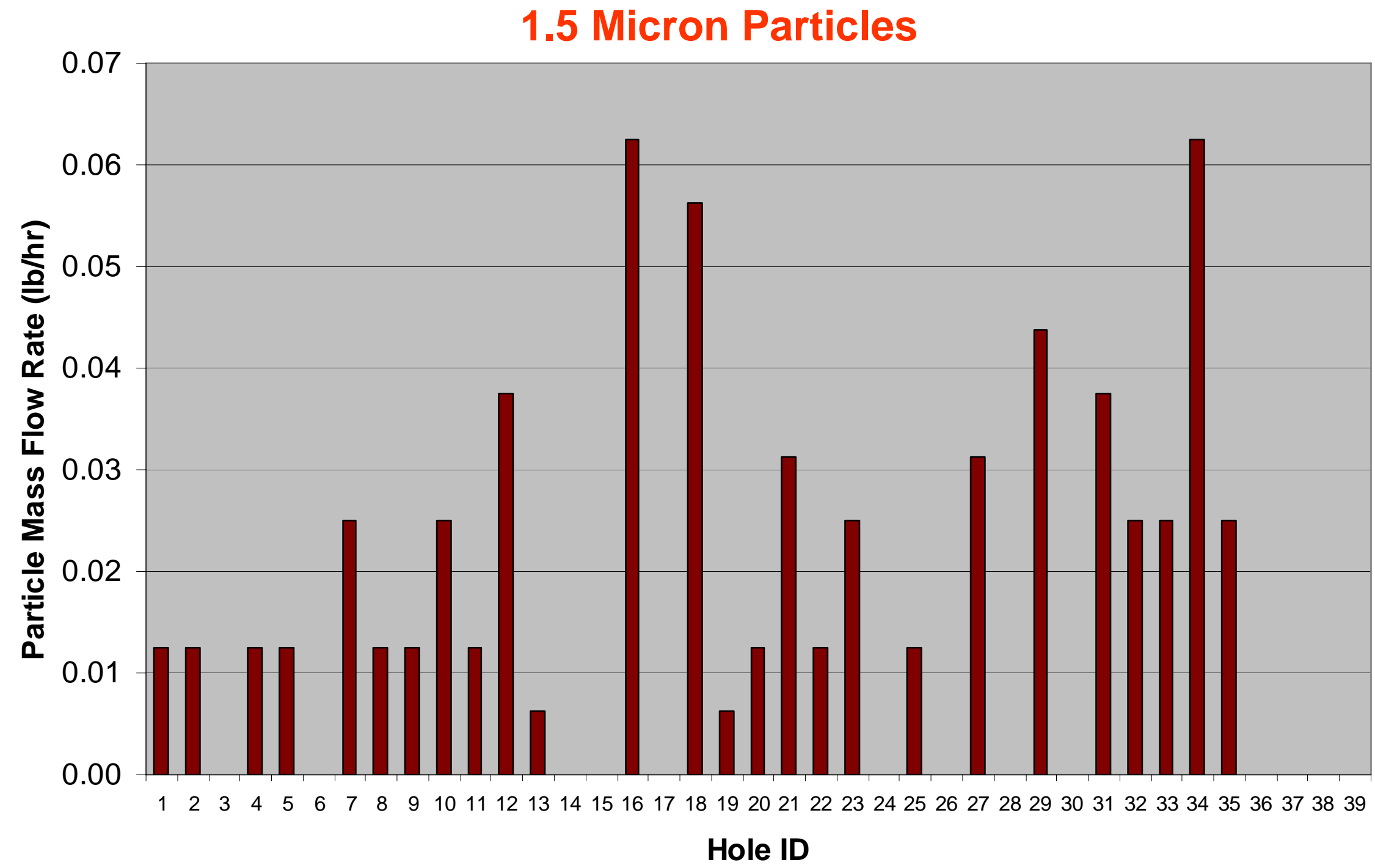




\section{Particle Flow Through Individual Holes}

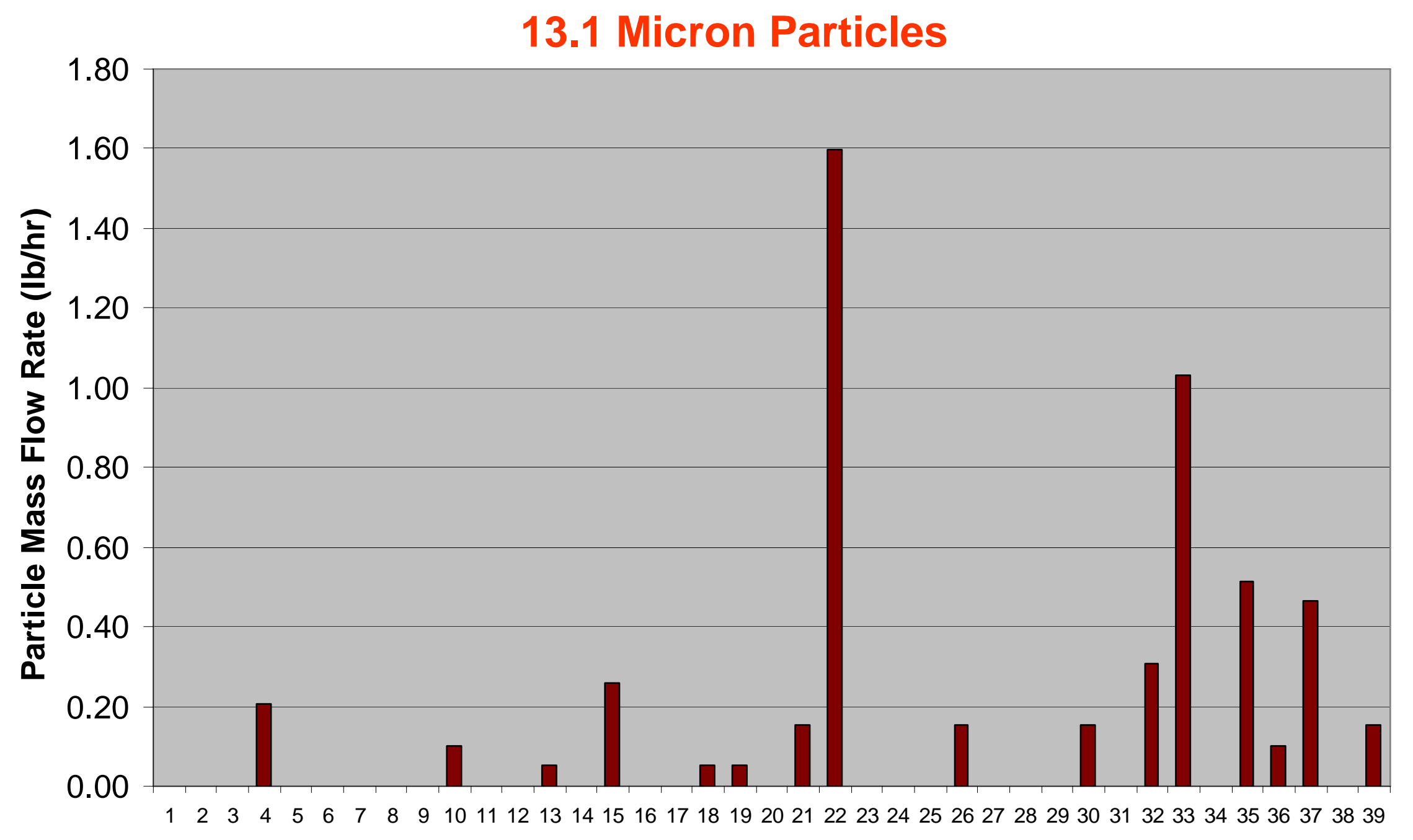

Hole ID 


\section{Particle Flow Through Individual Holes}

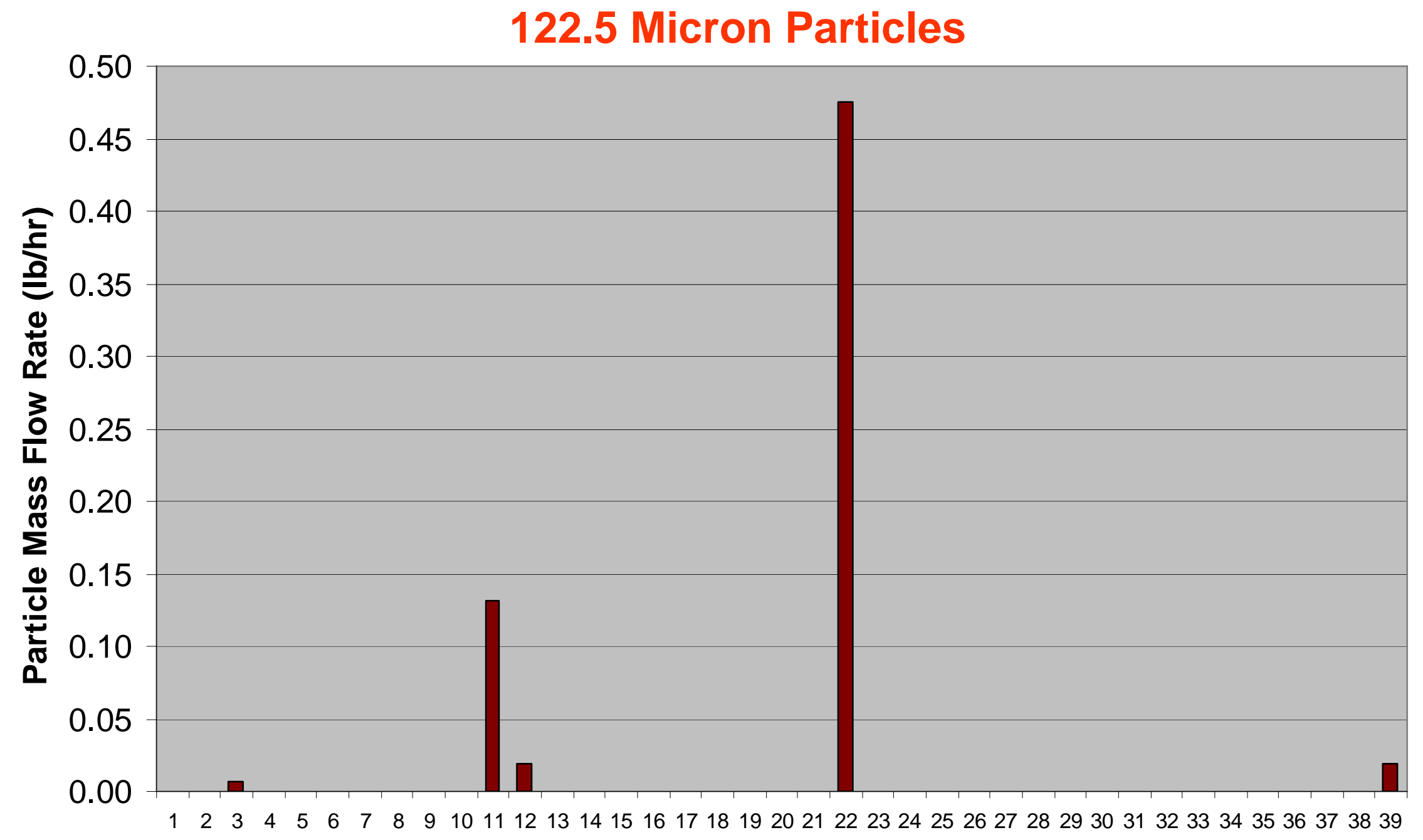

Hole ID 


\section{Particle Flow Through Individual Lances}

\section{All Sizes}

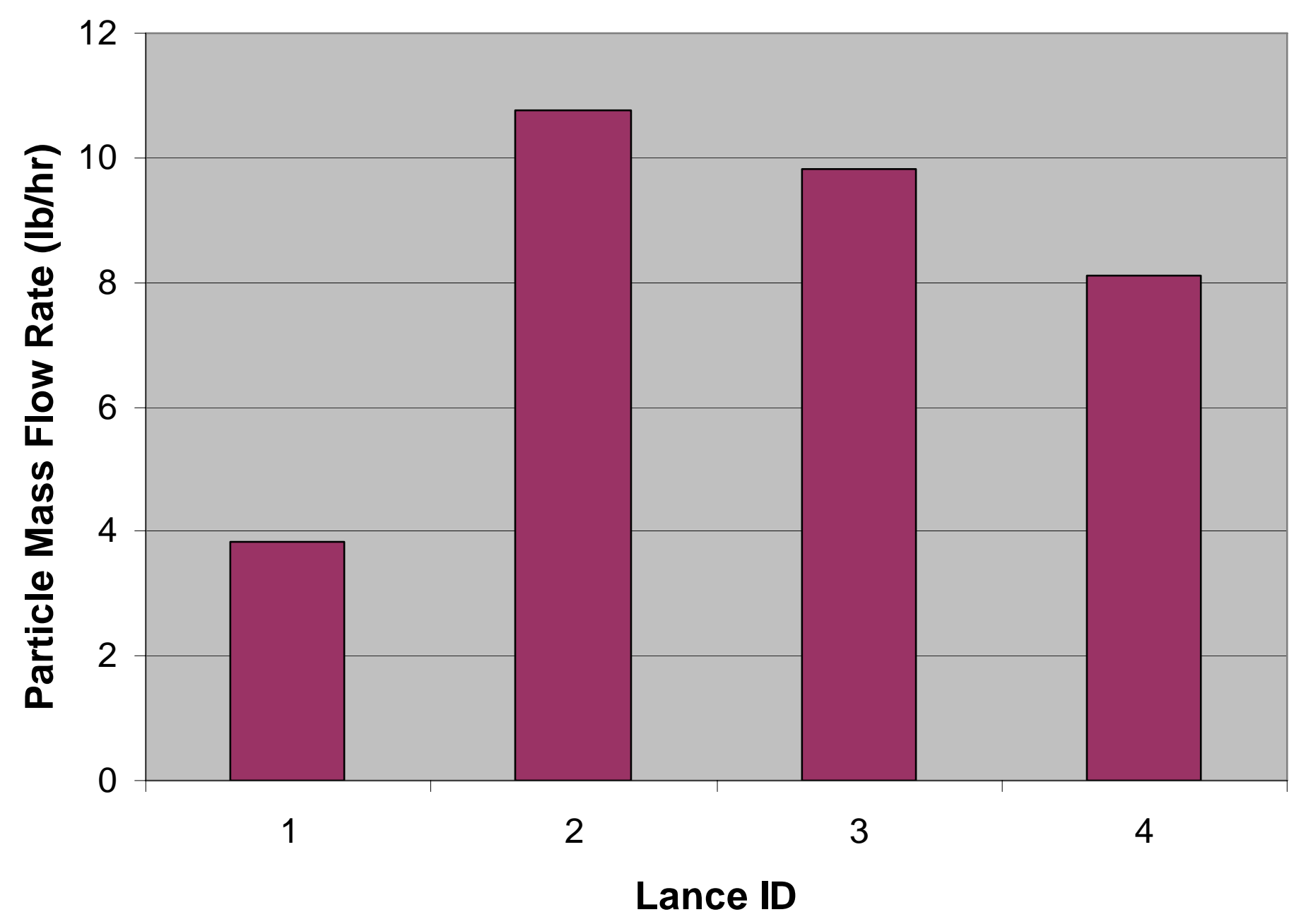




\section{Summary}

- Carrier air flows through 39 holes are quite evenly distributed

» Holes in Lance 4 have higher air flow due fewer holes drilled along the lance height

- Particle flows through 39 holes are severely biased

» Holes near the bottom end of the lances have much higher particle flows

» Large particles tend to leave the holes at the bottom end of the lances

»Small particles are more likely to leave the holes at higher elevations than large particles

»Among 4 lances, Lance 1 has the lowest particle flow

» The bottom holes on lances 1, 2, and 3 (holes 11, 22, 33) have high particle flow but low air flow indicating a potential for pluggage 


\section{APPENDIX E2: REI, Lance Modeling-August 11, 2006}




\section{CFD Modeling of TOXECON II at Independence}

ESP ACI Model Results

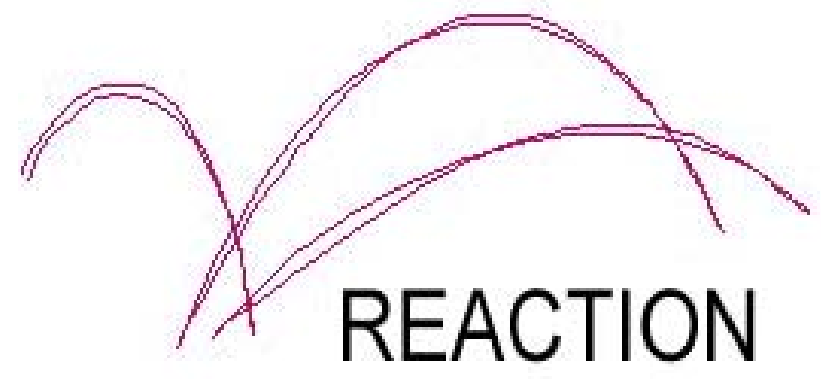

ENGINEERING

INTERNATIONAL

August 11, 2006 


\section{ESP Models}

- ESP flow model is for $1 / 2$ of one ESP box

- ESP flow model extends from ESP inlet duct to outlet duct including inlet and outlet plenums and associated turning vanes

- Activated carbon injection grid located between $2^{\text {nd }}$ and $3^{\text {rd }}$ ESP fields

- $\mathrm{ACl}$ is simulated within the ESP $\mathrm{ACl}$ model

- Flue gas flow field from the ESP flow model is mapped to inlet of ESP $\mathrm{ACl}$ model between $1^{\text {st }}$ and $2^{\text {nd }}$ ESP fields

- Results of $\mathrm{ACl}$ grid model were mapped into the ESP $\mathrm{ACl}$ model to predict dispersion of carbon

» Four injection lances were modeled in detail in the injection grid model

» Symmetry was assumed in order to specify carbon injection rates through all 16 lances (156 holes) within $1 / 2$ of the ESP box
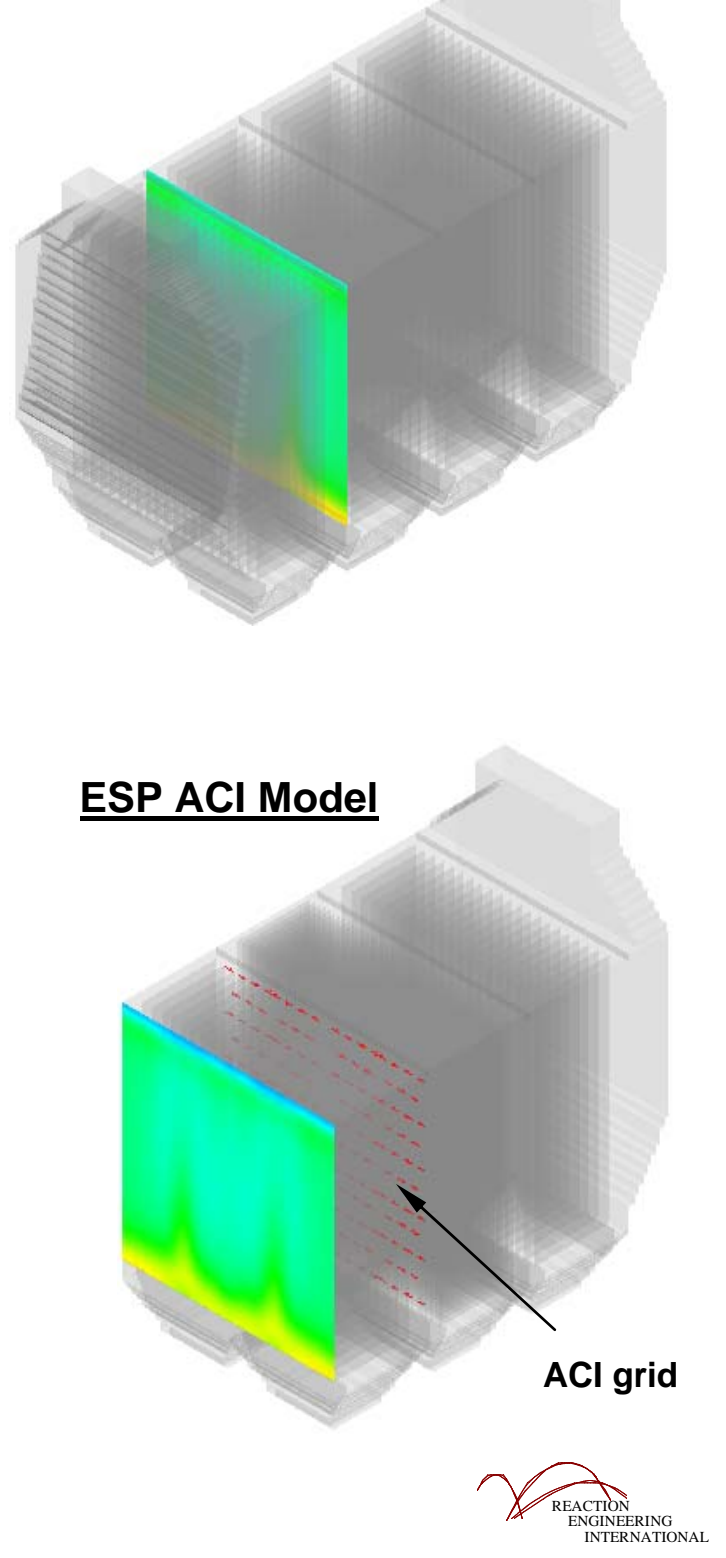


\section{Case Descriptions}

- Case 1: Uniform particle flow distribution among 156 holes

- Case 2: ACl grid model results

- Case 3: Smoothed distribution from ACl grid model results 


\section{Particle Flow Through Injection Grid}

Case 2

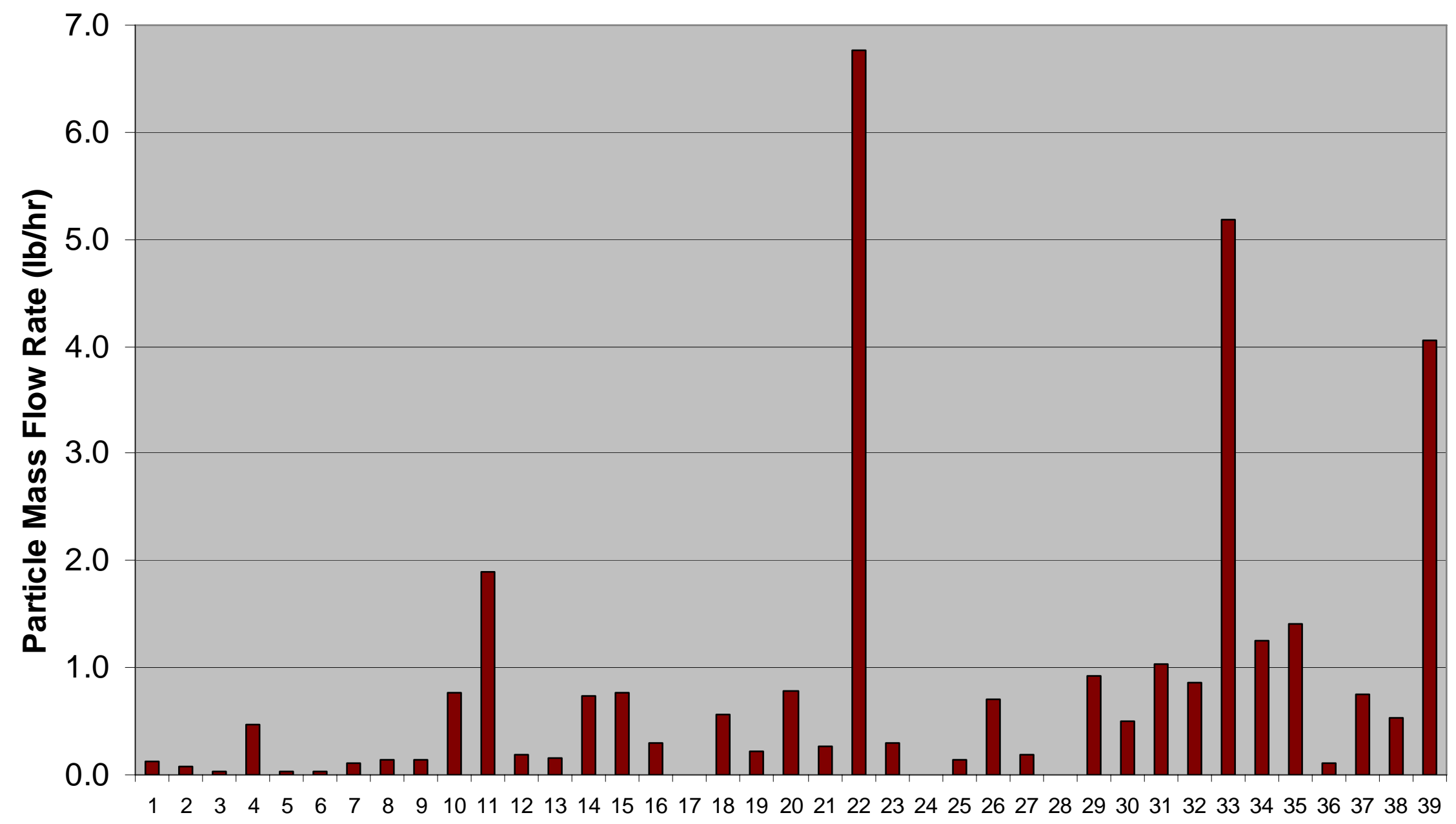

Hole ID 


\section{Particle Flow Through Injection Grid}

Case 3

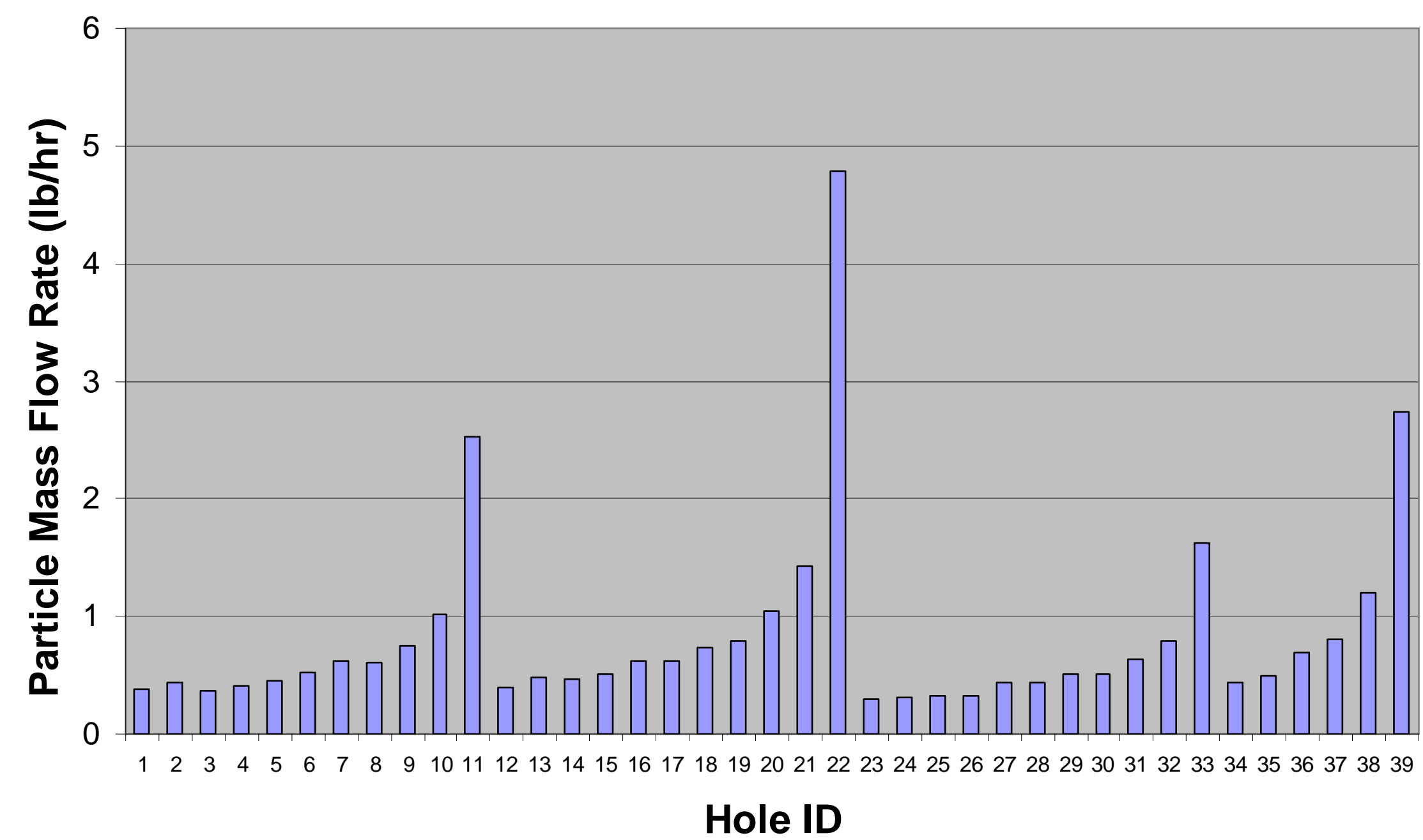




\section{Mapping ESP Flow Model Results}

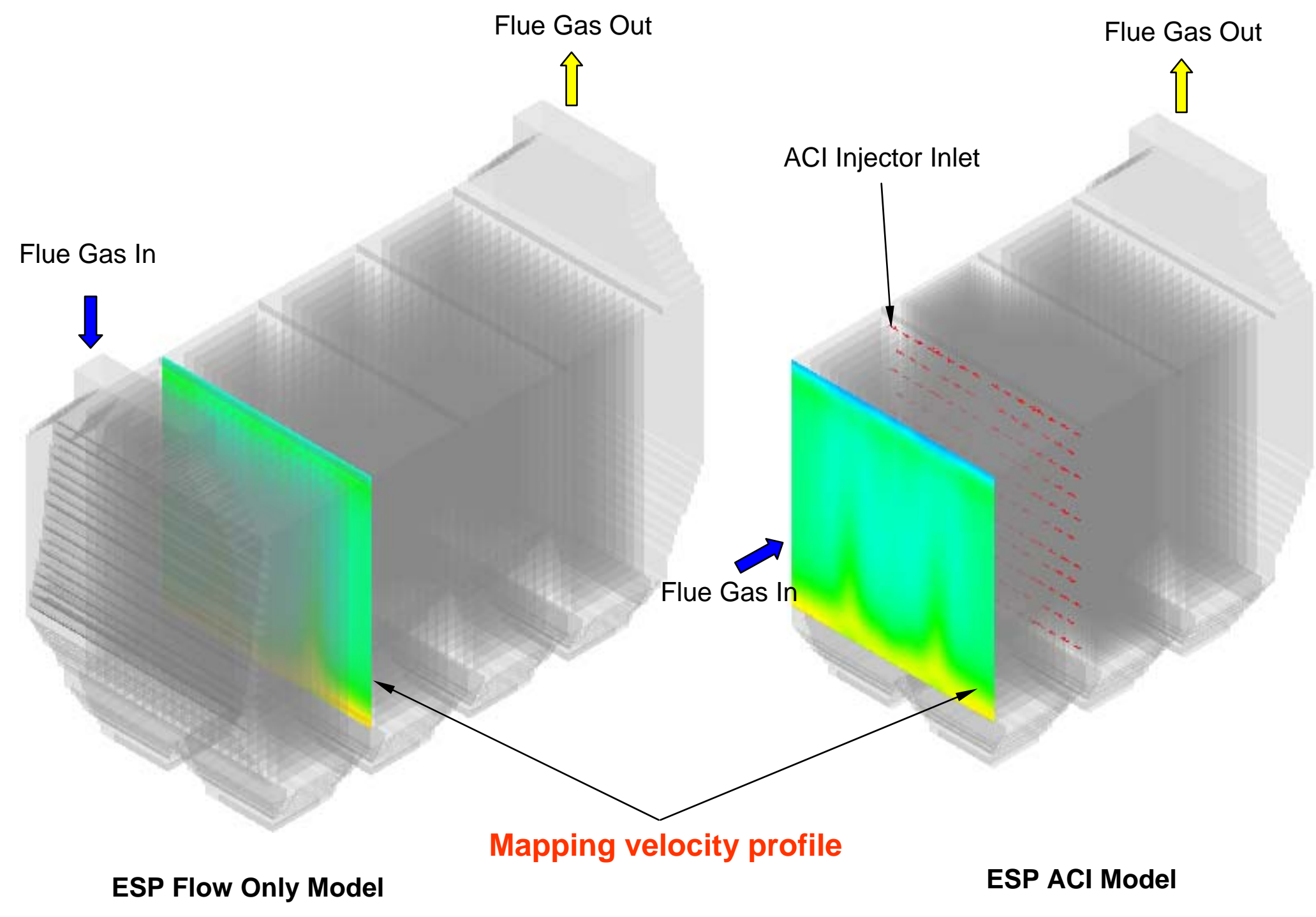




\section{Computational Model (ESP ACI)}
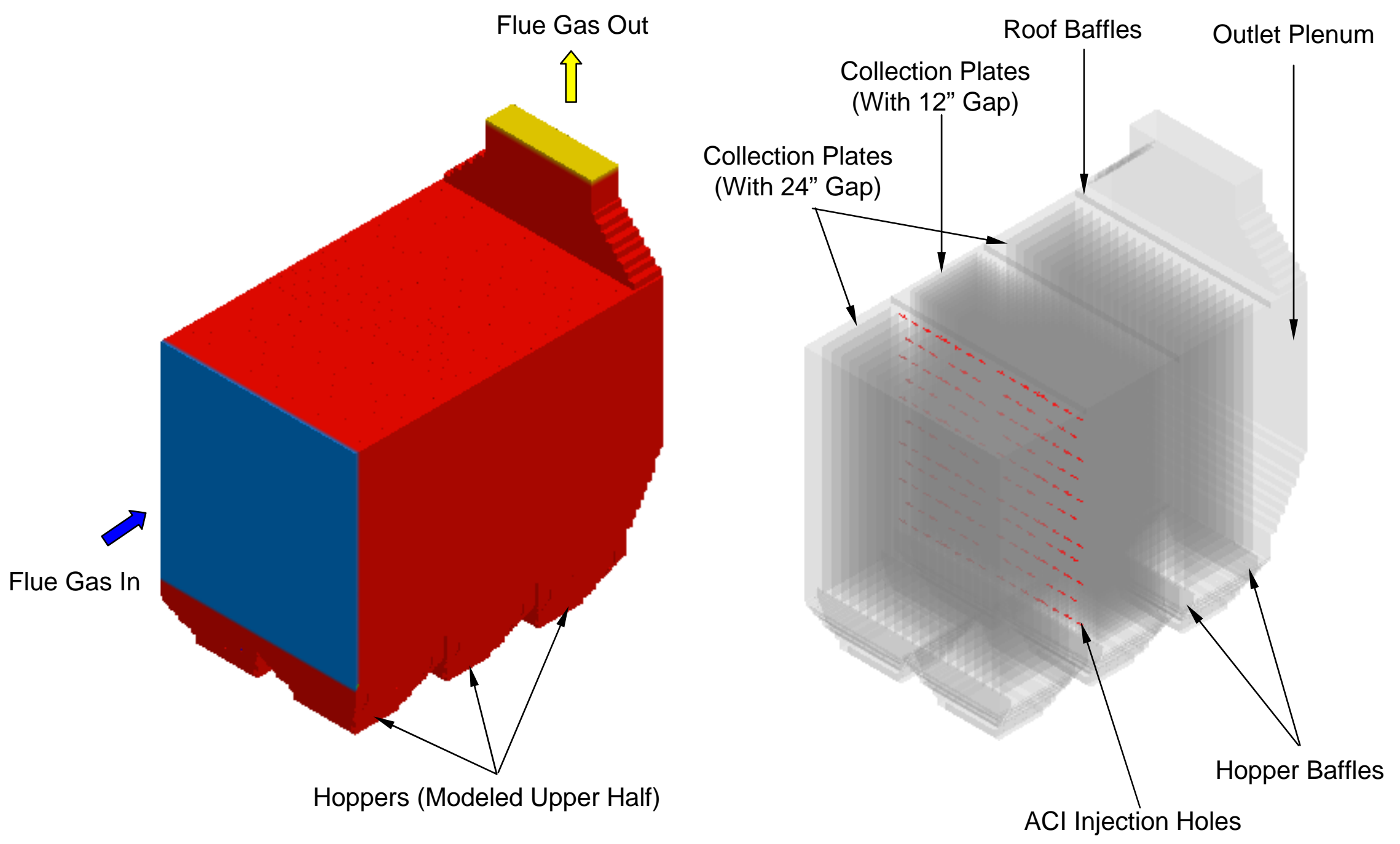


\section{Contour Plot Locations}

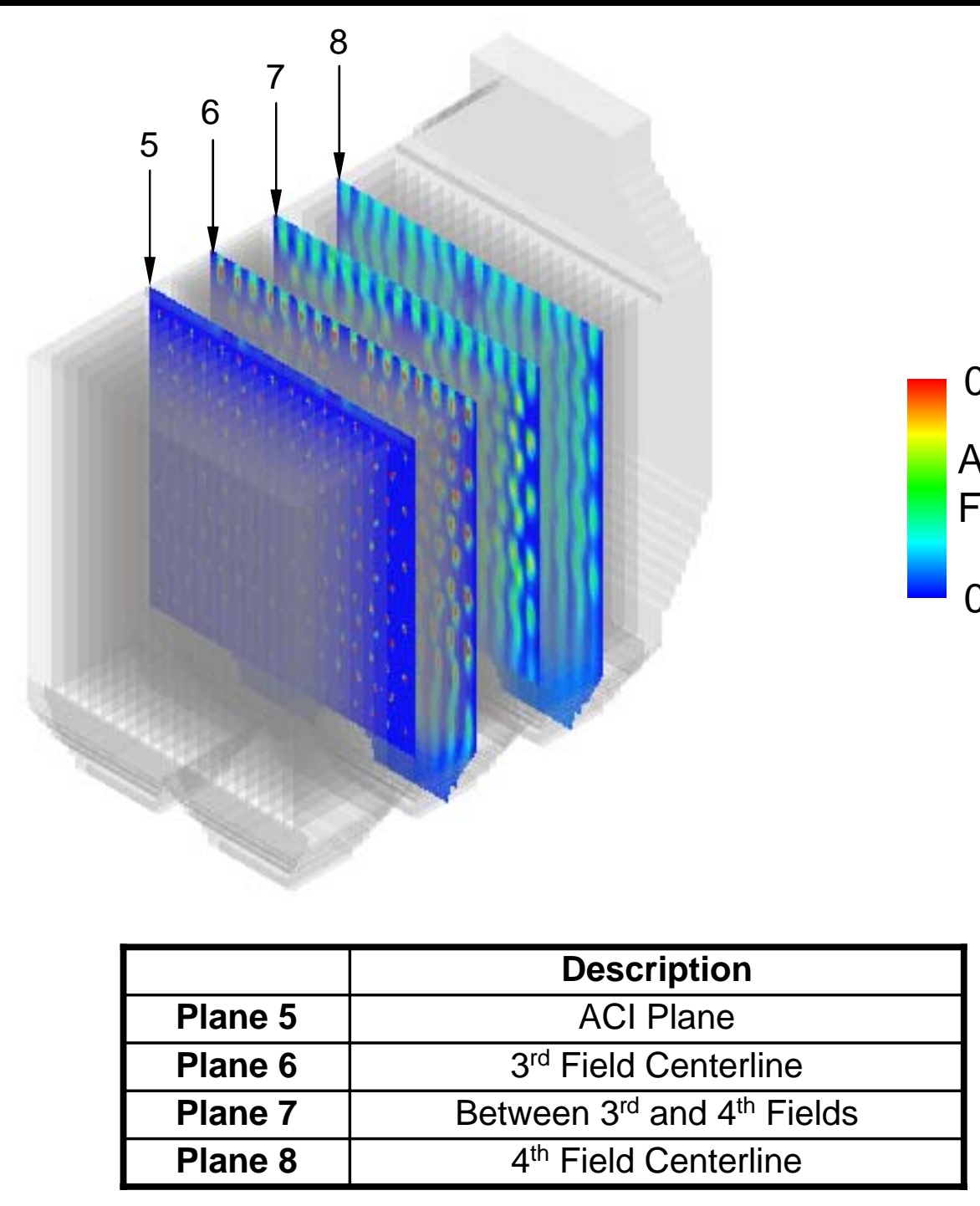




\section{Standard Deviation of Particle Bulk Density}

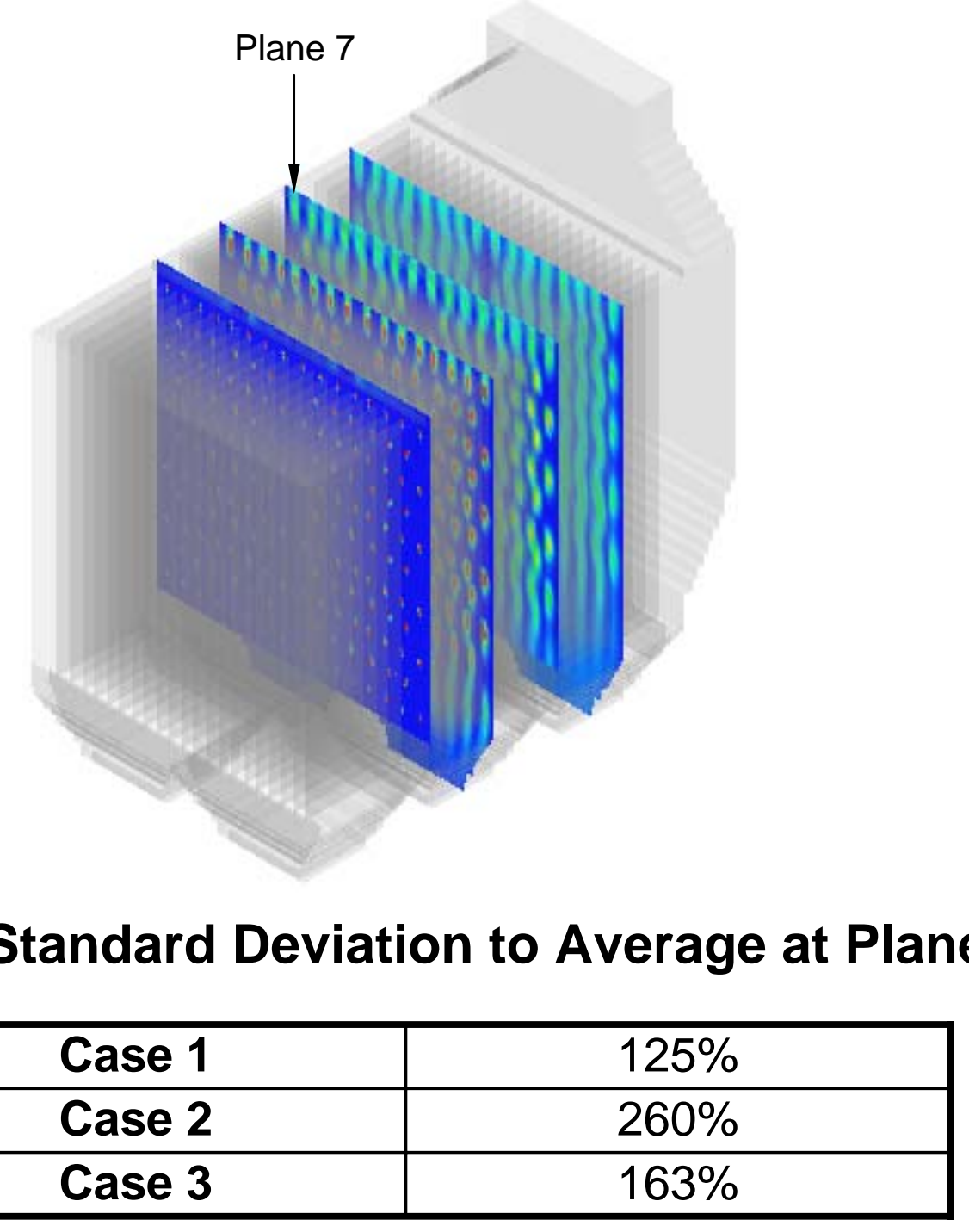




\section{Mass Fraction of Carrier Air (Case 1)}

Predicted air flow distributions for cases 1, 2, and 3 are nearly identical
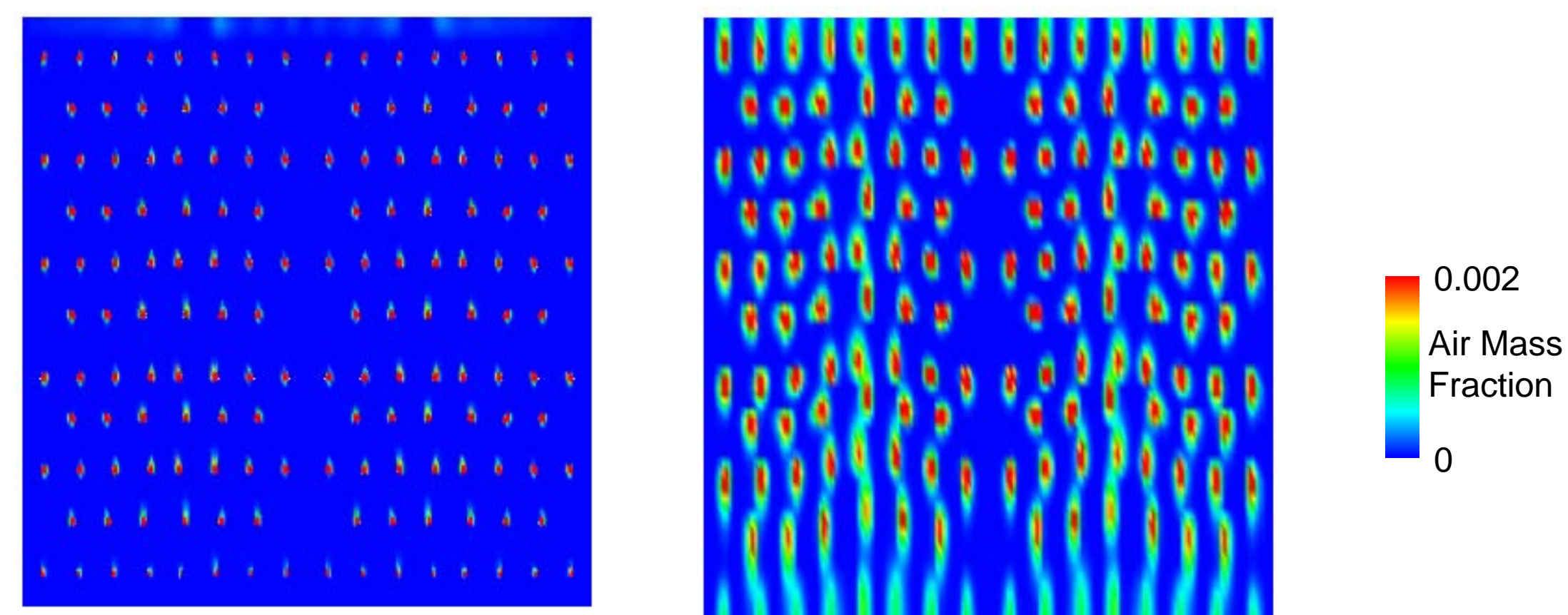

Plane 5

Plane 6 


\section{Mass Fraction of Carrier Air (Case 1)}

Predicted air flow distributions for cases 1, 2, and 3 are nearly identical
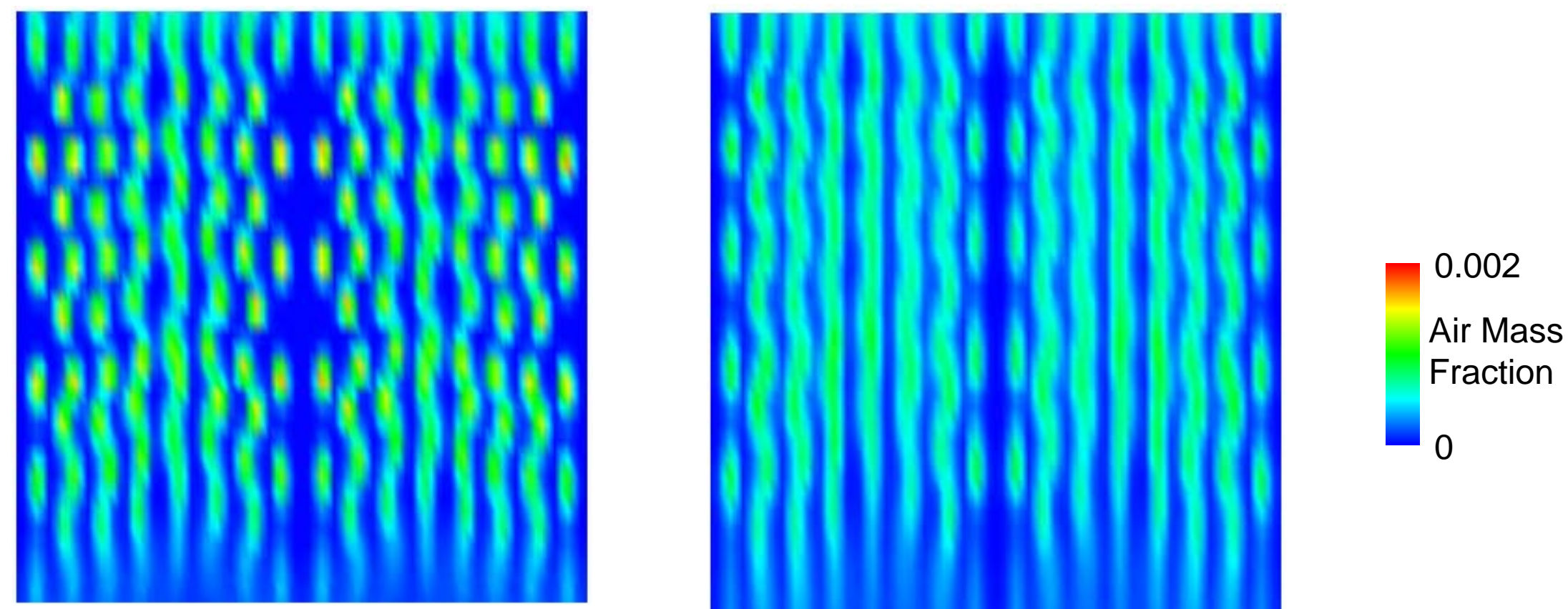

Plane 7

Plane 8

Channeling flow along ESP width

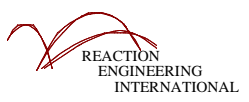




\section{Particle Bulk Density (Case 1)}
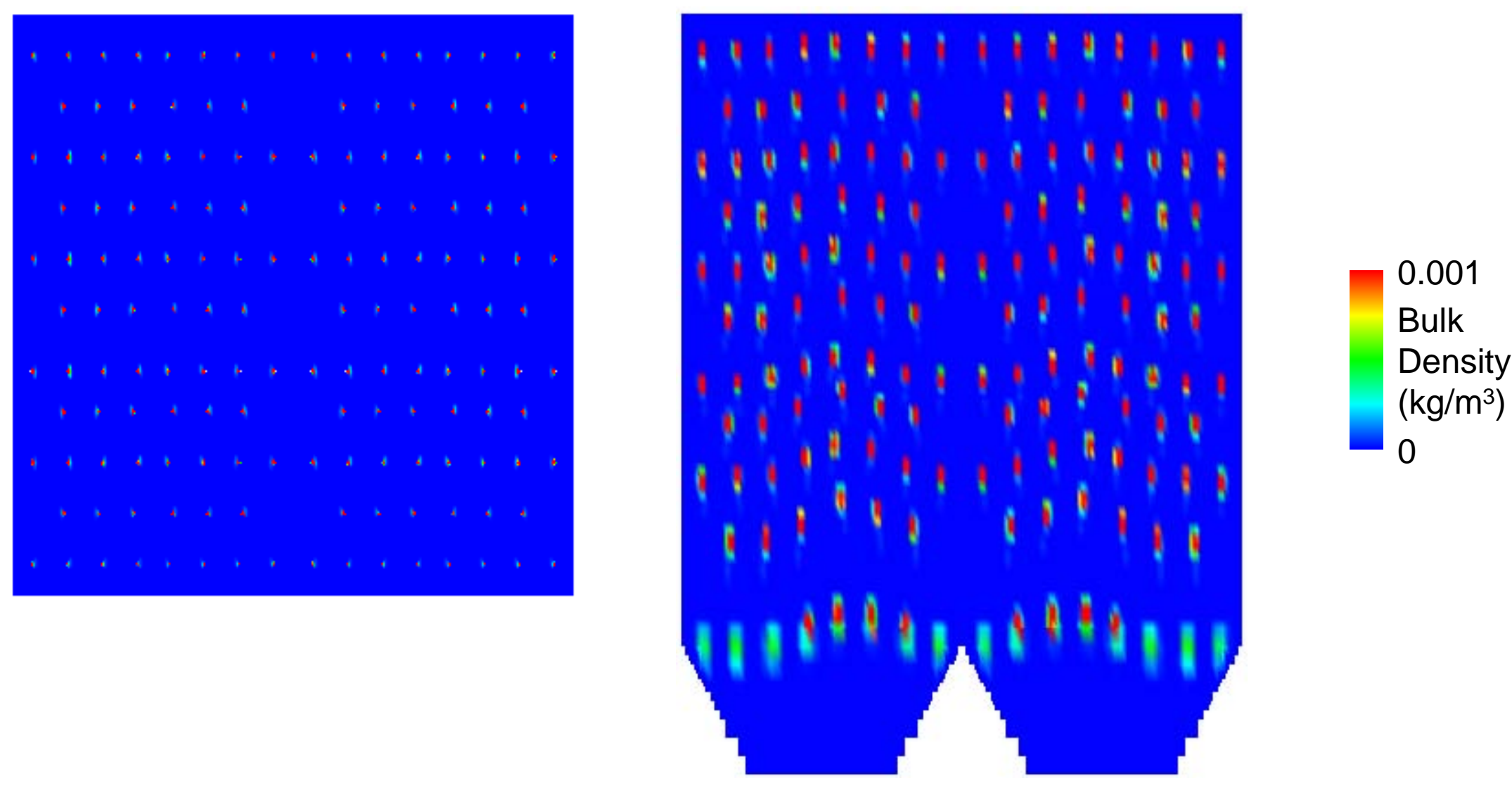

Plane 5

Plane 6

Particles not well dispersed 


\section{Particle Bulk Density (Case 1)}
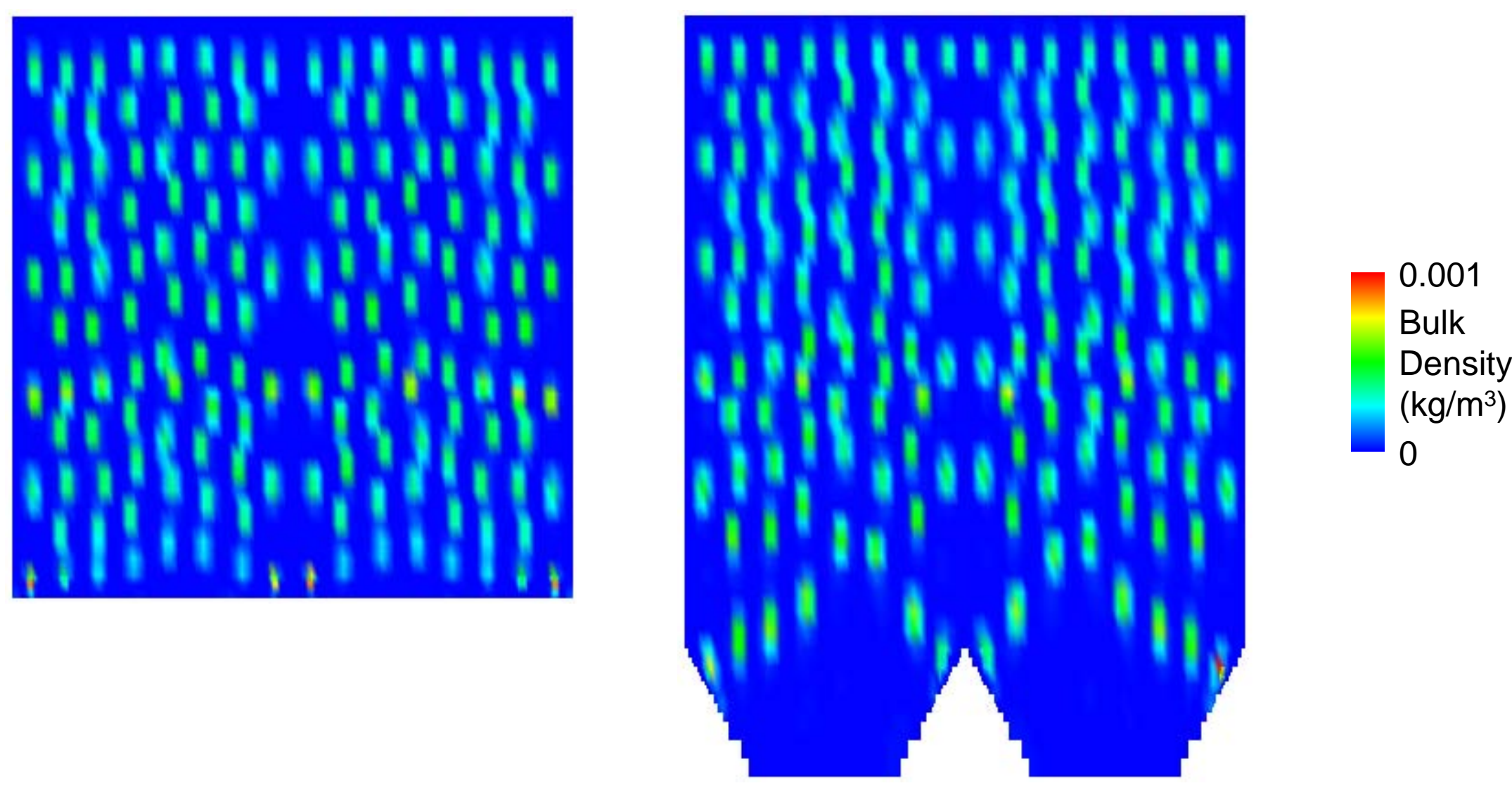

Plane 7

Plane 8

Particles not well dispersed 


\section{Particle Bulk Density (Case 2)}
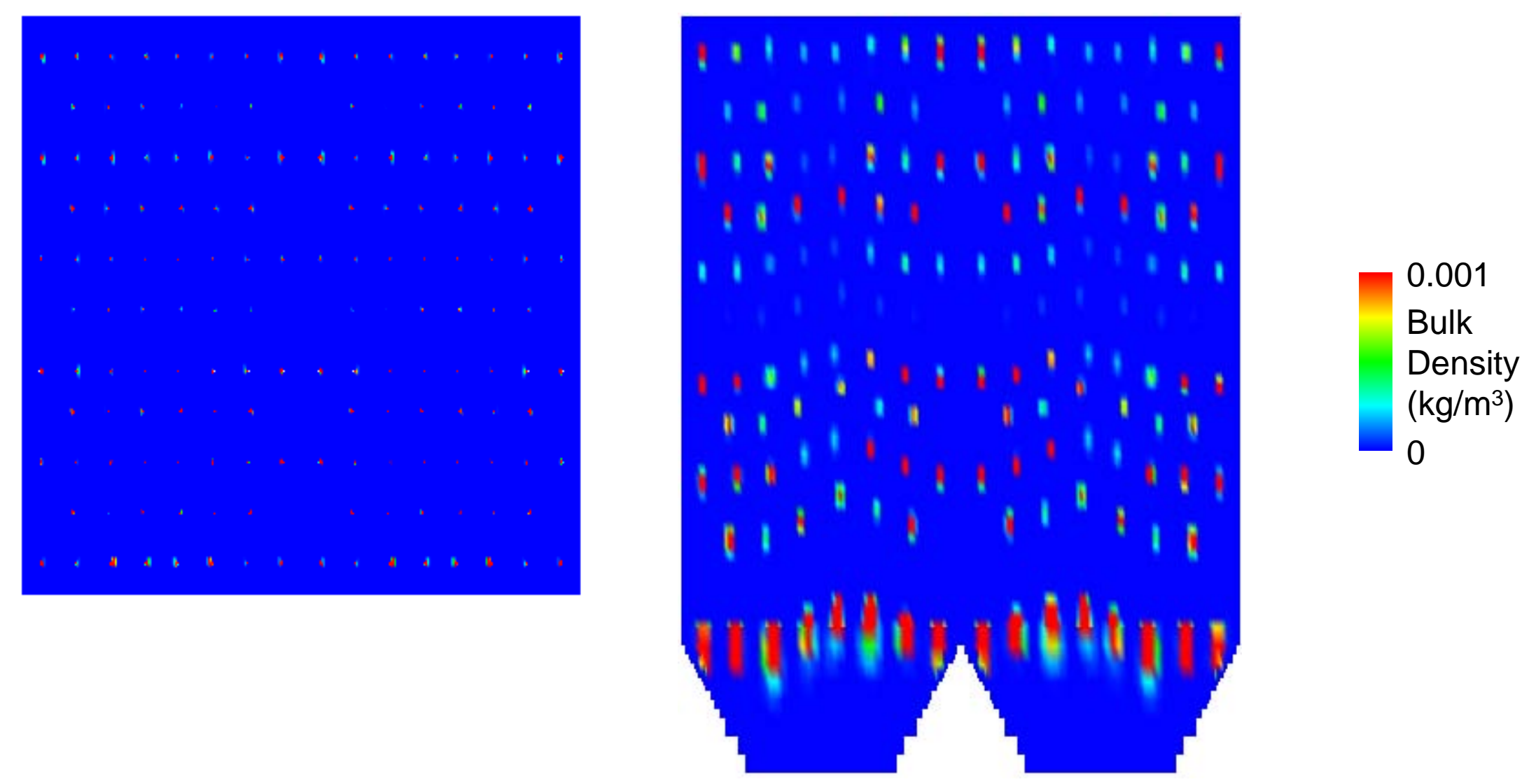

Plane 5

Plane 6

More particles near hoppers 


\section{Particle Bulk Density (Case 2)}
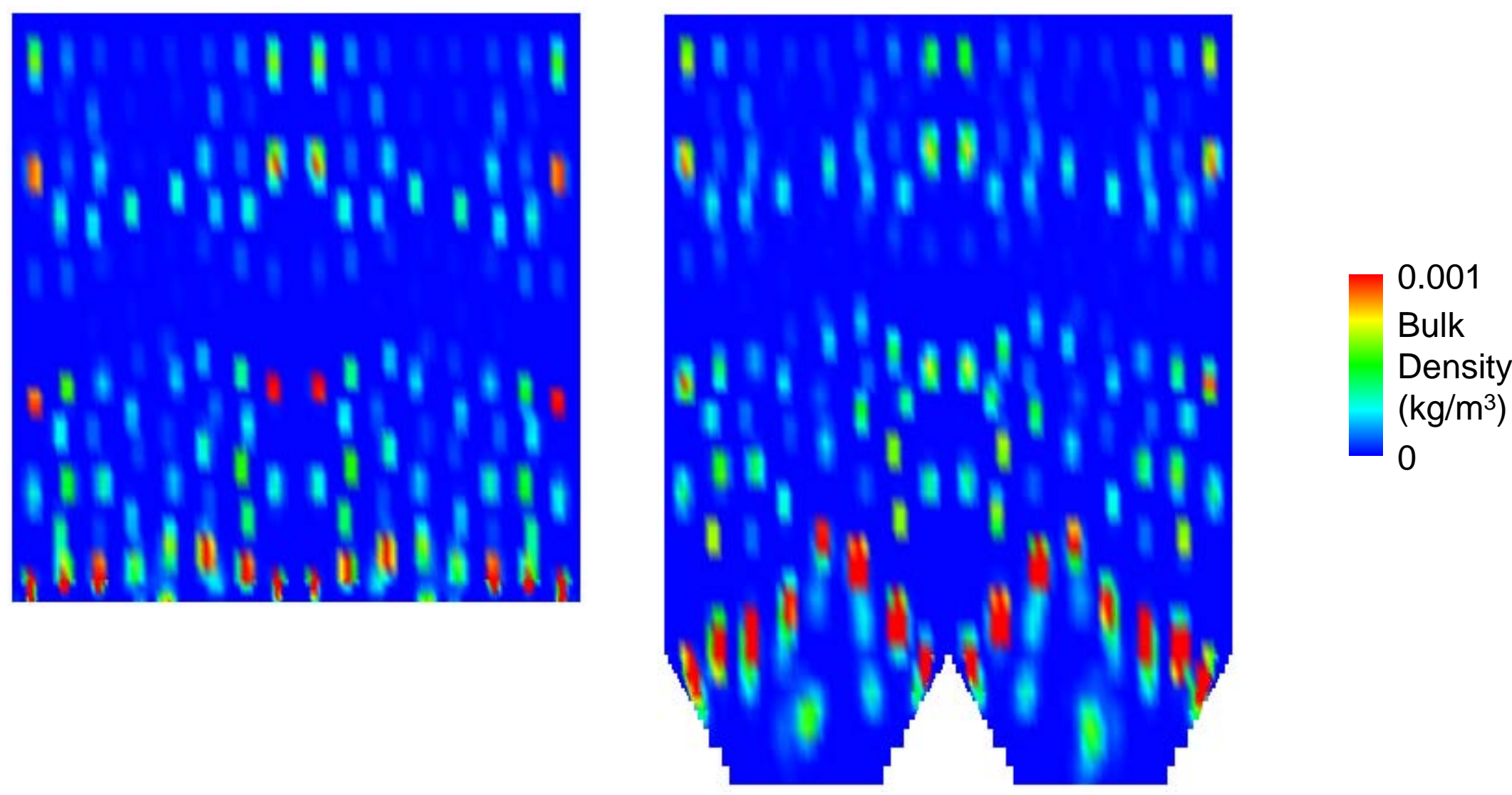

Plane 7

Plane 8

More particles near hoppers 


\section{Particle Bulk Density (Case 3)}
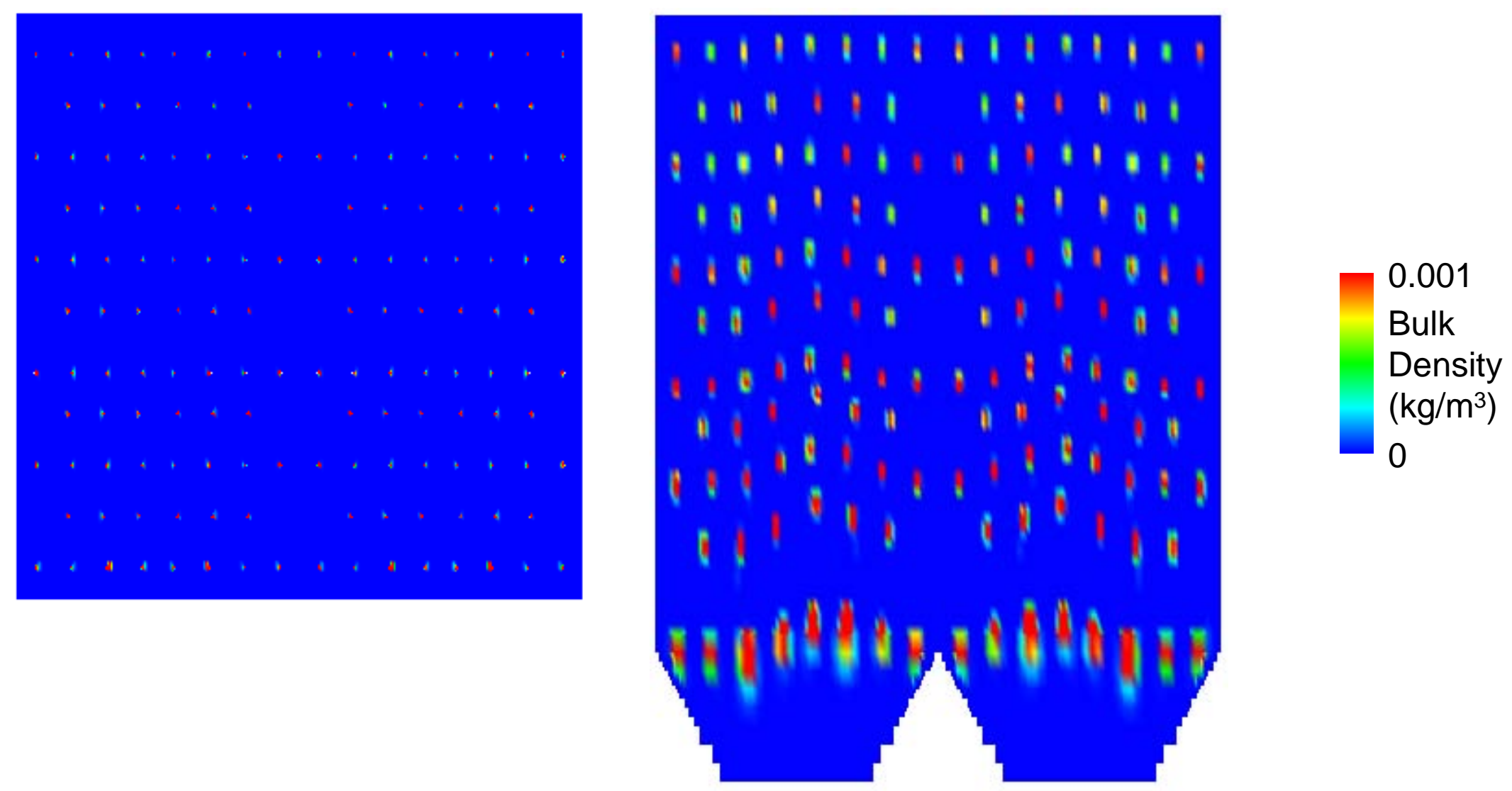

Plane 5

Plane 6

Particles concentrated near hoppers 


\section{Particle Bulk Density (Case 3)}
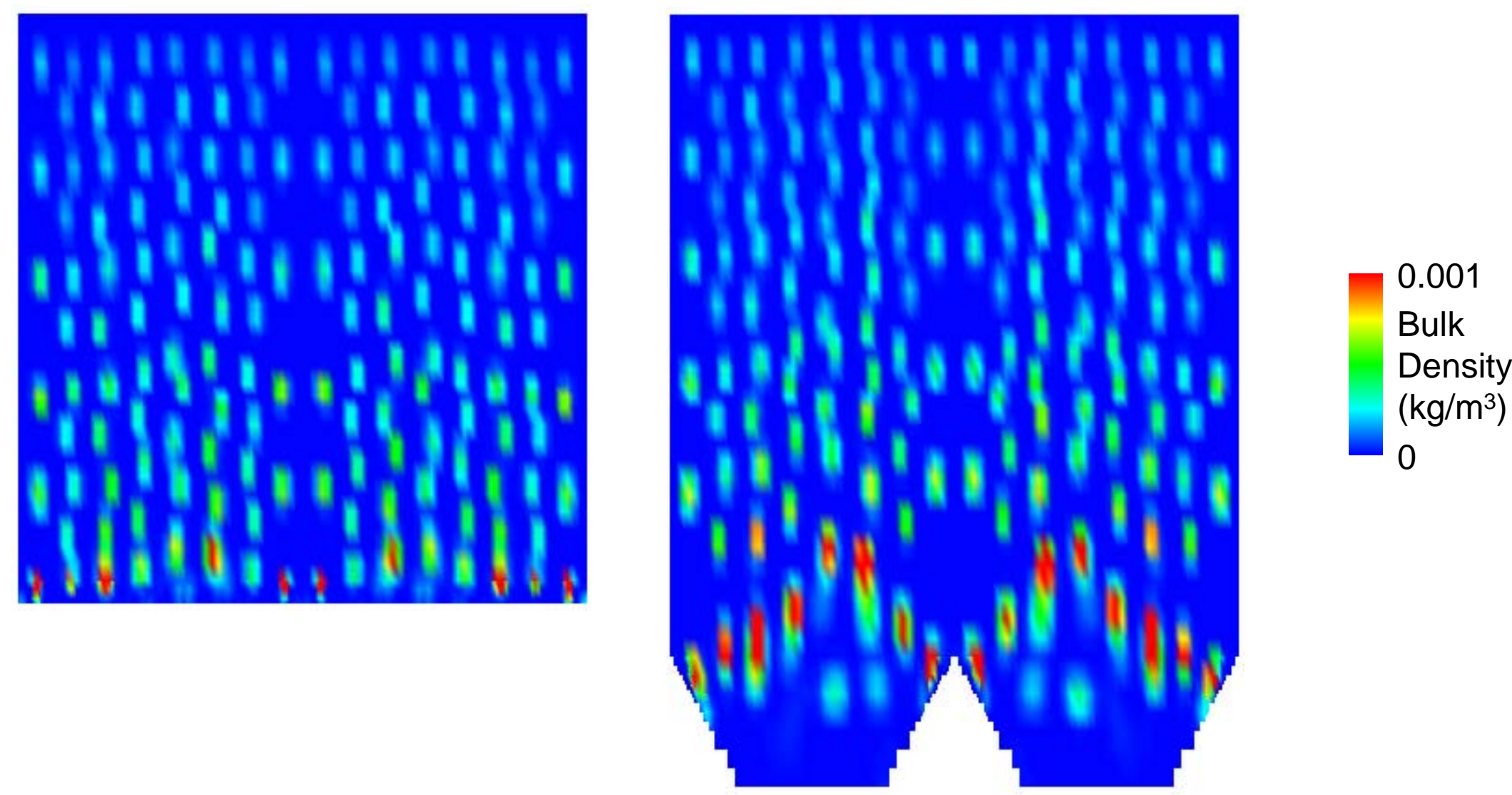

Plane 7

Plane 8

Particle distribution smoother than Case 2 


\section{Particle Average Trajectory (Plan View)}

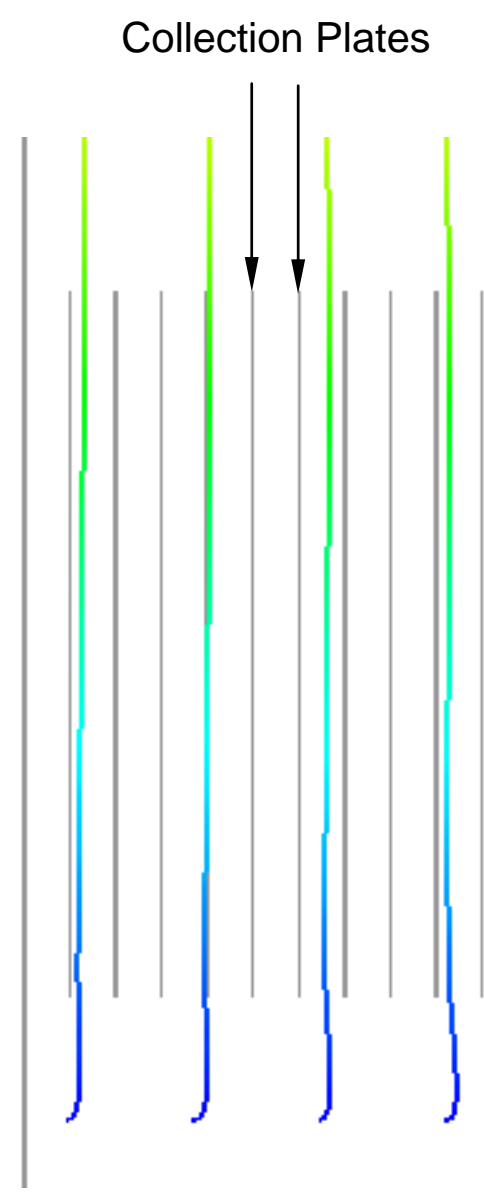

4.8 micron
Gap that has limited particles

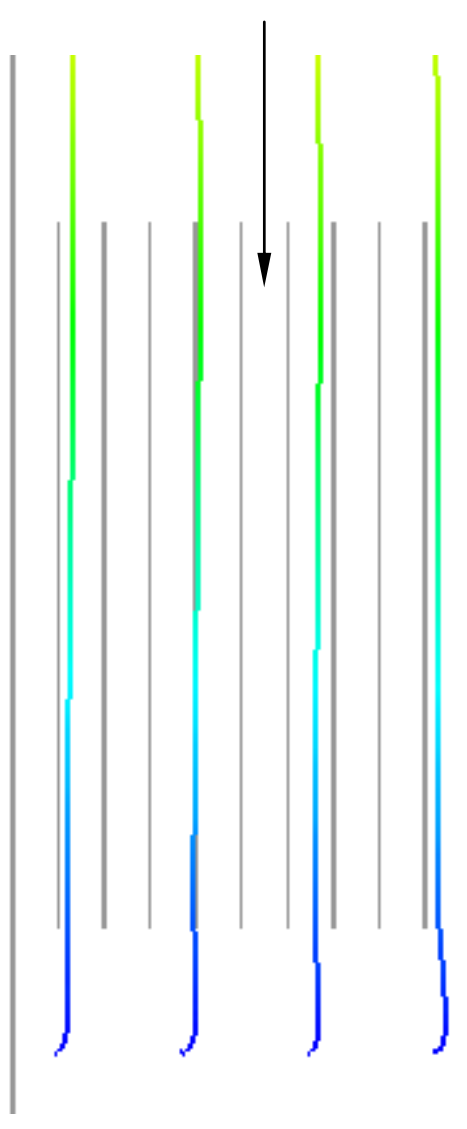

19 micron
Slightly deeper penetration for large particles

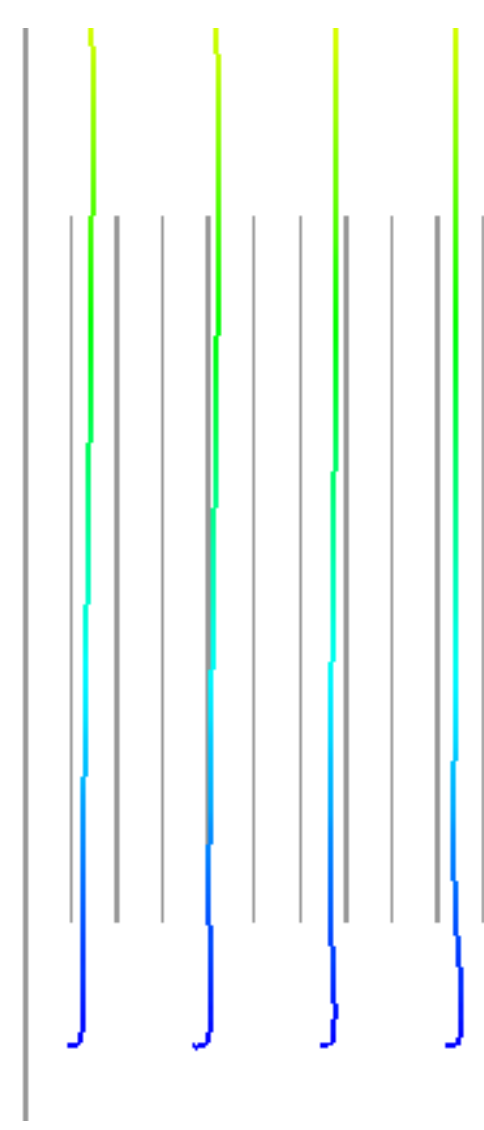

74.9 micron
Hole Locations

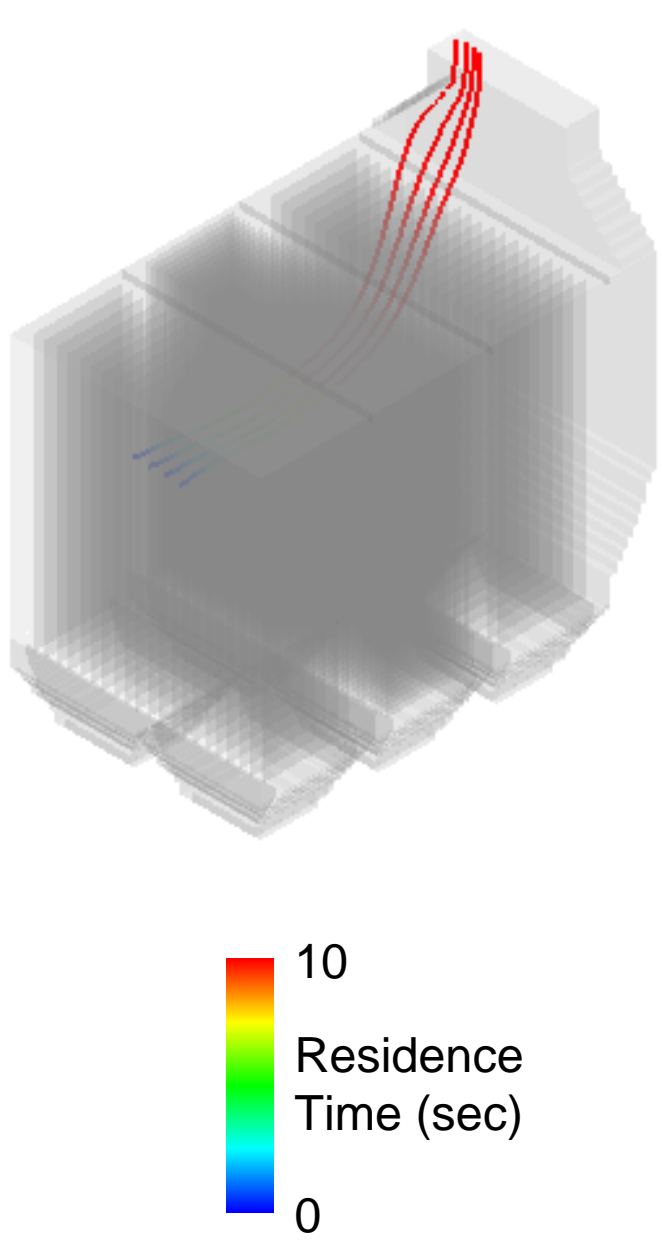




\section{Particle Trajectory (Case 1)}

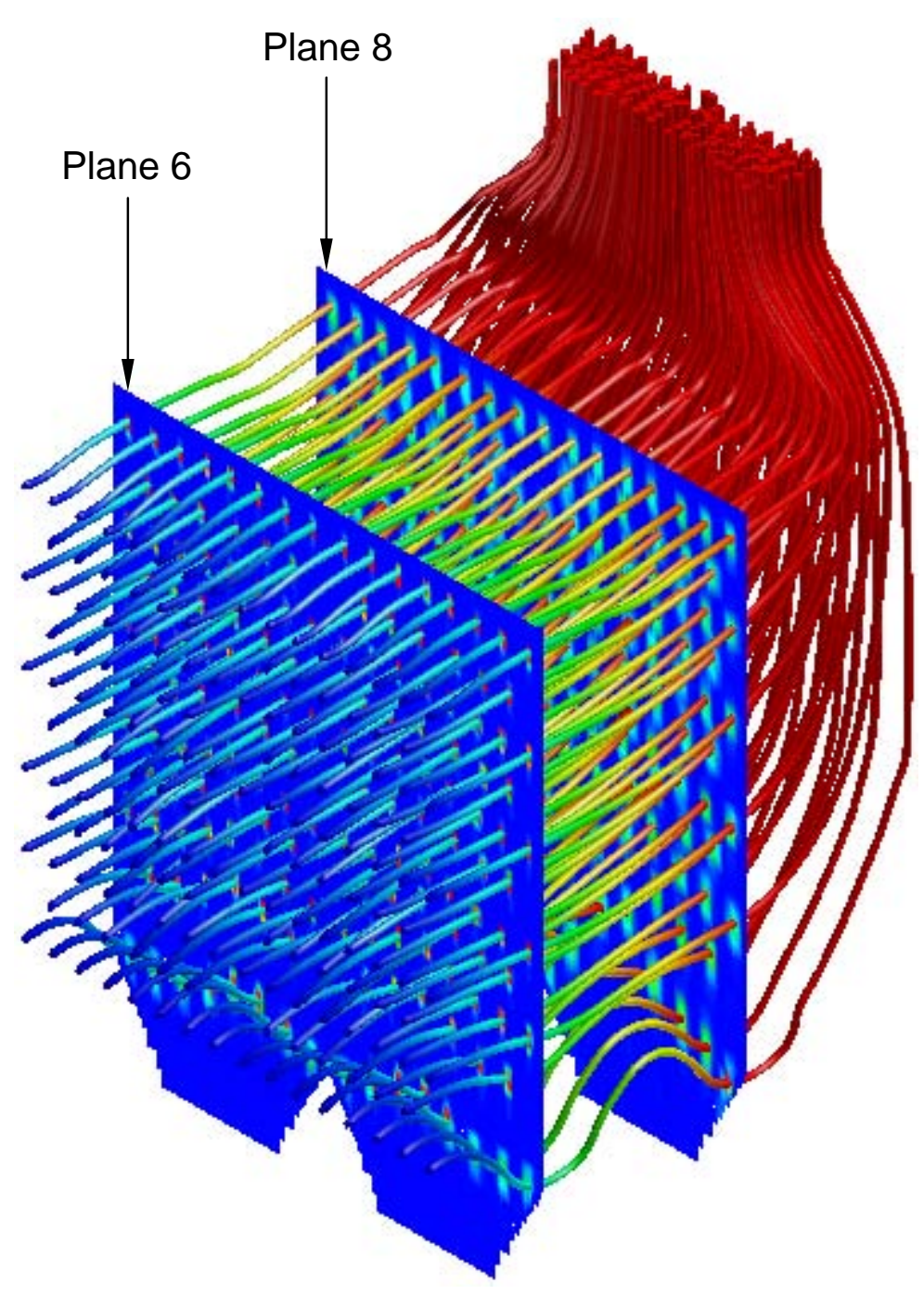

4.8 micron

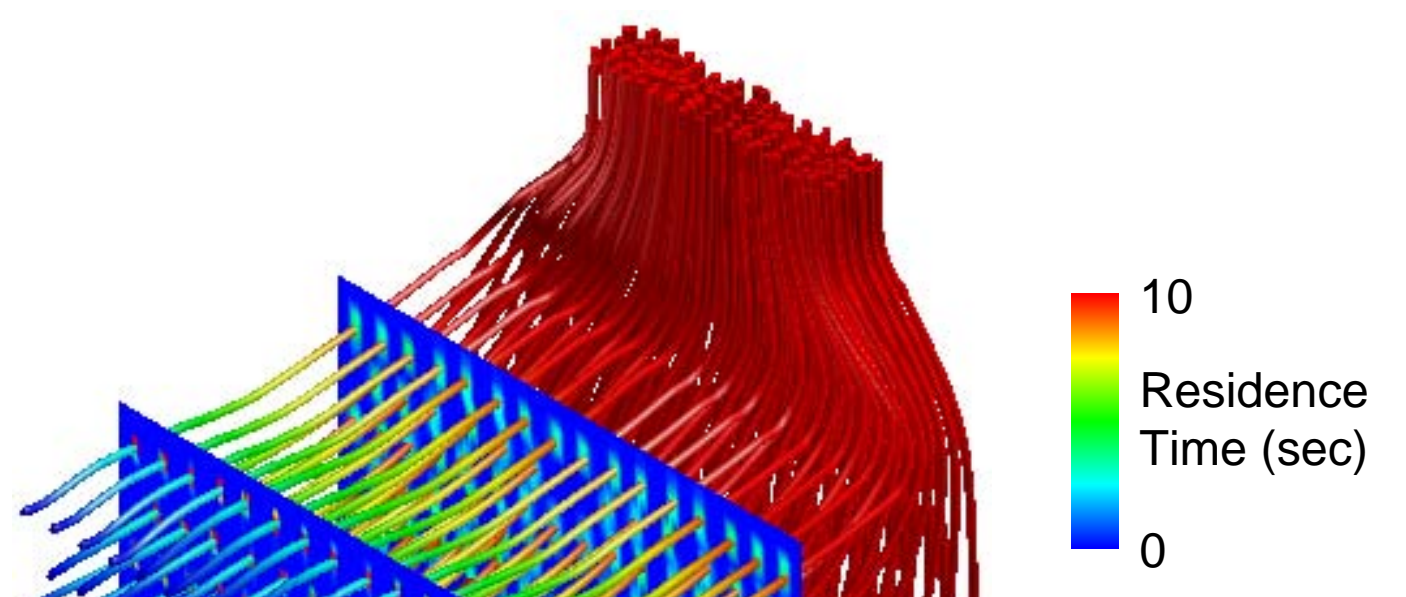




\section{Particle Trajectory (Case 2)}

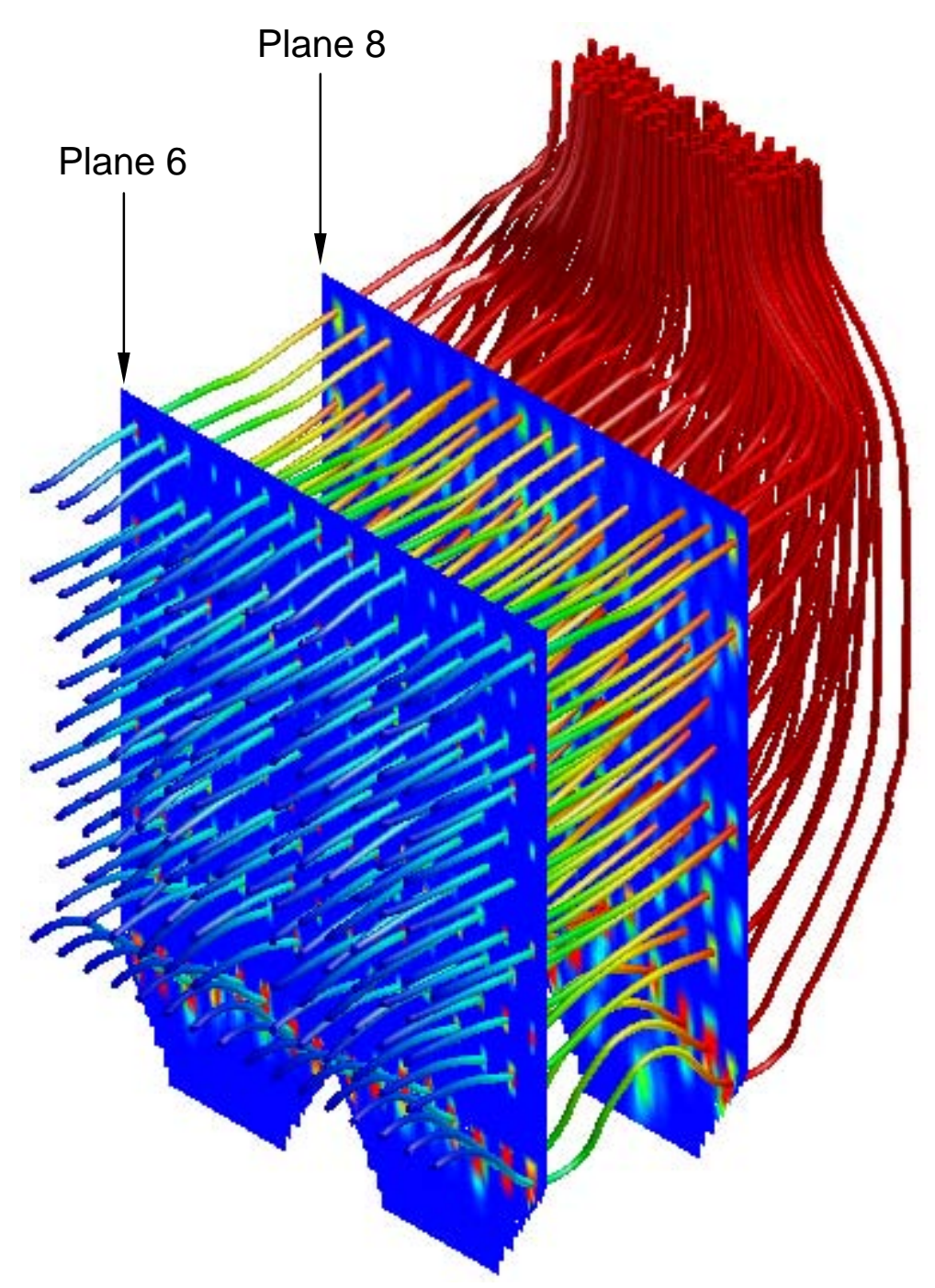

4.8 micron

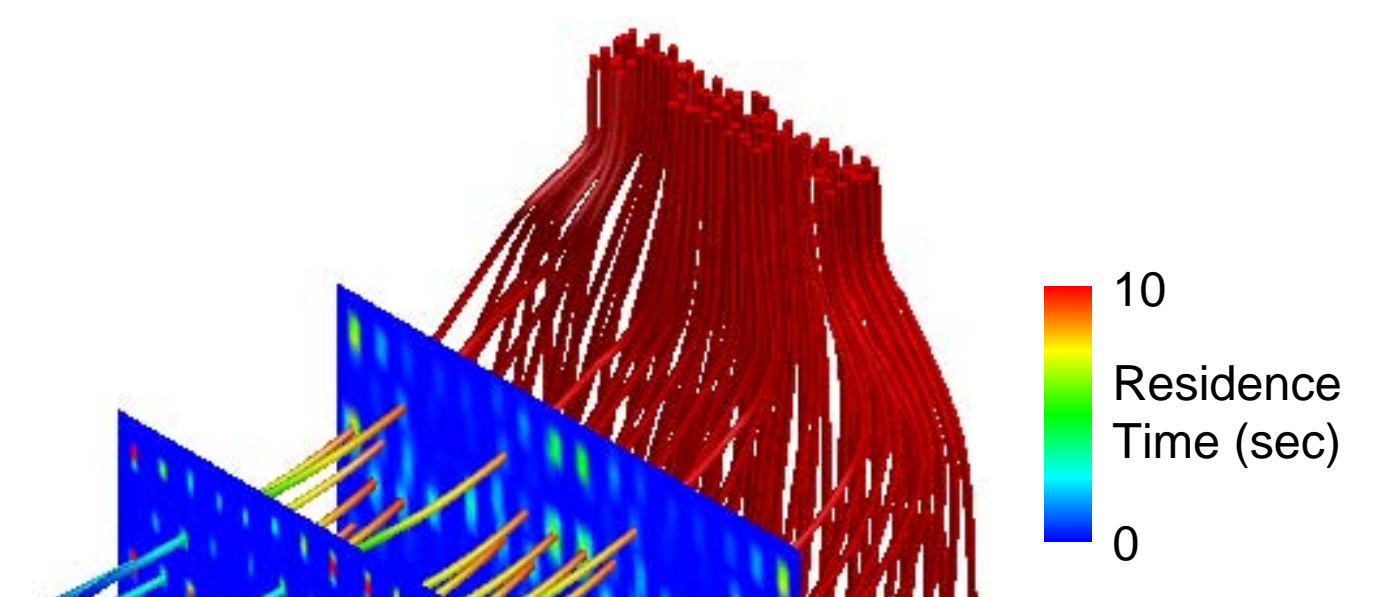

Toxecon II at Independence

19 micron

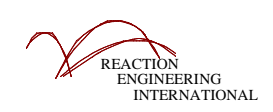




\section{Particle Trajectory (Case 3)}

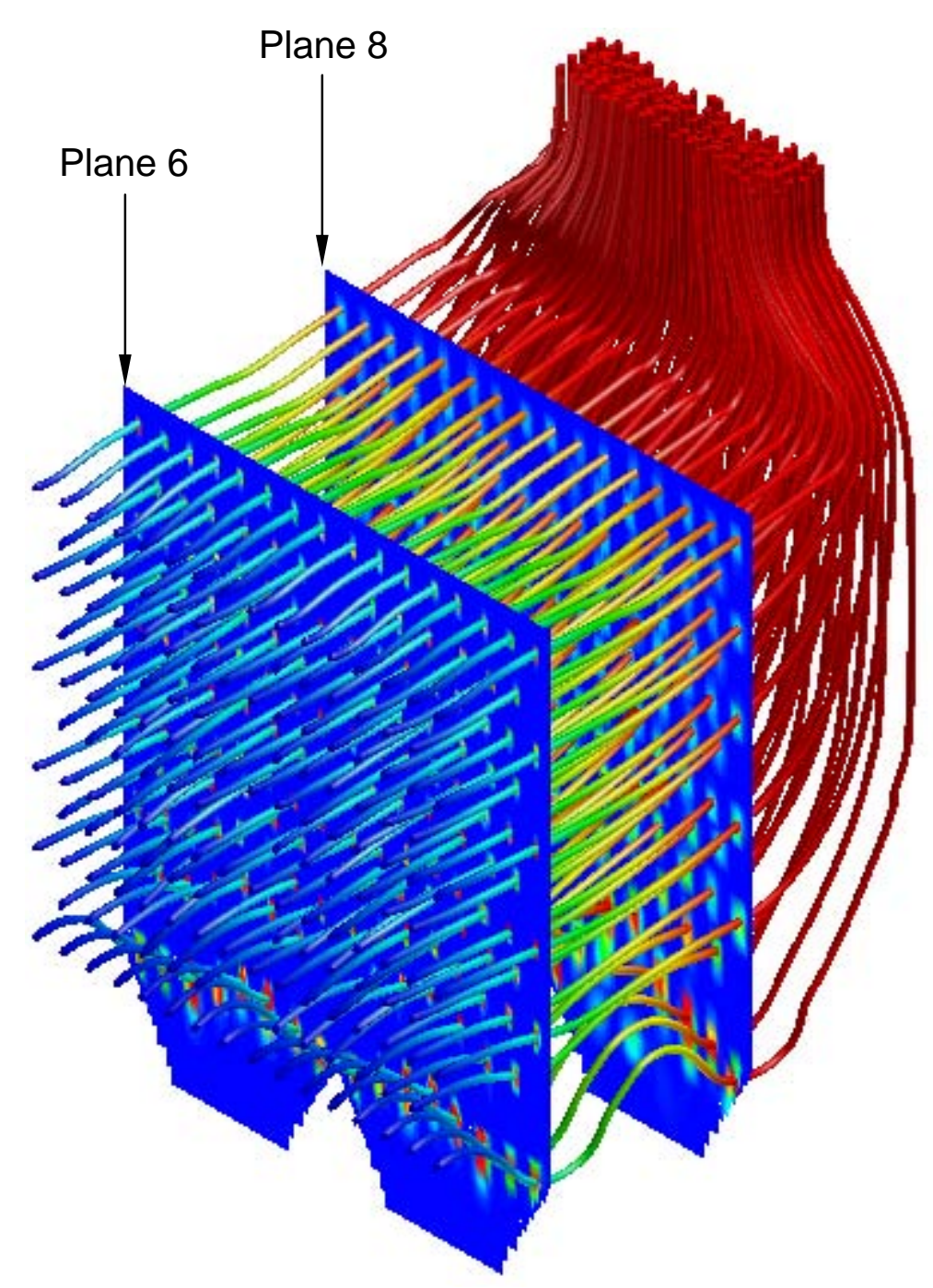

4.8 micron

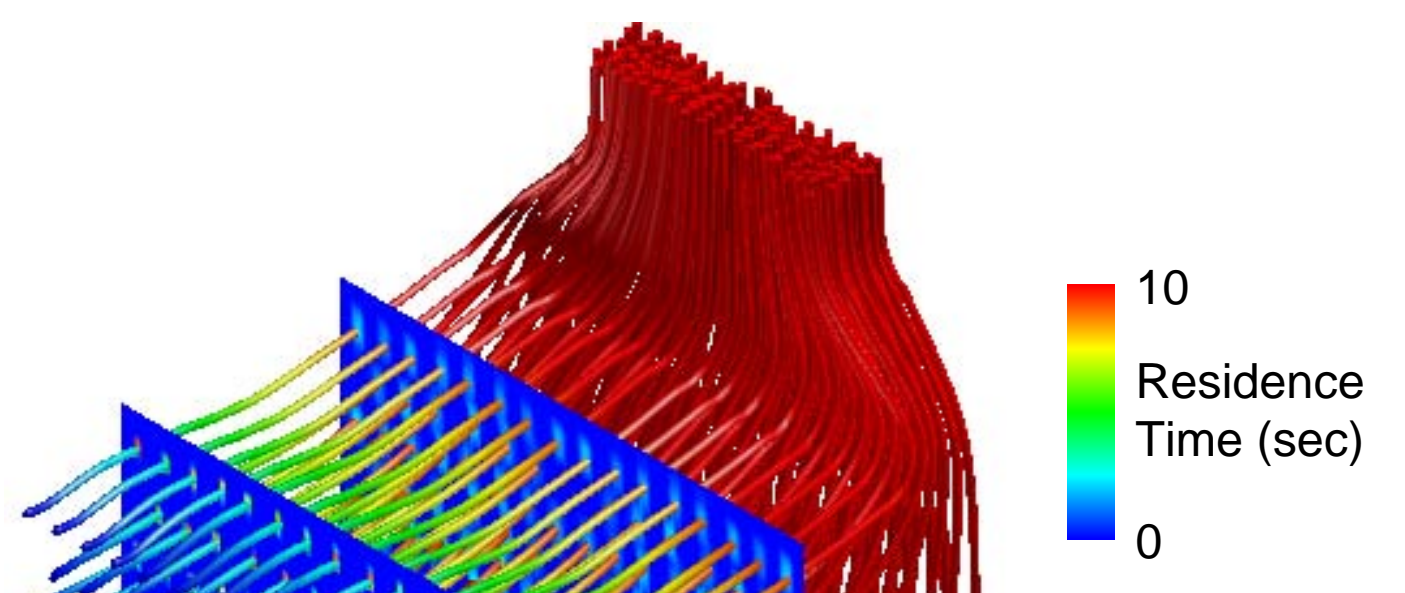

19 micron

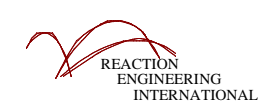




\section{Conclusions}

- Carrier air does not mix well with flue gas

» There are low air concentration gaps due to existence of collection plates

- Particle distribution has negligible effect on carrier air distribution inside ESP

»All three cases show very similar air concentration predictions

- Activated carbon particles are not well dispersed in the $3^{\text {rd }}$ and $4^{\text {th }}$ fields

»Very limited particle penetration along ESP width

» Some gaps between collection plates have almost no particles entering

- Higher particle bulk densities are predicted near hopper in Cases 2 and 3

» The hole at the bottom of each lance has higher particle flow rate, especially for large particles (mapped from grid model)

»Flue gas tends to enter hoppers and so are the particles 
APPENDIX E3: REI, Lance Modeling-September 14, 2006 


\section{CFD Modeling of TOXECON II at Independence}

\section{Domed Nozzle Model}

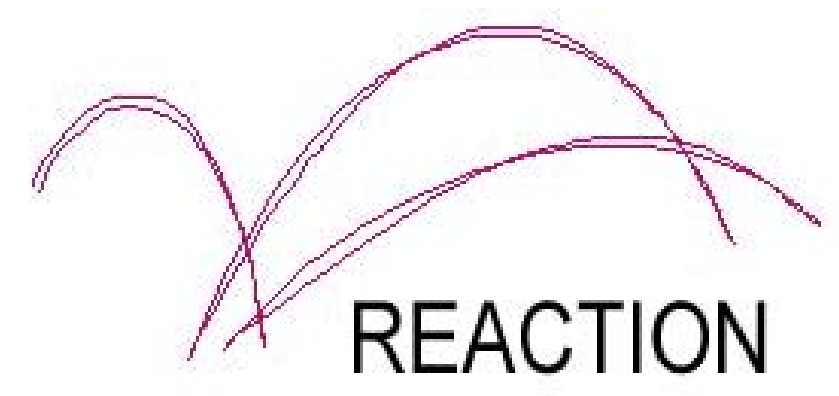

ENGINEERING

INTERNATIONAL

Sept. 14, 2006 


\section{Computational Model}

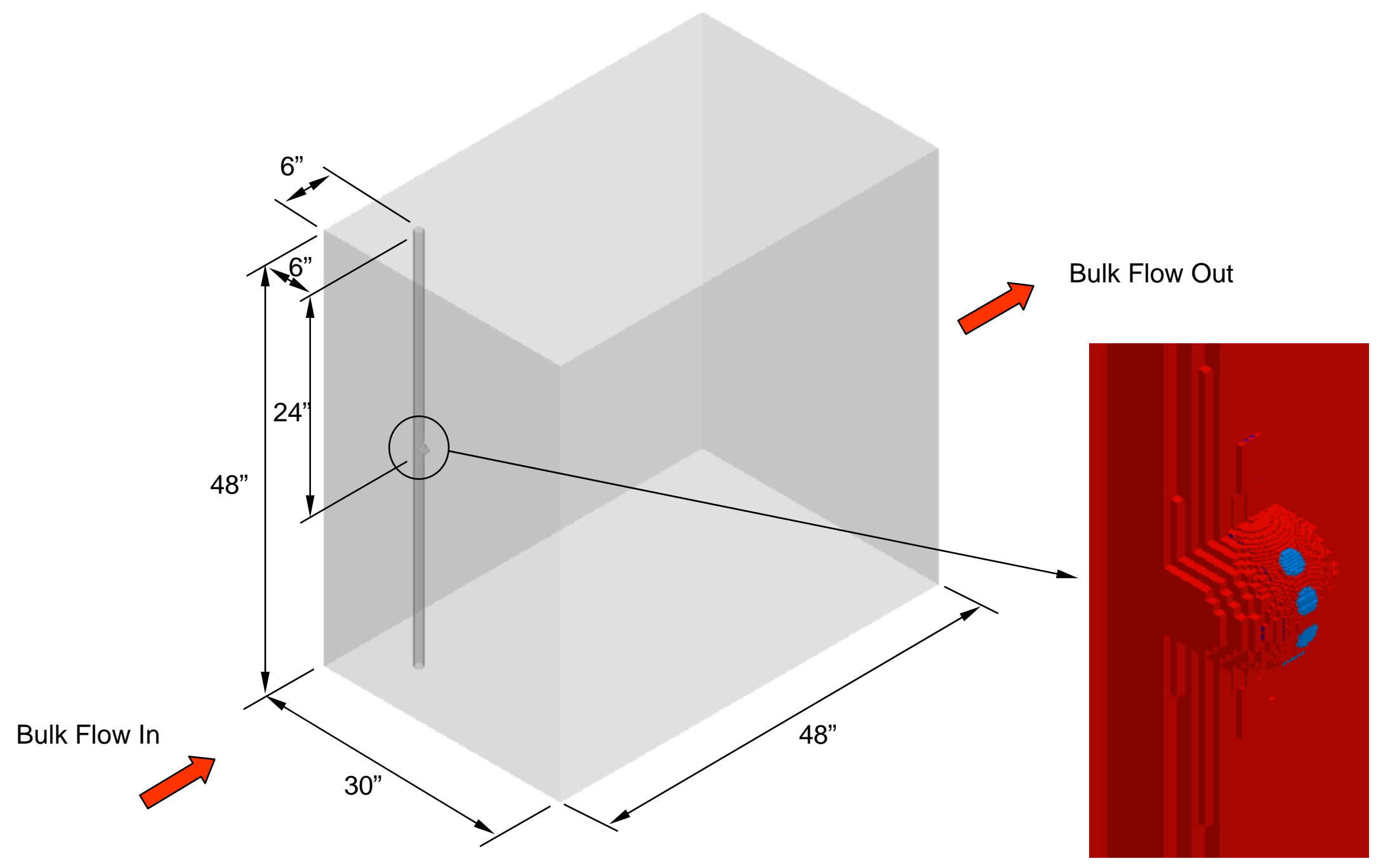




\section{Model Inputs}

- Bulk flow velocity: $4 \mathrm{ft} / \mathrm{s}$

- Flue gas temperature: $300^{\circ} \mathrm{F}$

- Pipe O.D.: 1.0625"

- Hole diameter: 0.16"

- Number of holes: 4

- Angle between holes: $30^{\circ}$

- Air jet velocity: $84.4 \mathrm{ft} / \mathrm{s}$

- Air temperature: $110^{\circ} \mathrm{F}$ 


\section{Locations of Contour Plots}

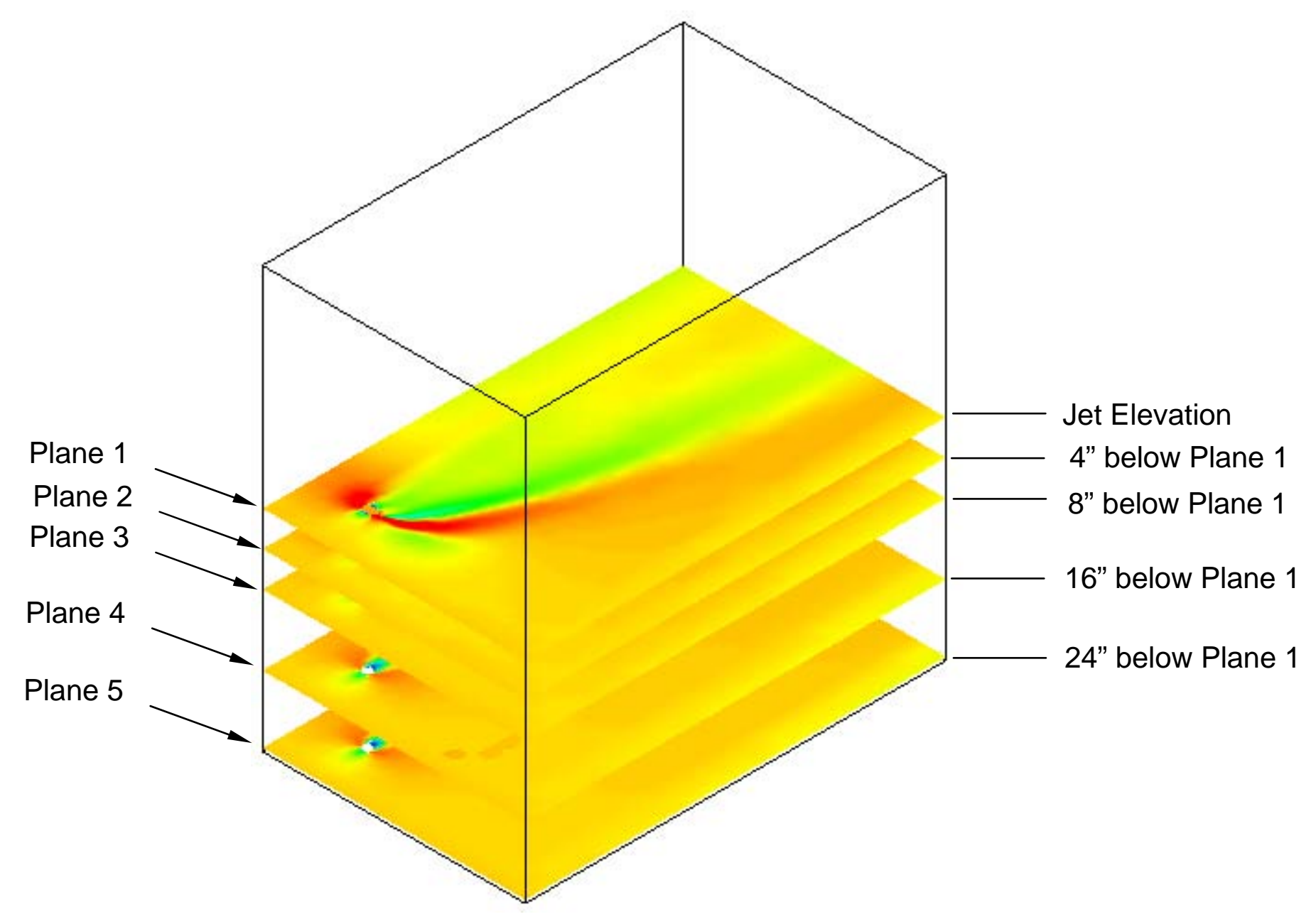




\section{Locations of Contour Plots}

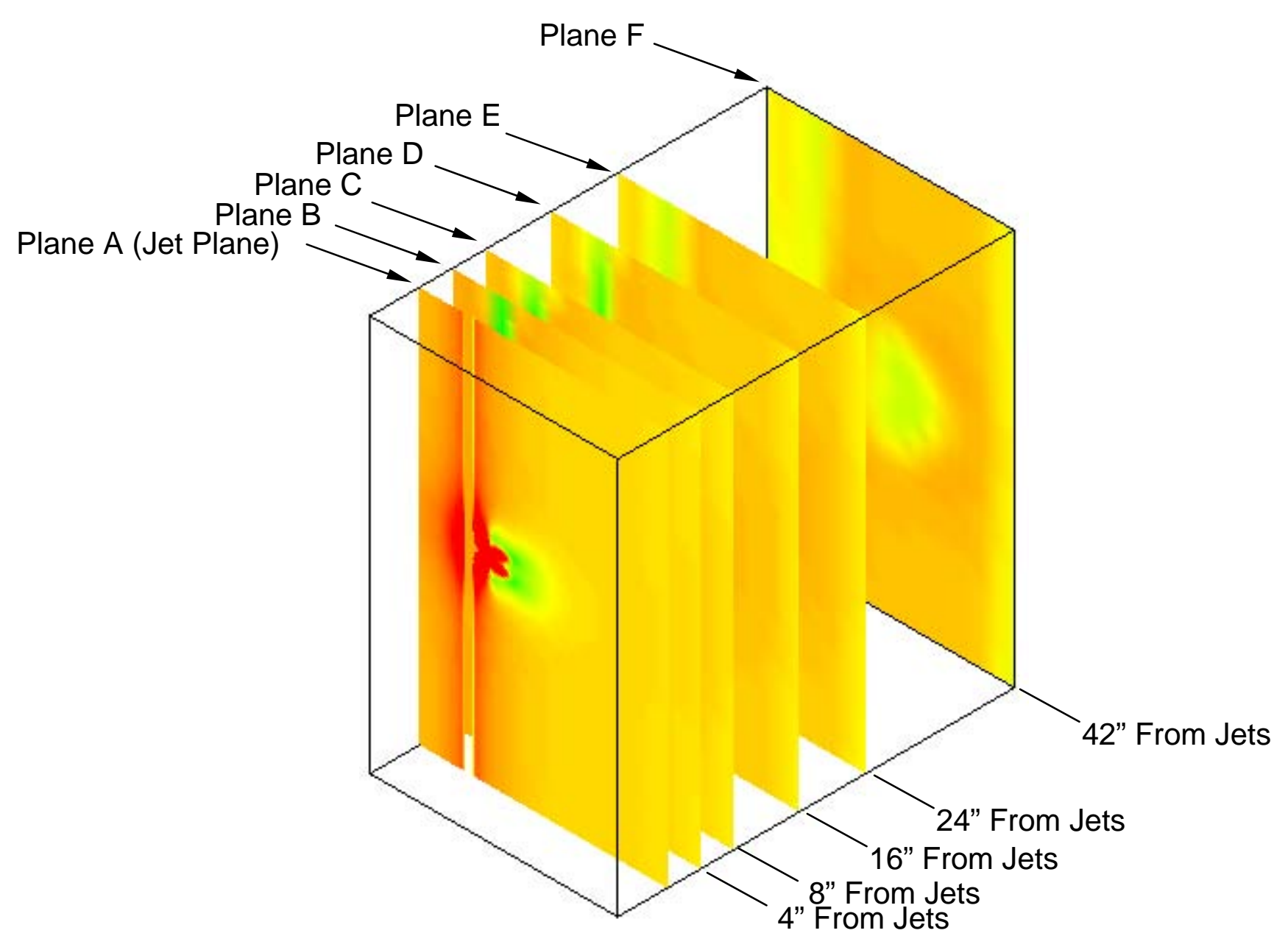




\section{Velocity Magnitude}

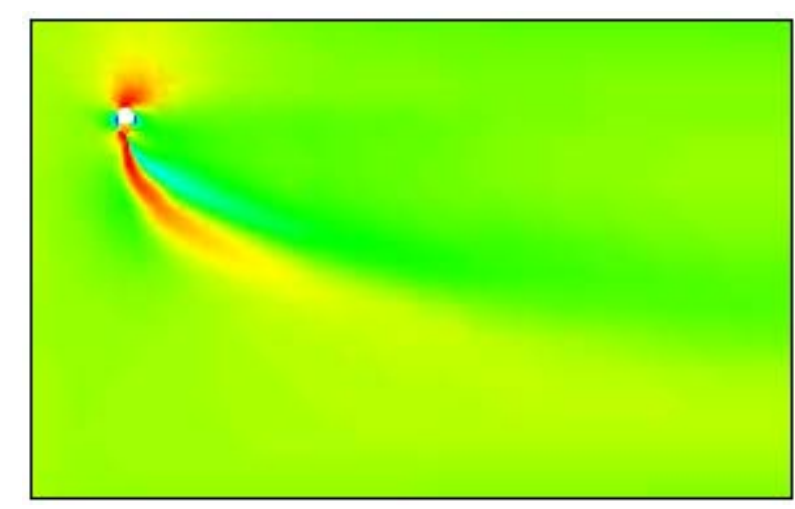

Plane 1

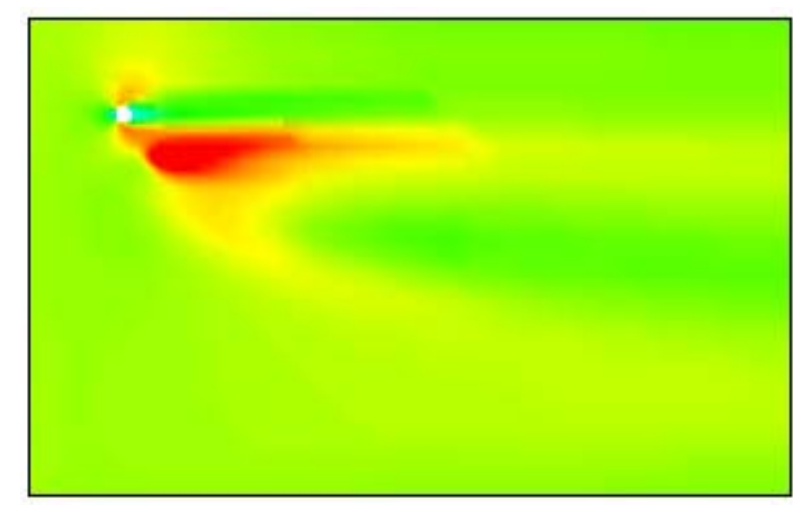

Plane 2

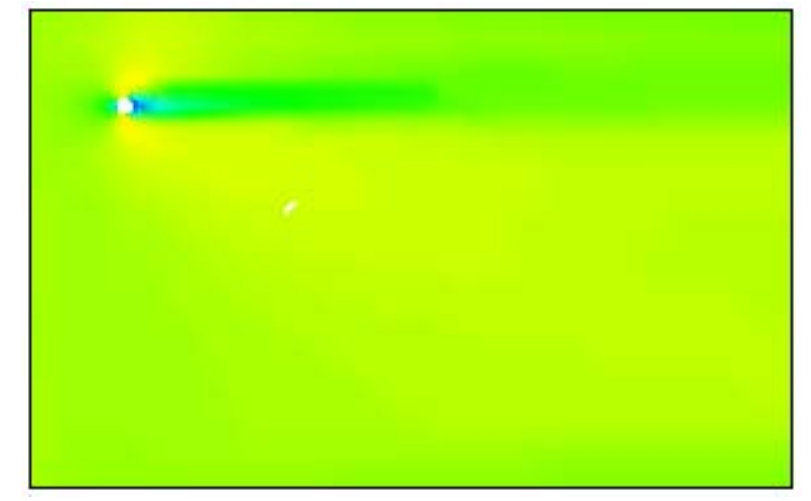

Plane 3

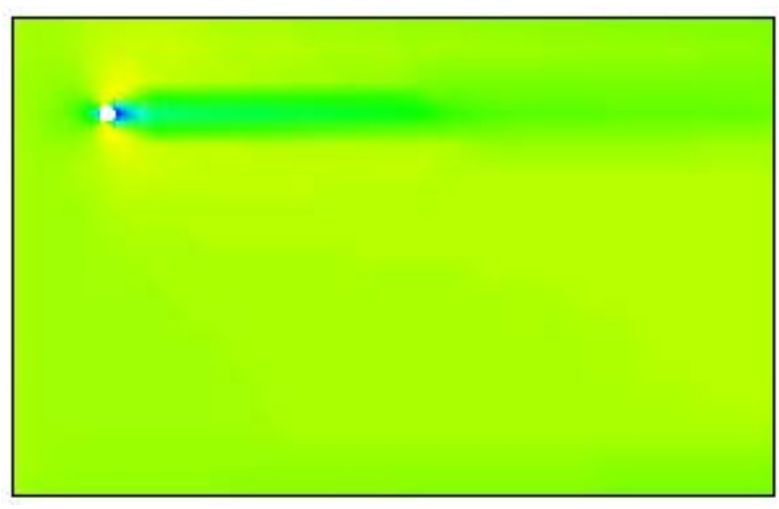

Plane 4

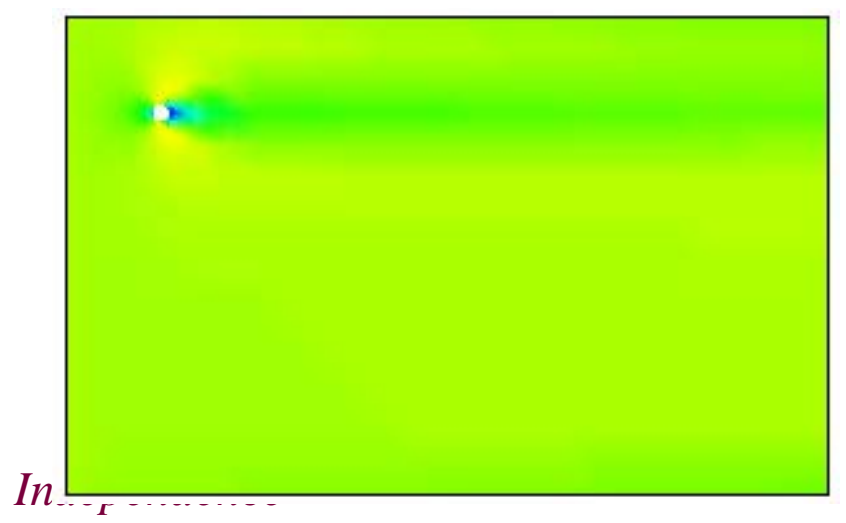

Plane 5
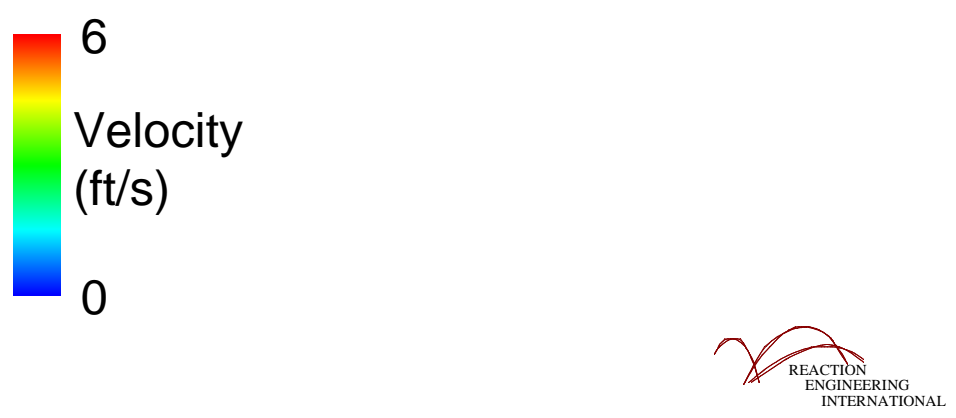


\section{Velocity Magnitude}

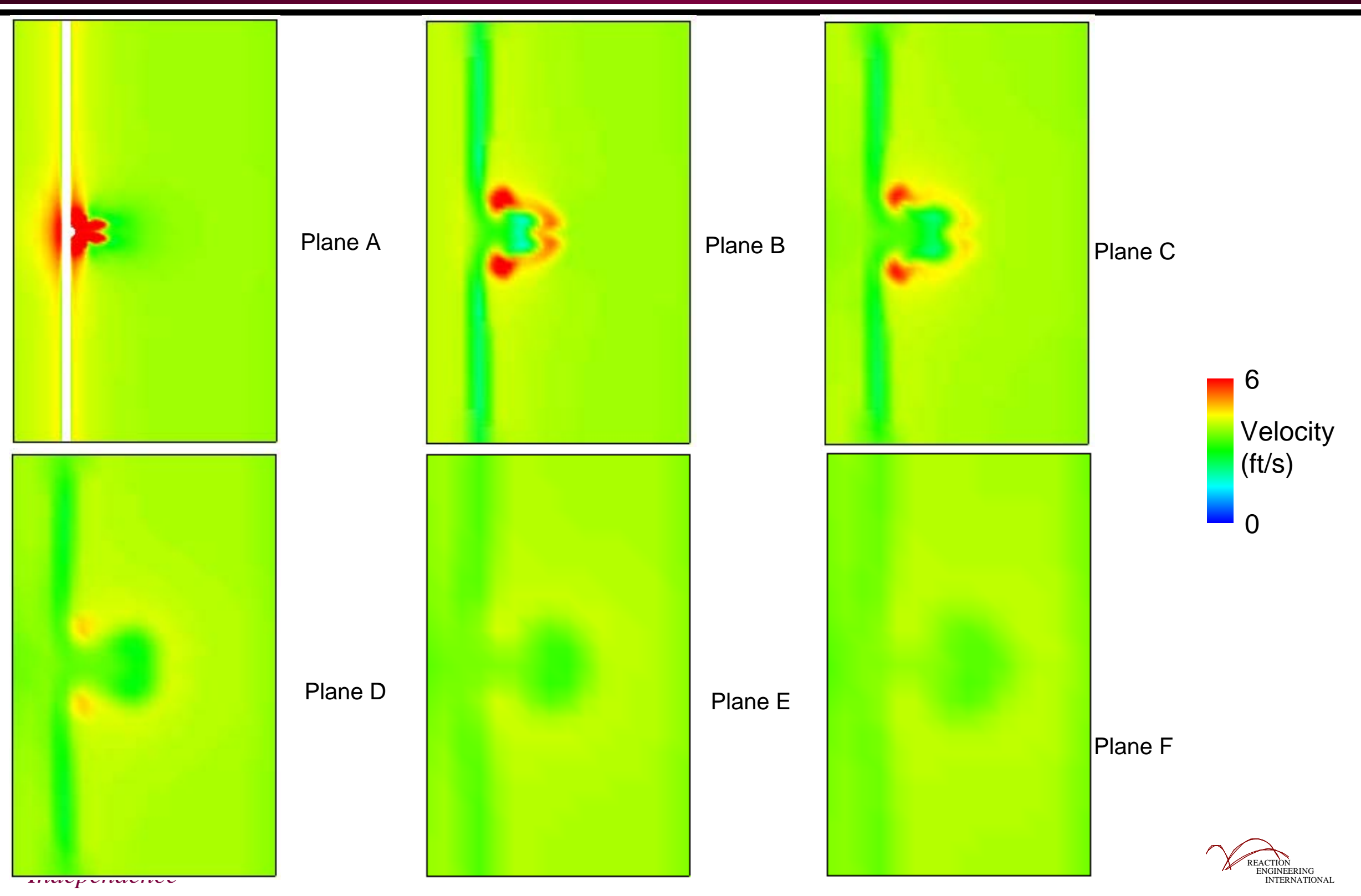




\section{Mass Fraction of Carrier Air}

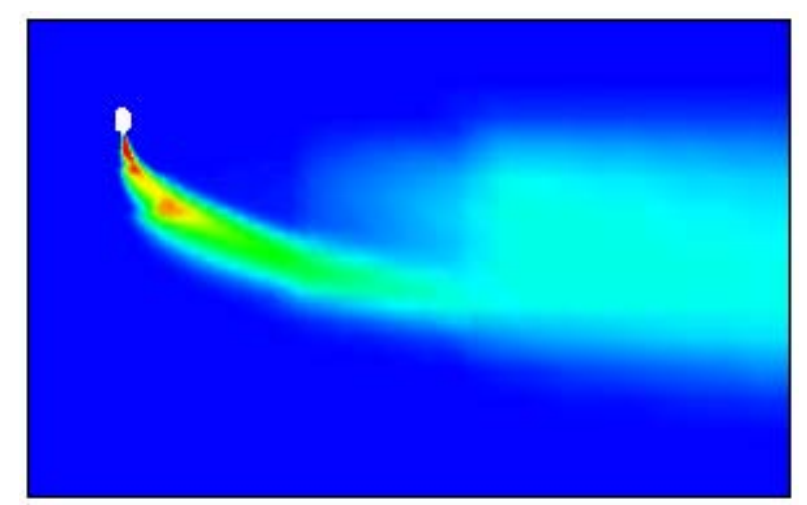

Plane 1

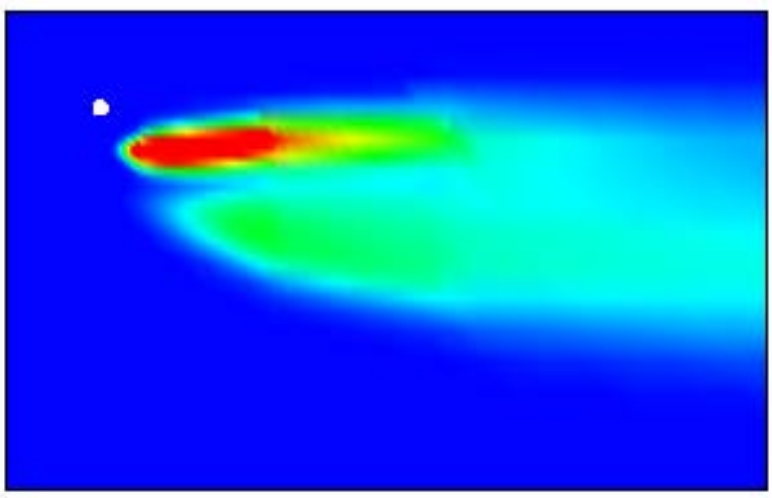

Plane 2

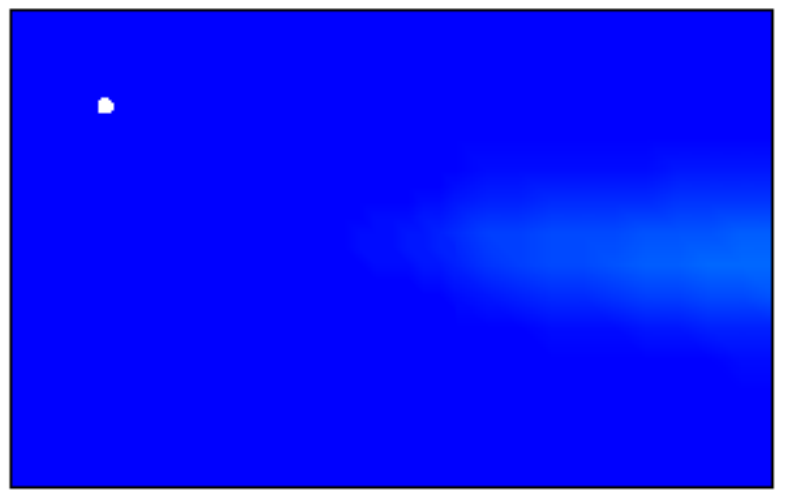

Plane 3

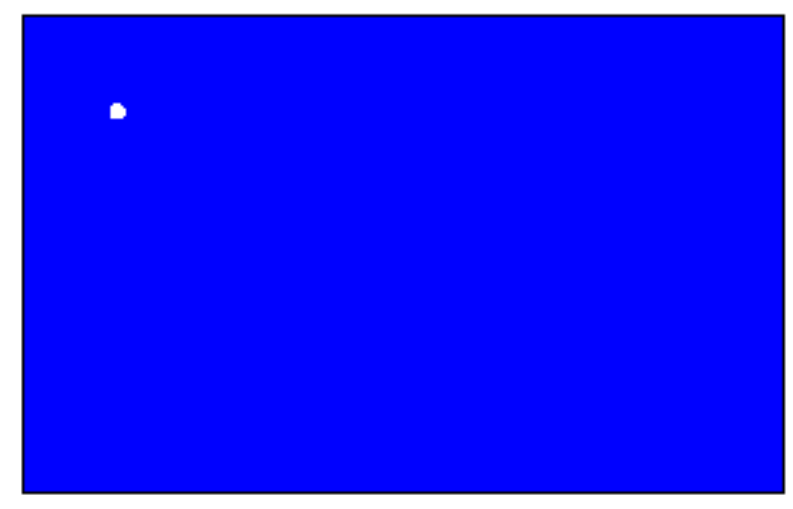

Plane 4

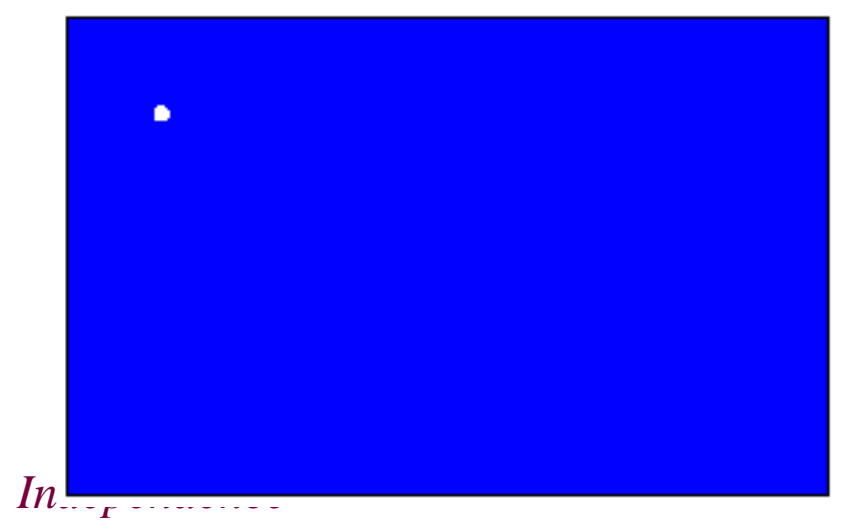

Plane 5

$5 \%$

Mass Fraction

of Air

0 


\section{Mass Fraction of Carrier Air}

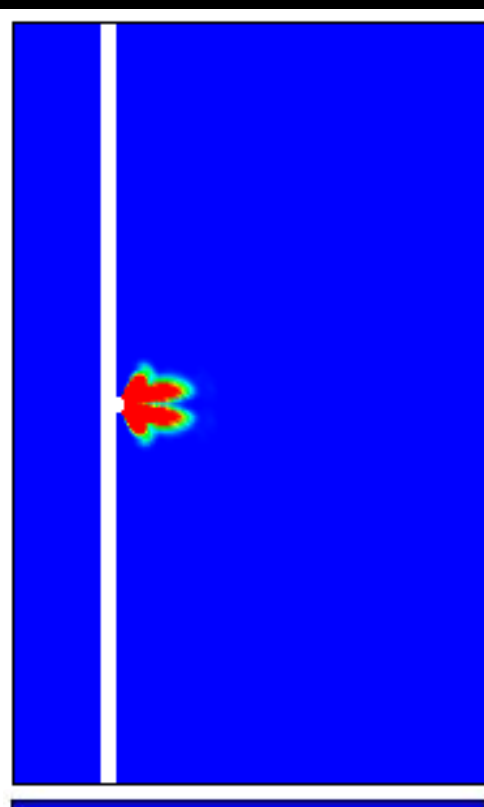

Plane A

Plane B

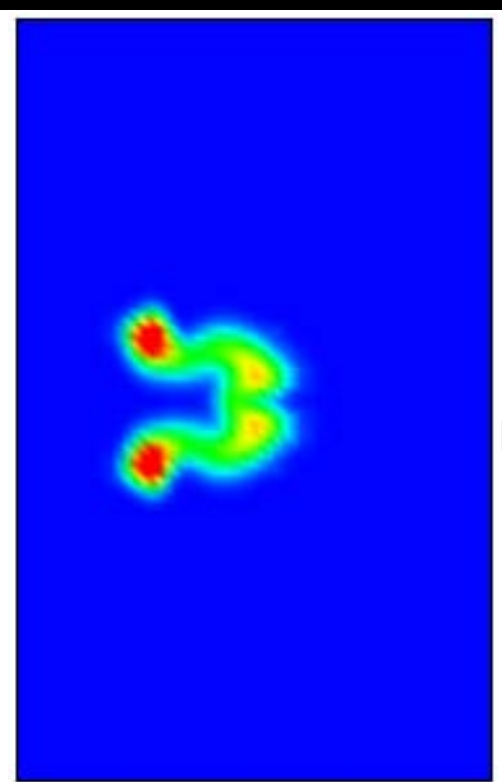

Plane C

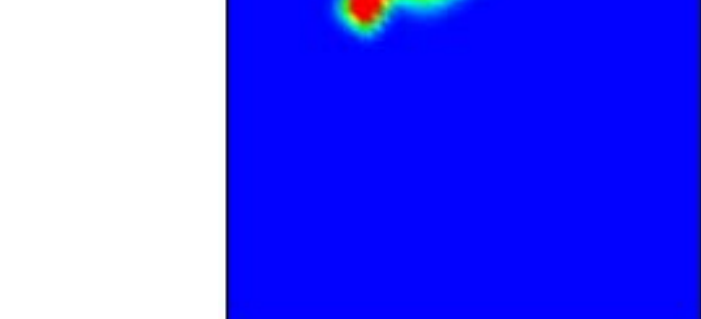

Plane D

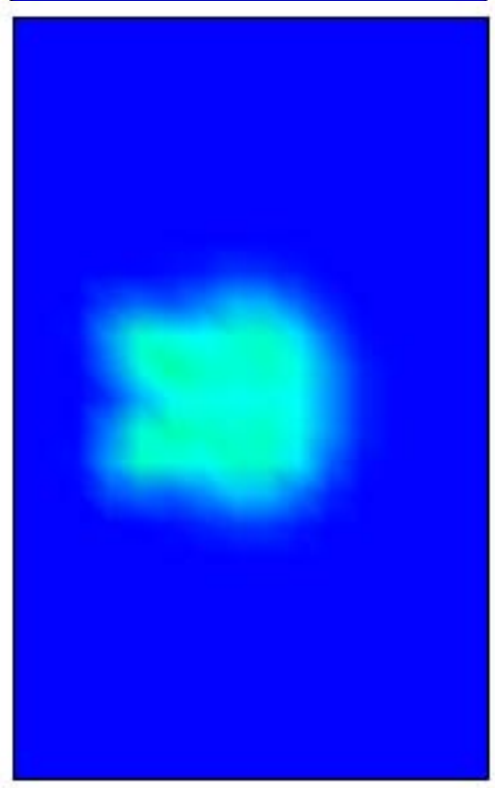

Plane E

$5 \%$
Mass Fraction
of Air
0

Plane F

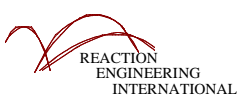


APPENDIX E4: NELS, ESP Flow Modeling-December 2006 


$$
\begin{gathered}
\text { ADA - ES INC } \\
\text { Littleton, Colorado }
\end{gathered}
$$

\author{
AIR FLOW MODEL STUDY \\ ENTERGY, INDEPENDENCE STATION UNIT 2 ESP \\ TOXICON II PROJECT, CARBON INJECTION
}

\title{
FINAL REPORT
}

ADA-ES Purchase Order No. AD06 - 448

NELS Project No. P119.06

\author{
NELS CONSULTING SERVICES, INC. \\ ST. CATHARINES, ONTARIO, CANADA \\ (905) 6822969
}

December 2006 


\section{ABSTRACT}

This report describes the airflow model study of the unit 2 electrostatic precipitator (PAC) carbon injection grid at the Independence Station located near Batesville, Arizona.

The objectives achieved by the study were as follows:

(a) To determine the existing configuration precipitator velocity distributions at the outlet of the first, second, third and last fields of collector curtains.

(b) To determine the existing configuration PAC injection grid distribution.

(c) To determine in the NELS wind tunnel the lance injection distribution using a full-scale section.

(d) To determine the existing configuration precipitator system pressure loss. 


\section{TABLE OF CONTENTS}

ABSTRACT ii

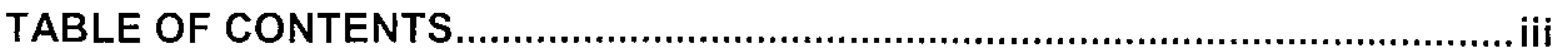

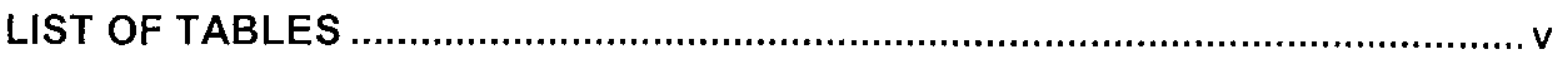

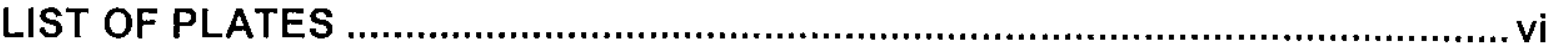

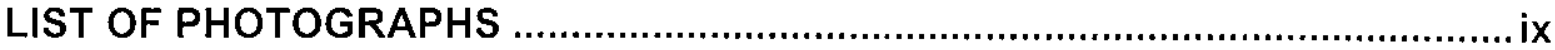

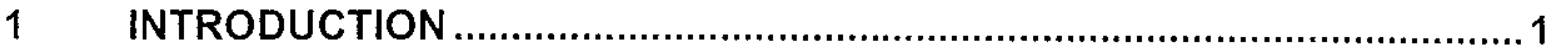

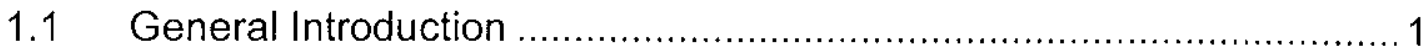

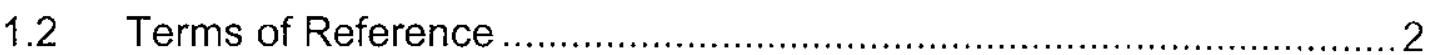

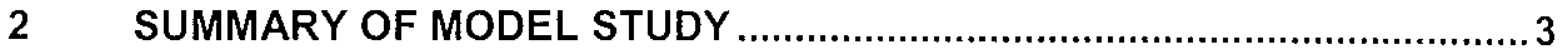

3 DESCRIPTION OF FULL SIZE INSTALLATION ........................................

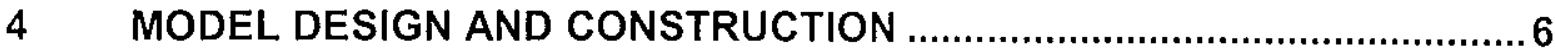

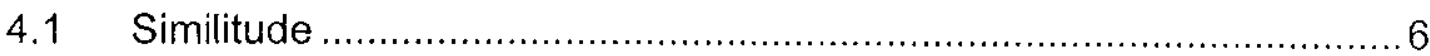

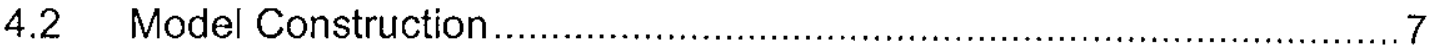

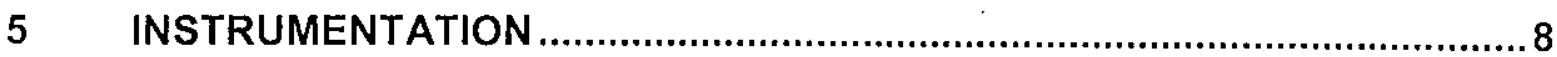

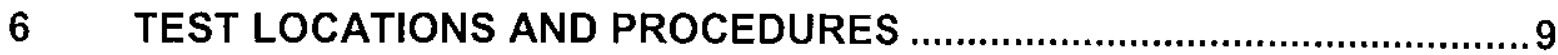

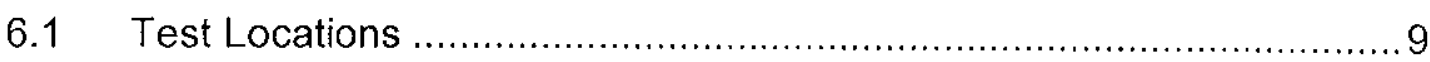

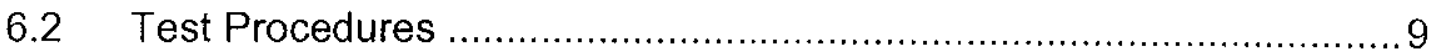

$7 \quad$ MODEL STUDY TEST RESULTS .......................................................... 11

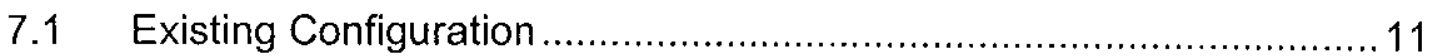

7.2 CO Distribution - Existing Configuration ........................................ 12

7.3 Wind Tunnel Observations - Existing Configuration ........................... 13

7.4 Precipitator Model Visual And Video................................................. 14 


\section{TABLE OF CONTENTS}

8 DISCUSSION AND RECOMMENDATIONS

TABLES

PLATES

PHOTOGRAPHS 


\section{LIST OF TABLES}

\begin{tabular}{ll}
\hline \hline Number & Title \\
1 & List of Drawings \\
2 & Full Size Installation - Design and Gas Flow Parameters \\
3 & Comparison between Full Size and Model \\
4 & Summary of Flow Scaling Parameters \\
5 & Powdered Activated Carbon (PAC) Injection Scaling Theory \\
6 & Description of Test Locations \\
7 & $\begin{array}{l}\text { Precipitator Gas Passage Test Locations } \\
8\end{array}$ \\
$\begin{array}{l}\text { Summary of Volume Flows } \\
\text { Precipitator Inlet Ducts T1L \& T1R to Outlet Duct T8 } \\
\text { Existing Configuration }\end{array}$ \\
9
\end{tabular}




\section{LIST OF PLATES}

\begin{tabular}{|c|c|}
\hline Number & Title \\
\hline 1 & $\begin{array}{l}\text { General Model Arrangement } \\
\text { And Test Locations } \\
\text { Plan And Elevation }\end{array}$ \\
\hline 2 & $\begin{array}{l}\text { General Model Arrangement } \\
\text { And Test Locations } \\
\text { Plan }\end{array}$ \\
\hline 3 & $\begin{array}{l}\text { General Arrangement } \\
\text { Precipitator } \\
\text { Plan And Elevations }\end{array}$ \\
\hline 4 & $\begin{array}{l}\text { General Arrangement } \\
\text { Precipitator And Ducting } \\
\text { Elevation }\end{array}$ \\
\hline 5 & $\begin{array}{l}\text { Details } \\
\text { Precipitator Inlet Plenum } \\
\text { Elevation }\end{array}$ \\
\hline 6 & $\begin{array}{l}\text { Arrangement \& Details } \\
\text { Inlet Plenum Turning Vanes } \\
\text { Existing Configuration }\end{array}$ \\
\hline 7 & $\begin{array}{l}\text { Details } \\
\text { Precipitator Outlet Plenum } \\
\text { Elevation }\end{array}$ \\
\hline 8 & $\begin{array}{l}\text { Details } \\
\text { Inlet \& Outlet Plenums } \\
\text { Truss Details }\end{array}$ \\
\hline 9 & $\begin{array}{l}\text { Details } \\
\text { Precipitator Inlet Duct } \\
\text { And Turning Vanes }\end{array}$ \\
\hline 10 & $\begin{array}{l}\text { Details } \\
\text { Precipitator Outlet Duct } \\
\text { And Turning Vanes }\end{array}$ \\
\hline 11 & $\begin{array}{l}\text { General Arrangement } \\
\text { Carbon Injection Grid } \\
\text { Elevation }\end{array}$ \\
\hline
\end{tabular}




\section{LIST OF PLATES}

Number Title

12

General Arrangement

Carbon Injection Grid Location

Elevation - Between $2^{\text {nd }} \& 3^{\text {rd }}$ Field

13

General Arrangement

Wind Tunnel Injection Grid

Elevation

14

Normalized Velocity Distribution

Inlet Duct to Precipitator, Location T1L

Existing Configuration

15 Normalized Velocity Distribution

Inlet Duct to Precipitator, Location T1R

Existing Configuration

$16 \quad$ Normalized Velocity Distribution

Inlet Duct to Precipitator, Location T2L

Existing Configuration

17 Normalized Velocity Distribution

Inlet Duct to Precipitator, Location T2R

Existing Configuration

18 Normalized Velocity Distribution

Outlet of First Field, Location T3

Existing Configuration

19 Normalized Velocity Distribution

Outlet of Second Field, Location T4

Existing Configuration

20 Normalized Velocity Distribution

Outlet of Third Field, Location T5

Existing Configuration

$21 \quad$ Normalized Velocity Distribution

Outlet of Last Field, Location T6

Existing Configuration

$22 \quad$ Normalized Velocity Distribution

Outlet Duct from Precipitator, Location T7

Existing Configuration 


\section{LIST OF PLATES}

Number Title

23 Normalized Velocity Distribution

Outlet Duct from Precipitator, Location T8

Existing Configuration

24 Carbon Monoxide Distribution

Middle of Third Field, Location T5A

Existing Configuration

25 Carbon Monoxide Distribution

Middle of Last Field, Location T6A

Existing Configuration 


\section{LIST OF PHOTOGRAPHS}

\begin{tabular}{ll}
\hline \hline Number & Title \\
A & General Model Arrangement \\
B & Bottom of Inlet Plenum \\
C & Vanes Upstream of The Inlet Plenum \\
D & Inlet Duct Vanes \\
E & (PAC) Injection Lance Arrangement \\
F & Existing Outlet Plenum
\end{tabular}




\section{INTRODUCTION}

\subsection{General Introduction}

Detailed in this report are the findings of the airflow model study conducted for the existing unit 2-precipitator installation at Entergy's, Independence Station located near Batesville, Arizona.

NELS Consulting Services Inc. conducted this study for ADA - ES Inc of Littleton, Colorado.

The unit 2 cold side precipitator, which was converted from a hot side installation, cleans particulate emissions from a coal-fired boiler. The existing precipitator installation has four casings that were supplied by CE Walther Pollution Control. The casings are paired with one pair above the other forming a double deck arrangement.

A $1 / 12$ scale air flow model of half of a lower precipitator casing as shown on Plates 1 and 2, Photographs $A$ to $F$ and the accompanying video of the unit 2 precipitator with associated inlet and outlet ductwork was constructed and tested to determine the PAC injection distribution, precipitator velocity distribution and system pressure loss. 


\subsection{Terms of Reference}

The objectives of the study were as follows:

a) To measure precipitator velocity distributions at the outlet of the first, second, third and last fields of collector curtains.

b) To measure the PAC injection grid distribution at the middle of the third and last field using $\mathrm{CO}$ gas as a tracer.

c) To measure system pressure loss.

d) To video in the NELS wind tunnel the injection distributions using a fullscale section of the lance grid with similar precipitator cross flow. 


\section{SUMMARY OF MODEL STUDY}

It was established by the model study that velocity distributions in the precipitator at the outlet of the first, second, third and fourth fields were not within the ICAC criteria stating that $85 \%$ and $99 \%$ of the measured velocities shall be less than 1.15 and 1.40 times the mean velocity.

Velocity distributions at the outlet of the first and second fields had $72.1 \%$, $94.2 \%$ and $77.1 \%, 99.2 \%$ of the velocities within 1.15 and 1.40 times the mean as shown on Plates 18 and 19. At the outlet of the third field the distribution measured improved slightly with $83.8 \%$ and $100.0 \%$ within 1.15 and 1.40 as shown on Plate 20. Further downstream at the outlet of the fourth field the distribution measured had $68.8 \%, 77.9 \%$ less than 1.15 and 1.40 and was severely skewed with high velocities towards the top as shown on Plate 21. NELS believes that high velocities along the lower collection area of the field, bottom of the opening and bottom of the collecting plates are a cause for particulate re-entrainment, opacity spiking and reduced overall collection efficiency.

Inlet and outlet ductwork velocity distributions were satisfactory and acceptable as shown on Plates 14 to 17,22 and 23.

In the $1 / 12^{\text {th }}$ scale model a test of the (PAC) injection lances as shown on Plates 11 and 12 was conducted by using $\mathrm{CO}$ gas as a tracer and measuring the distributions in the middle of the third and fourth fields as shown on Plates 24 and 25. The CO concentrations measured had RMS deviations of $111.5 \%$ and $68.8 \%$ with high to low cycling across the width of the test plane and higher concentrations being skewed towards the top.

Wind tunnel testing of the (PAC) lances as shown on Plate 13 were conducted by injecting bulk smoke and recording the observations on DVD. Having an orifice acfm of 0.735 (3,340 fpm), smoke can be seen exiting the lance and penetrating past half of the distance between two lances however no wind tunnel cross flow had been introduced. With the wind tunnel fan running and set for $4 \mathrm{ft} / \mathrm{s}$ cross flow, observations at the lances show the smoke path had folded with the direction of the cross flow and the distance of injection between lances was reduced considerably. With increased lance flow to $2.695 \mathrm{acfm}(12,250 \mathrm{fpm})$, observations 
show an improved distance of injection to about mid way between two lances was achieved with the $4 \mathrm{ft} / \mathrm{s}$ wind tunnel cross flow. The injection penetration had increased to about three quarters of the distance between lances when the orifice rate was increased to $3.675 \mathrm{acfm}(16,704 \mathrm{fpm})$.

From this study it can be concluded that modifications to the existing inlet plenum flow devices are necessary to achieve a velocity distribution that would be acceptable to ICAC flow criteria however NELS believes a reduction in high velocities in the lower section of the ESP causing particulate re-entrainment would be more beneficial but the resulting distribution may not be within the $85 \%, 99 \%$ recommendations. The (PAC) injection distribution should improve with a more uniform inlet flow upstream and with an increase in the lance orifice acfm flow but the extent of the inlet plenum modifications is unknown. The addition of a variable porosity screen in the outlet plenum would improve the velocity distribution and the lance distribution through the fourth field but an increase in system pressure loss would occur.

Further testing of the model to investigate these modifications would only be applicable to the lower precipitator casings and the evaluation of the upper precipitator casings may show a different outcome. Because the installation is a double deck arrangement, flow balance and dust loading to individual precipitators maybe biased. 


\section{DESCRIPTION OF FULL SIZE INSTALLATION}

Details of the existing unit 2 precipitator installation at Entergy's, Independence Station were supplied on the drawings listed in Table 1. The general arrangement of half of a lower precipitator casing is shown on Plates 1 and 2. The associated design and gas flow requirements for half of the full size precipitator casing are detailed in Table 2.

The unit 2 installation has four precipitator casings that clean particle emissions from an $800 \mathrm{MW}$ coal fired boiler. Each casing has two chambers that are 4 fields deep and each field has 42 gas passages that are $48^{\prime}$ tall. The existing precipitators are arranged as pairs with one pair situated above the other as a double deck arrangement and had operated as a hot side installation but was converted to a cold side system.

The unit is part of the EPRI Toxecon II test project. The test project consists of injecting a carbon sorbent into the precipitator gas flow between the second and third collecting fields to control Mercury emissions. The injection grid for half of a precipitator casing consists of two trains having 8 lances for each train that extend down for $42^{\prime}-21 / 2$ " between the second and third collecting fields.

Based on the information contained in the supplied drawings, the model design as shown in Photographs $A$ to $F$ and the accompanying video was developed, the simplified layouts shown on Plates 3 to 13 were drawn and the relevant gas flow parameters determined as described in Section 4. 


\section{MODEL DESIGN AND CONSTRUCTION}

\subsection{Similitude}

In gas flow modeling, it is theoretically necessary to simultaneously maintain geometric, kinematic and dynamic similarity. However, such a condition is not possible under modeling conditions. Therefore, this airflow model study was conducted using a geometric linear scale factor of $1 / 12$ and a velocity scale of $1 / 1$. These particular scaling factors were chosen based on the following rationale:

1. When a sufficiently large geometric scaling factor is used, the most critical aspect of geometric similarity is satisfied and the reliability of measurement can be ensured. A linear scale of $1 / 12$ was determined to be sufficient to satisfy this criterion and yet economical to build and test.

2. Kinematic similarity is dependent upon the Reynolds Number $(R e)$ and has no significant influence if this number is maintained above an approximate value of 20000 (i.e., the minimum Re to ensure fully turbulent flow conditions). Based on the velocities used in the system the typical Reynolds Numbers for the full size installation and the model were $5.91 \times 10^{5}$ and $9.16 \times 10^{4}$ respectively, thus satisfying the above criterion. As fully turbulent flow conditions were maintained in this study, velocity distributions and pressure losses can be accurately measured in the model and predictions made for the full size installation.

3. As this particular model study was based upon uniform air density, the forces of gravity are negligible in comparison with inertia forces. Therefore, it was not essential to maintain dynamic similarity during the airflow testing.

A comparison between the full size installation and the model is detailed in Table 3 and the necessary airflow scaling parameters listed in Tables 4 and 5 , respectively. 


\subsection{Model Construction}

A $1 / 12$ scale three dimensional model, geometrically similar to half of a typical precipitator casing of the full size unit 2 installation at Entergy's, Independence Station, as detailed on Plates 3 to 13 , shown in Photographs $A$ to $F$ and video was constructed from $1 / 4$ inch plexiglass. The model of the installation extends from a point upstream of the precipitator inlet plenum, through half of a typical casing to the outlet plenum and to a point downstream in the outlet ductwork.

The precipitator section included:

i) all gas distribution devices made from sheet metal and plastic

ii) internal trusses made from plastic and wood doweling

iii) all collecting plates simulated by flat sheets

iv) all hoppers and baffles

v) PAC injection grid having 16 lances

A full-scale section of the injection grid was constructed and installed in the NELS wind tunnel.

The wind tunnel lance grid included:

vi) single header from half of a train with 4 outlets

vii) 4 lances

Specified internal structural members considered significant to the gas flow were included in the precipitator model. The velocity distribution upstream of the model limits was considered uniform for this study. One single inlet centrifugal fan driven by a 30-hp motor provided flow through the model, which was operated under negative pressure.

A 30 " positive pressure blower provided flow to the wind tunnel lances. A double inlet centrifugal fan driven by a 200-hp motor, which was operated under negative pressure, provided wind tunnel flow. Flow entering the tunnel was considered uniform. 


\section{INSTRUMENTATION}

The airflow velocities and pressures in the model were measured using the following equipment:

(a) Ductwork Velocities

Pitot static tube (AMCA Std. 210-74, ASHRAE Std. 5175) or 'S' type Pitot static tube 12 inches long by $2.3 \mathrm{~mm}$ diameter, with an Airflow Testing Mark 5 inclined manometer, range 0 to $0.5,1,2,10$ and 20 inches of water.

(b) Total Pressures

Total pressure hole of the Pitot static tube and inclined manometer as in (a) above.

(c) Volume Flow

The volume flow through the model section was monitored using test locations with sufficiently uniform velocity distributions as indicated by a Root Mean Square Deviation (RMS) of less than $15 \%$ of the mean velocity.

(d) Precipitator Gas Passage Velocities

Thermo-Systems Inc. (TSI) constant temperature linearized anemometer Model No. 1054-B1 with a TSI Model 1051-1 monitor/power supply with a slosyn present indexer for continuous traversing and a Heathkit SR-201A chart recorder. This equipment was calibrated throughout the testing on a ThermoSystems Inc. Model 1125 Calibrator and against the pitot static tube using the NELS wind tunnel.

(e) Air Flow

The airflow patterns in the model were identified with:

- Smoke Generator.

- Digital video camera.

(f) CO Distributions

MultiGas Model MG140 Carbon Monoxide Analyzer

Range 0 - 1000 ppm

Resolution 1 ppm

Accuracy $+/-1 \%$ of full scale 


\section{TEST LOCATIONS AND PROCEDURES}

\subsection{Test Locations}

The test locations used in the precipitator model study shown on Plates 1 and 2 are described in Tables 6 and 7. Velocity and total pressure distributions were measured at each test location within the model. These test positions were selected such that the flow patterns within the ductwork and precipitator could be accurately evaluated and the associated pressure losses calculated. Specifically, these test locations were chosen such that the influence of changes in flow direction and magnitude, imparted by directional and size variations, could be quantified and qualified.

\subsection{Test Procedures}

The precipitator model was operated under negative pressures at velocities similar to those in the operating installation to ensure that representative flow patterns were maintained (i.e. Reynolds Number greater than 20 000). Air velocities and total pressures were measured at the centres of equal areas across each duct section where the minimum number of measurements was determined by,

\section{No. of Test Points $=1.5 \times \sqrt{A}$}

Where $A=$ cross section area $\left(\mathrm{ft}^{2}\right)$ of duct (full size dimensions).

The airflow velocities and pressure distributions at each test location within the inlet and outlet ductwork were evaluated based on a traverse of measurements across the duct using the Pitot tube and manometer as described in Section 5 (a and b). The velocity distribution within the precipitator was evaluated based on continuous airflow velocity traverses taken in every third gas passage across the fields using the equipment described in Section 5(d). Within each test plane (gas passage), 16 velocity measurements distributed over 17 equal spaces of the precipitator opening, listed in Table 7 and shown on Plates 1 and 2, were determined from these continuous traces. The velocities within the projected inlet and outlet openings were used to determine the average velocity, RMS deviation 
and statistical percentages. Velocities at the top of collecting plates (TP), top of opening (TO), bottom of opening (BO), and bottom of collecting plates (BP) were measured to evaluate gas flow anomalies outside the effective collection zone.

To evaluate the performance of the (PAC), the as designed carbon injection system was installed in the model. The full size carbon injection system was modeled to scale using brass tubing. The tracer gas, $(\mathrm{CO})$, to each lance manifold was balanced using a control valve so that each manifold had an equal amount to represent the equivalent amount of carbon. The model scaling was based on the momentum ratio between the gas flow and the carbon injection lances for the full size and scale model as shown in Table 5.

In the wind tunnel a single header (half of one train) with 4 lances was installed to represent the upper section. A blower with 30" of water lift and operating under positive pressure was connected to the lance header. Wind tunnel flow representing the precipitator gas flow was set at $4 \mathrm{ft}$ per second. Smoke was injected near the blower and observations of the lance injection were recorded on video. 


\section{MODEL STUDY TEST RESULTS}

\subsection{Existing Configuration}

The existing configuration of Entergy's, Independence Station unit 2 precipitator as shown on Plates 1 to 10 , was tested from the inlet duct, locations T1L and $T 1 R$, to the outlet duct, location $T 8$ and their respective velocity distributions are shown on Plates 14 to 23 and further summarized in Tables 8 to 10 .

Flow entering the model at locations $T 1 L$ and T1R was uniform and acceptable as shown on Plates 14 and 15. The inlet ducting then turns down towards the precipitator inlet plenum and the velocity distributions measured at locations, T2L and T2R, remained acceptable as shown on Plates 16 and 17.

At the outlet of the first field, location T3, the velocity distribution measured had $72.1 \%$ and $94.2 \%$ of the velocities less than 1.15 and 1.40 times the mean velocity as shown on Plate 18. ICAC recommends that $85 \%$ and $99 \%$ of the measured velocities shall be less than 1.15 and 1.40 times the mean velocity. Velocities are higher across the upper half of the collecting field, rows 1 to 8 then lower between rows 9 to 12 and then increase higher between rows 13 to 16 as shown on the Row Average graph. The side-to-side flow balance to the gas passages across the collecting field width slowly cycles high to low as shown on the Column Average graph. NELS believes that high velocities across the bottom three rows $(14,15 \& 16)$, bottom of the opening and bottom of the collector plates are a cause for particle re-entrainment, opacity spiking and reduced overall collection efficiency.

Further downstream at the outlet of the second field, location T4 the distribution improved slightly having $77.1 \%$ and $99.2 \%$ of the measured velocities less than 1.15 and 1.40 times the mean velocity as shown on Plate 19. Flow to the upper half, rows 1 to 8 has decreased slightly with an increase in flow between rows 9 to 12 and high flow remaining across the bottom between rows 13 to 16 as shown on the Row Average graph. The side to side flow balance has improved however gas passage 22 shows low flow near the structural column at the chamber centerline as shown on the Column Average graph. Velocities across rows 14, 15, 16 and bottom of the opening remained high and unacceptable. 
At the outlet of the third field, location T5, the distribution improved with respect to ICAC recommendations having $83.8 \%$ and $100.0 \%$ of the measured velocities less than 1.15 and 1.40 times the mean velocity as shown on Plate 20 . Flow to the upper half of the collecting field between rows 1 to 8 has increased. Rows 9 to 12 remained similar as before and flow between rows 13 to 16 was reduced as shown by the Row Average graph. The Column Average graph shows an acceptable flow balance side to side with the localized low gas passage 22 near the structural column.

At the outlet of the last field, location T6, the velocity distribution has $68.8 \%$ and $77.9 \%$ of the velocities within 1.15 and 1.40 times the mean velocity as shown on Plate 21. The Row Average graph shows a skewed trend towards the top of the collecting field with an extremely high band of velocities in rows 1 to 6 and decreasing flow towards the bottom of the opening and bottom of the collector plates. Flow balance side to side across the collecting field gas passages shown by the Column Average graph was similar as before. With this velocity distribution, even though rows $14,15,16$, bottom of the opening and bottom of the collecting plates have low velocities with respect to NELS recommendations, opacity spiking and particle re-entrainment will occur and are caused by the higher velocities with a severely skewed distribution since the existing outlet plenum has no flow devices installed.

Flow exiting the last field of the precipitator turns up to the outlet ducting and the velocity distribution measured at location $T 7$ was acceptable and satisfactory as shown on Plate 22. The outlet ducting turns horizontal and the velocity distribution measured at location T8 was uniform as shown on Plate 23.

From the inlet duct, locations T1L and T1R, to the outlet duct location T8 the estimated pressure loss measured from the model was $1.80^{\prime \prime} \mathrm{H}_{2} \mathrm{O}$ for a flow of 482,169 acfm at $299^{\circ} \mathrm{F}$.

\subsection{CO Distribution - Existing Configuration}

With the precipitator configuration shown on Plates 3 to 10 the PAC injection grid as shown on Plates 11 and 12 was tested using carbon monoxide as a tracer gas to represent the carbon injection and the measured distributions are shown on 
Plates 24 and 25 and further summarized in Table 10. The model had both trains installed with tracer gas being injected and sampling of the distribution was taken in gas passages 6 to 16. Midway through the third field at location T5A, the CO distribution measured would not be consider uniform having a very high RMS deviation of $111.5 \%$ as shown on Plate 24 . The Column Average graph shows high and low cycles side to side across gas passages 6 to 16 with similar cycling in the Row Average graph. The higher $\mathrm{CO}$ concentration and poor distribution measured at location T5A maybe influenced by the velocity distribution within the precipitator. The lance itself may have an imbalance top to bottom however ADA-ES noted that their earlier test results show a reasonable balance over the length of their test lance was achieved.

Further downstream at location T6A, middle of the fourth field, the $\mathrm{CO}$ distribution measured has improved but again was not uniform with respect to the high RMS deviation of $68.8 \%$ as shown on Plate 25 . Side to side balance across the collecting field width has improved with reduced high to low cycling as shown by the Column Average graph. The CO distribution shown by the Row Average graph has shifted towards the upper half, rows 1 to 6 and again maybe influenced by the velocity distribution within the precipitator collecting plates.

\subsection{Wind Tunnel Observations - Existing Configuration}

A full scale version of four lances representing a portion of the top section of half of an injection train was constructed and installed in the NELS wind tunnel as shown on Plate 13 and the test observations were recorded on video.

Two lances outside of the tunnel were closed and flow from the positive pressure blower was set for orifice flows of $0.735 \mathrm{acfm}(3,340 \mathrm{fpm})$ for the lances inside of the tunnel test section. On the accompanying video, when bulk smoke was injected near the blower, observations in the tunnel show smoke had exited the lances past mid distance between the pair however there was no wind tunnel cross flow representing the precipitator gas flow. With the wind tunnel fan running and set for $4 \mathrm{ft} / \mathrm{s}$ cross flow, the positive blower lance flow unchanged, observations at the lances show the smoke path had folded with the direction of the cross flow and the distance of injection between lances seen earlier was reduced considerably. 
Flow to the lances was increased to 2.695 acfm $(12,250 \mathrm{fpm})$ exiting the orifices and the wind tunnel cross flow remained at $4 \mathrm{ft} / \mathrm{s}$. On the video smoke exiting the lance orifice had penetrated half of the distance between two lances before folding into the direction of cross flow. Flow to the lances was then increased to $3.675 \mathrm{acfm}(16,704 \mathrm{fpm})$ exiting the orifice and the wind tunnel cross flow remained at $4 \mathrm{ft} / \mathrm{s}$. Smoke penetration had reached about three quarters of the distance between lances before folding into the direction of the cross flow.

\subsection{Precipitator Model Visual and Video}

On the accompanying video the general model arrangement of the unit 2 existing precipitator can be seen. Bulk smoke was injected to show velocity paths and interactions through the system.

Observations along the bottom of the inlet plenum and first field show smoke jetting down below the collector curtains and into the hopper. Smoke was also observed exiting the hopper quickly near the walkway.

Bulk smoke observations with the third field removed show the flow had angled upwards. In the outlet plenum bulk smoke had cleared quickly along the upper section of the collecting field indicating higher velocities. 


\section{DISCUSSION AND RECOMMENDATIONS}

The model study of the existing unit 2 precipitator installation showed velocity distributions through the inlet and outlet ductwork, as shown on Plates 14 to 17,22 and 23, were satisfactory and acceptable. Velocity distributions measured at the outlet of the first, second, third and fourth fields were not within the ICAC flow criteria stating that $85 \%$ and $99 \%$ of the measured velocities shall be less than $115 \%$ and $140 \%$ of the mean velocity. Distributions at the outlet of the first and second fields had $72.1 \%, 94.2 \%$ and $77.1 \%, 99.2 \%$ of the velocities measured less than 1.15 and 1.40 times the mean velocity as shown on Plates 18 and 19. NELS believes that high velocities across the lower collection area of the field, bottom of the opening (BO) and bottom of the collector plates (BP) result in particulate re-entrainment, causing opacity spiking and an overall reduction in collection efficiency. At the outlet of the third field the distribution measured had $83.8 \%$ of the velocities less than 1.15 and slightly below the $85 \%$ limit with $100.0 \%$ of the velocities less than 1.40 times the mean as shown on Plate 20. The distribution measured further downstream at the outlet of the last field was severely skewed high towards the top of the field having $68.8 \%, 77.9 \%$ of the velocities less than 1.15 and 1.40 times the mean velocity as shown on Plate 21 . The existing outlet plenum had no flow devices installed and this type of velocity distribution would only cause increased particle reentrainment with opacity spiking.

Simulation of the (PAC) injection lances using tracer gas has shown the distribution downstream in the middle of the third and last fields was biased high towards the upper section of the collecting fields and cycled high to low across the collecting field width as shown on Plates 24 and 25 . High to low cycles across the width of the third field maybe caused by the internal bracing and velocity distribution upstream and by the close proximity of the gas passages near the lances. In the fourth field this high to low effect had reduced suggesting that some of the tracer gas concentration had shifted gas passages when exiting the third field and before entering the fourth field gas passages. Also the injection point coverage for the lance is short as seen in the full size wind tunnel test. 
The model and wind tunnel testing of the (PAC) lances have shown a possible poor injection of sorbent would occur. With precipitator cross flow, wind tunnel observations indicate more delivery air would be required to carry the sorbent further away from the lance orifices and into the precipitator gas flow. Model study testing has shown that the precipitator inlet flow devices maybe causing the sorbent injection to become biased towards the upper section of the collecting fields.

NELS believes that the existing inlet plenum velocity distribution having high unacceptable velocities along the bottom of the first and second collecting fields is a cause for particulate re-entrainment, opacity spiking and reduced collection efficiency. For the lower precipitator casings and assuming the inlet plenum and turning vanes shown on Plates 5 and 6 were relatively clear of ash buildup the velocity distribution entering the precipitator would be similar to the model however from NELS experience similar vane designs and plenums have shown ash builds up along the plenum floor and in the inside vane radius therefore reducing the horizontal turning ability causing gas flow to angle downward and increasing higher velocities along the bottom of the collection fields and into the hoppers. The existing outlet plenum with no flow devices is a problem area by itself having high velocities along the top section of the last collecting field and again causing particulate reentrainment and opacity spiking.

Modifications to the inlet plenum flow devices to improve the velocity distribution with respect to minimizing high velocities based on NELS experience and to minimize ash build up along the plenum floor upstream of the inlet screen. Investigate the installation of devices between the first and second fields to further reduce particulate re-entrainment. Investigate the modifications to the outlet plenum by installing a variable porosity screen to improve flow distribution and to reduce particulate re-entrainment however these modifications would add some pressure loss to the existing system. It is possible that by using the current model of half of the lower casing the above recommendations can be achieved however the complete precipitator installation may have additional problems with respect to an equal amount of flow and ash loading to the four casings and there maybe areas in the existing system for some pressure loss savings. 
P119.06 - ADA-ES INC, Entergy, Independence Station Unit 2, Toxicon II Carbon Injection

\section{TABLES}


Table 1: List of Drawings

\begin{tabular}{|c|c|c|c|}
\hline Drawing No. & Rev. & Title & Source \\
\hline \multicolumn{4}{|c|}{ Drawings Received by E-mail on February 23, 2006} \\
\hline 27-E19 & & $\begin{array}{l}\text { Inlet \& Outlet Vertical Duct } \\
\text { Turning Vanes }\end{array}$ & CE Walther \\
\hline $27-E 34$ & & $\begin{array}{lll}\text { PPTR Duct Baffles \& } \\
\text { Blanking Plates }\end{array}$ & CE Power Systems \\
\hline $27-E 7$ & & $\begin{array}{l}\text { Inlet \& Outlet Vertical Flue } \\
\text { Elevations \& Sections }\end{array}$ & Pollution Control \\
\hline 28-E13 & & Illegible & Illegible \\
\hline $28-E 3$ & & Illegible & Illegible \\
\hline $2-E 1-1$ & & Illegible & Illegible \\
\hline $04-7007-05-400$ & 1 & Toxecon II Injection Lance & ADA-ES \\
\hline $04-7007-05-401$ & 1 & Toxecon II Manifold Assembly & ADA-ES \\
\hline $04-7007-74-40$ & 1 & ESP Section 2-3 Gen Arrgt & ADA-ES \\
\hline $04-7007-74-41$ & 1 & $\begin{array}{l}\text { Entergy Independence } \\
\text { Toxecon II Standard Drawing } \\
\text { Dimensions }\end{array}$ & ADA-ES \\
\hline \multicolumn{4}{|c|}{ Drawings Received by E-mail on March 9, 2006} \\
\hline $1-\mathrm{e} 17 \ldots$ & & ESP Section PP & Pollution Control \\
\hline $1-e 12 \_1$ & & ESP Plans & Pollution Control \\
\hline 1-e13_1 & & ESP Side Elevation KK & Pollution Control \\
\hline $1-e 14 \_1$ & & ESP Outlet Elevation & Pollution Control \\
\hline $1-e 161$ & & ESP Section NN & Pollution Control \\
\hline $2-E 1$ & & Illegible & Illegible \\
\hline 3-E1_1 & & DE System Installation & Pollution Control \\
\hline \multicolumn{4}{|c|}{ Drawings Received by E-mail on June 26, 2006} \\
\hline $27-E 52$ & & $\begin{array}{l}\text { Field Work to Inlet Vertical } \\
\text { Duct (Add Turning Vanes) }\end{array}$ & CE Power Systems \\
\hline $28-E 13$ & & Inlet Plenum & CE Walther Inc. \\
\hline \multicolumn{4}{|c|}{ Drawings Received by E-mail on June 27, 2006} \\
\hline $28-E 11$ & & $\begin{array}{l}\text { Lower PPTR Inlet Plenum } \\
\text { Turning Vane Design }\end{array}$ & $\begin{array}{l}\text { Pollution Control } \\
\text { Walther }\end{array}$ \\
\hline $28-\mathrm{E} 12$ & & $\begin{array}{l}\text { Upper Inlet Transition Splitter } \\
\text { Vane Design }\end{array}$ & CE Walther Inc. \\
\hline $27-\mathrm{E} 36$ & & $\begin{array}{l}\text { Field Work @ Inlet Vertical } \\
\text { Duct Turning Vanes }\end{array}$ & CE Walther Inc. \\
\hline $27-E 37$ & & $\begin{array}{l}\text { Field Work to Outlet Duct } \\
\text { Turning Vanes }\end{array}$ & CE Power Systems \\
\hline \multicolumn{4}{|c|}{ Drawings Received by E-mail on June 28,2006} \\
\hline $28-\mathrm{E} 14$ & B & $\begin{array}{l}\text { Lower PPTR Inlet Plenum } \\
\text { Turning Vane Details }\end{array}$ & $\begin{array}{l}\text { Pollution Control } \\
\text { Walther }\end{array}$ \\
\hline $28-\mathrm{E} 15$ & & $\begin{array}{l}\text { Lower PPTR Inlet Transition } \\
\text { Splitter Vane Design }\end{array}$ & CE Walther Inc. \\
\hline
\end{tabular}


Table 1: Continued

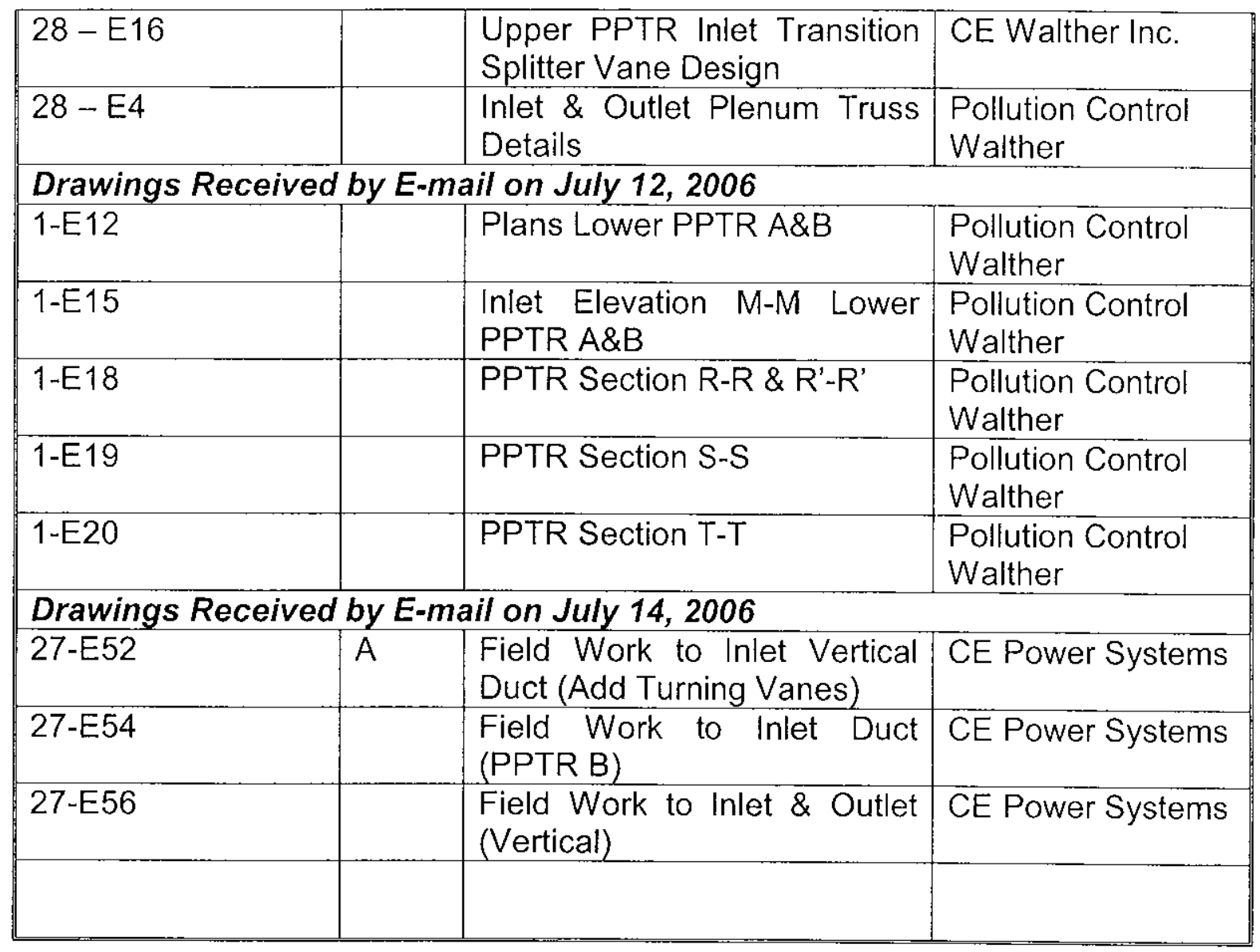


Table 2: Full Size Installation - Design and Gas Flow Parameters

\begin{tabular}{|l|l|}
\hline \multicolumn{2}{|c|}{ SYSTEM REQUIREMENTS } \\
\hline ESP Construction: Unit 2 & 4 \\
No. of Precipitator Casings & $2-$ Plenum \\
No. of Inlets per Casing & $1-$ Perforated Plate \\
No. of Screens per Inlet Plenum & $2-$ Plenum \\
No. of Outlets per Casing & None \\
No. of Screens per Outlet Nozzle & 2 \\
No. of Chambers per Casing & 4 \\
No. of Fields per Chamber & 43 \\
No. of Gas Passages per Chamber & $1^{\prime}-0^{\prime \prime}$ \\
Collecting Plate Spacing & $48^{\prime}-0^{\prime \prime}$ \\
Collecting Plate Height & $15^{\prime}-2^{\prime \prime}$ \\
Collecting Plate Depth & \\
Gas Flow Parameters & $4 \mathrm{ft} / \mathrm{s}^{\prime}$ \\
\hline Gas Passage Velocity & $482169 \mathrm{acfm}$ \\
Estimated Chamber Flow (44' $\left.\times 45^{\prime}-8^{\prime \prime}\right)$ & $299^{\circ} \mathrm{F}$ \\
Flue Gas Temperature & $0.0522 \mathrm{lb} / \mathrm{ft}^{3}$ \\
\hline Flue Gas Density &
\end{tabular}


P119.06 - ADA-ES INC, Entergy, Independence Station Unit 2, Toxicon II Carbon Injection

Table 3: Comparison between Full Size and Model

\begin{tabular}{|c|c|c|}
\hline Criteria & Full Size Installation & Model \\
\hline \multicolumn{3}{|l|}{ Construction: } \\
\hline Geometric Linear Scale & $1: 1$ & $1: 12$ \\
\hline \multicolumn{3}{|l|}{ Gas Flow: } \\
\hline Chamber Gas Flow (acfm) & 482,169 & 3348 \\
\hline Duct Velocity (T8)(fpm) & 3282 & 3282 \\
\hline Gas Temperature $\left({ }^{\circ} \mathrm{F}\right)$ & 299 & 70 \\
\hline Gas Density $\left(\mathrm{lbs} / \mathrm{ft}^{3}\right)$ & 0.0522 & 0.0747 \\
\hline
\end{tabular}


Table 4: Summary of Flow Scaling Parameters

\section{Reynolds Number, Re:}

Where: $V=$ velocity

$$
\operatorname{Re}=\frac{V D}{v}
$$

$D=$ characteristic length

$v=$ kinematic viscosity

Full Size Installation

$$
\begin{aligned}
V & =4.04 \mathrm{ft} / \mathrm{s} \text { (Location - inlet flange) } \\
D & =44.59 \mathrm{feet} \text { (equivalent duct diameter) } \\
\nu & =3.05 \times 10^{-4} \mathrm{ft}^{2} / \mathrm{s} \text { at } 299^{\circ} \mathrm{F} \\
\operatorname{Re}_{\text {Full Size }} & =\frac{4.04 \times 44.59}{3.05 \times 10^{-4}}=5.91 \times 10^{5}
\end{aligned}
$$

Model

$$
\begin{aligned}
& V=4.04 \mathrm{ft} / \mathrm{s} \text { (Location - inlet flange) } \\
& D=3.72 \text { feet (equivalent duct diameter) } \\
& v=1.64 \times 10^{-4} \mathrm{ft}^{2} / \mathrm{s} \text { at } 70^{\circ} \mathrm{F}
\end{aligned}
$$

$$
\operatorname{Re}_{\text {Model }}=\frac{4.04 \times 3.72}{1.64 \times 10^{-4}}=9.16 \times 10^{4}
$$

$$
\operatorname{Re}_{\text {Model }}=\frac{8.0 \times 2.84}{1.64 \times 10^{-4}}=1.38 \times 10^{5}
$$

The model Reynolds Number is above the minimum required value $(20000)$ for fully turbulent flow.

\section{Conversion of Model to Full Size Pressures}

$$
P_{\text {Full Size }}=P_{\text {Model }} x\left(\frac{Q_{\text {Full Size }}}{Q_{\text {Model }}}\right)^{2} x \frac{\rho_{\text {Gas Ful Size }}}{\rho_{\text {Air }} \text { Mollel }}
$$


Table 5: Powdered Activated Carbon (PAC) Injection Scaling Theory

The momentum ratio of the 'flue gas flow' to the 'PAC injection flow' should be the same when comparing the full size prototype to the model.

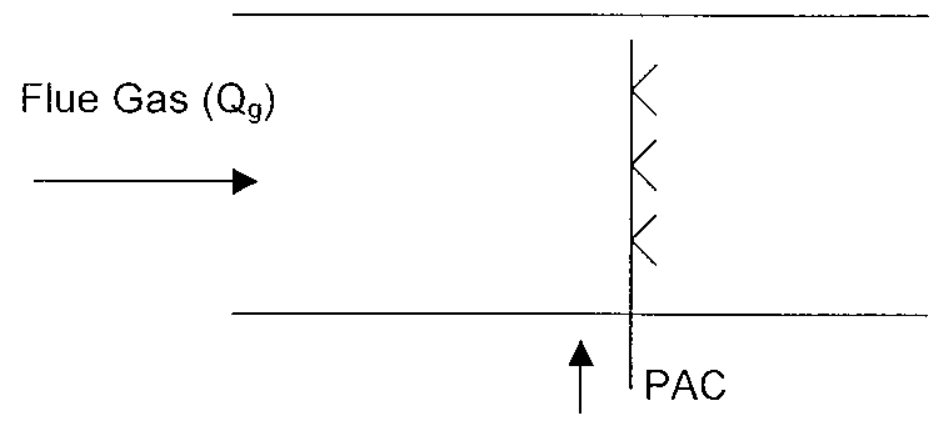

(16) Pipes

$$
\begin{aligned}
& \text { Flue } \operatorname{Gas}\left(Q_{g}\right) \\
& Q_{g}=482,169 \mathrm{acfm} \\
& T_{g}=299^{\circ} \mathrm{F} \\
& \rho_{g}=0.0522 \mathrm{lb} / \mathrm{ft}^{3}
\end{aligned}
$$

$\underline{\operatorname{PAC}\left(Q_{N}\right)}$

$Q_{N}=154 \mathrm{acfm}$ (77 acfm per train)

$\mathrm{m}_{\text {air }}=653 \mathrm{lb} / \mathrm{hr}$

$\mathrm{T}_{\mathrm{N}}=100^{\circ} \mathrm{F}, \rho_{\text {air }}=0.0707 \mathrm{lb} / \mathrm{ft}^{3}$

$\rho_{N}=0.0863 \mathrm{lb} / \mathrm{ft}^{3}$

Carbon $=144 \mathrm{lb} / \mathrm{hr}$

\section{Effective Density}

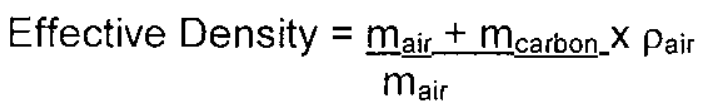

Effective Density $=\underline{653 \mathrm{lb} / \mathrm{hr}+144 \mathrm{lb} / \mathrm{hr}} \times 0.0707 \mathrm{lb} / \mathrm{ft}^{3}$

$$
653 \mathrm{lb} / \mathrm{hr}
$$

Effective Density $=0.0863 \mathrm{lb} / \mathrm{ft}^{3}$ 
Table 5: Continued

$$
\begin{aligned}
& \text { Prototype } \quad \text { Model } \\
& \frac{m_{N}{ }^{*} v_{N}}{m_{g}{ }^{*} v_{g}}=\frac{m_{N}{ }^{*} v_{N}}{m_{g}{ }^{*} v_{g}{ }^{\prime}} \\
& \frac{\rho_{N}{ }^{*} A_{N}{ }^{*} V_{N}{ }^{2}}{\rho_{g}{ }^{*} A_{g}{ }^{*} V_{g}{ }^{2}}=\frac{\rho_{N}{ }^{*} A_{N}{ }^{*} V_{N}{ }^{2}}{\rho_{g}{ }^{*} A_{g}{ }^{*}{ }^{*} V_{g}{ }^{2}} \\
& \frac{A_{N}{ }^{\prime}}{A_{N}}=\frac{A_{g}{ }^{\prime}}{A_{g}} \quad(\text { similar })(1 / 12)^{2} \\
& v_{\mathrm{g}}=v_{\mathrm{g}}{ }^{\prime} \quad \text { (precondition) } \\
& \rho_{\mathrm{g}}{ }^{\prime} \quad=\rho_{\mathrm{N}}{ }^{\prime} \quad \text { (tracer gas) }
\end{aligned}
$$

Therefore $\quad V_{N}{ }^{\prime}=\left(\rho_{N} / \rho_{g}\right)^{1 / 2 *} V_{N}$

$$
Q_{N}{ }^{\prime}=V_{N}{ }^{*} A_{N}{ }^{\prime}=\left(\rho_{N} / \rho_{g}\right)^{1 / 2 *} Q_{N}{ }^{*}\left(A_{N}{ }^{\prime} / A_{N}\right)
$$

Test Condition on the Model

$$
\begin{aligned}
& Q_{N}{ }^{\prime}=(0.0863 / 0.0522)^{1 / 2 *} 154 *(1 / 12)^{2} \\
& Q_{N}{ }^{\prime}=1.38 \mathrm{acfm}
\end{aligned}
$$

Therefore:

$$
Q_{N}{ }^{\prime}=0.000421 \times Q_{g}{ }^{\prime}
$$

The factor 0.000412 when multiplied by the $100 \%$ model flow rate will give the PAC + Air, flow rate. This value can be applied when operating the model at reduced flow rates.

\section{For Example:}

Max Model Flow Rate (typical) $=3,348$ acfm

$Q_{N}{ }^{\prime}=0.000412 \times Q_{g}{ }^{\prime}$

$Q_{N}{ }^{\prime}=0.000412 \times 3,348$

Therefore PAC Simulation Flow Set at $1.38 \mathrm{acfm}(82.8 \mathrm{cfh})$ 
Table 6: Description of Test Locations

\begin{tabular}{|c|c|}
\hline Test Location & Description \\
\hline \multicolumn{2}{|r|}{ Inlet Ducting } \\
\hline T1L \& T1R & Inlet Duct to Precipitator \\
\hline$T 2 L \& T 2 R$ & Inlet Duct to Precipitator \\
\hline \multicolumn{2}{|r|}{ Precipitator } \\
\hline T3 & Outlet of First Field \\
\hline T4 & Outlet of Second Field \\
\hline T5 & Outlet of Third Field \\
\hline T6 & Outlet of Last Field \\
\hline \multicolumn{2}{|r|}{ Outlet Ducting } \\
\hline T7 & Outlet Duct from Precipitator \\
\hline $\mathrm{T}^{*}$ & Outlet Duct from Precipitator ( Model Volume Control) \\
\hline
\end{tabular}

\section{Notes:}

*Model Volume Control

Refer to Plates 1 and 2 for test locations 
P119.06 - ADA-ES INC, Entergy, Independence Station Unit 2, Toxicon II Carbon Injection

Table 7: Precipitator Gas Passage Test Locations

\begin{tabular}{|c|l|}
\hline $\begin{array}{c}\text { Precipitator Test Location } \\
\text { (within each gas passage) }\end{array}$ & Description \\
\hline TP & Top of Collector Plates \\
\hline TO & Top of Precipitator Projected Opening \\
\hline 1 to 16 & $\begin{array}{l}17 \text { Evenly Spaced Measurements over the Height } \\
\text { of the Precipitator Projected Opening }\end{array}$ \\
\hline BO & Bottom of Precipitator Projected Opening \\
\hline BP & Bottom of Collector Plates \\
\hline
\end{tabular}

Notes:

Refer to Plates 1 and 2 for test locations 
P119.06 - ADA-ES INC, Entergy, Independence Station Unit 2, Toxicon II Carbon Injection

Table 8: Summary of Volume Flows

Precipitator Inlet Ducts T1L \& T1R to Outlet Duct T8

Existing Configuration

\begin{tabular}{|c|c|c|c|c|c|}
\hline Test Location & $\begin{array}{c}\text { Full Size } \\
\text { Area } \\
\left(\mathrm{ft}^{2} \mathbf{)}\right.\end{array}$ & $\begin{array}{c}\text { RMS } \\
\text { Deviation } \\
(\mathbf{\%})\end{array}$ & $\begin{array}{c}\text { Mean } \\
\text { Velocity } \\
(\mathbf{f p m})\end{array}$ & $\begin{array}{c}\text { Volume } \\
\text { Flow } \\
(\mathbf{a c f m})\end{array}$ & $\begin{array}{c}\text { Ref. } \\
\text { Plates }\end{array}$ \\
\hline $\mathrm{T} 1 \mathrm{~L}$ & 50.73 & 6.2 & 4614 & 234068 & 14 \\
\hline $\mathrm{T} 1 \mathrm{R}$ & 50.73 & 4.9 & 4599 & 233307 & 15 \\
\hline $\mathrm{T} 2 \mathrm{~L}$ & 50.73 & 10.1 & 4416 & 224024 & 16 \\
\hline $\mathrm{T} 2 \mathrm{R}$ & 50.73 & 9.2 & 4551 & 230872 & 17 \\
\hline $\mathrm{T} 7$ & 146.88 & 13.2 & 3361 & 493664 & 22 \\
\hline $\mathrm{T}^{*}$ & 146.88 & 6.1 & 3257 & 478388 & 23 \\
\hline & & & & & \\
\hline & & & & & \\
\hline
\end{tabular}

Notes:

\section{Existing Configuration}

*Model Volume Control

Refer to Plates 1 and 2 for test locations 
Table 9: Static and Total Pressures Determined from Model Precipitator Inlet Ducts T1L \& T1R to Outlet Duct T8 Existing Configuration

\begin{tabular}{|c|c|c|c|c|c|c|c|c|c|c|}
\hline \multirow{3}{*}{$\begin{array}{c}\text { Test } \\
\text { Location }\end{array}$} & \multicolumn{3}{|c|}{ Model Data } & \multicolumn{2}{|c|}{ Model $\times 0.706$} & \multirow{3}{*}{$\begin{array}{c}\text { Test } \\
\text { Increment } \\
\text { Location }\end{array}$} & \multicolumn{2}{|c|}{ Model $\triangle \mathrm{P}$} & \multicolumn{2}{|c|}{ Prototype $\Delta \mathrm{P}$} \\
\hline & Static & Velocity & Total & Static & Total & & Static & Total & Static & Total \\
\hline & \multicolumn{3}{|c|}{ (Inches of Water) } & \multicolumn{2}{|c|}{$\begin{array}{c}\text { (Inches of } \\
\text { Water) }\end{array}$} & & \multicolumn{2}{|c|}{$\begin{array}{c}\text { (Inches of } \\
\text { Water) }\end{array}$} & \multicolumn{2}{|c|}{$\begin{array}{c}\text { (Inches of } \\
\text { Water) }\end{array}$} \\
\hline T1L & -2.23 & 1.327 & -0.90 & -1.57 & -0.64 & $\mathrm{~T} 1 \mathrm{~L}-\mathrm{T} 2 \mathrm{~L}$ & 0.43 & 0.54 & 0.30 & 0.38 \\
\hline $\mathrm{T} 2 \mathrm{~L}$ & -2.66 & 1.215 & -1.44 & -1.87 & -1.02 & $\mathrm{~T} 2 \mathrm{~L}-\mathrm{T} 3$ & 0.21 & 1.43 & 0.16 & 1.01 \\
\hline $\mathrm{T} 1 \mathrm{R}$ & -2.23 & 1.318 & -0.91 & -1.57 & -0.64 & $\mathrm{~T} 1 \mathrm{R}-\mathrm{T} 2 \mathrm{R}$ & 0.50 & 0.53 & 0.36 & 0.38 \\
\hline $\mathrm{T} 2 \mathrm{R}$ & -2.73 & 1.291 & -1.44 & -1.93 & -1.02 & $\mathrm{~T} 2 \mathrm{R}-\mathrm{T} 3$ & 0.14 & 1.43 & 0.10 & 1.01 \\
\hline T3 & -2.87 & & -2.87 & -2.03 & -2.03 & $\mathrm{~T} 3-\mathrm{T} 6$ & 0.02 & 0.02 & 0.01 & 0.01 \\
\hline T6 & -2.89 & & -2.89 & -2.04 & -2.04 & $\mathrm{~T} 6-\mathrm{T} 7$ & 1.08 & 0.38 & 0.76 & 0.27 \\
\hline T7 & -3.97 & 0.704 & -3.27 & -2.80 & -2.31 & $\mathrm{~T} 7-\mathrm{T} 8$ & 0.15 & 0.19 & 0.11 & 0.13 \\
\hline \multirow[t]{4}{*}{ T8 } & -4.12 & 0.661 & -3.46 & -2.91 & -2.44 & & & & & \\
\hline & & & & & & $\mathrm{T} 1(\mathrm{~L} \& \mathrm{R})-\mathrm{T} 3$ & 0.64 & 1.97 & 0.46 & 1.39 \\
\hline & & & & & & $\mathrm{T} 6-\mathrm{T} 8$ & 1.23 & 0.57 & 0.87 & 0.40 \\
\hline & & & & & & $T 1(L \& R)-T 8$ & 1.89 & 2.56 & 1.34 & 1.80 \\
\hline & & & & & & & & & & \\
\hline & & & & & & & & & & \\
\hline
\end{tabular}

\section{Notes:}

\section{Existing Configuration}

Model flow representative of $478,388 \mathrm{acfm}$ at $62.0^{\circ} \mathrm{F}\left(\rho=0.0751 \mathrm{lb} / \mathrm{ft}^{3}\right)$

Prototype flow $=482,169 \mathrm{acfm}$ at $299^{\circ} \mathrm{F}\left(\rho=0.0522 \mathrm{lb} / \mathrm{ft}^{3}\right)$

Prototype $\Delta \mathrm{P}=$ Model $\Delta \mathrm{P} \times\left(\frac{\text { Prototype Flow }}{\text { Model Representative Flow }}\right)^{2} \times\left(\frac{\text { Prototype Gas Density }}{\text { Model Air Density }}\right)$

i.e. $\Delta P_{p}=\Delta P_{m} \times\left(\frac{482169}{478388}\right)^{2} \times\left(\frac{0.0522}{0.0751}\right)$

or $\Delta \mathrm{P}_{\text {Full Size }}=\Delta \mathrm{P}_{\text {Model }} \times 0.706$ (Correction Factor) 
Table 10: Summary of Precipitator Velocity \& CO Injection Distributions

\begin{tabular}{|c|c|c|c|c|c|c|}
\hline Test Location & $\begin{array}{c}\text { RMS Deviation } \\
\% \text { of Mean }\end{array}$ & \multicolumn{2}{|c|}{$\begin{array}{c}\% \text { Below } \\
115 \%\end{array}$} & \multicolumn{2}{|c|}{$\begin{array}{c}\% \text { Below } \\
140 \%\end{array}$} & $\begin{array}{c}\text { Ref. } \\
\text { Plates }\end{array}$ \\
\hline \multicolumn{7}{|c|}{ Existing Configuration Velocity Distributions } \\
\hline T3 & 25.5 & \multicolumn{2}{|c|}{72.1} & \multicolumn{2}{|c|}{94.2} & 18 \\
\hline T4 & 21.0 & \multicolumn{2}{|c|}{77.1} & \multicolumn{2}{|c|}{99.2} & 19 \\
\hline T5 & 17.9 & \multicolumn{2}{|c|}{83.8} & \multicolumn{2}{|c|}{100.0} & 20 \\
\hline T6 & 43.3 & \multicolumn{2}{|c|}{68.8} & \multicolumn{2}{|c|}{77.9} & 21 \\
\hline \multicolumn{7}{|c|}{ Existing Configuration (PAC) Injection Distributions } \\
\hline Test Location & $\begin{array}{c}\text { RMS Deviation } \\
\% \text { of Mean }\end{array}$ & $\begin{array}{l}\text { Mean } \\
\text { PPM }\end{array}$ & $\begin{array}{l}\%< \\
100\end{array}$ & $\begin{array}{c}\% \\
100-200\end{array}$ & $\begin{array}{l}\%> \\
200\end{array}$ & $\begin{array}{c}\text { Ref. } \\
\text { Plates }\end{array}$ \\
\hline T5A (Mid Field) & 111.5 & 156.4 & 64.4 & 17.4 & 18.2 & 24 \\
\hline T6A (Mid Field) & 68.8 & 80.0 & 50.4 & 38.8 & 10.7 & 25 \\
\hline
\end{tabular}

Notes:

Refer to Plates 1 and 2 for test locations 
P119.06 - ADA-ES INC, Entergy, Independence Station Unit 2, Toxicon II Carbon Injection

\section{PLATES}



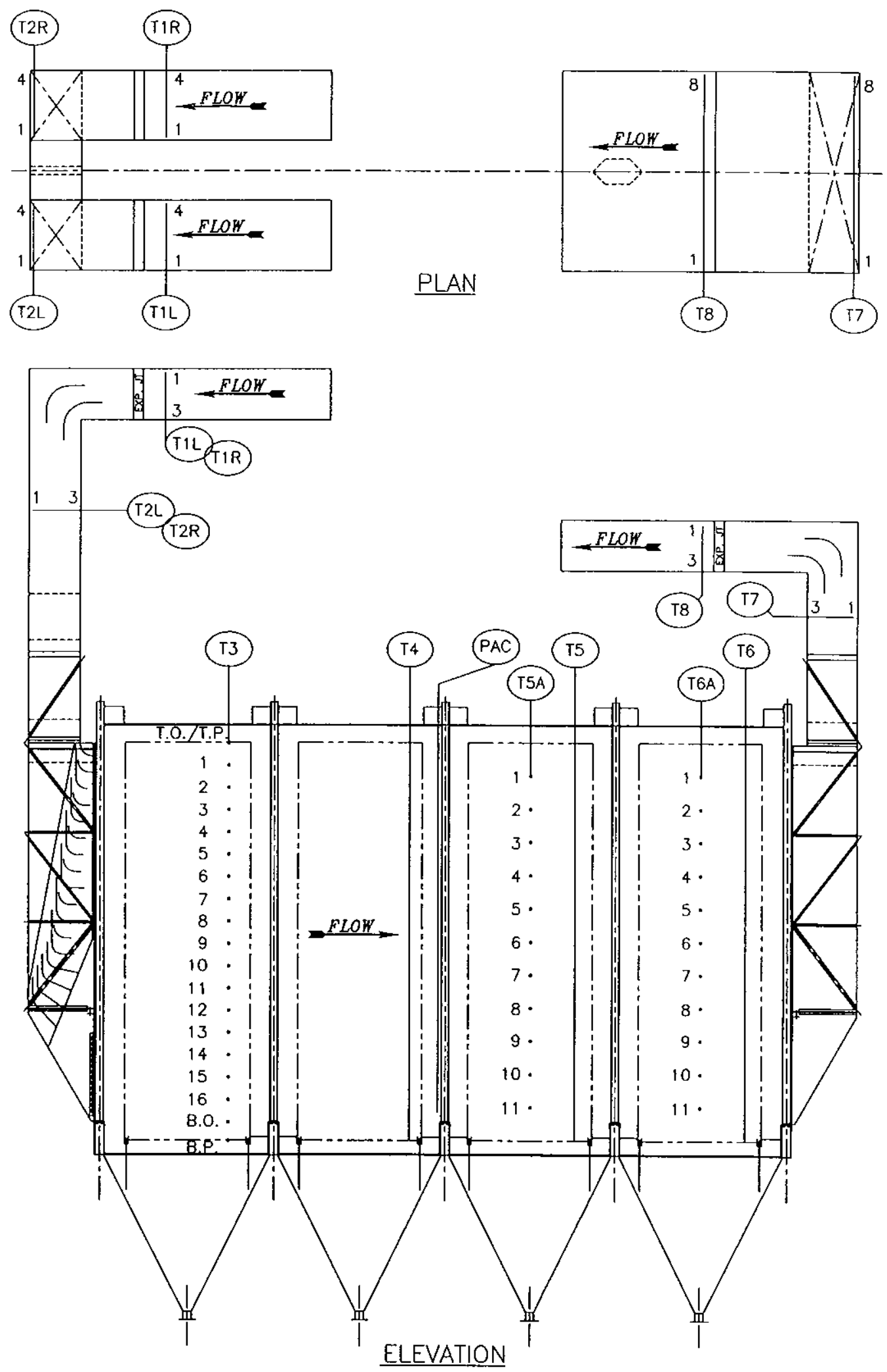

\begin{tabular}{|c|c|c|c|}
\hline & $\begin{array}{l}\text { ADA - ES INC } \\
\text { LITTLETON, COLORADO }\end{array}$ & DATE & DEC 2006 \\
\hline & \multirow{2}{*}{$\begin{array}{c}\text { ENTERGY, INDEPENDANCE STATION UNIT } 2 \text { ESP } \\
\text { TOXICON IIPROJECT, CARBON INJECTION } \\
\text { AIR FLOW MODEL STUDY }\end{array}$} & $D R . B Y$ & $D G$ \\
\hline & & $\begin{array}{l}\text { MODEL } \\
\text { SCALE }\end{array}$ & $1 / 12$ \\
\hline NCS INC. & \multirow{3}{*}{$\begin{array}{c}\text { GENERAL MODEL ARRNGEMENT } \\
\text { AND TEST LOCATIONS } \\
\text { PLAN AND ELEVATION }\end{array}$} & \multirow{3}{*}{\multicolumn{2}{|c|}{ PLATE No. }} \\
\hline$P 119.06$ & & & \\
\hline P11906-PL1 & & & \\
\hline
\end{tabular}




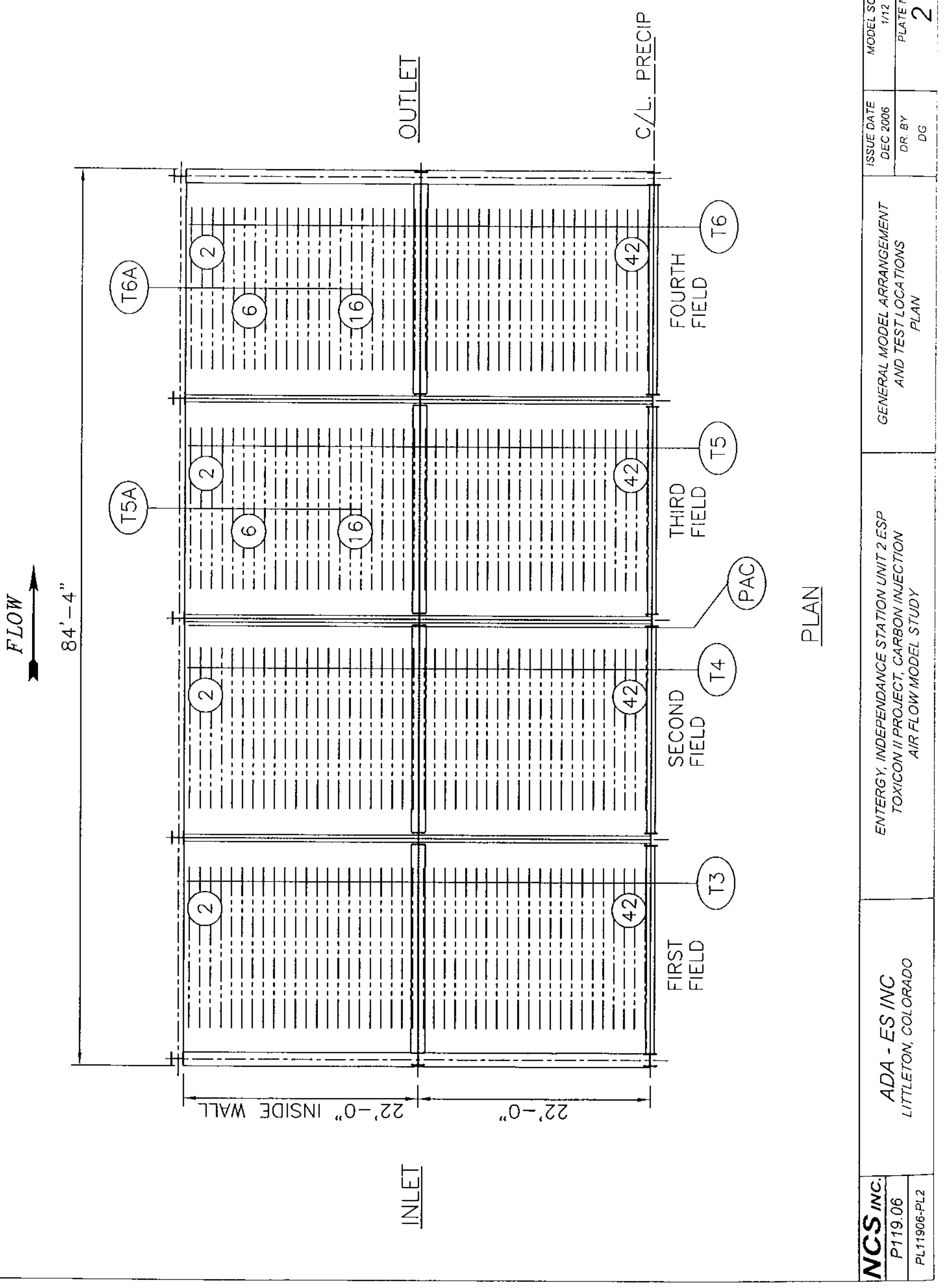




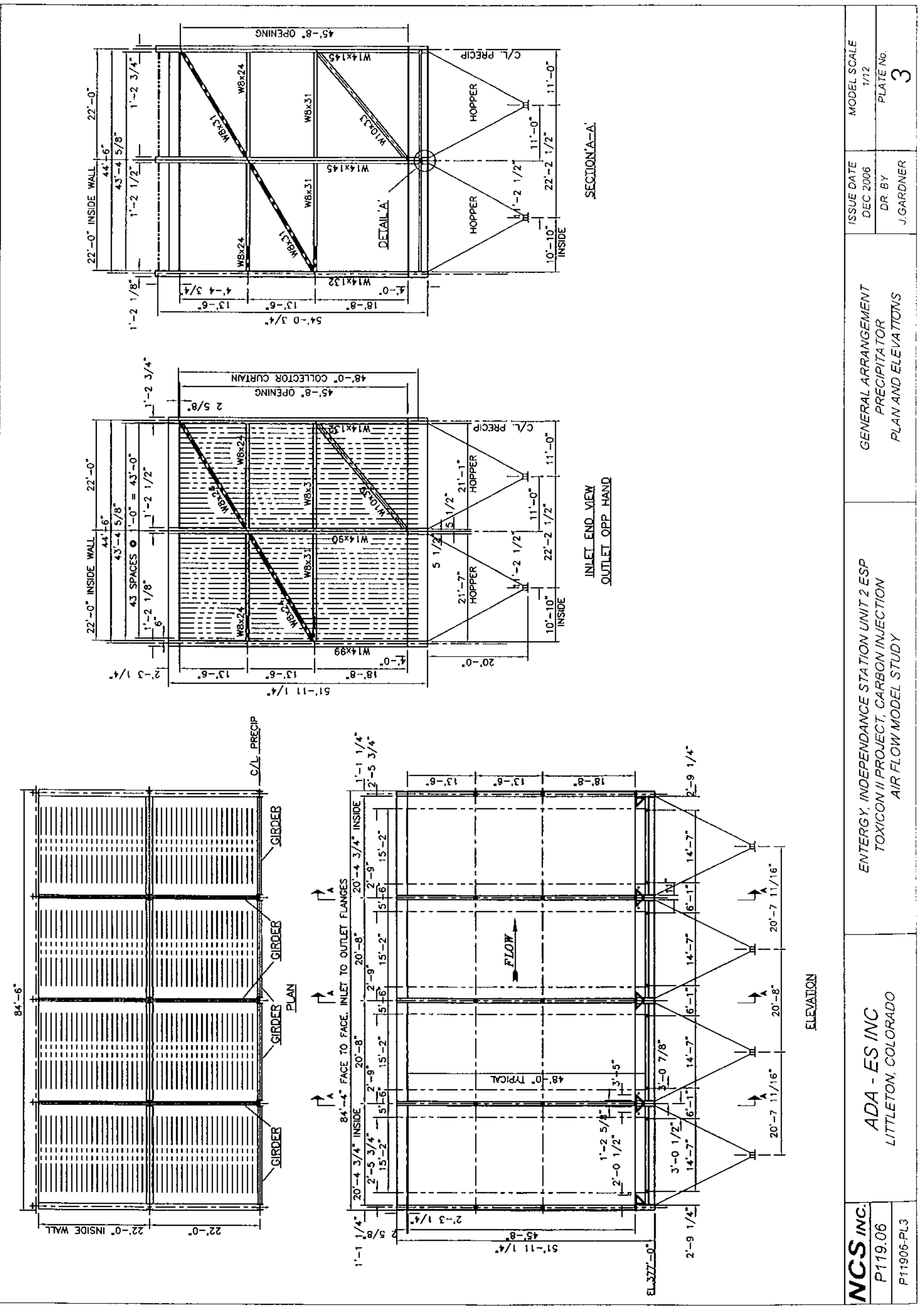




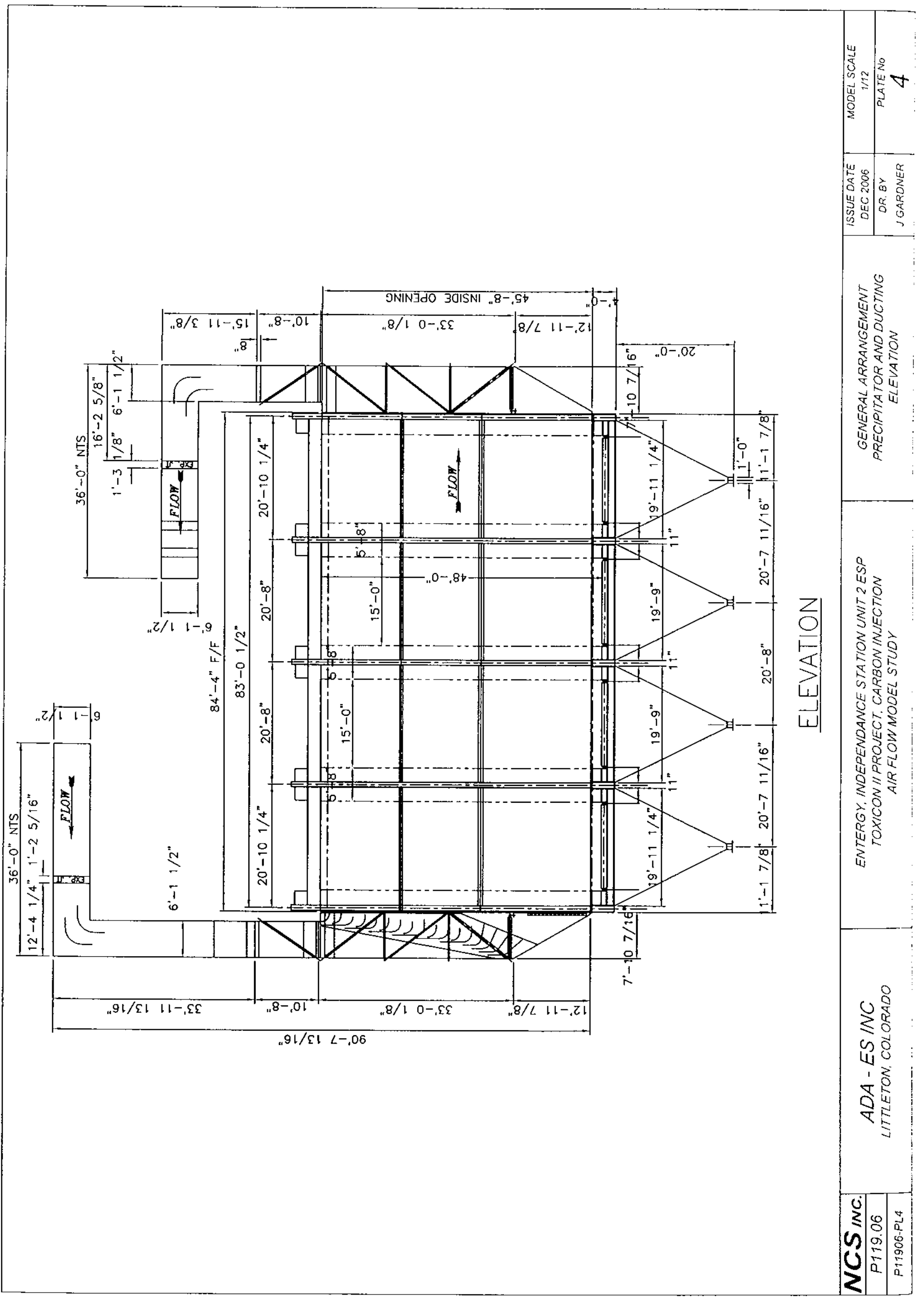




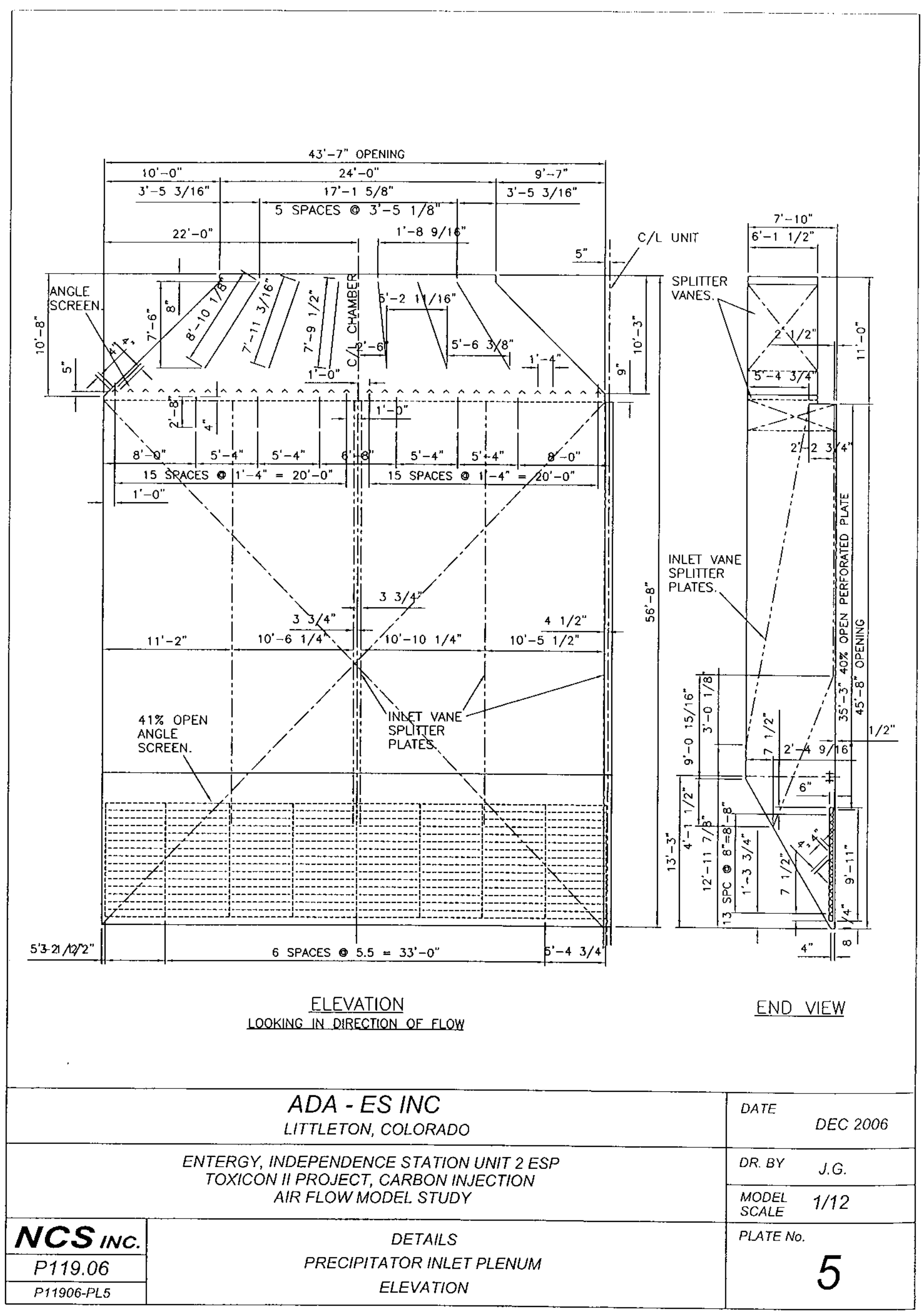




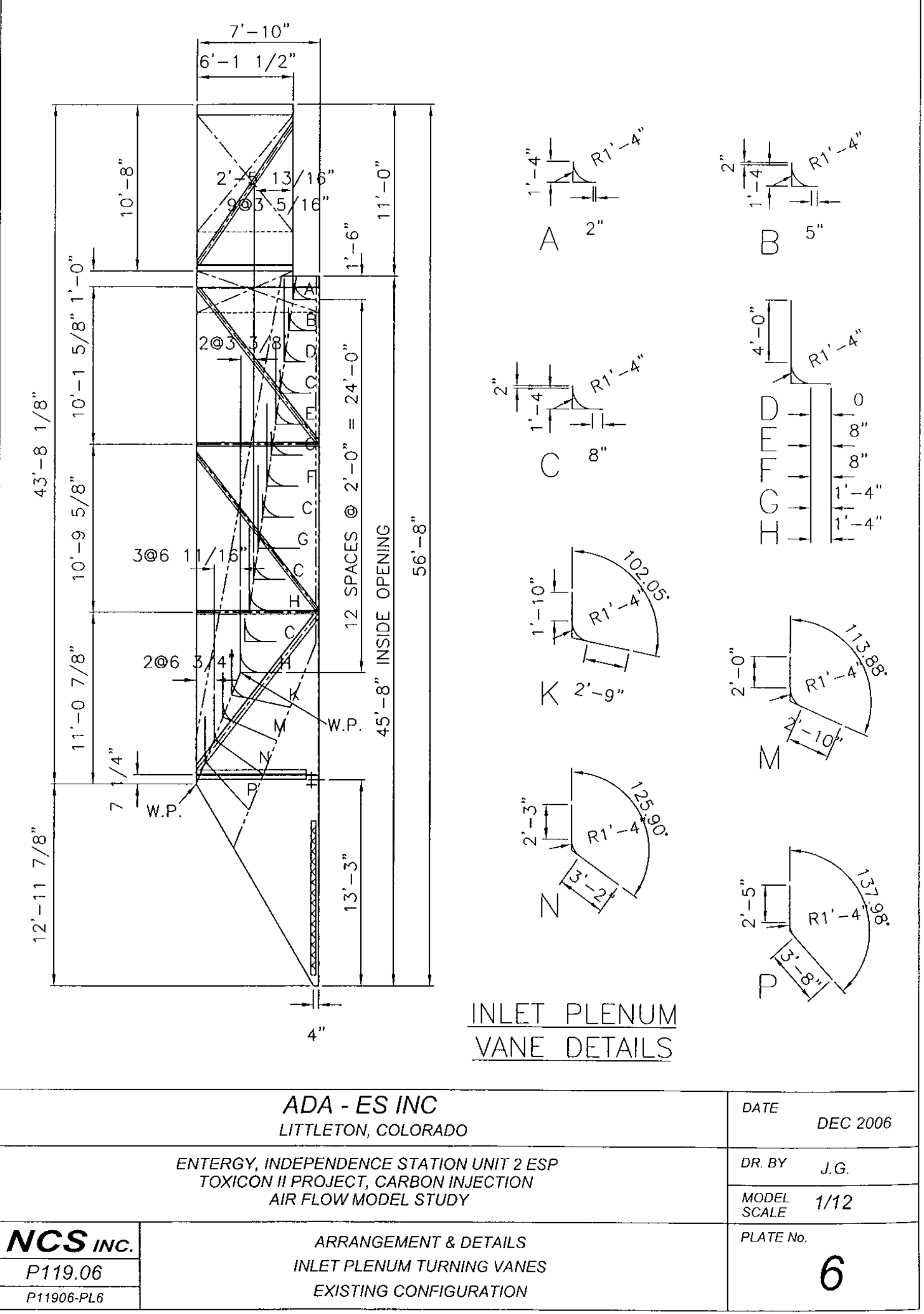




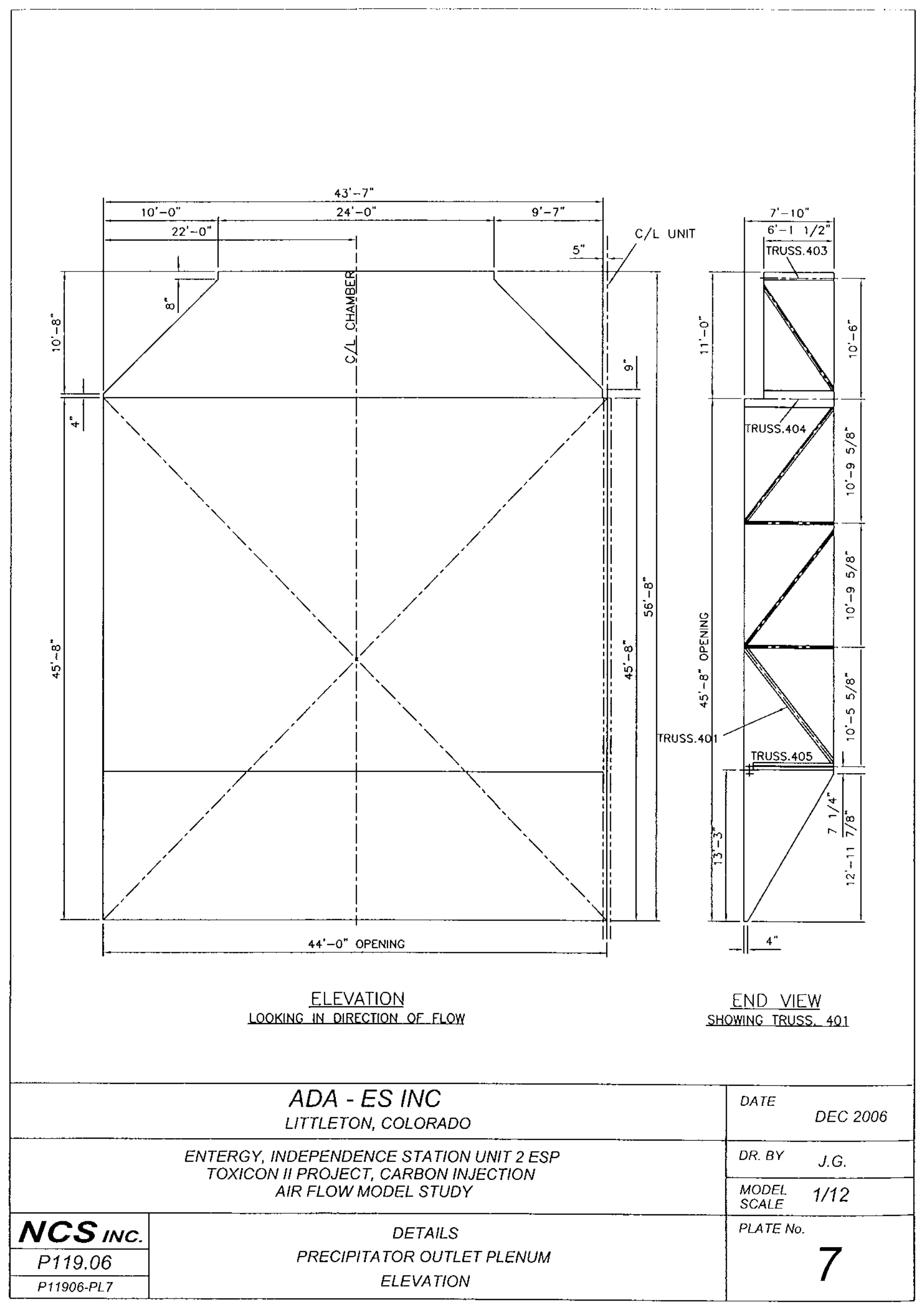



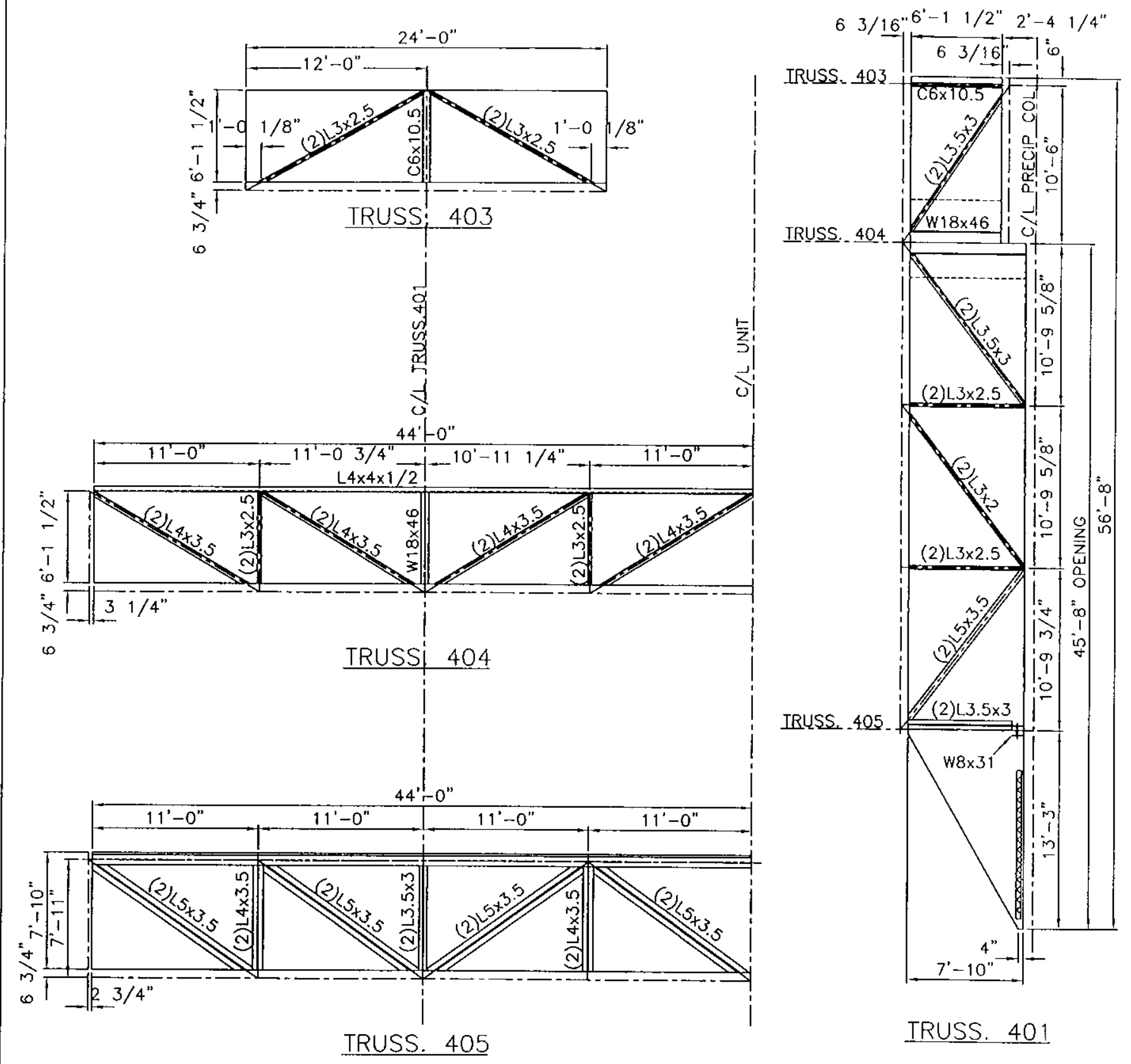

IRUSS. 401

\section{INLET TRUSS AS SHOWN \\ OUTLET TRUSS OPP HAND}

\begin{tabular}{|c|c|c|c|}
\hline & $\begin{array}{l}\text { ADA - ES INC } \\
\text { LITTLETON, COLORADO }\end{array}$ & DATE & DEC 2006 \\
\hline \multirow{2}{*}{\multicolumn{2}{|c|}{$\begin{array}{c}\text { ENTERGY, INDEPENDENCE STATION UNIT } 2 \text { ESP } \\
\text { TOXICONIPROJECT, CARBONINJECTION } \\
\text { AIR FLOW MODEL STUDY }\end{array}$}} & $D R, B Y$ & $J . G$ \\
\hline & & $\begin{array}{l}\text { MODEL } \\
\text { SCALEE }\end{array}$ & $1 / 12$ \\
\hline NCS INC. & \multirow{3}{*}{$\begin{array}{c}\text { DETAILS } \\
\text { INLET \& OUTLET PLENUMS } \\
\text { TRUSS DETAILS }\end{array}$} & PLATEN & \\
\hline$P 119.06$ & & \multirow{2}{*}{\multicolumn{2}{|c|}{8}} \\
\hline P11906-PL8 & & & \\
\hline
\end{tabular}




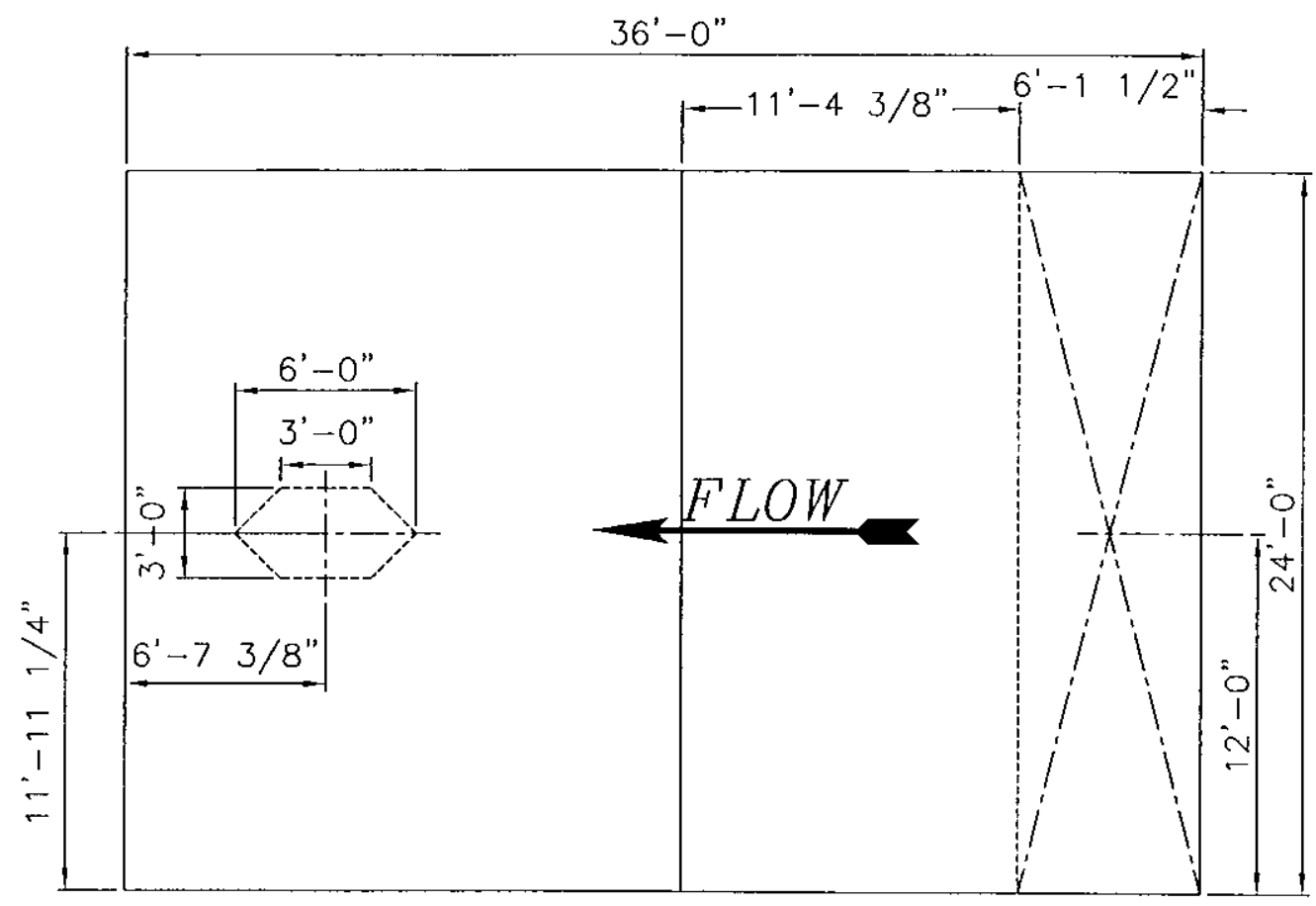

PLAN

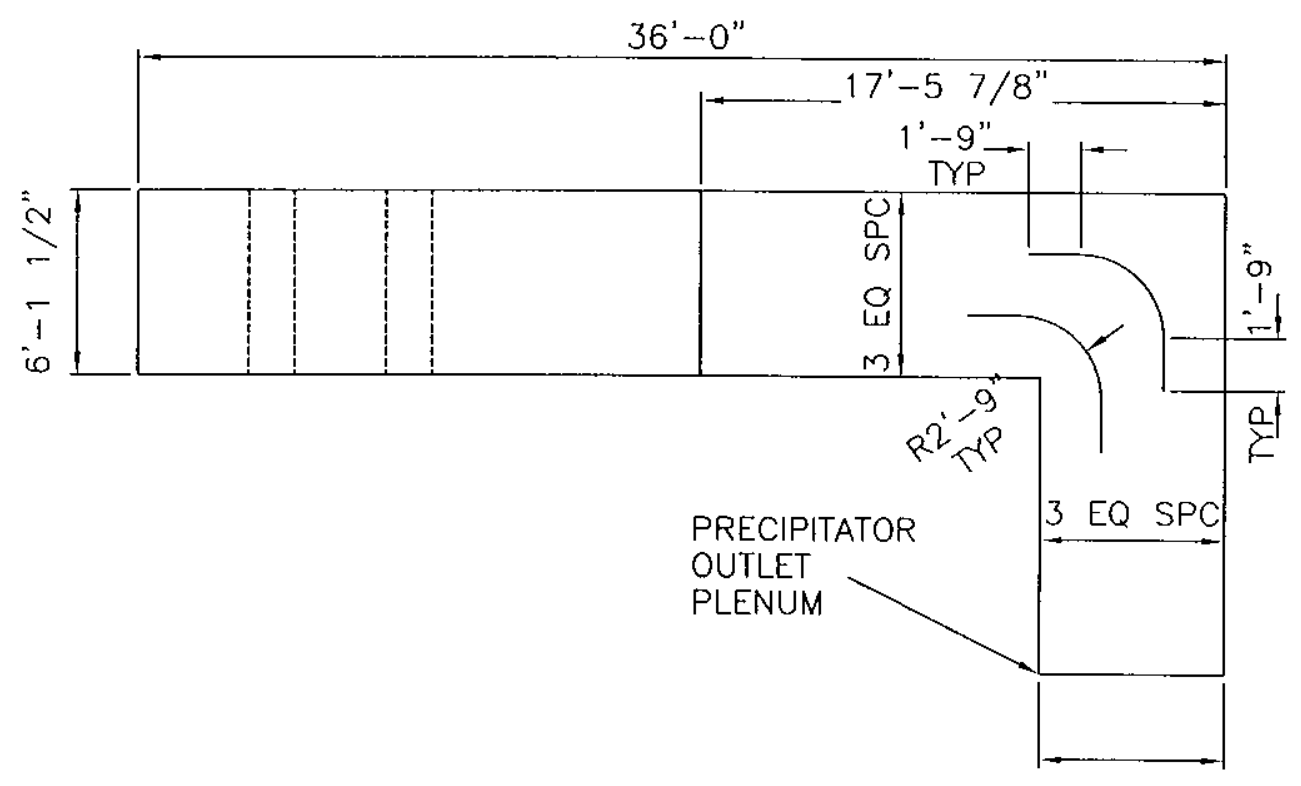

ELEVATION

$6^{\prime}-1 \quad 1 / 2 "$

\begin{tabular}{|c|c|c|c|}
\hline & $\begin{array}{l}\text { ADA - ES INC } \\
\text { LITTLETON, COLORADO }\end{array}$ & DATE & DEC 2006 \\
\hline \multirow{2}{*}{\multicolumn{2}{|c|}{$\begin{array}{c}\text { ENTERGY, INDEPENDENCE STATION UNIT } 2 \\
\text { TOXICON IIPROJECT, CARBON INJECTION } \\
\text { AIR FLOW MODEL STUDY }\end{array}$}} & $D R . B Y$ & J.G. \\
\hline & & $\begin{array}{l}\text { MODEL } \\
\text { SCALE }\end{array}$ & $1 / 12$ \\
\hline NCS INC. & \multirow{3}{*}{$\begin{array}{c}\text { DETAILS } \\
\text { PRECIPITATOR OUTLET DUCT } \\
\text { AND TURNING VANES }\end{array}$} & \multirow{3}{*}{\multicolumn{2}{|c|}{ PLATE No. }} \\
\hline$P 119.06$ & & & \\
\hline P11906-PL10 & & & \\
\hline
\end{tabular}




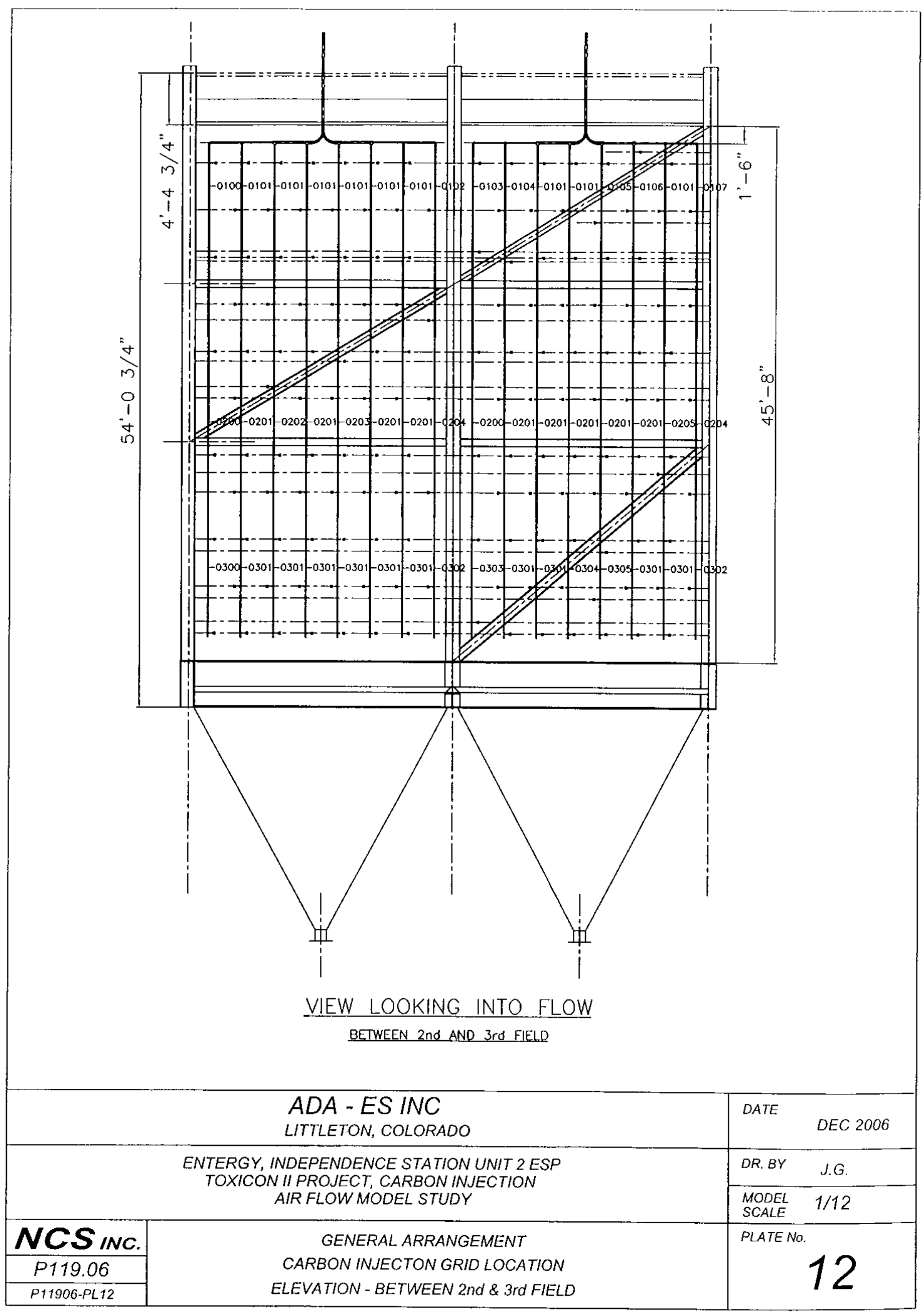




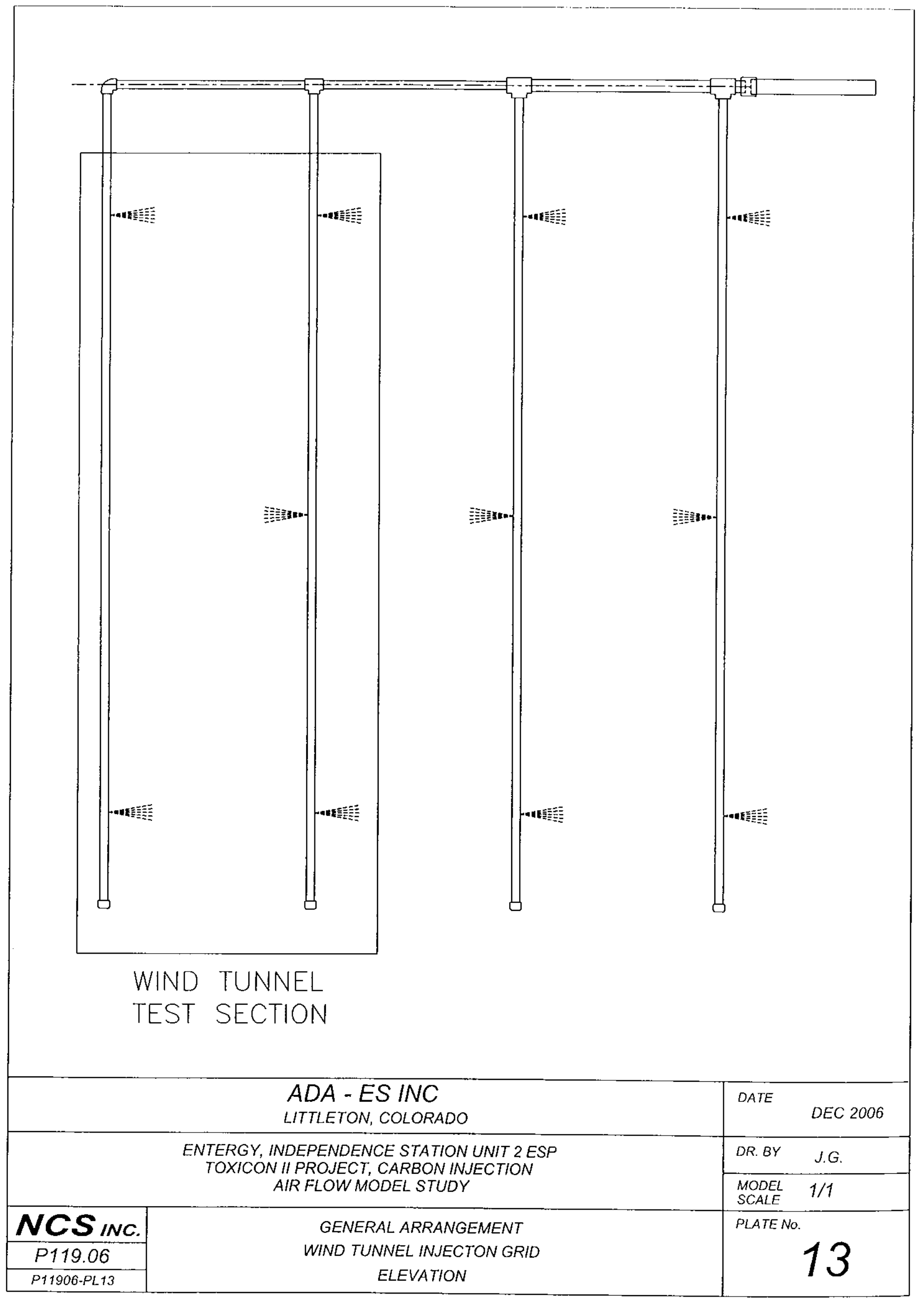




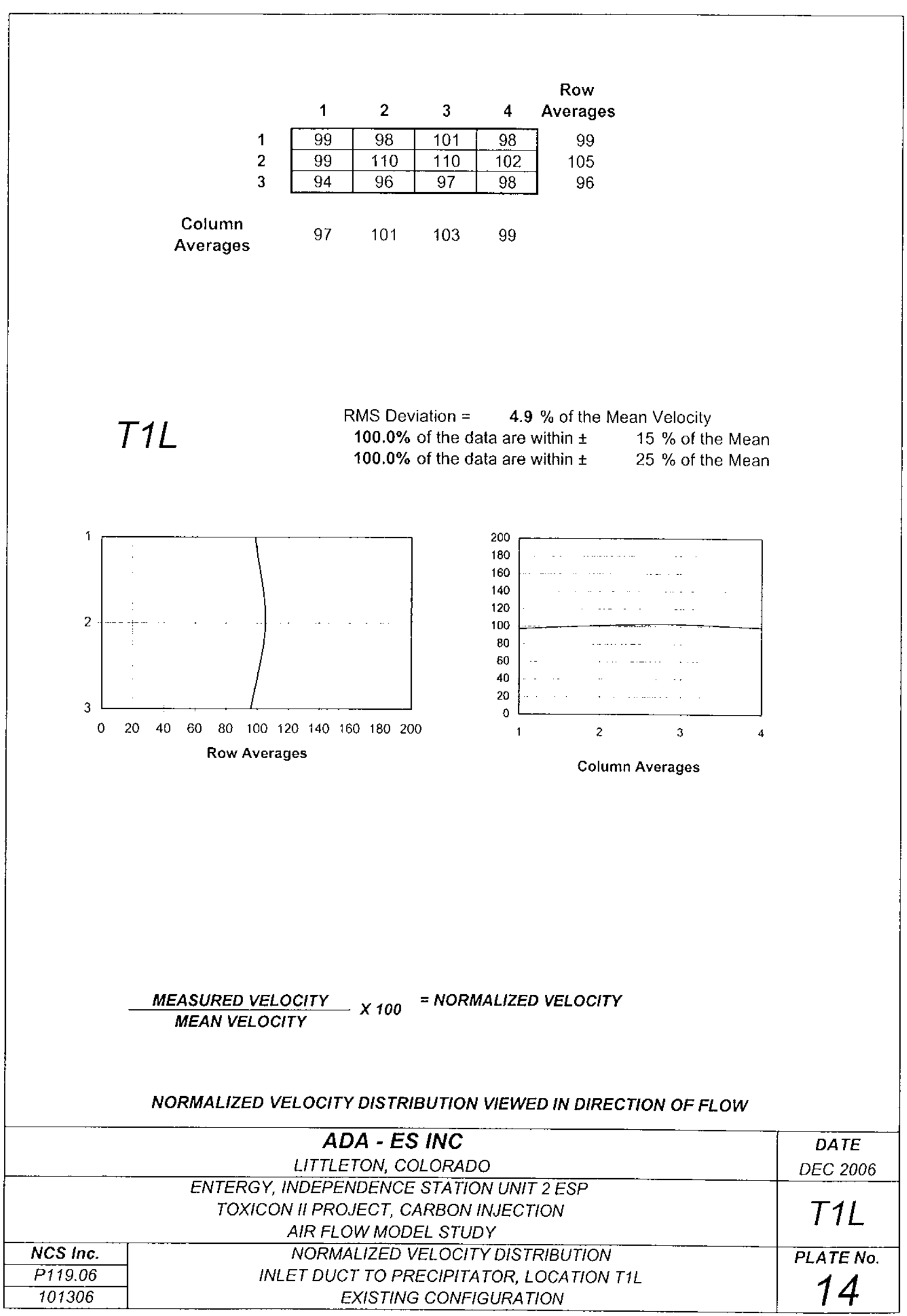




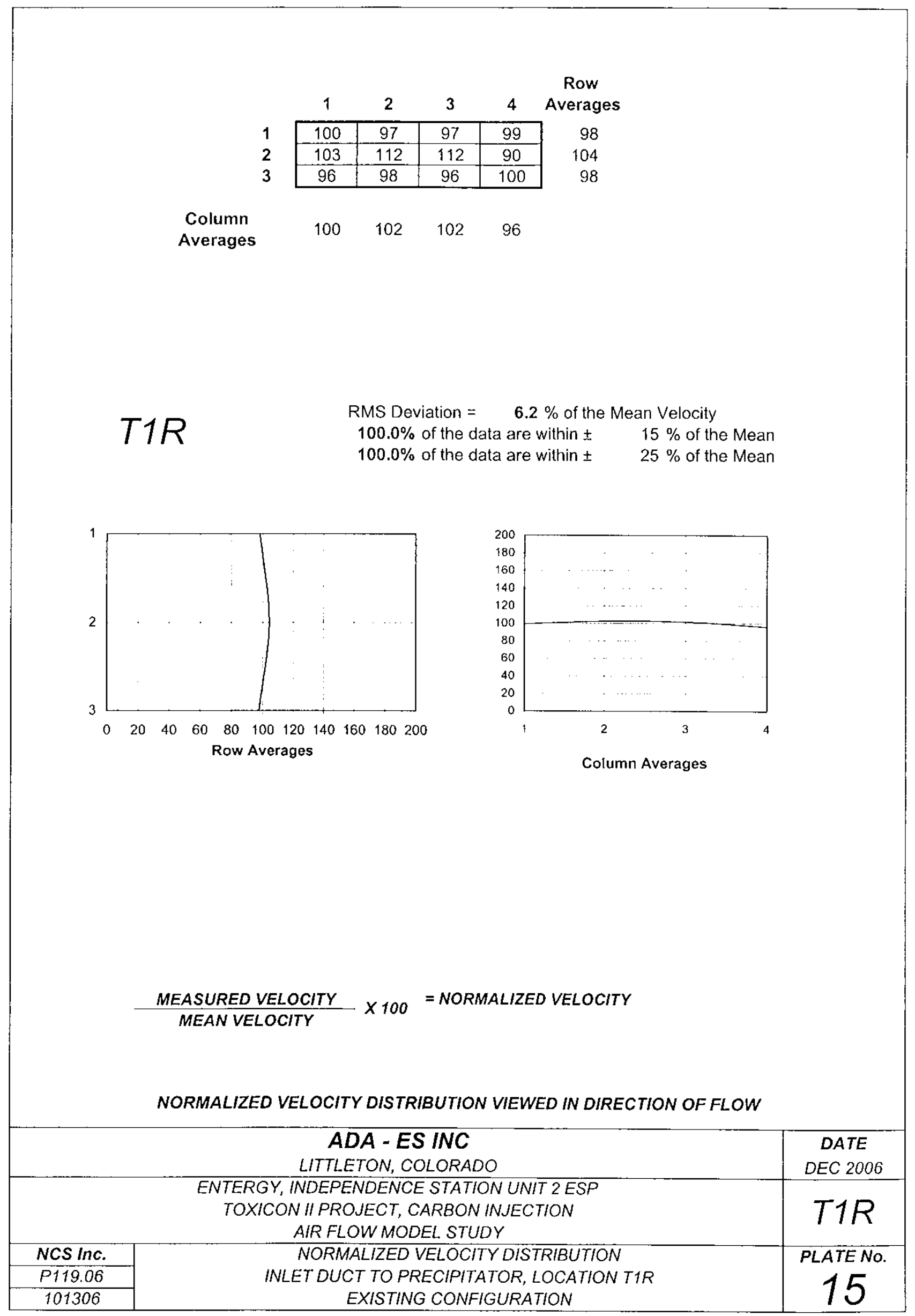




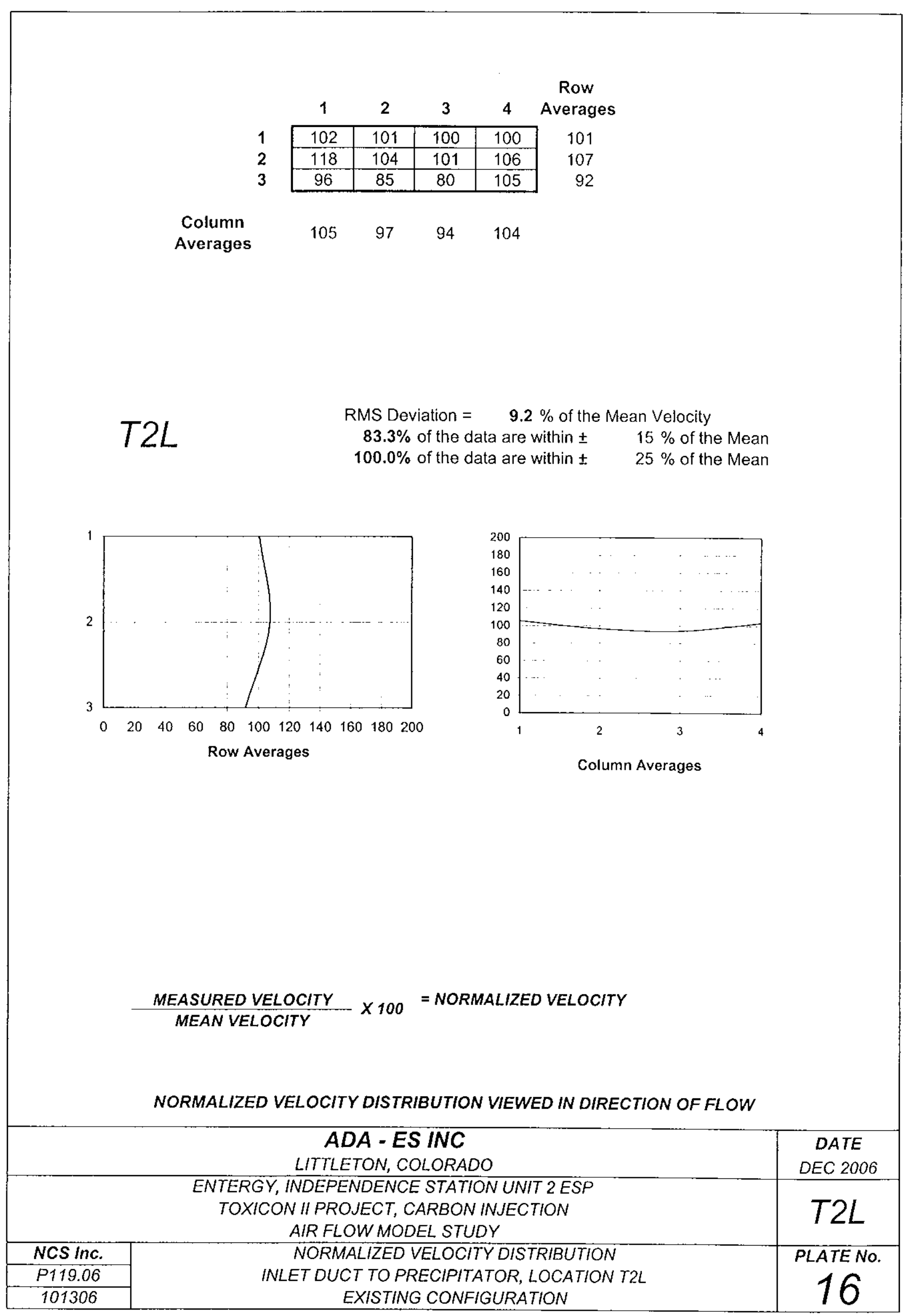




\begin{tabular}{|c|c|c|c|c|c|}
\hline & 1 & 2 & 3 & 4 & $\begin{array}{c}\text { Row } \\
\text { Averages }\end{array}$ \\
\hline 1 & 101 & 99 & 101 & 102 & 101 \\
\hline & 112 & 95 & 99 & 114 & 105 \\
\hline 3 & 106 & 80 & 82 & 109 & 94 \\
\hline
\end{tabular}

Column

Averages

$\begin{array}{llll}107 & 91 & 94 \quad 108\end{array}$

$T 2 R$

RMS Deviation $=10.1 \%$ of the Mean Velocity

$83.3 \%$ of the data are within $\pm \quad 15 \%$ of the Mean

$100.0 \%$ of the data are within $\pm \quad 25 \%$ of the Mean
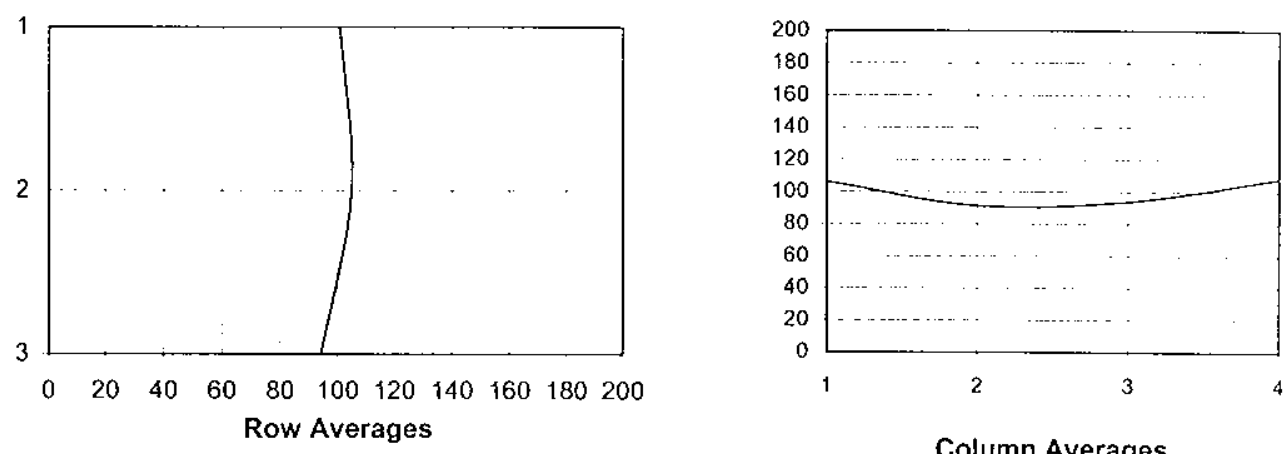

Column Averages

MEASURED VELOCITY $\times 100=$ NORMALIZED VELOCITY MEAN VELOCITY

NORMALIZED VELOCITY DISTRIBUTION VIEWED IN DIRECTION OF FLOW

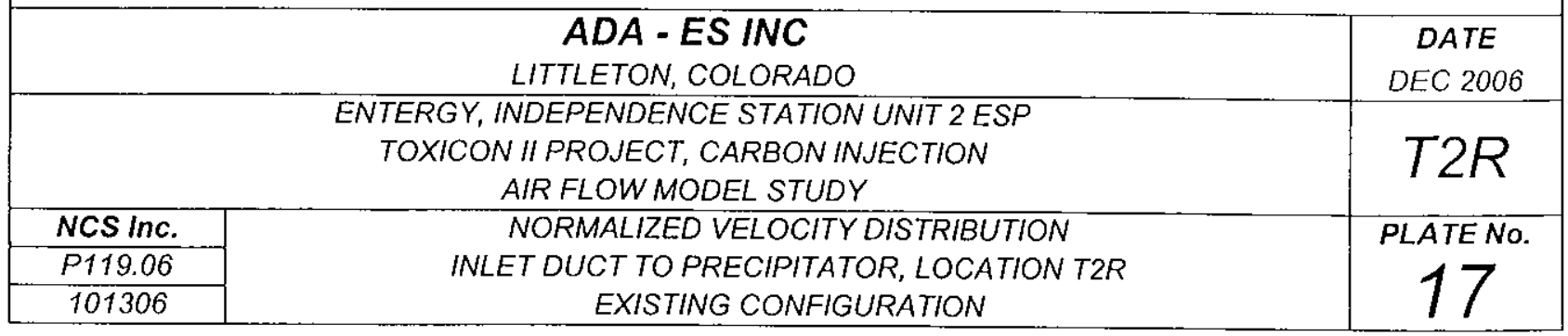




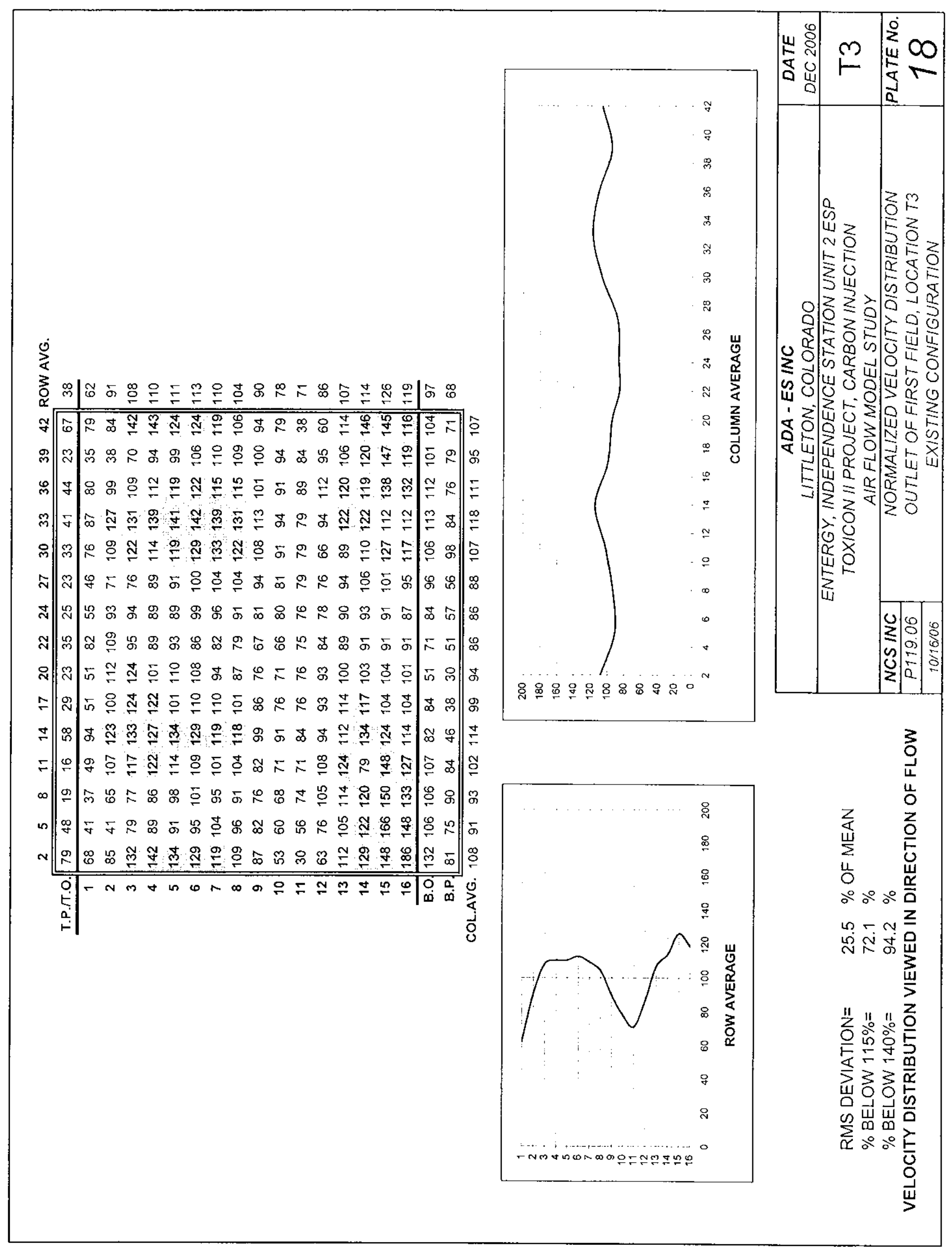




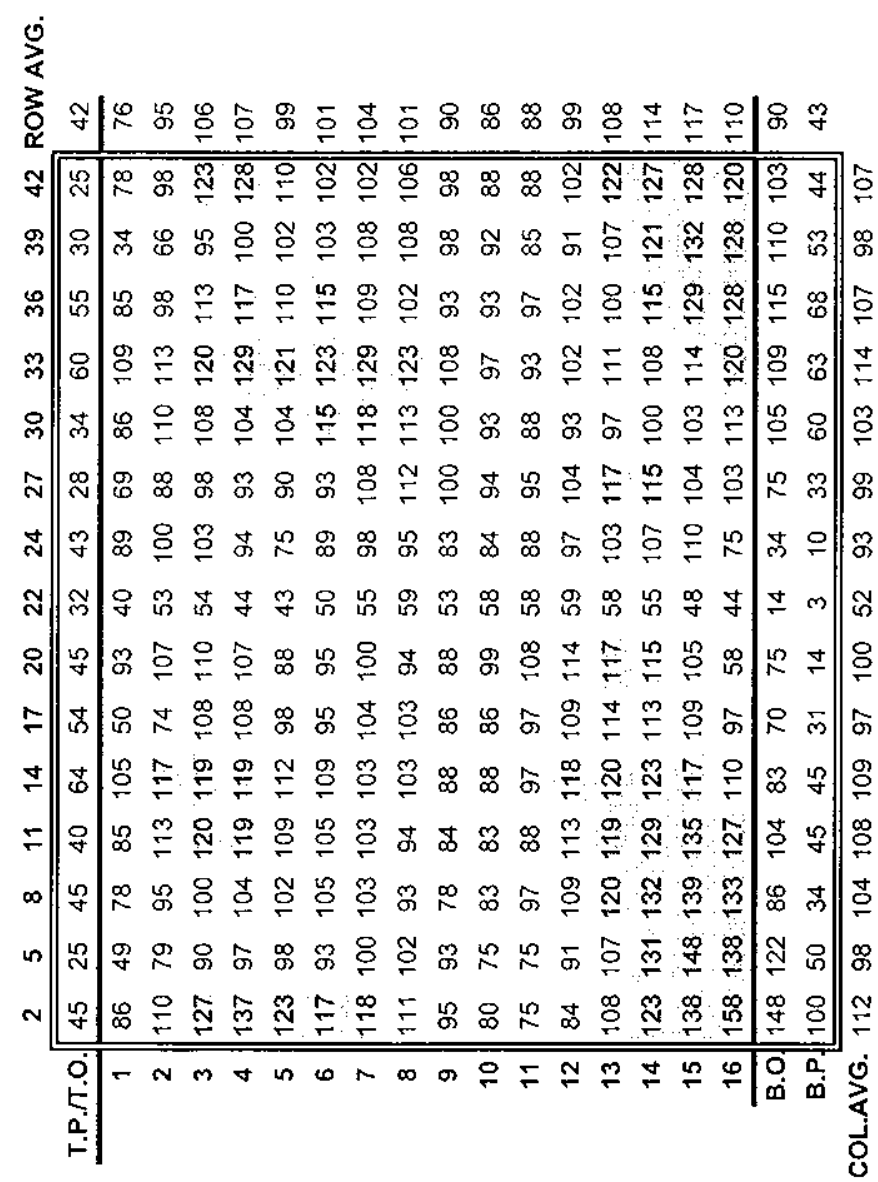



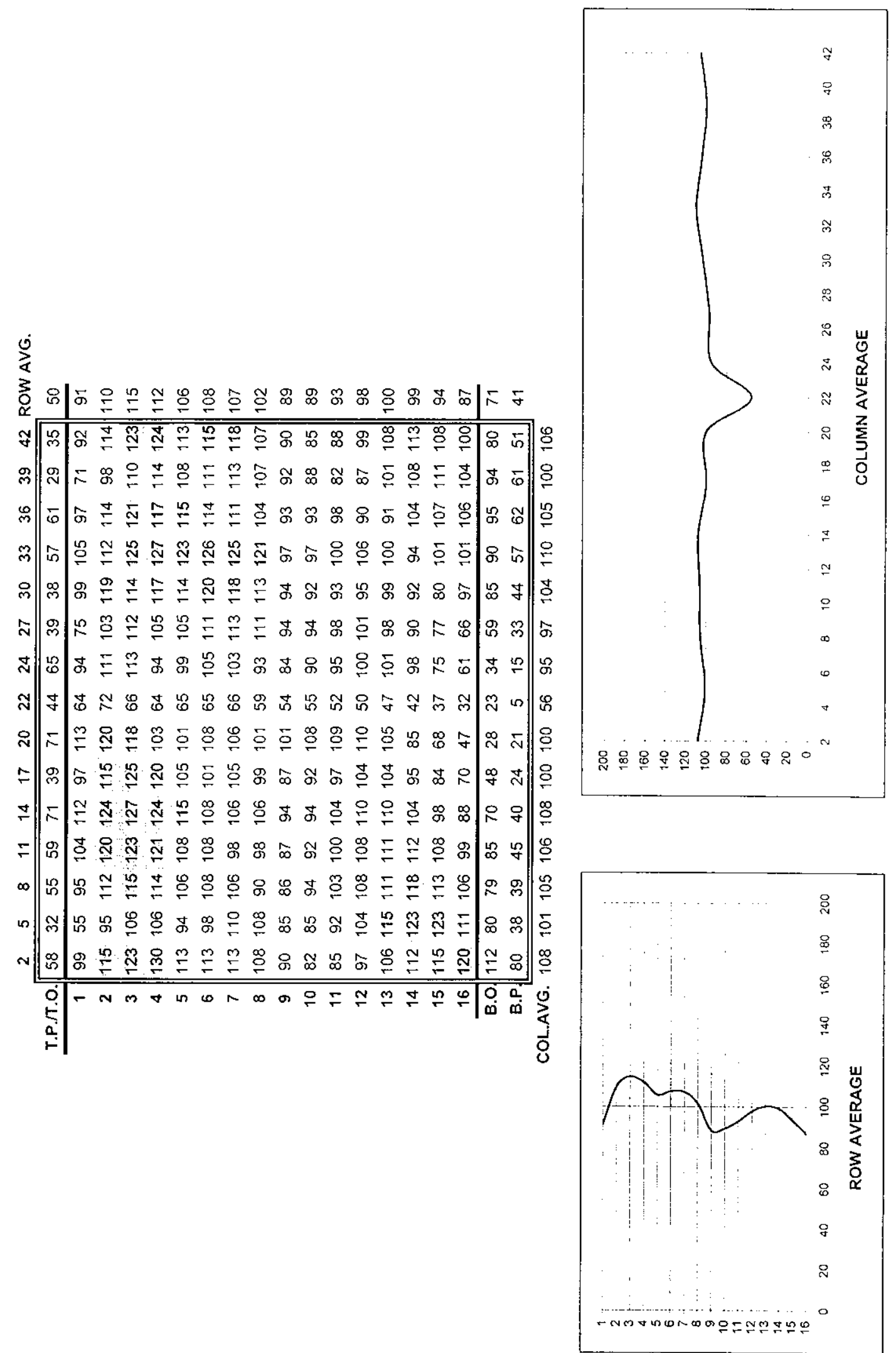

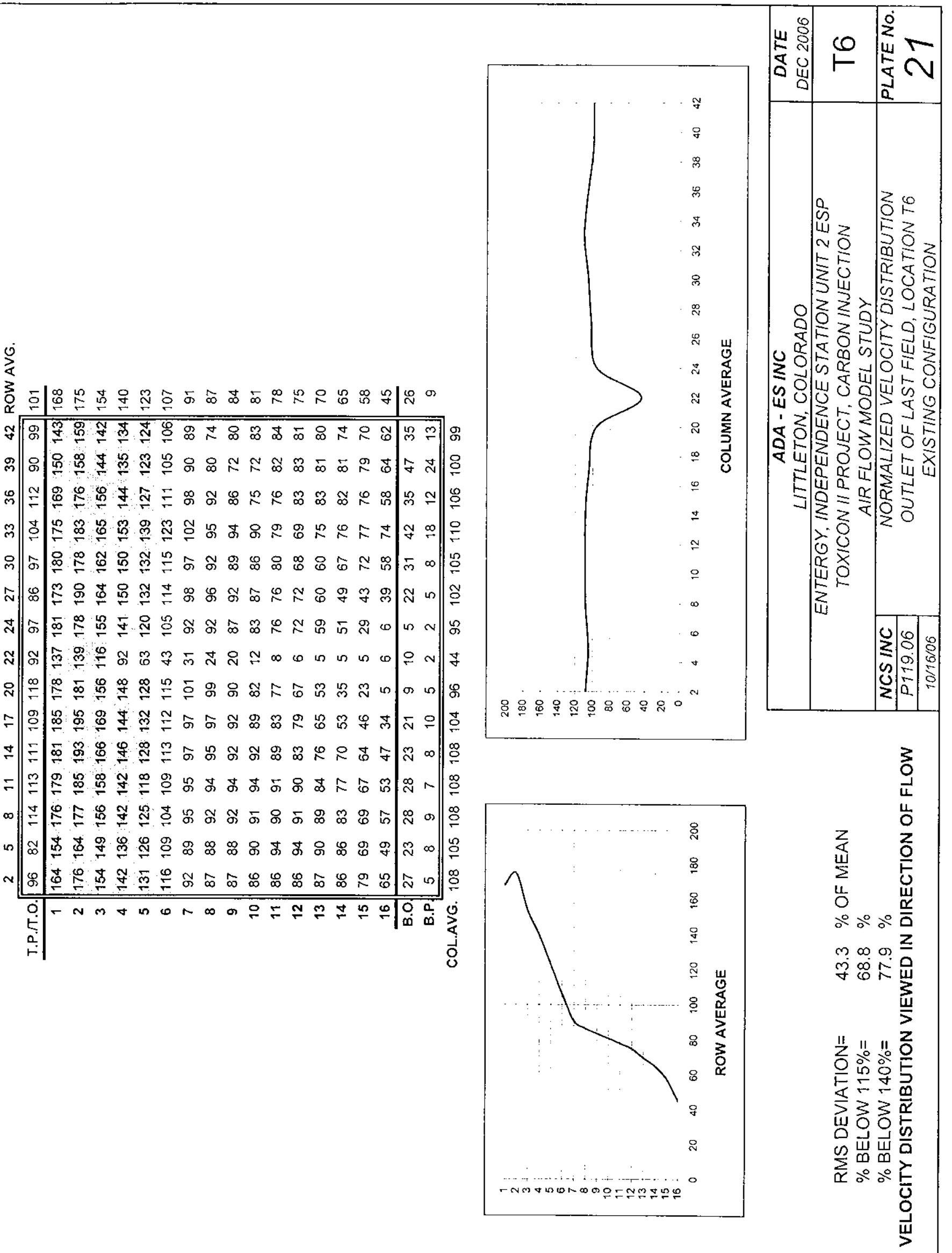


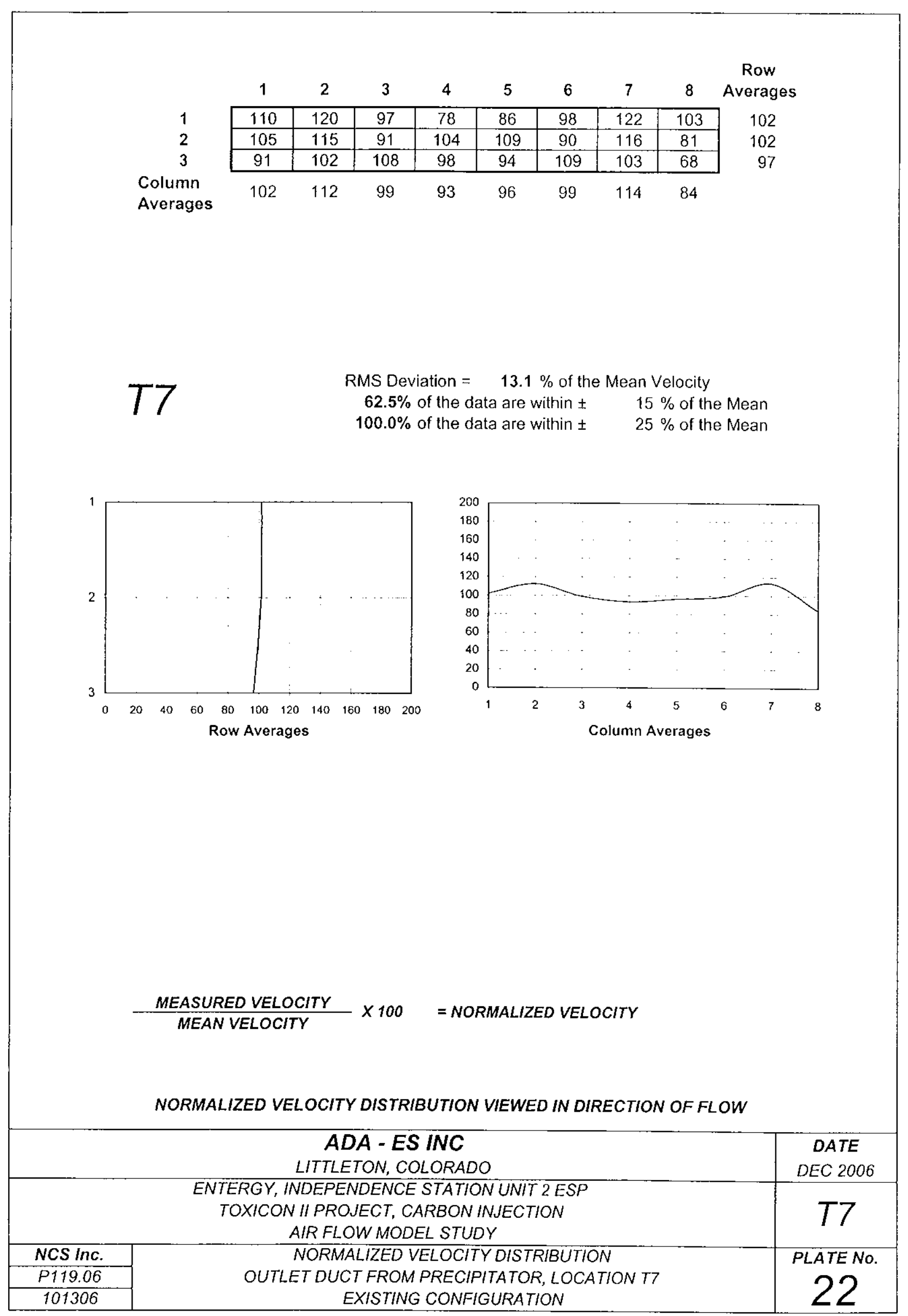




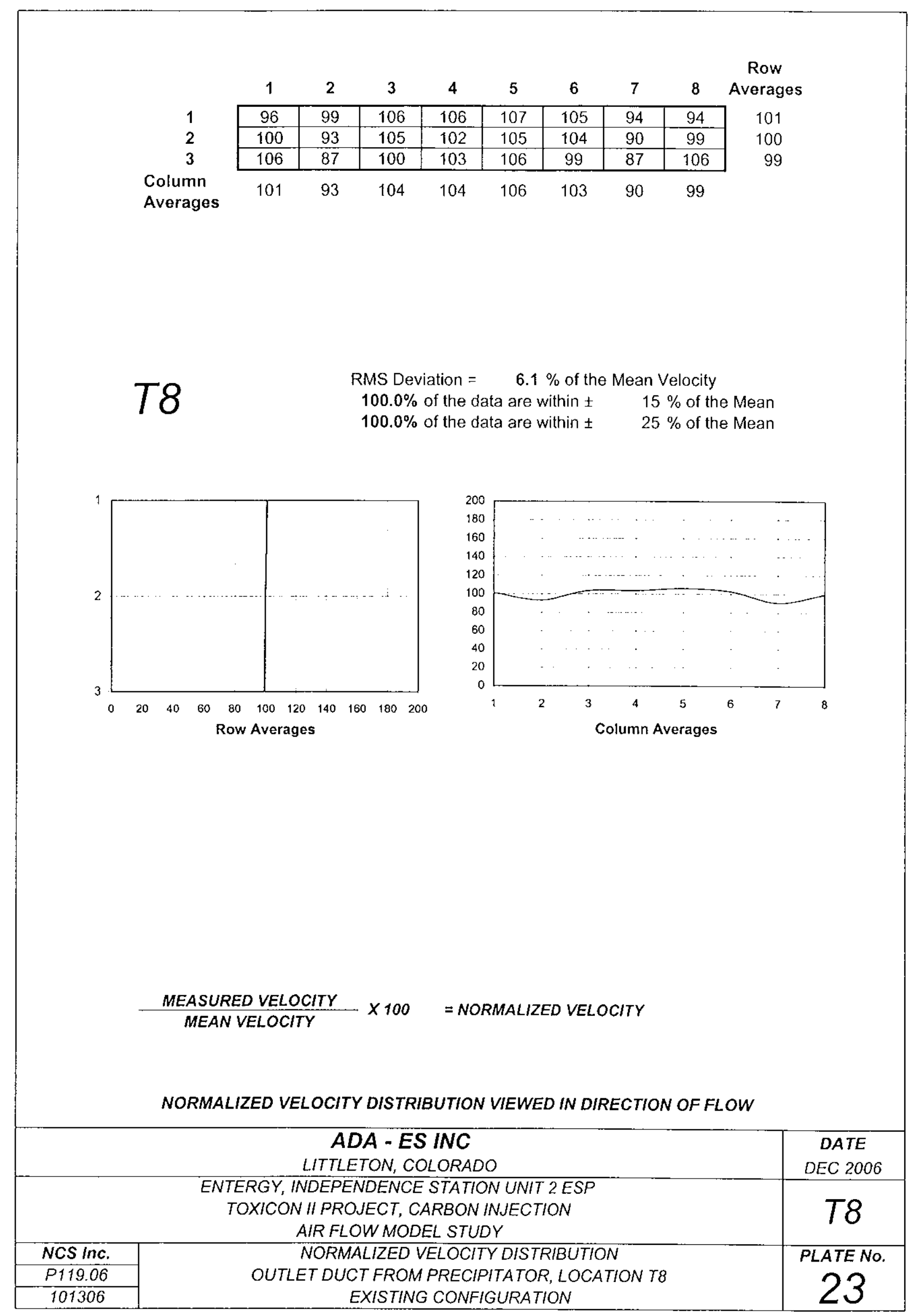


NORMALIZED GAS DISTRIBUTION

\begin{tabular}{|c|c|c|c|c|c|c|c|c|c|c|c|c|c|}
\hline & 6 & 7 & 8 & 9 & 10 & 11 & 12 & 13 & 14 & 15 & 16 & \multicolumn{2}{|c|}{ ROW AVG. } \\
\hline 1 & 2912 & 34 & 4286 & 13 & 69 & 60 & 6 & 107 & 11 & 15 & 113 & 91 & \\
\hline 2 & 176 & 15 & 285 & 116 & 7 & 256 & 120 & 10 & 235 & 62 & 17 & 118 & \\
\hline 3 & 16 & 73 & W:315 & 10 & 144 & 672063 & 10 & 6262 & 56 & 36 & W 3500 & 134 & \\
\hline 4 & 136 & 26 & 174 & 153 & 10 & 134 & 183 & 13 & 215 & 59 & 56 & 105 & \\
\hline 5 & 33 & 31 & 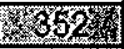 & 159 & 70 & 3280 & 66 & 2400 & 38 & 21 & 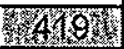 & 151 & \\
\hline 6 & 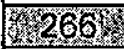 & 27 & 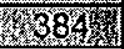 & 105 & 27 & 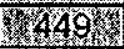 & 88 & 42 & 206027 & 63 & 99 & 183 & \\
\hline 7 & 38 & 20 & 38 & 37 & 8 & 26 & 20 & 31 & 21 & 34 & 22 & 27 & \\
\hline 8 & 848 & 74 & 30 & 2628 & 10 & 166 & 98 & 6 & 153 & 136 & 10 & 118 & \\
\hline 9 & 12 & 146 & 196 & 10 & 270 & 136 & 24 & 262,1 & 13 & 88 & 167 & 120 & \\
\hline 10 & 8 & 132 & 8 & 74 & 50 & 8 & 41 & 49 & 6 & 82 & 15 & 43 & \\
\hline 11[ & 4 & 15 & 6 & 9 & 12 & 6 & 7 & 20 & 5 & 13 & 8 & 9 & \\
\hline $\begin{array}{l}\text { COL. } \\
\text { AVG. }\end{array}$ & 121 & $\begin{array}{c}54 \\
4\end{array}$ & 189 & 86 & 62 & 152 & 60 & 95 & 110 & $\begin{array}{l}55 \\
4\end{array}$ & 116 & & \\
\hline & & $\sum_{0}^{5}$ & & Г & & & & & & 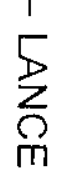 & & Whark & $\begin{array}{l}<100 \% \\
100-200 \% \\
>200 \%\end{array}$ \\
\hline
\end{tabular}
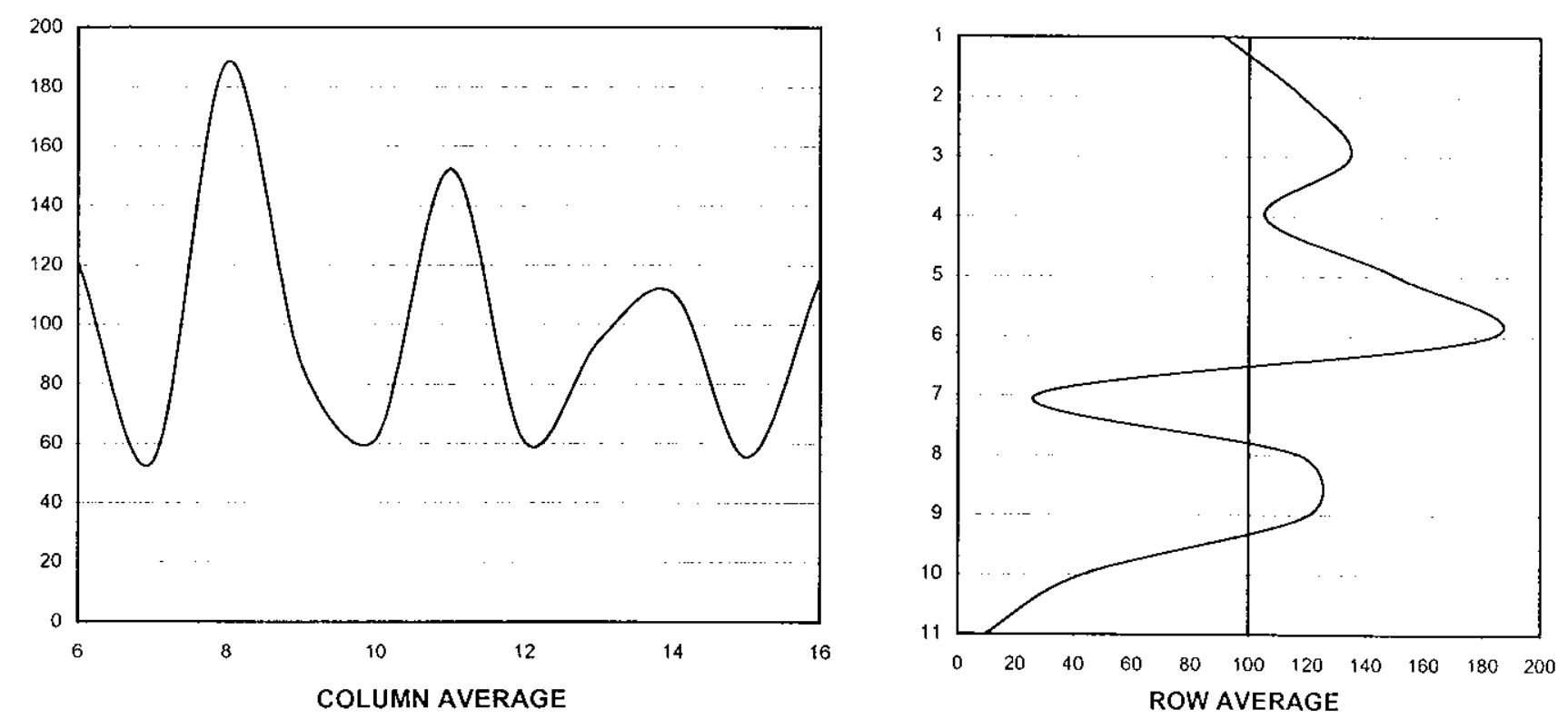

MEAN CO VALUE=

156.4 PPM

RMS DEVIATION=

$111.5 \%$ OF MEAN

GAS DISTRIBUTION VIEWED IN DIRECTION OF FLOW NORMALIZED GAS DISTRIBUTION EXPRESSED AS A PERCENT OF MEAN

$A D A$ - ES INC

DATE

LITTLETON, COLORADO

ENTERGY, INDEPENDENCE STATION UNIT 2 ESP TOXICON II PROJECT, CARBON INJECTION

AIR FLOW MODEL STUDY

NCS Inc. $\quad$ CARBON MONOXIDE DISTRIBUTION

P119.06

MIDDLE OF THIRD FIELD, LOCATION T5A 


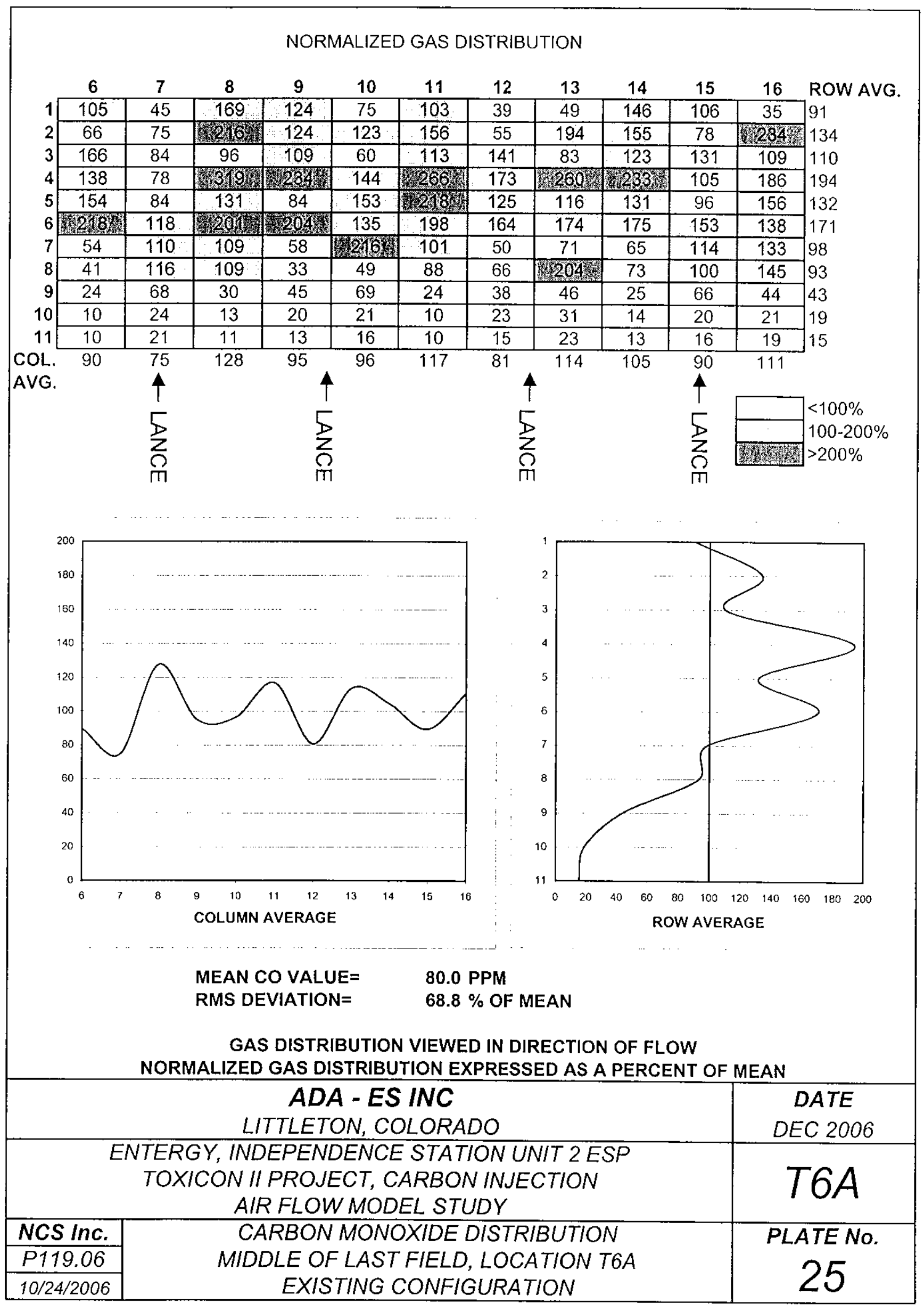




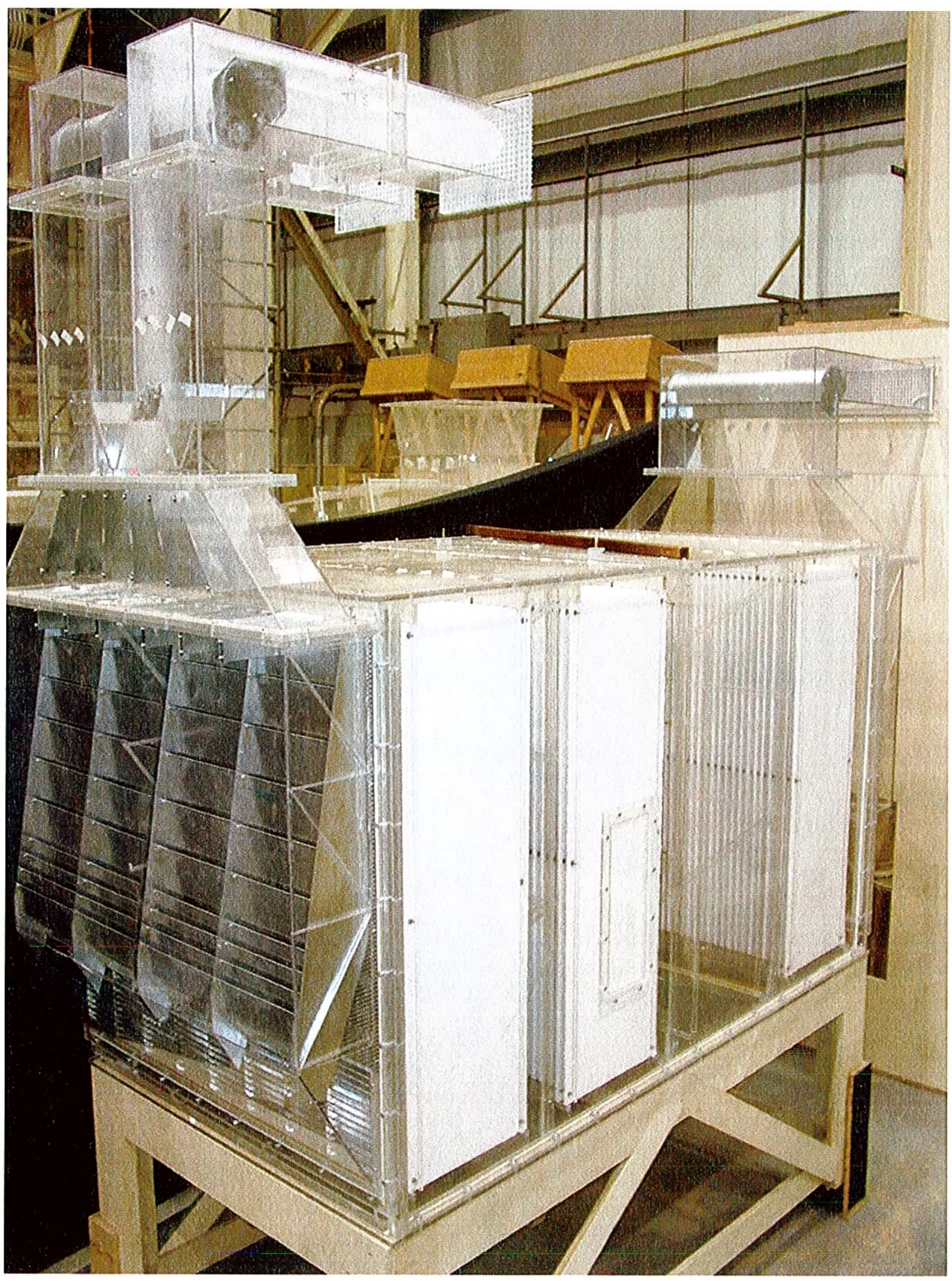

A - General Model Arrangement 
P119.06 - ADA-ES INC, Entergy, Independence Station Unit 2, Toxicon II Carbon Injection

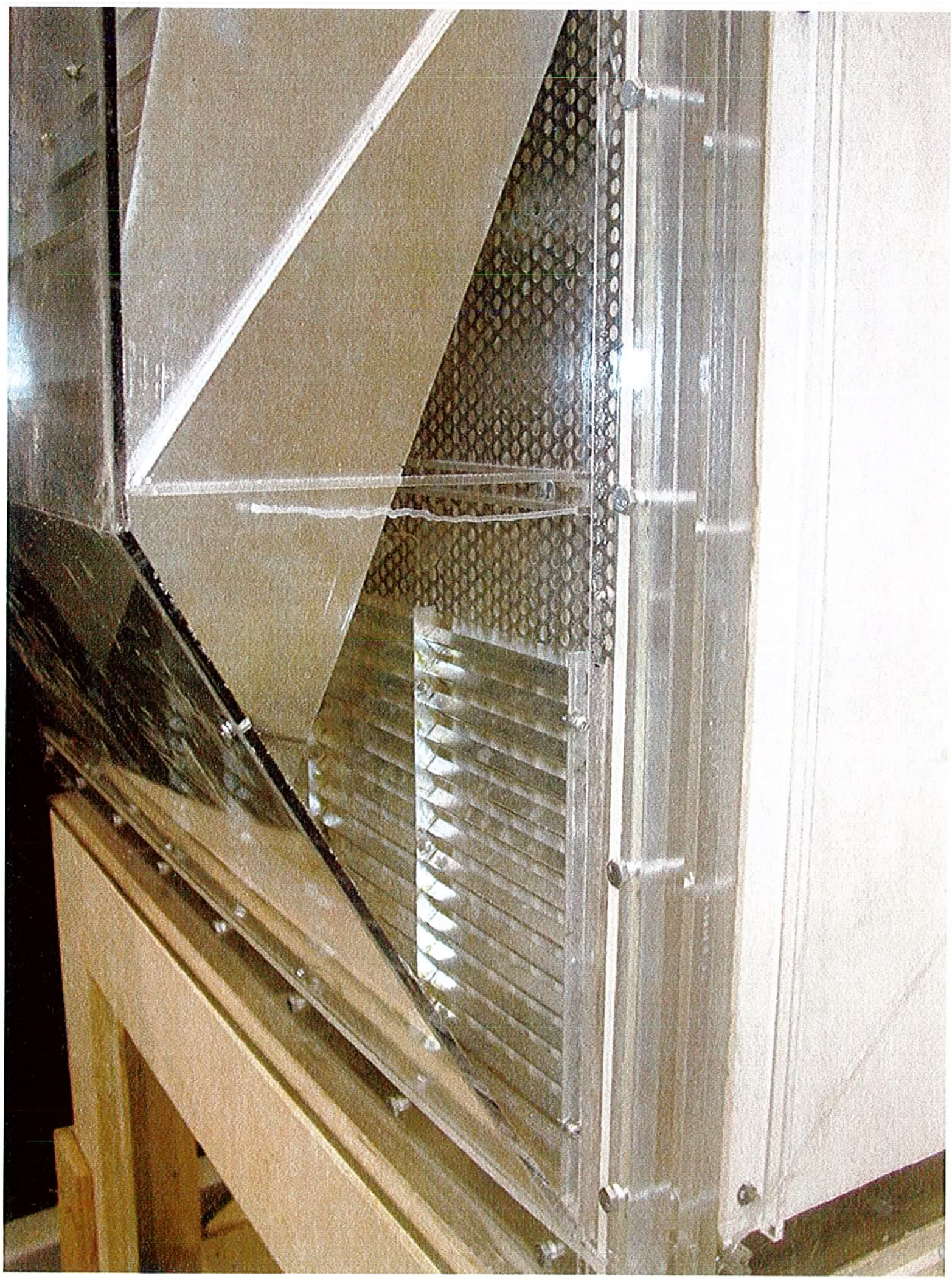

\section{B - Bottom of Inlet Plenum}


P119.06 - ADA-ES INC, Entergy, Independence Station Unit 2, Toxicon II Carbon Injection

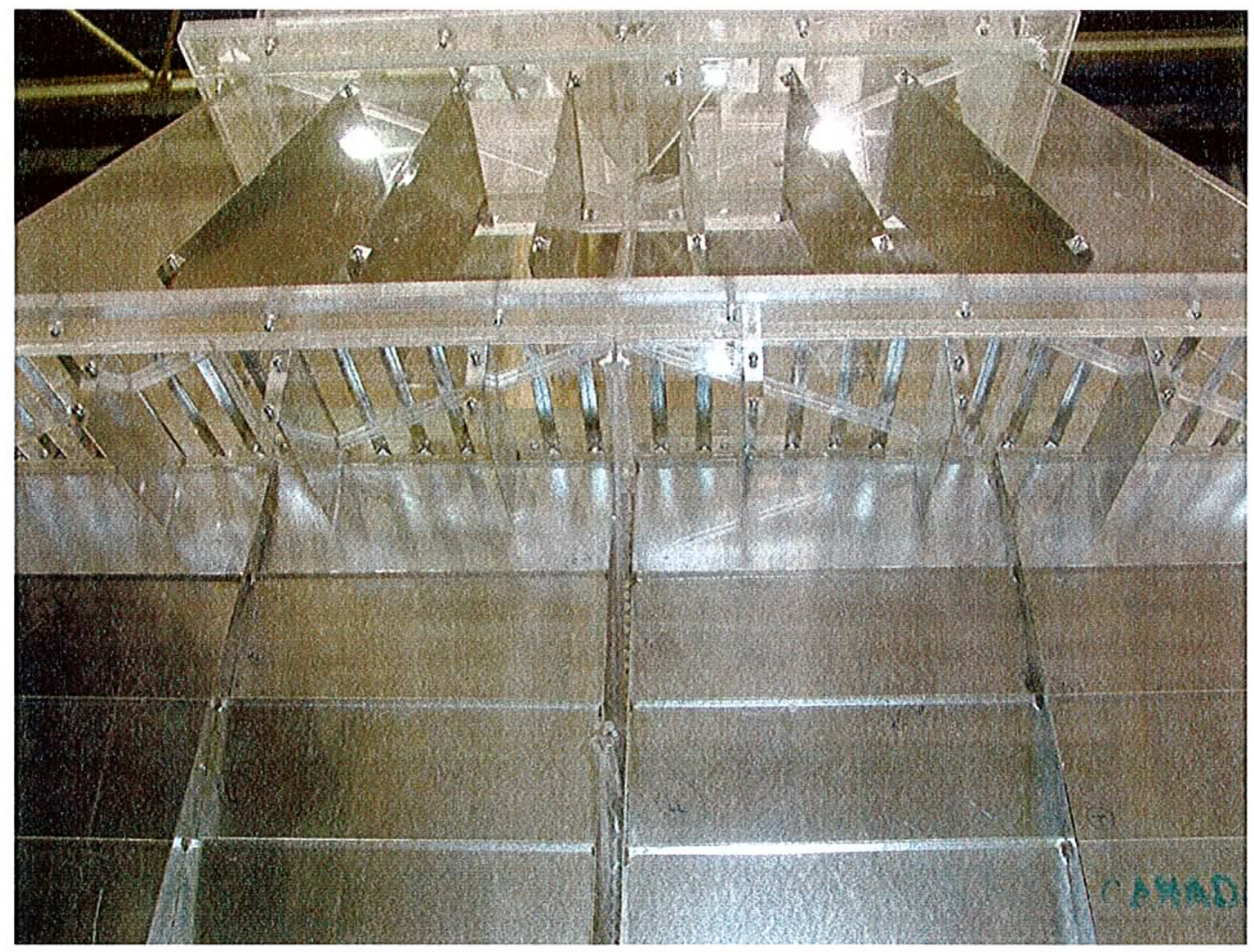

\section{C - Vanes Upstream of The Inlet Plenum}

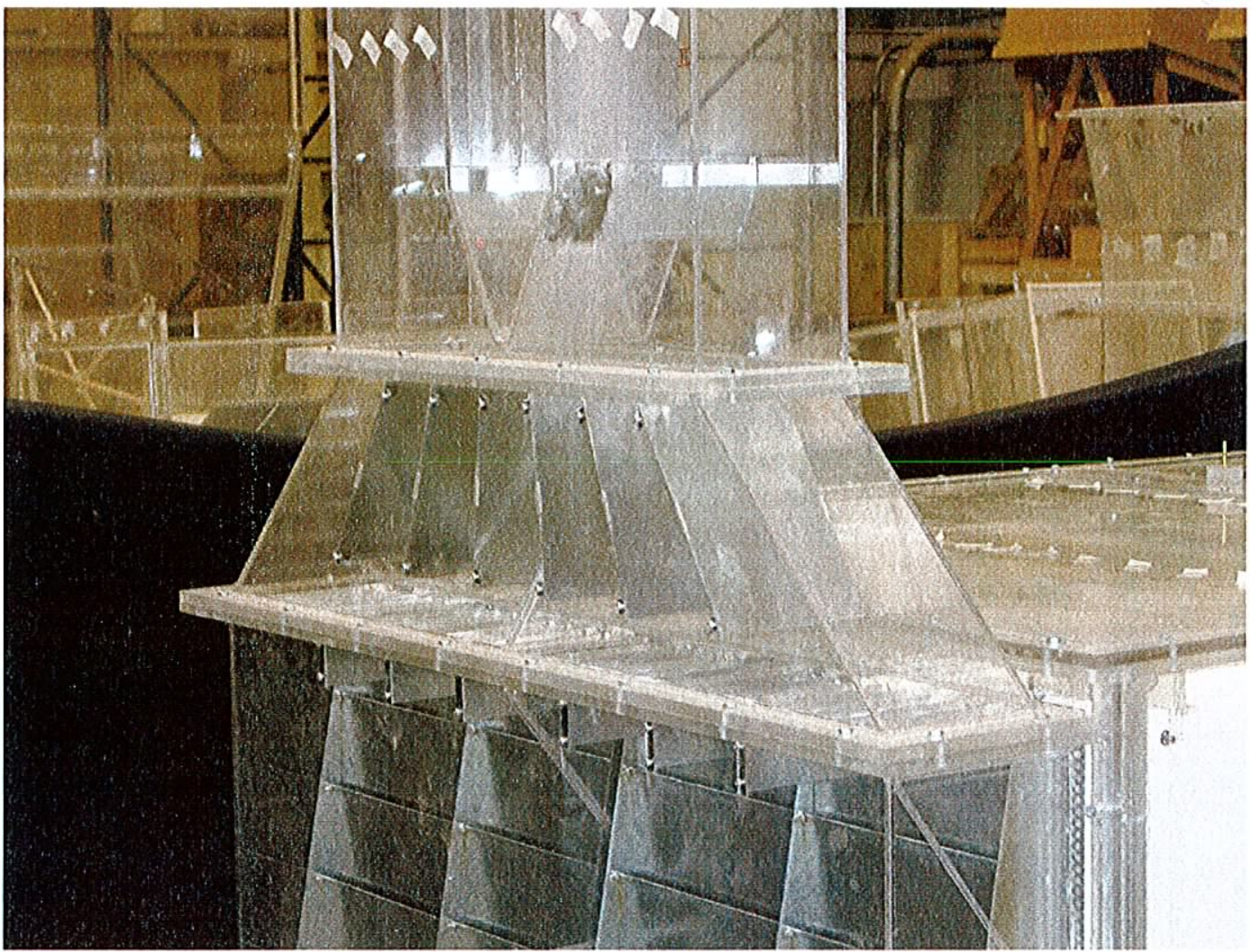

D - Inlet Duct Vanes 


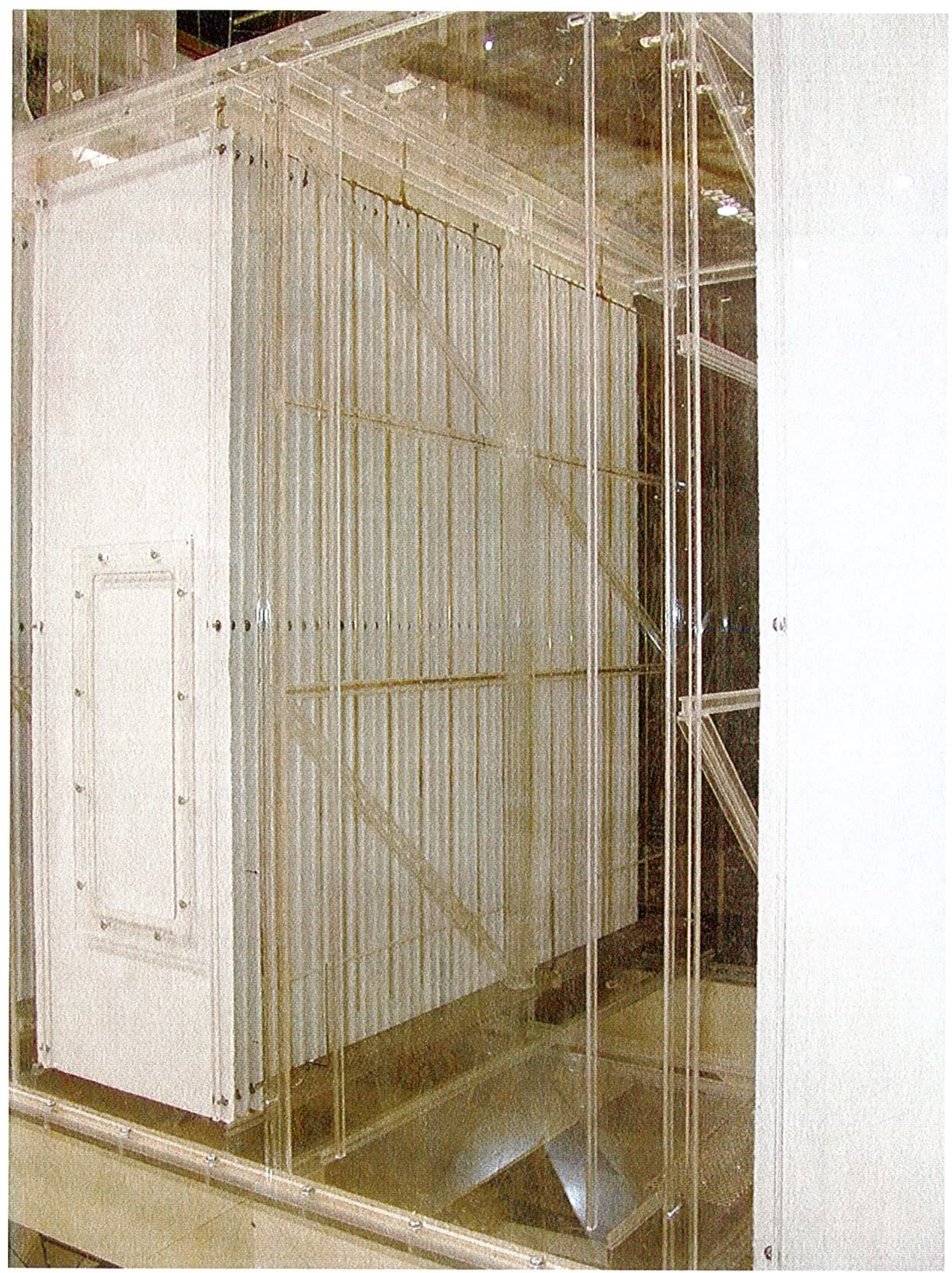

\section{E - (PAC) Injection Lance Arrangement}




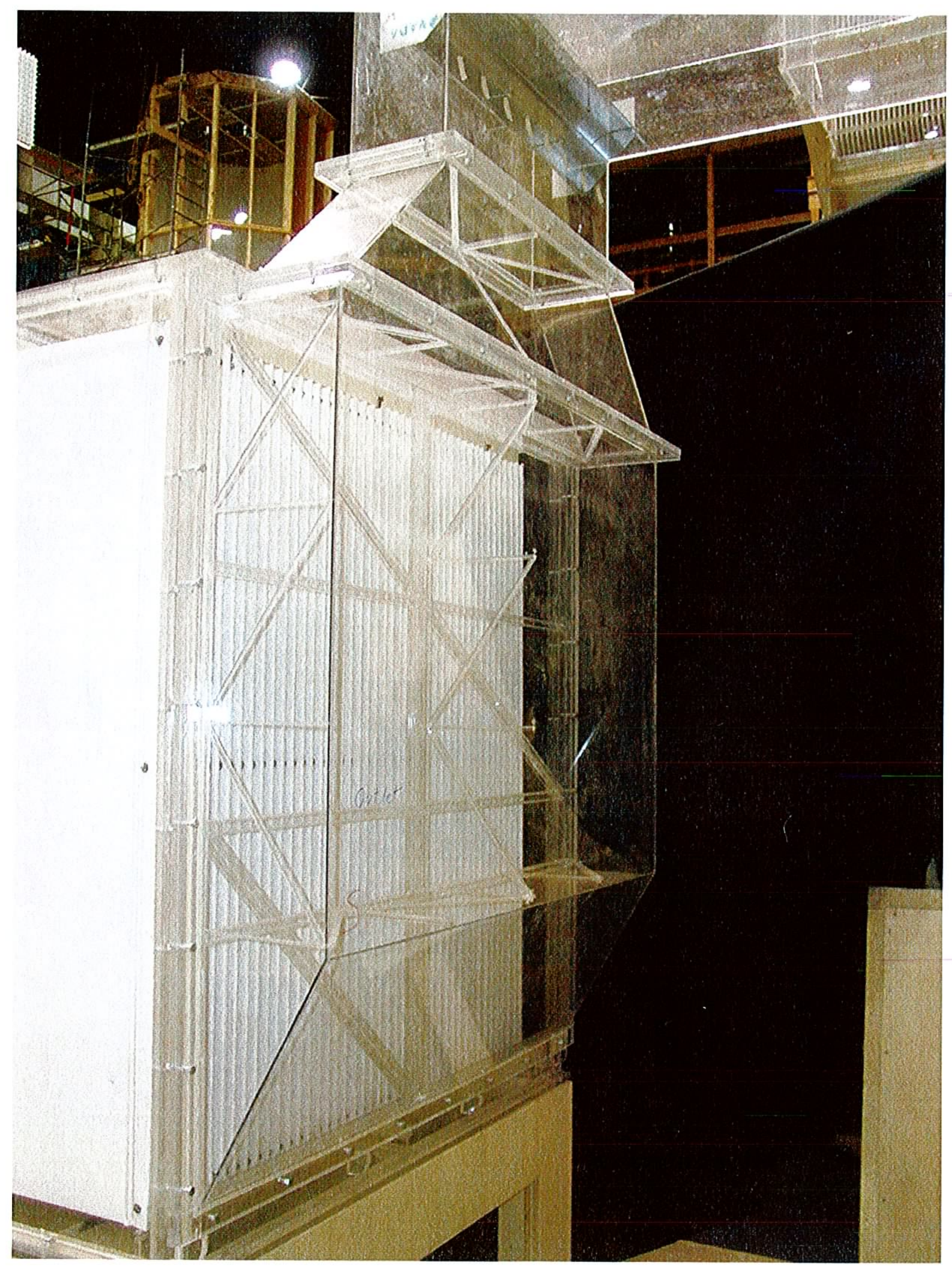

\section{F - Existing Outlet Plenum}




\section{APPENDIX F:}

Sample Collection and Management Plan 


\section{LABORATORY MERCURY FIELD EVALUATION}

Evaluation of Sorbent Injection for Mercury Control at Entergy Independence Station

Sample Collection and Management Plan

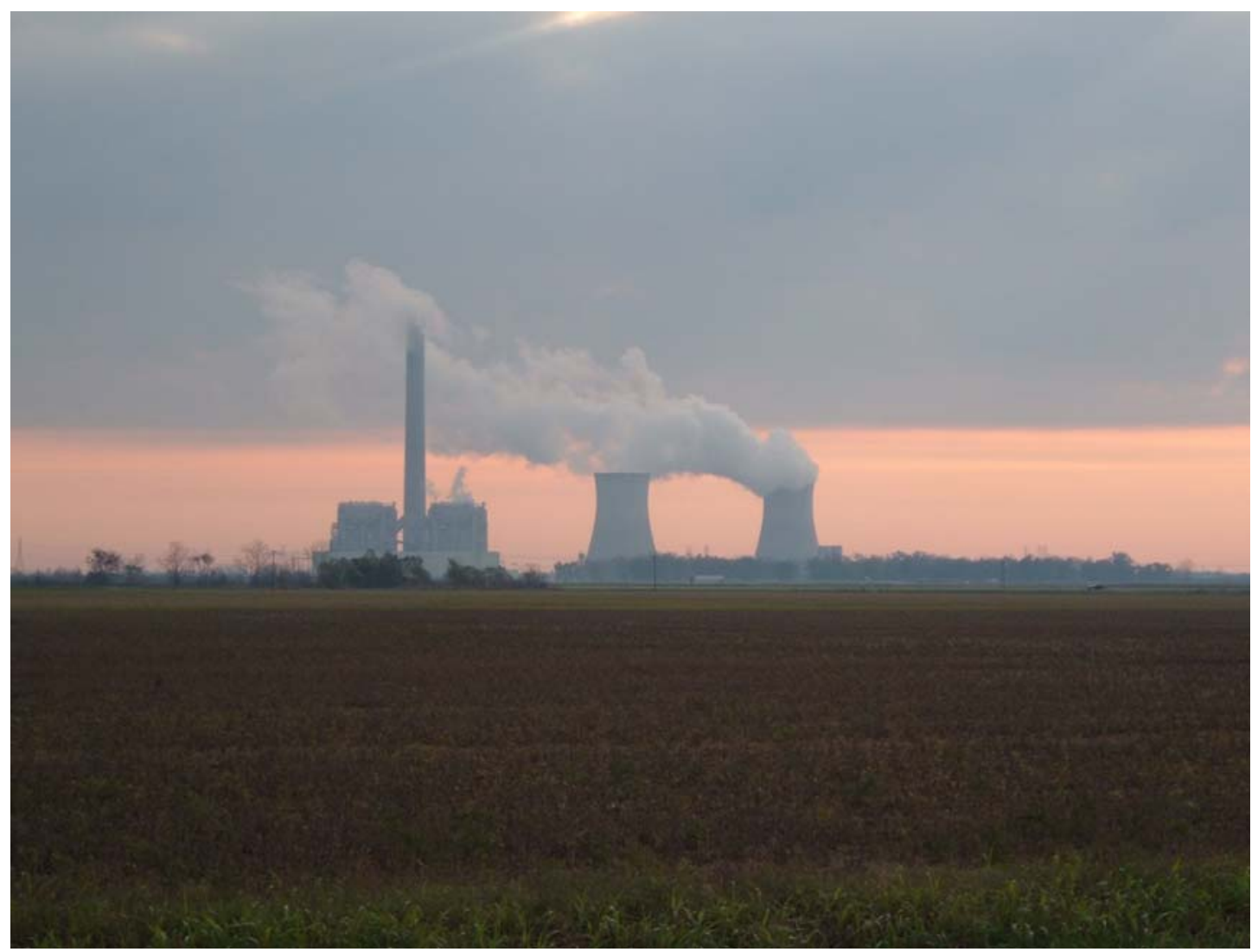

Prepared by:

ADA Environmental Solutions, Inc. 8100 SouthPark Way, Unit B Littleton, CO 80120

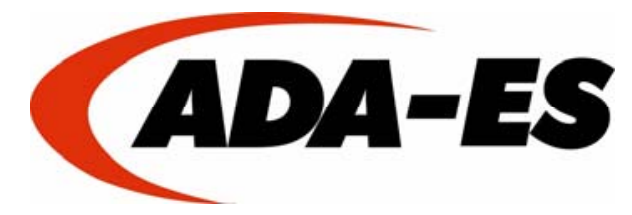

July 25, 2005 
ADA-ES, Inc. is conducting an evaluation looking at sorbent injection for mercury control at Entergy's Independence Station. The overall objective of this project is to determine the cost and effects of sorbent injection for control of mercury in stack emissions using a TOXECON II sorbent injection configuration.

During the evaluation, fuel samples and certain process byproducts will be collected for determination of mercury content, stability, and other analytes. Process byproduct of primary interest is fly ash; however, other process byproducts may also be collected.

Sample and data management are needed for tracking approximately 500 samples from various solid process streams at Independence Station. ADA-ES has developed a Sample and Data Management System (SDMS) that will store test data from the evaluation. These data can be used to generate reports, track sample history, and input results from laboratory analyses.

ADA-ES will also store plant operational data and other test data during the evaluation. Pertinent plant operating parameters will be logged electronically. For data control and security, full access will be limited to the project manager and site manager at ADA-ES and the sample manager. Operators collecting samples will be able to upload information to the database and print sample labels and Chainof-Custody forms. ADA-ES will include results with regularly issued reports to the test team.

\section{Sampling Locations}

Samples of various gaseous and solid process streams will be collected during the evaluation. Specific flue gas samples are not included in this document. Sampling locations for Independence Station Unit 2 are shown in Figure 1. 


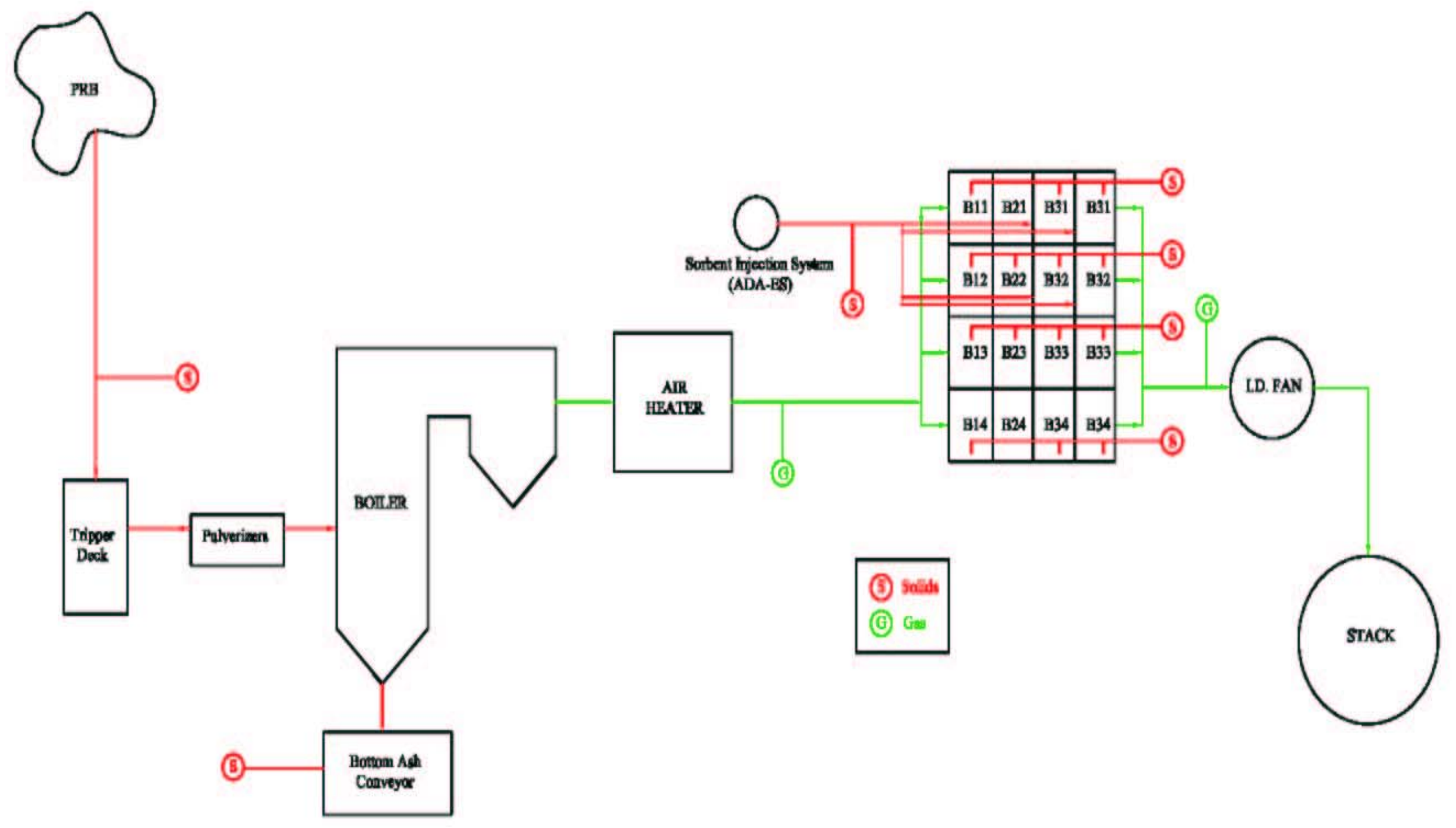

Figure 1. Independence Station Unit 2 Configuration and Sampling Locations. 


\section{Sample Collection}

Coal and combustion byproducts will be collected during the mercury control evaluation. Samples will be segregated by the test condition (baseline, each parametric test, and longterm test). Collecting a representative sample is the primary objective of the sampling strategy. Representative samples will be collected only under stable and normal operating conditions unless otherwise directed by ADA-ES personnel.

\section{Sample Streams}

Coal Samples - Daily grab samples will be collected from the coal conveyor that feeds the unit 2 coal silos. Independence plant personnel will collect the sample between 5:00 am and 11:00 am, thus representing the coal fired between 10:00am and 4:00pm. ADA-ES will provide the sample schedule and sample bottles.

ESP Fly Ash - Grab samples of ash will be collected from the ESP hoppers each day of testing. Samples will be segregated by the test condition (baseline, each parametric test, and long-term test). The samples will be stored in 1-liter or 5-gallon sample containers for shipping to the analytical laboratories.

The schedule indicates sampling from multiple rows on both the control side and test side of the ESP. These samples will be used to determine if stratification exists throughout the system and to compare ash properties of the test side with the control side. A sketch showing the hoppers from the ESP is shown in Figure 2. The shaded hoppers indicate the hoppers from which fly ash samples will be collected.

During testing, the ESP hoppers should be emptied around 10:00am with ash samples being collected at 3:00 pm. This will ensure the sample collected represents the ash collected by the ESP during the test period which typically starts at 10am and ends around 5:00pm. ADA will coordinate ash sampling and hopper emptying activities with plant operations. 


\begin{tabular}{|c|c|c|c|}
\hline \multicolumn{2}{|c|}{ Gas Flow $\sqrt{ }$} \\
\hline B14* & B13* & B12* & B11* \\
\hline B24 & B23* & B22* & B21* \\
\hline B34 & B33* & B32* & B31* \\
\hline B44 & B43* & B42* & B41* \\
\hline
\end{tabular}

*Sampled Hopper

Figure 2. ESP Hopper Layout and Sampling Locations.

If possible, plant personnel may collect a fly ash sample inside the ESP at the end of the long-term testing period. This sample should be collected from any surface structures (e.g., ledges, corners) that are capable of holding fly ash material in place for a long period of time. This sample should be exposed to coal-derived flue gas for long periods of time. This sample will be analyzed for metals content (e.g., Hg, As, Se) to help determine if these toxics accumulate over time and surpass any recommended exposure limits. 
Table 1. Tentative Sampling Schedule.

\begin{tabular}{|c|c|c|c|}
\hline $\begin{array}{c}\text { Test } \\
\text { Condition }\end{array}$ & Type & Frequency & $\begin{array}{l}\text { Volume } \\
\text { Collected }\end{array}$ \\
\hline \multirow[b]{2}{*}{ Baseline } & Coal & Daily & 2 liters \\
\hline & ESP Ash & $\begin{array}{l}\text { Daily: } \\
\text { One Hopper Each Row on Test Side, } \\
\text { Inlet Hopper on Control Side (B12, } \\
\text { B22, B32, B42, B13) } \\
2 \text { samples per week: } \\
\text { All Hoppers on Test Side, All inlet } \\
\text { Hoppers and one Hopper each row } \\
\text { on Control Side (B11, B21, B31, } \\
\text { B41, B14, B23, B33, B43) }\end{array}$ & $\begin{array}{l}1 \text { liter } \\
1 \text { liter }\end{array}$ \\
\hline \multirow[b]{2}{*}{ Parametric } & Coal & Daily & 2 liters \\
\hline & ESP Ash & $\begin{array}{l}\text { Daily: } \\
\text { One Hopper Each Row on Test Side, } \\
\text { Inlet Hopper on Control Side (B12, } \\
\text { B22, B32, B42, B13) } \\
2 \text { samples per week: } \\
\text { All Hoppers on Test Side, One } \\
\text { Hopper each row on Control Side } \\
\text { (B11, B21, B31, B41, B23, B33, } \\
\text { B43) }\end{array}$ & $\begin{array}{l}1 \text { liter } \\
1 \text { liter }\end{array}$ \\
\hline \multirow[b]{2}{*}{$\begin{array}{l}\text { Long- } \\
\text { Term }\end{array}$} & Coal & Daily & 2 liters \\
\hline & ESP Ash & $\begin{array}{l}\text { Daily: } \\
\text { One Hopper Each Row on Test } \\
\text { Side, Inlet Hopper on Control Side } \\
\text { (B12, B22, B32, B42, B13) } \\
2 \text { samples per week: } \\
\text { All Hoppers on Test Side, One } \\
\text { Hopper each row on Control Side } \\
\text { (B11, B21, B31, B41, B23, B33, } \\
\text { B43) }\end{array}$ & $\begin{array}{l}1 \text { liter } \\
1 \text { liter }\end{array}$ \\
\hline
\end{tabular}




\section{Sample Management Strategy}

During the mercury control evaluation, Independence plant personnel, as directed by ADAES, will collect the coal samples. ADA-ES personnel will collect the fly ash samples. ADAES will deliver a sampling schedule, which shows the sampling times, volume, and specific samples to collect during each testing day. A sample management flow chart is shown in Figure 3.

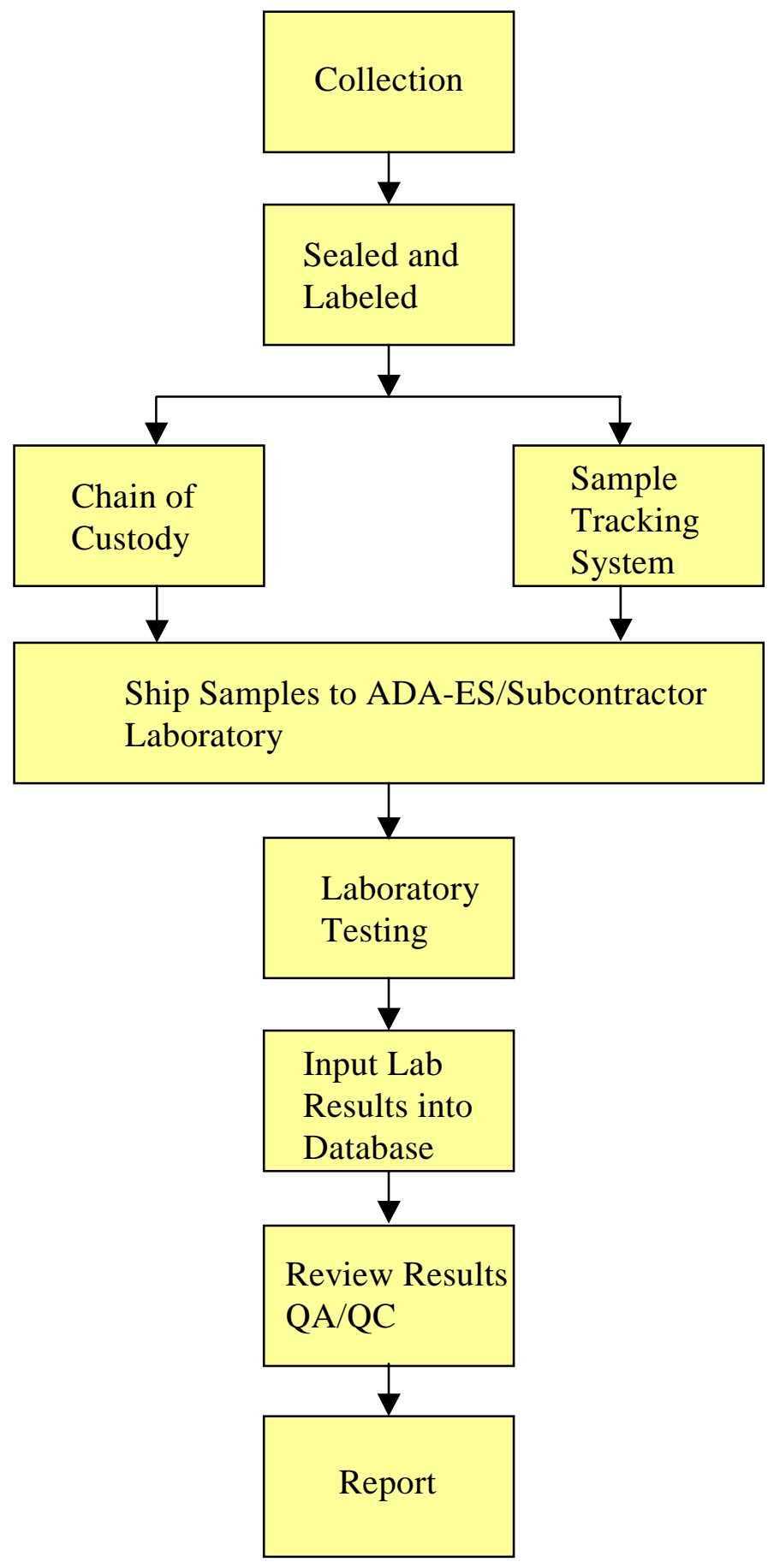




\section{Figure 3. Sample Management Flowchart.}

Once the samples have been collected, they will be delivered to ADA-ES personnel to be sealed and labeled. The samples will be logged into a database and given a sample identification number. Authorized project team members will have access to the database to see which samples have been collected and are available for testing.

Once the samples have been sealed and labeled, ADA-ES personnel will generate a Chain-of Custody (COC) form to be delivered with each shipment of samples. The COC will be used for sample tracking and identification. Although ADA-ES will not enforce the strict COC procedures (e.g., signatures to release sample custody, controlled access), all pertinent information will be recorded.

The samples, along with a COC, will be shipped to the ADA-ES laboratory for storage. Once received, ADA-ES will identify samples for mercury, and other, analyses. Other analyses will include ultimate and proximate analyses for coal, elemental analyses for coal and ash samples (including chlorine and fluorine contents), and size distribution analyses for sorbent samples.

\section{Sample Analysis}

Although previous tests from other programs have shown that the byproducts mixed with activated carbon are highly stable, it is important to continue evaluating these byproducts for each condition using well-established and documented techniques, and new techniques designed to perform even more robust analyses of the byproducts. Additional ash samples will be collected and archived for other tests, including tests requested by EPA, DOE, and independent companies approved by DOE. No samples will be shipped to outside firms without prior approval of Entergy and DOE.

Standard leaching test methods conducted on the fly ash samples will include the Toxicity Characteristic Leaching Procedure (TCLP, SW846-1311) and the synthetic groundwater leaching procedure (SGLP). Solid and liquid samples will be collected and analyzed according to the methods as prescribed in Table 2 . If a chemically treated sorbent is chosen for long-term tests, leaching of the chemical used in the treatment process will be reviewed.

The final series of tests are optional, based on whether a determination is made that additional analyses are needed for purposes of troubleshooting or for gaining additional insight into control options. For example, it may be desirable to determine the size and composition of the ash for certain applications. These analyses will provide information on the impacts of mercury control on ash properties. The properties have a significant impact on the performance of combustion and environmental control systems. 
Table 2. Summary of Byproduct and Waste Characterization Testing

\begin{tabular}{|c|l|l|l|}
\hline Series & Test Purpose & Test Method & Comments \\
\hline 1 & Ash Disposal & TCLP (SW846-1311) & $\begin{array}{l}\text { Measures leachable Hg, As, Ba, Cd, } \\
\text { Cr, Pb, Se, Ag }\end{array}$ \\
\hline 2 & $\begin{array}{l}\text { Environmental } \\
\text { Ltability - }\end{array}$ & EERC SGLP & $\begin{array}{l}\text { Measures leachable Hg at 18 hours, } \\
\text { 2 weeks, and 4 weeks }\end{array}$ \\
\hline 3 & Special Testing & Various & $\begin{array}{l}\text { As needed for troubleshooting or site- } \\
\text { specific information needs }\end{array}$ \\
\hline
\end{tabular}

Once the laboratory testing is complete, results will be logged into the SDMS. Authorized project team members will have access to the database to view the results. A report will be generated summarizing results from the sample analyses.

\section{Flue Gas Samples}

Flue gas measurements will be made at the locations indicated on Figure 1. Flue gas analyses include Ontario Hydros, Method 17, and Method 26a. Hg analyzers and sorbent trap method tests (STM) will also be used at selected locations measuring near-real-time vapor-phase mercury concentrations in the flue gas. 
Table 3. Sampling and Analytical Matrix.

\begin{tabular}{|c|c|c|c|}
\hline Sampling Location & Sample/Type & Sampling Method & Analytical Method \\
\hline \multirow[t]{5}{*}{ ESP Inlet } & Speciated Mercury & Ontario Hydro & $\begin{array}{l}\text { EPA SW } 8467470 \text { cold vapor atomic absorption spectrometry } \\
\text { (CVAAS) }\end{array}$ \\
\hline & $\mathrm{HBr}, \mathrm{HCl}, \mathrm{HF}, \mathrm{BR}_{2}, \mathrm{CL}_{2}$ & M26a & Ion chromatography per the promulgated EPA Method 26a \\
\hline & Particulate Matter & M17 & Gravimetrically \\
\hline & $\mathrm{Hg}$ & STM & EPA Method 1631 \\
\hline & Total/Elemental Mercury & Continuous & AF or AA -Analysis \\
\hline \multirow[t]{5}{*}{ ESP Outlet } & Speciated Mercury & Ontario Hydro & $\begin{array}{l}\text { EPA SW } 8467470 \text { cold vapor atomic absorption spectrometry } \\
\text { (CVAAS) }\end{array}$ \\
\hline & $\mathrm{HBr}, \mathrm{HCl}, \mathrm{HF}, \mathrm{BR}_{2}, \mathrm{CL}_{2}$ & M26a & Ion chromatography per the promulgated EPA Method 26a \\
\hline & Particulate Matter & M17 & Gravimetrically \\
\hline & $\mathrm{Hg}$ & STM & EPA Method 1631 \\
\hline & Total/Elemental Mercury & Continuous & AF or AA-Analysis \\
\hline \multirow[t]{6}{*}{ Coal Fuel to Boiler } & $\mathrm{Hg}$ & Grab Sample & ASTM D6414-99 or 01 \\
\hline & $\mathrm{Cl}$ & Grab Sample & Modified ASTM D5808 (Oxidative Hydrolysis Microcoulometry) \\
\hline & $\mathrm{F}$ & Grab Sample & TBD \\
\hline & Ultimate Analysis & Grab Sample & \\
\hline & Proximate Analysis & Grab Sample & \\
\hline & Trace Metals & Grab Sample & \\
\hline \multirow[t]{5}{*}{ Bottom Ash, Fly Ash } & $\mathrm{Hg}$ & Grab Sample & ASTM D6414-99 or 01 \\
\hline & $\mathrm{Cl}$ & Grab Sample & Modified ASTM D5808 (Oxidative Hydrolysis Microcoulometry) \\
\hline & LOI / Carbon Content & Grab Sample & ASTM C311-04 \\
\hline & Leaching & Grab Sample & TCLP, SW846-1311, SGLP \\
\hline & Trace Metals & Grab Sample & \\
\hline
\end{tabular}

